\title{
COMPATIBILITY OF REFRIGERANTS AND LUBRICANTS WITH MOTOR MATERIALS
}

Effects of Refrigerant-Lubricant Exposures on Motor Materials

Volume III

Robert Doerr and Stephen Kujak

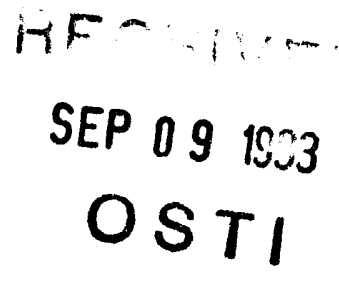

The Trane Company

3600 Pammel Creek Road

La Crosse, Wisconsin 54601-7599

May 1993

Prepared for

The Air-Conditioning and Refrigeration Technology Institute

Under

ARTI MCLR Project Number 650-50400

ate.FCO2-81CE23810: Matorials

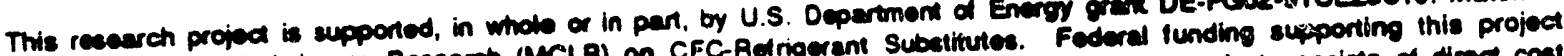

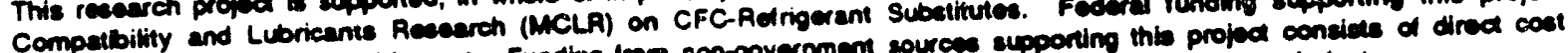

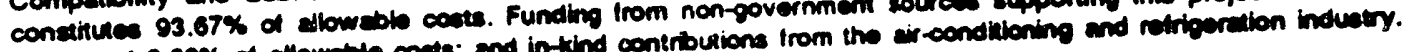




\title{
DISCLAIMER
}

The U.S. Department of Energy's and the air-conditioning industry's support for the Materials Compatibility and Lubricants Research (MCLR) program does not constitute an endorsement by the U.S Department of Energy, nor by the air-conditioning and refrigeration industry, of the views expressed herein.

\section{NOTICE}

This report was prepared as an account of work sponsored by the United States Government. Neither the United States nor the Department of Energy, nor the AirConditioning and Refrigeration Technology Institute, nor any of their employees, nor any of their contractors, subcontractors, or their employees, makes any warranty, expressed or implied, or assumes any legal liability or responsibility for the accuracy, completeness, or usefulness of any information, apparatus, product or process disclosed or represents that its use would not infringe privately-owned rights.

\author{
COPYRIGHT NOTICE \\ (for journal publication submissions)
}

By acceptance of this article, the publisher and/or recipient acknowledges the rights of the U.S. Government and the Air-Conditioning and Refrigeration Technology Institute, Inc. (ARTI) rights to retain a nonexclusive, royalty-free license in and to any copyrights covering this paper. 


\title{
COMPATIBILITY OF REFRIGERANTS AND LUBRICANTS WITH MOTOR MATERIALS
}

\author{
Refrigerant-Lubricant Exposure Data \\ Volume III
}

Because of the large scope of this project and the large amount of data recorded, the final report is divided into four volumes.

Volume III contains all the recorded measurements from the tests on the motor materials after exposure to the 17 refrigerant-lubricant combinations and to nitrogen at the same temperatures. The motor materials are identified by the codes listed on page iv. A letter coding system was used to identify the motor materials in the data tables. Data for each pure refrigerant-lubricant are listed in a separate appendix.

Volume I contains the abstract, scope, discussion of results, charts of motor material compatibility, test procedures, material identifications and 84 pages of data summary tables. This volume provides results of the study and other information of interest to most users of the information.

Volume II contains all the recorded measurements from the tests on the motor materials after exposures to the 11 pure refrigerants and to nitrogen at the same temperatures.

Volume IV contains the photographs of motor materials after exposures to pure refrigerants and to refrigerant-lubricant combinations. 


\section{TABLE OF CONTENTS}

Effects of Refrigerant-Lubricant Exposures on Motor Materials Volume III

CODE CHART FOR MOTOR MATERIAL EXPOSURES

UNITS OF MEASURE

ABBREVIATIONS

APPENDIXES(Experimental data for 500 hour exposures to)*

Appendix $A$

Nitrogen

Appendix $B$

Appendix $C$

HCFC-22/Witco 3GS, ISO-32

HCFC-124/Shrieve Zerol-150, ISO-32

Appendix D

HCFC-1 142b/Shrieve Zerol-150, ISO-32

Appendix $E$ HFC-152a/Shrieve Zerol-150, ISO-32

Appendix $F$

Appendix $G$

Appendix $\mathrm{H}$

Appendix 1

Appendix $J$

Appendix $\mathrm{K}$

Appendix $L$

Appendix $M$

Appendix $\mathrm{N}$

Appendix $O$

Appendix $P$

Appendix $Q$

Appendix $\mathbf{R}$

HFC-134a/ICl Emkarate RL244, ISO-22

HFC-134a/Henkel-Emery 2927, ISO-32

HFC-32/Henkel-Emery 2927, ISO-32

HFC-125/Henkel-Emery 2927, ISO-32

HFC-143a/Henkel-Emery 2927, ISO-32

HFC-134/Henkel-Emery 2927, ISO-32

HFC-245ca/Henkel-Emery 2927, ISO-32

HFC-134a/ICI Emkarox VG32, ISO-32

HFC-32/ICI Emkarox VG32, ISO-32

HFC-125/ICI Emkarox VG32, ISO-32

HFC-134a/Allied Signal BRL-150, ISO-32

HFC-125/Allied Signal BRL-150, ISO-32

HFC-134a/Dow P-425, ISO-32

\begin{tabular}{l} 
Pages \\
\hline iv \\
v \\
vi
\end{tabular}

$A-1$ to $A-18$

$B-1$ to $B-18$

C-1 to $C-18$

D-1 to $D-18$

$\mathrm{E}-1$ to $\mathrm{E}-18$

$\mathrm{F}-1$ to $\mathrm{F}-18$

G-1 to $\mathrm{G}-18$

$\mathrm{H}-1$ to $\mathrm{H}-18$

$1-1$ to $\mid-18$

$\mathrm{J}-1$ to $\mathrm{J}-18$

$\mathrm{K}-1$ to $\mathrm{K}-18$

$L-1$ to $L-18$

$M-1$ to $M-18$

$\mathrm{N}-1$ to $\mathrm{N}-18$

0-1 to 0-18

$P-1$ to $P-18$

$\mathrm{Q}-1$ to $\mathrm{Q}-18$

$R-1$ to $R-18$

${ }^{\star}$ All refrigerant-lubricant exposures and this nitrogen exposure were conducted at $127^{\circ} \mathrm{C}\left(260^{\circ} \mathrm{F}\right)$. 


\section{Code Chart For Motor Material Exposures}

\section{Magnet Wire}

Code

A -Modified polyester overcoated with polyamide imide, as described in Section MW 73 of NEMA Standard MW 1000.

B - Modified polyester overcoated with polyamide imide and epoxy saturated glass as described in Section MW 73 and MW 46 of NEMA Standard MW 1000.

C - Polyester imide over coated with polyamide imide.

\section{Varnishes}

\section{Code}

A $-U-475 E H$ solvent epoxy

B -Y-390PG solvent epoxy-phenolic

C -ER-610 93\% solids epoxy

D - Y- $-833100 \%$ solids VPI epoxy

E -923 solvent epoxy

$F$-Isopoxy 800 water-borne epoxy

\section{Sheet Insulation, Slot Liners and Phase Separators}

\section{Code}

A -Nomex/Mylar/Nomex

B -Dacron/Mylar/Dacron

C - Mylar MO

D -Nomex 410

E -Nomex Mica 418

F - Melinex 228

\section{Spiral Wraceed Sleeving Insulation}

Code
A -Nomex
B - Mylar
C -Nomex/Mylar

\section{Lead Wire Insulation}

Code
A -Dacron/Mylar/Dacron
B - - Cucron/Teflon/Mylar/Dacron

\section{Tapes}

Code

A - Heat Cleaned Glass

B - Heat Shrinkable Braided Polyester

C -Permacel P247 glass/acrylic

\section{Tie Cords}

Code

A-Polyester

\#1-after 500 hour exposure to refrigerant or refrigerant/lubricant. \#2-after 500 hour exposure plus 24 hours at $127^{\circ} \mathrm{C}\left(302^{\circ} \mathrm{F}\right)$. 


\section{Units of Measure}

Magnet Wire

Test Performed

Weight Change

Burnout Strength

Dielectric Strength

Varnish

Test Performed

Weight Change

Bond Strength

Sheet Insulation

Test Performed

Weight Change

Tensile Strength

Elongation

Dielectric Strength

Spiral Wrapped Sleeving

Test Performed

Weight Change

Lead Wire

Test Performed

Weight Change

Dielectric Strength

Tapes and Tie Cords

Test Performed

Weight Change

Break Load Strength

Elongation

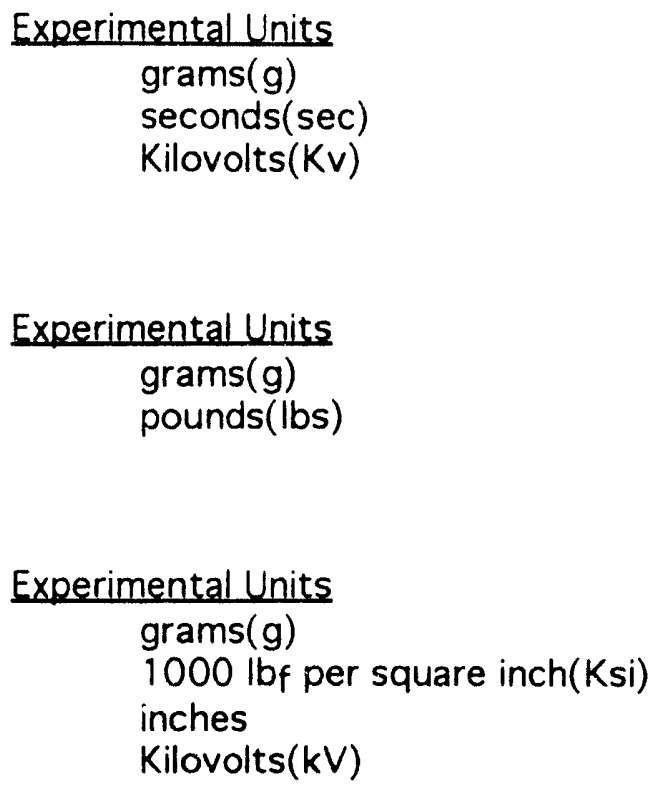

Experimental Units

$\operatorname{grams}(\mathrm{g})$

pounds(lbs)

Experimental Units

$\operatorname{grams}(\mathrm{g})$

$1000 \mathrm{lbf}$ per square inch(Ksi)

inches

Kilovolts(kV)

Experimental Units

$\operatorname{grams}(\mathrm{g})$

Experimental Units

grams $(\mathrm{g})$

Kilovolts(kV)

Experimental Units

$\operatorname{grams}(\mathrm{g})$

pounds(lbs)

inches 


\section{Abbreviations}

$W T=$ weight

EXP $=$ exposed or experimental

BRN OUT=burnout

VIS=visual inspection

DIE=dielectric

VARN=varnish

BND STRENGTH=bond strength

COIL=helical coil

FLEX=flexibility test

$\mathrm{N} / \mathrm{C}=$ no change 


\section{Appendix A}

Experimental Data for Nitrogen Exposure at $126^{\circ} \mathrm{C}\left(260^{\circ} \mathrm{F}\right)$ 


\begin{tabular}{|c|c|c|c|c|c|c|c|c|c|c|}
\hline & 500 HRS IN & Nitrogen & t $260 \mathrm{~F}$ & & & & & & & \\
\hline ID & $W T$ & EXPWT & $\begin{array}{c}\text { WT \% } \\
\text { CHANGE }\end{array}$ & EXP VIS & $\begin{array}{c}\text { BASE BRN } \\
\text { OUT } \\
\text { (AVE) }\end{array}$ & $\begin{array}{c}\text { EXPRRN } \\
\text { OUT }\end{array}$ & $\begin{array}{c}\text { BRN OUT } \\
\% \\
\text { CHANGE }\end{array}$ & $\begin{array}{c}\text { BASE DIE } \\
\text { (AVE) }\end{array}$ & EXP DIE & $\begin{array}{c}\text { DIE \% } \\
\text { CHANGE }\end{array}$ \\
\hline A1 & 21.1038 & 21.1050 & $0.006 \%$ & $\mathrm{~N} / \mathrm{C}$ & 576 & 514 & & 15.80 & 14.59 & \\
\hline & & & & & 576 & 544 & $-5.3 \%$ & 15.80 & 16.21 & $-2.5 \%$ \\
\hline & & & & & 576 & 578 & & 15.80 & lost & \\
\hline B1 & 21.6679 & 21.6712 & $0.015 \%$ & $\mathrm{~N} / \mathrm{C}$ & 736 & 723 & & 11.62 & 11.01 & \\
\hline & & & & & 736 & 688 & $-3.3 \%$ & 11.62 & 10.60 & $-6.9 \%$ \\
\hline & & & & & 736 & 724 & & 11.62 & 10.86 & \\
\hline C1 & 19.0279 & 19.0299 & $0.011 \%$ & $\mathrm{~N} / \mathrm{C}$ & 579 & 612 & & 16.58 & 10.97 & \\
\hline & & & & & 579 & 601 & $4.8 \%$ & 16.58 & 13.70 & $-21.4 \%$ \\
\hline & & & & & 579 & 608 & & 16.58 & 14.44 & \\
\hline & Nitrogen--> & 24 HRS@ & $302 F$ & & & & & & & \\
\hline A2 & 20.4637 & 20.4635 & $-0.001 \%$ & $\mathrm{~N} / \mathrm{C}$ & 576 & 559 & & 15.80 & 13.69 & \\
\hline & & & & & 576 & 554 & $-3.2 \%$ & 15.80 & 15.69 & $-5.2 \%$ \\
\hline & & & & & 576 & 559 & & 15.80 & 15.54 & \\
\hline B2 & 22.2279 & 22.2298 & $0.009 \%$ & $\mathrm{~N} / \mathrm{C}$ & 736 & 722 & & 11.62 & 11.09 & \\
\hline & & & & & 736 & 722 & $-1.9 \%$ & 11.62 & 9.40 & $-8.1 \%$ \\
\hline & & & & & 736 & 722 & & 11.62 & 11.53 & \\
\hline $\mathrm{C} 2$ & 19.2909 & 19.2911 & $0.001 \%$ & $\mathrm{~N} / \mathrm{C}$ & 579 & 608 & & 16.58 & 14.74 & \\
\hline & & & & & 579 & 608 & $3.9 \%$ & 16.58 & 11.58 & $-15.7 \%$ \\
\hline & & & & & 579 & 589 & & 16.58 & 15.63 & \\
\hline
\end{tabular}


500 HOURS IN Nitrogen at $260 \mathrm{~F}$

\begin{tabular}{|c|c|c|c|c|c|c|c|c|c|c|c|}
\hline & VARN & $\begin{array}{l}\text { TWISTEED } \\
\text { PAIR WT }\end{array}$ & $\begin{array}{c}\text { EXPT } \\
\text { PAIR WT }\end{array}$ & $\begin{array}{c}\text { WT \% } \\
\text { CHANGE }\end{array}$ & $\begin{array}{l}\text { EXP } \\
\text { VIS }\end{array}$ & BASE DIE & EXP DIE & $\begin{array}{c}\text { DIE \% } \\
\text { CHANGE }\end{array}$ & $\begin{array}{c}\text { BASE } \\
\text { BUPN } \\
\text { OUT }\end{array}$ & $\begin{array}{c}\text { EXP } \\
\text { BURN } \\
\text { OUT }\end{array}$ & $\begin{array}{c}\text { BRNOUT } \\
\% \\
\text { CHANGE }\end{array}$ \\
\hline \multirow{3}{*}{$\begin{array}{l}P \\
O \\
L\end{array}$} & \multirow{3}{*}{$\begin{array}{l}U-475 \\
A 1\end{array}$} & \multirow[t]{3}{*}{18.3479} & \multirow[t]{3}{*}{18.3542} & \multirow[t]{3}{*}{$0.034 \%$} & $\mathrm{~N} / \mathrm{C}$ & 16.24 & 6.67 & & 430 & 435 & \\
\hline & & & & & & 16.24 & 5.54 & $-53.53 \%$ & 430 & 443 & $3.80 \%$ \\
\hline & & & & & & 16.24 & 10.43 & & 430 & 461 & \\
\hline \multirow{3}{*}{$\begin{array}{l}Y \\
E \\
S\end{array}$} & \multirow{3}{*}{$\begin{array}{l}Y-390 \\
B 1\end{array}$} & \multirow[t]{3}{*}{23.1853} & \multirow[t]{3}{*}{23.1794} & \multirow[t]{3}{*}{$-0.025 \%$} & $\mathrm{~N} / \mathrm{C}$ & 18.77 & 6.06 & & 510 & 700 & \\
\hline & & & & & & 18.77 & 12.30 & $-32.05 \%$ & 510 & 597 & $11.76 \%$ \\
\hline & & & & & & 18.77 & 19.90 & & 510 & 413 & \\
\hline \multirow{4}{*}{$\begin{array}{l}T \\
E \\
R\end{array}$} & ER-610 & \multirow[t]{3}{*}{20.7498} & \multirow[t]{3}{*}{20.7519} & \multirow[t]{3}{*}{$0.010 \%$} & $\mathrm{~N} / \mathrm{C}$ & 15.57 & 13.34 & & 442 & 432 & \\
\hline & \multirow{2}{*}{ C1 } & & & & & 15.57 & 9.30 & $-19.14 \%$ & 442 & 422 & $-4.07 \%$ \\
\hline & & & & & & 15.57 & 15.13 & & 442 & 418 & \\
\hline & \multirow{3}{*}{$\begin{array}{l}Y-833 \\
D 1\end{array}$} & \multirow[t]{3}{*}{18.6978} & \multirow[t]{3}{*}{18.6996} & \multirow[t]{3}{*}{$0.010 \%$} & $\mathrm{~N} / \mathrm{C}$ & 12.04 & 12.38 & & 578 & 554 & \\
\hline \multirow{2}{*}{$\begin{array}{l}P \\
O\end{array}$} & & & & & & 12.04 & 11.67 & $1.00 \%$ & 578 & 557 & $-4.50 \%$ \\
\hline & & & & & & 12.04 & 12.43 & & 578 & 545 & \\
\hline \multirow{3}{*}{$\begin{array}{l}L \\
Y \\
A\end{array}$} & \multirow{3}{*}{$\begin{array}{l}923 \\
E 1\end{array}$} & \multirow[t]{3}{*}{21.4609} & \multirow[t]{3}{*}{21.4605} & $-0.002 \%$ & $\mathrm{~N} / \mathrm{C}$ & 16.76 & 12.16 & & 606 & 549 & \\
\hline & & & & & & 16.76 & 5.43 & $-43.56 \%$ & 606 & 548 & $.7 .59 \%$ \\
\hline & & & & & & 16.76 & 10.79 & & 606 & 583 & \\
\hline$M$ & 150.800 & 18.7122 & 18.7124 & $0.001 \%$ & $\mathrm{~N} / \mathrm{C}$ & 19.08 & 13.45 & & 580 & 460 & \\
\hline 1 & F1 & & & & & 19.08 & 9.55 & $.34 .07 \%$ & 580 & 581 & $.1: .72 \%$ \\
\hline$D$ & & & & & & 19.08 & 14.74 & & 580 & 495 & \\
\hline$E$ & & Nitrogen-.. & $->24$ hour & $s$ at 302 & $\mathrm{~F}$ & & & & & & \\
\hline & $U-475$ & 18.4726 & 18.4748 & $0.012 \%$ & $\mathrm{~N} / \mathrm{C}$ & 16.24 & 3.85 & & 430 & 456 & \\
\hline 1 & A 2 & & & & & 16.24 & 12.43 & $-37.85 \%$ & 430 & 562 & $15.43 \%$ \\
\hline$M$ & & & & & & 16.24 & 14.00 & & 430 & 471 & \\
\hline 1 & $Y-390$ & 21.2974 & 21.2950 & $-0.011 \%$ & $\mathrm{~N} / \mathrm{C}$ & 18.77 & 11.97 & & 510 & 653 & \\
\hline$D$ & $\mathrm{~B} 2$ & & & & & 18.77 & 16.21 & $-22.61 \%$ & 510 & 624 & $17.84 \%$ \\
\hline$E$ & & & & & & 18.77 & 15.40 & & 510 & 526 & \\
\hline & ER-610 & 20.4942 & 20.4948 & $0.003 \%$ & $\mathrm{~N} / \mathrm{C}$ & 15.57 & 14.32 & & 442 & 416 & \\
\hline & $\mathrm{C} 2$ & & & & & 15.57 & 15.19 & $-9.89 \%$ & 442 & 434 & $-5.51 \%$ \\
\hline & & & & & & 15.57 & 12.58 & & 442 & 403 & \\
\hline & $Y-833$ & 19.6798 & 19.6809 & $0.006 \%$ & $\mathrm{~N} / \mathrm{C}$ & 12.04 & 12.23 & & 578 & 578 & \\
\hline & D2 & & & & & 12.04 & 13.47 & $1.91 \%$ & 578 & 561 & $.1 .33 \%$ \\
\hline & & & & & & 12.04 & 11.11 & & 578 & 572 & \\
\hline & 923 & 21.6455 & 21.6440 & $-0.007 \%$ & $\mathrm{~N} / \mathrm{C}$ & 16.76 & 12.17 & & 606 & 608 & \\
\hline & E2 & & & & & 16.76 & 15.71 & $.20 .54 \%$ & 606 & 577 & $-2.48 \%$ \\
\hline & & & & & & 16.76 & 12.07 & & 606 & 588 & \\
\hline & $1 \mathrm{SO}-800$ & 18.3483 & 18.3439 & $-0.024 \%$ & $\mathrm{~N} / \mathrm{C}$ & 19.08 & 14.53 & & 580 & 572 & \\
\hline & $\mathrm{F} 2$ & & & & & 19.08 & 7.69 & $-48.43 \%$ & 580 & 543 & $-3.68 \%$ \\
\hline & & & & & & 19.08 & 7.30 & & 580 & 561 & \\
\hline
\end{tabular}




\begin{tabular}{|c|c|c|c|c|c|c|c|c|c|c|c|}
\hline & $\begin{array}{l}\text { OO HOUR } \\
\text { VARN }\end{array}$ & $\begin{array}{l}\text { S IN Nitrog } \\
\text { TWISTED } \\
\text { PAIR WT }\end{array}$ & $\begin{array}{c}\text { en at } 260 \\
\text { EXP T } \\
\text { PAIR WT }\end{array}$ & $\begin{array}{c}\text { WT \% } \\
\text { CHANGE }\end{array}$ & $\begin{array}{l}\text { EXP } \\
\text { VIS }\end{array}$ & $\begin{array}{c}\text { BASE } \\
\text { DIE }\end{array}$ & $\begin{array}{l}\text { EXP } \\
\text { DIE }\end{array}$ & $\begin{array}{c}\text { DIE \% } \\
\text { CHANGE }\end{array}$ & $\begin{array}{l}\text { BASE } \\
\text { BURN } \\
\text { OUT }\end{array}$ & $\begin{array}{l}\text { EXP } \\
\text { BUPN } \\
\text { OUT }\end{array}$ & $\begin{array}{c}\text { BRNOUT } \\
\% \\
\text { CHANGE }\end{array}$ \\
\hline \multirow{3}{*}{$\begin{array}{l}P \\
O \\
L\end{array}$} & \multirow{3}{*}{$\begin{array}{l}\text { U. } 475 \\
\text { A } 1 \\
\end{array}$} & \multirow[t]{3}{*}{22.8475} & \multirow[t]{3}{*}{22.8512} & \multirow[t]{3}{*}{$0.02 \%$} & $N / C$ & 13.32 & 12.23 & & 746 & 737 & \\
\hline & & & & & & 13.32 & 12.03 & $.8 .93 \%$ & 746 & 728 & $-1.79 \%$ \\
\hline & & & & & & 13.32 & 12.13 & & 746 & 733 & \\
\hline \multirow{3}{*}{$\begin{array}{l}Y \\
E \\
S\end{array}$} & \multirow{3}{*}{$\begin{array}{l}Y-390 \\
B 1\end{array}$} & \multirow[t]{3}{*}{21.4228} & \multirow[t]{3}{*}{21.4243} & \multirow[t]{3}{*}{$0.01 \%$} & $\mathrm{~N} / \mathrm{C}$ & 12.28 & 11.89 & & 755 & 733 & \\
\hline & & & & & & 12.28 & 12.83 & $-2.85 \%$ & 755 & 727 & $-3.09 \%$ \\
\hline & & & & & & 12.28 & 11.07 & & 755 & 735 & \\
\hline \multirow{4}{*}{$\begin{array}{l}T \\
E \\
R\end{array}$} & ER-610 & \multirow[t]{3}{*}{21.8850} & \multirow[t]{3}{*}{21.8880} & \multirow[t]{3}{*}{$0.01 \%$} & $\mathrm{~N} / \mathrm{C}$ & 12.73 & 12.40 & & 734 & 723 & \\
\hline & \multirow{2}{*}{ C1 } & & & & & 12.73 & 6.83 & $-4.87 \%$ & 734 & 728 & $-1.04 \%$ \\
\hline & & & & & & 12.73 & 11.82 & & 734 & 728 & \\
\hline & \multirow{3}{*}{$\begin{array}{l}Y-833 \\
D 1\end{array}$} & \multirow[t]{3}{*}{20.0934} & \multirow[t]{3}{*}{20.0956} & \multirow[t]{3}{*}{$0.01 \%$} & $\mathrm{~N} / \mathrm{C}$ & 12.49 & 11.87 & & 734 & 732 & \\
\hline \multirow{2}{*}{$\begin{array}{l}P \\
O\end{array}$} & & & & & & 12.49 & 11.97 & $.6 .00 \%$ & 734 & 735 & $-0.36 \%$ \\
\hline & & & & & & 12.49 & 11.38 & & 734 & 727 & \\
\hline \multirow{3}{*}{$\begin{array}{l}L \\
Y \\
A\end{array}$} & \multirow{3}{*}{$\begin{array}{l}923 \\
E 1\end{array}$} & \multirow[t]{3}{*}{23.6462} & 23.6453 & $0.00 \%$ & $\mathrm{~N} / \mathrm{C}$ & 14.38 & 12.66 & & 742 & 728 & \\
\hline & & & & & & 14.38 & 12.67 & $-14.74 \%$ & 742 & 734 & $-1.44 \%$ \\
\hline & & & & & & 14.38 & 11.45 & & 742 & 732 & \\
\hline$M$ & $150-800$ & 20.1280 & 20.1272 & $0.00 \%$ & $\mathrm{~N} / \mathrm{C}$ & 12.29 & 11.94 & & 747 & 734 & \\
\hline 1 & $F 1$ & & & & & 12.29 & 12.37 & $-2.01 \%$ & 747 & 729 & $-2.23 \%$ \\
\hline D & & & & $\cdot$ & & 12.29 & 11.82 & & 747 & 728 & \\
\hline$E$ & & Nitrogen & $>24 \mathrm{hol}$ & s@302 & $F$ & & & & & & \\
\hline & $U-475$ & 23.0498 & 23.0463 & $-0.02 \%$ & $\mathrm{~N} / \mathrm{C}$ & 13.32 & 11.61 & & 746 & 723 & \\
\hline 1 & $A 2$ & & & & & 13.32 & 12.40 & $-10.89 \%$ & 746 & 723 & $-3.08 \%$ \\
\hline M & & & & & & 13.32 & 11.60 & & 746 & 723 & \\
\hline 1 & $Y-390$ & 21.1739 & 21.1771 & $0.02 \%$ & $\mathrm{~N} / \mathrm{C}$ & 12.28 & 9.60 & & 755 & 723 & \\
\hline$D$ & B2 & & & & & 12.28 & 10.78 & $-15.36 \%$ & 755 & 722 & $-4.30 \%$ \\
\hline$E$ & & & & & & 12.28 & 10.80 & & 755 & 723 & \\
\hline & ER-610 & 22.1851 & 22.1829 & $-0.01 \%$ & $\mathrm{~N} / \mathrm{C}$ & 12.73 & 10.49 & & 734 & 727 & \\
\hline$E$ & $\mathrm{C}_{2}$ & & & & & 12.73 & 12.10 & $-9.87 \%$ & 734 & 723 & $-1.14 \%$ \\
\hline$P$ & & & & & & 12.73 & 11.83 & & 734 & 727 & \\
\hline 0 & $Y-833$ & 20.1326 & 20.1315 & $-0.01 \%$ & $\mathrm{~N} / \mathrm{C}$ & 12.49 & 10.15 & & 734 & 723 & \\
\hline$x$ & $\mathrm{D} 2$ & & & & & 12.49 & 9.89 & $-13.18 \%$ & 734 & 723 & $-1.50 \%$ \\
\hline Y & & & & & & 12.49 & 12.49 & & 734 & 723 & \\
\hline & 923 & 23.3100 & 23.3045 & $-0.02 \%$ & $\mathrm{~N} / \mathrm{C}$ & 14.38 & 12.10 & & 742 & 723 & \\
\hline G & E2 & & & & & 14.38 & 11.52 & $-18.24 \%$ & 742 & 723 & $-2.56 \%$ \\
\hline$L$ & & & & & & 14.38 & 11.65 & & 742 & 723 & \\
\hline A & ISO-800 & 22.6942 & 22.6919 & $.0 .01 \%$ & & 12.29 & 9.60 & & 747 & 723 & \\
\hline$S$ & $\mathrm{~F} 2$ & & & & $\mathrm{~N} / \mathrm{C}$ & 12.29 & 10.49 & $-17.93 \%$ & 747 & 723 & $-3.21 \%$ \\
\hline$S$ & & & & & & 12.29 & 10.17 & & 747 & 723 & \\
\hline
\end{tabular}


500 HOURS IN Nitrogen at $260 \mathrm{~F}$

\begin{tabular}{|c|c|c|c|c|c|c|c|c|c|c|c|}
\hline & VARN & $\begin{array}{l}\text { TWISTED } \\
\text { PAIR WT }\end{array}$ & $\begin{array}{c}\text { EXPT } \\
\text { PAIR WT }\end{array}$ & $\begin{array}{c}\text { WT \% } \\
\text { CHANGE }\end{array}$ & $\begin{array}{l}\text { EXP } \\
\text { VIS }\end{array}$ & $\begin{array}{l}\text { BASE } \\
\text { DIE }\end{array}$ & $\begin{array}{l}\text { EXP } \\
\text { DIE }\end{array}$ & $\begin{array}{c}\text { DIE \% } \\
\text { CHANGE }\end{array}$ & \begin{tabular}{|l|} 
BASE \\
BUPN \\
OUT
\end{tabular} & $\begin{array}{c}\text { EXP } \\
\text { BURN } \\
\text { OUT }\end{array}$ & $\begin{array}{c}\text { BRNOUT } \\
\% \\
\text { CHANGE }\end{array}$ \\
\hline \multirow{3}{*}{$\begin{array}{l}P \\
O \\
L\end{array}$} & \multirow{3}{*}{$\begin{array}{l}J-475 \\
\text { A } 1\end{array}$} & \multirow[t]{3}{*}{21.5063} & \multirow[t]{3}{*}{21.5128} & \multirow[t]{3}{*}{$0.030 \%$} & $\mathrm{~N} / \mathrm{C}$ & 15.10 & 13.17 & & 469 & 538 & \\
\hline & & & & & & 15.10 & 13.64 & $-16.40 \%$ & 469 & 505 & $6.11 \%$ \\
\hline & & & & & & 15.10 & 11.06 & & 469 & 450 & \\
\hline \multirow{3}{*}{$\begin{array}{l}E \\
S\end{array}$} & \multirow{3}{*}{$\begin{array}{l}-390 \\
B 1\end{array}$} & \multirow[t]{3}{*}{21.6169} & \multirow[t]{3}{*}{21.6199} & \multirow[t]{3}{*}{$0.014 \%$} & $\mathrm{~N} / \mathrm{C}$ & 18.24 & 12.18 & & 473 & 613 & \\
\hline & & & & & & 18.24 & 4.87 & $-35.00 \%$ & 473 & 480 & $14.45 \%$ \\
\hline & & & & & & 18.24 & 18.52 & & 473 & 531 & \\
\hline \multirow{4}{*}{$\begin{array}{l}T \\
E \\
R\end{array}$} & ER-610 & \multirow[t]{3}{*}{20.4960} & \multirow[t]{3}{*}{20.4982} & \multirow[t]{3}{*}{$0.011 \%$} & $\mathrm{~N} / \mathrm{C}$ & 14.53 & 13.60 & & 494 & 419 & \\
\hline & \multirow{2}{*}{ C1 } & & & & & 14.53 & 12.96 & $-16.27 \%$ & 494 & 419 & $-11.94 \%$ \\
\hline & & & & & & \begin{tabular}{|l|}
14.53 \\
\end{tabular} & 9.94 & & 494 & 467 & \\
\hline & \multirow{3}{*}{$\begin{array}{l}\mathrm{Y}-833 \\
\mathrm{D} 1\end{array}$} & \multirow[t]{3}{*}{19.7961} & \multirow[t]{3}{*}{19.7990} & \multirow[t]{3}{*}{$0.015 \%$} & $\mathrm{~N} / \mathrm{C}$ & 11.38 & 15.66 & & 557 & 532 & \\
\hline & & & & & & 11.38 & 12.92 & $16.87 \%$ & 557 & 485 & $-7.06 \%$ \\
\hline M & & & & & & 11.38 & 11.32 & & 557 & 536 & \\
\hline \multirow{4}{*}{$\begin{array}{l}D \\
D\end{array}$} & \multirow{3}{*}{$\begin{array}{l}923 \\
\text { E1 }\end{array}$} & \multirow[t]{3}{*}{20.6379} & \multirow[t]{3}{*}{20.6362} & \multirow[t]{3}{*}{$-0.008 \%$} & $\mathrm{~N} / \mathrm{C}$ & 15.85 & 6.57 & & 503 & 475 & \\
\hline & & & & & & 15.85 & 3.50 & $.42 .78 \%$ & 503 & 551 & $7.82 \%$ \\
\hline & & & & & & 15.85 & 17.14 & & 503 & 601 & \\
\hline & ISO-800 & 20.1979 & 20.1963 & $-0.008 \%$ & $\mathrm{~N} / \mathrm{C}$ & 14.75 & 16.27 & & 632 & 580 & \\
\hline$P$ & F1 & & & & & 14.75 & 14.46 & $4.09 \%$ & 632 & 596 & $-7.01 \%$ \\
\hline כ & & & & & & 14.75 & 15.33 & & 632 & 587 & \\
\hline & & Nitrogen - & $\rightarrow 24$ houl & s@302 & $F$ & & & & & & \\
\hline Y & $U-475$ & 20.1467 & 20.1455 & $-0.006 \%$ & $N / C$ & 15.10 & 12.33 & & 469 & 436 & \\
\hline$A$ & $A 2$ & & & & & 15.10 & 15.90 & $.5 .47 \%$ & 469 & 366 & $-19.33 \%$ \\
\hline$M$ & & & & & & 15.10 & 14.59 & & 469 & 333 & \\
\hline & $Y-390$ & 20.9674 & 20.9627 & $-0.022 \%$ & $\mathrm{~N} / \mathrm{C}$ & 18.24 & 18.95 & & 473 & 445 & \\
\hline D & $B 2$ & & & & & 18.24 & 20.00 & $2.11 \%$ & 473 & 469 & $-3.31 \%$ \\
\hline 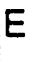 & & & & & & 18.24 & 17.25 & & 473 & 458 & \\
\hline & ER-610 & 19.2992 & 19.2960 & $-0.017 \%$ & $\mathrm{~N} / \mathrm{C}$ & 14.53 & 7.29 & & 494 & 425 & \\
\hline & $\mathrm{C} 2$ & & & & & 14.53 & 9.89 & $-45.61 \%$ & 494 & 473 & $-10.53 \%$ \\
\hline M & & & & & & 14.53 & 6.53 & & 494 & 428 & \\
\hline 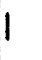 & $Y-833$ & 20.7858 & 20.7872 & $0.007 \%$ & $\mathrm{~N} / \mathrm{C}$ & 11.38 & 12.31 & & 557 & 573 & \\
\hline$D$ & $D 2$ & & & & & 11.38 & 10.96 & $9.67 \%$ & 557 & 474 & $-3.65 \%$ \\
\hline$E$ & & & & & & 11.38 & 14.17 & & 557 & 563 & \\
\hline & 923 & 17.3115 & 17.3100 & $-0.009 \%$ & $\mathrm{~N} / \mathrm{C}$ & 15.85 & 10.63 & & 503 & 487 & \\
\hline & E2 & & & & & 15.85 & 11.14 & $-32.03 \%$ & 503 & 473 & $-3.51 \%$ \\
\hline & & & & & & 15.85 & 10.55 & & 503 & 496 & \\
\hline & ISO-800 & 20.5725 & 20.5725 & $0.000 \%$ & $\mathrm{~N} / \mathrm{C}$ & 14.75 & 14.57 & & 632 & 585 & \\
\hline & $\mathrm{F} 2$ & & & & & 14.75 & 15.94 & $-1.31 \%$ & 632 & 568 & $-9.49 \%$ \\
\hline & & & & & & 14.75 & 13.16 & & 632 & 563 & \\
\hline
\end{tabular}




\begin{tabular}{|c|c|c|c|c|c|c|c|c|}
\hline & & 500 HRS & IN Nitroger & @ 260 F & & & & \\
\hline & VARN & $\begin{array}{l}\text { COIL } \\
W T\end{array}$ & $\begin{array}{c}\text { EXP COIL } \\
W T\end{array}$ & $\begin{array}{c}\text { WT \% } \\
\text { CHANGE }\end{array}$ & EXP VIS & $\begin{array}{c}\text { BASE } \\
\text { BND STR } \\
\text { (AVE) }\end{array}$ & $\begin{array}{c}\text { EXPBND } \\
\text { STR }\end{array}$ & $\begin{array}{c}\text { BND STR } \\
\% \\
\text { CHANGE }\end{array}$ \\
\hline$P$ & $U-475$ & 39.0844 & 39.0930 & $0.022^{\circ}$ & $\mathrm{N} / \mathrm{C}$ & 73.73 & 42.77 & \\
\hline 0 & A 1 & & & & & 73.73 & 53.55 & $-29.92 \%$ \\
\hline$L$ & & & & & & 73.73 & 58.70 & \\
\hline$Y$ & $Y-390$ & 37.2579 & 37.2601 & $0.006 \%$ & $\mathrm{~N} / \mathrm{C}$ & 43.78 & 22.62 & \\
\hline$E$ & B1 & & & & & 43.78 & 25.00 & $-42.83 \%$ \\
\hline$S$ & & & & & & 43.78 & 27.47 & \\
\hline$T$ & ER-610 & 38.6224 & 38.6296 & $0.019 \%$ & $\mathrm{~N} / \mathrm{C}$ & 51.81 & 20.50 & \\
\hline$E$ & C1 & & & & & 51.81 & 42.27 & $.36 .61 \%$ \\
\hline R & & & & & & 51.81 & 35.75 & \\
\hline & $Y-833$ & 37.4889 & 37.4958 & $0.018 \%$ & $N / C$ & 9.85 & 13.42 & \\
\hline$P$ & D1 & & & & & 9.85 & 11.42 & $-5.79 \%$ \\
\hline 0 & & & & & & 9.85 & 3.00 & \\
\hline L & 923 & $38.6 \div 94$ & 38.6209 & $0.004 \%$ & $\mathrm{~N} / \mathrm{C}$ & 41.28 & 25.55 & \\
\hline$Y$ & E1 & & & & & 41.28 & 26.67 & $-40.94 \%$ \\
\hline A & & & & & & 41.28 & 20.92 & \\
\hline M & $150-800$ & 36.8827 & 36.8844 & $0.005 \%$ & $N / C$ & 45.01 & 19.37 & \\
\hline & F1 & & & & & 45.01 & 16.90 & $.59 .71 \%$ \\
\hline D & & & & & & 45.01 & 21.95 & \\
\hline$E$ & & Nitrogen & $60>24$ & IRS $302 \mathrm{~F}$ & & & & \\
\hline & $U-475$ & 39.5820 & 39.5825 & $0.001 \%$ & $N / C$ & 73.73 & 83.40 & \\
\hline | & A2 & & & & & 73.73 & 61.60 & $-8.28 \%$ \\
\hline$M$ & & & & & & 73.73 & 73.65 & \\
\hline I & $Y-390$ & 38.2884 & 38.2846 & $-0.010 \%$ & N/C & 43.78 & 32.57 & \\
\hline D & $B 2$ & & & & & 43.78 & 28.72 & $-29.96 \%$ \\
\hline E & & & & & & 43.78 & 30.70 & \\
\hline & ER-610 & $38.33: 7$ & 38.3419 & $0.006 \%$ & $N / C$ & 51.81 & 49.17 & \\
\hline & C2 & & & & & 51.81 & 38.62 & $.11 .12 \%$ \\
\hline & & & & & & 51.81 & 50.35 & \\
\hline & $Y-833$ & 36.9484 & 36.9531 & $0.013 \%$ & $N / C$ & 9.85 & 13.30 & \\
\hline & D2 & & & & & 9.85 & 14.12 & $31.03 \%$ \\
\hline & & & & & & 9.85 & 11.30 & \\
\hline & 923 & 36.5332 & 36.5323 & $.0 .002 \%$ & N/C & 41.28 & 25.50 & \\
\hline & E2 & & & & & 41.28 & 26.30 & $-35.04 \%$ \\
\hline & & & & & & 41.28 & 28.65 & \\
\hline & ISO-800 & 37.6092 & 37.6105 & $0.003 \%$ & $\mathrm{~N} / \mathrm{C}$ & 45.01 & 31.17 & \\
\hline & F2 & & & & & 45.01 & 31.35 & $-28.37 \%$ \\
\hline & & & & & & 45.01 & 34.20 & \\
\hline
\end{tabular}




\begin{tabular}{|c|c|c|c|c|c|c|c|c|}
\hline & & $500 \mathrm{HRS}$ & IN Nitro & $n$ at $260 \mathrm{~F}$ & & & & \\
\hline & VARN & $\begin{array}{l}\text { COIL } \\
W T\end{array}$ & $\begin{array}{c}\text { Exp Coil } \\
w t\end{array}$ & $\begin{array}{c}\text { WT \% } \\
\text { CHANGE }\end{array}$ & EXP VIS & $\begin{array}{c}\text { BASE } \\
\text { BND STR } \\
\text { (AVE) }\end{array}$ & $\begin{array}{c}\text { EXP BND } \\
\text { STR }\end{array}$ & $\begin{array}{c}\text { BND STR } \\
\% \\
\text { CHANGE }\end{array}$ \\
\hline $\mathrm{P}$ & U-475 & 36.8961 & 36.9066 & $0.028 \%$ & $\mathrm{~N} / \mathrm{C}$ & 40.14 & 30.17 & \\
\hline 0 & A 1 & & & & & 40.14 & 25.15 & $-30.31 \%$ \\
\hline$L$ & & & & & & 40.14 & 28.60 & \\
\hline Y & $Y-390$ & 36.2001 & 36.2051 & $0.014 \%$ & $\mathrm{~N} / \mathrm{C}$ & 36.12 & 25.92 & \\
\hline $\mathbf{E}$ & B1 & & & & & 36.12 & 32.60 & $-11.11 \%$ \\
\hline $\mathrm{S}$ & & & & & & 36.12 & 37.80 & \\
\hline$T$ & ER-610 & 35.5126 & 35.5150 & $0.007 \%$ & $\mathrm{~N} / \mathrm{C}$ & 35.96 & 23.00 & \\
\hline$E$ & $C_{1}$ & & & & & 35.96 & 23.90 & $-36.76 \%$ \\
\hline $\mathbf{R}$ & & & & & & 35.96 & 21.32 & \\
\hline & $Y-833$ & 36.9060 & 36.9192 & $0.036 \%$ & $\mathrm{~N} / \mathrm{C}$ & 33.14 & 18.40 & \\
\hline$P$ & D1 & & & & & 33.14 & 20.37 & $-43.45 \%$ \\
\hline 0 & & & & & & 33.14 & 17.45 & \\
\hline$L$ & 923 & 36.2460 & 36.2468 & $0.002 \% \|$ & $\mathrm{N} / \mathrm{C}$ & 40.52 & 33.52 & \\
\hline Y & E1 & & & & & 40.52 & 28.72 & $-26.57 \%$ \\
\hline A & & & & & & 40.52 & 27.02 & \\
\hline$M$ & ISO-800 & 35.4661 & 35.4717 & $0.016 \%$ & $\mathrm{~N} / \mathrm{C}$ & 20.20 & 17.55 & \\
\hline 1 & $F 1$ & & & & & 20.20 & 15.65 & $-12.51 \%$ \\
\hline D & & & & & & 20.20 & 19.82 & \\
\hline $\mathbf{E}$ & & Nitrogen & $>24 \mathrm{HRS}$ & $302 \mathrm{~F}$ & & & & \\
\hline & $U .475$ & 37.7676 & 37.7712 & $0.010 \%$ & $N / C$ & 40.14 & 25.05 & \\
\hline 1 & A2 & & & & & 40.14 & 28.37 & $-35.54 \%$ \\
\hline$M$ & & & & & & 40.14 & 24.20 & \\
\hline I & $Y-390$ & 36.1450 & 36.1451 & $0.000 \%$ & $N, C$ & 36.12 & 31.62 & \\
\hline D & B2 & & & & & 36.12 & 30.20 & $-10.95 \%$ \\
\hline E & & & & & & 36.12 & 34.67 & \\
\hline & ER-610 & 36.9622 & 36.9600 & $-0.006 \%$ & $\mathrm{~N} / \mathrm{C}$ & 35.96 & 23.77 & \\
\hline $\mathbf{E}$ & C2 & & & & & 35.96 & 32.70 & $-24.00 \%$ \\
\hline$P$ & & & & & & 35.96 & 25.52 & \\
\hline 0 & $Y-833$ & 36.5969 & 36.6068 & $0.027 \%$ & $\mathrm{~N} / \mathrm{C}$ & 33.14 & 19.52 & \\
\hline$x$ & D2 & & & & & 33.14 & 18.15 & $-44.54 \%$ \\
\hline Y & & & & & & 33.14 & 17.47 & \\
\hline & 923 & 36.3908 & 36.3862 & $-0.013 \%$ & $\mathrm{~N} / \mathrm{C}$ & 40.52 & 36.60 & \\
\hline G & E2 & & & & & 40.52 & 36.12 & $-16.61 \%$ \\
\hline$L$ & & & & & & 40.52 & 28.65 & \\
\hline A & $150-800$ & 36.3733 & 36.3742 & $0.002 \%$ & $\mathrm{~N} / \mathrm{C}$ & 20.20 & 9.15 & \\
\hline S & F2 & & & & & 20.20 & 18.85 & $-25.74 \%$ \\
\hline S & & & & & & 20.20 & 17.00 & \\
\hline
\end{tabular}




\begin{tabular}{|c|c|c|c|c|c|c|c|c|}
\hline & & 500 HRS & IN Nitros & $260 \mathrm{~F}$ & & & & \\
\hline & VARN & $\begin{array}{l}\text { COIL } \\
W T\end{array}$ & $\begin{array}{c}\text { EXP COIL } \\
W T\end{array}$ & $\begin{array}{l}\text { WT \% } \\
\text { CHANGE }\end{array}$ & EXP VIS & $\begin{array}{c}\text { BASE } \\
\text { BND STR } \\
(\text { AVE })\end{array}$ & $\begin{array}{c}\text { EXPBND } \\
\text { STR }\end{array}$ & $\begin{array}{c}\text { BND STR } \\
\% \\
\text { CHANGE }\end{array}$ \\
\hline$P$ & $U-475$ & 39.1167 & 39.1219 & $0.013 \%$ & $\mathrm{~N} / \mathrm{C}$ & 51.21 & 21.37 & \\
\hline 0 & A1 & & & & & 51.21 & 24.40 & $-56.60 \%$ \\
\hline$L$ & & & & & & 51.21 & 20.90 & \\
\hline$Y$ & $Y-390$ & 38.7438 & 38.7434 & $-0.001 \%$ & $\mathrm{~N} / \mathrm{C}$ & 50.72 & 26.17 & \\
\hline$E$ & B1 & & & & & 50.72 & 27.02 & $-50.97 \%$ \\
\hline $\mathrm{S}$ & & & & & & 50.72 & 21.42 & \\
\hline$T$ & ER-610 & 37.3327 & 37.3372 & $0.012 \%$ & $\mathrm{~N} / \mathrm{C}$ & 58.33 & 52.97 & \\
\hline$E$ & $C_{1}$ & & & & & 58.33 & 44.42 & $-24.26 \%$ \\
\hline$R$ & & & & & & 58.33 & 35.15 & \\
\hline & $Y-833$ & 37.1885 & 37.1953 & $0.018 \%$ & $\mathrm{~N} / \mathrm{C}$ & 5.84 & 12.50 & \\
\hline 1 & D1 & & & & & 5.84 & 13.05 & $109.76 \%$ \\
\hline$M$ & & & & & & 5.84 & 11.20 & \\
\hline | & 923 & 38.3342 & 38.3357 & $0.004 \%$ & $\mathrm{~N} / \mathrm{C}$ & 49.26 & 27.32 & \\
\hline D & E1 & & & & & 49.26 & 26.80 & $-49.90 \%$ \\
\hline$E$ & & & & & & 49.26 & 19.92 & \\
\hline & ISO-800 & 38.1312 & 38.1365 & $0.014 \%$ & $\mathrm{~N} / \mathrm{C}$ & 36.08 & 23.52 & \\
\hline$P$ & $F 1$ & & & & & 36.08 & 22.70 & $-37.80 \%$ \\
\hline 0 & & & & & & 36.08 & 21.10 & \\
\hline L & & Nitrogen & $>24 \mathrm{HRS}$ & $302 \mathrm{~F}$ & & & & \\
\hline$Y$ & $U-475$ & 37.6244 & 37.6220 & $-0.006 \%$ & $\mathrm{~N} / \mathrm{C}$ & 51.21 & 51.72 & \\
\hline A & $A 2$ & & & & & 51.21 & 67.82 & $15.66 \%$ \\
\hline$M$ & & & & & & 51.21 & 58.15 & \\
\hline | & $Y-390$ & 37.7500 & 37.7460 & $-0.011 \%$ & $\mathrm{~N} / \mathrm{C}$ & 50.72 & 25.12 & \\
\hline$D$ & B2 & & & & & 50.72 & 29.32 & $-45.64 \%$ \\
\hline 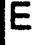 & & & & & & 50.72 & 28.27 & \\
\hline & ER-610 & 37.1831 & 37.1786 & $-0.012 \%$ & $\mathrm{~N} / \mathrm{C}$ & 58.33 & 69.85 & \\
\hline I & $\mathrm{C} 2$ & & & & & 58.33 & 51.25 & $3.70 \%$ \\
\hline$M$ & & & & & & 58.33 & 60.37 & \\
\hline 1 & $Y-833$ & 37.7129 & 37.7155 & $0.007 \%$ & $N / C$ & 5.84 & 12.37 & \\
\hline$D$ & D2 & & & & & 5.84 & 12.00 & $125.57 \%$ \\
\hline$E$ & & & & & & 5.84 & 15.15 & \\
\hline & 923 & 38.7550 & 38.7490 & $-0.015 \%$ & $\mathrm{~N} / \mathrm{C}$ & 49.26 & 29.25 & \\
\hline & E2 & & & & & 49.26 & 33.62 & $-37.66 \%$ \\
\hline & & & & & & 49.26 & 29.25 & \\
\hline & ISO-800 & 37.7538 & 37.7515 & $-0.006 \%$ & $N / C$ & 36.08 & 47.67 & \\
\hline & F2 & & & & & 36.08 & 24.50 & $11.87 \%$ \\
\hline & & & & & & 36.08 & 48.92 & \\
\hline
\end{tabular}




\begin{tabular}{|c|c|c|c|c|c|}
\hline & 500 HRS IN & Vitrogen @ & $260 \mathrm{~F}$ & & \\
\hline ID & WT & EXPWT & $\begin{array}{l}\text { WT\% } \\
\text { CHANGE }\end{array}$ & EXPVS & EXPFLEX \\
\hline A.1 & 5.3677 & 5.3693 & $0.030 \%$ & $\mathrm{~N} / \mathrm{C}$ & \\
\hline & & & & & YES \\
\hline B1 & 5.668 & 56693 & $0023 \%$ & $N / C$ & \\
\hline & & & & & YES \\
\hline & & & & & \\
\hline C1 & 5.4165 & 5.4142 & $-0.042 \%$ & $\mathrm{~N} / \mathrm{C}$ & \\
\hline & & & & & YES \\
\hline & Nitrogen & $302 F 24 \mathrm{~h}$ & & & \\
\hline A2 & 4.9404 & 4.9381 & $.0 .047 \%$ & $\mathrm{~N} / \mathrm{C}$ & \\
\hline & & & & & YES \\
\hline & & & & & \\
\hline$B 2$ & 5.4959 & 5.4938 & $-0.038 \%$ & $\mathrm{~N} / \mathrm{C}$ & \\
\hline & & & & & YES \\
\hline $\mathrm{C} 2$ & 5.9803 & 5.98 & $-0.005 \%$ & $\mathrm{~N} / \mathrm{C}$ & \\
\hline & & & & & YES \\
\hline & & & & & \\
\hline
\end{tabular}




\begin{tabular}{|c|c|c|c|c|c|c|c|}
\hline & $500 \mathrm{hrs}$ & in Nitrogen & at $260 \mathrm{~F}$ & & & & \\
\hline & VARN & WIREWT & $\begin{array}{c}\text { EXPWRE } \\
W T\end{array}$ & $\begin{array}{c}\text { WT \% } \\
\text { CHANGE }\end{array}$ & $\begin{array}{l}\text { EXP } \\
\text { VIS }\end{array}$ & $\begin{array}{l}\text { EXP } \\
\text { FLEX }\end{array}$ & \\
\hline$P$ & U-475 & 5.7063 & 5.7087 & $0.042 \%$ & $\mathrm{~N} / \mathrm{C}$ & & \\
\hline 0 & A 1 & & & & & NO & \\
\hline L & & & & & & & \\
\hline Y & $Y-390$ & 6.2283 & 6.2272 & $.0 .018 \%$ & $\mathrm{~N} / \mathrm{C}$ & & \\
\hline$E$ & B1 & & & & & NO & \\
\hline & & & & & & & \\
\hline$T$ & ER-610 & 5.8323 & 5.8332 & $0.015 \%$ & $\mathrm{~N} / \mathrm{C}$ & & \\
\hline E & & & & & & $\mathrm{NO}$ & \\
\hline & $Y-833$ & 57952 & 57054 & 00020 & N/C & & \\
\hline $\mathrm{P}$ & & & & & & YES-1 & \\
\hline o & & & & & & & \\
\hline L & 923 & 6.0210 & 6.0213 & $0.005 \%$ & $\mathrm{~N} / \mathrm{C}$ & & \\
\hline Y & E1 & & & & & NO & \\
\hline A & & & & & & & \\
\hline M & $150-800$ & 6.0178 & 6.0173 & $-0.008 \%$ & $\mathrm{~N} / \mathrm{C}$ & & \\
\hline 1 & & & & & & NO & \\
\hline D & & & & & & & \\
\hline$E$ & & Nitrogen -> & 24 HRS @ & $302 \mathrm{~F}$ & & & \\
\hline & U-475 & 5.9720 & 5.9709 & $0.018 \%$ & $N / C$ & & \\
\hline 1 & A2 & & & & & NO & \\
\hline $\mathrm{M}$ & & & & & & & \\
\hline 1 & $(Y-=)$ & 5.9340 & 5.9308 & $.0 .054 \%$ & $\mathrm{~N} / \mathrm{C}$ & & \\
\hline D & & & & & & NO & \\
\hline$E$ & & & & & & & \\
\hline & ER-610 & 5.6010 & 5.6007 & $-0.005 \%$ & $N / C$ & & \\
\hline & & & & & & NO & \\
\hline & $Y-833$ & 56635 & 56618 & $.0030 \%$ & $N / C$ & & \\
\hline & $\mathrm{D} 2$ & & & & & YES-1 & \\
\hline & & & & & & & \\
\hline & 923 & 5.8101 & 5.8066 & $.0 .060 \%$ & $\mathrm{~N} / \mathrm{C}$ & & \\
\hline & E2 & & & & & NO & \\
\hline & & & & & & & \\
\hline & ISO-800 & 5.1321 & 5.1299 & $.0 .043 \%$ & $\mathrm{~N} / \mathrm{C}$ & & \\
\hline & & & & & & NO & \\
\hline & & & & & & & \\
\hline
\end{tabular}




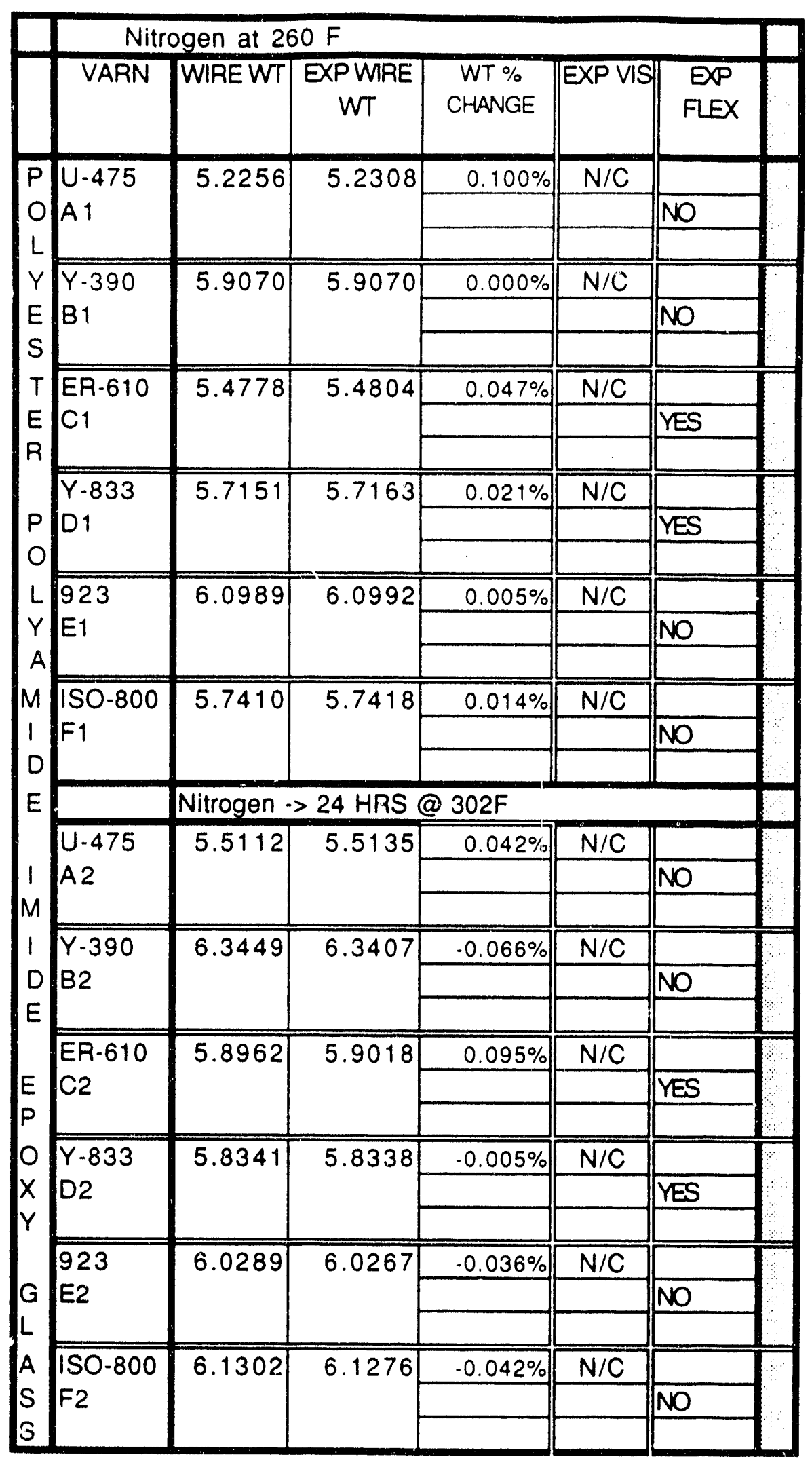




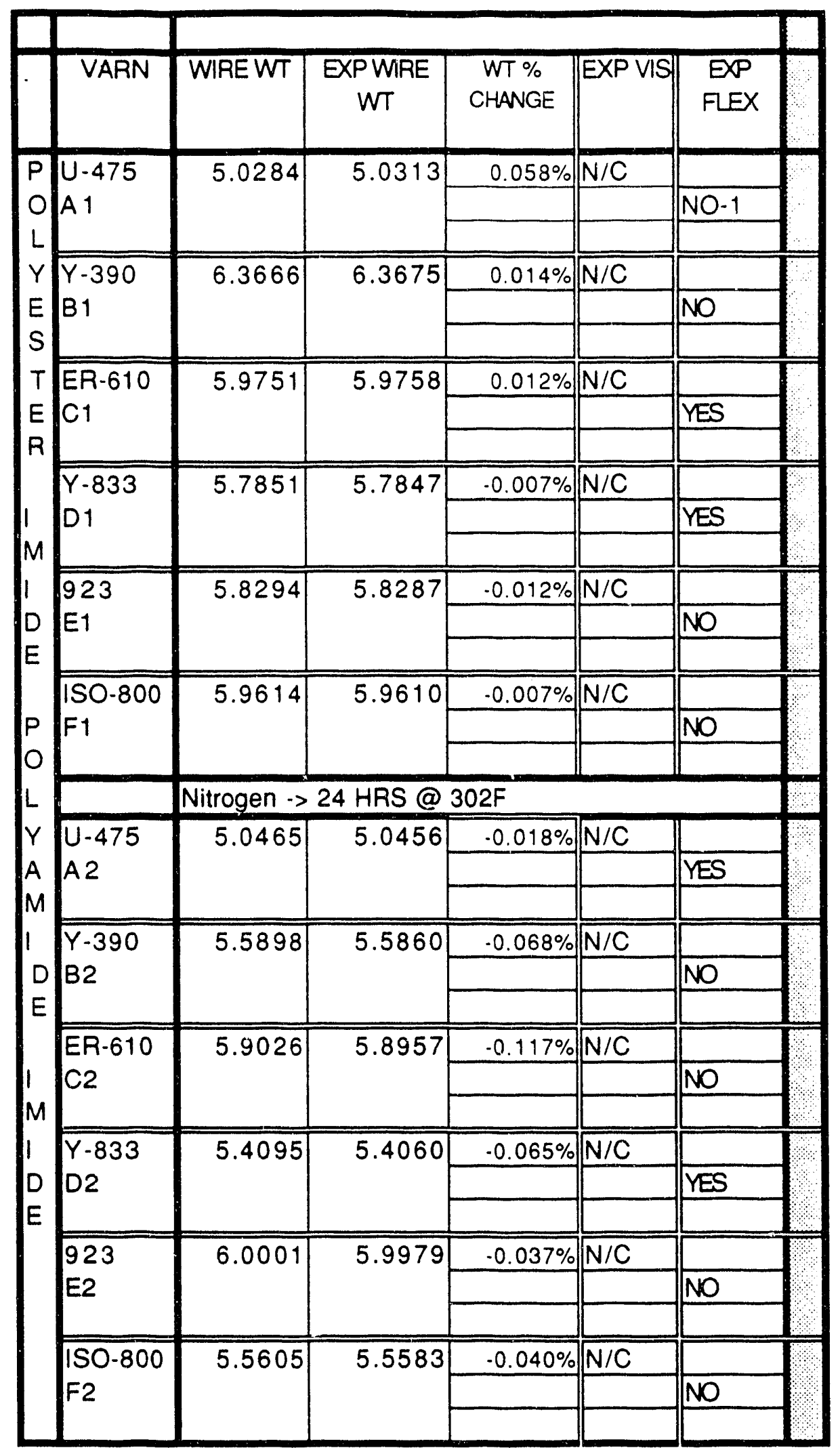




\begin{tabular}{|c|c|c|c|c|c|}
\hline \multicolumn{6}{|c|}{500 HRS IN Nitrogen @ 260 F } \\
\hline ID & WT & EXPWT & $\begin{array}{c}\text { WT \% } \\
\text { CHANGE }\end{array}$ & EXP VIS & EXPFLEX \\
\hline $\begin{array}{l}\text { U-475 } \\
\text { A1 }\end{array}$ & 2.8657 & 2.8203 & $-1.58 \%$ & $\mathrm{~N} / \mathrm{C}$ & $\mathrm{N} / \mathrm{C}$ \\
\hline $\begin{array}{l}Y-390 \\
B 1\end{array}$ & 2.3480 & 2.3132 & $1.48 \%$ & \begin{tabular}{|l|} 
Slightly \\
Warped \\
\end{tabular} & $\mathrm{N} / \mathrm{C}$ \\
\hline $\begin{array}{l}E R-610 \\
C 1\end{array}$ & 2.4281 & 2.4274 & $-0.03 \%$ & $\mathrm{~N} / \mathrm{C}$ & $\mathrm{N} / \mathrm{C}$ \\
\hline $\begin{array}{l}Y-833 \\
D 1\end{array}$ & 2.9013 & 2.9195 & $0.63 \%$ & $\mathrm{~N} / \mathrm{C}$ & $\mathrm{N} / \mathrm{C}$ \\
\hline $\begin{array}{l}923 \\
E 1\end{array}$ & 2.0774 & 2.0492 & $-1.36 \%$ & $\begin{array}{l}\text { slightly } \\
\text { warped } \\
\end{array}$ & $\mathrm{N} / \mathrm{C}$ \\
\hline $\begin{array}{l}\text { ISO-800 } \\
\text { F1 }\end{array}$ & 2.0598 & 1.9941 & $-3.19 \%$ & \begin{tabular}{|l} 
Slightly \\
Warped \\
\end{tabular} & $\mathrm{N} / \mathrm{C}$ \\
\hline & Nitrogen 26 & $\mathrm{~F}$ at $->302 \mathrm{~F}$ & 24 HRS & & \\
\hline $\begin{array}{l}\text { U-475 } \\
\text { A2 }\end{array}$ & 2.8981 & 2.8621 & $-1.24 \%$ & $\mathrm{~N} / \mathrm{C}$ & $\mathrm{N} / \mathrm{C}$ \\
\hline$Y-390$ & 2.7299 & 2.6786 & $-1.88 \%$ & & $\mathrm{~N} / \mathrm{C}$ \\
\hline B2 & & & & $\begin{array}{l}\text { Slightly } \\
\text { warped }\end{array}$ & \\
\hline $\begin{array}{l}\text { ER-610 } \\
\text { C2 }\end{array}$ & 2.3703 & 2.3588 & $-0.49 \%$ & Darkened & $\mathrm{N} / \mathrm{C}$ \\
\hline $\begin{array}{l}Y-833 \\
D 2\end{array}$ & 3.5321 & 3.5552 & $0.65 \%$ & $\mathrm{~N} / \mathrm{C}$ & $\mathrm{N} / \mathrm{C}$ \\
\hline $\begin{array}{l}923 \\
\text { E2 }\end{array}$ & 2.3378 & 2.3068 & $-1.33 \%$ & $\begin{array}{l}\text { Slightly } \\
\text { Warped } \\
\end{array}$ & $\mathrm{N} / \mathrm{C}$ \\
\hline $150-800$ & 2.1550 & 2.0885 & $-3.09 \%$ & & $\mathrm{~N} / \mathrm{C}$ \\
\hline$F 2$ & & & & $\frac{\text { Slightly }}{\text { warped }}$ & \\
\hline
\end{tabular}




\begin{tabular}{|c|c|c|c|c|c|c|c|c|c|}
\hline \multicolumn{10}{|c|}{500 HR IN NITROGEN@ $260 \mathrm{~F}$} \\
\hline $\mathrm{ID}$ & WT & EXPWT & $\begin{array}{c}\text { WT \% } \\
\text { CHANGE }\end{array}$ & $\begin{array}{l}\text { SAMPLE } \\
\text { WIDTH }\end{array}$ & $\begin{array}{l}\text { BREAK } \\
\text { LOAD }\end{array}$ & $\begin{array}{c}\text { SAMPLE } \\
\text { THICKNESS } \\
\text { Mils }\end{array}$ & $\begin{array}{c}\text { TENSILE } \\
\text { STR } \\
\text { BASE }\end{array}$ & $\begin{array}{l}\text { TENSILE } \\
\text { STREXP }\end{array}$ & $\begin{array}{c}\% \\
\text { CHANGE } \\
\text { TENSILE }\end{array}$ \\
\hline \multirow{3}{*}{$\begin{array}{l}\mathrm{NO} / \mathrm{MY} / \mathrm{NO} \\
\mathrm{A} 1\end{array}$} & \multirow{3}{*}{8.2896} & \multirow[t]{3}{*}{8.2953} & \multirow[t]{3}{*}{$0.07 \%$} & 0.490 & 92.05 & 21 & 17.4 & 8.95 & $\therefore$ \\
\hline & & & & 0.355 & 64.20 & 21 & 17.4 & 8.61 & $-49.55 \%$ \\
\hline & & & & 0.378 & 133.00 & 21 & 17.4 & 16.75 & \\
\hline \multirow{3}{*}{$\begin{array}{l}\mathrm{DA} / \mathrm{MY} / \mathrm{DA} \\
\mathrm{B} 1\end{array}$} & \multirow{3}{*}{7.6508} & \multirow[t]{3}{*}{7.6543} & \multirow[t]{3}{*}{$0.05 \%$} & 0.495 & 125.70 & 22 & 13.7 & 11.54 & \\
\hline & & & & 0.470 & 127.80 & 22. & 13.7 & 12.36 & $-8.56 \%$ \\
\hline & & & & 0.377 & 105.30 & 22 & 13.7 & 12.70 & \\
\hline \multirow{3}{*}{$\begin{array}{l}\text { MYLAR MO } \\
C 1\end{array}$} & \multirow{3}{*}{3.7184} & \multirow[t]{3}{*}{3.7225} & \multirow{3}{*}{$0.11 \%$} & 0.394 & 64.10 & 10 & 21.71 & 16.27 & \\
\hline & & & & 0.475 & 79.10 & 10 & $2 i .7$ & 16.65 & $-24.04 \%$ \\
\hline & & & & 0.428 & 70.75 & 10 & 21.7 & 16.53 & \\
\hline \multirow{3}{*}{$\begin{array}{l}\text { NO } 410 \\
\text { D1 }\end{array}$} & \multirow{3}{*}{3.4136} & \multirow{3}{*}{3.4211} & \multirow[t]{3}{*}{$0.22 \%$} & 0.424 & 82.50 & 11 & 18.7 & 17.69 & \\
\hline & & & & 0.481 & 97.20 & 11 & 18.7 & 18.37 & $-8.95 \%$ \\
\hline & & & & 0.515 & 85.10 & 11 & 18.7 & 15.02 & \\
\hline \multirow{3}{*}{$\begin{array}{l}\text { NO MI } 418 \\
\text { E1 }\end{array}$} & \multirow[t]{3}{*}{3.6602} & \multirow[t]{3}{*}{3.6698} & \multirow[t]{3}{*}{$0.26 \%$} & 0.488 & 30.95 & 9 & 7.5 & 7.05 & \\
\hline & & & & 0.482 & 30.95 & 9 & 7.5 & 7.13 & $-5.86 \%$ \\
\hline & & & & 0.515 & 32.45 & 9 & 7.5 & 7.00 & \\
\hline \multirow{3}{*}{$\begin{array}{l}\text { MEL } 228 \\
\text { F1 }\end{array}$} & \multirow[t]{3}{*}{4.3084} & \multirow[t]{3}{*}{4.3130} & \multirow[t]{3}{*}{$0.11 \%$} & 0.475 & 82.60 & 10 & 21.7 & 17.39 & \\
\hline & & & & 0.475 & 80.90 & 10 & 21.7 & 17.03 & $-21.53 \%$ \\
\hline & & & & 0.491 & 81.80 & 10 & 21.7 & 16.66 & \\
\hline & & $500 \mathrm{HRS}$ & IN NITRC & OGEN@ & $260 F->2$ & 24 HRS@ & $02 \mathrm{~F}$ & & \\
\hline $\mathrm{NO} / \mathrm{MY} / \mathrm{NO}$ & 7.5739 & 7.6150 & $0.54 \%$ & 0.409 & 144.40 & 23 & 17.4 & 15.35 & \\
\hline A2 & & & & 0.363 & 129.30 & 23 & 17.4 & 15.49 & $-1 ? .59 \%$ \\
\hline & & & & 0.430 & 146.30 & 23 & 17.4 & 14.79 & \\
\hline$D A / M Y / D A$ & 7.8608 & 7.8626 & $0.02 \%$ & 0.430 & 120.80 & 22 & 13.7 & 12.77 & \\
\hline B2 & & & & 0.450 & 127.30 & 22 & 13.7 & 12.86 & $-11.41 \%$ \\
\hline & & & & 0.460 & 109.10 & 22 & 13.7 & 10.78 & \\
\hline MYLARMO & 4.1170 & 4.1232 & $0.15 \%$ & 0.477 & 79.55 & 10 & 21.7 & 16.68 & \\
\hline $\mathrm{C} 2$ & & & & 0.492 & 83.10 & 10 & 21.7 & 16.89 & $.24 .40 \%$ \\
\hline & & & & 0.462 & 72.30 & 10 & 21.7 & 15.65 & \\
\hline NOMEX 410 & 3.6320 & 3.6848 & $1.45 \%$ & 0.443 & 81.05 & 11 & 18.7 & 16.63 & \\
\hline D2 & & & & 0.509 & 109.90 & 11 & 18.7 & 19.63 & $-4.04 \%$ \\
\hline & & & & 0.491 & 94.90 & 11 & 18.7 & 17.57 & \\
\hline NO/MI 418 & 3.8369 & 3.8656 & $0.75 \%$ & 0.485 & 28.90 & 10 & 7.5 & 5.96 & \\
\hline E2 & & & & 0.502 & 30.20 & 10 & 7.5 & 6.02 & $.19 .71 \%$ \\
\hline & & & & 0.518 & 31.55 & 10 & 7.5 & 6.09 & \\
\hline MEL 228 & 4.3790 & 4.3845 & $0.13 \%$ & 0.491 & 86.05 & 11 & 21.7 & 15.93 & \\
\hline & & & & 0.524 & 91.00 & 11 & 21.7 & 15.79 & $-30.19 \%$ \\
\hline & & & & 0.411 & 62.05 & 11 & 21.7 & 13.72 & \\
\hline
\end{tabular}




\begin{tabular}{|c|c|c|c|c|c|c|c|c|}
\hline & 500 hour & in Nit & $n @ 26$ & $27^{\circ} \mathrm{C}$ & & & & \\
\hline ID & $\begin{array}{c}\text { STRETCH } \\
\text { (inch) }\end{array}$ & $\begin{array}{c}\% \\
\text { ELONG }\end{array}$ & $\begin{array}{l}\text { BASE } \\
\text { ELONG } \\
\text { (AVE) }\end{array}$ & $\begin{array}{l}\text { ELONG \% } \\
\text { CHANGE }\end{array}$ & \begin{tabular}{|c} 
BASE \\
DIE \\
(AVE)
\end{tabular} & EXPDIE & $\begin{array}{c}\text { DIE \% } \\
\text { CHANGE }\end{array}$ & $\begin{array}{l}\text { VISUAL } \\
\text { EPP }\end{array}$ \\
\hline NO/MY/NO & 0.10 & $2.5 \%$ & $20.0 \%$ & & $>18.97$ & flash & & $N / C$ \\
\hline & 0.10 & $2.5 \%$ & $20.0 \%$ & $.87 .5 \%$ & $\geq 18.97$ & flash & flash & \\
\hline & 0.52 & $13.0 \%$ & $20.0 \%$ & & $>18.97$ & flash & & \\
\hline DA/MY/DA & 0.42 & $21.0 \%$ & $46.0 \%$ & & $>15.27$ & flash & & ightly \\
\hline & 0.46 & $23.0 \%$ & $46.0 \%$ & $-50.0 \%$ & $>15.27$ & flash & flash & \\
\hline & 0.42 & $21.0 \%$ & $46.0 \%$ & & $>15.27$ & flash & & yellowed \\
\hline MYLAR MO & 0.12 & $6.0 \%$ & $131.0 \%$ & & $>14.91$ & flash & & $N / C$ \\
\hline & 0.14 & $7.0 \%$ & $131.0 \%$ & $-95.0 \%$ & $>14.91$ & flash & flash & \\
\hline & 0.13 & $6.5 \%$ & $131.0 \%$ & & $>14.91$ & flash & & \\
\hline NO 410 & 0.41 & $10.3 \%$ & $17.0 \%$ & & 10.67 & 9.81 & & $N / C$ \\
\hline & 0.51 & $12.8 \%$ & $17.0 \%$ & $.43 .6 \%$ & 10.67 & 10.41 & $.5 .6 \%$ & \\
\hline & 0.23 & $5.8 \%$ & $17.0 \%$ & & 10.67 & 9.99 & & \\
\hline NO MI 418 & 0.05 & $1.3 \%$ & $4.0 \%$ & & 10.23 & 9.33 & & $\mathrm{~N} / \mathrm{C}$ \\
\hline & 0.05 & $1.3 \%$ & $4.0 \%$ & $.70 .8 \%$ & 10.23 & 9.87 & $-6.3 \%$ & \\
\hline & 0.04 & $1.0 \%$ & $4.0 \%$ & & 10.23 & 9.57 & & \\
\hline MEL 228 & 0.13 & $6.5 \%$ & $160.0 \%$ & & $>14.22$ & flash & & $N / C$ \\
\hline & 0.13 & $6.5 \%$ & $160.0 \%$ & $.96 .4 \%$ & $>14.22$ & flash & flash & \\
\hline & 0.10 & $5.0 \%$ & $160.0 \%$ & & $>14.22$ & flash & & \\
\hline & After 500 & hour $\theta$ & osure pl & Is a $24 \mathrm{~h}$ & nour airba & ake at 15 & 300 & F) \\
\hline NO/MY/NO & 0.34 & $8.5 \%$ & $20.0 \%$ & & $>18.97$ & flash & & $\mathrm{N} / \mathrm{C}$ \\
\hline A2 & 0.25 & $6.3 \%$ & $20.0 \%$ & $.66 .7 \%$ & $>18.97$ & flash & flash & \\
\hline & 0.21 & $5.3 \%$ & $20.0 \%$ & & $>18.97$ & flash & & \\
\hline $\mathrm{DA} / \mathrm{MY} / \mathrm{DA}$ & 0.49 & $24.5 \%$ & $46.0 \%$ & & $>15.27$ & flash & & Slightly \\
\hline & 0.50 & $25.0 \%$ & $46.0 \%$ & $.54 .3 \%$ & $>15.27$ & flash & flash & warped \\
\hline & 0.42 & $21.0 \%$ & $46.0 \%$ & & $>15.27$ & flash & & \\
\hline MYLAR MO & 0.13 & $6.5 \%$ & $131.0 \%$ & & $>14.91$ & flash & & $\mathrm{N} / \mathrm{C}$ \\
\hline $\mathrm{C} 2$ & 0.13 & $6.5 \%$ & $131.0 \%$ & $.95 .4 \%$ & $>14.91$ & flash & flash & \\
\hline & 0.11 & $5.5 \%$ & $131.0 \%$ & & $>14.91$ & flash & & \\
\hline NOMEX $41 \mathrm{~d}$ & 0.19 & $4.8 \%$ & $17.0 \%$ & & \begin{tabular}{|l|}
10.67 \\
\end{tabular} & 11.15 & & $N / C$ \\
\hline D2 & 0.27 & $6.8 \%$ & $17.0 \%$ & $.54 .4 \%$ & 10.67 & 10.89 & $-1.8 \%$ & \\
\hline & 0.47 & $11.8 \%$ & $17.0 \%$ & & 10.67 & 9.40 & & \\
\hline NO/MI 418 & 0.05 & $1.3 \%$ & $4.0 \%$ & & 10.23 & 9.52 & & $N / C$ \\
\hline E2 & 0.05 & $1.3 \%$ & $4.0 \%$ & $.68 .8 \%$ & 10.23 & 9.83 & $.4 .6 \%$ & \\
\hline & 0.05 & $1.3 \%$ & $4.0 \%$ & & 10.23 & 9.94 & & \\
\hline MEL 228 & 0.11 & $5.5 \%$ & $160.0 \%$ & & $>14.22$ & flash & & $\mathrm{N} / \mathrm{C}$ \\
\hline $\mathrm{F} 2$ & 0.13 & $6.5 \%$ & $160.0 \%$ & $-96.8 \%$ & $\geq 14.22$ & flash & flash & \\
\hline & 0.07 & $3.5 \%$ & $160.0 \%$ & & $>14.22$ & flash & & \\
\hline
\end{tabular}


SLEEVING

\begin{tabular}{|c|c|c|c|c|}
\hline & \multicolumn{4}{|c|}{500 HRS IN Nitrogen@260F } \\
\hline & WT & EXPWT & $\begin{array}{c}\% \\
\text { CHANGE }\end{array}$ & EXP VIS \\
\hline $\begin{array}{l}\text { A } 1 \\
\text { NOMEX }\end{array}$ & 0.5426 & 0.5441 & $0.28 \%$ & $\begin{array}{l}\text { Discolored } \\
\text { see Photo }\end{array}$ \\
\hline $\begin{array}{l}B 1 \\
M Y A R\end{array}$ & 0.5060 & 0.5057 & $-0.06 \%$ & \begin{tabular}{|l|} 
Brittle \\
See Photo \\
\end{tabular} \\
\hline \multirow[t]{2}{*}{$\begin{array}{l}\text { C1 } \\
\text { NOMY }\end{array}$} & 0.3959 & 0.3963 & $0.10 \%$ & \begin{tabular}{||c|} 
Outside \\
Brittle \\
see photo \\
\end{tabular} \\
\hline & \multicolumn{4}{|c|}{ Nitrogen@260F....>24 hrs@302F } \\
\hline $\begin{array}{l}\text { A2 } \\
\text { NOMEX }\end{array}$ & 0.5492 & 0.5449 & $-0.78 \%$ & \begin{tabular}{||c|} 
Color \\
lightened \\
see Photos
\end{tabular} \\
\hline $\begin{array}{l}\text { B2 } \\
\text { MYLAR }\end{array}$ & 0.5190 & 0.5142 & $-0.92 \%$ & $\begin{array}{l}\mid \text { Darkened } \\
\text { Brittle } \\
\text { "see photo }\end{array}$ \\
\hline $\begin{array}{l}\text { C2 } \\
\text { NOMY }\end{array}$ & 0.4021 & 0.3981 & $-0.99 \%$ & $\begin{array}{l}\text { Darkened } \\
\text { Brittle } \\
\text { "see photo }\end{array}$ \\
\hline
\end{tabular}




\begin{tabular}{|c|c|c|c|c|c|c|c|c|c|}
\hline & 500 HRS & N NITRO & EN@260 & & & & & & \\
\hline$D$ & WT & EXPWT & $\begin{array}{c}\text { WT \% } \\
\text { CHANGE }\end{array}$ & $\begin{array}{l}\text { BREAK } \\
\text { LOAD } \\
\text { (AVE) }\end{array}$ & $\begin{array}{c}\text { BREAK } \\
\text { LOAD } \\
\text { EPP }\end{array}$ & $\begin{array}{c}\% \\
\text { CHANGE } \\
\text { BRK } \\
\text { LOAD }\end{array}$ & $\begin{array}{l}\text { STRETCH } \\
\text { (INCH) }\end{array}$ & \%ELONG & EXP VIS \\
\hline$A 1$ & 1.7369 & 1.7300 & $-0.40 \%$ & 39.02 & 43.40 & & 0.04 & $2.00 \%$ & $\mathrm{~N} / \mathrm{C}$ \\
\hline Glass & & & & 39.02 & 48.55 & $5.42 \%$ & 0.04 & $2.00 \%$ & \\
\hline & & & & 39.02 & 31.45 & & 0.04 & $2.00 \%$ & \\
\hline $\mathrm{B1}$ & 0.7091 & 0.7096 & $0.07 \%$ & 56.12 & 43.50 & & 0.30 & $15.00 \%$ & $\mathrm{~N} / \mathrm{C}$ \\
\hline Polyester & & & & 56.12 & 38.10 & $27.30 \%$ & 0.24 & $12.00 \%$ & \\
\hline & & & & 56.12 & LOST & & LOST & lost & \\
\hline $\mathrm{C} 1$ & 1.6459 & 1.6376 & $-0.50 \%$ & 88.50 & 91.45 & & 0.09 & $4.50 \%$ & \\
\hline Permacel & & & & 88.50 & 87.55 & $6.48 \%$ & 0.08 & $4.00 \%$ & \\
\hline & & & & 88.50 & 103.70 & & 0.08 & $4.00 \%$ & \\
\hline & & 500 HRS & in NITROG & $E N->302$ & IF $24 \mathrm{HRS}$ & & & & \\
\hline$A 2$ & 1.4994 & 1.4958 & $-0.24 \%$ & 39.02 & 46.00 & & 0.04 & $2.00 \%$ & $N / C$ \\
\hline Glass & & & & 39.02 & 55.70 & $14.77 \%$ & 0.07 & $3.50 \%$ & \\
\hline & & & & 39.02 & 32.65 & & 0.04 & $2.00 \%$ & \\
\hline & 0.6781 & 0.6782 & $0.01 \%$ & 56.12 & 41.50 & & 0.29 & $14.50 \%$ & $\mathrm{~N} / \mathrm{C}$ \\
\hline Polyester & & & & 56.12 & 37.10 & $-29.14 \%$ & 0.39 & $19.50 \%$ & \\
\hline & & & & 56.12 & 40.70 & & 0.32 & $16.00 \%$ & \\
\hline & 1.6451 & 1.5769 & $-4.15 \%$ & 88.50 & 58.55 & & 0.05 & $2.50 \%$ & Darkened \\
\hline Permacel & & & & 88.50 & 109.10 & $-11.41 \%$ & 0.10 & $5.00 \%$ & \\
\hline & & & & 88.50 & 67.55 & & 0.05 & $2.50 \%$ & \\
\hline
\end{tabular}


TIE CORDS

\begin{tabular}{|c|c|c|c|c|c|c|c|c|c|}
\hline \multicolumn{10}{|c|}{500 HRS IN NITROGEN @ $260 \mathrm{~F}$} \\
\hline ID & WT & EXPWT & $\begin{array}{l}\text { WT \% } \\
\text { CHANGE }\end{array}$ & $\begin{array}{l}\text { EXP } \\
\text { VIS }\end{array}$ & $\begin{array}{l}\text { BREAK } \\
\text { LOAD } \\
\text { (AVE) }\end{array}$ & $\begin{array}{c}\text { BREAK } \\
\text { LOAD } \\
\text { EXP } \\
(\text { ave })\end{array}$ & $\begin{array}{c}\% \\
\text { CHANGE } \\
\text { BPK } \\
\text { LOAD }\end{array}$ & $\begin{array}{c}\text { STRETCH } \\
\text { (Inch) }\end{array}$ & $\begin{array}{c}\% \\
\text { ELONG }\end{array}$ \\
\hline \multirow[t]{3}{*}{ A1 1} & \multirow[t]{3}{*}{0.2617} & \multirow[t]{3}{*}{0.2611} & \multirow[t]{3}{*}{$-0.23 \%$} & $\mathrm{~N} / \mathrm{C}$ & 28.36 & 25.05 & & 0.31 & $15.5 \%$ \\
\hline & & & & & 28.36 & 25.10 & $.11 .14 \%$ & 0.34 & $17.0 \%$ \\
\hline & & & & & 28.36 & 25.45 & & 0.36 & $18.0 \%$ \\
\hline & \multicolumn{9}{|c|}{500 HRS IN NITROGEN $->24$ HRS @ 302F } \\
\hline \multirow[t]{3}{*}{$\overline{A 2}$} & \multirow[t]{3}{*}{0.2853} & \multirow[t]{3}{*}{0.2852} & \multirow[t]{3}{*}{$-0.035 \%$} & $N / C$ & 28.36 & 29.10 & & 0.35 & $17.5 \%$ \\
\hline & & & & & 28.36 & 29.55 & $0.49 \%$ & 0.34 & $17.0 \%$ \\
\hline & & & & & 28.36 & 26.85 & & 0.36 & $18.0 \%$ \\
\hline
\end{tabular}




\begin{tabular}{|c|c|c|c|c|c|c|c|}
\hline & $500 \mathrm{HRS}$ IN & trogen@2 & $0 \mathrm{~F}$ & & & & \\
\hline ID & WT & EXPWT & $\begin{array}{c}\text { WT \% } \\
\text { CHANGE }\end{array}$ & EXP VIS & $\begin{array}{c}\text { BASE DIE } \\
\text { (AVE) }\end{array}$ & EXPDIE & $\begin{array}{c}\text { DIE\% } \\
\text { CHANGE }\end{array}$ \\
\hline DMD & 4.0361 & 4.0362 & $0.00 \%$ & $\mathrm{~N} / \mathrm{C}$ & 9.61 & 10.37 & \\
\hline A1 & & & & & 9.61 & 9.64 & $3.02 \%$ \\
\hline & & & & & 9.61 & 9.69 & \\
\hline DTMD & 4.2147 & 4.2147 & $0.00 \%$ & $\mathrm{~N} / \mathrm{C}$ & 9.95 & 10.67 & \\
\hline B1 & & & & & 9.95 & 7.89 & $-12.23 \%$ \\
\hline & & & & : & 9.95 & 7.64 & \\
\hline & Nitrogen@2 & $F \cdot>24 H$ & 3S@302 & & & & \\
\hline DMD & 4.0556 & 4.0556 & $0.00 \%$ & - & 9.61 & 8.85 & \\
\hline A2 & & & & & 9.61 & 8.90 & $-9.05 \%$ \\
\hline & & & & & 9.61 & 8.47 & \\
\hline DTMD & 4.2947 & 4.2929 & $-0.04 \%$ & & 9.95 & 9.23 & \\
\hline $\mathrm{B2}$ & & & & & 9.95 & 9.99 & $-7.91 \%$ \\
\hline & & & & & 9.95 & 8.27 & \\
\hline
\end{tabular}




\section{Appendix B}

\section{Experimental Data for HCFC-22/Witco 3GS Exposure at $126^{\circ} \mathrm{C}\left(260^{\circ} \mathrm{F}\right)$}




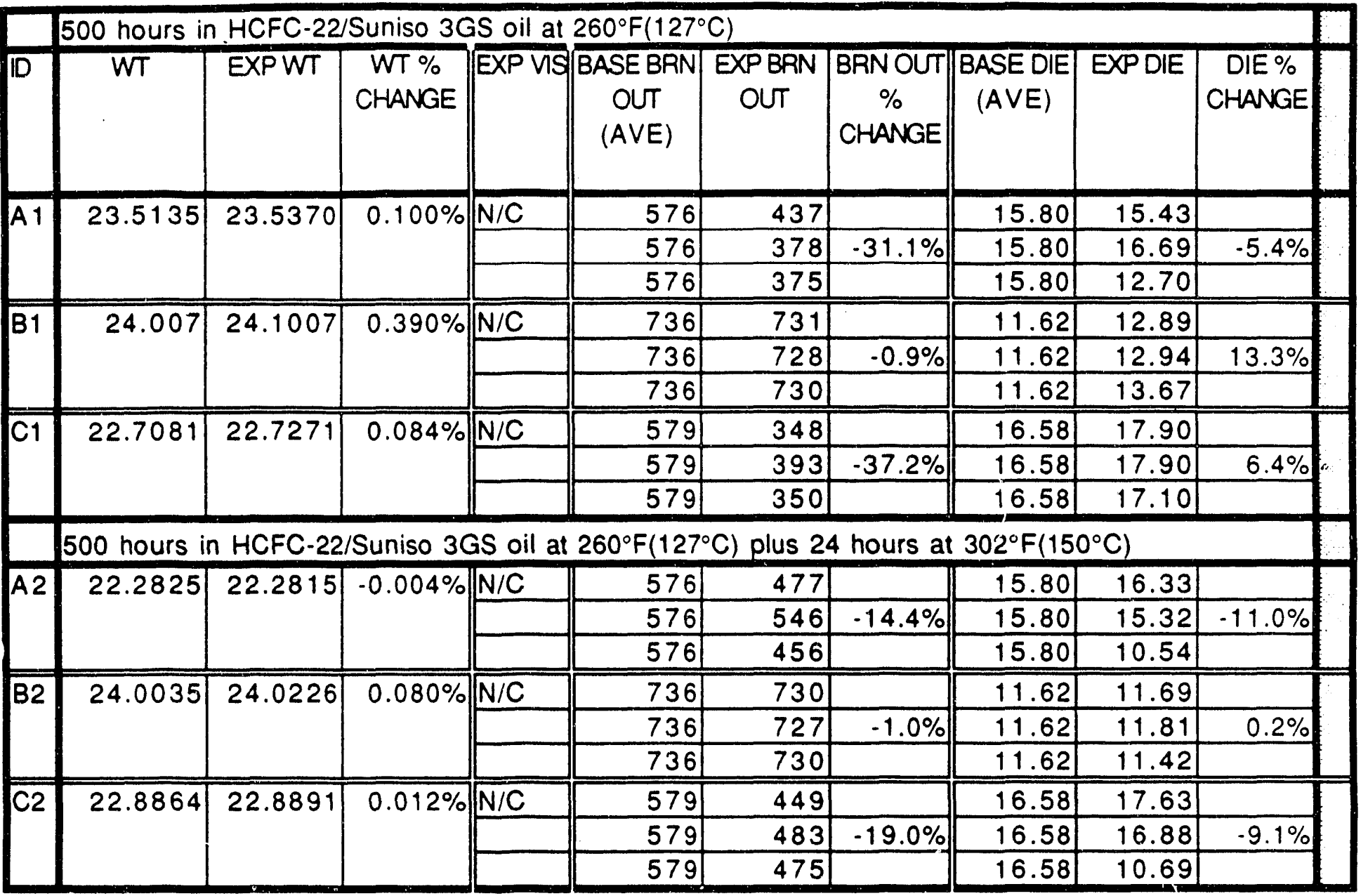


TWISTED PAIRS/WIRE AWITH VARNISH-HCFC-22/Suniso 3GS oil @260 $\mathrm{F}\left(127^{\circ} \mathrm{C}\right)$

\begin{tabular}{|c|c|c|c|c|c|c|c|c|c|c|c|}
\hline & & \multicolumn{10}{|c|}{500 hours in HCFC-22/Suniso 3GS oil @ 260 $\mathrm{F}\left(127^{\circ} \mathrm{C}\right)$} \\
\hline & VARN & $\begin{array}{l}\text { TWISTED } \\
\text { PAIR WT }\end{array}$ & $\begin{array}{l}\text { Exp Pair } \\
\text { weight }\end{array}$ & $\begin{array}{c}\text { WT \% } \\
\text { CHANGE }\end{array}$ & $\begin{array}{l}\text { EXP } \\
\text { VIS }\end{array}$ & |BASE DIE & EXPDIE & $\begin{array}{c}\text { DIE \% } \\
\text { CHANGE }\end{array}$ & $\begin{array}{c}\text { BASE } \\
\text { BUPN } \\
\text { OUT }\end{array}$ & $\begin{array}{l}\text { EXP } \\
\text { BURN } \\
\text { OUT }\end{array}$ & $\begin{array}{c}\text { BRNOUT } \\
\% \\
\text { CHANGE }\end{array}$ \\
\hline \multirow{3}{*}{$\begin{array}{l}P \\
O \\
L\end{array}$} & \multirow{3}{*}{$\begin{array}{l}U-475 \\
\text { A } 1 \\
\end{array}$} & \multirow[t]{3}{*}{22.5250} & \multirow[t]{3}{*}{22.6049} & \multirow[t]{3}{*}{$0.355 \%$} & $\mathrm{~N} / \mathrm{C}$ & 16.24 & 13.81 & & 430 & 325 & \\
\hline & & & & & & 16.24 & 17.43 & $.11 .45 \%$ & 430 & 332 & $-20.78 \%$ \\
\hline & & & & & & 16.24 & 11.90 & & 430 & 365 & \\
\hline Y & \multirow{3}{*}{$\begin{array}{l}Y-390 \\
B 1\end{array}$} & \multirow[t]{3}{*}{22.2260} & \multirow[t]{3}{*}{22.3107} & \multirow[t]{3}{*}{$0.381 \%$} & $\mathrm{~N} / \mathrm{C}$ & 18.77 & 16.10 & & 510 & 391 & \\
\hline$E$ & & & & & & 18.77 & 12.74 & $-13.27 \%$ & 510 & 399 & $-22.48 \%$ \\
\hline S & & & & & & 18.77 & 20.00 & & 510 & 396 & \\
\hline \multirow{4}{*}{$\begin{array}{l}T \\
E \\
R\end{array}$} & ER-610 & \multirow[t]{3}{*}{22.4122} & \multirow[t]{3}{*}{22.4600} & \multirow[t]{3}{*}{$0.213 \%$} & $\mathrm{~N} / \mathrm{C}$ & 15.57 & 13.51 & & 442 & 270 & \\
\hline & \multirow{2}{*}{ C1 } & & & & & 15.57 & 16.62 & $-2.72 \%$ & 442 & 292 & $.37 .93 \%$ \\
\hline & & & & & & 15.57 & 15.31 & & 442 & 261 & \\
\hline \multirow{3}{*}{\multicolumn{2}{|c|}{$\begin{array}{l}Y-833 \\
D 1\end{array}$}} & \multirow[t]{3}{*}{20.9834} & \multirow[t]{3}{*}{20.9936} & \multirow[t]{3}{*}{$0.049 \%$} & $\mathrm{~N} / \mathrm{C}$ & 12.04 & 11.23 & & 578 & 414 & \\
\hline & & & & & & 12.04 & 11.15 & $0.19 \%$ & 578 & 370 & $-33.22 \%$ \\
\hline & & & & & & 12.04 & 13.81 & & 578 & 374 & \\
\hline \multirow{3}{*}{$\begin{array}{l}L \\
Y \\
A\end{array}$} & \multirow{3}{*}{$\begin{array}{l}923 \\
E 1 \\
\end{array}$} & \multirow[t]{3}{*}{23.1388} & \multirow[t]{3}{*}{23.2430} & $0.450 \%$ & $\mathrm{~N} / \mathrm{C}$ & 16.76 & 12.46 & & 606 & 391 & \\
\hline & & & & & & 16.76 & 7.37 & $-49.90 \%$ & 606 & 389 & $-35.64 \%$ \\
\hline & & & & & & 16.76 & 5.36 & & 606 & broke & \\
\hline$M$ & $150-800$ & 21.5467 & 21.5929 & $0.214 \%$ & $\mathrm{~N} / \mathrm{C}$ & 19.08 & 17.17 & & 580 & 388 & \\
\hline 1 & & & & & & 19.08 & 15.82 & $-13.56 \%$ & 580 & 393 & $-33.68 \%$ \\
\hline$D$ & & & & & & 19.08 & 16.49 & & 580 & 373 & \\
\hline $\mathbf{E}$ & & 500 hours & in HCFC. & 22/Sunis & $3 G S$ & oil @ 260 & ${ }^{\circ} \mathrm{F}\left(127^{\circ} \mathrm{C}\right)$ & plus 24 & ours@ & $302^{\circ} \mathrm{F}(1$ & $\left.50^{\circ} \mathrm{C}\right)$ \\
\hline & $U .475$ & 21.9872 & 21.9968 & $0.044 \%$ & $\mathrm{~N} / \mathrm{C}$ & \begin{tabular}{|l|}
16.24 \\
\end{tabular} & 16.37 & & 430 & 283 & \\
\hline 1 & $A 2$ & & & & & 16.24 & 15.52 & $-4.89 \%$ & 430 & 242 & $-41.24 \%$ \\
\hline$M$ & & & & & & 16.24 & 14.45 & & 430 & 233 & \\
\hline 1 & $Y-390$ & 22.1704 & 22.1910 & $0.093 \%$ & $\mathrm{~N} / \mathrm{C}$ & 18.77 & 15.87 & & $5 ; 0$ & 500 & \\
\hline$D$ & & & & & & 18.77 & 19.85 & $-8.22 \%$ & 510 & 392 & $-15.69 \%$ \\
\hline$E$ & & & & & & 18.77 & 15.96 & & 510 & 398 & \\
\hline & ER-610 & 22.7480 & 22.7555 & $0.033 \%$ & $\mathrm{~N} / \mathrm{C}$ & 15.57 & 13.39 & & 442 & 341 & \\
\hline & $\mathrm{C} 2$ & & & & & 15.57 & 15.02 & $-11.56 \%$ & 442 & 315 & $-27.15 \%$ \\
\hline & & & & & & 15.57 & 12.90 & & 442 & 310 & \\
\hline & $Y-833$ & 21.2636 & 21.2466 & $-0.080 \%$ & $\mathrm{~N} / \mathrm{C}$ & 12.04 & 12.06 & & 578 & 380 & \\
\hline & D2 & & & & & 12.04 & 11.64 & $-0.94 \%$ & 578 & 382 & $-32.87 \%$ \\
\hline & & & & & & 12.04 & 12.08 & & 578 & 402 & \\
\hline & 923 & 22.8186 & 22.8406 & $0.096 \%$ & $\mathrm{~N} / \mathrm{C}$ & 16.76 & 12.82 & & 606 & 550 & \\
\hline & E2 & & & & & 16.76 & \begin{tabular}{|l|}
10.99 \\
\end{tabular} & $-28.97 \%$ & 606 & 404 & $-25.80 \%$ \\
\hline & & & & & & 16.76 & broke & & 606 & 395 & \\
\hline & $150-800$ & 21.6972 & 21.7068 & $0.044 \%$ & $\mathrm{~N} / \mathrm{C}$ & 19.08 & \begin{tabular}{|l|}
14.63 \\
\end{tabular} & & 580 & 290 & \\
\hline & $\mathrm{F} 2$ & & & & & 19.08 & 17.20 & $-22.10 \%$ & 580 & 262 & $.51 .84 \%$ \\
\hline & & & & & & 19.08 & 12.76 & & 580 & 286 & \\
\hline
\end{tabular}


TWISTED PAIRSMIRE BMITH VARNISH -HCFC.22/Suniso 3GS oil @260 $\mathrm{F}\left(127^{\circ} \mathrm{C}\right)$

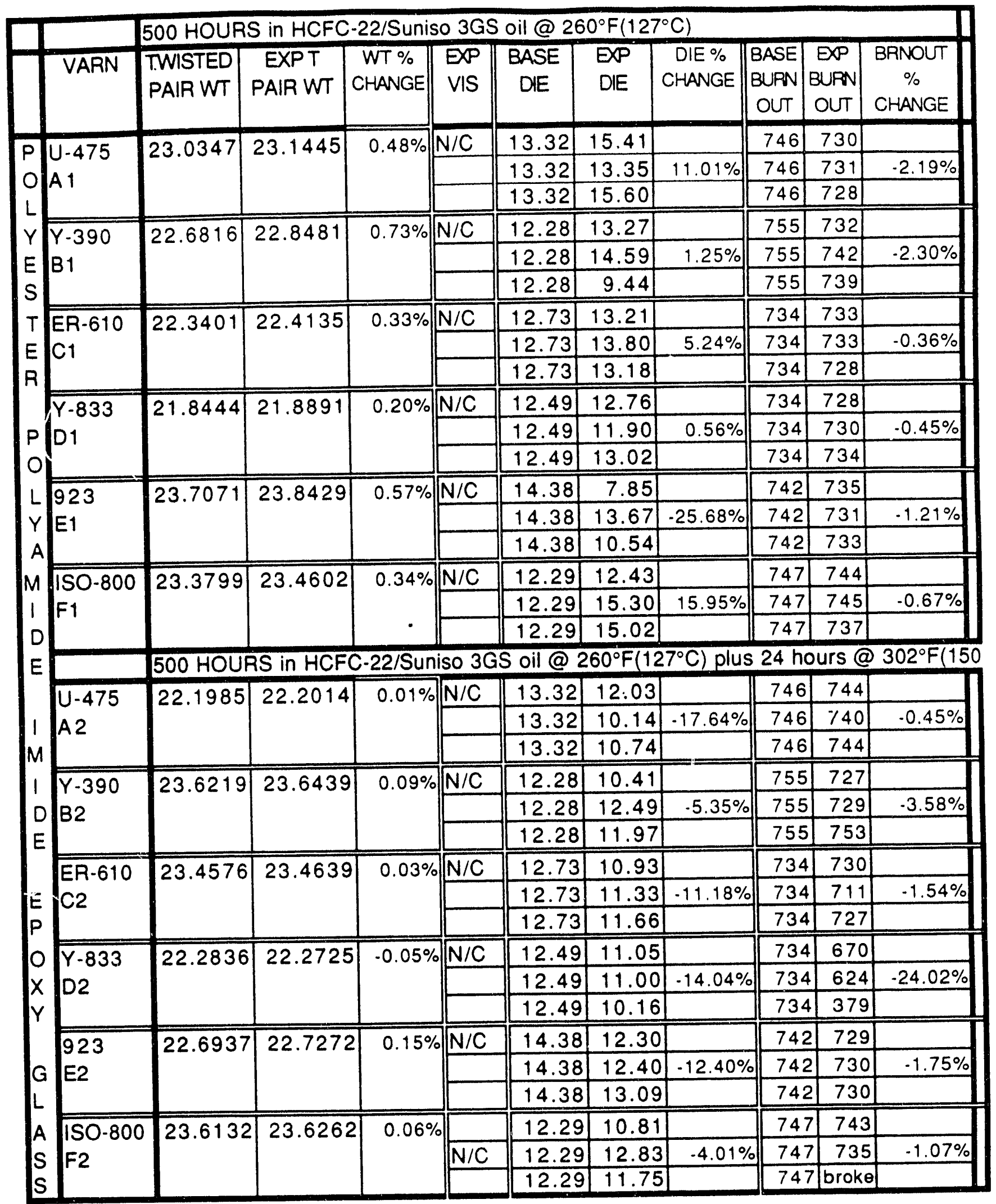


TWISTED PAIRSMIRE CMITH VARNISH-HCFC-22/SUniso 3GS @ $260^{\circ} \mathrm{F}\left(127^{\circ} \mathrm{C}\right)$

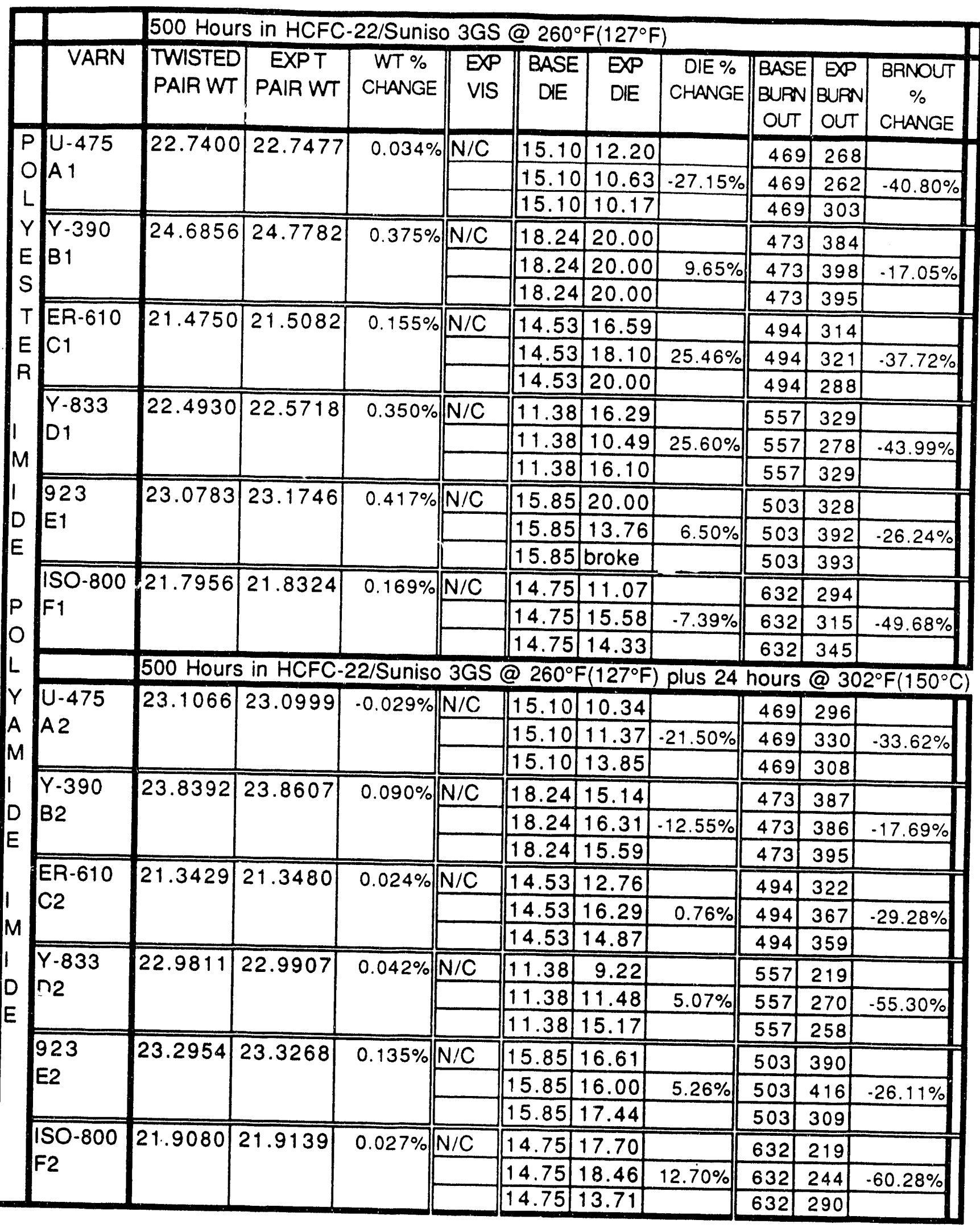


HELICAL COILSMIRE A--.HCFC-22/Suniso 3GS oil @260'F(127'C)

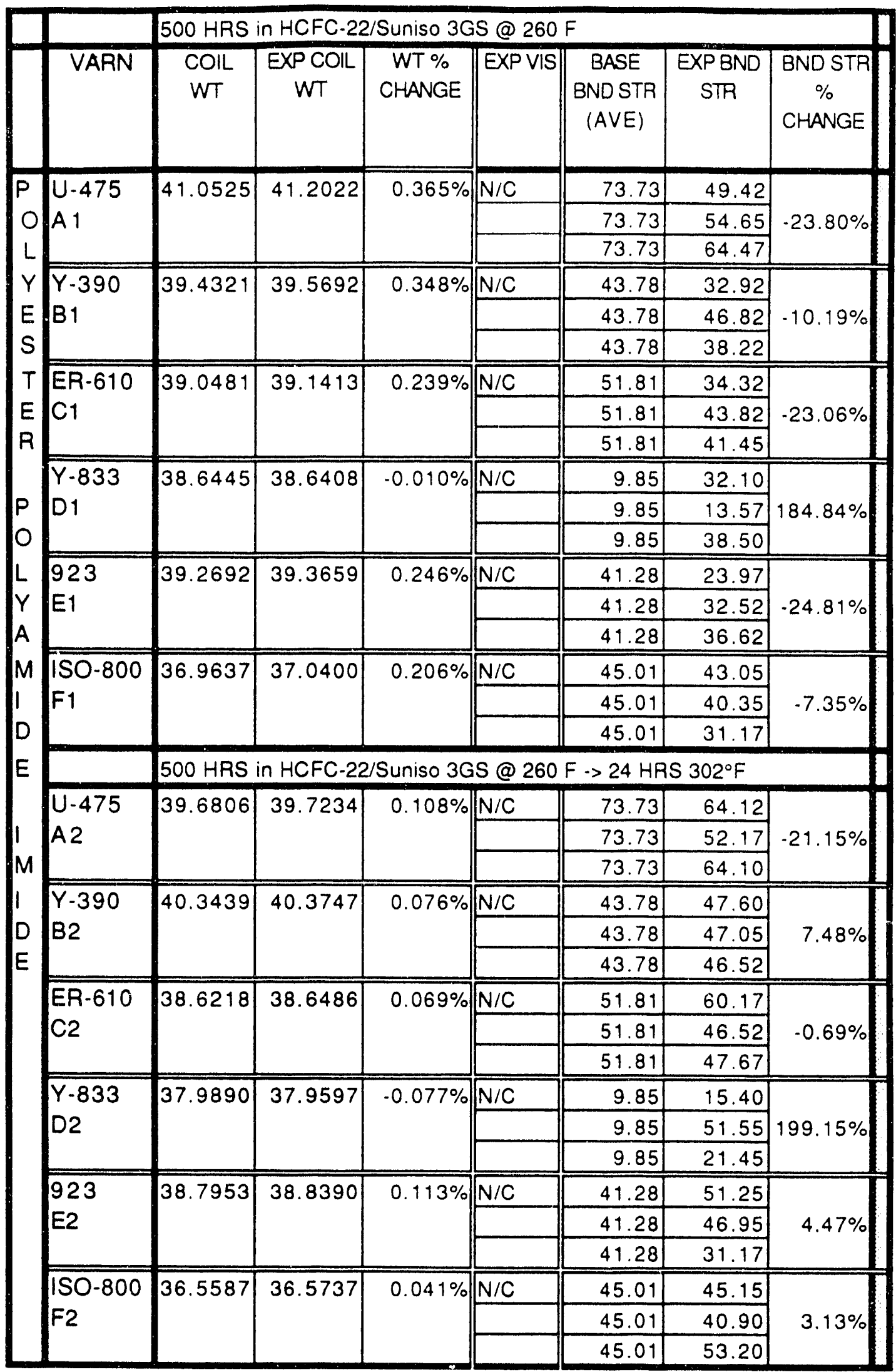




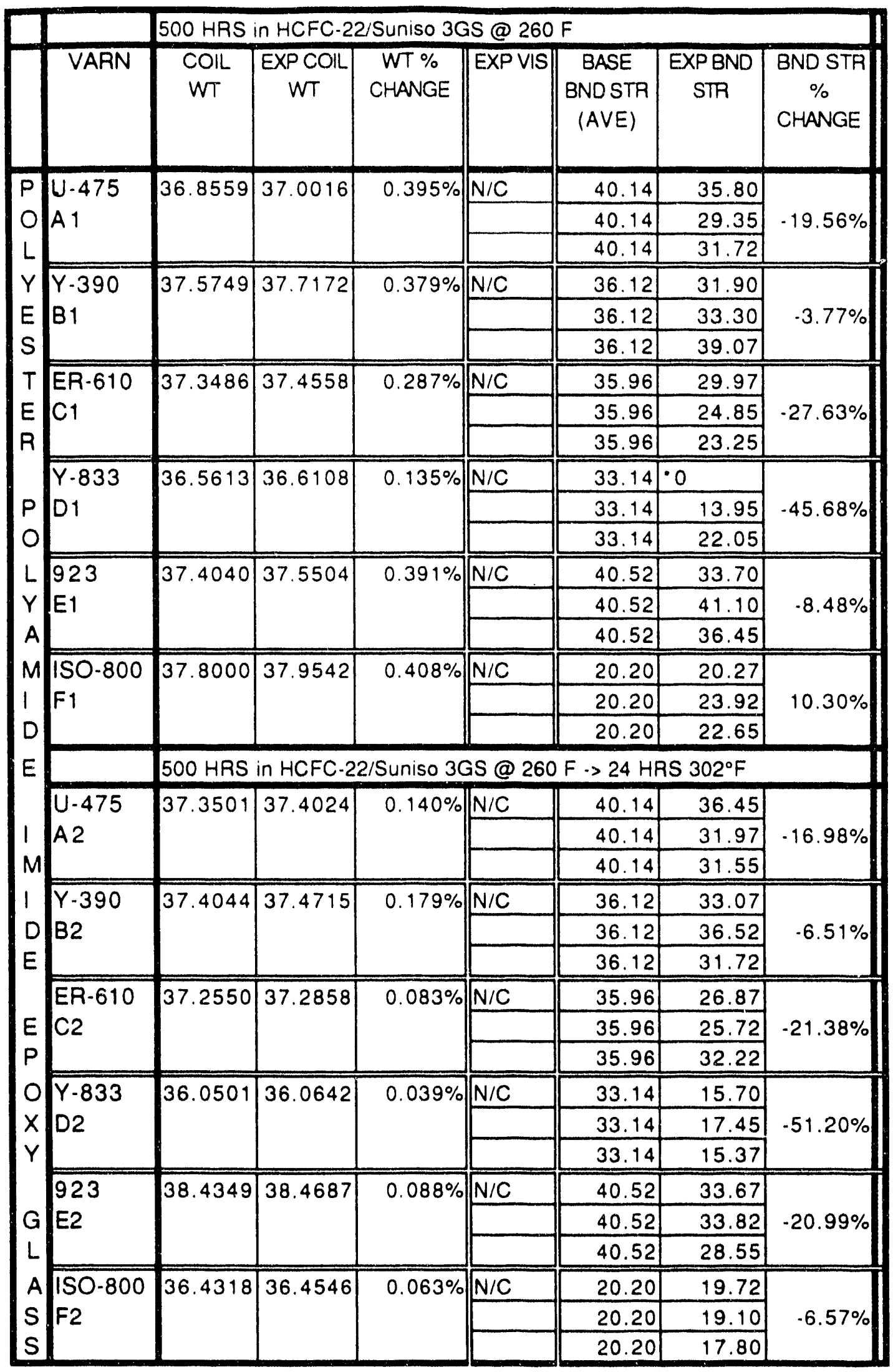




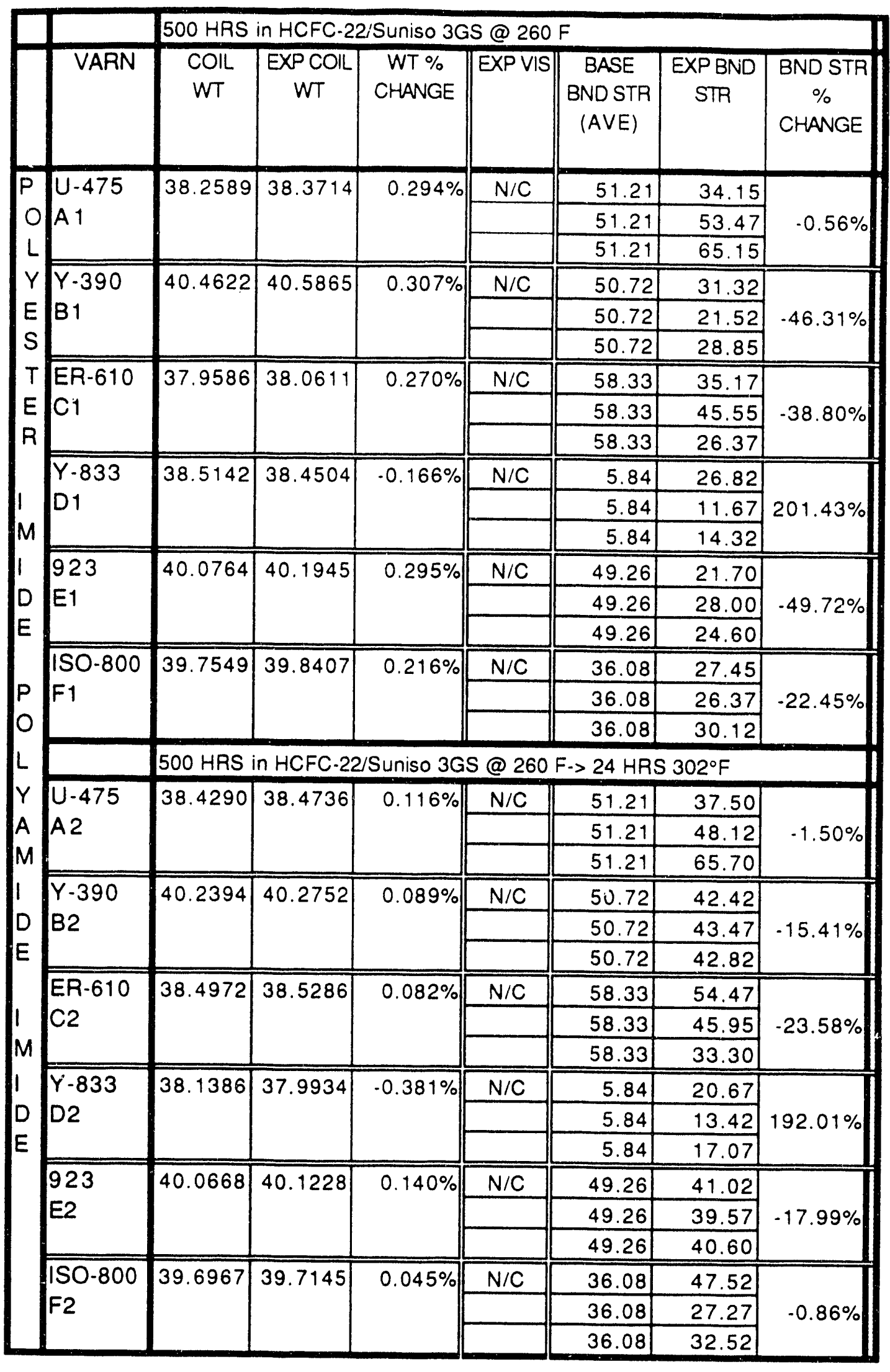




\begin{tabular}{|c|c|c|c|c|c|}
\hline & $500 \mathrm{HRS}$ in & HCFC-22/ & niso 3GS & (c) 250 & (1) \\
\hline ID & $W_{T}$ & EXPWT & $\begin{array}{c}\text { WT \% } \\
\text { CHANGE }\end{array}$ & EXPVIS & EXPFLEX \\
\hline A1 & 4.2337 & 4.2342 & $0.012 \%$ & $\mathrm{~N} / \mathrm{C}$ & \\
\hline & & & & & YES \\
\hline $\mathrm{B} 1_{1}$ & 61380 & 61643 & $0414 \%$ & $N / C$ & \\
\hline & & & & & YES \\
\hline & & & & & \\
\hline C1 & 5.5328 & 5.5383 & $0.099 \%$ & $\mathrm{~N} / \mathrm{C}$ & \\
\hline & & & & & YES \\
\hline & & & & & \\
\hline & $500 \mathrm{HRS}$ & HCFC. 22 & Suniso $3 G S-$ & $>302^{\circ} \mathrm{F} \mathrm{fc}$ & I 24 HRS \\
\hline$A 2$ & 4.2951 & 4.2928 & $-0.054 \%$ & $\mathrm{~N} / \mathrm{C}$ & \\
\hline & & & & & YES \\
\hline 82 & 61086 & 6.1197 & $0.182 \%$ & $N / C$ & \\
\hline & & & & & YES \\
\hline & & & & & \\
\hline $\mathrm{C} 2$ & 5.6362 & 5.6371 & $0.016 \%$ & $\mathrm{~N} / \mathrm{C}$ & \\
\hline & & & & & YES \\
\hline
\end{tabular}




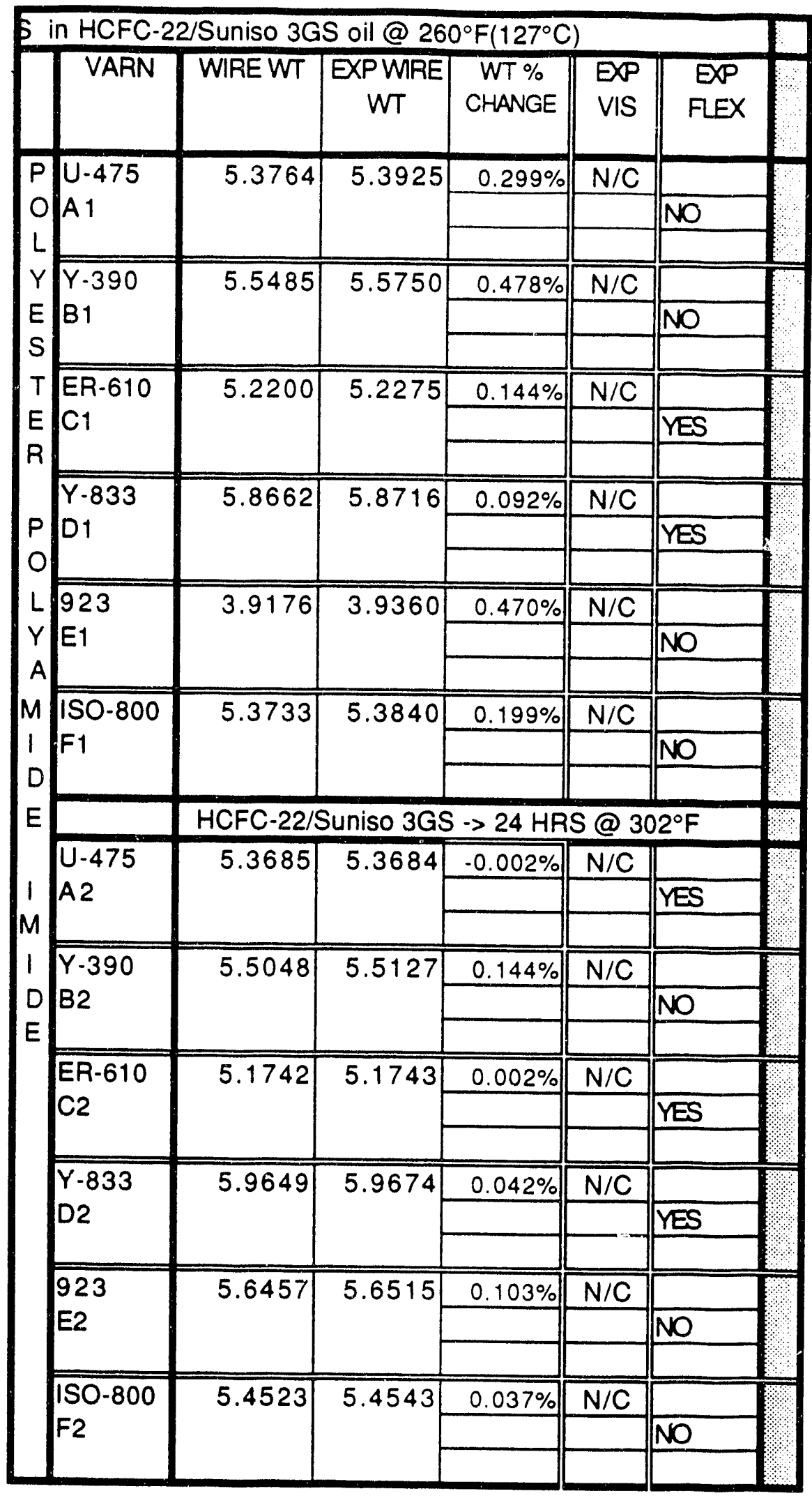




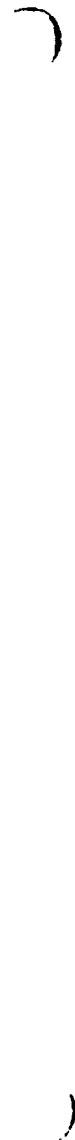

\begin{tabular}{|c|c|c|c|c|c|c|c|}
\hline & & $500 \mathrm{HRS}$ & in $\mathrm{HCFC}-2$ & ISuniso $3 C$ & is oil@ & $260^{\circ} \mathrm{Fl}$ & $77^{\circ}$ \\
\hline & VARN & WIREWT & $\begin{array}{c}\text { EXPWIRE } \\
W T\end{array}$ & $\begin{array}{l}\text { WT \% } \\
\text { CHANGE }\end{array}$ & EXP VIS & \begin{tabular}{|l} 
EXP \\
FLEX
\end{tabular} & \\
\hline $\mathrm{P}$ & U-475 & 5.4995 & 5.5191 & $0.356 \%$ & $\mathrm{~N} / \mathrm{C}$ & & \\
\hline 이 & & & & & & No & \\
\hline L & & & & & & & \\
\hline Y & $Y-390$ & 5.8081 & 5.8414 & $0.573 \%$ & $\mathrm{~N} / \mathrm{C}$ & & \\
\hline E & & & & & & NO & \\
\hline$T$ & & & & & & & \\
\hline E & $\begin{array}{l}\text { ER-610 } \\
\text { C1 }\end{array}$ & 5.4412 & 5.4533 & $0.222 \%$ & $\mathrm{~N} / \mathrm{C}$ & & \\
\hline$R$ & & & & & & & \\
\hline & $Y-8.33$ & 5.6020 & 5.6167 & $0.262 \%$ & $\mathrm{~N} / \mathrm{C}$ & & \\
\hline $\mathrm{P}$ & & & & & & YES & \\
\hline 0 & & & & & & & \\
\hline L & 923 & 5.5039 & 5.5485 & $0.810 \%$ & $\mathrm{~N} / \mathrm{C}$ & & \\
\hline Y & E1 & & & & & NO & \\
\hline A & & & & & & & \\
\hline$M$ & $150-800$ & 5.4714 & 5.4866 & $0.278 \%$ & $N / C$ & & \\
\hline 1 & & & & & & $\mathrm{NO}$ & \\
\hline$D$ & & & & & & & \\
\hline$E$ & & HCFC-22 & 2/Suniso $3 G$ & @ 260 26 & $\left(127^{\circ} \mathrm{C}\right)$ & & \\
\hline & U-475 & 5.6013 & 5.6000 & $-0.023 \%$ & $\mathrm{~N} / \mathrm{C}$ & & \\
\hline 1 & & & & & & NO & \\
\hline M & & & & & & & \\
\hline 1 & $Y-390$ & 5.7672 & 5.7729 & $0.099 \%$ & $\mathrm{~N} / \mathrm{C}$ & & \\
\hline D & B2 & & & & & No & \\
\hline & ER-610 & 5.5532 & 5.5561 & $0.052 \%$ & $\mathrm{~N} / \mathrm{C}$ & & \\
\hline$E$ & & & & & & NO & \\
\hline$P$ & & & & & & & \\
\hline 0 & $Y-833$ & 5.6848 & 5.6895 & $0.083 \%$ & $\mathrm{~N} / \mathrm{C}$ & & \\
\hline$x$ & & & & & & YES & \\
\hline & 923 & 5.5288 & 5.5444 & $0.282 \%$ & $\mathrm{~N} / \mathrm{C}$ & & \\
\hline$G$ & E2 & & & & & NO & \\
\hline L & & & & & & & \\
\hline$A$ & ISO-800 & 5.5583 & 5.5619 & $0.065 \%$ & $\mathrm{~N} / \mathrm{C}$ & & \\
\hline$S$ & & & & & & NO & \\
\hline
\end{tabular}




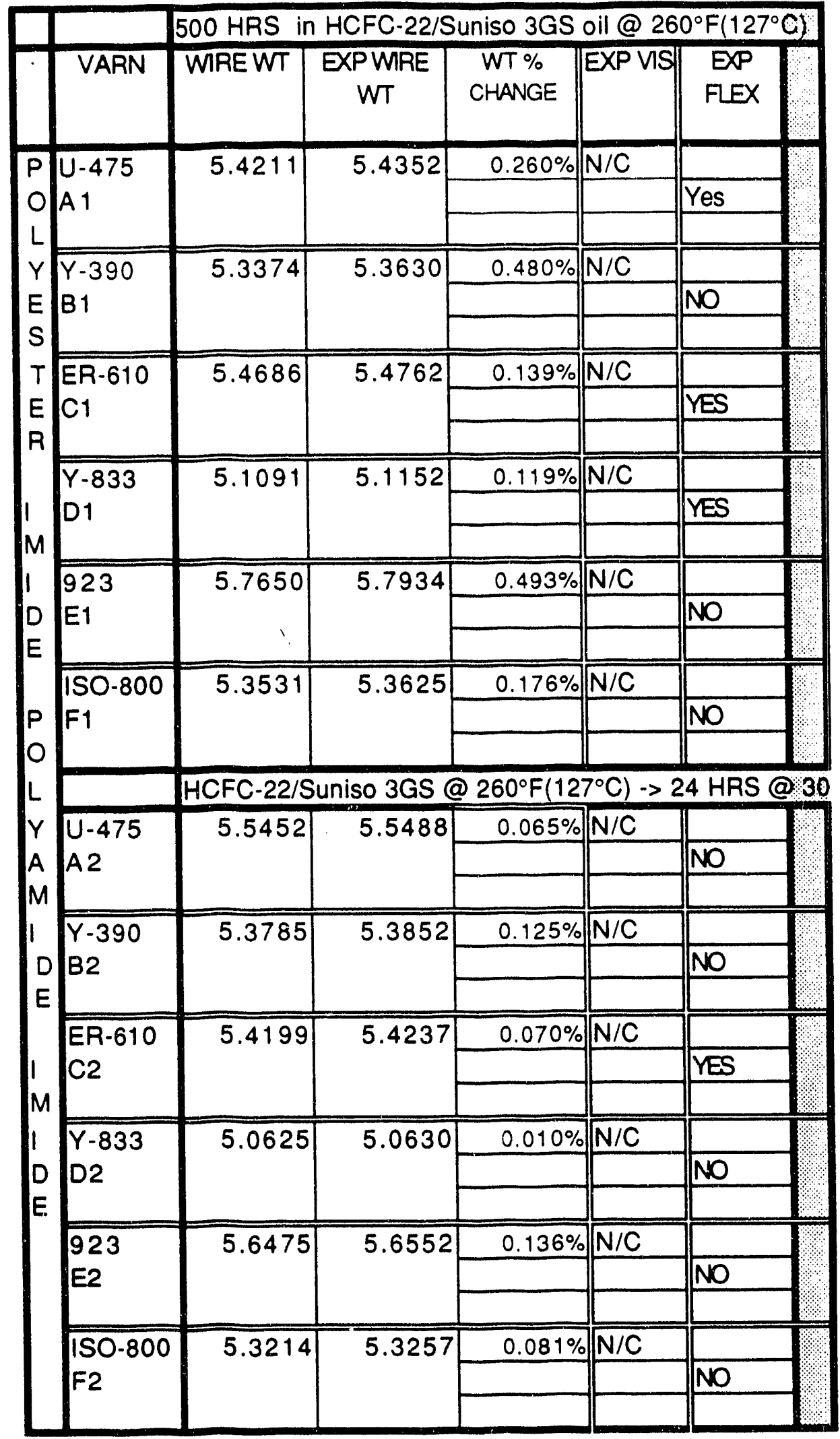


Varnish Disks-...HCFC-22/Suniso 3GS Oil

\begin{tabular}{|c|c|c|c|c|c|}
\hline \multicolumn{6}{|c|}{500 HRS IN R-22/Suniso 3Gs @ 260 F } \\
\hline ID & WT & EXPWT & $\begin{array}{c}\text { WT \% } \\
\text { CHANGE }\end{array}$ & EXP VIS & EXPFLEX \\
\hline $\begin{array}{l}U-475 \\
\text { A } 1\end{array}$ & 2.2186 & 2.3196 & $4.55 \%$ & $N / C$ & $\mathrm{~N} / \mathrm{C}$ \\
\hline $\begin{array}{l}Y-390 \\
B 1\end{array}$ & 2.0067 & 2.1010 & $4.70 \%$ & $\begin{array}{l}\text { slightly } \\
\text { warped }\end{array}$ & $\mathrm{N} / \mathrm{C}$ \\
\hline ER-610 & 1.9738 & 2.0926 & $6.02 \%$ & $\mathrm{~N} / \mathrm{C}$ & $N / C$ \\
\hline $\begin{array}{l}Y-833 \\
D 1\end{array}$ & 2.1735 & 2.2576 & $3.87 \%$ & $\mathrm{~N} / \mathrm{C}$ & $\mathrm{N} / \mathrm{C}$ \\
\hline $\begin{array}{l}923 \\
E 1\end{array}$ & 1.5719 & 1.5816 & $0.62 \%$ & $N / C$ & $N / C$ \\
\hline $150-800$ & 1.6531 & 1.6038 & $-2.98 \%$ & & $\mathrm{~N} / \mathrm{C}$ \\
\hline & & & & warped & \\
\hline & R22/Suniso & $3 G S$ at $\cdots>$ & 4 hour at 3 & $302^{\circ} \mathrm{F}$. & \\
\hline U.475 & 1.9055 & 1.9181 & $0.66 \%$ & darkened & $\mathrm{N} / \mathrm{C}$ \\
\hline $\begin{array}{l}Y \cdot 390 \\
B 2\end{array}$ & 1.9896 & 1.9580 & $-1.59 \%$ & $\begin{array}{l}\text { slightly } \\
\text { warped }\end{array}$ & $\mathrm{N} / \mathrm{C}$ \\
\hline ER-610 & 2.4213 & 2.4799 & $2.42 \%$ & darkened & $\mathrm{N} / \mathrm{C}$ \\
\hline$C 2$ & & & & & \\
\hline Y-833 & 2.5593 & 2.5927 & $1.31 \% \|$ & $\mathrm{N} / \mathrm{C}$ & $\mathrm{N} / \mathrm{C}$ \\
\hline D2 & & & & & \\
\hline 923 & 1.5617 & 1.5363 & $-1.63 \%$ & & \\
\hline E2 & & & & $\mathrm{N} / \mathrm{C}$ & $\mathrm{N} / \mathrm{C}$ \\
\hline ISO-800 & 1.4199 & 1.3553 & $-4.55 \%$ & very & \\
\hline F2 & & & & warped & $N / C$ \\
\hline
\end{tabular}




\begin{tabular}{|c|c|c|c|c|c|c|c|c|c|}
\hline \multicolumn{10}{|c|}{500 HRS in HCFC-22/Suniso 3GS @ 260 F } \\
\hline 10 & WT & EXPWT & $\begin{array}{c}\text { WT \% } \\
\text { CHANGE }\end{array}$ & $\begin{array}{l}\text { SAMPLE } \\
\text { WIDTH }\end{array}$ & $\begin{array}{l}\text { BREAK } \\
\text { LOAD }\end{array}$ & $\begin{array}{c}\text { SAMPLE } \\
\text { THICKNESS } \\
\text { Mils }\end{array}$ & $\begin{array}{c}\text { TENSILE } \\
\text { STR } \\
\text { BASE } \\
\end{array}$ & $\begin{array}{l}\text { TENSILE } \\
\text { STREXP }\end{array}$ & $\begin{array}{c}\% \text { CHANGE } \\
\text { TENSILE }\end{array}$ \\
\hline \multirow{3}{*}{$\begin{array}{l}\text { NO/MY/NO } \\
\text { A1 }\end{array}$} & \multirow{3}{*}{4.8759} & \multirow[t]{3}{*}{5.4523} & \multirow[t]{3}{*}{$11.82 \%$} & 0.335 & 53.45 & 21 & 17.4 & 7.60 & \\
\hline & & & & 0.376 & 62.27 & 21 & 17.4 & 7.89 & $.54 .64 \%$ \\
\hline & & & & 0.496 & 85.37 & 21 & 17.4 & 8.20 & \\
\hline \multirow{3}{*}{$\begin{array}{l}\text { DA/MY/DA } \\
\text { B1 }\end{array}$} & \multirow[t]{3}{*}{4.1338} & \multirow[t]{3}{*}{4.2629} & \multirow[t]{3}{*}{$3.12 \%$} & 0.398 & 14.00 & 21 & 13.7 & 1.68 & \\
\hline & & & & 0.385 & 14.00 & 21 & 13.7 & 1.73 & $-88.16 \%$ \\
\hline & & & & 0.429 & 13.15 & 21 & 13.7 & 1.46 & \\
\hline \multirow{3}{*}{$\begin{array}{l}\text { MYLAR MO } \\
\text { C1 }\end{array}$} & \multirow[t]{3}{*}{2.2119} & \multirow[t]{3}{*}{2.2635} & \multirow[t]{3}{*}{$2.33 \%$} & 0.390 & 0.00 & 10 & 21.7 & 0.00 & \\
\hline & & & & 0.450 & 0.00 & 10 & 21.7 & 0.00 & $.100 .00 \%$ \\
\hline & & & & 0.429 & 0.00 & 10 & 21.7 & 0.00 & \\
\hline \multirow{3}{*}{$\begin{array}{l}\text { NO } 410 \\
\text { D1 }\end{array}$} & \multirow[t]{3}{*}{2.2203} & 2.5243 & $13.69 \%$ & 0.456 & 76.20 & 10 & 18.7 & 16.71 & \\
\hline & & & & 0.430 & 77.52 & 10 & 18.7 & 18.03 & $-5.47 \%$ \\
\hline & & & & 0.495 & 90.55 & 10 & 18.7 & 18.29 & \\
\hline NO MI 418 & 2.3705 & 2.9645 & $25.06 \%$ & 0.485 & 28.62 & 10 & 7.5 & 5.90 & \\
\hline E1 & & & & 0.501 & 29.10 & 10 & 7.5 & 5.81 & $-20.74 \%$ \\
\hline & & & & 0.489 & 29.95 & 10 & 7.5 & 6.12 & \\
\hline MEL 228 & 2.4124 & 2.5093 & $4.02 \%$ & 0.498 & 0.00 & 10 & 21.7 & 0.00 & \\
\hline $\mathrm{F} 1$ & & & & 0.438 & 0.00 & 10 & 21.7 & 0.00 & $-100.00 \%$ \\
\hline & & & & 0.487 & 0.00 & 10 & 21.7 & 0.00 & \\
\hline & & 500 HRS & $\mathrm{S}$ in $\mathrm{HCF}$ & ;-22/Sunis & o 3GS@ & $260 \mathrm{~F}->24$ & HRS@3 & $02^{\circ} \mathrm{F}$ & \\
\hline NO/MY/NO & 4.3581 & 4.5743 & $4.96 \%$ & 0.560 & 21.05 & 21 & 17.4 & 1.79 & \\
\hline$A 2$ & & & & 0.480 & 82.05 & 21 & 17.4 & 8.14 & $.69 .86 \%$ \\
\hline & & & & 0.512 & 62.40 & 21 & 17.4 & 5.80 & \\
\hline $\mathrm{DA} / \mathrm{MY} / \mathrm{DA}$ & 4.3440 & 4.3395 & $-0.10 \%$ & 0.332 & 12.00 & 21 & 13.7 & 1.72 & \\
\hline $\mathrm{B2}$ & & & & 0.415 & 18.00 & 21 & 13.7 & 2.07 & $.85 .61 \%$ \\
\hline & & & & 0.398 & 17.80 & 21 & 13.7 & 2.13 & \\
\hline MYLARMO & 2.2041 & 2.2105 & $0.29 \%$ & 0.401 & 0.00 & 10 & 21.7 & 0.00 & \\
\hline $\mathrm{C} 2$ & & & & 0.405 & 0.00 & 10 & 21.7 & 0.00 & $-100.00 \%$ \\
\hline & & & & 0.418 & 0.00 & 10 & 21.7 & 0.00 & \\
\hline NOMEX 410 & 2.0491 & 2.1781 & $6.30 \%$ & 0.505 & 94.02 & 10 & 18.7 & 18.62 & \\
\hline D2 & & & & 0.509 & 94.32 & 10 & 18.7 & 18.53 & $0.52 \%$ \\
\hline & & & & 0.468 & 90.05 & 10 & 18.7 & 19.24 & \\
\hline $\mathrm{NO} / \mathrm{MI} 418$ & 2.6279 & 2.9851 & $13.59 \%$ & 0.509 & 28.60 & 10 & 7.5 & 5.62 & \\
\hline E2 & & & & 0.502 & 26.90 & 10 & 7.5 & 5.36 & $-27.13 \%$ \\
\hline & & & & 0.502 & 27.20 & 10 & 7.5 & 5.42 & \\
\hline MEL 228 & 2.2579 & 2.3071 & $2.18 \%$ & 0.448 & 0.00 & 10 & 21.7 & 0.00 & \\
\hline & & & & 0.475 & 0.00 & 10 & 21.7 & 0.00 & $-100.00 \%$ \\
\hline & & & & 0.468 & 0.00 & 10 & 21.7 & $0.0 \mathrm{C}$ & \\
\hline
\end{tabular}




\begin{tabular}{|c|c|c|c|c|c|c|c|c|}
\hline $\begin{array}{c}500 \mathrm{HRS} \\
10\end{array}$ & \begin{tabular}{|} 
EAfter 500 \\
$\begin{array}{c}\text { STF }=\mathrm{TCH} \\
\text { (inch) }\end{array}$
\end{tabular} & $\begin{array}{c}\text { hour e } \\
\% \\
\text { ELONG }\end{array}$ & $\begin{array}{l}\text { posure to } \\
\text { BASE } \\
\text { ELONG } \\
\text { (AVE) }\end{array}$ & $\begin{array}{l}\text { Nitrogen } \\
\text { ELONG \% } \\
\text { CHANGE }\end{array}$ & $\begin{array}{c}\text { BASE } \\
\text { DIE } \\
\text { (AVE) }\end{array}$ & EXP DIE & $\begin{array}{c}\text { DIE \% } \\
\text { CHANGE }\end{array}$ & VISUAL EXP \\
\hline \multirow{3}{*}{$\begin{array}{l}\text { NO/MY/NO } \\
\text { A1 }\end{array}$} & 0.14 & $3.5 \%$ & $20.0 \%$ & & $>18.97$ & $>18.88$ & & \multirow{3}{*}{$\mid \begin{array}{l}\text { Mylar brittle } \\
\text { discolored } \\
\text { yellowed }\end{array}$} \\
\hline & 0.19 & $4.8 \%$ & $20.0 \%$ & $.79 .4 \%$ & $>18.97$ & $>18.13$ & flash & \\
\hline & 0.17 & $4.3 \%$ & $20.0 \%$ & & $>18.97$ & $>18.35$ & & \\
\hline \multirow{3}{*}{$\begin{array}{l}D A / M Y / D A \\
B 1\end{array}$} & 0.00 & $0.0 \%$ & $46.0 \%$ & & $>15.27$ & $>12.71$ & & \multirow{3}{*}{$\begin{array}{l}\text { Mylar Brittle } \\
\text { Dielectric went } \\
\text { through crack }\end{array}$} \\
\hline & 0.00 & $0.0 \%$ & $46.0 \%$ & $.99 .3 \%$ & $>15.27$ & $>13.91$ & flash & \\
\hline & 0.02 & $1.0 \%$ & $46.0 \%$ & & $>15.27$ & 11.39 & & \\
\hline \multirow{3}{*}{$\begin{array}{l}\text { MYLAR MO } \\
\text { C1 }\end{array}$} & 0.00 & $0.0 \%$ & $131.0 \%$ & & $>14.91$ & $\geq 12.41$ & & \multirow{3}{*}{$\begin{array}{l}\text { Mylar brittle } \\
\text { discolored } \\
\text { yellowed }\end{array}$} \\
\hline & 0.00 & $0.0 \%$ & $131.0 \%$ & $-100.0 \%$ & $>14.91$ & $>12.57$ & flash & \\
\hline & 0.00 & $0.0 \%$ & $131.0 \%$ & & $>14.91$ & $>12.39$ & & \\
\hline \multirow{3}{*}{$\begin{array}{l}\text { NO } 410 \\
\text { D1 }\end{array}$} & 0.18 & $4.5 \%$ & $17.0 \%$ & & 10.67 & $>12.29$ & & \multirow{3}{*}{$\begin{array}{l}\text { oil in nomex } \\
\text { raised dielectric }\end{array}$} \\
\hline & 0.22 & $5.5 \%$ & $17.0 \%$ & $-69.6 \%$ & 10.67 & $>12.47$ & flash & \\
\hline & 0.22 & $5.5 \%$ & $17.0 \%$ & & 10.67 & $>12.03$ & & \\
\hline \multirow{3}{*}{$\begin{array}{l}\text { NO Ml } 418 \\
\text { E1 }\end{array}$} & 0.05 & $1.3 \%$ & $4.0 \%$ & & 10.23 & 11.79 & & \multirow{3}{*}{ oil in insulation } \\
\hline & 0.04 & $1.0 \%$ & $4.0 \%$ & $-77.1 \%$ & 10.23 & 12.23 & $18.1 \%$ & \\
\hline & 0.02 & $0.5 \%$ & $4.0 \%$ & & 10.23 & 12.24 & & \\
\hline \multirow{4}{*}{$\begin{array}{l}\text { MEL } 228 \\
\text { F1 }\end{array}$} & 0.00 & $0.0 \%$ & $160.0 \%$ & & $>14.22$ & $>12.45$ & & \multirow{3}{*}{$\begin{array}{l}\text { Brittle,discolored } \\
\text { yellowed }\end{array}$} \\
\hline & 0.00 & $0.0 \%$ & $160.0 \%$ & $.100 .0 \%$ & $>14.22$ & $\geq 13.52$ & flash & \\
\hline & 0.00 & $0.0 \%$ & $160.0 \%$ & & $>14.22$ & $=13.13$ & & \\
\hline & \multicolumn{7}{|c|}{ After 500 hour exposure plus a 24 hourairbake at 15} & F) \\
\hline \multirow{3}{*}{$\begin{array}{l}\text { NO/MY/NO } \\
\text { A2 }\end{array}$} & 0.02 & $0.5 \%$ & $20.0 \%$ & & $>18.97$ & 11.68 & & \multirow{3}{*}{$\begin{array}{l}\text { darkened } \\
\text { delamination } \\
\text { dielectric failed }\end{array}$} \\
\hline & 0.34 & $8.5 \%$ & $20.0 \%$ & $.78 .3 \%$ & $>18.97$ & 12.36 & flash & \\
\hline & 0.16 & $4.0 \%$ & $20.0 \%$ & & $>18.97$ & 16.72 & & \\
\hline \multirow{3}{*}{$\begin{array}{l}\text { DA/MY/DA } \\
\text { B2 }\end{array}$} & 0.03 & $1.5 \%$ & $46.0 \%$ & & $>15.27$ & $\geq 16.09$ & & \multirow{3}{*}{$\begin{array}{l}\text { darkened } \\
\text { delamination } \\
\text { mylar brown }\end{array}$} \\
\hline & 0.04 & $2.0 \%$ & $46.0 \%$ & $.96 .0 \%$ & $>15.27$ & $\geq 15.23$ & flash & \\
\hline & 0.04 & $2.0 \%$ & $46.0 \%$ & & -15.27 & $>14.34$ & & \\
\hline \multirow{3}{*}{$\begin{array}{l}\text { MYLAR MO } \\
\text { C2 }\end{array}$} & 0.00 & $0.0 \%$ & $131.0 \%$ & & $>14.91$ & $>13.04$ & & \multirow{3}{*}{$\begin{array}{l}\text { brittle, mylar } \\
\text { brown }\end{array}$} \\
\hline & 0.00 & $0.0 \%$ & $131.0 \%$ & $.100 .0 \%$ & $>14.91$ & $>12.24$ & flash & \\
\hline & 0.00 & $0.0 \%$ & $131.0 \%$ & & $\geq 14.91$ & $>13.27$ & & \\
\hline \multirow{3}{*}{$\begin{array}{l}\text { NOMEX } 410 \\
\text { D2 }\end{array}$} & 0.16 & $4.0 \%$ & $17.0 \%$ & & 10.67 & 10.13 & & \multirow{3}{*}{$\sqrt{N / C}$} \\
\hline & 0.16 & $4.0 \%$ & $17.0 \%$ & $.77 .0 \%$ & 10.67 & 9.93 & $-3.8 \%$ & \\
\hline & 0.15 & $3.8 \%$ & $17.0 \%$ & & 10.67 & 10.72 & & \\
\hline \multirow{3}{*}{$\begin{array}{l}\text { NO/MI } 418 \\
\text { E2 }\end{array}$} & 0.04 & $1.0 \%$ & $4.0 \%$ & & 10.23 & 10.33 & & \multirow{3}{*}{$\mathrm{N} / \mathrm{C}$} \\
\hline & 0.03 & $0.8 \%$ & $4.0 \%$ & $.75 .0 \%$ & 10.23 & 9.29 & $-0.8 \%$ & \\
\hline & 0.05 & $1.3 \%$ & $4.0 \%$ & & 10.23 & 10.81 & & \\
\hline \multirow{3}{*}{$\begin{array}{l}\text { MEL } 228 \\
\text { F2 }\end{array}$} & 0.00 & $0.0 \%$ & $160.0 \%$ & & $>14.22$ & $>12.9$ & & mylar brown \\
\hline & 0.00 & $0.0 \%$ & $160.0 \%$ & $-100.0 \%$ & $>14.22$ & $>13.17$ & flash & brittle \\
\hline & 0.00 & $0.0 \%$ & $160.0 \%$ & & $>14.22$ & $>13.03$ & & \\
\hline
\end{tabular}




\begin{tabular}{|c|c|c|c|c|}
\hline & \multicolumn{4}{|c|}{500 HRS in HCFC-22/Suniso 3GS oil @ 260 $\mathrm{F}$} \\
\hline & WT & EXPWT & $\begin{array}{c}\% \\
\text { CHANGE }\end{array}$ & EXPVIS \\
\hline NOMEX & $9.5 ? 7 ?$ & 0.6294 & An naor. & $\begin{array}{l}\text { Able to null layars } \\
\text { of nomex apart }\end{array}$ \\
\hline $\begin{array}{l}\text { B1 } \\
\text { MYLAR }\end{array}$ & 0.5126 & 0.5294 & $3.28 \%$ & $\begin{array}{c}\text { Able to pul layers } \\
\text { of mylar apart }\end{array}$ \\
\hline C1 & 0.3928 & 0.4186 & $6.57 \%$ & $\mathrm{~N} / \mathrm{C}$ \\
\hline & HCFC-22/Su & iso $3 G S$ oil & @ 260 $\mathrm{F}$. & $-->24$ hrs @302F \\
\hline $\begin{array}{l}\text { A2 } \\
\text { NOMEX }\end{array}$ & 0.5510 & 0.5803 & $5.32 \%$ & $\begin{array}{c}\text { Nomex turned } \\
\text { darker, may be } \\
\text { due to oil. }\end{array}$ \\
\hline $\begin{array}{l}\text { B2 } \\
\text { MYLAR }\end{array}$ & 0.4835 & 0.4843 & $0.17 \%$ & \begin{tabular}{|c|} 
darkened \\
some pockets in \\
mylar, pulled away \\
\end{tabular} \\
\hline $\begin{array}{l}\text { C2 } \\
\text { NO/MY }\end{array}$ & 0.3925 & 0.3981 & $1.43 \%$ & \begin{tabular}{|c|} 
darkened \\
some pockets in \\
mylar, pulled away
\end{tabular} \\
\hline
\end{tabular}




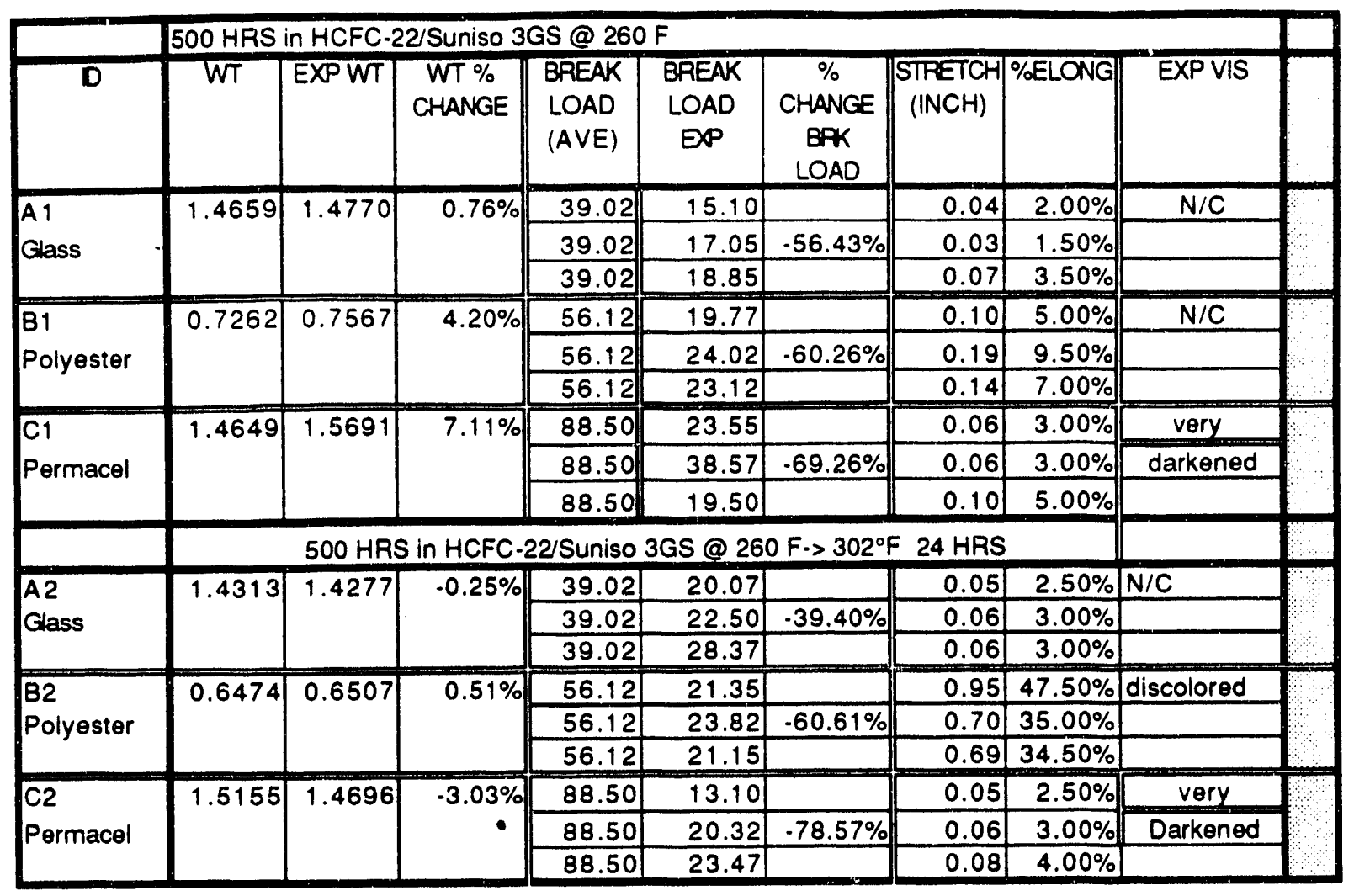


TIE CORD--HCFC-22/Suniso 3GS oil @ 260 $\mathrm{F}\left(127^{\circ} \mathrm{C}\right)$

\begin{tabular}{|c|c|c|c|c|c|c|c|c|c|}
\hline \multicolumn{10}{|c|}{500 HRS IN R.22/Suniso 3GS @ 260 F } \\
\hline ID & WT & EXPWT & $\begin{array}{c}\text { WT \% } \\
\text { CHANGE }\end{array}$ & \begin{tabular}{|l} 
EXP \\
VIS
\end{tabular} & $\begin{array}{l}\text { BREAK } \\
\text { LOAD } \\
\text { (AVE) }\end{array}$ & $\begin{array}{l}\text { BREAK } \\
\text { LOAD } \\
\text { EXP } \\
(\text { ave })\end{array}$ & $\begin{array}{c}\% \\
\text { CHANGE } \\
\text { BPK } \\
\text { LOAD }\end{array}$ & $\begin{array}{c}\text { STRETCH } \\
\text { (Inch) }\end{array}$ & $\begin{array}{c}\% \\
\text { ELONG }\end{array}$ \\
\hline \multirow[t]{4}{*}{ A 1} & \multirow[t]{3}{*}{0.2523} & \multirow[t]{3}{*}{0.2579} & \multirow[t]{3}{*}{$2.22 \%$} & $N / C$ & 28.36 & 22.25 & & 0.40 & $20.0 \%$ \\
\hline & & & & & 28.36 & 23.17 & \multirow{2}{*}{\begin{tabular}{|l|l|}
7 & $-20.97 \%$ \\
2 & \\
\end{tabular}} & 0.43 & $21.5 \%$ \\
\hline & & & & & 28.36 & 21.82 & & 0.42 & $21.0 \%$ \\
\hline & \multicolumn{9}{|c|}{500 HRS IN R-22/Suniso 3GS $>24$ HRS @ 302 $\mathrm{F}$} \\
\hline \multirow[t]{3}{*}{ A2 } & \multirow[t]{3}{*}{0.2404} & \multirow[t]{3}{*}{0.2405} & \multirow[t]{3}{*}{$0.042 \%$} & $\mathrm{~N} / \mathrm{C}$ & 28.36 & 22.55 & & 0.47 & $23.5 \%$ \\
\hline & & & & & 28.36 & 22.15 & $-21.76 \%$ & 0.45 & $22.5 \%$ \\
\hline & & & & & 28.36 & 21.87 & & 0.45 & $22.5 \%$ \\
\hline
\end{tabular}


LEAD WIRE INSULATION-HCFC-22/Suniso 3GS @ 260 $\mathrm{F}\left(127^{\circ} \mathrm{C}\right)$

\begin{tabular}{|l|r|r|r||r|r|r|r|}
\hline & 500 HRS IN HCFC-22/Suniso 3GS @ 260 & & & \\
\hline ID & WT & EXPWT & $\begin{array}{c}\text { WT \% } \\
\text { CHANGE }\end{array}$ & EXPVIS & $\begin{array}{r}\text { BASE DIE } \\
\text { (AVE) }\end{array}$ & EXPDIE & $\begin{array}{c}\text { DIE\% } \\
\text { CHANGE }\end{array}$ \\
\hline DMD & 3.9593 & 4.1507 & $4.83 \%$ & N/C & 9.61 & 8.27 & \\
\hline A1 & & & & & 9.61 & 8.81 & $-8.84 \%$ \\
\hline \hline DTMD & 4.2976 & 4.4731 & $4.08 \%$ & N/C & 9.91 & 9.20 & \\
\hline B1 & & & & & 9.95 & 11.99 & $11.06 \%$ \\
\hline & & & & & 9.95 & 10.85 & \\
\hline & & & & & & & \\
& & & & & & & \\
\hline DMD & 4.0622 & 4.0740 & $0.29 \%$ & darkened & 9.61 & 8.27 & \\
\hline A2 & & & & & 9.61 & 10.13 & $-1.01 \%$ \\
\hline & & & & & 9.61 & 10.14 & \\
\hline \hline DTMD & 4.3780 & 4.2444 & $-3.05 \%$ & darkened & 9.95 & 11.54 & \\
\hline B2 & & & & & 9.95 & 12.78 & $22.81 \%$ \\
\hline & & & & & 9.95 & 12.34 & \\
\hline
\end{tabular}




\section{Appendix C}

Experimental Data for HCFC-124/Shrieve Zerol-150 Exposure at $127^{\circ} \mathrm{C}\left(260^{\circ} \mathrm{F}\right)$ 


\begin{tabular}{|c|c|c|c|c|c|c|c|c|c|c|}
\hline 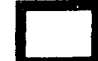 & \multicolumn{10}{|c|}{500 hours in HCFC-124/Shrieve Zerol-150 oil at $260^{\circ} \mathrm{F}\left(127^{\circ} \mathrm{C}\right)$} \\
\hline ID & WT & EXPWT & $\begin{array}{c}\text { WT \% } \\
\text { CHANGE }\end{array}$ & EXP VIS & $\begin{array}{c}\text { BASE BRN } \\
\text { OUT } \\
(\text { AVE })\end{array}$ & $\begin{array}{c}\text { EXP BRN } \\
\text { DUT }\end{array}$ & $\begin{array}{c}\text { BRN OUT } \\
\% \\
\text { CHANGE }\end{array}$ & $\begin{array}{c}\text { BASE DIE } \\
\text { (AVE) }\end{array}$ & EXP DIE & $\begin{array}{c}\text { DIE \% } \\
\text { CHANGE }\end{array}$ \\
\hline \multirow[t]{3}{*}{ A1 } & \multirow[t]{3}{*}{22.7381} & \multirow[t]{3}{*}{22.7460} & \multirow[t]{3}{*}{$0.035 \%$} & $N / C$ & 576 & 370 & & 15.80 & 13.40 & \\
\hline & & & & & 576 & 419 & $-30.6 \%$ & 15.80 & 14.94 & $-5.0 \%$ \\
\hline & & & & & 576 & 411 & & 15.80 & 16.67 & \\
\hline \multirow[t]{3}{*}{ B1 } & \multirow[t]{3}{*}{27.103} & \multirow[t]{3}{*}{27.1693} & \multirow[t]{3}{*}{$0.245 \%$} & $\mathrm{~N} / \mathrm{C}$ & 736 & 735 & & 11.62 & 12.94 & \\
\hline & & & & & 736 & 696 & $-2.1 \%$ & 11.62 & 11.63 & $6.5 \%$ \\
\hline & & & & & 736 & 730 & & 11.62 & 12.54 & \\
\hline \multirow{3}{*}{ C1 } & \multirow[t]{3}{*}{23.9575} & \multirow[t]{3}{*}{23.9641} & \multirow[t]{3}{*}{$0.028 \%$} & $\mathrm{~N} / \mathrm{C}$ & 579 & 228 & & 16.58 & 16.54 & \\
\hline & & & & & 579 & 217 & $-61.8 \%$ & 16.58 & 11.23 & $-18.6 \%$ \\
\hline & & & & & 579 & 218 & & 16.58 & 12.70 & \\
\hline & \multicolumn{10}{|c|}{500 hours in HCFC-124/Shrieve Zerol- 150 oil at $260^{\circ} \mathrm{F}\left(127^{\circ} \mathrm{C}\right)$ plus 24 hours at $302^{\circ} \mathrm{F}\left(150^{\circ} \mathrm{C}\right)$} \\
\hline \multirow[t]{3}{*}{ A2 } & \multirow[t]{3}{*}{22.3776} & \multirow[t]{3}{*}{22.3812} & \multirow[t]{3}{*}{$0.016 \%$} & $\mathrm{~N} / \mathrm{C}$ & 576 & 416 & & 15.80 & 15.64 & \\
\hline & & & & & 576 & 526 & $-14.7 \%$ & 15.80 & 15.61 & $-1.4 \%$ \\
\hline & & & & & 576 & 532 & & 15.80 & 15.49 & \\
\hline \multirow[t]{3}{*}{$B 2$} & \multirow[t]{3}{*}{27.1936} & \multirow[t]{3}{*}{27.2069} & \multirow[t]{3}{*}{$0.049 \%$} & $\mathrm{~N} / \mathrm{C}$ & 736 & 731 & & 11.62 & 11.31 & \\
\hline & & & & & 736 & 734 & $-2.1 \%$ & 11.62 & 11.16 & $-3.6 \%$ \\
\hline & & & & & 736 & 697 & & 11.62 & 11.15 & \\
\hline \multirow[t]{3}{*}{$\mathrm{C} 2$} & \multirow[t]{3}{*}{24.4878} & \multirow[t]{3}{*}{24.4921} & \multirow[t]{3}{*}{$0.018 \%$} & $\mathrm{~N} / \mathrm{C}$ & 579 & 421 & & 16.58 & 18.04 & \\
\hline & & & & & 579 & 464 & $-22.6 \%$ & 16.58 & 17.65 & $-0.1 \%$ \\
\hline & & & & & 579 & 459 & & 16.58 & 14.01 & \\
\hline
\end{tabular}




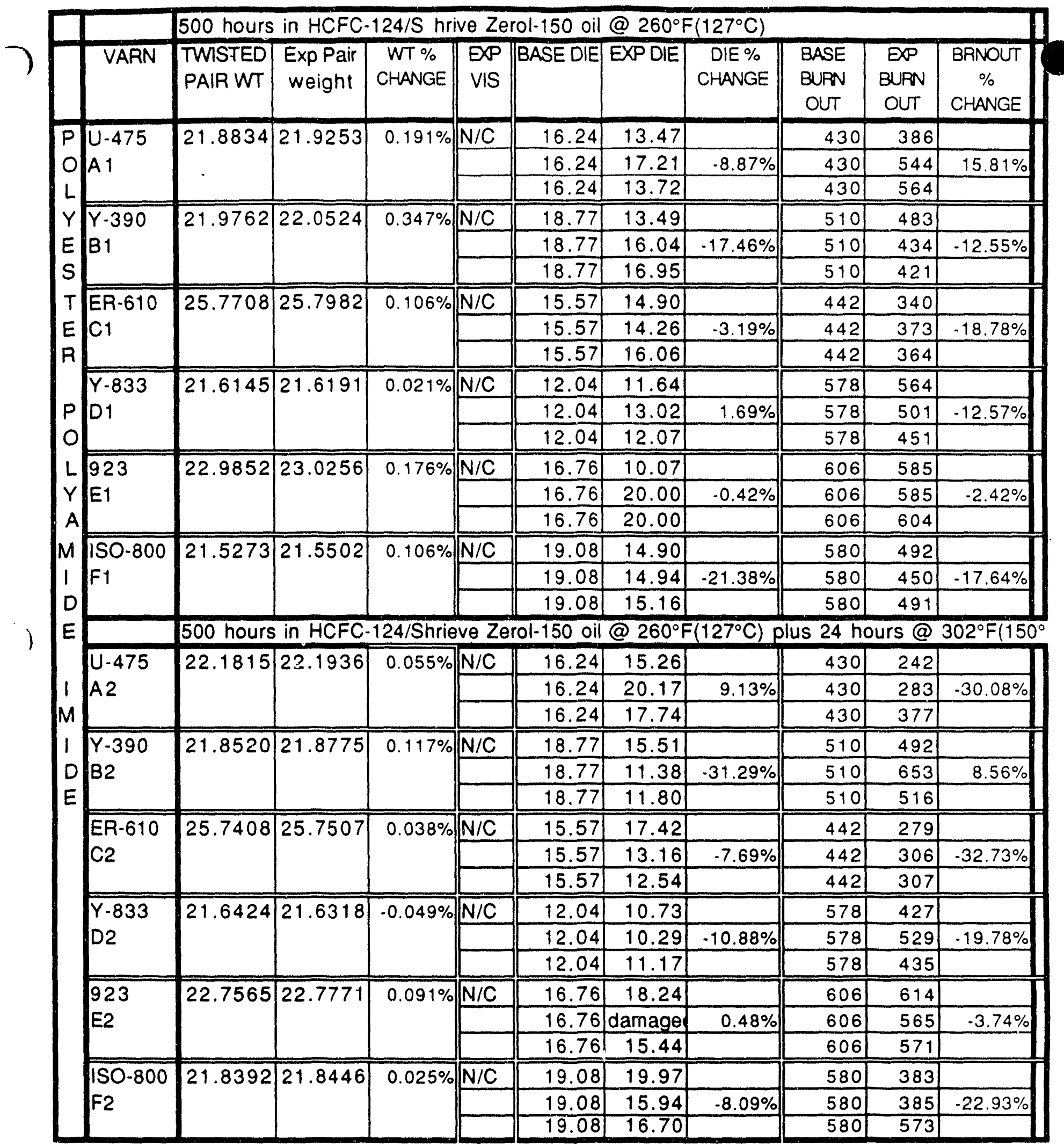


TWISTED PAIRSNIRE BNITH VARNISH -HCFC-124/S hrieve Zerol-150 oil @260 $\mathrm{F}\left(127^{\circ} \mathrm{C}\right)$

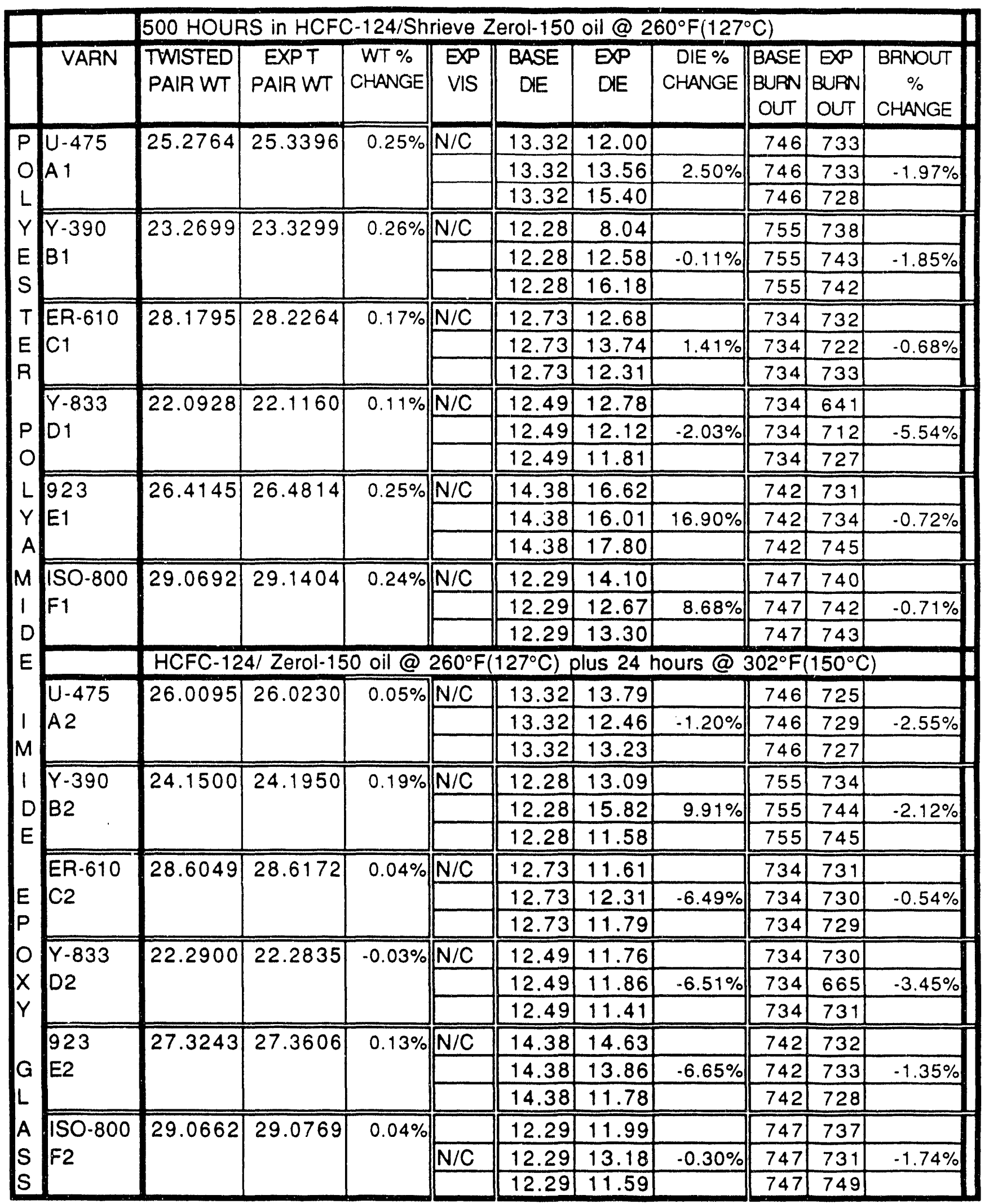


TWISTED PAIRS WIRE CNITH VARNISH-HCFC-124/S hrieve Zerol-150 @ 260 F $\left(127^{\circ} \mathrm{C}\right)$

500 Hours in HCFC-124/S hrieve Zerol-150 @ 260 $\mathrm{F}\left(127^{\circ} \mathrm{F}\right)$

\begin{tabular}{|c|c|c|c|c|c|c|c|c|c|c|}
\hline VARN & $\begin{array}{l}\text { TWISTED } \\
\text { PAIR WT }\end{array}$ & \begin{tabular}{|c|} 
EXPT \\
PAIR WT
\end{tabular} & $\begin{array}{c}\text { WT \% } \\
\text { CHANGE }\end{array}$ & $\begin{array}{l}\text { EXP } \\
\text { VIS }\end{array}$ & $\begin{array}{l}\text { BASE } \\
\text { DIE }\end{array}$ & $\begin{array}{l}\text { EXP } \\
\text { DIE }\end{array}$ & $\begin{array}{c}\text { DIE \% } \\
\text { CHANGE }\end{array}$ & $\begin{array}{l}\text { BASE } \\
\text { BURN } \\
\text { OUT }\end{array}$ & \begin{tabular}{|l|} 
EXP \\
BUPN \\
OUT
\end{tabular} & $\begin{array}{c}\text { BRNOUT } \\
\% \\
\text { CHANGE }\end{array}$ \\
\hline \multirow{3}{*}{$\begin{array}{l}U-475 \\
A 1\end{array}$} & 23.8490 & 23.8555 & $0.027 \%$ & $\mathrm{~N} / \mathrm{C}$ & 15.10 & 11.37 & & 469 & 527 & \\
\hline & & & & & 15.10 & 10.24 & $.25 .03 \%$ & 469 & 327 & $2.06 \%$ \\
\hline & & & & & 15.10 & 12.35 & & 469 & 582 & \\
\hline \multirow{3}{*}{$\begin{array}{l}Y-390 \\
B 1\end{array}$} & 26.9354 & 26.9828 & $0.176 \%$ & $\mathrm{~N} / \mathrm{C}$ & 18.24 & 20.00 & & 473 & 408 & \\
\hline & & & & & 18.24 & 20.00 & $2.16 \%$ & 473 & 473 & $-9.87 \%$ \\
\hline & & & & & 18.24 & 15.90 & & 473 & 398 & \\
\hline \multirow{3}{*}{$\begin{array}{l}\text { ER-610 } \\
\text { C1 }\end{array}$} & 25.6281 & 25.6033 & $-0.097 \%$ & $\mathrm{~N} / \mathrm{C}$ & 14.53 & 18.77 & & 494 & 558 & \\
\hline & & & & & 14.53 & 18.03 & $20.60 \%$ & 494 & 473 & $-0.94 \%$ \\
\hline & & & & & 14.53 & 15.77 & & 494 & 437 & \\
\hline \multirow{3}{*}{$\begin{array}{l}Y-833 \\
D 1\end{array}$} & 26.0443 & 26.0544 & $0.039 \%$ & $\mathrm{~N} / \mathrm{C}$ & 11.38 & 12.75 & & 557 & 318 & \\
\hline & & & & & 11.38 & 12.81 & $15.82 \%$ & 557 & 349 & $-39.74 \%$ \\
\hline & & & & & 11.38 & 13.98 & & 557 & 340 & \\
\hline \multirow{3}{*}{$\begin{array}{l}923 \\
E 1\end{array}$} & 23.3496 & 23.4045 & $0.235 \%$ & $\mathrm{~N} / \mathrm{C}$ & 15.85 & 13.90 & & 503 & 560 & \\
\hline & & & & & 15.85 & 13.77 & $.12 .16 \%$ & 503 & 403 & $-9.54 \%$ \\
\hline & & & & & 15.85 & 14.10 & & 503 & 402 & \\
\hline \multirow{3}{*}{$\begin{array}{l}\text { ISO-800 } \\
\text { F1 }\end{array}$} & 22.2677 & 22.2835 & $0.071 \%$ & $\mathrm{~N} / \mathrm{C}$ & 14.75 & 15.48 & & 632 & 370 & \\
\hline & & & & & 14.75 & 16.61 & $9.04 \%$ & 632 & 486 & $-27.74 \%$ \\
\hline & & & & & 14.75 & 16.16 & & 632 & 514 & \\
\hline & HCFC-12 & 24/S hriev & Zerol-15 & 0 @ 2 & $0^{\circ} \mathrm{F}$ plu & s 24 ho & Lis es 00 & ${ }^{\circ} \mathrm{F}(1$ & $\left.50^{\circ} \mathrm{C}\right)$ & \\
\hline \multirow{3}{*}{$\begin{array}{l}U-475 \\
A 2\end{array}$} & 23.8799 & 23.8659 & $-0.059 \%$ & $\mathrm{~N} / \mathrm{C}$ & 15.10 & 10.86 & & 469 & 449 & \\
\hline & & & & & 15.10 & 11.22 & $-23.80 \%$ & 469 & 505 & $0.28 \%$ \\
\hline & & & & & 15.10 & 12.44 & & 469 & 457 & \\
\hline \multirow{3}{*}{$\begin{array}{l}Y-390 \\
B 2\end{array}$} & 26.1871 & 26.2125 & $0.097 \%$ & $\mathrm{~N} / \mathrm{C}$ & 18.24 & 19.75 & & 473 & 458 & \\
\hline & & & & & 18.24 & 19.70 & $8.83 \%$ & 473 & 428 & $-3.52 \%$ \\
\hline & & & & & 18.24 & 20.00 & & 473 & 483 & \\
\hline \multirow{3}{*}{$\begin{array}{l}\text { ER-610 } \\
\text { C2 }\end{array}$} & 25.9784 & 25.9475 & $-0.119 \%$ & $\mathrm{~N} / \mathrm{C}$ & 14.53 & 11.10 & & 494 & 511 & \\
\hline & & & & & 14.53 & 12.44 & $-22.85 \%$ & 494 & 409 & $-3.24 \%$ \\
\hline & & & & & 14.53 & 10.09 & & 494 & 514 & \\
\hline \multirow{3}{*}{$\begin{array}{l}Y-833 \\
D 2\end{array}$} & 25.7970 & 25.7914 & $-0.022 \%$ & $\mathrm{~N} / \mathrm{C}$ & 11.38 & 16.14 & & 557 & 368 & \\
\hline & & & & & 11.38 & 20.00 & $51.64 \%$ & 557 & 409 & $-34.53 \%$ \\
\hline & & & & & 11.38 & 15.63 & & 557 & 317 & \\
\hline \multirow{3}{*}{$\begin{array}{l}923 \\
\text { E2 }\end{array}$} & 23.2933 & 23.3268 & $0.144 \%$ & $\mathrm{~N} / \mathrm{C}$ & 15.85 & 20.00 & & 503 & 450 & \\
\hline & & & & & 15.85 & 12.71 & $6.04 \%$ & 503 & 462 & $-9.48 \%$ \\
\hline & & & & & 15.85 & 17.71 & & 503 & 454 & \\
\hline \multirow{3}{*}{$\begin{array}{l}\text { ISO-800 } \\
\text { F2 }\end{array}$} & 22.2815 & 22.2877 & $0.028 \%$ & $\mathrm{~N} / \mathrm{C}$ & 14.75 & 20.00 & & 632 & 621 & \\
\hline & & & & & 14.75 & 17.48 & $24.14 \%$ & 632 & 561 & $-14.50 \%$ \\
\hline & & & & & 14.75 & 17.45 & & 632 & 439 & \\
\hline
\end{tabular}




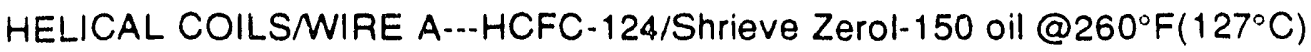

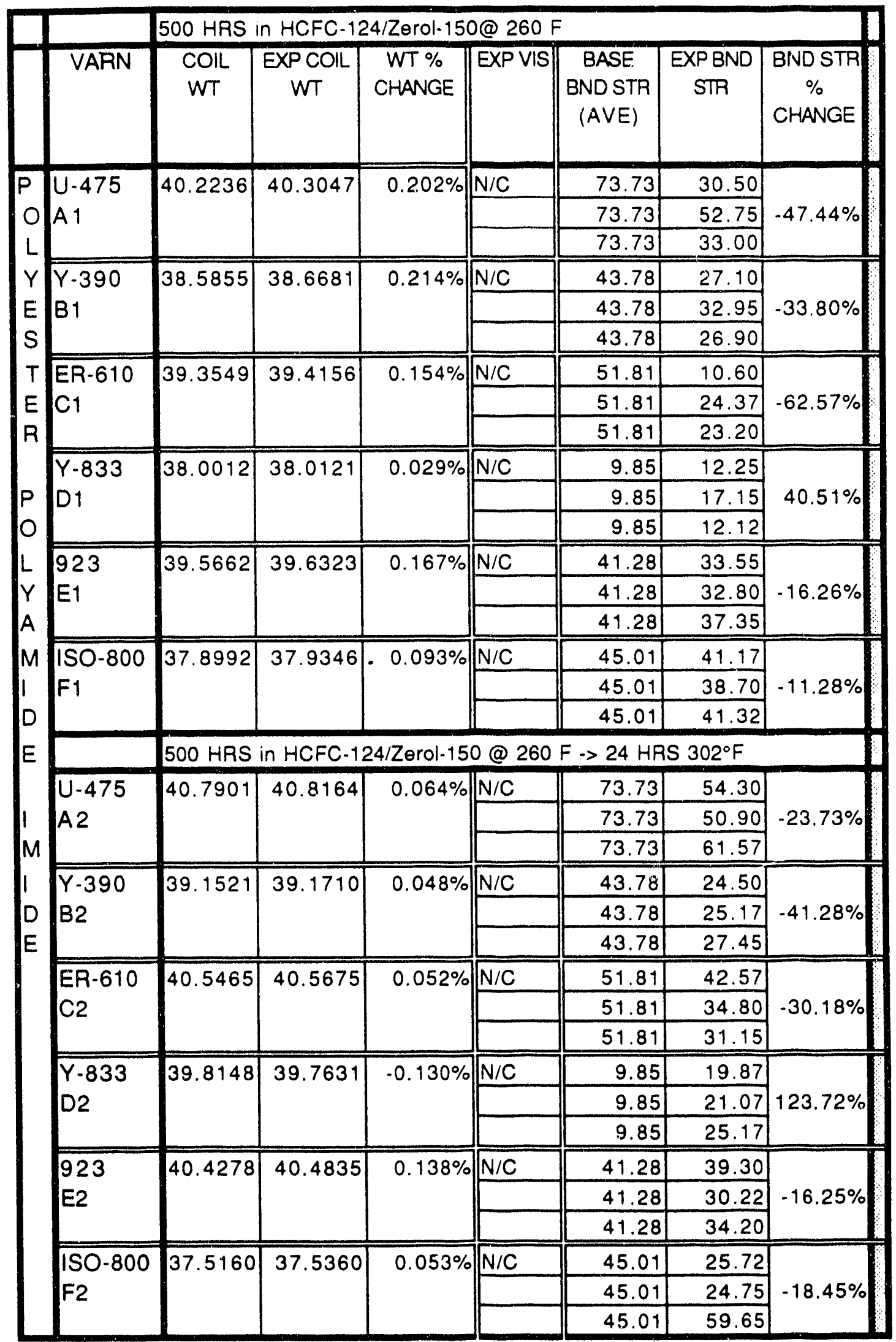


HELICAL COILSMIRE B-HCFC-124/Shrieve Zerol-150 Oil

\begin{tabular}{|c|c|c|c|c|c|c|c|c|}
\hline & & $500 \mathrm{HRS}$ & in $\mathrm{HCFC}$ - 1 & $24 /$ Shrieve & Zerol-150 & @ $260 \mathrm{~F}$ & & \\
\hline & VARN & $\begin{array}{l}\text { COIL } \\
\text { WT }\end{array}$ & $\begin{array}{c}\text { EXP COIL } \\
W T\end{array}$ & $\begin{array}{c}\text { WT \% } \\
\text { CHANGE }\end{array}$ & EXPVIS & $\begin{array}{c}\text { BASE } \\
\text { BND STR } \\
(A \vee E)\end{array}$ & $\begin{array}{c}\text { EXPBND } \\
\text { STR }\end{array}$ & $\begin{array}{c}\text { BND STR } \\
\% \\
\text { CHANGE }\end{array}$ \\
\hline$P$ & $U-475$ & 37.4514 & 37.5548 & $0.276 \%$ & $N / C$ & 40.14 & 41.40 & \\
\hline 0 & A 1 & & & & & 40.14 & 31.12 & $-16.77 \%$ \\
\hline$L$ & & & & & & 40.14 & 27.70 & \\
\hline Y & $Y-390$ & 37.8148 & 37.9263 & $0.295 \%$ & $\mathrm{~N} / \mathrm{C}$ & 36.12 & 36.30 & \\
\hline$E$ & B1 & & & & & 36.12 & 35.87 & $.1 .01 \%$ \\
\hline $\mathbf{S}$ & & & & & & 36.12 & 35.10 & \\
\hline$T$ & ER-610 & 37.8819 & 37.9738 & $0.243 \%$ & $N / C$ & 35.96 & 25.92 & \\
\hline$E$ & C1 & & & & & 35.96 & 24.30 & $-27.57 \%$ \\
\hline$R$ & & & & & & 35.96 & 27.92 & \\
\hline & $Y-833$ & 36.7933 & 36.8216 & $0.077 \%$ & $\mathrm{~N} / \mathrm{C}$ & 33.14 & 18.22 & \\
\hline$P$ & D1 & & & & & 33.14 & 21.25 & $-45.09 \%$ \\
\hline 0 & & & & & & 33.14 & 15.12 & \\
\hline$L$ & 923 & 37.4935 & 37.5530 & $0.159 \%$ & $\mathrm{~N} / \mathrm{C}$ & 40.52 & 31.62 & \\
\hline Y & E1 & & & & & 40.52 & 42.82 & $-11.45 \%$ \\
\hline A & & & & & & 40.52 & 33.20 & \\
\hline$M$ & $150-800$ & 36.9535 & 37.0199 & $0.180 \%$ & $N / C$ & 20.20 & 18.02 & \\
\hline 1 & $F 1$ & & & & & 20.20 & 20.92 & $.3 .56 \%$ \\
\hline D & & & & & & 20.20 & 19.50 & \\
\hline $\mathbf{E}$ & & $500 \mathrm{HRS}$ & in $\mathrm{HCFC}-1$ & $24 /$ Zerol-15 & $30 @ 260$ & $F .>24 \mathrm{HR}$ & $\mathrm{S} 302^{\circ} \mathrm{F}$ & \\
\hline & $U-475$ & 37.9410 & 37.9782 & $0.098 \%$ & $\mathrm{~N} / \mathrm{C}$ & 40.14 & 28.70 & \\
\hline 1 & $A 2$ & & & & & 40.14 & 35.17 & $-19.83 \%$ \\
\hline$M$ & & & & & & 40.14 & 32.67 & \\
\hline 1 & $Y-390$ & 37.2453 & 37.3014 & $0.151 \%$ & $\mathrm{~N} / \mathrm{C}$ & 36.12 & 30.12 & \\
\hline$D$ & B2 & & & & & 36.12 & 35.40 & $-11.20 \%$ \\
\hline$E$ & & & & & & 36.12 & 30.70 & \\
\hline & ER-610 & 37.8732 & 37.8985 & $0.067 \%$ & $\mathrm{~N} / \mathrm{C}$ & 35.96 & 24.47 & \\
\hline$E$ & $\mathrm{C} 2$ & & & & & 35.96 & 26.22 & $.20 .99 \%$ \\
\hline$P$ & & & & & & 35.96 & 34.55 & \\
\hline 0 & $Y-833$ & 36.7758 & 36.7699 & $-0.016 \%$ & $\mathrm{~N} / \mathrm{C}$ & 33.14 & 18.92 & \\
\hline$x$ & D2 & & & & & 33.14 & 16.65 & $.38 .02 \%$ \\
\hline Y & & & & & & 33.14 & 26.05 & \\
\hline & 923 & 38.0573 & 38.0962 & $0.102 \%$ & $\mathrm{~N} / \mathrm{C}$ & 40.52 & 23.25 & \\
\hline G & E2 & & & & & 40.52 & 29.37 & $-29.55 \%$ \\
\hline L & & & & & & 40.52 & 33.02 & \\
\hline A & ISO-800 & 38.1027 & 38.1164 & $0.036 \%$ & $\mathrm{~N} / \mathrm{C}$ & 20.20 & 18.77 & \\
\hline$S$ & $F 2$ & & & & & 20.20 & 18.42 & $-6.42 \%$ \\
\hline$S$ & & & & & & 20.20 & 19.52 & \\
\hline
\end{tabular}


HELICAL COILSMIRE C.HCFC-124/Shrieve Zerol-150 oil @ 260 $\mathrm{F}\left(127^{\circ} \mathrm{C}\right)$

\begin{tabular}{|c|c|c|c|c|c|c|c|c|}
\hline & & $500 \mathrm{HRS}$ & in $\mathrm{HCFC}$ & 24/Shrieve $Z$ & Zer & $60 \mathrm{~F}$ & & \\
\hline & VARN & $\begin{array}{l}\text { COIL } \\
W T\end{array}$ & $\begin{array}{c}\text { EXP COIL } \\
W T\end{array}$ & $\begin{array}{c}\text { WT \% } \\
\text { CHANGE }\end{array}$ & EXP VIS & $\begin{array}{c}\text { BASE } \\
\text { BND STR } \\
\text { (AVE) }\end{array}$ & $\begin{array}{c}\text { EXPBND } \\
\text { STR }\end{array}$ & $\begin{array}{c}\text { BND STR } \\
\% \\
\text { CHANGE }\end{array}$ \\
\hline$P$ & U.475 & 37.7929 & 37.8676 & $0.198 \%$ & $\mathrm{~N} / \mathrm{C}$ & 51.21 & 23.17 & \\
\hline 0 & A 1 & & & & & 51.21 & 46.90 & $-27.10 \%$ \\
\hline$L$ & & & & & & 51.21 & 41.92 & \\
\hline$Y$ & Y-390 & 40.7938 & 40.8402 & $0.114 \%$ & $\mathrm{~N} / \mathrm{C}$ & 50.72 & 26.35 & \\
\hline$E$ & B1 & & & & & 50.72 & 29.77 & $-46.59 \%$ \\
\hline S & & & & & & 50.72 & 25.15 & \\
\hline $\mathrm{T}$ & ER-610 & 38.4619 & 38.5300 & $0.177 \%$ & $\mathrm{~N} / \mathrm{C}$ & 58.33 & 18.52 & \\
\hline$E$ & C1 & & & & & 58.33 & 20.17 & $-56.82 \%$ \\
\hline R & & & & & & 58.33 & 36.87 & \\
\hline & $Y-833$ & 38.5156 & 38.4274 & $-0.229 \%$ & $\mathrm{~N} / \mathrm{C}$ & 5.84 & 11.25 & \\
\hline I & D1 & & & & & 5.84 & 11.07 & $99.43 \%$ \\
\hline$M$ & & & & & & 5.84 & 12.62 & \\
\hline I & 923 & 40.3966 & 40.4652 & $0.170 \%$ & $\mathrm{~N} / \mathrm{C}$ & 49.26 & 28.27 & \\
\hline$D$ & E1 & & & & & 49.26 & 26.45 & $-46.89 \%$ \\
\hline E & & & & & & 49.26 & 23.77 & \\
\hline & ISO-800 & 39.8049 & 39.8481 & $0.109 \%$ & $\mathrm{~N} / \mathrm{C}$ & 36.08 & 40.32 & \\
\hline$P$ & F1 & & & & & 36.08 & 33.22 & $4.78 \%$ \\
\hline 0 & & & & & & 36.08 & 39.87 & \\
\hline L & & $500 \mathrm{HRS}$ & in HCFC-1 & $24 /$ Shrieve 2 & Zerol-150 & @ 260 F. & 24 HRS & $02^{\circ} \mathrm{F}$ \\
\hline Y & $U-475$ & 37.1777 & 37.2032 & $0.069 \%$ & $\mathrm{~N} / \mathrm{C}$ & 51.21 & 69.65 & \\
\hline A & A 2 & & & & & 51.21 & 68.37 & $37.76 \%$ \\
\hline$M$ & & & & & & 51.21 & 73.62 & \\
\hline I & $Y-390$ & 38.9310 & 38.9581 & $0.070 \%$ & $\mathrm{~N} / \mathrm{C}$ & 50.72 & 31.60 & \\
\hline D & B2 & & & & & 50.72 & 27.22 & $-44.90 \%$ \\
\hline $\mathrm{E}$ & & & & & & 50.72 & 25.02 & \\
\hline & ER-610 & 37.9620 & 37.9830 & $0.055 \%$ & $\mathrm{~N} / \mathrm{C}$ & 58.33 & 31.60 & \\
\hline 1 & C2 & & & & & 58.33 & 25.85 & $-38.74 \%$ \\
\hline M & & & & & & 58.33 & 49.75 & \\
\hline 1 & $Y-833$ & 39.2567 & 39.1607 & $-0.245 \%$ & $\mathrm{~N} / \mathrm{C}$ & 5.84 & 10.50 & \\
\hline D & D2 & & & & & 5.84 & 11.95 & $123.00 \%$ \\
\hline$E$ & & & & & & 5.84 & 16.62 & \\
\hline & 923 & 39.3561 & 39.3926 & $0.093 \%$ & $\mathrm{~N} / \mathrm{C}$ & 49.26 & 22.35 & \\
\hline & E2 & & & & & 49.26 & 26.35 & $-49.95 \%$ \\
\hline & & & & & & 49.26 & 25.27 & \\
\hline & $150-800$ & 39.7532 & 39.7609 & $0.019 \%$ & $\mathrm{~N} / \mathrm{C}$ & 36.08 & 56.80 & \\
\hline & F2 & & & & & 36.08 & 30.60 & $21.32 \%$ \\
\hline & & & & & & 36.08 & 43.92 & \\
\hline
\end{tabular}


500 HRS in HCFC.124/Zerol-150 oil @ 260 $\mathrm{F}\left(127^{\circ} \mathrm{C}\right)$

\begin{tabular}{|c|c|c|c|c|c|}
\hline ID & WT & EXPWT & $\begin{array}{c}\text { WT \% } \\
\text { CHANGE }\end{array}$ & EXP VS & EXPFLEX \\
\hline \multirow[t]{2}{*}{ A1 } & \multirow[t]{2}{*}{3.4228} & \multirow[t]{2}{*}{3.426} & \multirow[t]{2}{*}{$0.093 \%$} & $\mathrm{~N} / \mathrm{C}$ & \\
\hline & & & & & YES \\
\hline \multirow[t]{2}{*}{$B 1$} & \multirow[t]{2}{*}{3.7686} & \multirow[t]{2}{*}{3.776} & \multirow[t]{2}{*}{$0.196 \%$} & $\mathrm{~N} / \mathrm{C}$ & \\
\hline & & & & & YES \\
\hline \multirow[t]{4}{*}{ C1 } & \multirow[t]{3}{*}{3.372} & \multirow[t]{3}{*}{3.3748} & \multirow[t]{3}{*}{$0.083 \%$} & $\mathrm{~N} / \mathrm{C}$ & \\
\hline & & & & & YES \\
\hline & & & & & \\
\hline & \multicolumn{5}{|c|}{$500 \mathrm{HRS}$ in HCFC-124/Zerol-150-> $302^{\circ} \mathrm{F}$ for $24 \mathrm{HRS}$} \\
\hline \multirow[t]{2}{*}{ A2 } & \multirow[t]{2}{*}{3.4972} & \multirow[t]{2}{*}{3.4971} & \multirow[t]{3}{*}{$-0.003 \%$} & $\mathrm{~N} / \mathrm{C}$ & \\
\hline & & & & & YES \\
\hline & & & & & \\
\hline \multirow[t]{2}{*}{$\mathrm{B2}$} & \multirow[t]{2}{*}{3.7452} & \multirow[t]{2}{*}{3.7473} & \multirow[t]{3}{*}{$0.056 \%$} & $\mathrm{~N} / \mathrm{C}$ & \\
\hline & & & & & YES \\
\hline & & & & & \\
\hline \multirow[t]{3}{*}{$\mathrm{C} 2$} & \multirow[t]{3}{*}{3.453} & \multirow[t]{3}{*}{3.451} & \multirow[t]{3}{*}{$-0.058 \%$} & $\mathrm{~N} / \mathrm{C}$ & \\
\hline & & & & & YES \\
\hline & & & & & \\
\hline
\end{tabular}




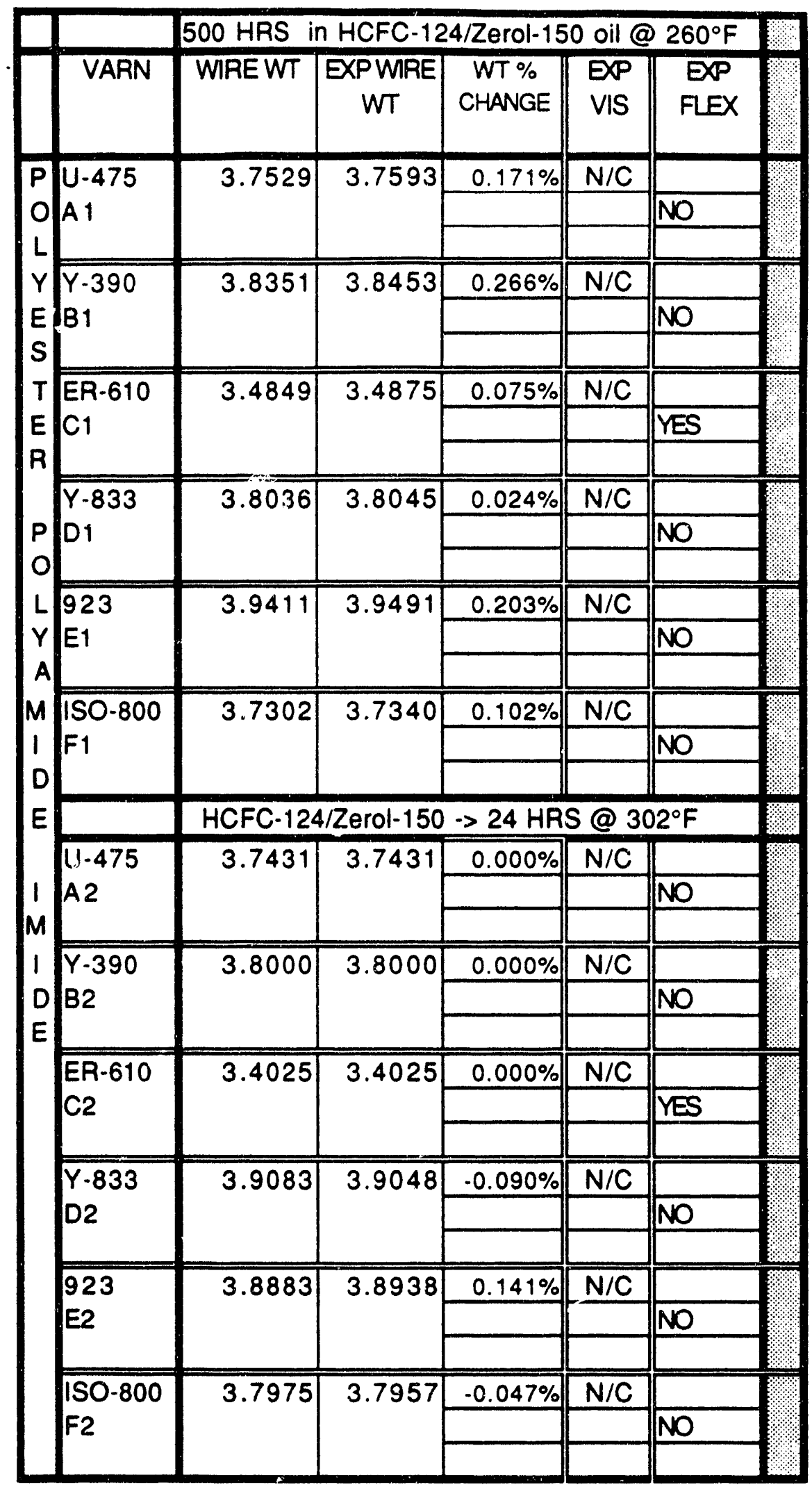




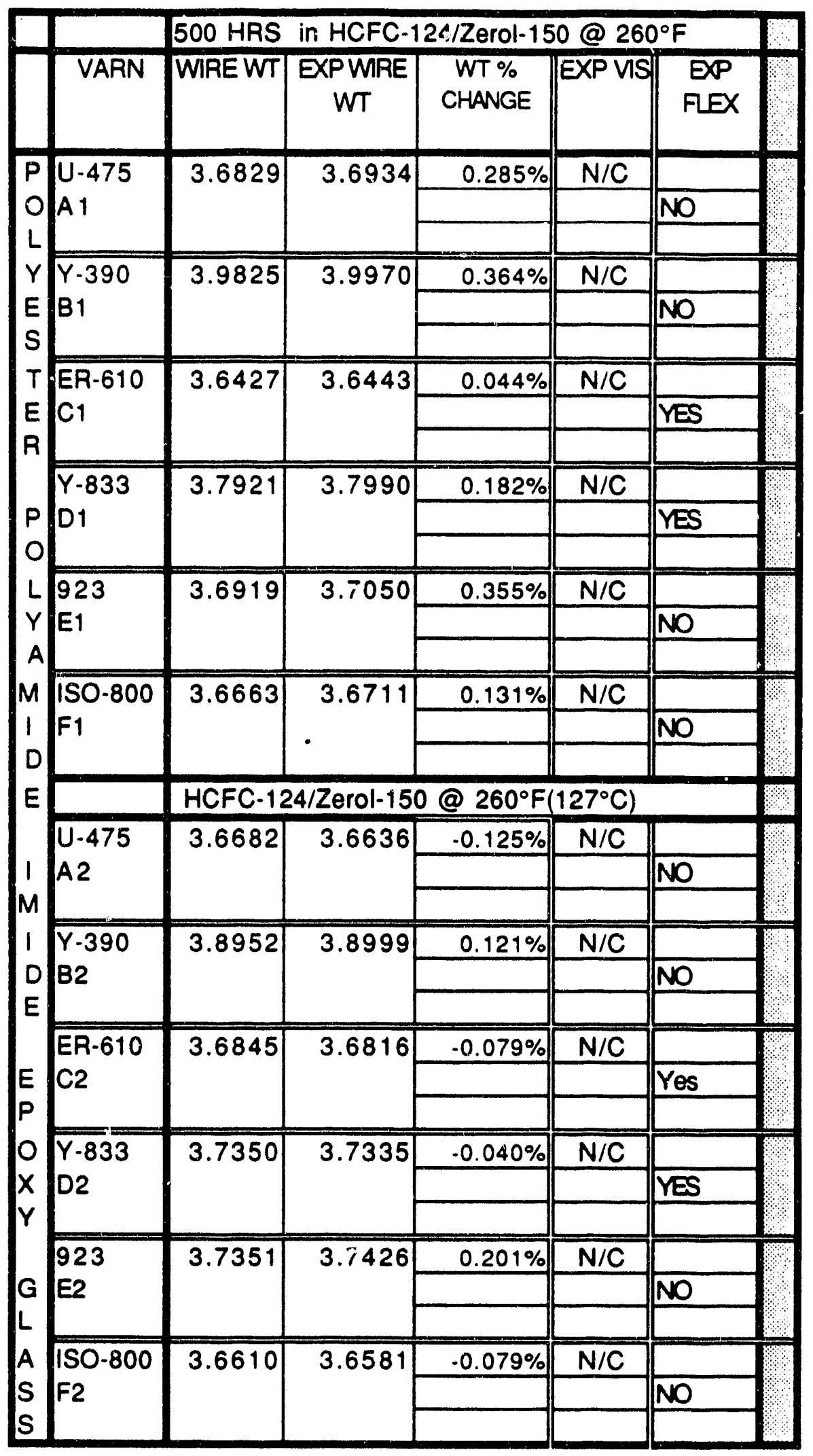




\begin{tabular}{|c|c|c|c|c|c|c|c|}
\hline & & 500 HRS & $n$ HCFC-124 & Zerol-150 & @ 260 & $=\left(127^{\circ} \mathrm{C}\right)$ & \\
\hline & VARN & WREWT & $\begin{array}{c}\text { EXPWRE } \\
W T\end{array}$ & \begin{tabular}{c||} 
WT \% \\
CHANGE
\end{tabular} & EXPVS| & \begin{tabular}{|l} 
EXP \\
FEEX
\end{tabular} & \\
\hline$P$ & $U-475$ & 3.5397 & 3.5479 & $0.232 \%$ & $\mathrm{~N} / \mathrm{C}$ & & \\
\hline 0 & & & & & & No & \\
\hline$L$ & & & & & & & \\
\hline $\mathrm{Y}$ & Y.390 & 3.6475 & 3.6562 & $0.239 \%$ & $\mathrm{~N} / \mathrm{C}$ & & \\
\hline$E$ & & & & & & NO & \\
\hline 5 & & & & & & & \\
\hline $\begin{array}{l}T \\
E\end{array}$ & ER-610 & 3.5756 & 3.5768 & $0.034 \%$ & $\mathrm{~N} / \mathrm{C}$ & & \\
\hline $\begin{array}{l}E \\
R\end{array}$ & & & & & & NO & \\
\hline & $Y-833$ & 3.4621 & 3.4629 & $0.023 \%$ & $\mathrm{~N} / \mathrm{C}$ & & \\
\hline I & D1 & & & & & YES & \\
\hline$M$ & & & & & & & \\
\hline 1 & 923 & 3.8174 & 3.8245 & $0.186 \%$ & $\mathrm{~N} / \mathrm{C}$ & & \\
\hline D & E1 & & & & & NO & \\
\hline E & ISO-800 & 3.4873 & 3.4850 & $-0.066 \%$ & $\mathrm{~N} / \mathrm{C}$ & & \\
\hline$P$ & & & & & & NO & \\
\hline 0 & & & & & & & \\
\hline L & & HCFC-124 & Zerol-150@ & $260^{\circ} \mathrm{F}>2$ & 4 HRS & @ $302 F$ & \\
\hline$Y$ & U-475 & 3.5612 & 3.5587 & $.0 .070 \%$ & $\mathrm{~N} / \mathrm{C}$ & & \\
\hline A & & & & & & YES & \\
\hline$M$ & & & & & & & \\
\hline 1 & $Y-390$ & 3.5843 & 3.5873 & $0.084 \%$ & $N / C$ & & \\
\hline$D$ & & & & & & NO & \\
\hline & ER-610 & 3.5677 & 3.5651 & $.0 .073 \%$ & $\mathrm{~N} / \mathrm{C}$ & & \\
\hline 1 & & & & & & NO & \\
\hline M & & & & & & & \\
\hline 1 & $Y \cdot 833$ & 3.4743 & 3.4708 & $-0.101 \%$ & $N / C$ & & \\
\hline D & & & & & & YES & \\
\hline & & 3.8380 & 3.8432 & $0.135 \%$ & $\mathrm{~N} / \mathrm{C}$ & & \\
\hline & E2 & & & & & NO & \\
\hline & & & & & & & \\
\hline & ISO-800 & 3.4888 & 3.4884 & $-0.011 \%$ & $\mathrm{~N} / \mathrm{C}$ & $\ln n$ & \\
\hline & & & & & & & \\
\hline
\end{tabular}




\begin{tabular}{|c|c|c|c|c|c|}
\hline & 500 HRS II & R-124/Zerol & -150 Oil @ & 260 & \\
\hline ID & WT & EXPWT & $\begin{array}{l}\text { WT \% } \\
\text { CHANGE }\end{array}$ & EXPVS & EXPFLEX \\
\hline $\begin{array}{l}\text { U-475 } \\
\text { A } 1\end{array}$ & 2.0977 & 2.1397 & $2.00 \%$ & $N / C$ & $N / C$ \\
\hline $\begin{array}{l}Y-390 \\
B 1\end{array}$ & 2.2072 & 2.2085 & $0.06 \%$ & $\frac{\text { slightly }}{\text { warped }}$ & $N / C$ \\
\hline ER-610 & 1.9784 & 2.0847 & $5.37 \%$ & surface & $\mathrm{N} / \mathrm{C}$ \\
\hline & & & & $\frac{\text { appears }}{\text { cloudv }}$ & \\
\hline$Y-833$ & 2.2822 & 2.3490 & $2.93 \%$ & $N / C$ & $\mathrm{~N} / \mathrm{C}$ \\
\hline W & & & & & \\
\hline 923 & 1.5897 & 1.6035 & $0.87 \%$ & $N / C$ & $N / C$ \\
\hline & & & & & \\
\hline ISO-800 & 1.3500 & 1.3320 & $-1.33 \%$ & slightly & $\mathrm{N} / \mathrm{C}$ \\
\hline & & & & warped & \\
\hline & R-124/Zero & 150 at $\cdots>2$ & 4 hour at & $302^{\circ} \mathrm{F}$ & \\
\hline$U-475$ & 2.4054 & 2.4058 & $0.02 \%$ & darkened & $N / C$ \\
\hline A2 & & & & & \\
\hline$Y-390$ & 20235 & 00140 & $0.450 / 4$ & Cliahtly & $\sqrt{\text { Non }}$ \\
\hline B2 & $.0<<0$ & 2.0170 & 0.4070 & $\frac{\text { olliginy }}{\text { warped }}$ & IN/C \\
\hline ER-610 & 18220 & 18532 & $171 \%$ & darkened & $N / C$ \\
\hline & & & & same as & \\
\hline & & & & above & \\
\hline$Y \cdot 833$ & 2.3713 & 2.4086 & $1.57 \%$ & slightly & $N / C$ \\
\hline D2 & & & & warped & \\
\hline 923 & 2.0330 & 2.0418 & $0.43 \%$ & & \\
\hline E2 & & & & darkened & $\mathrm{N} / \mathrm{C}$ \\
\hline ISO-800 & 1.5173 & 1.1207 & $-571 \%$ & eliahtly & \\
\hline & 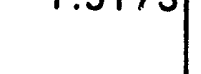 & 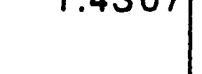 & 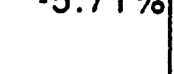 & warped & $\mathrm{N} / \mathrm{C}$ \\
\hline & & & & & \\
\hline
\end{tabular}




\begin{tabular}{|c|c|c|c|c|c|c|c|c|c|}
\hline \multicolumn{10}{|l|}{ 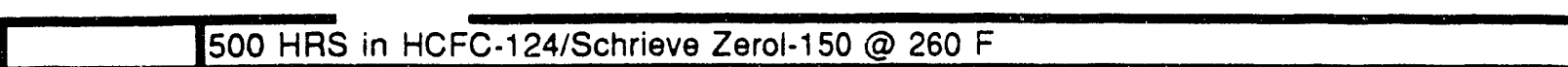 } \\
\hline ID & WT & EXPWT & $\begin{array}{c}\text { WT \% } \\
\text { CHANGE }\end{array}$ & SAMPLE & $\begin{array}{l}\text { BREAK } \\
\text { LOAD }\end{array}$ & $\begin{array}{c}\text { SAMPLE } \\
\text { THICKNESS } \\
M i L_{S}\end{array}$ & $\begin{array}{c}\text { TENSILE } \\
\text { STIR } \\
\text { BASE }\end{array}$ & $\begin{array}{l}\text { TENSILE } \\
\text { STREXP }\end{array}$ & $\begin{array}{c}\% \text { CHANGE } \\
\text { TENSILE }\end{array}$ \\
\hline \multirow{3}{*}{$\begin{array}{l}\text { NO/MY/NO } \\
\text { A1 }\end{array}$} & \multirow[t]{3}{*}{7.3592} & \multirow[t]{3}{*}{7.8442} & \multirow[t]{3}{*}{$6.59 \%$} & 0.330 & 61.17 & 21 & 17.4 & 8.83 & \\
\hline & & & & 0.420 & 73.20 & 21 & 17.4 & 8.30 & $.50 .51 \%$ \\
\hline & & & & 0.495 & 90.52 & 21 & 17.4 & 8.71 & \\
\hline \multirow{3}{*}{$\begin{array}{l}\mathrm{DA} / \mathrm{MY} / \mathrm{DA} \\
\mathrm{B} 1\end{array}$} & \multirow[t]{3}{*}{7.1013} & \multirow[t]{3}{*}{7.1815} & \multirow[t]{3}{*}{$1.13 \%$} & 0.360 & 16.02 & 21 & 13.7 & 2.12 & \\
\hline & & & & 0.380 & 15.95 & 21 & 13.7 & 2.00 & $.84 .82 \%$ \\
\hline & & & & 0.375 & 16.70 & 21 & 13.7 & 2.12 & \\
\hline \multirow{3}{*}{$\begin{array}{l}\text { MYLAR MO } \\
\text { C1 }\end{array}$} & \multirow[t]{3}{*}{3.7728} & \multirow[t]{3}{*}{3.8664} & \multirow[t]{3}{*}{$2.48 \%$} & 0.430 & 0.00 & 10 & 21.7 & 0.00 & \\
\hline & & & & 0.445 & 0.00 & 10 & 21.7 & 0.00 & $-100.00 \%$ \\
\hline & & & & 0.450 & 0.00 & 10 & 21.7 & 0.00 & \\
\hline \multirow{3}{*}{$\begin{array}{l}\text { NO } 410 \\
\text { D1 }\end{array}$} & \multirow[t]{3}{*}{3.7410} & \multirow[t]{3}{*}{4.1766} & \multirow[t]{3}{*}{$11.64 \%$} & 0.450 & 82.72 & 10 & 18.7 & 18.38 & \\
\hline & & & & 0.525 & 102.40 & 10 & 18.7 & 19.50 & $1.88 \%$ \\
\hline & & & & 0.507 & 97.70 & 10 & 18.7 & 19.27 & \\
\hline \multirow{3}{*}{$\begin{array}{l}\text { NO MI } 418 \\
\text { E1 }\end{array}$} & \multirow[t]{3}{*}{3.7877} & \multirow[t]{3}{*}{4.7988} & \multirow[t]{3}{*}{$26.69 \%$} & 0.488 & 35.90 & 9 & 7.5 & 8.17 & \\
\hline & & & & 0.485 & 33.17 & 9 & 7.5 & 7.60 & $5.11 \%$ \\
\hline & & & & 0.500 & 35.45 & 9 & 7.5 & 7.88 & \\
\hline \multirow{3}{*}{$\begin{array}{l}\text { MEL } 228 \\
F 1\end{array}$} & \multirow[t]{3}{*}{4.2637} & \multirow[t]{3}{*}{4.3157} & \multirow[t]{3}{*}{$1.22 \%$} & 0.420 & 0.00 & 10 & 21.7 & 0.00 & \\
\hline & & & & 0.435 & 0.00 & 10 & 21.7 & 0.00 & $-100.00 \%$ \\
\hline & & & & 0.512 & 0.00 & 10 & 21.7 & 0.00 & \\
\hline & 500 HRS & $S$ in $H C F$ & C-124/Scl & hieve Zerc & 1-150@ & $260 F->24$ & HRS@3 & $02^{\circ} \mathrm{F}$ & \\
\hline NO/MY/NO & 6.9426 & 7.0459 & $1.49 \%$ & 0.381 & 68.15 & 21 & 17.4 & 8.52 & \\
\hline A2 & & & & 0.430 & 73.25 & 21 & 17.4 & 8.11 & $.54 .98 \%$ \\
\hline & & & & 0.480 & 69.25 & 21 & 17.4 & 6.87 & \\
\hline$D A / M Y / D A$ & 6.8327 & 6.8054 & $-0.40 \%$ & 0.430 & 19.02 & 21 & 13.7 & 2.11 & \\
\hline $\mathrm{B} 2$ & & & & 0.380 & 2.10 & 21 & 13.7 & 0.26 & $-93.21 \%$ \\
\hline & & & & 0.390 & 3.45 & 21 & 13.7 & 0.42 & \\
\hline MYAR MO & 3.9026 & 3.9084 & $0.15 \%$ & 0.398 & 0.00 & 10 & 21.7 & 0.00 & \\
\hline $\mathrm{C} 2$ & & & & 0.395 & 0.00 & 10 & 21.7 & 0.00 & $.100 .00 \%$ \\
\hline & & & & 0.436 & 0.00 & 10 & 21.7 & 0.00 & \\
\hline NOMEX 410 & 3.8099 & 3.9891 & $4.70 \%$ & 0.475 & 93.55 & 10 & 18.7 & 19.69 & \\
\hline D2 & & & & 0.518 & 100.00 & 10 & 18.7 & 19.31 & $4.09 \%$ \\
\hline & & & & 0.495 & 96.00 & 10 & 18.7 & 19.39 & \\
\hline NO/MI 418 & 3.8599 & 4.1392 & $7.24 \%$ & 0.475 & 28.05 & 10 & 7.5 & 5.91 & \\
\hline E2 & & & & 0.518 & 27.85 & 10 & 7.5 & 5.38 & $-24.00 \%$ \\
\hline & & & & 0.495 & 28.80 & 10 & 7.5 & 5.82 & \\
\hline MEL 228 & 4.2999 & 4.3136 & $0.32 \%$ & 0.455 & 0.00 & 10 & 21.7 & 0.00 & \\
\hline $\mathrm{F} 2$ & & & & 0.436 & 0.00 & 10 & 21.7 & 0.00 & $-100.00 \%$ \\
\hline & & & & 0.416 & 0.00 & 10 & 21.7 & 0.00 & \\
\hline
\end{tabular}




\begin{tabular}{|c|c|c|c|c|c|c|c|c|}
\hline \multicolumn{8}{|c|}{ After 500 hour exposure at $260^{\circ} \mathrm{F}$} & \multirow[b]{2}{*}{ VISUAL EXP } \\
\hline ID & $\begin{array}{c}\text { STRETCH } \\
\text { (inch) }\end{array}$ & $\begin{array}{c}\% \\
\text { ELONG }\end{array}$ & $\begin{array}{l}\text { BASE } \\
\text { ELONG } \\
\text { (AVE) }\end{array}$ & \begin{tabular}{|l} 
ELONG \% \\
CHANGE
\end{tabular} & $\begin{array}{c}\text { BASE } \\
\text { DIE } \\
\text { (AVE) }\end{array}$ & EXPDIE & \begin{tabular}{|c|} 
DIE \% \\
CHANGE
\end{tabular} & \\
\hline \multirow{3}{*}{$\begin{array}{l}\text { NO/MY/NO } \\
\text { A1 }\end{array}$} & 0.40 & $10.0 \%$ & $20.0 \%$ & & $>18.97$ & $>13.91$ & & \multirow{3}{*}{$\begin{array}{l}\text { Mylar brittle } \\
\text { discolored } \\
\text { yellowed }\end{array}$} \\
\hline & 0.33 & $8.3 \%$ & $20.0 \%$ & $-54.4 \%$ & $>18.97$ & $>14.91$ & flash & \\
\hline & 0.41 & $10.3 \%$ & $20.0 \%$ & & $>18.97$ & $>16.50$ & & \\
\hline \multirow{3}{*}{$\begin{array}{l}\text { DA/MY/DA } \\
B 1\end{array}$} & 0.02 & $1.0 \%$ & $46.0 \%$ & & $>15.27$ & $>14.07$ & & \multirow[t]{3}{*}{ Mylar Brittle } \\
\hline & 0.01 & $0.5 \%$ & $46.0 \%$ & $-98.2 \%$ & $>15.27$ & $>15.59$ & flash & \\
\hline & 0.02 & $1.0 \%$ & $46.0 \%$ & & $>15.27$ & $>15.82$ & & \\
\hline \multirow{3}{*}{$\begin{array}{l}\text { MYLAR MO } \\
\mathrm{C} 1\end{array}$} & 0.00 & $0.0 \%$ & $131.0 \%$ & & $>14.91$ & $>12.30$ & & \multirow{3}{*}{$\begin{array}{l}\text { Mylar brittle } \\
\text { discolored } \\
\text { yellowed }\end{array}$} \\
\hline & 0.00 & $0.0 \%$ & $131.0 \%$ & $.100 .0 \%$ & $>14.91$ & $\geq 12.97$ & flash & \\
\hline & 0.00 & $0.0 \%$ & $131.0 \%$ & & $>14.91$ & $>11.44$ & & \\
\hline \multirow{3}{*}{$\begin{array}{l}\text { NO } 410 \\
\text { D1 }\end{array}$} & 0.32 & $8.0 \%$ & $17.0 \%$ & & 10.67 & $>11.34$ & & \multirow{3}{*}{$\begin{array}{l}\text { oil in nomex } \\
\text { raised dielectric }\end{array}$} \\
\hline & 0.43 & $10.8 \%$ & $17.0 \%$ & $.45 .1 \%$ & 10.67 & $>12.44$ & flash & \\
\hline & 0.37 & $9.3 \%$ & $17.0 \%$ & & 10.67 & $>12.29$ & & \\
\hline \multirow{3}{*}{$\begin{array}{l}\text { NO MI } 418 \\
\text { E1 }\end{array}$} & 0.05 & $1.3 \%$ & $4.0 \%$ & & 10.23 & 11.65 & & \multirow{3}{*}{ oil in insulation } \\
\hline & 0.04 & $1.0 \%$ & $4.0 \%$ & $.72 .9 \%$ & 10.23 & 12.25 & $14.0 \%$ & \\
\hline & 0.04 & $1.0 \%$ & $4.0 \%$ & & 10.23 & 11.10 & & \\
\hline \multirow{4}{*}{$\begin{array}{l}\text { MEL } 228 \\
\text { F1 }\end{array}$} & 0.00 & $0.0 \%$ & $160.0 \%$ & & $>14.22$ & $>12.82$ & & \multirow{3}{*}{$\begin{array}{l}\text { Brittle,discolored } \\
\text { yellowed }\end{array}$} \\
\hline & 0.00 & $0.0 \%$ & $160.0 \%$ & $.100 .0 \%$ & $>14.22$ & $>12.75$ & flash & \\
\hline & 0.00 & $0.0 \%$ & $160.0 \%$ & & $>14.22$ & $>12.45$ & & \\
\hline & \multicolumn{7}{|c|}{ After 500 hour exposure plus a 24 hour air bake at 150} & $\left.{ }^{\circ} \mathrm{F}\right)$ \\
\hline \multirow{3}{*}{$\begin{array}{l}\text { NO/MY/NO } \\
\text { A2 }\end{array}$} & 0.34 & \begin{tabular}{|l|}
$8.5 \%$ \\
\end{tabular} & $20.0 \%$ & & $>18.97$ & $>15.99$ & & \multirow{3}{*}{$\begin{array}{l}\text { darkened } \\
\text { delamination }\end{array}$} \\
\hline & 0.35 & $8.8 \%$ & $20.0 \%$ & $.57 .5 \%$ & $>18.97$ & $>16.47$ & flash & \\
\hline & 0.33 & $8.3 \%$ & $20.0 \%$ & & $>18.97$ & $>17.7$ & & \\
\hline \multirow{3}{*}{$\begin{array}{l}D A / M Y / D A \\
B 2\end{array}$} & 0.03 & $1.5 \%$ & $46.0 \%$ & & $>15.27$ & $>15.33$ & & \multirow{3}{*}{$\begin{array}{l}\text { darkened } \\
\text { delamination } \\
\text { mylar brown }\end{array}$} \\
\hline & 0.04 & $2.0 \%$ & $46.0 \%$ & $-96.7 \%$ & $>15.27$ & $>13.11$ & flash & \\
\hline & 0.02 & $1.0 \%$ & $46.0 \%$ & & $>15.27$ & $>14.15$ & & \\
\hline \multirow{3}{*}{$\begin{array}{l}\text { MYLAR MO } \\
\text { C2 }\end{array}$} & 0.00 & $0.0 \%$ & $131.0 \%$ & & $>14.91$ & $>12.71$ & & \multirow{3}{*}{$\begin{array}{l}\text { brittle, mylar } \\
\text { brown }\end{array}$} \\
\hline & 0.00 & $0.0 \%$ & $131.0 \%$ & $-100.0 \%$ & $>14.91$ & $>13.07$ & flash & \\
\hline & 0.00 & $0.0 \%$ & $131.0 \%$ & & $>14.91$ & $>12.31$ & & \\
\hline \multirow{3}{*}{$\begin{array}{l}\text { NOMEX 410 } \\
\text { D2 }\end{array}$} & 0.39 & $9.8 \%$ & $17.0 \%$ & & 10.67 & 10.53 & & \multirow{3}{*}{$\mathrm{N} / \mathrm{C}$} \\
\hline & 0.40 & $10.0 \%$ & $17.0 \%$ & $-40.7 \%$ & 10.67 & 10.57 & $-3.2 \%$ & \\
\hline & 0.42 & $10.5 \%$ & $17.0 \%$ & & 10.67 & 9.87 & & \\
\hline \multirow{3}{*}{$\begin{array}{l}\text { NO/MI } 418 \\
\text { E2 }\end{array}$} & 0.07 & $1.8 \%$ & $4.0 \%$ & & 10.23 & 9.91 & & \multirow{3}{*}{$N / C$} \\
\hline & 0.04 & $1.0 \%$ & $4.0 \%$ & $-66.7 \%$ & 10.23 & 8.76 & $-9.5 \%$ & \\
\hline & 0.05 & $1.3 \%$ & $4.0 \%$ & & 10.23 & 9.10 & & \\
\hline MEL 228 & 0.00 & $0.0 \%$ & $160.0 \%$ & & $\geq 14.22$ & $>12.53$ & & mylar brown \\
\hline F2 & 0.00 & $0.0 \%$ & $160.0 \%$ & $-100.0 \%$ & $>14.22$ & $>13.10$ & flash & brittle \\
\hline & 0.00 & $0.0 \%$ & $160.0 \%$ & & $>14.22$ & $>12.77$ & & \\
\hline
\end{tabular}


SLEEVING--HCFC-124/Shrieve Zerol-150 oil @260 $\mathrm{F}\left(127^{\circ} \mathrm{C}\right)$

\begin{tabular}{|c|c|c|c|c|}
\hline & 500 HRS in 1 & CFC-124/S & rieve Zer & |-150 oil @260F \\
\hline & $\overline{W T}$ & EXPWT & $\begin{array}{c}\% \\
\text { CHANGE }\end{array}$ & EXPVIS \\
\hline A 1 & 0.5439 & 0.6095 & $12.06 \%$ & Darkened, Nomex discolored \\
\hline NOMEX & & & & black \\
\hline & & & & see photo \\
\hline & 0.4787 & 0.4877 & $1.88 \%$ & \\
\hline MYAR & & & & $\mathrm{N} / \mathrm{C}$ \\
\hline C1 & 0.4000 & 0.4180 & $4.50 \%$ & Black discoloration of the \\
\hline NO/MY & & & & nomex inside \\
\hline & & & & see photo \\
\hline & HCFC-124/S & rieve Zerol- & 150 oil @ & $60^{\circ} \mathrm{F}$ plus24 hrs@302 F \\
\hline & 0.5371 & 0.5514 & $2.66 \%$ & $\mathrm{~N} / \mathrm{C}$ \\
\hline NOMEX & & & & same condition as above \\
\hline & 0.4700 & 0.4673 & $-0.57 \%$ & darkened \\
\hline MYAR & & & & some pockets in \\
\hline & & & & mylar, pulled away \\
\hline $\mathrm{C} 2$ & 0.3982 & 0.3995 & $0.33 \%$ & $\mathrm{~N} / \mathrm{C}$ \\
\hline NO/MY & & & & same condition as above \\
\hline & & & & see photo \\
\hline
\end{tabular}




\begin{tabular}{|c|c|c|c|c|c|c|c|c|c|}
\hline & $500 \mathrm{HRS}$ & in $\mathrm{HCFC}$. & $124 /$ Shriev & Zerol-15 & @ 260 F & & & & \\
\hline D & WT & EXP WT & $\begin{array}{c}\text { WT \% } \\
\text { CHANGE }\end{array}$ & $\begin{array}{l}\text { BREAK } \\
\text { LOAD } \\
\text { (AVE) }\end{array}$ & $\begin{array}{l}\text { BREAK } \\
\text { LOAD } \\
\text { EAP }\end{array}$ & $\begin{array}{c}\% \\
\text { CHANGE } \\
\text { BAK } \\
\text { LOAD }\end{array}$ & $\mid \begin{array}{c}\text { STRETCH } \\
\text { (INCH) }\end{array}$ & $\%$ ELONG & EXP VIS \\
\hline A 1 & 1.5114 & 1.5141 & $0.18 \%$ & 39.02 & 53.50 & & 0.06 & $3.00 \%$ & $\mathrm{~N} / \mathrm{C}$ \\
\hline Glass & & & & 39.02 & 62.12 & $35.95 \%$ & 0.06 & $3.00 \%$ & \\
\hline & & & & 39.02 & 43.52 & & 0.05 & $2.50 \%$ & \\
\hline B1 & 0.7275 & 0.7429 & $2.12 \%$ & 56.12 & 26.02 & & 0.06 & $3.00 \%$ & $\mathrm{~N} / \mathrm{C}$ \\
\hline Polyester & & & & 56.12 & 25.22 & $-53.93 \%$ & 0.05 & $2.50 \%$ & \\
\hline & & & & 56.12 & 26.32 & & 0.06 & $3.00 \%$ & \\
\hline C1 & 1.6171 & 1.9128 & $18.29 \%$ & 88.50 & 65.82 & & 0.12 & $6.00 \%$ & very \\
\hline Permacel & & & & 88.50 & 44.62 & $-36.39 \%$ & 0.21 & $10.50 \%$ & darkened \\
\hline & & & & 88.50 & 58.45 & & 0.16 & $8.00 \%$ & \\
\hline & & $500 \mathrm{HR}$ & $\mathrm{S}$ in $\mathrm{HCFC}$. & 24/Shrie & e Zerol-15 & $0 @ 260$ F & $->302^{\circ} \mathrm{F}$ & 24 HRS & \\
\hline & 1.4230 & 1.4222 & $-0.06 \%$ & 39.02 & 64.07 & & 0.06 & $3.00 \%$ & $\mathrm{~N} / \mathrm{C}$ \\
\hline Class & & & & 39.02 & 54.55 & $55.89 \%$ & 0.06 & $3.00 \%$ & \\
\hline & & & & 39.02 & 63.87 & & 0.06 & $3.00 \%$ & \\
\hline & 0.6620 & 0.6654 & $0.51 \%$ & 56.12 & 20.87 & & 0.06 & $3.00 \%$ & discolored \\
\hline Polyester & & & & 56.12 & 19.90 & $.69 .57 \%$ & 0.04 & $2.00 \%$ & \\
\hline & & & & 56.12 & 10.47 & & 0.10 & $5.00 \%$ & \\
\hline $\mathrm{C2}$ & 1.5531 & 1.4890 & $-4.13 \%$ & 88.50 & 52.35 & & 0.10 & $5.00 \%$ & very \\
\hline Permacel & & & & 88.50 & 68.37 & $-19.91 \%$ & 0.10 & $5.00 \%$ & Darkened \\
\hline & & & & 88.50 & 91.92 & & 0.13 & $6.50 \%$ & \\
\hline
\end{tabular}


TIE CORD--HCFC-124/S .inieve Zerol-150 oil @ 260 $\mathrm{F}\left(127^{\circ} \mathrm{C}\right)$

\begin{tabular}{|c|c|c|c|c|c|c|c|c|c|}
\hline \multicolumn{10}{|c|}{500 HRS IN R-124/Shrieve Zerol-150 @ 260 F } \\
\hline ID & WT & EXPWT & $\begin{array}{c}\text { WT \% } \\
\text { CHANGE }\end{array}$ & \begin{tabular}{|l|} 
EXP \\
VIS
\end{tabular} & $\begin{array}{l}\text { BREAK } \\
\text { LOAD } \\
\text { (AVE) }\end{array}$ & $\begin{array}{c}\text { BREAK } \\
\text { LOAD } \\
\text { EXP } \\
(\text { ave })\end{array}$ & $\begin{array}{c}\% \\
\text { CHANGE } \\
\text { BAK } \\
\text { LOAD }\end{array}$ & $\begin{array}{c}\text { STRETCH } \\
\text { (Inch) }\end{array}$ & $\begin{array}{c}\% \\
\text { ELONG }\end{array}$ \\
\hline \multirow[t]{4}{*}{ A1 } & \multirow[t]{3}{*}{0.2131} & \multirow[t]{3}{*}{0.2148} & \multirow[t]{3}{*}{$0.80 \%$} & $\mathrm{~N} / \mathrm{C}$ & 28.36 & 38.87 & & 0.45 & $22.5 \%$ \\
\hline & & & & & 28.36 & 37.02 & \multirow[t]{2}{*}{$32.18 \%$} & 0.43 & $21.5 \%$ \\
\hline & & & & & 28.36 & 36.57 & & 0.48 & $24.0 \%$ \\
\hline & \multicolumn{9}{|c|}{500 HRS IN R-124/S hrieve Zerol-150 -> 24 HRS @ 302 $\mathrm{F}$} \\
\hline \multirow[t]{3}{*}{ A2 } & \multirow[t]{3}{*}{0.2347} & \multirow[t]{3}{*}{0.2339} & \multirow[t]{3}{*}{$-0.341 \%$} & $\mathrm{~N} / \mathrm{C}$ & 28.36 & 33.42 & & 0.42 & $21.0 \%$ \\
\hline & & & & & 28.36 & 34.40 & $15.86 \%$ & 0.63 & $31.5 \%$ \\
\hline & & & & & 28.36 & 30.75 & & 0.47 & $23.5 \%$ \\
\hline
\end{tabular}




\begin{tabular}{|c|c|c|c|c|c|c|c|}
\hline & 500 HRS IN & CFC. $124 / \mathrm{Sc}$ & chrieve Zer & rol-150@ & $260^{\circ} \mathrm{F}$ & & \\
\hline ID & $\cdot \quad W T$ & EXPWT & $\begin{array}{c}\text { WT \% } \\
\text { CHANGE }\end{array}$ & EXP VIS & $\begin{array}{c}\text { BASE DIE } \\
\text { (AVE) }\end{array}$ & EXPDIE & $\begin{array}{c}\text { DIE\% } \\
\text { CHANGE }\end{array}$ \\
\hline DMD & 4.0187 & 4.1877 & $4.21 \%$ & $\mathrm{~N} / \mathrm{C}$ & 9.61 & 8.64 & \\
\hline A1 & & & & & 9.61 & 7.68 & $-17.93 \%$ \\
\hline & & & & & 9.61 & 7.34 & \\
\hline DTMD & 4.3659 & 4.5285 & $3.72 \%$ & $\mathrm{~N} / \mathrm{C}$ & 9.95 & 11.23 & \\
\hline$B 1$ & & & & & 9.95 & 13.34 & $30.42 \%$ \\
\hline & & & & & 9.95 & 14.36 & \\
\hline & HCFC-124/Sc & rieve Zerol- & 150@26 & $0^{\circ} \mathrm{F} \rightarrow 24$ & JRS@ @ 30 & & \\
\hline DMD & 4.0743 & 4.1148 & $0.99 \%$ & darkened & 9.61 & 9.97 & \\
\hline A2 & & & & & 9.61 & 10.10 & $1.70 \%$ \\
\hline & & & & & 9.61 & 9.25 & \\
\hline DTMD & 4.3351 & 4.3729 & $0.87 \%$ & darkened & 9.95 & 12.41 & \\
\hline $\mathrm{B2}$ & & & & & 9.95 & 10.97 & $20.67 \%$ \\
\hline & & & & & 9.95 & 12.64 & \\
\hline
\end{tabular}




\section{Appendix D}

\section{Experimental Data for HCFC-142b/Shrieve Zerol-150 Exposure at $127^{\circ} \mathrm{C}\left(260^{\circ} \mathrm{F}\right)$}




\begin{tabular}{|c|c|c|c|c|c|c|c|c|c|c|}
\hline & 500 hours & ICL & - & Lerol- & 50 oil & $F(127$ & & & & \\
\hline ID & WT & EXPWT & $\begin{array}{c}\text { WT \% } \\
\text { CHANGE }\end{array}$ & EXP VIS & $\begin{array}{c}\text { BASE BRN } \\
\text { OUT } \\
\text { (AVE) }\end{array}$ & $\begin{array}{c}\text { EXPBRN } \\
\text { OUT }\end{array}$ & $\begin{array}{c}\text { BRN OUTT } \\
\% \\
\text { CHANGE }\end{array}$ & $\begin{array}{c}\text { BASE DIE } \\
(A \vee E)\end{array}$ & EXP DIE & $\begin{array}{c}\text { DIE \% } \\
\text { CHANGE }\end{array}$ \\
\hline A1 & 21.892 & 21.9106 & $0.085 \%$ & $\mathrm{~N} / \mathrm{C}$ & 576 & 317 & & 15.80 & 16.13 & \\
\hline & & & & & 576 & 390 & $-37.3 \%$ & 15.80 & 12.17 & $-19.2 \%$ \\
\hline & & & & & 576 & 376 & & 15.80 & 10.00 & \\
\hline B1 & 27.3851 & 27.4600 & $0.274 \%$ & $\mathrm{~N} / \mathrm{C}$ & 736 & 732 & & 11.62 & 13.10 & \\
\hline & & & & & 736 & 617 & $-6.7 \%$ & 11.62 & 11.96 & $8.3 \%$ \\
\hline & & & & & 736 & 711 & & 11.62 & 12.68 & \\
\hline$C_{1}$ & 23.9242 & 23.9430 & $0.079 \%$ & $\mathrm{~N} / \mathrm{C}$ & 579 & 286 & & 16.58 & 15.37 & \\
\hline & & & & & 579 & 334 & $-53.1 \%$ & 16.58 & 10.95 & $-16.8 \%$ \\
\hline & & & & & 579 & 195 & & 16.58 & 15.08 & \\
\hline & 500 hours i & HCFC-14 & 2b/Shrieve & Zerol-1 & 50 oil at 26 & $0^{\circ} \mathrm{F}\left(127^{\circ} \mathrm{C}\right.$ & C) plus 24 & hours at & $302^{\circ} \mathrm{F}(15 \mathrm{C}$ & $\left.0^{\circ} \mathrm{C}\right)$ \\
\hline A2 & 21.8597 & 21.8680 & $0.038 \%$ & $\mathrm{~N} / \mathrm{C}$ & 576 & 391 & & 15.80 & 16.39 & \\
\hline & & & & & 576 & 393 & $-34.5 \%$ & 15.80 & 15.77 & $-3.1 \%$ \\
\hline & & & & & 576 & 348 & & 15.80 & 13.79 & \\
\hline $\mathrm{B} 2$ & 27.7826 & 27.8017 & $0.069 \%$ & $\mathrm{~N} / \mathrm{C}$ & 736 & 729 & & 11.62 & 10.54 & \\
\hline & & & & & 736 & 738 & $-0.4 \%$ & 11.62 & 11.03 & $-6.1 \%$ \\
\hline & & & & & 736 & 732 & & 11.62 & 11.16 & \\
\hline $\mathrm{C} 2$ & 23.5414 & 23.5523 & $0.046 \%$ & $\mathrm{~N} / \mathrm{C}$ & 579 & 390 & & 16.58 & 12.72 & \\
\hline & & & & & 579 & 230 & $-45.8 \%$ & 16.58 & 16.16 & $-10.9 \%$ \\
\hline & & & & & 579 & 322 & & 16.58 & 15.46 & \\
\hline
\end{tabular}


TWISTED PAIRSMIRE AWITH VARNISH-HCFC-142b/Shrieve Zerol-150 oil @260 $\mathrm{F}\left(127^{\circ} \mathrm{C}\right)$

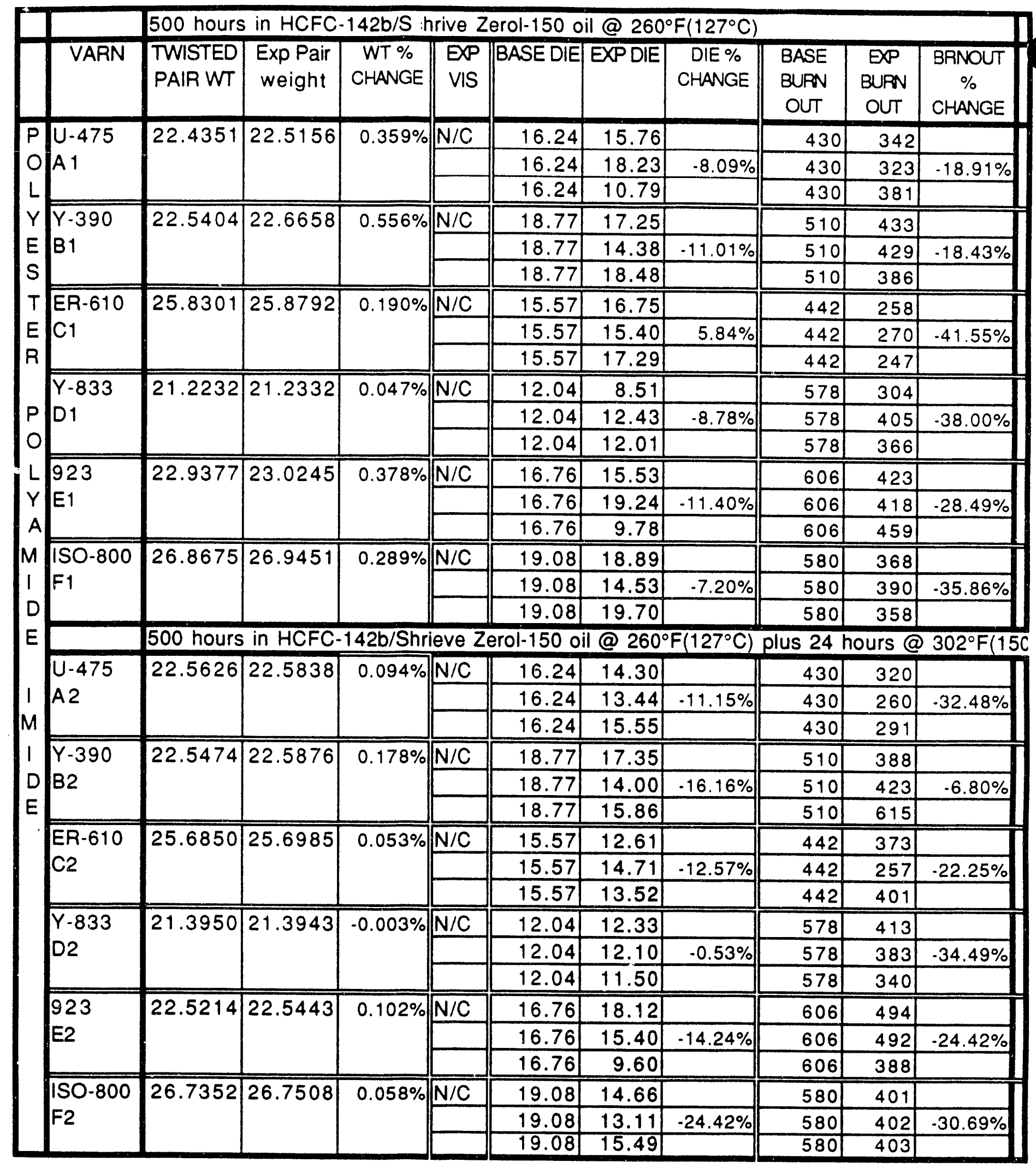


TWISTED PAIRS/WIRE BNITH VARNISH -HCFC-142b/S hrieve Zerol-150 oil @260․ $\left(127^{\circ} \mathrm{C}\right)$

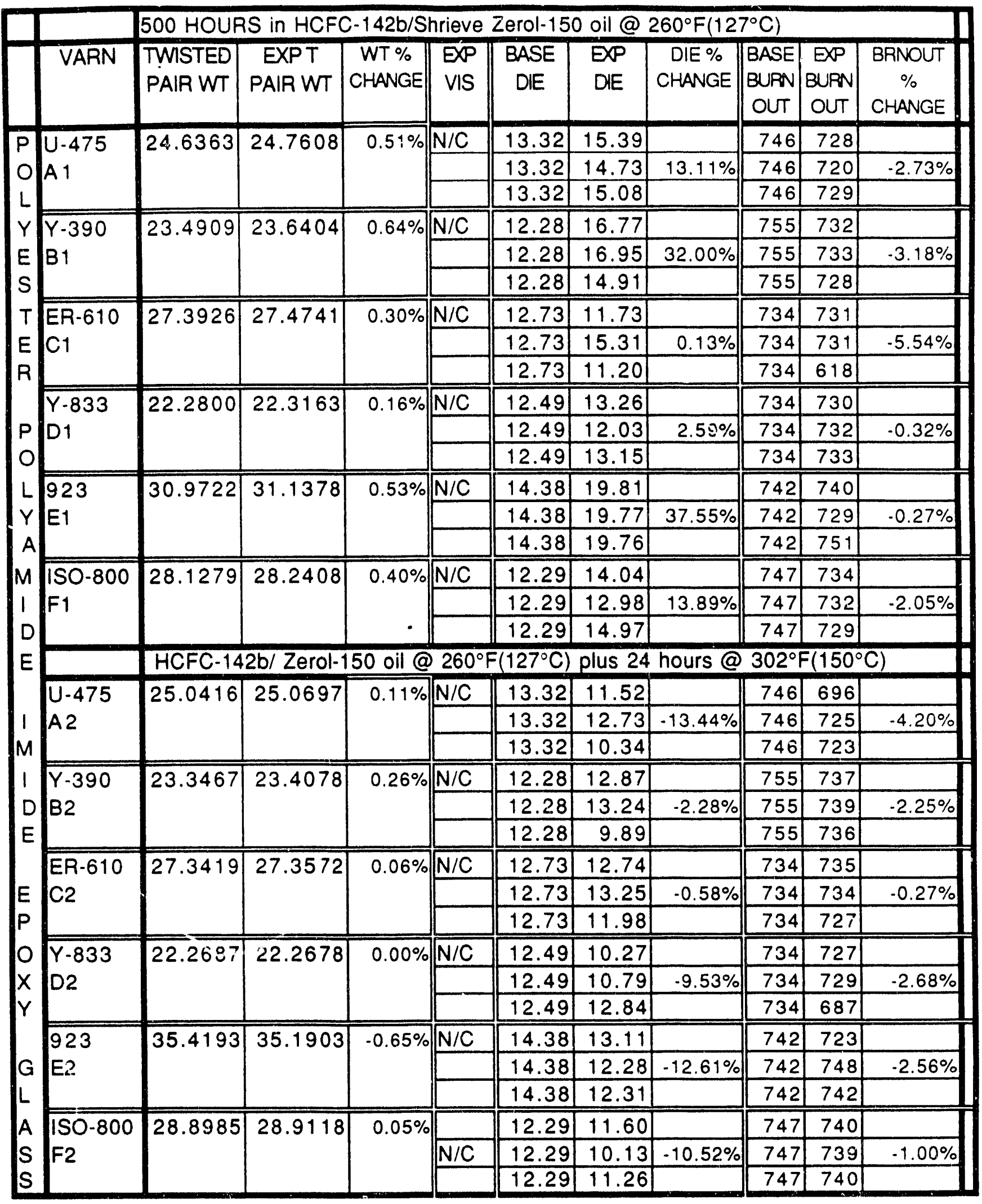


TWISTED PAIRSMIRE CNITH VARNISH-HCFC-142b/S hrieve Zerol-150 @ 260 $\mathrm{F}\left(127^{\circ} \mathrm{C}\right)$

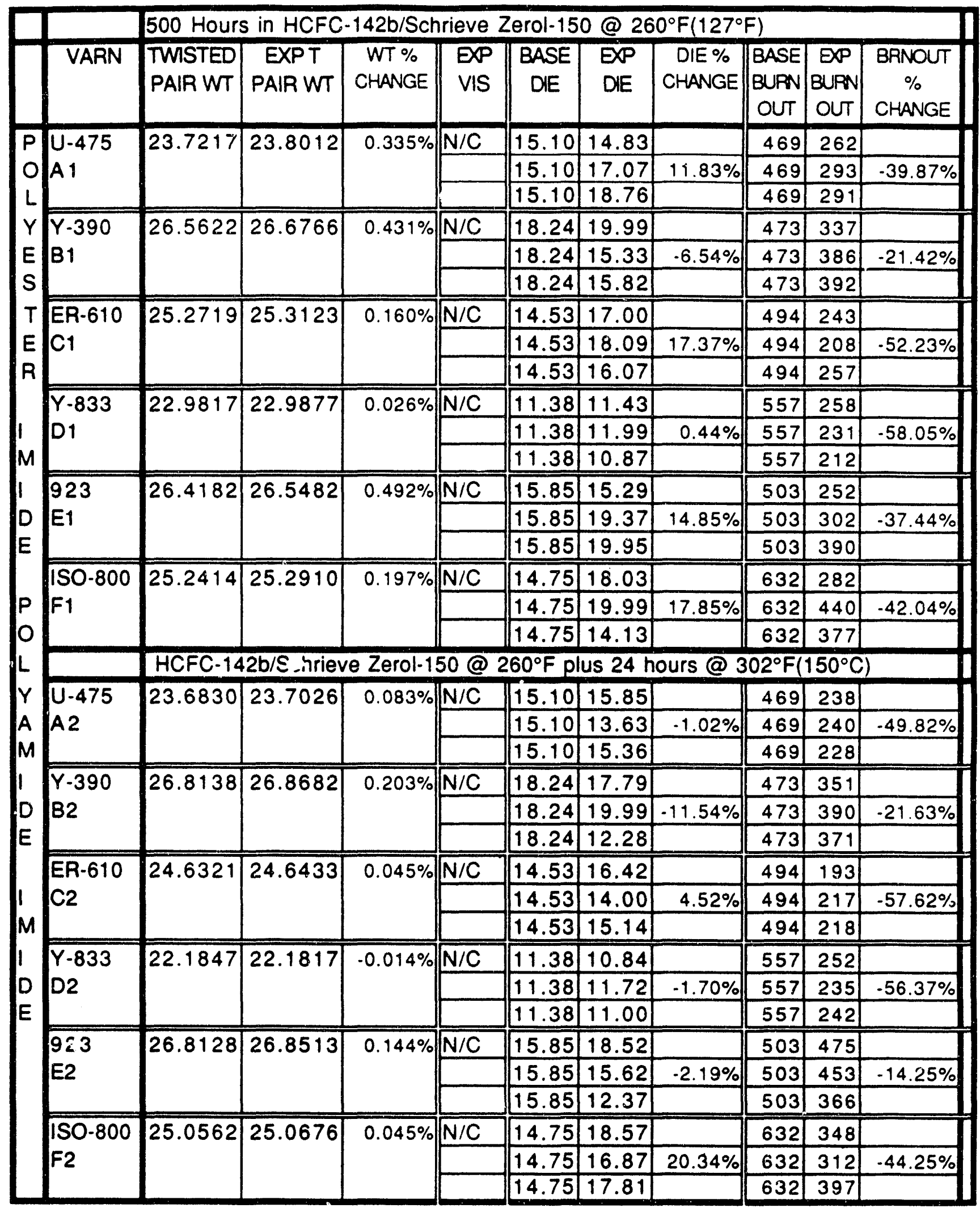


HELICAL COILSMIRE A-.-HCFC-142b/Shrieve Zerol-150 oil @260 F $\left(127^{\circ} \mathrm{C}\right)$

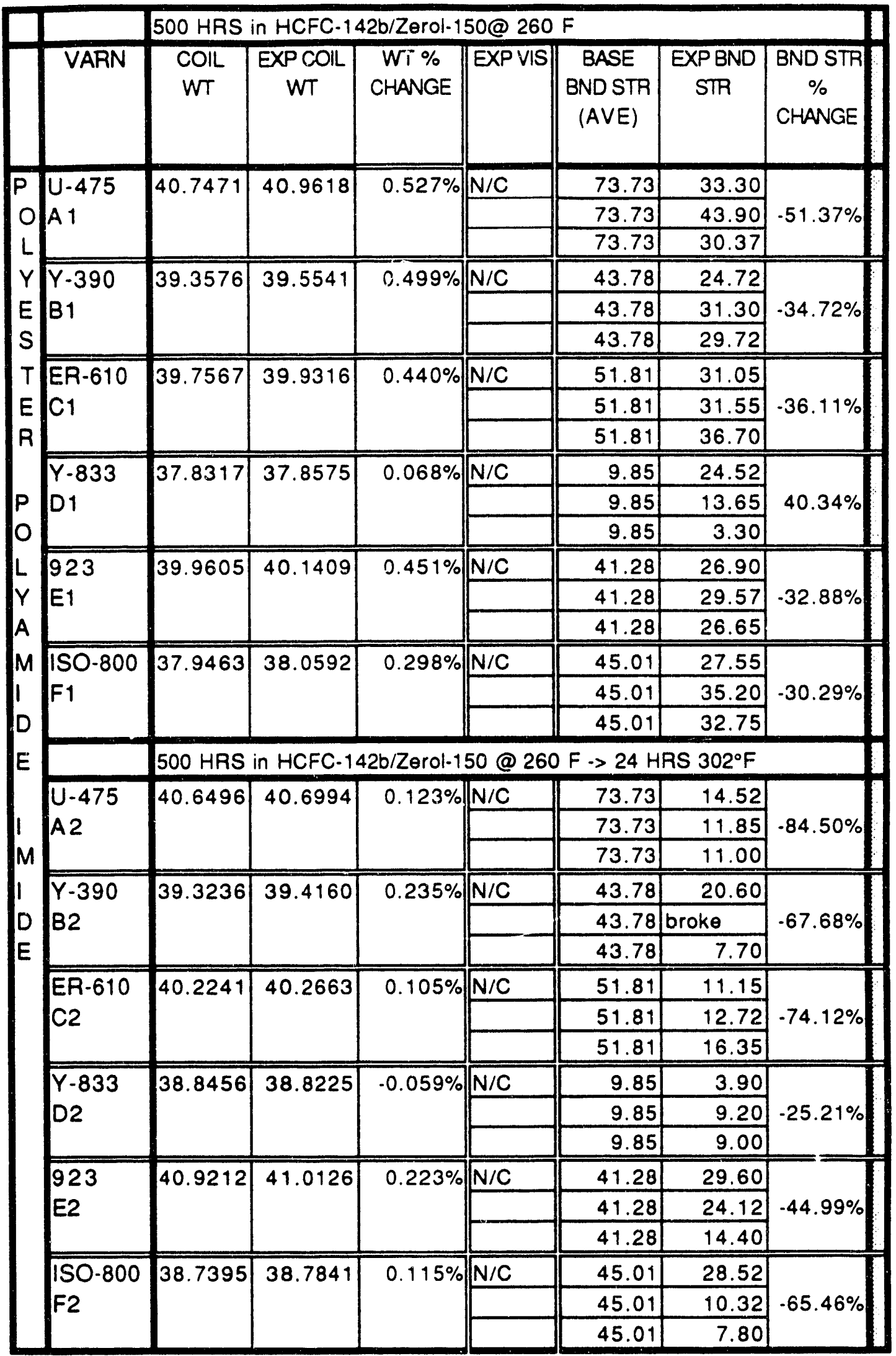


HELICAL COILSMIRE B-HCFC-142b/Shrieve Zerol-150 Oil

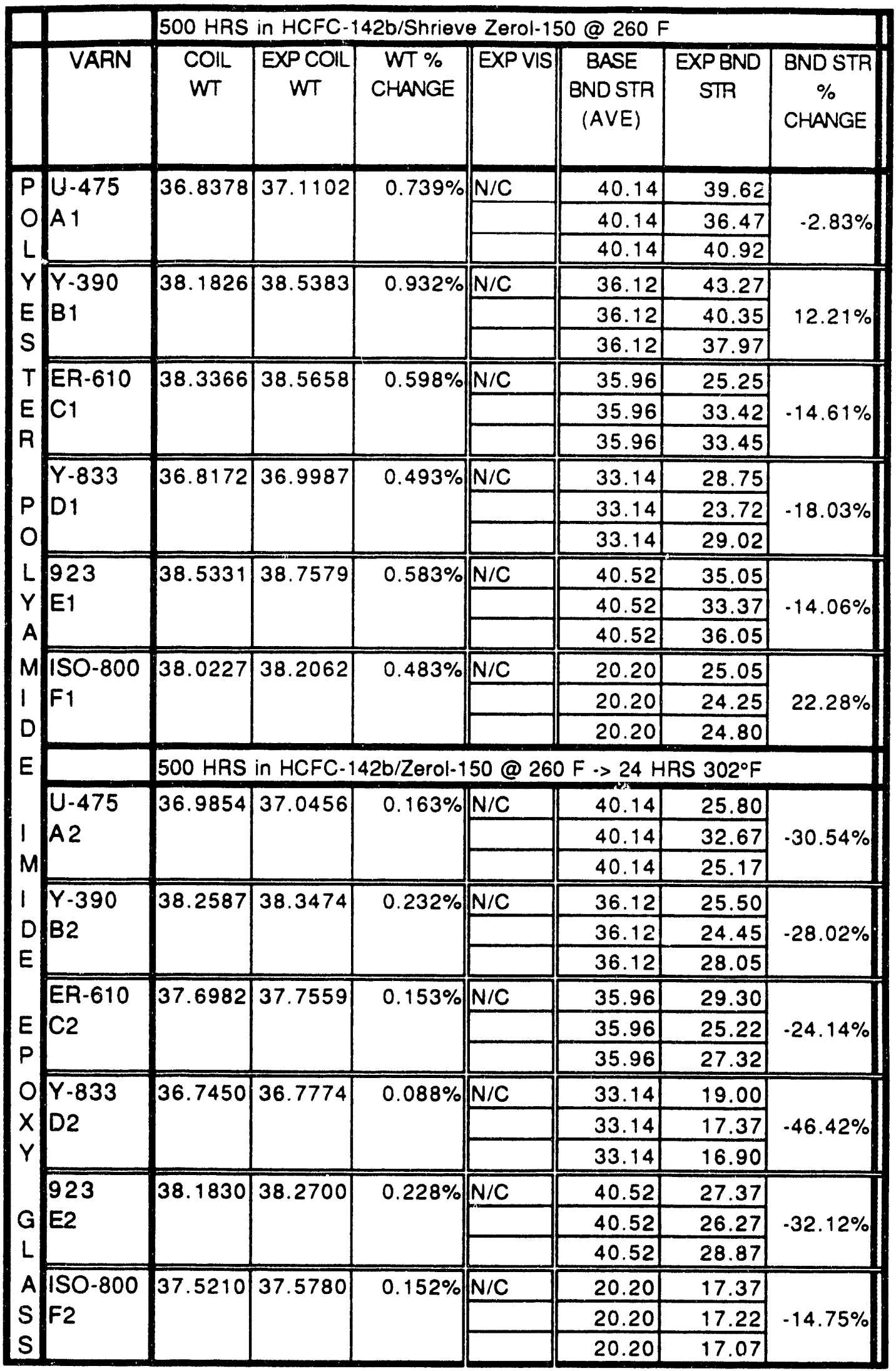


HELICAL COILS/WIRE C-HCFC-142b/Shrieve Zerol-150 oil @ $260^{\circ} \mathrm{F}\left(127^{\circ} \mathrm{C}\right)$

\begin{tabular}{|c|c|c|c|c|c|c|c|c|}
\hline & & 500 HRS i & in HCFC. & $2 b /$ Shriev & Zerol-1 & $60 \mathrm{~F}$ & & \\
\hline & VARN & $\begin{array}{l}\text { COIL } \\
\text { WT }\end{array}$ & $\begin{array}{c}\text { EXP COIL } \\
W T\end{array}$ & $\begin{array}{c}\text { WT \% } \\
\text { CHANGE }\end{array}$ & EXPVIS| & $\begin{array}{c}\text { BASE } \\
\text { BND STR } \\
\text { (AVE) }\end{array}$ & $\begin{array}{c}\text { EXPBND } \\
\text { STR }\end{array}$ & $\begin{array}{c}\text { BND STR } \\
\% \\
\text { CHANGE }\end{array}$ \\
\hline $\mathbf{P}$ & $U-475$ & $|38.1970|$ & 38.3834 & $0.488 \%$ & $\mathrm{~N} / \mathrm{C}$ & 51.21 & 62.92 & \\
\hline 0 & A1 1 & & & & & 51.21 & 36.87 & $-7.41 \%$ \\
\hline L & & & & & & 51.21 & 42.45 & \\
\hline$Y$ & $Y-390$ & $39.4390 \mid$ & 39.6051 & $0.421 \%$ & $\mathrm{~N} / \mathrm{C}$ & 50.72 & 25.55 & \\
\hline$E$ & B1 & & & & & 50.72 & 23.87 & $-50.39 \%$ \\
\hline $\mathrm{s}$ & & & & & & 50.72 & 26.07 & \\
\hline $\mathrm{T}$ & ER-610 & 38.0862 & 38.2452 & $0.417 \%$ & $\mathrm{~N} / \mathrm{C}$ & 58.33 & 34.40 & \\
\hline$E$ & C1 & & & & & 58.33 & 22.25 & $.47 .13 \%$ \\
\hline$R$ & & & & & & 58.33 & 35.87 & \\
\hline & $Y-833$ & 38.5008 & 38.4147 & $-0.224 \%$ & $\mathrm{~N} / \mathrm{C}$ & 5.84 & broken & \\
\hline 1 & D1 & & & & & 5.84 & broken & $62.67 \%$ \\
\hline$M$ & & & & & & 5.84 & 9.50 & \\
\hline 1 & 923 & 39.8348 & 40.0153 & $0.453 \%$ & $\mathrm{~N} / \mathrm{C}$ & 49.26 & 20.92 & \\
\hline$D$ & E1 & & & & & 49.26 & 25.02 & $-55.87 \%$ \\
\hline E & & & & & & 49.26 & 19.27 & \\
\hline & $150-800$ & 39.7158 & 39.8353 & $0.301 \%$ & $\mathrm{~N} / \mathrm{C}$ & 36.08 & 26.10 & \\
\hline $\mathbf{P}$ & $F_{1}$ & & & & & 36.08 & 25.27 & $.25 .50 \%$ \\
\hline 0 & & & & & & 36.08 & 29.27 & \\
\hline L & & 500 HRS & in HCFC- 1 & $+2 \mathrm{~b} /$ Shrieve & Zerol-15 & $0 @ 260 \mathrm{~F}$. & $>24 \mathrm{HRS}$ & $302^{\circ} \mathrm{F}$ \\
\hline Y & $U-475$ & 37.3945 & 37.4482 & $0.144 \%$ & $\mathrm{~N} / \mathrm{C}$ & 51.21 & 6.00 & \\
\hline A & $A 2$ & & & & & 51.21 & 12.37 & $-81.16 \%$ \\
\hline$M$ & & & & & & 51.21 & 10.57 & \\
\hline 1 & $Y-390$ & 40.8246 & 40.9084 & $0.205 \%$ & $\mathrm{~N} / \mathrm{C}$ & 50.72 & 12.90 & \\
\hline D & B2 & & & & & 50.72 & 8.60 & $.71 .33 \%$ \\
\hline$E$ & & & & & & 50.72 & 22.12 & \\
\hline & ER-610 & 38.1005 & 38.1480 & $0.125 \%$ & $\mathrm{~N} / \mathrm{C}$ & 58.33 & 9.90 & \\
\hline 1 & C2 & & & & & 58.33 & 11.82 & $.73 .06 \%$ \\
\hline$M$ & & & & & & 58.33 & 25.42 & \\
\hline 1 & $Y-833$ & 38.6826 & 38.5539 & $-0.333 \%$ & $\mathrm{~N} / \mathrm{C}$ & 5.84 & 11.45 & \\
\hline D & D2 & & & & & 5.84 & 18.95 & $151.43 \%$ \\
\hline $\mathbf{E}$ & & & & & & 5.84 & 13.65 & \\
\hline & 923 & 39.8603 & 39.9482 & $0.221 \%$ & $\mathrm{~N} / \mathrm{C}$ & 49.26 & 23.50 & \\
\hline & E2 & & & & & 49.26 & 26.27 & $-49.88 \%$ \\
\hline & & & & & & 49.26 & 24.30 & \\
\hline & ISO-800 & 39.2767 & 39.3214 & $0.114 \%$ & $\mathrm{~N} / \mathrm{C}$ & 36.08 & 30.20 & \\
\hline & F2 & & & & & 36.08 & 32.90 & $-17.06 \%$ \\
\hline & & & & & & 36.08 & 26.67 & \\
\hline
\end{tabular}


SINGLE MAG WIRE WITHOUT VARNISH-HCFC-142b/S hrieve Zerol-150@ $260^{\circ} \mathrm{F}\left(127^{\circ} \mathrm{C}\right)$ )

\begin{tabular}{|c|c|c|c|c|c|}
\hline \multicolumn{6}{|c|}{500 HRS in HCFC-142b/Zerol-150 oil @ 260 $0^{\circ} \mathrm{F}\left(127^{\circ} \mathrm{C}\right)$} \\
\hline ID & WT & EXPWT & $\begin{array}{c}\text { WT \% } \\
\text { CHANGE }\end{array}$ & EXPVS & EXPFLEX \\
\hline \multirow{2}{*}{ A1 } & \multirow[t]{2}{*}{3.6171} & \multirow[t]{2}{*}{3.62} & \multirow[t]{2}{*}{$0.080 \%$} & $\mathrm{~N} / \mathrm{C}$ & \\
\hline & & & & & YES \\
\hline \multirow[t]{2}{*}{ B1 } & \multirow[t]{2}{*}{3.3665} & \multirow{2}{*}{3.3756} & \multirow[t]{2}{*}{$0.270 \%$} & $\mathrm{~N} / \mathrm{C}$ & \\
\hline & & & & & YES \\
\hline \multirow[t]{2}{*}{$C_{1}$} & \multirow[t]{2}{*}{3.8304} & \multirow[t]{2}{*}{3.8328} & \multirow[t]{2}{*}{$0.063 \%$} & $\mathrm{~N} / \mathrm{C}$ & \\
\hline & & & & & YES \\
\hline \multicolumn{6}{|c|}{ 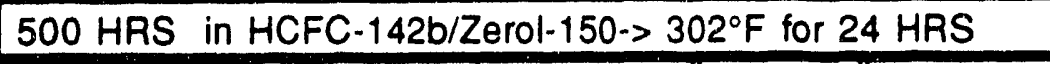 } \\
\hline \multirow[t]{2}{*}{ A2 } & \multirow[t]{2}{*}{3.7416} & \multirow[t]{2}{*}{3.7419} & \multirow[t]{2}{*}{$0.008 \%$} & $\mathrm{~N} / \mathrm{C}$ & \\
\hline & & & & & YES \\
\hline \multirow[t]{2}{*}{ B2 } & \multirow[t]{2}{*}{3.3361} & \multirow[t]{2}{*}{3.3362} & \multirow[t]{2}{*}{$0.003 \%$} & $\mathrm{~N} / \mathrm{C}$ & \\
\hline & & & & & YES \\
\hline \multirow[t]{3}{*}{$\mathrm{C2}$} & \multirow[t]{3}{*}{3.806} & \multirow{3}{*}{3.805} & \multirow[t]{3}{*}{$-0.026 \%$} & $\mathrm{~N} / \mathrm{C}$ & \\
\hline & & & & & YES \\
\hline & & & & & \\
\hline
\end{tabular}




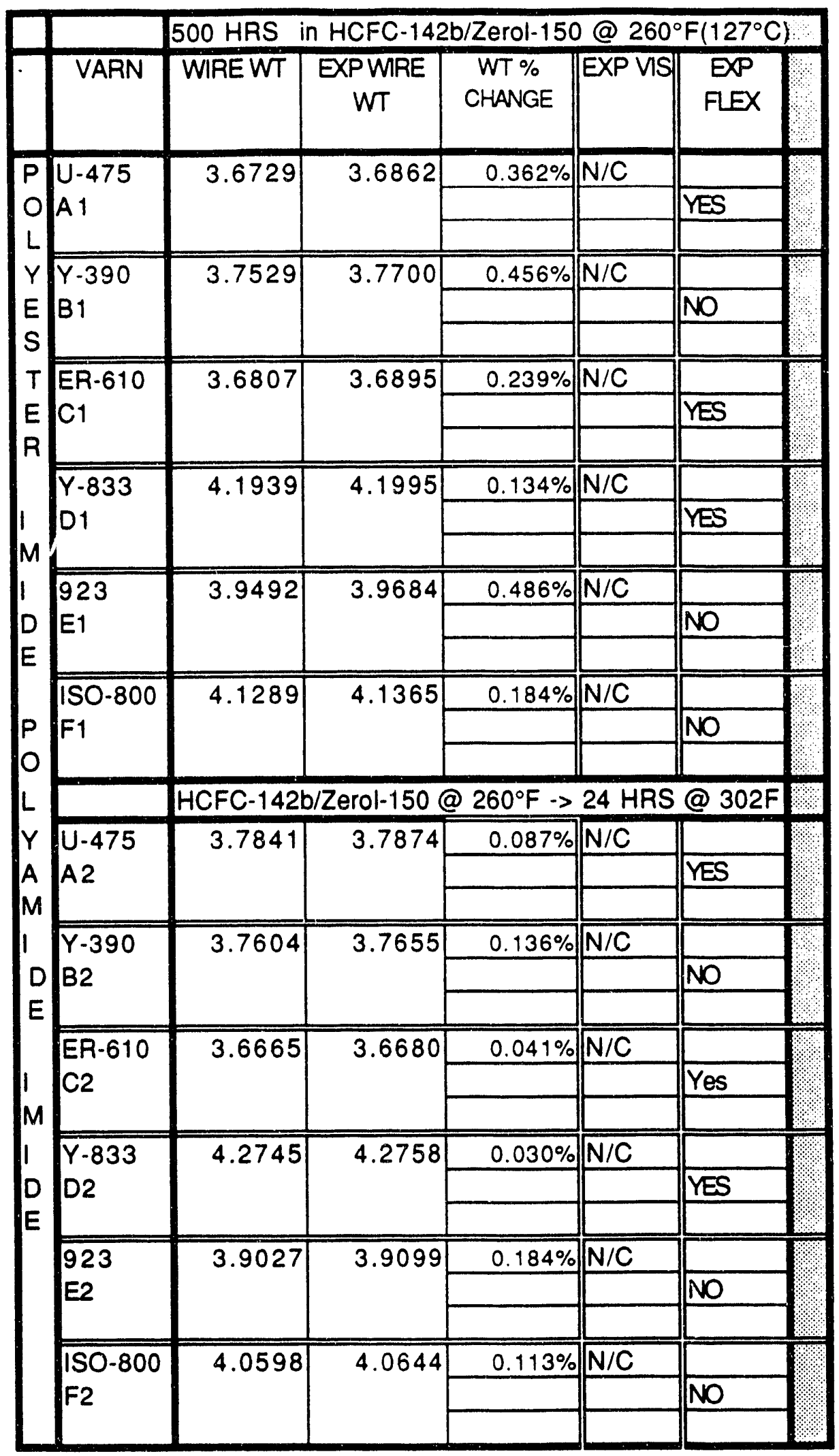




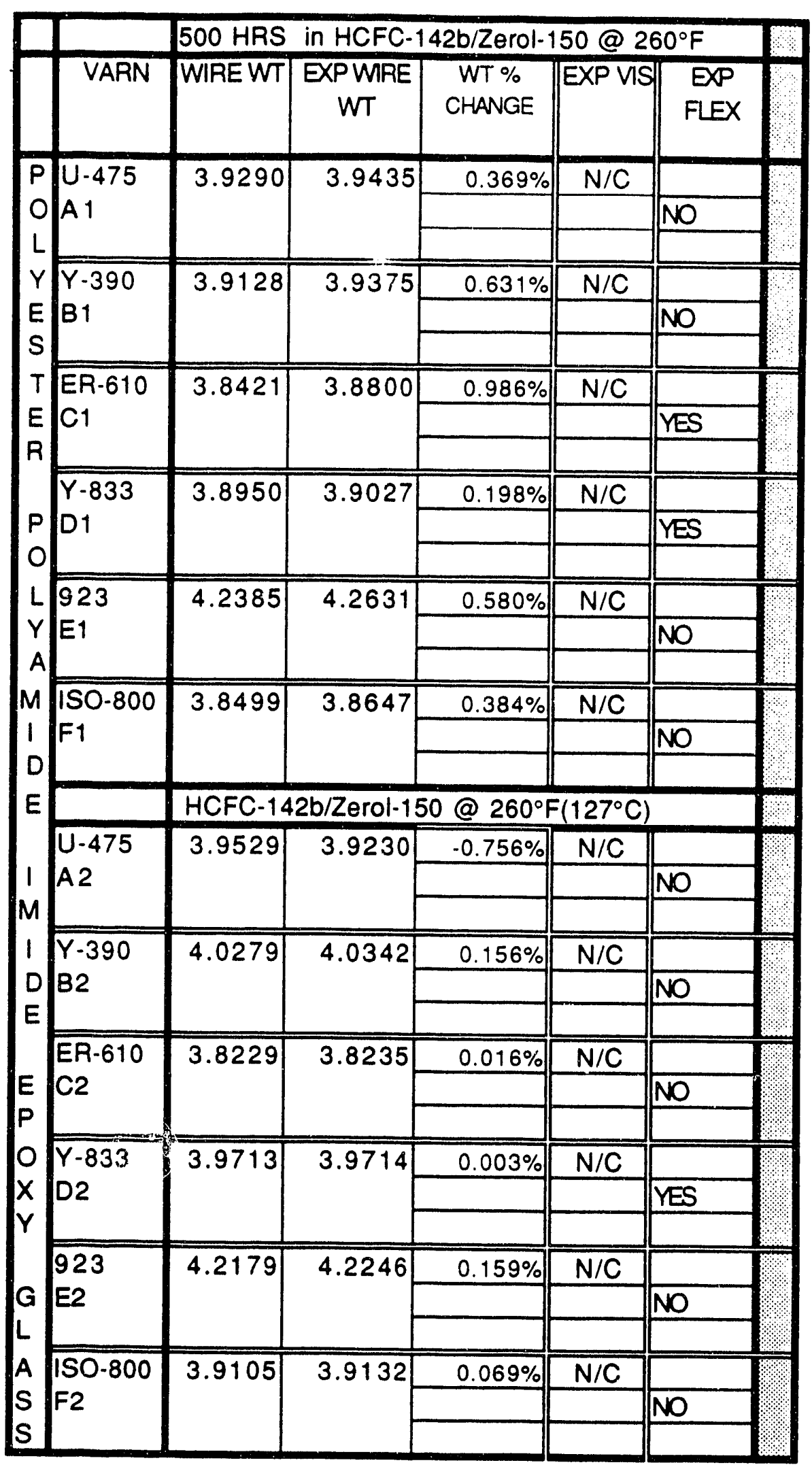




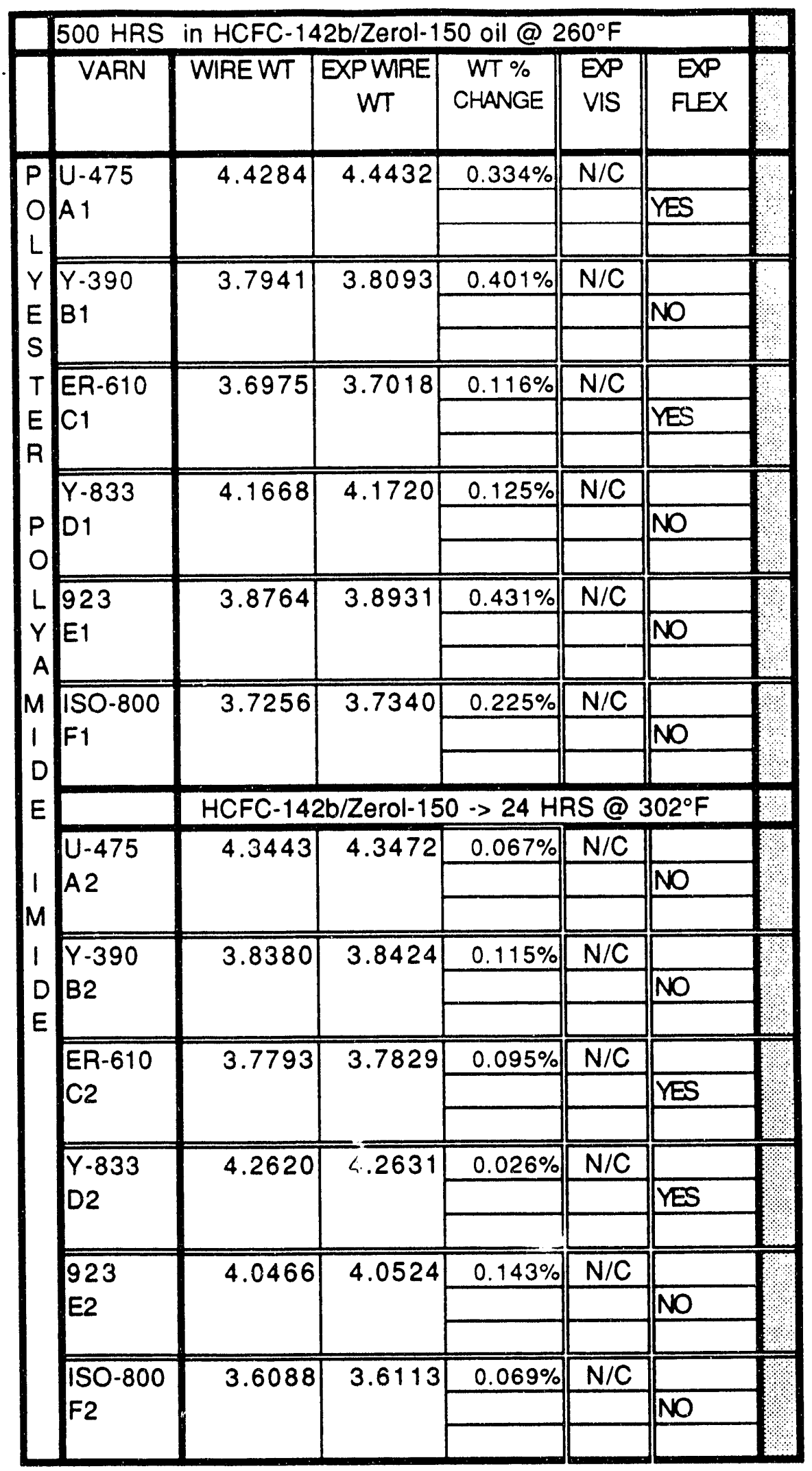




\begin{tabular}{|c|c|c|c|c|c|}
\hline \multicolumn{6}{|c|}{500 HRS IN R-142b/Zerol-150 Oil @ 260 $\mathrm{F}$} \\
\hline ID & WT & EXPWT & $\begin{array}{c}\text { WT \% } \\
\text { CHANGE }\end{array}$ & EXP VIS & EXPFLEX \\
\hline $\begin{array}{l}\text { U. } 475 \\
\text { A1 }\end{array}$ & 2.4993 & 2.6242 & $5.00 \%$ & $\mathrm{~N} / \mathrm{C}$ & $\mathrm{N} / \mathrm{C}$ \\
\hline $\begin{array}{l}Y-390 \\
B 1\end{array}$ & 2.2531 & 2.3947 & $6.28 \%$ & $\begin{array}{l}\text { slightly } \\
\text { warped }\end{array}$ & $\mathrm{N} / \mathrm{C}$ \\
\hline $\begin{array}{l}\text { ER-610 } \\
\text { C1 }\end{array}$ & 2.4949 & 2.7669 & $10.90 \%$ & \begin{tabular}{|l} 
Rough \\
Surface \\
\end{tabular} & $\mathrm{N} / \mathrm{C}$ \\
\hline $\begin{array}{l}Y-833 \\
D 1\end{array}$ & 1.8570 & 1.9812 & $6.69 \%$ & \begin{tabular}{|l} 
Slightly \\
warped
\end{tabular} & $\mathrm{N} / \mathrm{C}$ \\
\hline $\begin{array}{l}923 \\
E 1\end{array}$ & 1.3171 & 1.3941 & $5.85 \%$ & \begin{tabular}{|l} 
Rough \\
surface \\
\end{tabular} & $\mathrm{N} / \mathrm{C}$ \\
\hline $\begin{array}{l}\text { ISO-800 } \\
F_{1}\end{array}$ & 1.3864 & 1.3738 & $-0.91 \%$ & \begin{tabular}{|l} 
Slightly \\
warped \\
\end{tabular} & $\mathrm{N} / \mathrm{C}$ \\
\hline & R-142b/Zer & -150 at $\cdots$ & 24 hour a & $302^{\circ} \mathrm{F}$. & \\
\hline $\begin{array}{l}U-475 \\
A 2\end{array}$ & 2.0993 & 2.1372 & $1.81 \%$ & darkened & $N / C$ \\
\hline $\begin{array}{l}Y-390 \\
B 2\end{array}$ & 1.6439 & 1.6768 & $2.00 \%$ & \begin{tabular}{|l|} 
slightly \\
warped \\
darkened \\
\end{tabular} & $\mathrm{N} / \mathrm{C}$ \\
\hline $\begin{array}{l}\text { ER-610 } \\
\text { C2 }\end{array}$ & 2.3679 & 2.4183 & $2.13 \%$ & $\begin{array}{l}\text { darkened } \\
\text { Rough } \\
\text { Surface } \\
\end{array}$ & $N / C$ \\
\hline $\begin{array}{l}Y-833 \\
D 2\end{array}$ & 2.2175 & 2.3144 & $4.37 \%$ & $\begin{array}{l}\text { slightly } \\
\text { warped } \\
\text { pockets }\end{array}$ & $\mathrm{N} / \mathrm{C}$ \\
\hline $\begin{array}{l}923 \\
\text { E2 }\end{array}$ & 1.5177 & 1.5568 & $2.58 \%$ & \begin{tabular}{|l|} 
Rough \\
surface \\
darkened \\
\end{tabular} & $\mathrm{N} / \mathrm{C}$ \\
\hline $\begin{array}{l}\text { ISO-800 } \\
\text { F2 }\end{array}$ & 1.2454 & 1.1927 & $-4.23 \%$ & \begin{tabular}{|l|} 
slightly \\
warped \\
darkened \\
\end{tabular} & $\mathrm{N} / \mathrm{C}$ \\
\hline
\end{tabular}




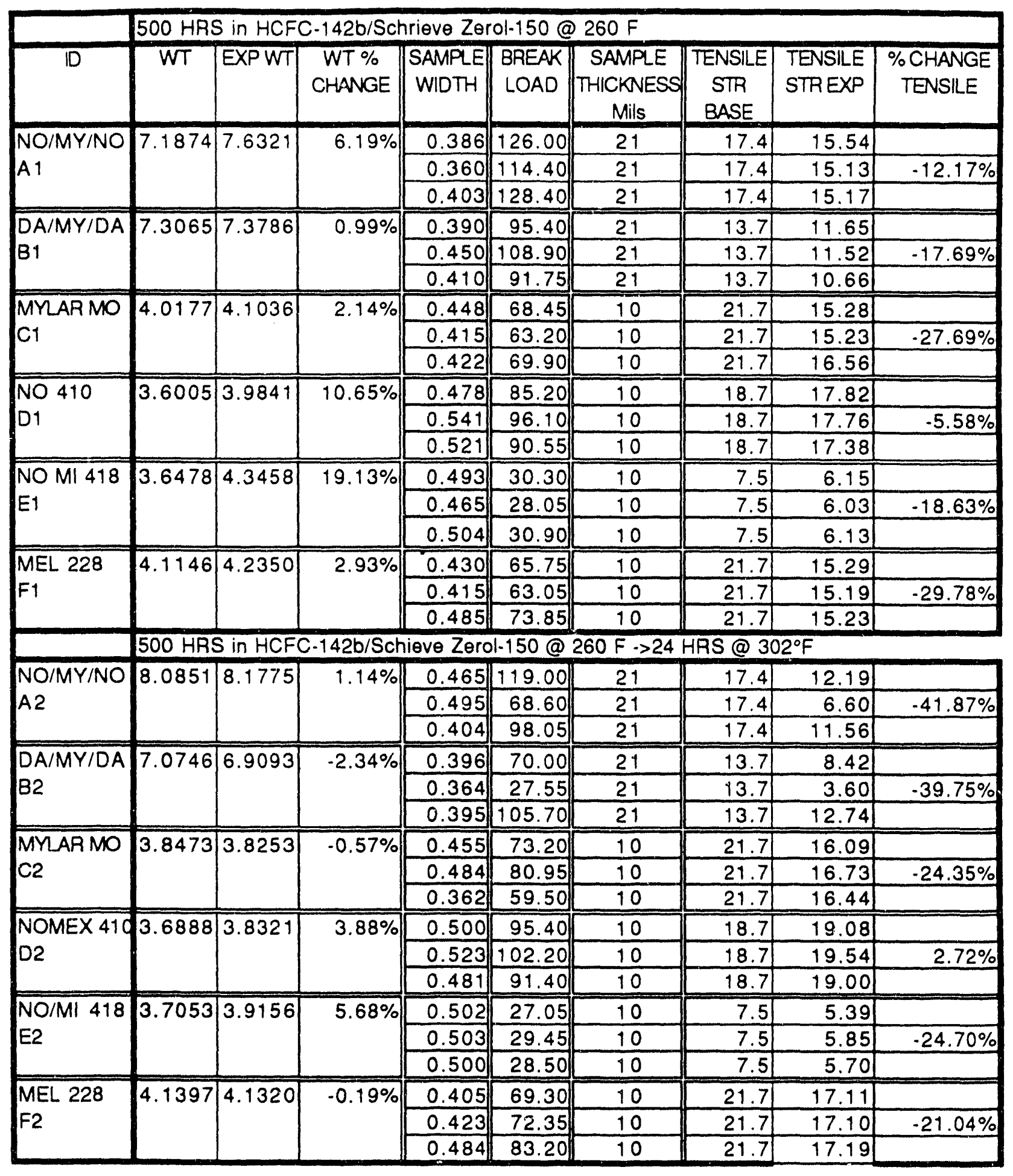




\begin{tabular}{|c|c|c|c|c|c|c|c|c|}
\hline & \multicolumn{7}{|c|}{ Results after 500 hour exposure } & \multirow[b]{2}{*}{ VISUAL EXP } \\
\hline$\overline{D D}$ & $\begin{array}{c}\text { STRETCH } \\
\text { (inch) }\end{array}$ & $\begin{array}{c}\% \\
\text { ELONG }\end{array}$ & $\begin{array}{c}\text { BASE } \\
\text { ELONG } \\
\text { (AVE) } \\
\end{array}$ & $\left|\begin{array}{l}\text { ELONG \% } \\
\text { CHANGE }\end{array}\right|$ & \begin{tabular}{|c|c|} 
BASE \\
DEE \\
$(A V E)$
\end{tabular} & EXP DIE & $\mid \begin{array}{c}\text { DIE \% } \\
\text { CHANGE }\end{array}$ & \\
\hline \multirow{3}{*}{$\begin{array}{l}\text { NO/MY/NO } \\
\text { A1 }\end{array}$} & 0.44 & $11.0 \%$ & $20.0 \%$ & & $>18.97$ & flash & & \multirow{3}{*}{$\begin{array}{l}\text { Major delamination } \\
\text { N/C appearance }\end{array}$} \\
\hline & 0.37 & $9.3 \%$ & $20.0 \%$ & $.49 .4 \%$ & $>18.97$ & flash & flash & \\
\hline & 0.38 & $9.5 \%$ & $20.0 \%$ & & $>18.97$ & flash & & \\
\hline \multirow{3}{*}{$\begin{array}{l}\mathrm{DA} / \mathrm{MY} / \mathrm{DA} \\
\mathrm{B} 1\end{array}$} & 0.35 & $17.5 \%$ & $46.0 \%$ & & $>15.27$ & flash & & \multirow{3}{*}{\begin{tabular}{|l} 
Discolored \\
yellowed \\
slightly warped
\end{tabular}} \\
\hline & 0.34 & $17.0 \%$ & $46.0 \%$ & $.64 .5 \%$ & $>15.27$ & flash & flash & \\
\hline & 0.29 & $14.5 \%$ & $46.0 \%$ & & $>15.27$ & flash & & \\
\hline \multirow{3}{*}{$\begin{array}{l}\text { MYLAR MO } \\
C_{1}\end{array}$} & 0.74 & $37.0 \%$ & $131.0 \%$ & & $>14.91$ & flash & & \multirow[t]{3}{*}{$\mathrm{N} / \mathrm{C}$} \\
\hline & 0.74 & $37.0 \%$ & $131.0 \%$ & $.72 .0 \%$ & $>14.91$ & flash & flash & \\
\hline & 0.72 & $36.0 \%$ & $131.0 \%$ & & $>14.91$ & flash & & \\
\hline \multirow{3}{*}{$\begin{array}{l}\text { NO } 410 \\
\text { D1 }\end{array}$} & 0.27 & $6.8 \%$ & $17.0 \%$ & & 10.67 & 10.53 & & \multirow{3}{*}{$N / C$} \\
\hline & 0.33 & $8.3 \%$ & $17.0 \%$ & $-56.4 \%$ & 10.67 & 10.72 & $0.4 \%$ & \\
\hline & 0.29 & $7.3 \%$ & $17.0 \%$ & & 10.67 & 10.90 & & \\
\hline \multirow{3}{*}{$\begin{array}{l}\text { NO MI } 418 \\
\text { E1 }\end{array}$} & 0.03 & $0.8 \%$ & $4.0 \%$ & & 10.23 & 11.27 & & \multirow[t]{3}{*}{$N / C$} \\
\hline & 0.04 & $1.0 \%$ & $4.0 \%$ & $.79 .2 \%$ & 10.23 & 11.47 & flash & \\
\hline & 0.03 & $0.8 \%$ & $4.0 \%$ & & 10.23 & 11.14 & & \\
\hline \multirow{4}{*}{$\begin{array}{l}\text { MEL } 228 \\
\text { F1 }\end{array}$} & 0.34 & $17.0 \%$ & $160.0 \%$ & & $>14.22$ & flash & & \multirow{3}{*}{$\begin{array}{l}\text { Originally white } \\
\text { lightly } \\
\text { yellowed }\end{array}$} \\
\hline & 0.34 & $17.0 \%$ & $160.0 \%$ & $.89 .2 \%$ & $>14.22$ & flash & flash & \\
\hline & 0.36 & $18.0 \%$ & $160.0 \%$ & & $>14.22$ & flash & & \\
\hline & \multicolumn{8}{|c|}{ After 500 hour exposure plus 24 hour air bake at $150^{\circ} \mathrm{C}\left(302^{\circ} \mathrm{F}\right)$} \\
\hline \multirow{3}{*}{$\begin{array}{l}\text { NO/MY/NO } \\
\text { A2 }\end{array}$} & 0.12 & $3.0 \%$ & $20.0 \%$ & & $>18.97$ & flash & & \multirow{3}{*}{$\begin{array}{l}\text { Major } \\
\text { delamination }\end{array}$} \\
\hline & 0.07 & $1.8 \%$ & $20.0 \%$ & $-87.5 \%$ & $>18.97$ & flash & flash & \\
\hline & 0.11 & $2.8 \%$ & $20.0 \%$ & & $>18.97$ & flash & & \\
\hline \multirow{3}{*}{$\begin{array}{l}D A / M Y / D A \\
B 2\end{array}$} & 0.05 & $2.5 \%$ & $46.0 \%$ & & $>15.27$ & flash & & \multirow{3}{*}{$\begin{array}{l}\text { darkened } \\
\text { slightly } \\
\text { warped }\end{array}$} \\
\hline & 0.03 & $1.5 \%$ & $46.0 \%$ & $.84 .4 \%$ & $>15.27$ & flash & flash & \\
\hline & 0.35 & $17.5 \%$ & $46.0 \%$ & & $>15.27$ & flash & & \\
\hline \multirow{3}{*}{$\begin{array}{l}\text { MYLAR MO } \\
\mathrm{C2}\end{array}$} & 0.16 & $8.0 \%$ & $131.0 \%$ & & $\geq 14.91$ & flash & & \multirow{3}{*}{$\begin{array}{l}\text { slightly } \\
\text { darkened } \\
\text { yellowed }\end{array}$} \\
\hline & 0.14 & $7.0 \%$ & $131.0 \%$ & $.94 .4 \%$ & $\geq 14.91$ & flash & flash & \\
\hline & 0.14 & $7.0 \%$ & $131.0 \%$ & & $>14.91$ & flash & & \\
\hline \multirow{3}{*}{$\begin{array}{l}\text { VOMEX 410 } \\
\text { D2 }\end{array}$} & 0.33 & $8.3 \%$ & $17.0 \%$ & & 10.67 & 11.01 & & \multirow{3}{*}{$N / C$} \\
\hline & 0.39 & $9.8 \%$ & $17.0 \%$ & $.48 .5 \%$ & 10.67 & 10.29 & $3.4 \%$ & \\
\hline & 0.33 & $8.3 \%$ & $17.0 \%$ & & 10.67 & 11.79 & & \\
\hline \multirow{3}{*}{$\begin{array}{l}\text { VO/MI } 418 \\
=2\end{array}$} & 0.05 & $1.3 \%$ & $4.0 \%$ & & 10.23 & 10.53 & & \multirow{3}{*}{$N / C$} \\
\hline & 0.06 & $1.5 \%$ & $4.0 \%$ & $.64 .6 \%$ & 10.23 & 10.45 & $1.1 \%$ & \\
\hline & 0.06 & $1.5 \%$ & $4.0 \%$ & & 10.23 & 10.05 & & \\
\hline MEL 228 & 0.14 & $7.0 \%$ & $160.0 \%$ & & $>14.22$ & flash & & \\
\hline $\mathrm{F} 2$ & 0.14 & $7.0 \%$ & $160.0 \%$ & $-95.6 \%$ & $>14.22$ & flash & flash & \\
\hline & 0.14 & $7.0 \%$ & $160.0 \%$ & & $>14.22$ & flash & & Hyellowed \\
\hline
\end{tabular}




\begin{tabular}{|c|c|c|c|c|c|c|c|c|c|}
\hline & 00 HRS & in $\mathrm{HCFC}$. & $142 \mathrm{~b} /$ Shrie & e Zerol-1 & $0 @ 260$ & & & & \\
\hline ID & WT & EXPWT & $\begin{array}{c}\text { WT \% } \\
\text { CHANGE }\end{array}$ & $\begin{array}{l}\text { BREAK } \\
\text { LOAD } \\
\text { (AVE) }\end{array}$ & $\begin{array}{c}\text { BREAK } \\
\text { LOAD } \\
\text { EXP }\end{array}$ & $\begin{array}{c}\% \\
\text { CHANGE } \\
\text { BPK } \\
\text { LOAD }\end{array}$ & $\begin{array}{l}\text { STRETCH } \\
(\mathrm{INCH})\end{array}$ & $\%$ ELONG & EXPVIS \\
\hline$A_{1}$ & 1.6060 & 1.6042 & $-0.11 \%$ & 39.02 & 58.55 & & 0.12 & $6.00 \%$ & $\mathrm{~N} / \mathrm{C}$ \\
\hline Glass & & & & 39.02 & 61.45 & $49.51 \%$ & 0.07 & $3.50 \%$ & \\
\hline & & & & 39.02 & 55.02 & & 0.05 & $2.50 \%$ & \\
\hline 81 & 0.6950 & 0.7046 & $1.38 \%$ & 56.12 & 50.25 & & 0.46 & $23.00 \%$ & $\mathrm{~N} / \mathrm{C}$ \\
\hline Polyester & & & & 56.12 & 53.60 & $.7 .52 \%$ & 0.56 & $28.00 \%$ & \\
\hline & & & & 56.12 & 51.85 & & 0.49 & $24.50 \%$ & \\
\hline $\mathrm{C}_{1}$ & 1.6322 & 1.7563 & $7.60 \%$ & 88.50 & 67.40 & & 0.11 & $5.50 \%$ & \\
\hline Permacel & & & & 88.50 & 102.30 & $-1.07 \%$ & 0.15 & $7.50 \%$ & darkened \\
\hline & & & & 88.50 & 92.97 & & 0.15 & $7.50 \%$ & \\
\hline & $500 \mathrm{HR}$ & 3 in $\mathrm{HCFC}$ & $-142 b / S h r i$ & ve Zerol- & $50 @ 260$ & $\mathrm{~F} \cdot>302^{\circ} \mathrm{F}$ & $24 \mathrm{HRS}$ & & \\
\hline$A 2$ & 1.5441 & 1.5429 & $-0.08 \%$ & 39.02 & 55.77 & & 0.06 & $3.00 \%$ & $\mathrm{~N} / \mathrm{C}$ \\
\hline Glass & & & & 39.02 & 65.72 & $47.16 \%$ & 0.07 & $3.50 \%$ & \\
\hline & & & & 39.02 & 50.77 & & 0.06 & $3.00 \%$ & \\
\hline 82 & 0.6278 & 0.6205 & $-1.16 \%$ & 56.12 & 50.05 & & 0.51 & $25.50 \%$ & $\mathrm{~N} / \mathrm{C}$ \\
\hline Polyester & & & & 56.12 & 50.70 & $-9.66 \%$ & 0.50 & $25.00 \%$ & \\
\hline & & & & 56.12 & 51.35 & & 0.53 & $26.50 \%$ & \\
\hline $\mathrm{C} 2$ & 1.4392 & 1.3409 & $-6.83 \%$ & 88.50 & 65.65 & & 0.13 & $6.57 \%$ & \\
\hline Permacel & & & & 88.50 & 88.25 & $-16.15 \%$ & 0.11 & $5 . \therefore \ldots$ & Darkened \\
\hline & & & & 88.50 & 68.72 & & 0.10 & $5.00 \%$ & \\
\hline
\end{tabular}




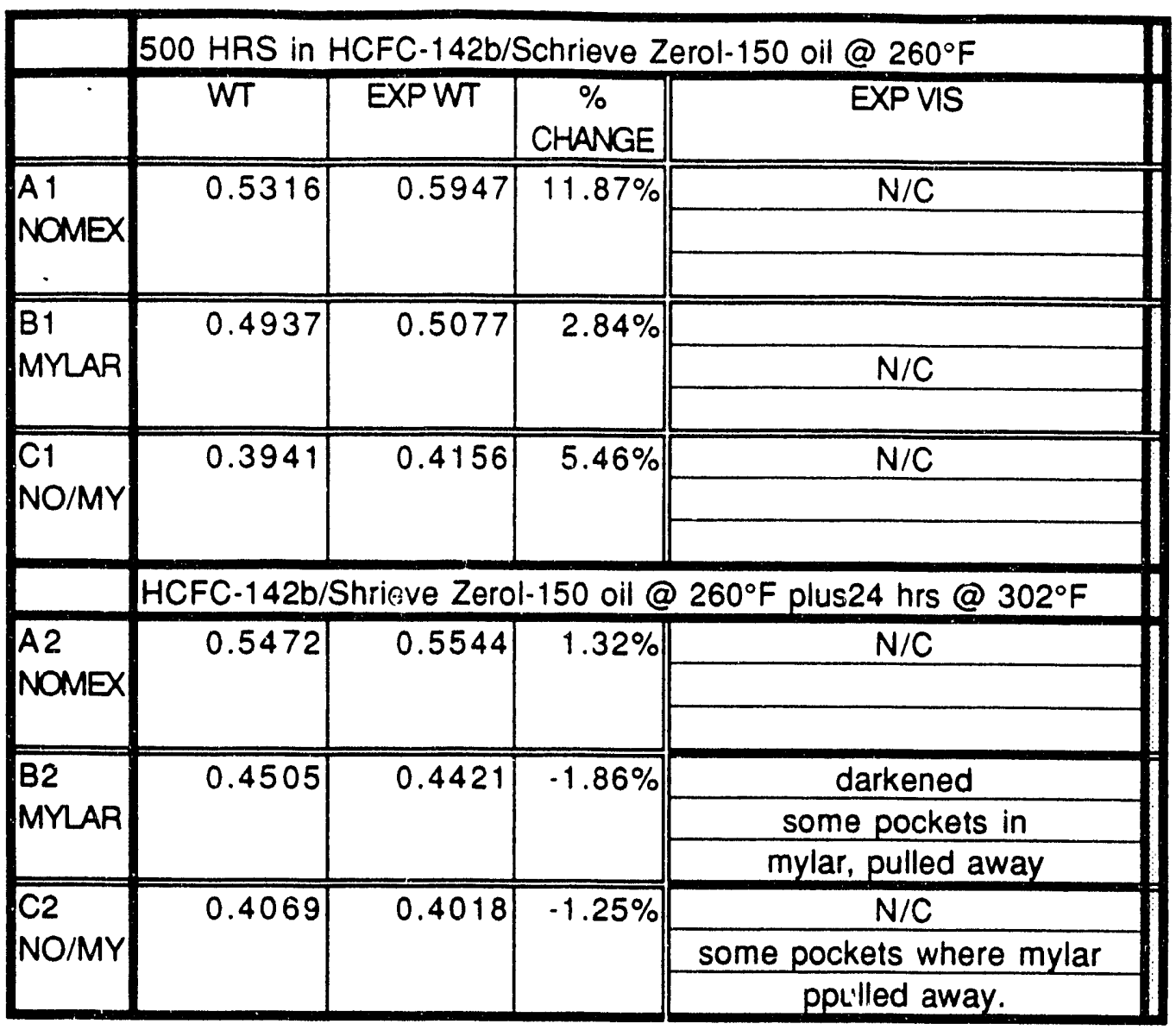


TIE CORD--HCFC-142b/Schrieve Zerol-150 oil @ 260 $\mathrm{F}\left(127^{\circ} \mathrm{C}\right)$

\begin{tabular}{|c|c|c|c|c|c|c|c|c|c|}
\hline \multicolumn{10}{|c|}{500 HRS IN R-142b/Shrieve Zerol-150 @ 260 F } \\
\hline ID & WT & EXPWT & $\begin{array}{l}\text { WT \% } \\
\text { CHANGE }\end{array}$ & $\begin{array}{l}\text { EXP } \\
\text { VIS }\end{array}$ & $\begin{array}{l}\text { BREAK } \\
\text { LOAD } \\
\text { (AVE) }\end{array}$ & $\begin{array}{c}\text { BREAK } \\
\text { LOAD } \\
\text { EXP } \\
\text { (ave) }\end{array}$ & $\begin{array}{c}\% \\
\text { CHANGE } \\
\text { BRK } \\
\text { LOAD }\end{array}$ & $\begin{array}{c}\text { STRETCH } \\
\text { (Inch) }\end{array}$ & $\begin{array}{c}\% \\
\text { ELONG }\end{array}$ \\
\hline \multirow[t]{4}{*}{ A 1} & \multirow[t]{3}{*}{0.2530} & \multirow[t]{3}{*}{0.2535} & \multirow[t]{3}{*}{$0.20 \%$} & $\mathrm{~N} / \mathrm{C}$ & 28.36 & 28.32 & & 0.42 & $21.0 \%$ \\
\hline & & & & & 28.36 & 32.80 & $15.27 \%$ & 0.47 & $23.5 \%$ \\
\hline & & & & & 28.36 & 36.95 & & 0.59 & $29.5 \%$ \\
\hline & \multicolumn{9}{|c|}{500 HRS IN R-142b/Schrieve Zerol-150 -> 24 HRS @ 302 F } \\
\hline \multirow[t]{3}{*}{ A2 } & \multirow[t]{3}{*}{0.2622} & \multirow[t]{3}{*}{0.2665} & \multirow[t]{3}{*}{$1.640 \%$} & $\mathrm{~N} / \mathrm{C}$ & 28.36 & 29.35 & & 0.41 & $20.5 \%$ \\
\hline & & & & & 28.36 & 31.92 & $9.24 \%$ & 0.47 & $23.5 \%$ \\
\hline & & & & & 28.36 & 31.67 & & 0.49 & $24.5 \%$ \\
\hline
\end{tabular}


LEAD WIRE INSULATION-HCFC-142b/S hrieve Zerol-150@260 $\mathrm{F}\left(127^{\circ} \mathrm{C}\right)$

\begin{tabular}{|c|c|c|c|c|c|c|c|}
\hline & 500 HRS IN & CFC-142b/S & Lhrieve Z & erol-150@ & $260^{\circ} \mathrm{F}$ & & \\
\hline D & WT & EXPWT & $\begin{array}{c}\text { WT \% } \\
\text { CHANGE }\end{array}$ & EXP VIS & $\begin{array}{c}\text { BASE DIE } \\
\text { (AVE) }\end{array}$ & EXPDIE & $\begin{array}{c}\text { DIE\% } \\
\text { CHANGE }\end{array}$ \\
\hline DMD & 4.2530 & 4.3681 & $2.71 \%$ & $\mathrm{~N} / \mathrm{C}$ & 9.61 & 11.23 & \\
\hline A1 & & & & & 9.61 & 12.60 & $27.19 \%$ \\
\hline & & & & & 9.61 & 12.84 & \\
\hline DTMD & 3.9554 & 4.0779 & $3.10 \%$ & $\mathrm{~N} / \mathrm{C}$ & 9.95 & 7.50 & \\
\hline B1 & & & & & 9.95 & 8.76 & $-13.57 \%$ \\
\hline & & & & & 9.95 & 9.54 & \\
\hline & HCFC- $142 \mathrm{~b} / \mathrm{s}$ & irieve Zero & 1-150@2 & $60^{\circ} \mathrm{F} \rightarrow 24$ & HRS@3 & $02^{\circ} \mathrm{F}$ & \\
\hline DMD & 4.3640 & 4.4051 & $0.94 \%$ & $\mathrm{~N} / \mathrm{C}$ & 9.61 & 10.96 & \\
\hline$A 2$ & & & & & 9.61 & 12.23 & $29.69 \%$ \\
\hline & & & & & 9.61 & 14.20 & \\
\hline DTMD & 4.1186 & 4.1495 & $0.75 \%$ & $\mathrm{~N} / \mathrm{C}$ & 9.95 & 9.04 & \\
\hline $\mathrm{B} 2$ & & & & & 9.95 & 9.78 & $-7.20 \%$ \\
\hline & & & & & 9.95 & 8.88 & \\
\hline
\end{tabular}




\section{Appendix E}

\section{Experimental Data for HFC-152a/Shrieve Zerol-150 Exposure at $127^{\circ} \mathrm{C}\left(260^{\circ} \mathrm{F}\right)$}




\begin{tabular}{|c|c|c|c|c|c|c|c|c|c|c|}
\hline & 500 hours i & HFC -15 & Shrieve & Zerol-15 & 0 oil at 26 & $=\left(127^{\circ} \mathrm{C}\right)$ & & & & \\
\hline ID & WT & EXPWT & $\begin{array}{c}\text { WT \% } \\
\text { CHANGE }\end{array}$ & EXP VIS & $\begin{array}{c}\text { BASE BRN } \\
\text { OUT } \\
\text { (AVE) }\end{array}$ & $\begin{array}{c}\text { EXP BRN } \\
\text { OUT }\end{array}$ & $\begin{array}{c}\text { BRN OUT } \\
\% \\
\text { CHANGE }\end{array}$ & $\begin{array}{c}\text { BASE DIE } \\
\text { (AVE) }\end{array}$ & EXP DIE & $\begin{array}{c}\text { DIE \% } \\
\text { CHANGE }\end{array}$ \\
\hline A 1 & 22.0238 & 22.0404 & $0.075 \%$ & $\mathrm{~N} / \mathrm{C}$ & 576 & 559 & & 15.80 & 14.33 & \\
\hline & & & & & 576 & 532 & $-13.1 \%$ & 15.80 & 15.81 & $-4.9 \%$ \\
\hline & & & & & 576 & 411 & & 15.80 & 14.96 & \\
\hline$B 1$ & 24.7692 & 24.8485 & $0.320 \%$ & $\mathrm{~N} / \mathrm{C}$ & 736 & 735 & & 11.62 & 8.65 & \\
\hline & & & & & 736 & 733 & $-0.3 \%$ & 11.62 & 12.33 & $-4.3 \%$ \\
\hline & & & & & 736 & 733 & & 11.62 & 12.39 & \\
\hline $\mathrm{C1}$ & 25.7615 & 25.7765 & $0.058 \%$ & $\mathrm{~N} / \mathrm{C}$ & 579 & 578 & & 16.58 & 17.88 & \\
\hline & & & & & 579 & 573 & $-1.0 \%$ & 16.58 & 17.08 & $7.1 \%$ \\
\hline & & & & & 579 & 568 & & 16.58 & 18.31 & \\
\hline & 500 hours i & HFC-152 & a/Shrieve & Zerol-15 & 0 oil at 260 & ${ }^{\circ} \mathrm{F}\left(127^{\circ} \mathrm{C}\right)$ & plus 24 & hours at 3 & $2^{\circ} \mathrm{F}\left(150^{\circ}\right.$ & \\
\hline A2 & 21.45 & 21.2715 & $-0.832 \%$ & $\mathrm{~N} / \mathrm{C}$ & 576 & 567 & & 15.80 & 13.78 & \\
\hline & & & & & 576 & 532 & $-3.8 \%$ & 15.80 & 15.62 & $-9.2 \%$ \\
\hline & & & & & 576 & 564 & & 15.80 & 13.66 & \\
\hline $\mathrm{B} 2$ & 25.2056 & 25.2265 & $0.083 \%$ & $\mathrm{~N} / \mathrm{C}$ & 736 & 736 & & 11.62 & 11.16 & \\
\hline & & & & & 736 & 727 & $-0.5 \%$ & 11.62 & 11.24 & $-2.7 \%$ \\
\hline & & & & & 736 & 735 & & 11.62 & 11.51 & \\
\hline $\mathrm{C} 2$ & 25.1082 & 25.1155 & $0.029 \%$ & $\mathrm{~N} / \mathrm{C}$ & 579 & 463 & & 16.58 & 11.00 & \\
\hline & & & & & 579 & 554 & $-8.9 \%$ & 16.58 & 15.29 & $-17.9 \%$ \\
\hline & & & & & 579 & 566 & & 16.58 & 14.57 & \\
\hline
\end{tabular}


TWISTED PAIRSMIRE AWITH VARNISH-HFC-152a/Shrieve Zerol-150 oil @260 $\mathrm{F}\left(127^{\circ} \mathrm{C}\right)$

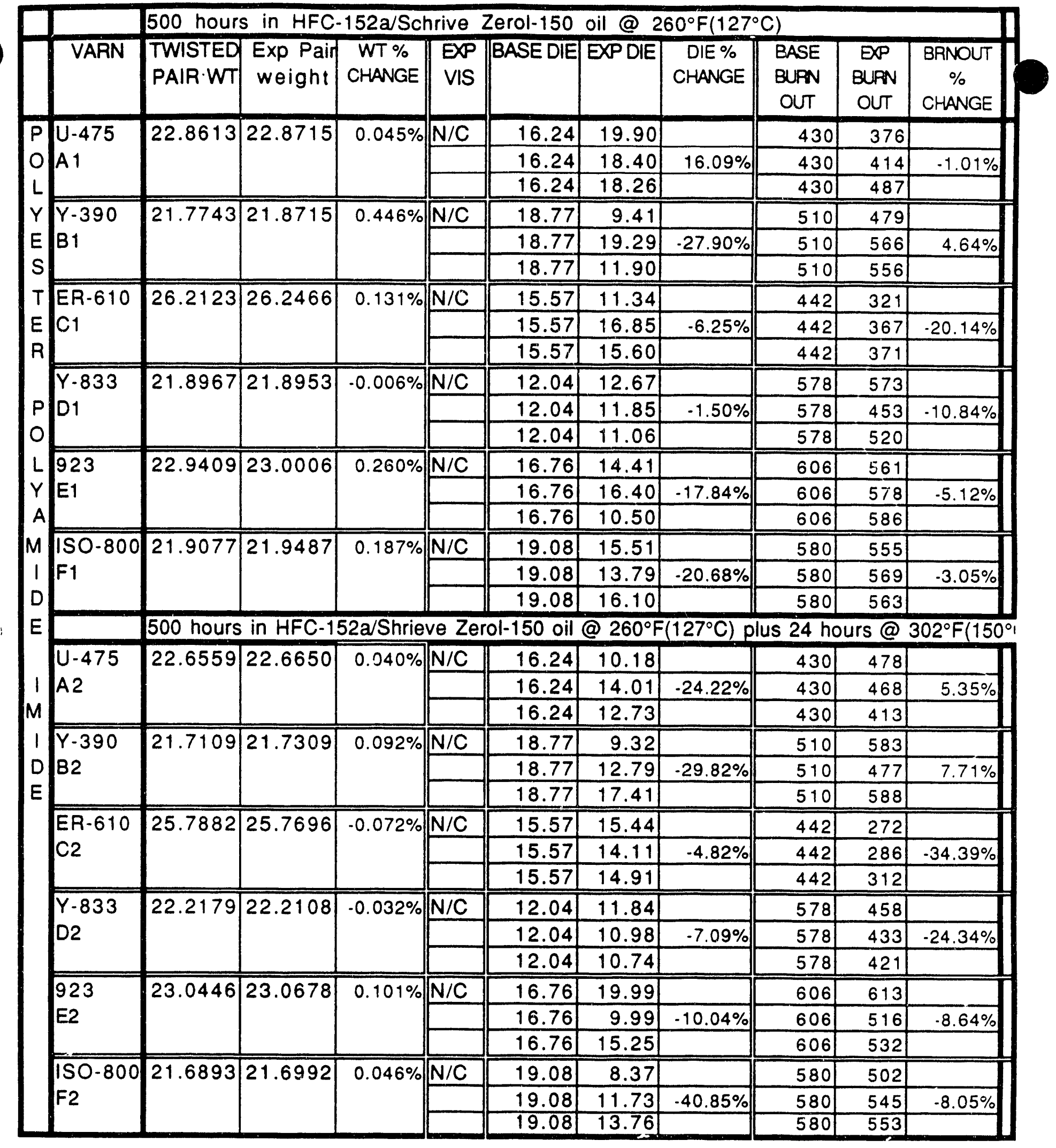


TWISTED PAIRS/WIRE BNITH VARNISH -HFC-152a/S hrieve Zerol-150 oil @260 $\mathrm{F}\left(127^{\circ} \mathrm{C}\right)$

\begin{tabular}{|c|c|c|c|c|c|c|c|c|c|c|c|}
\hline & & \multicolumn{10}{|c|}{500 HOURS in HFC-152a/Shrieve Zerol-150 oil @ $260^{\circ} \mathrm{F}\left(127^{\circ} \mathrm{C}\right)$} \\
\hline & VARN & $\begin{array}{l}\text { TWSTED } \\
\text { PAIR WT }\end{array}$ & $\begin{array}{c}\text { EXPT } \\
\text { PAIR WT }\end{array}$ & $\begin{array}{c}\text { WT \% } \\
\text { CHANGE }\end{array}$ & $\begin{array}{l}\text { EXP } \\
\text { VIS }\end{array}$ & $\begin{array}{c}\text { BASE } \\
\text { DAE }\end{array}$ & $\begin{array}{l}\text { EXP } \\
\text { DIE }\end{array}$ & $\begin{array}{c}\text { DIE \% } \\
\text { CHANGE }\end{array}$ & $\begin{array}{l}\text { BASE } \\
\text { BUPN } \\
\text { OUT } \\
\end{array}$ & $\begin{array}{c}\text { EXP } \\
\text { BUPN } \\
\text { OUT }\end{array}$ & $\begin{array}{c}\text { BRNOUT } \\
\% \\
\text { CHANGE }\end{array}$ \\
\hline \multirow{3}{*}{$\begin{array}{l}P \\
O \\
L\end{array}$} & \multirow{3}{*}{$\begin{array}{l}\text { U-475 } \\
\text { A1 }\end{array}$} & \multirow[t]{3}{*}{25.1300} & \multirow[t]{3}{*}{25.2080} & \multirow[t]{3}{*}{$0.31 \%$} & $\mathrm{~N} / \mathrm{C}$ & 13.32 & 13.21 & & 746 & 729 & \\
\hline & & & & & & 13.32 & 12.40 & $-5.73 \%$ & 746 & 731 & $-2.06 \%$ \\
\hline & & & & & & 13.32 & 12.06 & & 746 & 732 & \\
\hline \multirow{3}{*}{$\begin{array}{l}Y \\
E \\
S\end{array}$} & \multirow{3}{*}{$\begin{array}{l}\text { Y-390 } \\
\text { B1 }\end{array}$} & \multirow[t]{3}{*}{23.4105} & \multirow[t]{3}{*}{23.5353} & \multirow[t]{3}{*}{$0.53 \%$} & $\mathrm{~N} / \mathrm{C}$ & 12.28 & 8.31 & & 755 & 667 & \\
\hline & & & & & & 12.28 & 11.72 & $-15.07 \%$ & 755 & 751 & $-4.72 \%$ \\
\hline & & & & & & 12.28 & 11.26 & & 755 & 740 & \\
\hline \multirow{4}{*}{$\begin{array}{l}T \\
E \\
R\end{array}$} & \multirow{3}{*}{$\begin{array}{l}\text { ER-610 } \\
\text { C1 }\end{array}$} & \multirow[t]{3}{*}{22.1904} & \multirow[t]{3}{*}{22.2091} & \multirow[t]{3}{*}{$0.08 \%$} & $\mathrm{~N} / \mathrm{C}$ & 12.73 & 10.78 & & 734 & 735 & \\
\hline & & & & & & 12.73 & 9.40 & $-23.57 \%$ & 734 & 729 & $-3.81 \%$ \\
\hline & & & & & & $12.7 \mathrm{a}$ & 9.01 & & 734 & 654 & \\
\hline \multirow{3}{*}{\multicolumn{2}{|c|}{$\begin{array}{lll}P & D \\
O & \end{array}$}} & \multirow[t]{3}{*}{21.1090} & \multirow[t]{3}{*}{21.1242} & \multirow[t]{3}{*}{$0.07 \%$} & $\mathrm{~N} / \mathrm{C}$ & 12.49 & 9.82 & & 734 & 727 & \\
\hline & & & & & & 12.49 & 8.24 & $-35.76 \%$ & 734 & 727 & $-0.32 \%$ \\
\hline & & & & & & 12.49 & 6.01 & & 734 & 741 & \\
\hline \multirow{3}{*}{$\begin{array}{l}L \\
Y \\
A\end{array}$} & \multirow{3}{*}{$\begin{array}{l}923 \\
E 1 \\
\end{array}$} & \multirow[t]{3}{*}{26.3348} & 26.4272 & $0.35 \%$ & $\mathrm{~N} / \mathrm{C}$ & 14.38 & 9.30 & & 742 & 736 & \\
\hline & & & & & & 14.38 & 16.34 & $-3.45 \%$ & 742 & 732 & $-0.40 \%$ \\
\hline & & & & & & 14.38 & 16.01 & & 742 & 749 & \\
\hline$M$ & $150-800$ & 28.7632 & 28.8543 & $0.32 \%$ & $\mathrm{~N} / \mathrm{C}$ & 12.29 & 12.97 & & 747 & 732 & \\
\hline 1 & $F 1$ & & & & & 12.29 & 13.73 & $9.03 \%$ & 747 & 741 & $-1.52 \%$ \\
\hline$D$ & & & & & & 12.29 & 13.50 & & 747 & 734 & \\
\hline$E$ & & HFC-152a & a/ Zerol-15 & 0 oil @ 2 & $260^{\circ} \mathrm{F}$ & $\left.27^{\circ} \mathrm{C}\right) \mathrm{p}$ & Ius $24 h$ & lours@3 & $302^{\circ} \mathrm{F}$ & $150^{\circ} \mathrm{C}$ & \\
\hline & $U-475$ & 25.3920 & 25.3974 & $0.02 \%$ & $\mathrm{~N} / \mathrm{C}$ & 13.32 & 12.61 & & 746 & 731 & \\
\hline 1 & $A 2$ & & & & & 13.32 & 10.90 & $-15.39 \%$ & 746 & 731 & $-1.83 \%$ \\
\hline$M$ & & & & & & 13.32 & 10.30 & & 746 & 735 & \\
\hline 1 & $Y-390$ & 23.5808 & 23.6052 & $0.10 \%$ & $N / C$ & 12.28 & 11.78 & & 755 & 739 & \\
\hline D & B2 & & & & & 12.28 & 11.90 & $-3.85 \%$ & 755 & 739 & $-2.12 \%$ \\
\hline$E$ & & & & & & 12.28 & 11.74 & & 755 & 736 & \\
\hline & ER-610 & 22.5380 & 22.5295 & $-0.04 \%$ & $\mathrm{~N} / \mathrm{C}$ & 12.73 & 11.63 & & 734 & 733 & \\
\hline$E$ & $\mathrm{C} 2$ & & & & & 12.73 & 11.66 & $-6.70 \%$ & 734 & 731 & $-0.36 \%$ \\
\hline$P$ & & & & & & 12.73 & 12.34 & & 734 & 730 & \\
\hline 0 & $Y-833$ & 22.1914 & 22.1895 & $-0.01 \%$ & $\mathrm{~N} / \mathrm{C}$ & 12.49 & 10.10 & & 734 & 731 & \\
\hline$x$ & D2 & & & & & 12.49 & 12.10 & -8.1, & 734 & 730 & $-0.59 \%$ \\
\hline$Y$ & & & & & & 12.49 & 12.23 & & 734 & 728 & \\
\hline & 923 & 26.6833 & 26.7014 & $0.07 \%$ & $\mathrm{~N} / \mathrm{C}$ & 14.38 & 15.76 & & 742 & 734 & \\
\hline$G$ & E2 & & & & & 14.38 & 14.10 & $0.95 \%$ & 742 & 740 & $-1.08 \%$ \\
\hline$L$ & & & & & & 14.38 & 13.69 & & 742 & 732 & \\
\hline A & $150-800$ & 29.0483 & 29.0681 & $0.07 \%$ & & 12.29 & 11.66 & & 747 & 749 & \\
\hline$S$ & $F 2$ & & & & $\mathrm{~N} / \mathrm{C}$ & 12.29 & 11.76 & $-3.96 \%$ & 747 & 742 & $-0.20 \%$ \\
\hline$S$ & & & & & & 12.29 & 11.99 & & 747 & 737 & \\
\hline
\end{tabular}




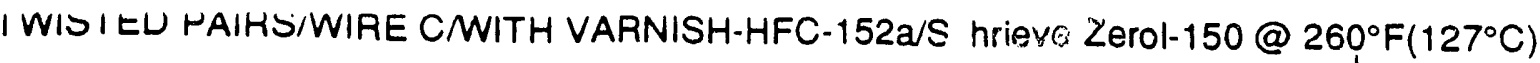

\begin{tabular}{|c|c|c|c|c|c|c|c|c|c|c|c|}
\hline & \multirow{2}{*}{ VARN } & \multicolumn{10}{|c|}{ 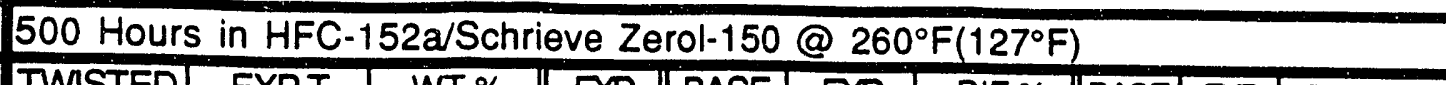 } \\
\hline & & $\begin{array}{l}\text { TWISTED } \\
\text { PAIR WT }\end{array}$ & $\begin{array}{c}\text { EXPT } \\
\text { PAIR WT }\end{array}$ & $\begin{array}{c}\text { WT \% } \\
\text { CHANGE }\end{array}$ & $\begin{array}{l}\text { EXP } \\
\text { VIS }\end{array}$ & $\begin{array}{c}\text { BASE } \\
\text { DIE }\end{array}$ & $\begin{array}{l}\text { EXP } \\
\text { DIE }\end{array}$ & $\begin{array}{c}\text { DIE \% } \\
\text { CHANGE }\end{array}$ & $\begin{array}{l}\text { BASE } \\
\text { BURN } \\
\text { OUT }\end{array}$ & \begin{tabular}{|c|} 
EXP \\
BUPN \\
OUT
\end{tabular} & $\begin{array}{c}\text { BRNOUT } \\
\% \\
\text { CHANGE }\end{array}$ \\
\hline \multirow{3}{*}{$\begin{array}{l}P \\
O \\
L\end{array}$} & \multirow{3}{*}{$\begin{array}{l}\text { U-475 } \\
\text { A } 1 \\
\end{array}$} & \multirow[t]{3}{*}{24.2691} & \multirow[t]{3}{*}{24.3104} & \multirow[t]{3}{*}{$0.170 \%$} & $\mathrm{~N} / \mathrm{C}$ & 15.10 & 11.99 & & 469 & 359 & \\
\hline & & & & & & 15.10 & 11.34 & $-25.17 \%$ & 469 & 456 & $-2.99 \%$ \\
\hline & & & & & & 15.10 & 10.57 & & 469 & 550 & \\
\hline \multirow{3}{*}{$\begin{array}{l}Y \\
E \\
S\end{array}$} & \multirow{3}{*}{$\begin{array}{l}\mathrm{Y}-390 \\
\mathrm{~B} 1\end{array}$} & \multirow[t]{3}{*}{25.0458} & \multirow[t]{3}{*}{25.1178} & \multirow[t]{3}{*}{$0.287 \%$} & $\mathrm{~N} / \mathrm{C}$ & 18.24 & 3.71 & & 473 & 522 & \\
\hline & & & & & & 18.24 & \begin{tabular}{|l|}
8.64 \\
\end{tabular} & \begin{tabular}{|l|}
$-40.90 \%$ \\
\end{tabular} & 473 & 569 & $13.04 \%$ \\
\hline & & & & & & 18.24 & 19.99 & & 473 & 513 & \\
\hline \multirow{4}{*}{$\begin{array}{l}T \\
E \\
R\end{array}$} & ER-610 & \multirow[t]{3}{*}{26.2063} & \multirow[t]{3}{*}{26.2349} & \multirow[t]{3}{*}{$0.109 \%$} & $\mathrm{~N} / \mathrm{C}$ & 14.53 & 15.30 & & 494 & 390 & \\
\hline & \multirow{2}{*}{ C1 } & & & & & 14.53 & 13.06 & $-0.02 \%$ & 494 & 387 & $-23.08 \%$ \\
\hline & & & & & & 14.53 & 15.22 & & 494 & 363 & \\
\hline & \multirow{3}{*}{$\begin{array}{l}Y-833 \\
D 1\end{array}$} & \multirow[t]{3}{*}{26.4181} & \multirow[t]{3}{*}{26.4070} & \multirow[t]{3}{*}{$-0.042 \%$} & $N / C$ & 11.38 & 14.34 & & 557 & 490 & \\
\hline \multirow{2}{*}{ M } & & & & & & 11.38 & 14.78 & $20.71 \%$ & 557 & 419 & $-10.89 \%$ \\
\hline & & & & & & 11.38 & 12.09 & & 557 & 580 & \\
\hline \multirow{3}{*}{$\begin{array}{l}1 \\
D \\
E\end{array}$} & 923 & 17.3814 & 17.4205 & $0.225 \%$ & $\mathrm{~N} / \mathrm{C}$ & 15.85 & 16.94 & & 503 & 515 & \\
\hline & E1 & & & & & 15.85 & 18.44 & $11.61 \%$ & 503 & 563 & $7.16 \%$ \\
\hline & & & & & & 15.85 & $N / A$ & & 503 & $N / A$ & \\
\hline & 150.800 & 22.3755 & 22.4164 & $0.183 \%$ & $\mathrm{~N} / \mathrm{C}$ & 14.75 & 18.34 & & 632 & 601 & \\
\hline$P$ & $\mathrm{~F} 1$ & & & & & 14.75 & 19.82 & $24.05 \%$ & 632 & 505 & $-9.49 \%$ \\
\hline 0 & & & & & & 14.75 & 16.73 & & 632 & 610 & \\
\hline$L$ & & HFC-152 & a/S hrieve & Zerol-150 & @ 26 & $0^{\circ} \mathrm{F}$ plu & s 24 ho & urs @ 30 & $\mathrm{~F}(15$ & $\left.50^{\circ} \mathrm{C}\right)$ & \\
\hline$Y$ & $U-475$ & 24.2312 & 24.2389 & $0.032 \%$ & $\mathrm{~N} / \mathrm{C}$ & 15.10 & 14.12 & & 469 & 548 & \\
\hline A & A2 & & & & & 15.10 & 14.66 & $-1.81 \%$ & 469 & 427 & $-3.55 \%$ \\
\hline$M$ & & & & & & 15.10 & 15.70 & & 469 & 382 & \\
\hline & $Y-390$ & 26.3111 & 26.3358 & $0.094 \%$ & $\mathrm{~N} / \mathrm{C}$ & 18.24 & 10.94 & & 473 & 640 & \\
\hline$D$ & B2 & & & & & 18.24 & 14.12 & $-18.39 \%$ & 473 & 443 & $6.55 \%$ \\
\hline$E$ & & & & & & 18.24 & 15.65 & & 473 & 429 & \\
\hline & ER-610 & 25.9247 & 25.9297 & $0.019 \%$ & $\mathrm{~N} / \mathrm{C}$ & 14.53 & 17.79 & & 494 & 309 & \\
\hline & C2 & & & & & 14.53 & 16.14 & $12.02 \%$ & 494 & 365 & $-26.79 \%$ \\
\hline$M$ & & & & & & 14.53 & 14.90 & & 494 & 411 & \\
\hline & Y-833 & 26.1411 & 26.1104 & $-0.117 \%$ & $\mathrm{~N} / \mathrm{C}$ & 11.38 & 16.73 & & 557 & 403 & \\
\hline$D$ & D2 & & & & & 11.38 & 15.63 & $31.52 \%$ & 557 & 540 & $-13.41 \%$ \\
\hline & & & & & & 11.38 & 12.54 & & 557 & 504 & \\
\hline & 923 & 24.4687 & 24.4817 & $0.053 \%$ & $\mathrm{~N} / \mathrm{C}$ & 15.85 & 13.47 & & 503 & 388 & \\
\hline & E2 & & & & & 15.85 & 12.77 & $-17.22 \%$ & 503 & 428 & $-17.16 \%$ \\
\hline & & & & & & 15.85 & $\mathrm{~N} / \mathrm{A}$ & & 503 & 434 & \\
\hline & ISO-800 & 22.4531 & 22.4634 & $0.046 \%$ & $\mathrm{~N} / \mathrm{C}$ & 14.75 & 17.43 & & 632 & 603 & \\
\hline & F2 & & & & & 14.75 & 13.50 & $5.92 \%$ & 632 & 686 & $2.27 \%$ \\
\hline & & & & & & 14.75 & 15.94 & & 632 & 650 & \\
\hline
\end{tabular}


HELICAL COILSMIRE A-...HFC-152a/Shrieve Zerol-150 oil @260 F(127\% C)

\begin{tabular}{|c|c|c|c|c|c|c|c|c|}
\hline & & $500 \mathrm{HR}$ & in HFC-1! & a/Zerol & $260 \mathrm{~F}$ & & & \\
\hline & VARN & $\begin{array}{l}\text { COIL } \\
W T\end{array}$ & $\begin{array}{c}\text { EXP COIL } \\
W T\end{array}$ & $\begin{array}{c}\text { WT \% } \\
\text { CHANGE }\end{array}$ & EXP VIS & $\begin{array}{c}\text { BASE } \\
\text { BND STR } \\
\text { (AVE) }\end{array}$ & $\begin{array}{c}\text { EXPBND } \\
\text { STR }\end{array}$ & $\begin{array}{c}\text { BND STR } \\
\% \\
\text { CHANGE }\end{array}$ \\
\hline$P$ & $U-475$ & 40.1082 & 40.2342 & $0.314 \%$ & $\mathrm{~N} / \mathrm{C}$ & 73.73 & 32.90 & \\
\hline 0 & A1 1 & & & & & 73.73 & 21.65 & $-64.49 \%$ \\
\hline$L$ & & & & & & 73.73 & 24.00 & \\
\hline$Y$ & $Y-390$ & 39.1315 & 39.2356 & $0.266 \%$ & N/C & 43.78 & 24.62 & \\
\hline E & B1 & & & & & 43.78 & 27.60 & $-42.23 \%$ \\
\hline$S$ & & & & & & 43.78 & 23.65 & \\
\hline$T$ & ER-610 & 39.1374 & 39.2436 & $0.271 \%$ & $\mathrm{~N} / \mathrm{C}$ & 51.81 & 22.20 & \\
\hline $\mathbf{E}$ & C1 & & & & & 51.81 & 24.25 & $-47.47 \%$ \\
\hline R & & & & & & 51.81 & 35.20 & \\
\hline & $Y-833$ & 38.5863 & 38.5725 & $-0.036 \%$ & N/C & 9.85 & 19.05 & \\
\hline$P$ & D1 & & & & & 9.85 & 11.32 & $78.24 \%$ \\
\hline 0 & & & & & & 9.85 & 22.30 & \\
\hline L & 923 & 40.1270 & 40.2713 & $0.360 \%$ & $\mathrm{~N} / \mathrm{C}$ & 41.28 & 34.90 & \\
\hline Y & E1 & & & & & 41.28 & 33.35 & $-20.22 \%$ \\
\hline A & & & & & & 41.28 & 30.55 & \\
\hline$M$ & ISO-800 & 37.5610 & 37.6263 & $0.174 \%$ & $\mathrm{~N} / \mathrm{C}$ & 45.01 & 42.85 & \\
\hline 1 & $F 1$ & & & & & 45.01 & 40.20 & $-7.74 \%$ \\
\hline$D$ & & & & & & 45.01 & 44.65 & \\
\hline$E$ & & $500 \mathrm{HRS}$ & in HFC-15? & a/Zerol-15c & 0 @ 260 & $\because>24 \mathrm{HR}$ & $\mathrm{S} 302^{\circ} \mathrm{F}$ & \\
\hline & $U-475$ & 40.0238 & 40.0674 & $0.109 \%$ & $N / C$ & 73.73 & 43.20 & \\
\hline I & A2 & & & & & 73.73 & 54.25 & $-42.80 \%$ \\
\hline$M$ & & & & & & 73.73 & 30.10 & \\
\hline 1 & $Y-390$ & $39.0847 \mid$ & 39.1312 & $0.119 \%$ & $\mathrm{~N} / \mathrm{C}$ & 43.78 & 22.05 & \\
\hline D & B2 & & & & & 43.78 & 18.60 & $-48.42 \%$ \\
\hline$E$ & & & & & & 43.78 & 27.10 & \\
\hline & ER-610 & 39.9705 & 39.9950 & $0.061 \%$ & $N / C$ & 51.81 & 18.55 & \\
\hline & C2 & & & & & 51.81 & 17.60 & $-70.08 \%$ \\
\hline & & & & & & 51.81 & 10.35 & \\
\hline & $Y-833$ & 38.8992 & 38.8374 & $-0.159 \%$ & $\mathrm{~N} / \mathrm{C}$ & 9.85 & 21.15 & \\
\hline & D2 & & & & & 9.85 & 11.90 & $79.26 \%$ \\
\hline & & & & & & 9.85 & 19.92 & \\
\hline & 923 & 39.8071 & 39.8618 & $0.137 \%$ & $\mathrm{~N} / \mathrm{C}$ & 41.28 & 16.25 & \\
\hline & E2 & & & & & 41.28 & 12.60 & $-61.67 \%$ \\
\hline & & & & & & 41.28 & 1862 & \\
\hline & ISO- 100 & 38.2487 & 38.2610 & $0.032 \%$ & $\mathrm{~N} / \mathrm{C}$ & 45.01 & 40.57 & \\
\hline & F2 & & & & & 45.01 & 44.97 & $-1.36 \%$ \\
\hline & & & & & & 45.01 & 47.65 & \\
\hline
\end{tabular}


HELICAL COILSMIRE B-HFC-152a/Shrieve Zerol-150 Oil

\begin{tabular}{|c|c|c|c|c|c|c|c|c|}
\hline & & $500 \mathrm{HRS}$ & in $\mathrm{HFC}-15$ & a/Shrieve & Zerol-150 & @ $260 \mathrm{~F}$ & & \\
\hline & VARN & $\begin{array}{l}\text { COIL } \\
\text { WT }\end{array}$ & $\begin{array}{c}\text { EXP COIL } \\
W T\end{array}$ & $\begin{array}{c}\text { WT \% } \\
\text { CHANGE }\end{array}$ & EXPVIS & $\begin{array}{c}\text { BASE } \\
\text { BND STR } \\
\text { (AVE) }\end{array}$ & $\begin{array}{c}\text { EXPBND } \\
\text { STR }\end{array}$ & $\begin{array}{c}\text { BND STR } \\
\% \\
\text { CHANGE }\end{array}$ \\
\hline $\mathrm{P}$ & $U-475$ & 38.2779 & 38.4450 & $0.437 \%$ & $\mathrm{~N} / \mathrm{C}$ & 40.14 & 33.77 & \\
\hline 0 & $A 1$ & & & & & 40.14 & 38.07 & $.8 .50 \%$ \\
\hline$L$ & & & & & & 40.14 & 38.35 & \\
\hline$Y$ & $Y-390$ & 38.5049 & 38.7153 & $0.546 \%$ & $N / C$ & 36.12 & 41.10 & \\
\hline$E$ & B1 & & & & & 36.12 & 39.57 & $5.70 \%$ \\
\hline S & & & & & & 36.12 & 33.87 & \\
\hline $\mathrm{T}$ & ER-610 & 37.3353 & 37.4632 & $0.343 \%$ & $\mathrm{~N} / \mathrm{C}$ & 35.96 & 31.22 & \\
\hline$E$ & C1 & & & & & 35.96 & 35.52 & $-1.04 \%$ \\
\hline $\mathbf{R}$ & & & & & & 35.96 & 40.02 & \\
\hline & $Y-833$ & 35.9631 & 36.0134 & $0.140 \%$ & $N / C$ & 33.14 & 25.32 & \\
\hline$P$ & D1 & & & & & 33.14 & 19.25 & $-31.63 \%$ \\
\hline O & & & & & & 33.14 & 23.40 & \\
\hline$L$ & 923 & 38.4021 & 38.5380 & $0.354 \%$ & $\mathrm{~N} / \mathrm{C}$ & 40.52 & 40.12 & \\
\hline $\mathrm{Y}$ & E1 & & & & & 40.52 & 39.72 & $-5.64 \%$ \\
\hline A & & & & & & 40.52 & 34.87 & \\
\hline M & ISO-800 & 37.6500 & 37.7471 & $0.258 \%$ & $\mathrm{~N} / \mathrm{C}$ & 20.20 & 20.57 & \\
\hline 1 & $F 1$ & & & & & 20.20 & 18.30 & $-9.46 \%$ \\
\hline$D$ & & & & & & 20.20 & 16.00 & \\
\hline$E$ & & 500 HRS & in HFC-15 & a/Zerol-15 & $0 @ 260$ & $F->24 \mathrm{HF}$ & $\mathrm{S} 302^{\circ} \mathrm{F}$ & \\
\hline & $U-475$ & 37.5655 & 37.6083 & $0.114 \%$ & $\mathrm{~N} / \mathrm{C}$ & 40.14 & 29.17 & \\
\hline 1 & A2 & & & & & 40.14 & 32.17 & $-25.39 \%$ \\
\hline$M$ & & & & & & 40.14 & 28.50 & \\
\hline 1 & $Y-390$ & 36.9782 & 37.0420 & $0.173 \%$ & $\mathrm{~N} / \mathrm{C}$ & 36.12 & 30.22 & \\
\hline D & B2 & & & & & 36.12 & 31.85 & $-12.75 \%$ \\
\hline E & & & & & & 36.12 & 32.47 & \\
\hline & ER-610 & 39.1917 & 39.2245 & $0.084 \%$ & $\mathrm{~N} / \mathrm{C}$ & 35.96 & 35.52 & \\
\hline$E$ & C2 & & & & & 35.96 & 30.07 & $-13.09 \%$ \\
\hline$P$ & & & & & & 35.96 & 28.17 & \\
\hline 0 & $Y-833$ & 36.5141 & 36.5203 & $0.017 \%$ & $\mathrm{~N} / \mathrm{C}$ & 33.14 & 17.72 & \\
\hline$x$ & D2 & & & & & 33.14 & 25.75 & $-39.23 \%$ \\
\hline Y & & & & & & 33.14 & 16.95 & \\
\hline & 923 & 38.0774 & 38.1289 & $0.135 \%$ & $N / C$ & 40.52 & 30.50 & \\
\hline G & E2 & & & & & 40.52 & 32.55 & $-20.78 \%$ \\
\hline L & & & & & & 40.52 & 33.25 & \\
\hline A & $1 S 0-800$ & 37.4183 & 37.4360 & $0.047 \%$ & $\mathrm{~N} / \mathrm{C}$ & 20.20 & 7.35 & \\
\hline 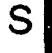 & $F 2$ & & & & & 20.20 & 19.82 & $-25.51 \%$ \\
\hline S & & & & & & 20.20 & 17.97 & \\
\hline
\end{tabular}




\begin{tabular}{|c|c|c|c|c|c|c|c|c|}
\hline & & 500 HRS & in HFC- 15 & a/Shrieve Z & Zerol-150 & @ 260 F & & \\
\hline & VARN & $\begin{array}{l}\text { COIL } \\
W T\end{array}$ & $\begin{array}{c}\text { EXP COIL } \\
W T\end{array}$ & $\begin{array}{c}\text { WT \% } \\
\text { CHANGE }\end{array}$ & EXP VIS & $\begin{array}{c}\text { BASE } \\
\text { BND STR } \\
\text { (AVE) }\end{array}$ & $\begin{array}{c}\text { EXPBND } \\
\text { STR }\end{array}$ & $\begin{array}{c}\text { BND STR } \\
\% \\
\text { CHANGE }\end{array}$ \\
\hline$P$ & $U-475$ & 38.8832 & 39.0090 & $0.324 \%$ & $\mathrm{~N} / \mathrm{C}$ & 51.21 & 21.75 & \\
\hline 0 & A 1 & & & & & 51.21 & 27.05 & $.54 .53 \%$ \\
\hline$L$ & & & & & & 51.21 & 21.05 & \\
\hline$Y$ & $Y-390$ & 40.2107 & 40.3165 & $0.263 \%$ & $\mathrm{~N} / \mathrm{C}$ & 50.72 & 24.17 & \\
\hline$E$ & B1 & & & & & 50.72 & 25.12 & $-53.48 \%$ \\
\hline$S$ & & & & & & 50.72 & 21.50 & \\
\hline$T$ & ER-610 & 36.3465 & 36.4387 & $0.254 \%$ & $N / C$ & 58.33 & 20.87 & \\
\hline$E$ & C1 & & & & & 58.33 & 43.67 & $-52.33 \%$ \\
\hline$R$ & & & & & & 58.33 & 18.87 & \\
\hline & $Y-833$ & 38.1322 & 38.0246 & $-0.282 \%$ & $\mathrm{~N} / \mathrm{C}$ & 5.84 & broken & \\
\hline 1 & D1 & & & & & 5.84 & broken & $89.21 \%$ \\
\hline$M$ & & & & & & 5.84 & 11.05 & \\
\hline 1 & 923 & 40.0291 & 40.1770 & $0.369 \%$ & $N / C$ & 49.26 & 25.15 & \\
\hline$D$ & E1 & & & & & 49.26 & 24.92 & $-49.36 \%$ \\
\hline$E$ & & & & & & 49.26 & 24.77 & \\
\hline & ISO-800 & 39.6188 & 39.6878 & $0.174 \%$ & $\mathrm{~N} / \mathrm{C}$ & 36.08 & 34.25 & \\
\hline$P$ & $F 1$ & & & & & 36.08 & 43.20 & $23.57 \%$ \\
\hline 0 & & & & & & 36.08 & 56.30 & \\
\hline L & & 500 HRS & in HFC-15e & a/Shrieve 2 & Zerol-150 & @ 260 F-> & 24 HRS 3 & $02^{\circ} \mathrm{F}$ \\
\hline$Y$ & U-475 & 37.5920 & 37.6256 & $0.089 \%$ & $\mathrm{~N} / \mathrm{C}$ & 51.21 & 24.05 & \\
\hline A & A2 & & & & & 51.21 & 27.30 & $-46.16 \%$ \\
\hline$M$ & & & & & & 51.21 & 31.37 & \\
\hline I & $Y-390$ & 40.0541 & 40.0987 & $0.111 \%$ & $\mathrm{~N} / \mathrm{C}$ & 50.72 & 32.95 & \\
\hline$D$ & B2 & & & & & 50.72 & 18.60 & $-51.09 \%$ \\
\hline$E$ & & & & & & 50.72 & 22.87 & \\
\hline & ER-610 & 37.1487 & 37.1738 & $0.068 \%$ & $\mathrm{~N} / \mathrm{C}$ & 58.33 & 47.75 & \\
\hline I & C2 & & & & & 58.33 & 58.97 & $-24.10 \%$ \\
\hline$M$ & & & & & & 58.33 & 26.10 & \\
\hline 1 & $Y-833$ & 37.6700 & 37.5218 & $-0.393 \%$ & $\mathrm{~N} / \mathrm{C}$ & 5.84 & 16.22 & \\
\hline D & D2 & & & & & 5.84 & 11.42 & $144.81 \%$ \\
\hline$E$ & & & & & & 5.84 & 15.25 & \\
\hline & 923 & 40.1700 & 40.2287 & $0.146 \%$ & $\mathrm{~N} / \mathrm{C}$ & 49.26 & 24.25 & \\
\hline & E2 & & & & & 49.26 & 22.80 & $-51.94 \%$ \\
\hline & & & & & & 49.26 & 23.97 & \\
\hline & ISO-800 & 39.7189 & 39.7377 & $0.047 \%$ & $\mathrm{~N} / \mathrm{C}$ & 36.08 & 54.27 & \\
\hline & F2 & & & & & 36.08 & 49.67 & $46.07 \%$ \\
\hline & & & & & & 36.08 & 54.17 & \\
\hline
\end{tabular}


SING' - NIr:-: 'NIRE WITHOUT VARNISH-HFC-152a/S hrieve Zerol-150 @ 260 $\left.\mathrm{F}\left(127^{\circ} \mathrm{C}\right)\right)$

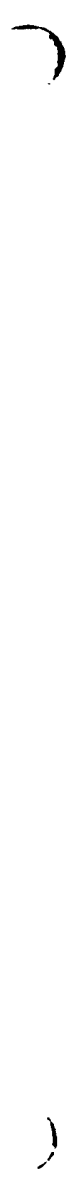

\begin{tabular}{|c|c|c|c|c|c|}
\hline & 500 HRS & HFC- $152 a$ & Zerol-150 o & @ 260 & $=\left(127^{\circ} \mathrm{C}\right)$ \\
\hline ID & WT & EXPWT & $\begin{array}{l}\text { WT \% } \\
\text { CHANGE }\end{array}$ & EXP VIS & EXPFLEX \\
\hline A1 1 & 3.8043 & 3.8086 & $0.113 \%$ & $\mathrm{~N} / \mathrm{C}$ & \\
\hline & & & & & YES \\
\hline B1 & 3.585 & 3.5983 & $0.371 \%$ & $N / C$ & \\
\hline & & & & & YES \\
\hline C1 & 36914 & 36956 & $0114 \%$ & $N / C$ & \\
\hline & & & & & YES \\
\hline & & & & & \\
\hline & 500 HRS & HCFC-15 & a/Zerol-150 & $>302^{\circ} \mathrm{F}$ & or 24 HRS \\
\hline A2 & 3.785 & 3.7891 & $0.108 \%$ & $N / C$ & \\
\hline & & & & & YES \\
\hline $\mathrm{B2}$ & 3.7148 & 3.72 & $0.140 \%$ & $\mathrm{~N} / \mathrm{C}$ & \\
\hline & & & & & YES \\
\hline $\mathrm{C} 2$ & 3.6329 & 3.6376 & $0.129 \%$ & $\mathrm{~N} / \mathrm{C}$ & \\
\hline & & & & & YES \\
\hline & & & & & \\
\hline
\end{tabular}




\begin{tabular}{|c|c|c|c|c|c|c|c|}
\hline & & 500 HRS & in HFC- 152 & a/Zerol-15 & 0 oil C & $260^{\circ} \mathrm{F}$ & \\
\hline & VARN & WREWT & $\begin{array}{c}\text { EXPWIRE } \\
\text { WT }\end{array}$ & $\begin{array}{c}\text { WT \% } \\
\text { CHANGE }\end{array}$ & $\begin{array}{l}\text { EXP } \\
\text { VIS }\end{array}$ & $\begin{array}{l}\text { EXP } \\
\text { FEX }\end{array}$ & \\
\hline$P$ & U-475 & 3.7634 & 3.7692 & $0.154 \%$ & $\mathrm{~N} / \mathrm{C}$ & & \\
\hline 0 & A 1 & & & & & NO & \\
\hline $\mathrm{Y}$ & $Y-390$ & 38171 & 38081 & 02000 & NOS & & \\
\hline$E$ & B1 & וזעם & $0.0<01$ & $0.280 \%$ & $10 / 2$ & NO & \\
\hline$s$ & & & & & & & \\
\hline$T$ & ER-610 & 3.7437 & 3.7468 & $0.083 \%$ & $\mathrm{~N} / \mathrm{C}$ & & \\
\hline E & & & & & & YES & \\
\hline & & & & & & & \\
\hline & Y.833 & 4.0218 & 4.0233 & $0.037 \%$ & $\mathrm{~N} / \mathrm{C}$ & & \\
\hline$P$ & D1 & & & & & YES & \\
\hline o & & & & & & & \\
\hline L & 923 & 3.8006 & 3.8145 & $0.366 \%$ & $\mathrm{~N} / \mathrm{C}$ & & \\
\hline Y & E1 & & & & & $\mathrm{NO}$ & \\
\hline A & & & & & & & \\
\hline$M$ & ISO-800 & 3.9367 & 3.9430 & $0.160 \%$ & $\mathrm{~N} / \mathrm{C}$ & & \\
\hline 1 & F1 & & & & & NO & \\
\hline$D$ & & & & & & & \\
\hline$E$ & & HFC-152i & /Zerol-150 & $\rightarrow 24 \mathrm{HRS}$ & @ 3 & $2^{\circ} \mathrm{F}$ & \\
\hline & U-475 & 3.6633 & 3.6636 & $0.008 \%$ & $\mathrm{~N} / \mathrm{C}$ & & \\
\hline 1 & A2 & & & & & NO & \\
\hline$M$ & & & & & & & \\
\hline 1 & $Y-390$ & 3.8383 & 3.8412 & $0.076 \%$ & $\mathrm{~N} / \mathrm{C}$ & & \\
\hline$D$ & & & & & & NO & \\
\hline$E$ & & & & & & & \\
\hline & ER-610 & 3.7610 & 3.7608 & $-0.005 \%$ & $\mathrm{~N} / \mathrm{C}$ & & \\
\hline & C2 & & & & & YES & \\
\hline & & & & & & & \\
\hline & Y-833 & 4.0131 & 4.0171 & $0.100 \%$ & $\mathrm{~N} / \mathrm{C}$ & & \\
\hline & D2 & & & & & YES & \\
\hline & 923 & 3.7855 & 3.7791 & $-0.169 \%$ & $\overline{N / C}$ & & \\
\hline & E2 & & & & & NO & \\
\hline & & & & & & & \\
\hline & ISO-800 & 3.9280 & 3.9307 & $0.069 \%$ & $\mathrm{~N} / \mathrm{C}$ & & \\
\hline & & & & & & NO & \\
\hline & & & & & & & \\
\hline
\end{tabular}




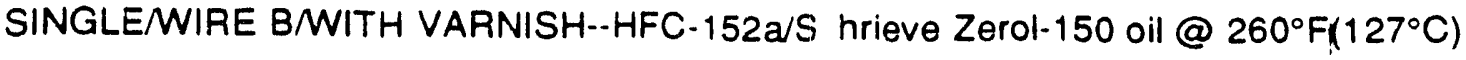

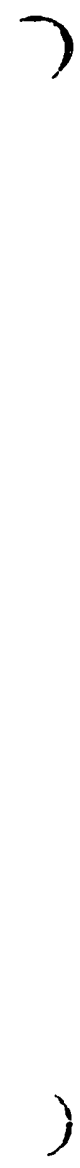

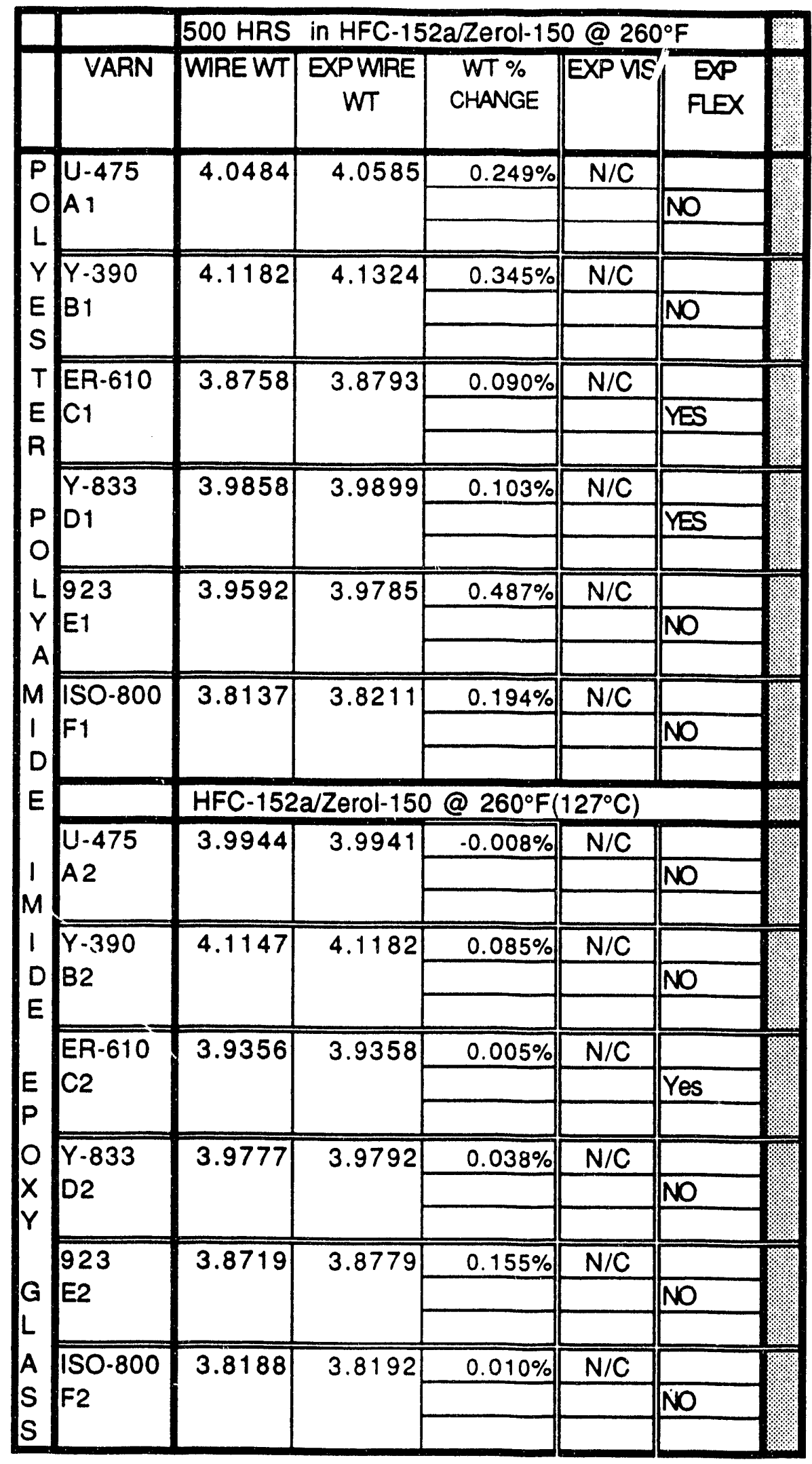




\begin{tabular}{|c|c|c|c|c|c|c|}
\hline & & 500 HRS i & in HFC-152a & Zerol-150 & @ 260 & $=\left(127^{\circ} \mathrm{C}\right)$ \\
\hline & VARN & WIREWT & $\begin{array}{c}\text { EXPWRE } \\
W T\end{array}$ & $\begin{array}{l}\text { WT \% } \\
\text { CHANGE }\end{array}$ & EXPVIS & $\begin{array}{l}\text { EXP } \\
\text { FEX }\end{array}$ \\
\hline$P \mid$ & U. 475 & 4.4751 & 4.4873 & $0.273 \%$ & $N / C$ & \\
\hline 0 & & & & & & YES \\
\hline 4 & & & & & & \\
\hline Y & Y-390 & 3.9869 & 3.9984 & $0.288 \%$ & $N / C$ & \\
\hline E & & & & & & NO \\
\hline s) & & & & & & \\
\hline TI & ER-610 & 3.6935 & 3.6977 & $0.114 \%$ & $N / C$ & \\
\hline$E$ & & & & & & YES \\
\hline & Y-833 & 3.5209 & 3.5261 & $0.148 \%$ & $\mathrm{~N} / \mathrm{C}$ & \\
\hline 1 & & & & & & No \\
\hline$M$ & & & & & & \\
\hline 1 & 923 & 3.9136 & 3.9259 & $0.314 \%$ & $N / C$ & \\
\hline D & E1 & & & & & NO \\
\hline$E$ & & & & & & \\
\hline & ISO-800 & 3.8411 & 3.8484 & $0.190 \%$ & $N / C$ & \\
\hline $\begin{array}{l}P \\
0\end{array}$ & & & & & & NO \\
\hline L & & HFC-152a/2 & Zerol-150@ & $260^{\circ} \mathrm{F} \cdot>2$ & 24 HRS & @ $302 \mathrm{~F}$ \\
\hline$Y$ & U-475 & 3.7035 & 3.7100 & $0.176 \%$ & $N / C$ & \\
\hline$A$ & A2 & & & & & No \\
\hline$M$ & & & & & & \\
\hline 1 & $Y-390$ & 4.0239 & 4.0302 & $0.157 \%$ & $N / C$ & \\
\hline$D$ & & & & & & NO \\
\hline & ER-610 & 3.6470 & 3.6467 & $-0.008 \%$ & $N / C$ & \\
\hline 1 & & & & & & Yes \\
\hline$M$ & & & & & & \\
\hline 1 & $Y-833$ & 3.5345 & 3.5332 & $-0.037 \%$ & $N / C$ & \\
\hline$D_{C}^{D} \quad-x$ & & & & & & YES \\
\hline & 923 & 4.0264 & 4.0310 & $0.114 \%$ & N/C & \\
\hline & E2 & & & & & NO \\
\hline & 150.800 & 3274 & 38770 & $0054 \%$ & N/C & \\
\hline & F2 & & & & & NO \\
\hline & & & & & & \\
\hline
\end{tabular}


Varnish Disks-..-HFC-152a/S hrieve Zerol-150 Oil I

7

500 HRS IN R-152a/Zerol-150 Oil @ 260 $\mathrm{F}$

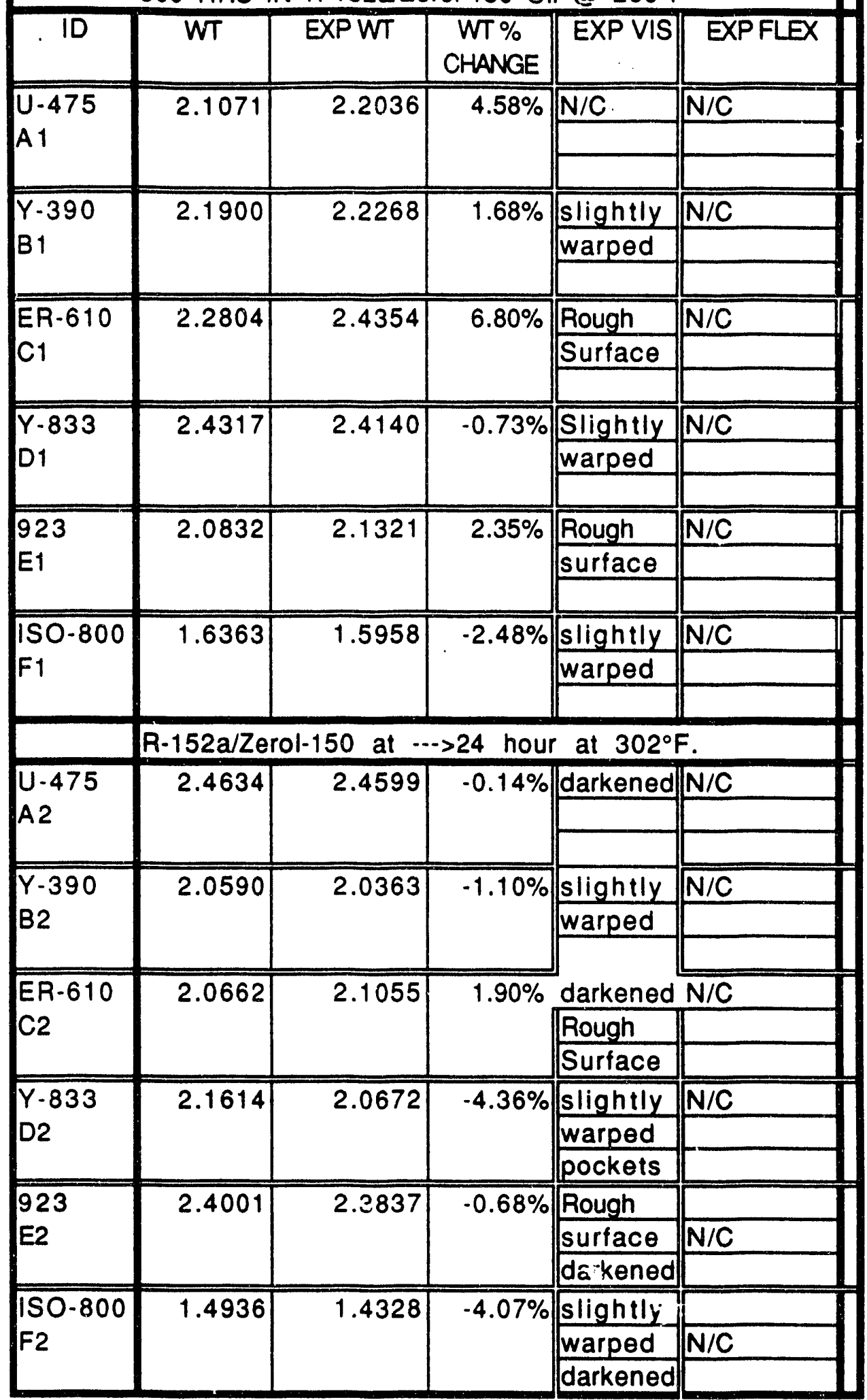




\begin{tabular}{|c|c|c|c|c|c|c|c|c|c|}
\hline \multirow{2}{*}{\begin{tabular}{|l|l} 
& 5 \\
$\mathrm{ID}$ &
\end{tabular}} & \multicolumn{9}{|c|}{$\begin{array}{l}500 \text { HRS in HFC-152a/Schrieve Zerol-150 @ 260 F } \\
\text { WT EXPWT WT \% ISAMPIEII BRFAK }\end{array}$} \\
\hline & $W T$ & EXPWT & $\begin{array}{c}\text { WT \% } \\
\text { CHANGE }\end{array}$ & $\| \begin{array}{l}\text { SAMPLE } \\
\text { WIDTH }\end{array}$ & $\begin{array}{l}\text { BREAK } \\
\text { LOAD }\end{array}$ & $\begin{array}{c}\text { SAMPLE } \\
\text { THICKNESS } \\
\text { Mils }\end{array}$ & $\begin{array}{c}\text { TENSILE } \\
\text { STR } \\
\text { BASE } \\
\end{array}$ & $\begin{array}{l}\text { TENSILE } \\
\text { STREXP }\end{array}$ & $\begin{array}{l}\% \text { CHANGE } \\
\text { TENSILE }\end{array}$ \\
\hline \multirow{3}{*}{$\begin{array}{l}\text { NO/MY/NO } \\
\text { A1 }\end{array}$} & 7.6582 & 8.1153 & $5.97 \%$ & 0.456 & 96.65 & 21 & 17.4 & 10.09 & \\
\hline & & & & 0.490 & 127.20 & 21 & 17.4 & 12.36 & $-37.59 \%$ \\
\hline & & & & 0.456 & 96.95 & 21 & 17.4 & 10.12 & \\
\hline \multirow{3}{*}{$\begin{array}{l}\text { DAIMYIDA } \\
\text { B1 }\end{array}$} & 7.6437 & 7.6283 & $.0 .20 \%$ & 0.318 & 81.05 & 21 & 13.7 & 12.14 & \\
\hline & & & & 0.491 & 98.80 & 21 & 13.7 & 9.58 & $-21.61 \%$ \\
\hline & & & & 0.479 & 105.60 & 21 & 13.7 & 10.50 & \\
\hline \multirow{3}{*}{$\begin{array}{l}\text { MYLAR MO } \\
\text { C1 }\end{array}$} & 3.6947 & 3.7124 & $0.48 \%$ & 0.385 & 56.10 & 10 & 21.7 & 14.57 & \\
\hline & & & & 0.475 & 71.95 & 10 & 21.7 & 15.15 & $-33.27 \%$ \\
\hline & & & & 0.493 & 67.65 & 10 & 21.7 & 13.72 & \\
\hline \multirow{3}{*}{$\begin{array}{l}\text { NO } 410 \\
\text { D1 }\end{array}$} & 3.7122 & 4.0842 & $10.02 \%$ & 0.438 & 78.00 & 10 & 18.7 & 17.81 & \\
\hline & & & & 0.513 & 88.20 & 10 & 18.7 & 17.19 & $-4.33 \%$ \\
\hline & & & & 0.496 & 92.60 & 10 & 18.7 & 18.67 & \\
\hline \multirow{3}{*}{$\begin{array}{l}\text { NO MI } 418 \\
\text { E1 }\end{array}$} & 3.7832 & 4.3420 & $14.77 \%$ & 0.490 & 27.70 & 10 & 7.5 & 5.65 & \\
\hline & & & & 0.507 & 29.75 & 10 & 7.5 & 5.87 & $-23.06 \%$ \\
\hline & & & & 0.525 & 30.40 & 10 & 7.5 & 5.79 & \\
\hline \multirow{4}{*}{$\begin{array}{l}\text { MEL } 228 \\
\text { F1 }\end{array}$} & 4.2582 & 4.3500 & $2.16 \%$ & 0.406 & 61.15 & 10 & 21.7 & 15.06 & \\
\hline & & & & 0.460 & 70.05 & 10 & 21.7 & 15.23 & $.27 .69 \%$ \\
\hline & & & & 0.476 & 79.90 & 10 & 21.7 & 16.79 & \\
\hline & $500 \mathrm{HRS}$ & $\mathrm{S}$ in $\mathrm{HFC}-1$ & $152 a / S c h i \theta$ & ve Zerol- & $150 @ 26$ & $30 \mathrm{~F}->24$ & @ 302 & & \\
\hline \multirow{3}{*}{$\begin{array}{l}\text { NO/MY/NO } \\
\text { A2 }\end{array}$} & 7.7568 & 7.9433 & $2.40 \%$ & 0.442 & 103.60 & 21 & 17.4 & 11.16 & \\
\hline & & & & 0.425 & 101.90 & 21 & 17.4 & 11.42 & $-36.32 \%$ \\
\hline & & & & 0.448 & 100.30 & 21 & 17.4 & 10.66 & \\
\hline \multirow{3}{*}{$\begin{array}{l}\text { DA/MYIDA } \\
\text { B2 }\end{array}$} & 7.1430 & 6.9705 & $-2.41 \%$ & 0.386 & 33.95 & 21 & 13.7 & 4.19 & \\
\hline & & & & 0.490 & 64.40 & 21 & 13.7 & 6.26 & $-63.83 \%$ \\
\hline & & & & 0.402 & 37.30 & 21 & 13.7 & 4.42 & \\
\hline \multirow{3}{*}{$\begin{array}{l}\text { MYAR MO } \\
\text { C2 }\end{array}$} & 3.7200 & 3.6844 & $-0.96 \%$ & 0.394 & 65.35 & 10 & 21.7 & 16.59 & \\
\hline & & & & 0.381 & 59.80 & 10 & 21.7 & 15.70 & $-24.95 \%$ \\
\hline & & & & 0.368 & 61.00 & 10 & 21.7 & 16.58 & \\
\hline \multirow{3}{*}{$\begin{array}{l}\text { NOMEX 410 } \\
\text { D2 }\end{array}$} & 3.8005 & 3.9461 & $3.83 \%$ & 0.444 & 80.20 & 10 & 18.7 & 18.06 & \\
\hline & & & & 0.492 & 90.80 & 10 & 18.7 & 18.46 & $-0.11 \%$ \\
\hline & & & & 0.520 & 101.50 & 10 & 18.7 & 19.52 & \\
\hline \multirow{3}{*}{$\begin{array}{l}\text { NO/MI } 418 \\
\text { E2 }\end{array}$} & 3.7850 & 4.0056 & $5.83 \%$ & 0.472 & 24.70 & 10 & 7.5 & 5.23 & \\
\hline & & & & 0.480 & 28.90 & 10 & 7.5 & 6.02 & $-24.53 \%$ \\
\hline & & & & 0.496 & 28.40 & 10 & 7.5 & 5.73 & \\
\hline \multirow{3}{*}{$\begin{array}{l}\text { MEL } 228 \\
\text { F2 }\end{array}$} & 4.0835 & 4.0826 & $-0.02 \%$ & 0.431 & 74.90 & 10 & 21.7 & 17.38 & \\
\hline & & & & 0.495 & 84.25 & 10 & 21.7 & 17.02 & $-21.07 \%$ \\
\hline & & & & 0.431 & 73.20 & 10 & 21.7 & 16.98 & \\
\hline
\end{tabular}




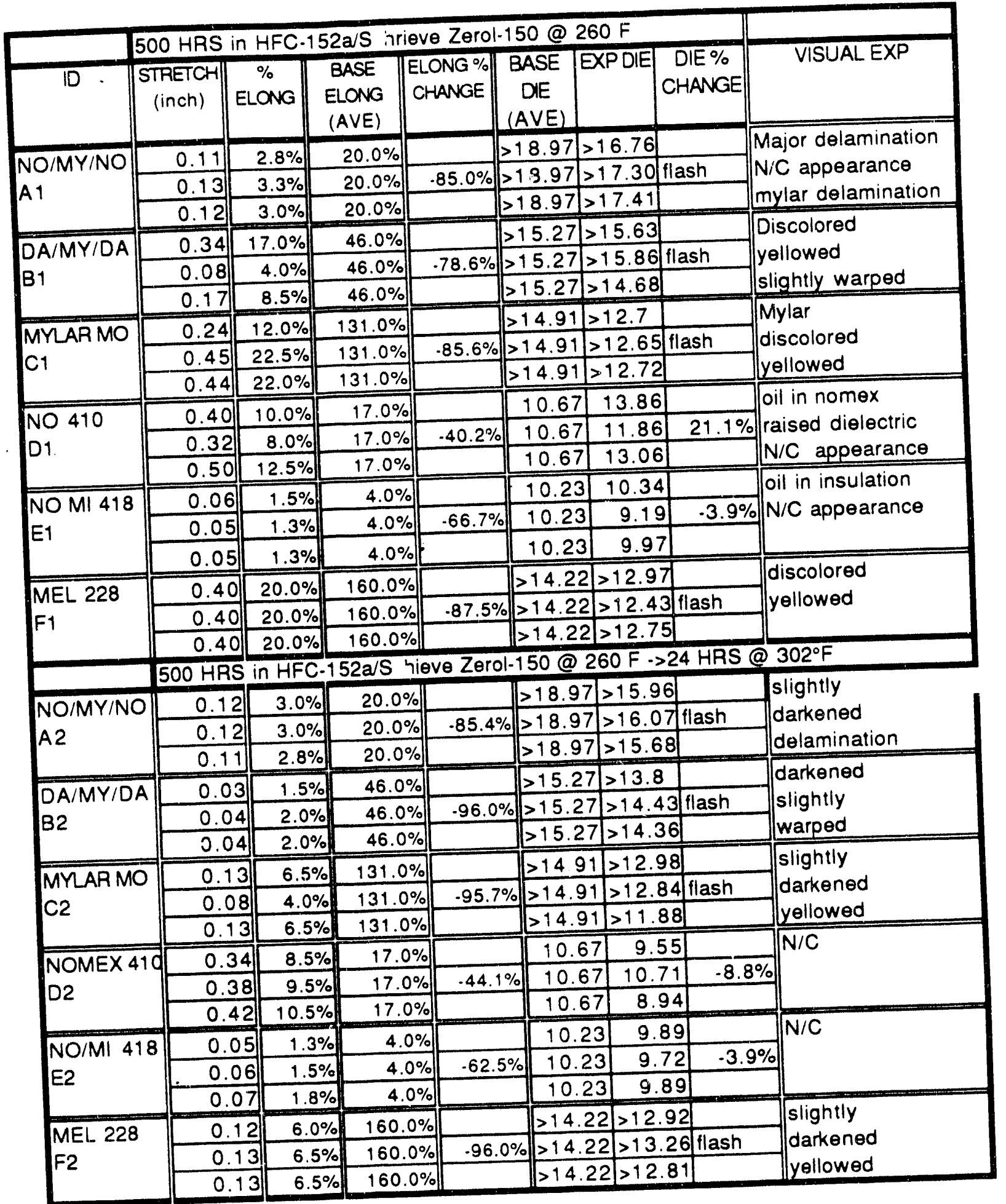




\begin{tabular}{|c|c|c|c|c|}
\hline & \multicolumn{4}{|c|}{500 HRS in HFC-152a/S hrieve Zerol-150 oil @ 260 F } \\
\hline & WT & EXPWT & $\begin{array}{c}\% \\
\text { CHANGE } \\
\end{array}$ & EXP VS \\
\hline \multirow{3}{*}{$\begin{array}{l}\text { A1 } \\
\text { NOMEX }\end{array}$} & \multirow[t]{3}{*}{0.5561} & \multirow[t]{3}{*}{0.6178} & \multirow[t]{3}{*}{$11.10 \%$} & Darkened, Nomex discolored \\
\hline & & & & black \\
\hline & & & & see photo \\
\hline \multirow{2}{*}{$\begin{array}{l}\text { B1 } \\
\text { MYLAR }\end{array}$} & \multirow[t]{2}{*}{0.5093} & \multirow[t]{2}{*}{0.5219} & \multirow[t]{2}{*}{$2.47 \%$} & \\
\hline & & & & $\mathrm{N} / \mathrm{C}$ \\
\hline \multirow{3}{*}{$\begin{array}{l}\mathrm{C} 1 \\
\mathrm{NO} / \mathrm{MY}\end{array}$} & \multirow[t]{3}{*}{0.3910} & \multirow[t]{3}{*}{0.4188} & \multirow[t]{3}{*}{$7.11 \%$} & Black discoloration of the \\
\hline & & & & nomex inside \\
\hline & & & & see photo \\
\hline & \multicolumn{4}{|c|}{ HFC-152a/Shrieve Zerol-150 oil @ 260 F plus24 hrs @ 302 ${ }^{\circ} \mathrm{F}$} \\
\hline \multirow{2}{*}{$\begin{array}{l}\text { A2 } \\
\text { NOMEX }\end{array}$} & \multirow[t]{2}{*}{0.5445} & \multirow[t]{2}{*}{0.5659} & \multirow[t]{2}{*}{$3.93 \%$} & $\mathrm{~N} / \mathrm{C}$ \\
\hline & & & & same condition as above \\
\hline \multirow{3}{*}{$\begin{array}{l}\text { B2 } \\
\text { MYLAR }\end{array}$} & \multirow[t]{3}{*}{0.4778} & \multirow[t]{3}{*}{0.4767} & \multirow[t]{3}{*}{$-0.23 \%$} & darkened \\
\hline & & & & some pockets in \\
\hline & & & & mylar, pulled away \\
\hline \multirow{3}{*}{$\begin{array}{l}\mathrm{C} 2 \\
\mathrm{NO} / \mathrm{MY}\end{array}$} & \multirow[t]{3}{*}{0.3999} & \multirow[t]{3}{*}{0.4017} & \multirow[t]{3}{*}{$0.45 \%$} & $\mathrm{~N} / \mathrm{C}$ \\
\hline & & & & same condition as above \\
\hline & & & & see photo \\
\hline
\end{tabular}




\begin{tabular}{|c|c|c|c|c|c|c|c|c|c|}
\hline & 500 HRS & in HFC-15 & 2a/Shrieve & Zerol-15 & @260 F & & & & \\
\hline ID & WT & EXPWT & $\begin{array}{c}\text { WT \% } \\
\text { CHANGE }\end{array}$ & $\begin{array}{l}\text { BREAK } \\
\text { LOAD } \\
\text { (AVE) }\end{array}$ & $\begin{array}{c}\text { BREAK } \\
\text { LOAD } \\
\text { DP }\end{array}$ & $\begin{array}{c}\% \\
\text { CHANGE: } \\
\text { BPK } \\
\text { LOAD }\end{array}$ & $\begin{array}{l}\text { STRETCH } \\
(\text { INCH) }\end{array}$ & $\%$ ELNG & EXP VIS \\
\hline$A 1$ & 1.4382 & 1.4466 & $0.58 \%$ & 39.02 & 55.60 & & 0.05 & $2.50 \%$ & N/C \\
\hline Glass & & & & 39.02 & 62.25 & $53.30 \%$ & 0.06 & $3.00 \%$ & \\
\hline & & & & 39.02 & 61.60 & & 0.06 & $3.00 \%$ & \\
\hline$B 1$ & 0.6805 & 0.6918 & $1.66 \%$ & 56.12 & 53.55 & & 0.49 & $24.50 \%$ & $\mathrm{~N} / \mathrm{C}$ \\
\hline Polyester & & & & 56.12 & 55.35 & $-4.73 \%$ & 0.53 & $26.50 \%$ & \\
\hline & & & & 56.12 & 51.50 & & 0.44 & $22.00 \%$ & \\
\hline $\mathrm{C}_{1}$ & 1.6600 & 1.7749 & $6.92 \%$ & 88.50 & 82.70 & & 0.15 & $7.50 \%$ & \\
\hline Permacel & & & & 88.50 & 101.00 & $4.12 \%$ & 0.14 & $7.00 \%$ & darkened \\
\hline & & & & 88.50 & 92.75 & & 0.14 & $7.00 \%$ & \\
\hline & $500 \mathrm{HR}$ & $S$ in HFC- & 52a/Shriel & e Zerol-15 & $0 @ 260 \mathrm{~F}$ & $\Rightarrow 302^{\circ} \mathrm{F}$ & $24 \mathrm{HRS}$ & & \\
\hline A2 & 1.3848 & 1.3857 & $0.06 \%$ & 39.02 & 48.25 & & 0.06 & $3.00 \%$ & $\mathrm{~N} / \mathrm{C}$ \\
\hline Glass & & & & 39.02 & 69.65 & $43.94 \%$ & 0.08 & $4.00 \%$ & \\
\hline & & & & 39.02 & 50.60 & & 0.07 & $3.50 \%$ & \\
\hline & 0.6400 & 0.6351 & $-0.77 \%$ & 56.12 & 41.00 & & 0.33 & $16.50 \%$ & discolored \\
\hline Polyester & & & & 56.12 & 42.80 & $-22.40 \%$ & 0.39 & $19.50 \%$ & \\
\hline & & & & 56.12 & 46.85 & & 0.47 & $23.50 \%$ & \\
\hline $\mathrm{C} 2$ & 1.4577 & 1.3456 & $-7.69 \%$ & 88.50 & 96.75 & & 0.16 & $8.00 \%$ & \\
\hline Permacel & & & & 88.50 & 43.75 & $-15.31 \%$ & 0.09 & $4.50 \%$ & Darkened \\
\hline & & & & 88.50 & 84.35 & & 0.13 & $6.50 \%$ & \\
\hline
\end{tabular}


TIE CORD--HFC-152a/S nrieve Zerol-150 oil @ 260 $0^{\circ} \mathrm{F}\left(127^{\circ} \mathrm{C}\right)$

\begin{tabular}{|c|c|c|c|c|c|c|c|c|c|}
\hline \multicolumn{10}{|c|}{500 HRS IN R-152a/Shrieve Zerol-150 @ 260\% F } \\
\hline ID & WT & EXPWT & $\begin{array}{c}\text { WT \% } \\
\text { CHANGE }\end{array}$ & $\begin{array}{l}E P \\
\text { VIS }\end{array}$ & $\begin{array}{l}\text { BREAK } \\
\text { LOAD } \\
\text { (AVE) }\end{array}$ & $\begin{array}{c}\text { BREAK } \\
\text { LOAD } \\
\text { EXP } \\
\text { (ave) }\end{array}$ & $\begin{array}{c}\% \\
\text { CHANGE } \\
\text { BRK } \\
\text { LOAD }\end{array}$ & $\begin{array}{c}\text { STRETCH } \\
\text { (lnch) }\end{array}$ & $\begin{array}{c}\% \\
\text { ELONG }\end{array}$ \\
\hline \multirow[t]{4}{*}{ A1 } & \multirow[t]{3}{*}{0.2625} & \multirow[t]{3}{*}{0.2663} & \multirow[t]{3}{*}{$1.45 \%$} & $N / C$ & 28.36 & 34.75 & & 0.45 & $22.5 \%$ \\
\hline & & & & & 28.36 & 37.45 & $29.82 \%$ & 0.48 & $24.0 \%$ \\
\hline & & & & & 28.36 & 38.25 & & 0.48 & $24.0 \%$ \\
\hline & \multicolumn{9}{|c|}{500 HRS IN R-152a/ } \\
\hline \multirow[t]{3}{*}{ A2 } & \multirow[t]{3}{*}{0.2620} & \multirow[t]{3}{*}{0.2612} & \multirow[t]{3}{*}{$-0.305 \%$} & $\mathrm{~N} / \mathrm{C}$ & 28.36 & 24.45 & & 0.41 & $20.5 \%$ \\
\hline & & & & & 28.36 & 28.25 & $1.49 \%$ & 0.47 & $23.5 \%$ \\
\hline & & & & & 28.36 & 33.65 & & 0.51 & $25.5 \%$ \\
\hline
\end{tabular}


LEAD WIRE INSULATION-HFC-152a/S hrieve Zerol-150@260 $\mathrm{F}\left(127^{\circ} \mathrm{C}\right)$

\begin{tabular}{|l|r|r|r||r||r|r|r|}
\hline & 500 HRS IN HFC-152a/S hrieve Zerol-150 @ $2600^{\circ} \mathrm{F}$ & & \\
\hline ID & WT & EXPWT & $\begin{array}{c}\text { WT \% } \\
\text { CHANGE }\end{array}$ & EXP VIS & $\begin{array}{r}\text { BASE DIE } \\
\text { (AVE) }\end{array}$ & EXP DIE & $\begin{array}{c}\text { DIE\% } \\
\text { CHANGE }\end{array}$ \\
\hline DMD & 4.3315 & 4.4631 & $3.04 \%$ & N/C & 9.61 & 13.17 & \\
\hline A1 & & & & & 9.61 & 13.34 & $35.31 \%$ \\
\hline & & & & & 9.61 & 12.50 & \\
\hline DTMD & 4.0187 & 4.1600 & $3.52 \%$ & N/C & 9.95 & 8.73 & \\
\hline B1 & & & & & 9.95 & 9.19 & $-18.49 \%$ \\
\hline & & & & & 9.95 & 6.41 & \\
\hline & & & & & & & \\
& & & & & & & \\
\hline DMD & 4.3686 & 4.3973 & $0.66 \%$ & N/C & 9.61 & 13.33 & \\
\hline A2 & & & & & 9.61 & 13.02 & $31.36 \%$ \\
\hline & & & & & 9.61 & 11.52 & \\
\hline \hline DTMD & 4.1010 & 4.1448 & $1.07 \%$ & N/C & 9.95 & 9.66 & \\
\hline B2 & & & & & 9.95 & 10.12 & $0.57 \%$ \\
\hline & & & & & 9.95 & 10.24 & \\
\hline
\end{tabular}




\section{Appendix F}

\section{Experimental Data for $\mathrm{HFC}-134 \mathrm{a} / \mathrm{ICI}$ Emkarate RL244 Exposure at $126^{\circ} \mathrm{C}\left(260^{\circ} \mathrm{F}\right)$}




\begin{tabular}{|c|c|c|c|c|c|c|c|c|c|c|}
\hline & 500 hours i & HFC-134 & Emkarat & e RL244 & oil at $260^{\circ}$ & $\mathrm{F}\left(127^{\circ} \mathrm{C}\right)$ & & & & \\
\hline ID & $W T$ & EXPWT & $\begin{array}{c}\text { WT \% } \\
\text { CHANGE }\end{array}$ & EXP VIS & $\begin{array}{c}\text { BASE BRN } \\
\text { OUT } \\
\text { (AVE) }\end{array}$ & $\begin{array}{c}\text { EXP BRN } \\
\text { OUT }\end{array}$ & $\begin{array}{c}\text { BRN OUT } \\
\% \\
\text { CHANGE }\end{array}$ & $\begin{array}{c}\text { BASE DIE } \\
\text { (AVE) }\end{array}$ & EXPDIE & $\begin{array}{c}\text { DIE \% } \\
\text { CHANGE }\end{array}$ \\
\hline A1 & 25.6686 & 25.6817 & $0.051 \%$ & $N / C$ & 576 & 522 & & 15.80 & 14.23 & \\
\hline & & & & & 576 & 517 & $-9.9 \%$ & 15.80 & 12.17 & $-16.6 \%$ \\
\hline & & & & & 576 & 518 & & 15.80 & 13.13 & \\
\hline B1 & 25.3778 & 25.4405 & $0.247 \%$ & $\mathrm{~N} / \mathrm{C}$ & 736 & 735 & & 11.62 & 14.00 & \\
\hline & & & & & 736 & 731 & $-0.2 \%$ & 11.62 & 16.61 & $28.0 \%$ \\
\hline & & & & & 736 & 738 & & 11.62 & 14.01 & \\
\hline $\mathrm{C} 1$ & 22.2264 & 22.2379 & $0.052 \%$ & $N / C$ & 579 & 446 & & 16.58 & 17.73 & \\
\hline & & & & & 579 & 464 & $-14.6 \%$ & 16.58 & 16.57 & $-0.6 \%$ \\
\hline & & & & & 579 & 573 & & 16.58 & 15.13 & \\
\hline & 500 hours i & HFC-134 & a/Emkarat & e RL244 & oil at $260^{\circ}$ & $\mathrm{F}\left(127^{\circ} \mathrm{C}\right)$ & plus $24 \mathrm{hc}$ & ours at 30 & $2^{\circ} \mathrm{F}\left(150^{\circ} \mathrm{C}\right.$ & \\
\hline$A \cdot 2$ & 25.8451 & 25.8650 & $0.077 \%$ & $N / C$ & \begin{tabular}{|l|}
576 \\
\end{tabular} & 558 & & 15.80 & 15.12 & \\
\hline & & & & & 576 & 511 & $-7.6 \%$ & 15.80 & 15.29 & $-11.0 \%$ \\
\hline & & & & & 576 & 527 & & 15.80 & 11.76 & \\
\hline B2 & 25.0496 & 25.0888 & $0.156 \%$ & $\mathrm{~N} / \mathrm{C}$ & 736 & 731 & & 11.62 & 13.10 & \\
\hline & & & & & 736 & 731 & $-0.7 \%$ & 11.62 & 11.76 & $13.4 \%$ \\
\hline & & & & & 736 & 731 & & 11.62 & 14.67 & \\
\hline $\mathrm{C} 2$ & 22.6674 & 22.6874 & $0.088 \%$ & $\mathrm{~N} / \mathrm{C}$ & 579 & 568 & & 16.58 & 14.86 & \\
\hline & & & & & 579 & 561 & $-3.9 \%$ & 16.58 & 17.06 & $-3.6 \%$ \\
\hline & & & & & 579 & 541 & & 16.58 & 16.03 & \\
\hline
\end{tabular}


TWISTED PAIRS/WIRE ANWITH VARNISH-HFC-134a/ICI Emkarate RL244 vil @260"F(127:C)

\begin{tabular}{|c|c|c|c|c|c|c|c|c|c|c|c|}
\hline & \multirow{2}{*}{ VARN } & \multicolumn{10}{|c|}{500 hours in HFC-134/Emkarate RL244 oil @260 $\mathrm{F}\left(127^{\circ} \mathrm{C}\right)$} \\
\hline & & $\begin{array}{l}\text { TWISTED } \\
\text { PAIR WT }\end{array}$ & $\begin{array}{c}\text { Exp Pair } \\
\text { weight }\end{array}$ & $\begin{array}{c}\text { WT \% } \\
\text { CHANGE }\end{array}$ & $\begin{array}{l}\text { EXP } \\
\text { VIS }\end{array}$ & BASE DIE & EXPDIE & $\begin{array}{c}\text { DIE \% } \\
\text { CHANGE }\end{array}$ & $\begin{array}{l}\text { BASE } \\
\text { BURN } \\
\text { OUT }\end{array}$ & $\begin{array}{l}\text { EXP } \\
\text { BUPN } \\
\text { OUT }\end{array}$ & $\begin{array}{c}\text { BRNOUT } \\
\% \\
\text { CHANGE }\end{array}$ \\
\hline \multirow{3}{*}{$\begin{array}{l}P \\
O \\
L\end{array}$} & \multirow{3}{*}{$\begin{array}{l}\text { U.475 } \\
\text { A1 } \\
\end{array}$} & \multirow[t]{3}{*}{22.8106} & \multirow[t]{3}{*}{22.8406} & \multirow[t]{3}{*}{$0.132 \%$} & $\mathrm{~N} / \mathrm{C}$ & 16.24 & 18.85 & & 430 & 378 & \\
\hline & & & & & & 16.24 & 17.70 & $16.05 \%$ & 430 & 377 & $2.87 \%$ \\
\hline & & & & & & 16.24 & 19.99 & & 430 & 572 & \\
\hline \multirow{3}{*}{$\begin{array}{l}Y \\
E \\
S\end{array}$} & \multirow{3}{*}{$\begin{array}{l}Y .390 \\
B 1\end{array}$} & \multirow[t]{3}{*}{25.2574} & \multirow[t]{3}{*}{25.1887} & \multirow[t]{3}{*}{$-0.272 \%$} & $\mathrm{~N} / \mathrm{C}$ & 18.77 & 14.07 & & 510 & 332 & \\
\hline & & & & & & 18.77 & 16.45 & $-21.81 \%$ & 510 & 391 & $-30.26 \%$ \\
\hline & & & & & & 18.77 & 13.51 & & 510 & 344 & \\
\hline \multirow{4}{*}{$\begin{array}{l}T \\
E \\
R\end{array}$} & \multirow{3}{*}{$\begin{array}{l}\text { ER-610 } \\
\text { C1 }\end{array}$} & \multirow[t]{3}{*}{25.9696} & \multirow[t]{3}{*}{26.0118} & \multirow[t]{3}{*}{$0.162 \%$} & $\mathrm{~N} / \mathrm{C}$ & 15.57 & 13.34 & & 442 & 362 & \\
\hline & & & & & & 15.57 & 13.95 & $-13.40 \%$ & 442 & 328 & $-19.08 \%$ \\
\hline & & & & & & 15.57 & 13.16 & & 442 & 383 & \\
\hline & \multirow{3}{*}{$\begin{array}{l}Y-833 \\
D 1\end{array}$} & \multirow[t]{3}{*}{21.4774} & \multirow[t]{3}{*}{21.4996} & \multirow[t]{3}{*}{$0.103 \%$} & $\mathrm{~N} / \mathrm{C}$ & 12.04 & 12.89 & & 578 & 566 & \\
\hline$P$ & & & & & & 12.04 & 16.30 & $17.66 \%$ & 578 & 469 & $.7 .96 \%$ \\
\hline 0 & & & & & & 12.04 & 13.31 & & 578 & 561 & \\
\hline \multirow{3}{*}{$\begin{array}{l}L \\
Y \\
A\end{array}$} & \multirow{3}{*}{$\begin{array}{l}923 \\
E 1\end{array}$} & 22.9761 & 23.0134 & $0.162 \%$ & $\mathrm{~N} / \mathrm{C}$ & 16.76 & 19.99 & & 606 & 470 & \\
\hline & & & & & & 16.76 & 17.81 & $12.71 \%$ & 606 & 558 & $-13.26 \%$ \\
\hline & & & & & & 16.76 & 18.87 & & 606 & 549 & \\
\hline$M$ & ISO-800 & 27.1394 & 27.2858 & $0.539 \%$ & $\mathrm{~N} / \mathrm{C}$ & 19.08 & 16.23 & & 580 & 593 & \\
\hline 1 & $F 1$ & & & & & 19.08 & 18.70 & $.4 .21 \%$ & 580 & 577 & $0.40 \%$ \\
\hline$D$ & & & & & & 19.08 & 19.90 & & 580 & 577 & \\
\hline$E$ & & 500 hours & in $\mathrm{HFC}-13$ & $34 \mathrm{a} / \mathrm{Emka}$ & irate $F$ & L244 oil @ & 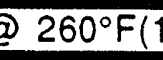 & $127^{\circ} \mathrm{C}$ ) ple & $24 \mathrm{hol}$ & rs@3 & $2^{\circ} F\left(150^{\circ}\right.$ \\
\hline & $U-475$ & 22.4812 & 22.5356 & $0.242 \%$ & $\mathrm{~N} / \mathrm{C}$ & 16.24 & 16.78 & & 430 & 333 & \\
\hline 1 & $A 2$ & & & & & 16.24 & 14.58 & $3.80 \%$ & 430 & 372 & $-16.28 \%$ \\
\hline M & & & & & & 16.24 & 19.24 & & 430 & 375 & \\
\hline I & $Y-390$ & 25.0748 & 25.0991 & $0.097 \%$ & $\mathrm{~N} / \mathrm{C}$ & 18.77 & 12.99 & & 510 & 372 & \\
\hline$D$ & B2 & & & & & 18.77 & 14.63 & $-23.76 \%$ & 510 & 385 & $-24.90 \%$ \\
\hline$E$ & & & & & & 18.77 & 15.31 & & 510 & 392 & \\
\hline & ER-610 & 25.8473 & 25.8745 & $0.105 \%$ & $\mathrm{~N} / \mathrm{C}$ & 15.57 & 15.20 & & 442 & 323 & \\
\hline & $\mathrm{C} 2$ & & & & & 15.57 & 11.67 & $-8.29 \%$ & 442 & 341 & $-24.06 \%$ \\
\hline & & & & & & 15.57 & 15.97 & & 442 & 343 & \\
\hline & $Y-833$ & 21.3866 & 21.4539 & $0.315 \%$ & $\mathrm{~N} / \mathrm{C}$ & 12.04 & 14.66 & & 578 & 477 & \\
\hline & D2 & & & & & 12.04 & 14.11 & $14.62 \%$ & 578 & 544 & $-11.59 \%$ \\
\hline & & & & & & 12.04 & 12.63 & & 578 & 512 & \\
\hline & 923 & 22.6679 & 22.7056 & $0.166 \%$ & $\mathrm{~N} / \mathrm{C}$ & 16.76 & 18.15 & & 606 & 557 & \\
\hline & E2 & & & & & 16.76 & 17.97 & $10.36 \%$ & 606 & 572 & $-9.30 \%$ \\
\hline & & & & & & 16.76 & 19.37 & & 606 & 520 & \\
\hline & ISO-800 & 26.7568 & 26.8549 & $0.367 \%$ & $\mathrm{~N} / \mathrm{C}$ & 19.08 & 18.42 & & 580 & 585 & \\
\hline & $\mathrm{F} 2$ & & & & & 19.08 & 17.53 & $-6.13 \%$ & 580 & 459 & $-6.09 \%$ \\
\hline & & & & & & 19.08 & 17.78 & & 580 & 590 & \\
\hline
\end{tabular}


TWISTED PAIRSNIRE BNITH VARNISH -HFC-134a/ICI Emkarate RL244 oil @260 $\mathrm{F}\left(127^{\circ} \mathrm{C}\right)$

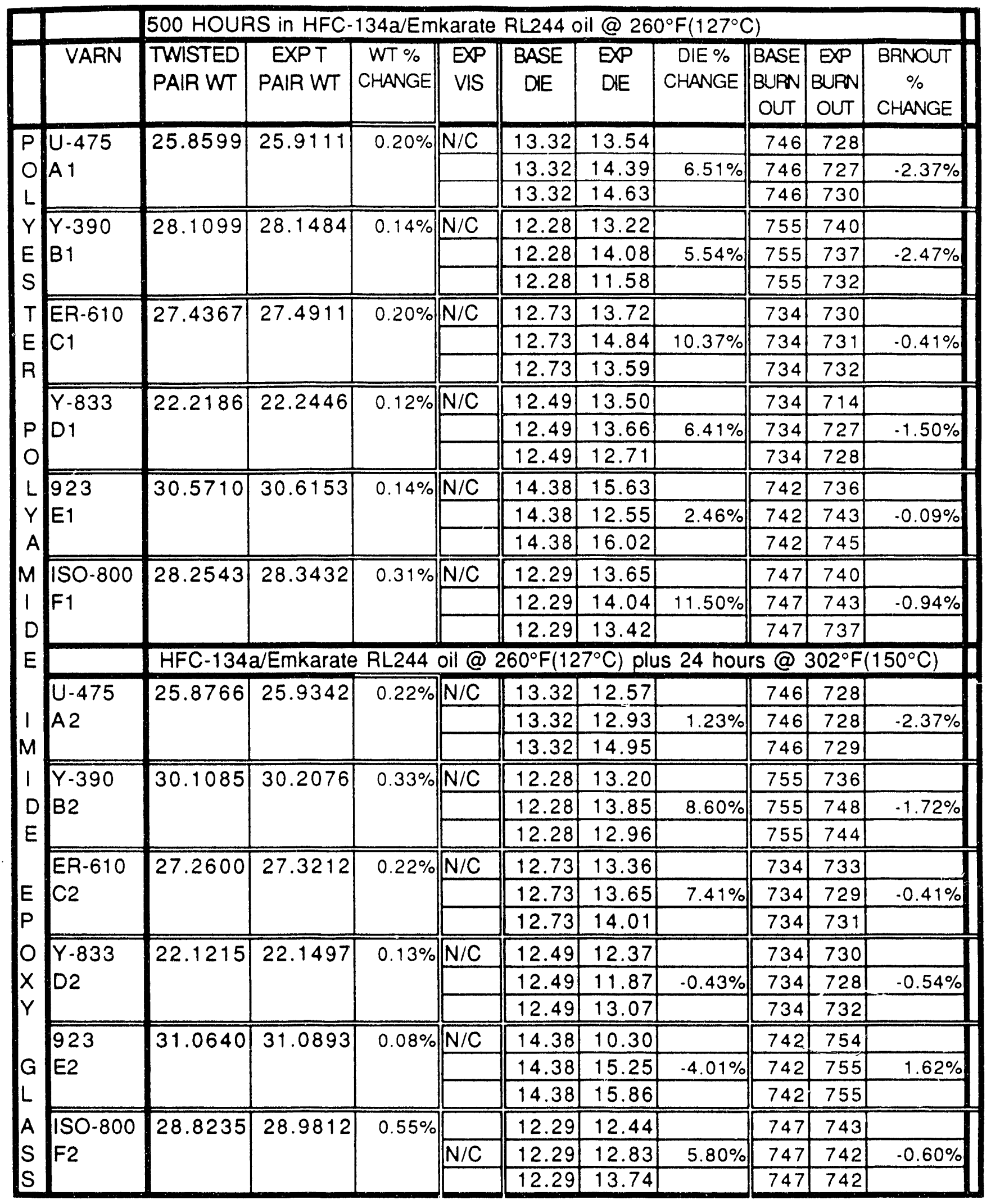


TWISTED PAIRS WIRE CMITH VAFNISH.HFC.134a/ICI Emkarate RL244@260 $\mathrm{F}\left(127^{\circ} \mathrm{C}\right)$

\begin{tabular}{|c|c|c|c|c|c|c|c|c|c|c|c|}
\hline & \multirow{2}{*}{ VARN } & \multicolumn{10}{|c|}{ 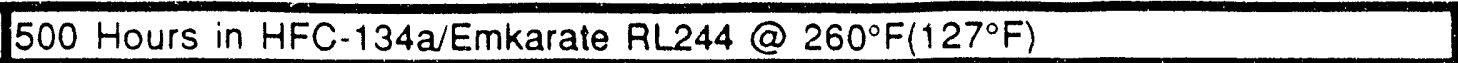 } \\
\hline & & $\begin{array}{l}\text { TWISTED } \\
\text { PAIR WT }\end{array}$ & $\begin{array}{c}\text { EXPT } \\
\text { PAIR WT }\end{array}$ & $\begin{array}{c}\text { WT \% } \\
\text { CHANGE }\end{array}$ & $\begin{array}{l}\text { EXP } \\
\text { VIS }\end{array}$ & $\begin{array}{c}\text { BASE } \\
\text { DIE }\end{array}$ & $\begin{array}{l}\text { EXP } \\
\text { DIE }\end{array}$ & $\begin{array}{c}\text { DIE \% } \\
\text { CHANGE }\end{array}$ & $\begin{array}{l}\text { BASE } \\
\text { BURN } \\
\text { OUT }\end{array}$ & \begin{tabular}{|l|} 
EXP \\
BUPN \\
OUT
\end{tabular} & $\begin{array}{c}\text { BRNOUT } \\
\% \\
\text { CHANGE }\end{array}$ \\
\hline \multirow{3}{*}{$\begin{array}{l}P \\
O \\
L\end{array}$} & \multirow{3}{*}{$\begin{array}{l}U-475 \\
\text { A } 1 \\
\end{array}$} & \multirow[t]{3}{*}{26.2922} & \multirow[t]{3}{*}{26.3264} & \multirow[t]{3}{*}{$0.130 \%$} & $\mathrm{~N} / \mathrm{C}$ & 15.10 & 16.28 & & 469 & 267 & \\
\hline & & & & & & 15.10 & 13.99 & $8.79 \%$ & 469 & 321 & $.35 .32 \%$ \\
\hline & & & & & & 15.10 & 19.01 & & 469 & 322 & \\
\hline \multirow{3}{*}{$\begin{array}{l}Y \\
E \\
S\end{array}$} & \multirow{3}{*}{$\begin{array}{l}Y-390 \\
B 1\end{array}$} & \multirow[t]{3}{*}{27.0214} & \multirow[t]{3}{*}{27.0640} & \multirow[t]{3}{*}{$0.158 \%$} & $\mathrm{~N} / \mathrm{C}$ & 18.24 & 12.84 & & 473 & 441 & \\
\hline & & & & & & 18.24 & 19.99 & $-10.54 \%$ & 473 & 386 & $-10.57 \%$ \\
\hline & & & & & & 18.24 & 16.12 & & 473 & 442 & \\
\hline \multirow{4}{*}{$\begin{array}{l}T \\
E \\
R\end{array}$} & ER-610 & \multirow[t]{3}{*}{22.9872} & \multirow[t]{3}{*}{23.0812} & \multirow[t]{3}{*}{$0.409 \%$} & $\mathrm{~N} / \mathrm{C}$ & 14.53 & 15.11 & & 494 & 421 & \\
\hline & \multirow{2}{*}{ C1 } & & & & & 14.53 & 6.96 & $-13.01 \%$ & 494 & 402 & $-16.53 \%$ \\
\hline & & & & & & 14.53 & 15.85 & & 494 & 414 & \\
\hline & \multirow{3}{*}{$\begin{array}{l}Y-833 \\
D 1\end{array}$} & \multirow[t]{3}{*}{25.7669} & \multirow[t]{3}{*}{25.7842} & \multirow[t]{3}{*}{$0.067 \%$} & $\mathrm{~N} / \mathrm{C}$ & 11.38 & 19.99 & & 557 & 390 & \\
\hline 1 & & & & & & 11.38 & 15.61 & $62.83 \%$ & 557 & 681 & $-12.57 \%$ \\
\hline$M$ & & & & & & 11.38 & 19.99 & & 557 & 390 & \\
\hline \multirow{4}{*}{$\begin{array}{l}I \\
D \\
E\end{array}$} & \multirow{3}{*}{$\begin{array}{l}923 \\
E 1\end{array}$} & 25.4722 & 25.4827 & $0.041 \%$ & $\mathrm{~N} / \mathrm{C}$ & 15.85 & 11.87 & & 503 & 555 & \\
\hline & & & & & & 15.85 & 11.79 & $-24.03 \%$ & 503 & 434 & $3.71 \%$ \\
\hline & & & & & & 15.85 & 12.46 & & 503 & 576 & \\
\hline & ISO-800 & 25.4310 & 25.4863 & $0.217 \%$ & $\mathrm{~N} / \mathrm{C}$ & 14.75 & 17.05 & & 632 & 575 & \\
\hline$P$ & $F 1$ & & & & & 14.75 & 16.13 & $15.86 \%$ & 632 & 632 & $-5.85 \%$ \\
\hline 0 & & & & & & 14.75 & 18.09 & & 632 & 578 & \\
\hline$L$ & & HFC -134 & IEmkaral & RL244@ & $260^{\circ} \mathrm{F}$ & plus 26 & 4 hours & @ $302^{\circ} \mathrm{F}$ & $\left(150^{\circ} \mathrm{C}\right.$ & & \\
\hline Y & $U .475$ & 26.6999 & 26.7340 & $0.128 \%$ & $\mathrm{~N} / \mathrm{C}$ & 15.10 & 18.61 & & 469 & 290 & \\
\hline A & A 2 & & & & & 15.10 & 17.91 & $24.46 \%$ & 469 & 346 & $-33.40 \%$ \\
\hline M & & & & & & 15.10 & 19.86 & & 469 & 301 & \\
\hline 1 & $Y-390$ & 26.4269 & 26.5024 & $0.286 \%$ & $\mathrm{~N} / \mathrm{C}$ & 18.24 & 18.45 & & 473 & 552 & \\
\hline$D$ & $\mathrm{~B} 2$ & & & & & 18.24 & 19.48 & $-0.99 \%$ & 473 & 527 & $9.16 \%$ \\
\hline$E$ & & & & & & 18.24 & 16.64 & & 473 & 470 & \\
\hline & ER-610 & 24.9227 & 24.9433 & $0.083 \%$ & $N / C$ & 14.53 & 4.27 & & 494 & 367 & \\
\hline 1 & $\mathrm{C} 2$ & & & & & 14.53 & 12.07 & $-23.40 \%$ & 494 & 435 & $-21.66 \%$ \\
\hline M & & & & & & 14.53 & 17.05 & & 494 & 359 & \\
\hline 1 & $\overline{Y-833}$ & 25.5671 & 25.5830 & $0.062 \%$ & $\mathrm{~N} / \mathrm{C}$ & 11.38 & 19.75 & & 557 & 548 & \\
\hline$D$ & D2 & & & & & 11.38 & 16.16 & $36.09 \%$ & 557 & 563 & $-8.62 \%$ \\
\hline$E$ & & & & & & 11.38 & 10.55 & & 557 & 416 & \\
\hline & 923 & 25.4331 & 25.4346 & $0.006 \%$ & $N / C$ & 15.85 & 13.76 & & 503 & 543 & \\
\hline & E2 & & & & & 15.85 & 16.31 & $-4.94 \%$ & 503 & 503 & $7.55 \%$ \\
\hline & & & & & & 15.85 & 15.13 & & 503 & 577 & \\
\hline & ISO-800 & 25.2502 & 25.2838 & $0.133 \%$ & $N / C$ & 14.75 & 19.22 & & 632 & 557 & \\
\hline & $F 2$ & & & & & 14.75 & 19.74 & $32.43 \%$ & 632 & 591 & $-8.54 \%$ \\
\hline & & & & & & 14.75 & 19.64 & & 632 & 586 & \\
\hline
\end{tabular}




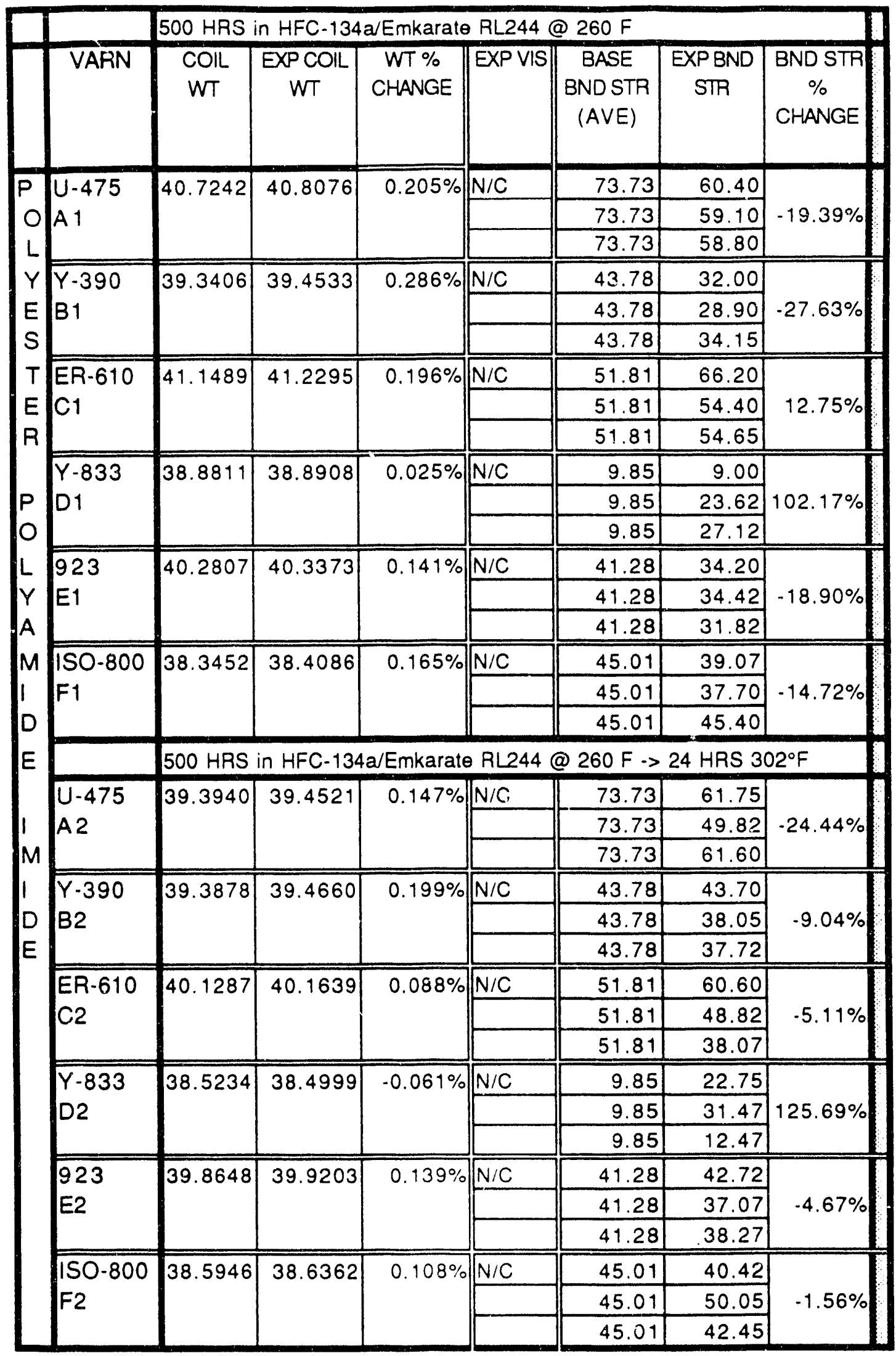




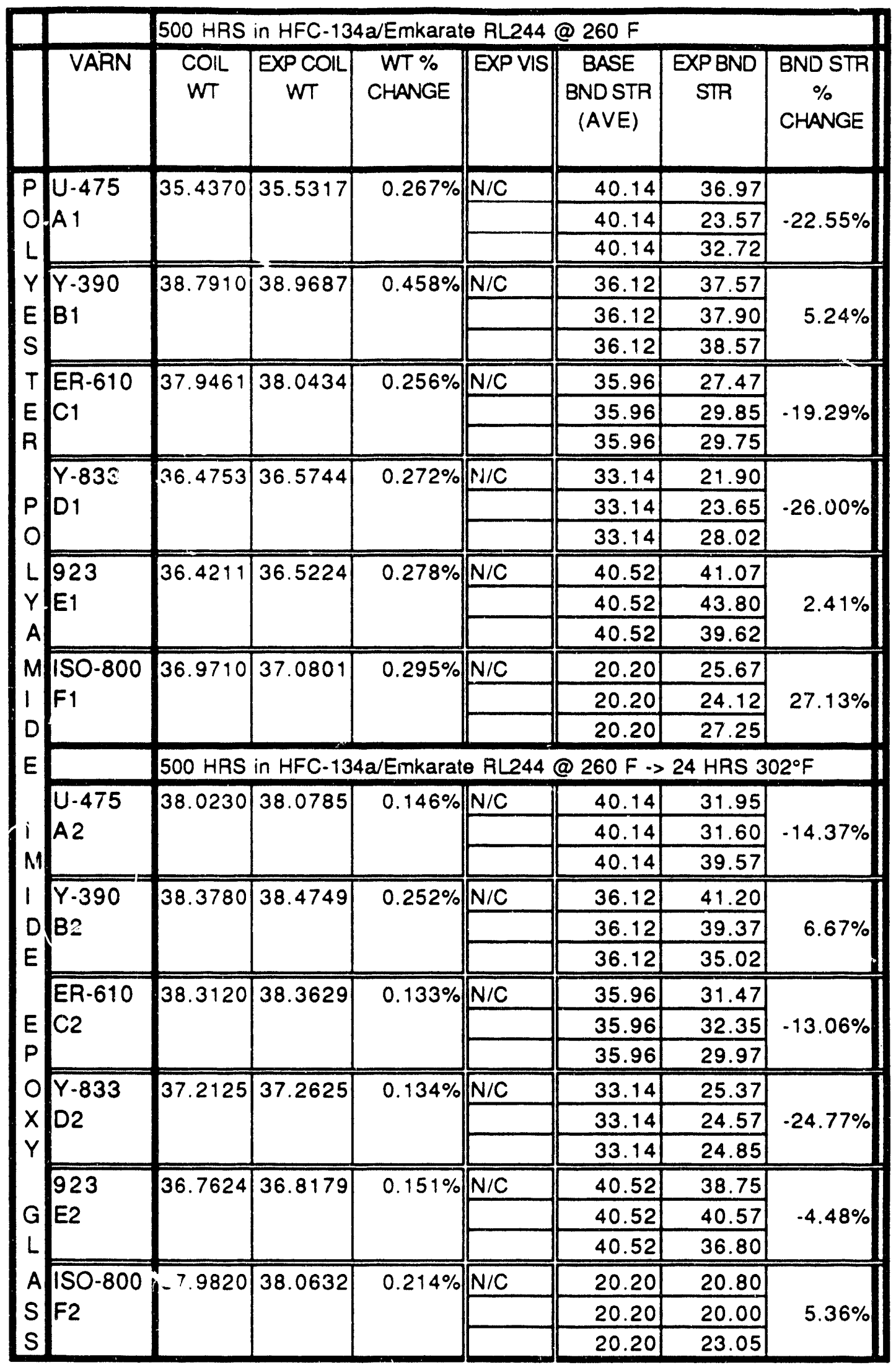




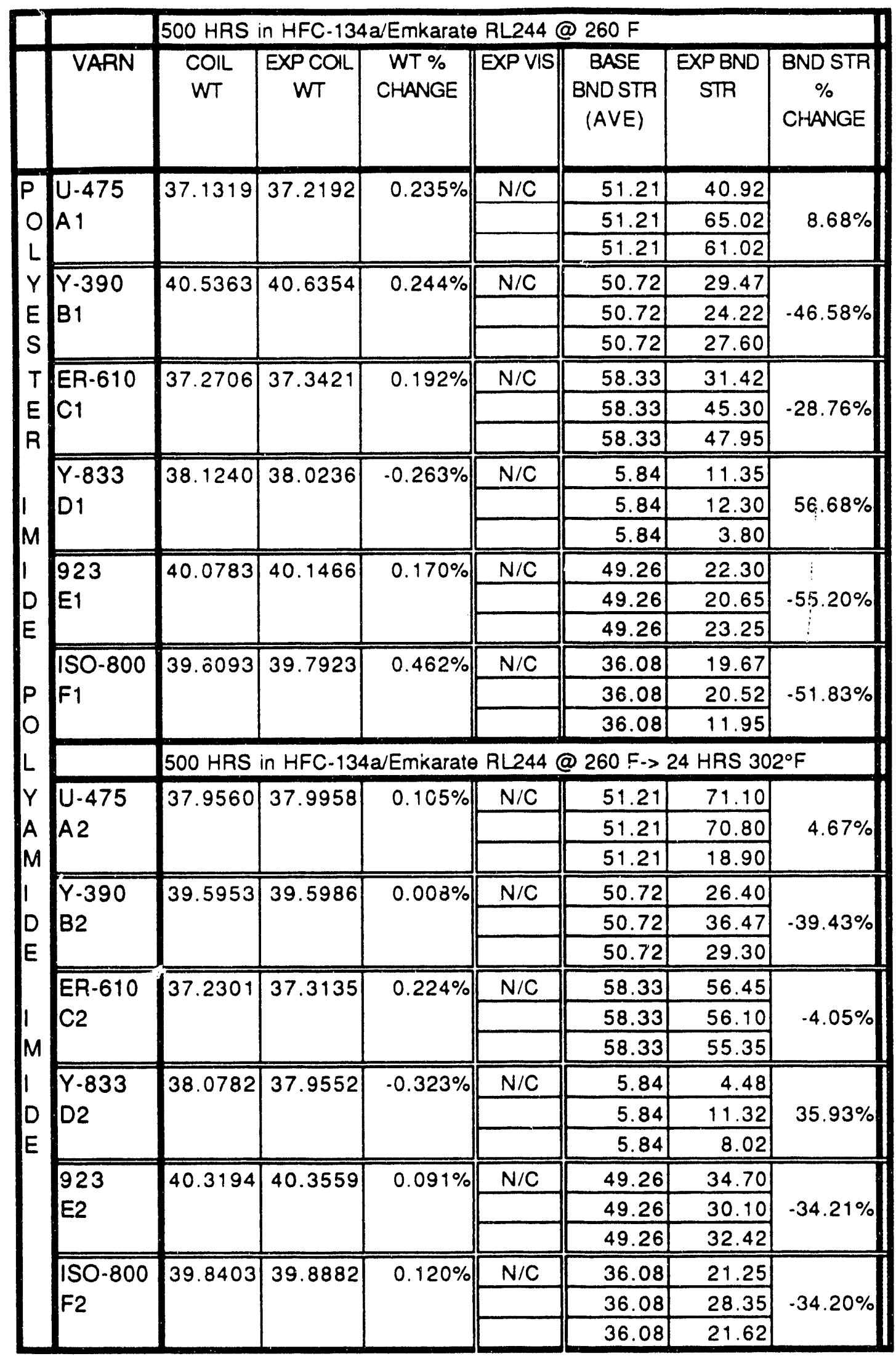


SINGLE MAG WIRE WITHOUT VARNISH.HFC-134a/ICI Emkarate RL244@260 $\mathrm{F}\left(127^{\circ} \mathrm{C}\right)$ )

7

\begin{tabular}{|c|c|c|c|c|c|}
\hline \multicolumn{6}{|c|}{$500 \mathrm{HRS}$ in HFC-134a/Emkarate RL244 oil @ 260 $\mathrm{F}\left(127^{\circ} \mathrm{C}\right)$} \\
\hline $\mathbf{D}$ & WT & EXPWT & $\begin{array}{c}\text { WT \% } \\
\text { CHANGE }\end{array}$ & EXP VIS & EXPFLEX \\
\hline \multirow[t]{2}{*}{ A1 } & \multirow[t]{2}{*}{3.939} & \multirow[t]{2}{*}{3.9492} & \multirow{2}{*}{$0.259 \%$} & $\mathrm{~N} / \mathrm{C}$ & \\
\hline & & & & & YES \\
\hline \multirow[t]{2}{*}{ B1 } & \multirow[t]{2}{*}{4.4094} & \multirow[t]{2}{*}{4.4273} & \multirow[t]{2}{*}{$0.406 \%$} & $\mathrm{~N} / \mathrm{C}$ & \\
\hline & & & & & YES \\
\hline & & & \multirow{3}{*}{$0.194 \%$} & & \\
\hline \multirow[t]{2}{*}{$\mathrm{C1}$} & \multirow[t]{2}{*}{3.9177} & \multirow[t]{2}{*}{3.9253} & & $\mathrm{~N} / \mathrm{C}$ & \\
\hline & & & & & YES \\
\hline \multicolumn{6}{|c|}{$500 \mathrm{HRS}$ in HFC-134a/Emkarate RL244-> $302^{\circ} \mathrm{F}$ for $24 \mathrm{HRS}$} \\
\hline \multirow[t]{2}{*}{ A2 } & \multirow[t]{2}{*}{3.9319} & \multirow[t]{2}{*}{3.9458} & \multirow[t]{2}{*}{$0.354 \%$} & $\mathrm{~N} / \mathrm{C}$ & \\
\hline & & & & & YES \\
\hline \multirow[t]{2}{*}{82} & \multirow[t]{2}{*}{4.3524} & \multirow[t]{2}{*}{4.3735} & \multirow[t]{2}{*}{$0.485 \%$} & $\mathrm{~N} / \mathrm{C}$ & \\
\hline & & & & & YES \\
\hline \multirow[t]{2}{*}{$\mathrm{C2}$} & \multirow[t]{2}{*}{3.9302} & \multirow[t]{2}{*}{3.9426} & \multirow{2}{*}{$0.316 \%$} & $\mathrm{~N} / \mathrm{C}$ & \\
\hline & & & & & YES \\
\hline
\end{tabular}




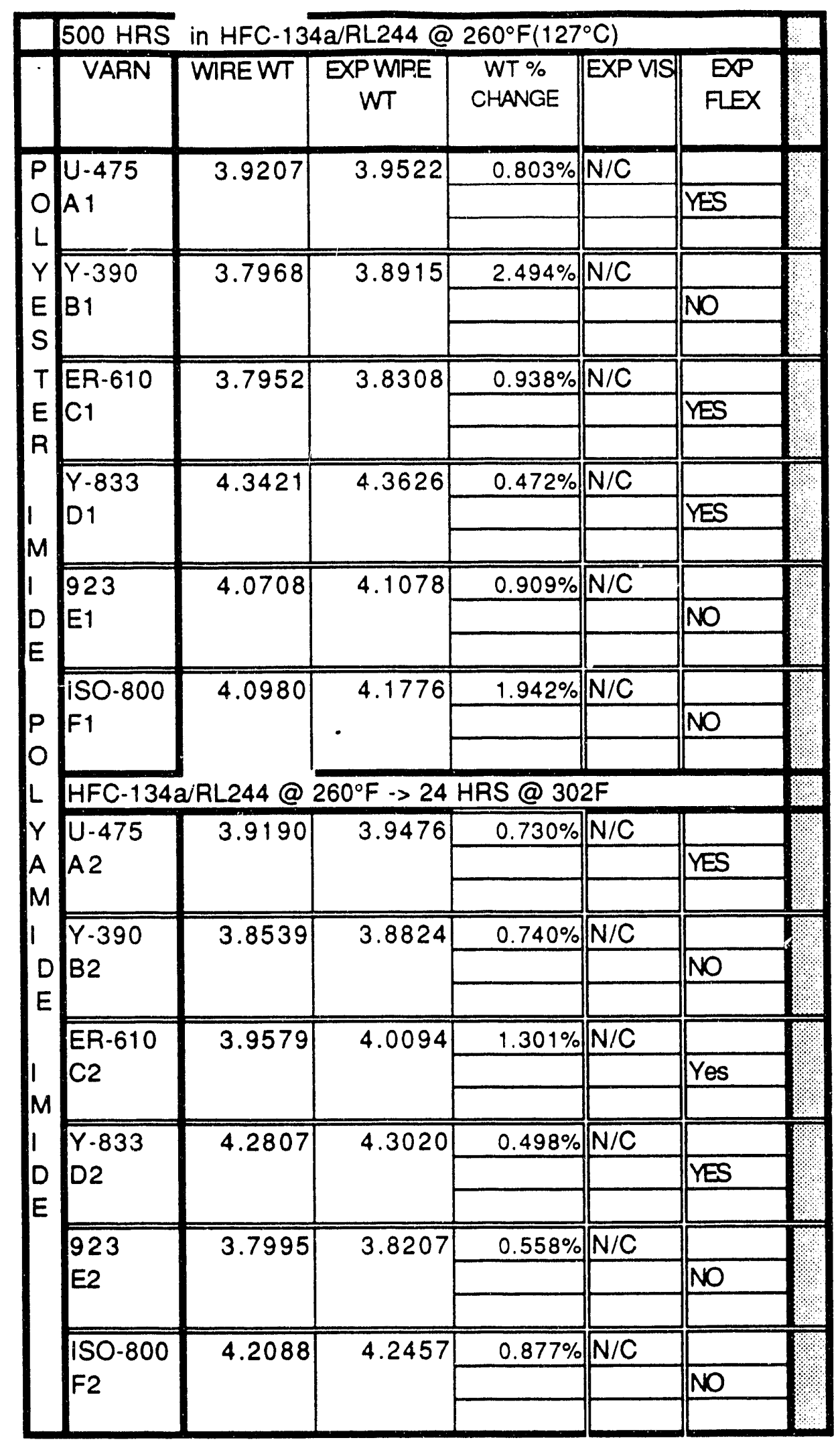




\section{ᄀ)}

\begin{tabular}{|c|c|c|c|c|c|c|}
\hline & & $500 \mathrm{HRS}$ & in $\mathrm{HFC}-13$ & a/Emkara & RL24 & @ 2 $260^{\circ}$ \\
\hline & VARN & WREWT & $\begin{array}{c}\text { EXPWIRE } \\
W T\end{array}$ & $\begin{array}{l}\text { WT \% } \\
\text { CHANGE }\end{array}$ & EXPVIS & $\begin{array}{l}\text { EXP } \\
\text { FEX }\end{array}$ \\
\hline $\mathbf{P}$ & $U .475$ & 4.3621 & 4.4156 & $1.226 \%$ & $\mathrm{~N} / \mathrm{C}$ & \\
\hline 0 & A 1 & & & & & NO \\
\hline Y & & 46095 & 46538 & $0961 \%$ & $N / C$ & \\
\hline$E$ & & & & & & NO \\
\hline $\mathrm{s}$ & & & & & & \\
\hline$T$ & ER-610 & 3.8093 & 3.8671 & $1.517 \%$ & $\mathrm{~N} / \mathrm{C}$ & \\
\hline$E$ & $c_{1}$ & & & & & YES \\
\hline & & 43010 & 43521 & $1167 \%$ & N/C & \\
\hline $\mathrm{P}$ & D1 & & & & & YES \\
\hline 0 & & & & & & \\
\hline $\mathrm{L}$ & 923 & 4.0331 & 4.0775 & $1.101 \%$ & $\mathrm{~N} / \mathrm{C}$ & \\
\hline$Y$ & E1 & & & & & NO \\
\hline${ }_{M}^{A}$ & $150-800$ & 37104 & 37738 & 17000 & Nur & \\
\hline 1 & & & & & & NO \\
\hline$D$ & & & & & & \\
\hline$E$ & & HFC-13 & a/Emkarate & RI244@ & $260^{\circ} \mathrm{F}(1$ & $\left.27^{\circ} \mathrm{C}\right)$ \\
\hline & $\mathrm{U}-475$ & 4.4611 & 4.4911 & $0.672 \%$ & $\mathrm{~N} / \mathrm{C}$ & \\
\hline 1 & A2 & & & & & NO \\
\hline$M$ & & & & & & \\
\hline 1 & $Y-390$ & 4.1843 & 4.2230 & $0.925 \%$ & $\mathrm{~N} / \mathrm{C}$ & \\
\hline D & B2 & & & & & NO \\
\hline$E$ & & & & & & \\
\hline & ER-610 & 3.9850 & 4.0357 & $1.272 \%$ & $\mathrm{~N} / \mathrm{C}$ & \\
\hline E & & & & & & YES \\
\hline & & & & & & \\
\hline 0 & $Y-833$ & 4.2665 & 4.2842 & $0.415 \%$ & $N / C$ & \\
\hline$x$ & D2 & & & & & YES \\
\hline$r$ & & & & & & \\
\hline & 923 & 4.1721 & 4.1972 & $0.602 \%$ & $\mathrm{~N} / \mathrm{C}$ & \\
\hline G & E2 & & & & & NO \\
\hline A & ISO-800 & 3.5970 & 3.6240 & $0.751 \%$ & $N / C$ & \\
\hline s & & & & & & NO \\
\hline & & & & & & \\
\hline
\end{tabular}




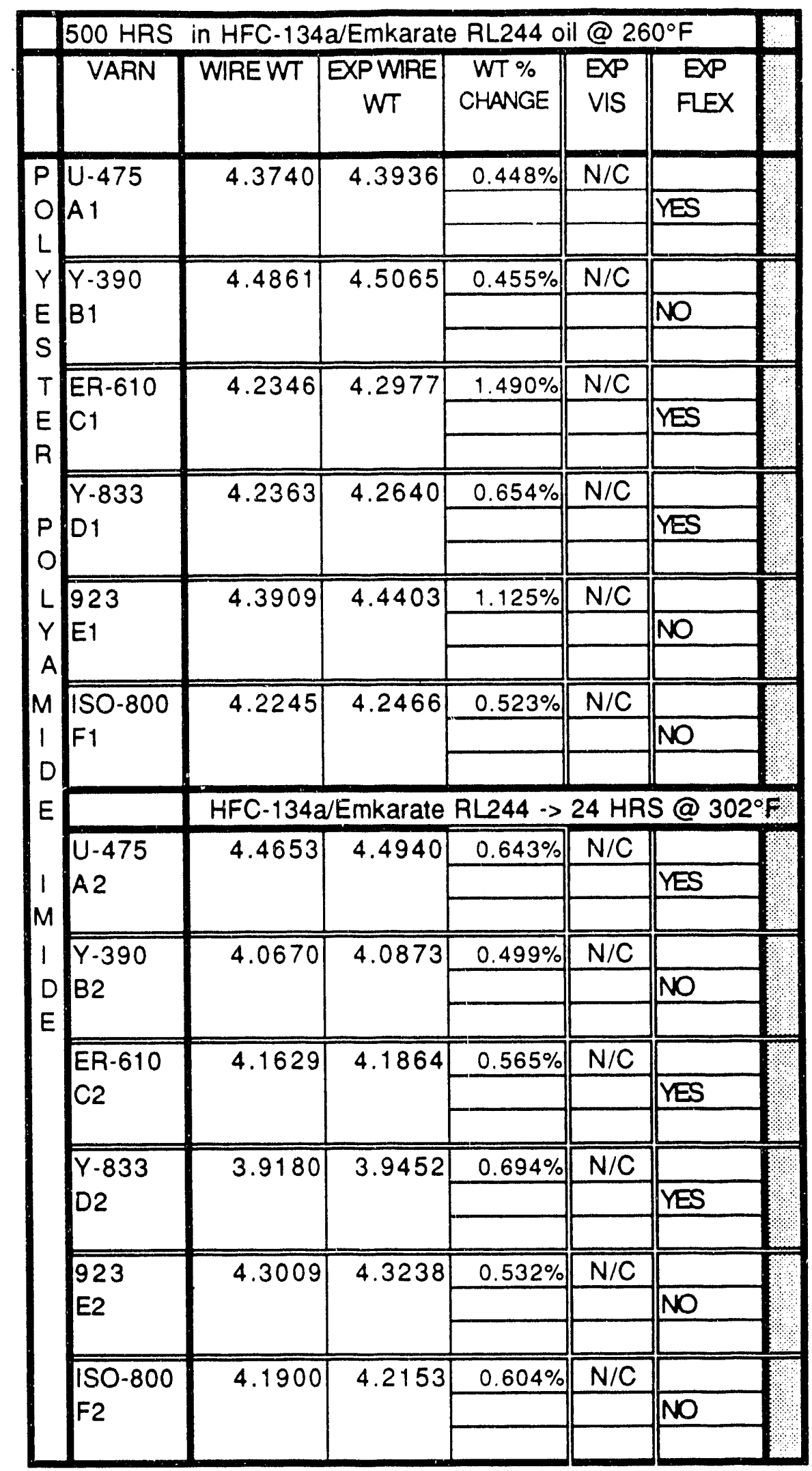




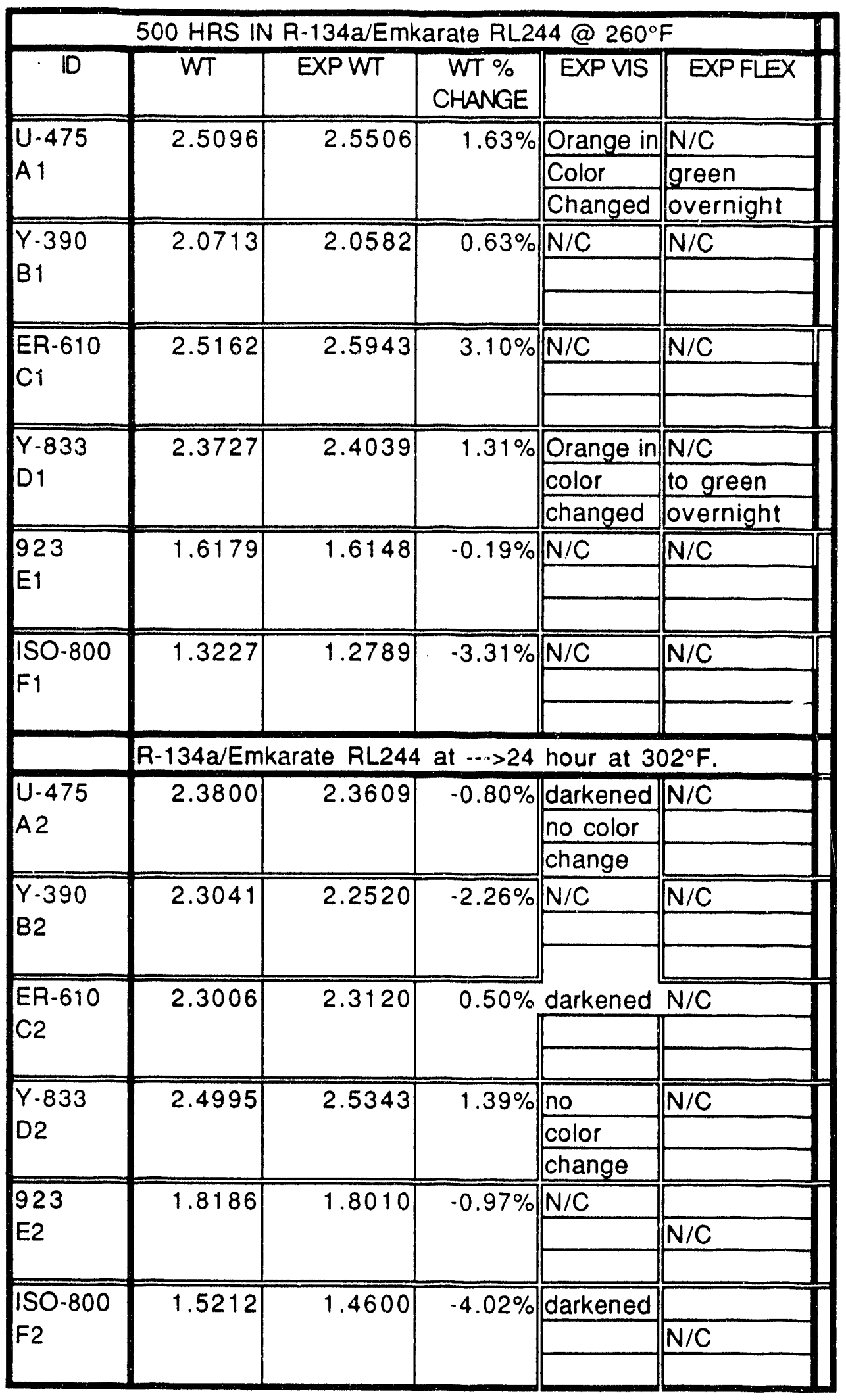




\begin{tabular}{|c|c|c|c|c|c|c|c|c|c|}
\hline & $500 \mathrm{HR}$ & in $\mathrm{HFC}$ & $4 \mathrm{a} / \mathrm{Em}$ & te RL & $4 @ 260$ & $O F$ & & & \\
\hline 10 & $W T$ & EXPWT & $\begin{array}{c}\text { WT \% } \\
\text { CHANGE }\end{array}$ & $\begin{array}{l}\text { SAMPLE| } \\
\text { WIDTH }\end{array}$ & $\begin{array}{l}\text { BREAK } \\
\text { LOAD }\end{array}$ & $\begin{array}{c}\text { SAMPLE } \\
\text { THICKNESS } \\
\text { Mils }\end{array}$ & $\begin{array}{c}\text { TENSILE } \\
\text { STR } \\
\text { BASE }\end{array}$ & $\begin{array}{l}\text { TENSILE } \\
\text { STREXP }\end{array}$ & $\begin{array}{c}\% \text { CHANGE } \\
\text { TENSILE }\end{array}$ \\
\hline NO/MY/NO & 7.3752 & 7.8988 & $7.10 \%$ & 0.433 & 152.80 & 21 & 17.4 & 16.80 & \\
\hline A1 & & & & 0.445 & 155.80 & 21 & 17.4 & 16.67 & $-7.31 \%$ \\
\hline & & & & 0.413 & 129.30 & 21 & 17.4 & 14.91 & \\
\hline$D A / M Y / D A$ & 7.5121 & 7.7963 & $3.78 \%$ & 0.368 & \begin{tabular}{|l|}
88.80 \\
\end{tabular} & 21 & 13.7 & 11.49 & \\
\hline B1 & & & & 0.401 & 105.40 & 21 & 13.7 & 12.52 & $-10.34 \%$ \\
\hline & & & & 0.386 & 104.10 & 21 & 13.7 & 12.84 & \\
\hline MYLAR MO & 3.9836 & 4.0443 & $1.52 \%$ & 0.449 & 76.60 & 10 & 21.7 & 17.06 & \\
\hline C1 & & & & 0.423 & 71.90 & 10 & 21.7 & 17.00 & $-20.66 \%$ \\
\hline & & & & 0.498 & 87.60 & 10 & 21.7 & 17.59 & \\
\hline NO 410 & 3.7038 & 4.1585 & $12.28 \%$ & 0.446 & 81.45 & 10 & 18.7 & 18.26 & \\
\hline D1 & & & & 0.468 & 80.85 & 10 & 18.7 & 17.28 & $-4.59 \%$ \\
\hline & & & & 0.484 & 87.05 & 10 & 18.7 & 17.99 & \\
\hline NO MI 418 & 3.6529 & 4.4321 & $21.33 \%$ & 0.503 & 28.10 & 10 & 7.5 & 5.59 & \\
\hline E1 & & & & 0.507 & 29.80 & 10 & 7.5 & 5.88 & $-23.76 \%$ \\
\hline & & & & 0.500 & 28.45 & 10 & 7.5 & 5.69 & \\
\hline MEL 228 & 4.2275 & 4.3047 & $1.83 \%$ & 0.458 & 73.50 & 10 & 21.7 & 16.05 & \\
\hline $\mathrm{F} 1$ & & & & 0.446 & 72.15 & 10 & 21.7 & 16.18 & $-26.15 \%$ \\
\hline & & & & 0.528 & 83.70 & 10 & 21.7 & 15.85 & \\
\hline & $500 \mathrm{HRS}$ & $\mathrm{S}$ in HFC- & 134a/Emka & arate RL2 & 141.90 & & & & \\
\hline NO/MY/NO & 7.2072 & 7.5181 & $4.31 \%$ & 0.418 & 141.90 & 21 & 17.4 & 16.17 & \\
\hline $\mathrm{A} 2$ & & & & 0.420 & 146.40 & 21 & 17.4 & 16.60 & $-5.55 \%$ \\
\hline & & & & 0.397 & 137.90 & 21 & 17.4 & 16.54 & \\
\hline$D A / M Y / D A$ & 7.3811 & $7.7396 \mid$ & $4.86 \%$ & 0.417 & 116.90 & 21 & 13.7 & 13.35 & \\
\hline B2 & & & & 0.394 & 108.50 & 21 & 13.7 & 13.11 & $-4.84 \%$ \\
\hline & & & & 0.375 & 99.60 & 21 & 13.7 & 12.65 & \\
\hline MYLAR MO & 3.9312 & 3.9587 & $0.70 \%$ & 0.446 & 69.40 & 10 & 21.7 & 15.56 & \\
\hline $\mathrm{C} 2$ & & & & 0.436 & 74.45 & 10 & 21.7 & 17.08 & $-23.36 \%$ \\
\hline & & & & 0.439 & 75.75 & 10 & 21.7 & 17.26 & \\
\hline NOMEX 410 & 3.6226 & 3.9332 & $8.57 \%$ & 0.488 & 93.15 & 10 & 18.7 & 19.09 & \\
\hline D2 & & & & 0.437 & 86.86 & 10 & 18.7 & 19.88 & $3.83 \%$ \\
\hline & & & & 0.511 & 98.55 & 10 & 18.7 & 1929 & \\
\hline $\mathrm{NO} / \mathrm{MI} 418$ & 3.8304 & 4.7518 & $24.05 \%$ & 0.480 & 27.30 & 10 & 7.5 & 5.69 & \\
\hline E2 & & & & 0.510 & 31.51 & 10 & 7.5 & 6.18 & $-20.69 \%$ \\
\hline & & & & 0.495 & 29.60 & 10 & 7.5 & 5.98 & \\
\hline MEL 228 & 4.1219 & 4.1445 & $0.55 \%$ & 0.455 & 76.20 & 10 & 21.7 & 16.75 & \\
\hline F2 & & & & 0.482 & 79.50 & 10 & 21.7 & 16.49 & $-23.77 \%$ \\
\hline & & & & 0.382 & 62.60 & 10 & 21.7 & 16.39 & \\
\hline
\end{tabular}




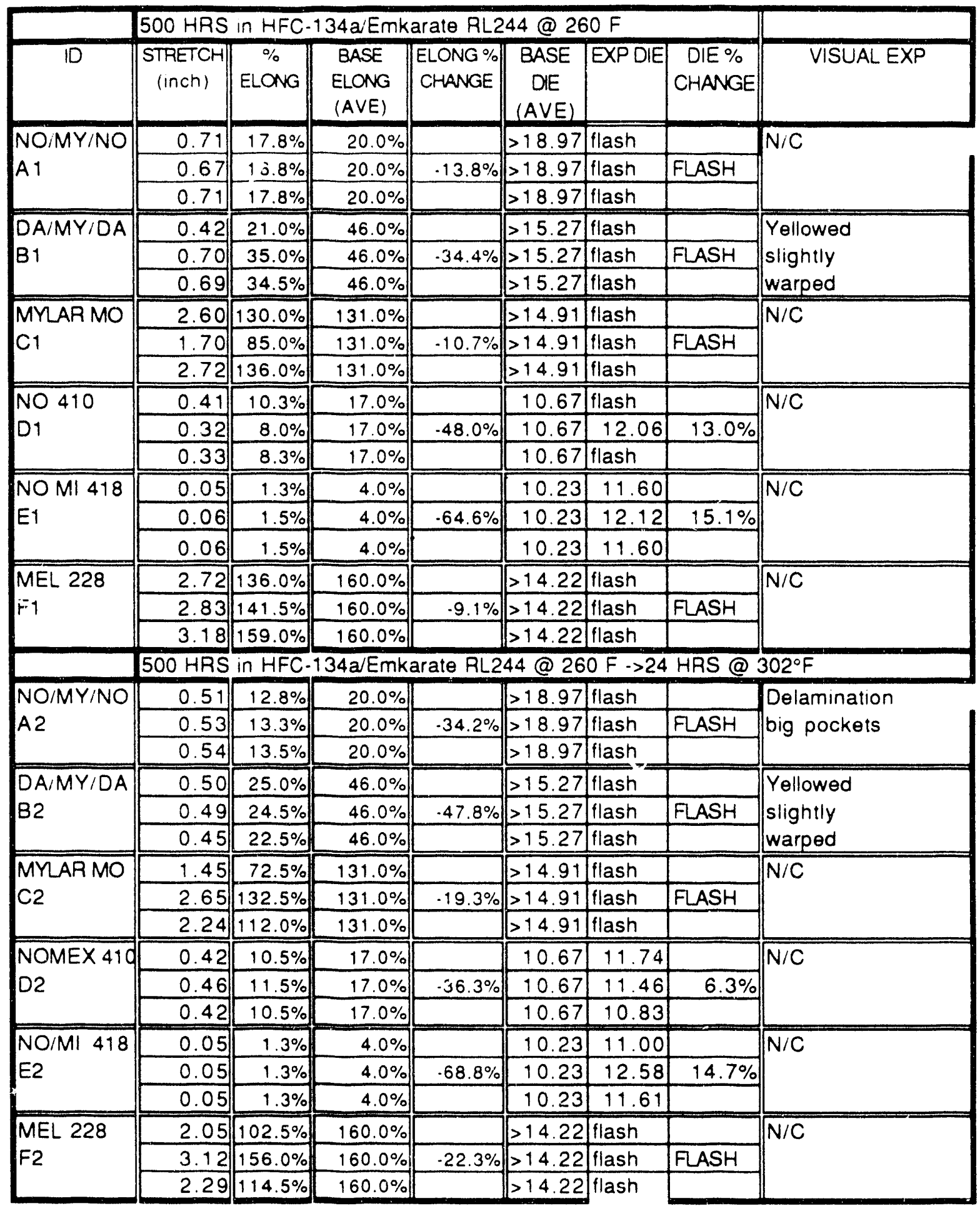


SLEEVING-.HFC-134a/ICI Emkarate RL244@260 $\mathrm{F}\left(127^{\circ} \mathrm{C}\right)$

\begin{tabular}{|c|c|c|c|c|}
\hline & \multicolumn{4}{|c|}{$500 \mathrm{HRS}$ in HFC-134a/Emkarate RL244@260 $\mathrm{F}$} \\
\hline & WT & EXPWT & $\begin{array}{c}\% \\
\text { CHANGE }\end{array}$ & EXP VIS \\
\hline & 0.5470 & 0.6347 & $16.03 \%$ & $\mathrm{~N} / \mathrm{C}$ \\
\hline B1 & 0.4930 & 0.5149 & $4.44 \%$ & $\mathrm{~N} / \mathrm{C}$ \\
\hline$C_{1}$ & 0.3887 & 0.4132 & $6.30 \%$ & $\mathrm{~N} / \mathrm{C}$ \\
\hline & HFC-134a/E & karate RL & 44 oil @ ? & ${ }^{\circ} \mathrm{F}$ plus24 hrs @ 302. F \\
\hline A2 & 0.5170 & 0.5778 & $11.76 \%$ & $\mathrm{~N} / \mathrm{C}$ \\
\hline & 0.3720 & 0.3819 & $2.66 \%$ & $\mathrm{~N} / \mathrm{C}$ \\
\hline $\mathrm{C} 2$ & 0.3615 & 0.3728 & $3.13 \%$ & $\mathrm{~N} / \mathrm{C}$ \\
\hline NO/MY & & & & some pockets where mylar \\
\hline & & & & pulled away. \\
\hline
\end{tabular}




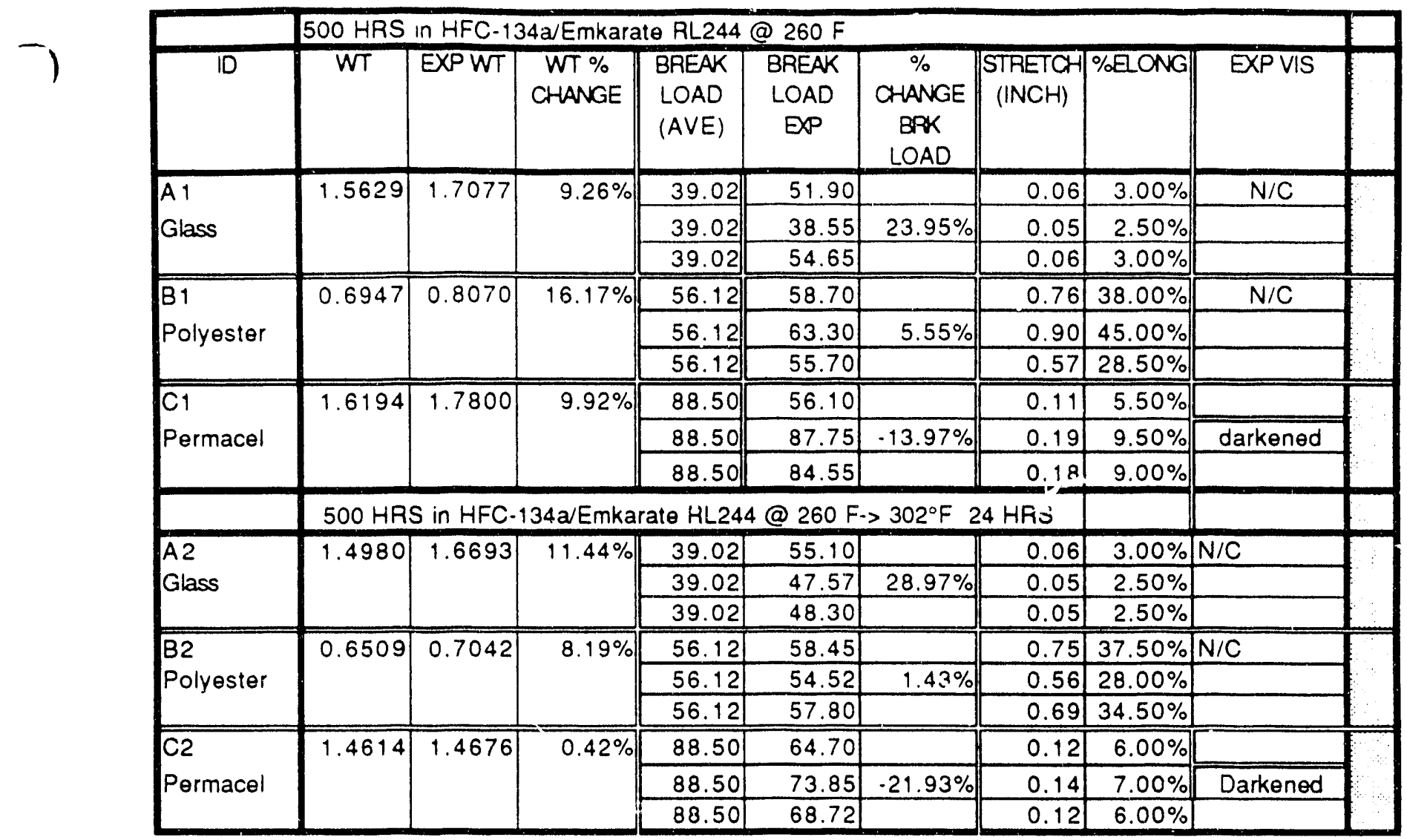


TIE CORD--HFC-134a/Emkarate RL244 oil @ $260^{\circ} \mathrm{F}\left(127^{\circ} \mathrm{C}\right)$

\begin{tabular}{|c|c|c|c|c|c|c|c|c|c|}
\hline \multicolumn{10}{|c|}{500 HRS IN R-134a/Emkarate RL244@260 $@$} \\
\hline ID & $W T$ & EXPWT & $\begin{array}{c}\text { WT \% } \\
\text { CHANGE }\end{array}$ & \begin{tabular}{|l|}
$E X P$ \\
VIS
\end{tabular} & $\begin{array}{l}\text { BREAK } \\
\text { LOAD } \\
\text { (AVE) }\end{array}$ & $\begin{array}{l}\text { BREAK } \\
\text { LOAD } \\
\text { EXP } \\
\text { (ave) }\end{array}$ & $\begin{array}{c}\% \\
\text { CHANGE } \\
\text { BRK } \\
\text { LOAD }\end{array}$ & $\begin{array}{c}\text { STRETCH } \\
\text { (Inch) }\end{array}$ & ELONG \\
\hline \multirow[t]{3}{*}{ A 1} & \multirow[t]{3}{*}{0.2803} & \multirow[t]{3}{*}{0.3297} & \multirow[t]{3}{*}{$17.62 \%$} & $\mathrm{~N} / \mathrm{C}$ & 28.36 & 36.25 & & 0.47 & $23.5 \%$ \\
\hline & & & & & 28.36 & 32.25 & $20.12 \%$ & 0.42 & $21.0 \%$ \\
\hline & & & & & 28.36 & 33.70 & & 0.44 & $22.0 \%$ \\
\hline & \multicolumn{9}{|c|}{500 HRS IN R-134a/Emkarate RL244 -> 24 HRS @ 302 $\mathrm{F}$} \\
\hline \multirow[t]{3}{*}{ A2 } & \multirow[t]{3}{*}{0.2708} & \multirow[t]{3}{*}{0.2927} & \multirow[t]{3}{*}{$8.087 \%$} & $\mathrm{~N} / \mathrm{C}$ & 28.36 & 31.07 & & 0.40 & $20.0 \%$ \\
\hline & & & & & 28.36 & 32.95 & $17.35 \%$ & 0.42 & $21.0 \%$ \\
\hline & & & & & 28.36 & 35.82 & & 0.44 & $22.0 \%$ \\
\hline
\end{tabular}




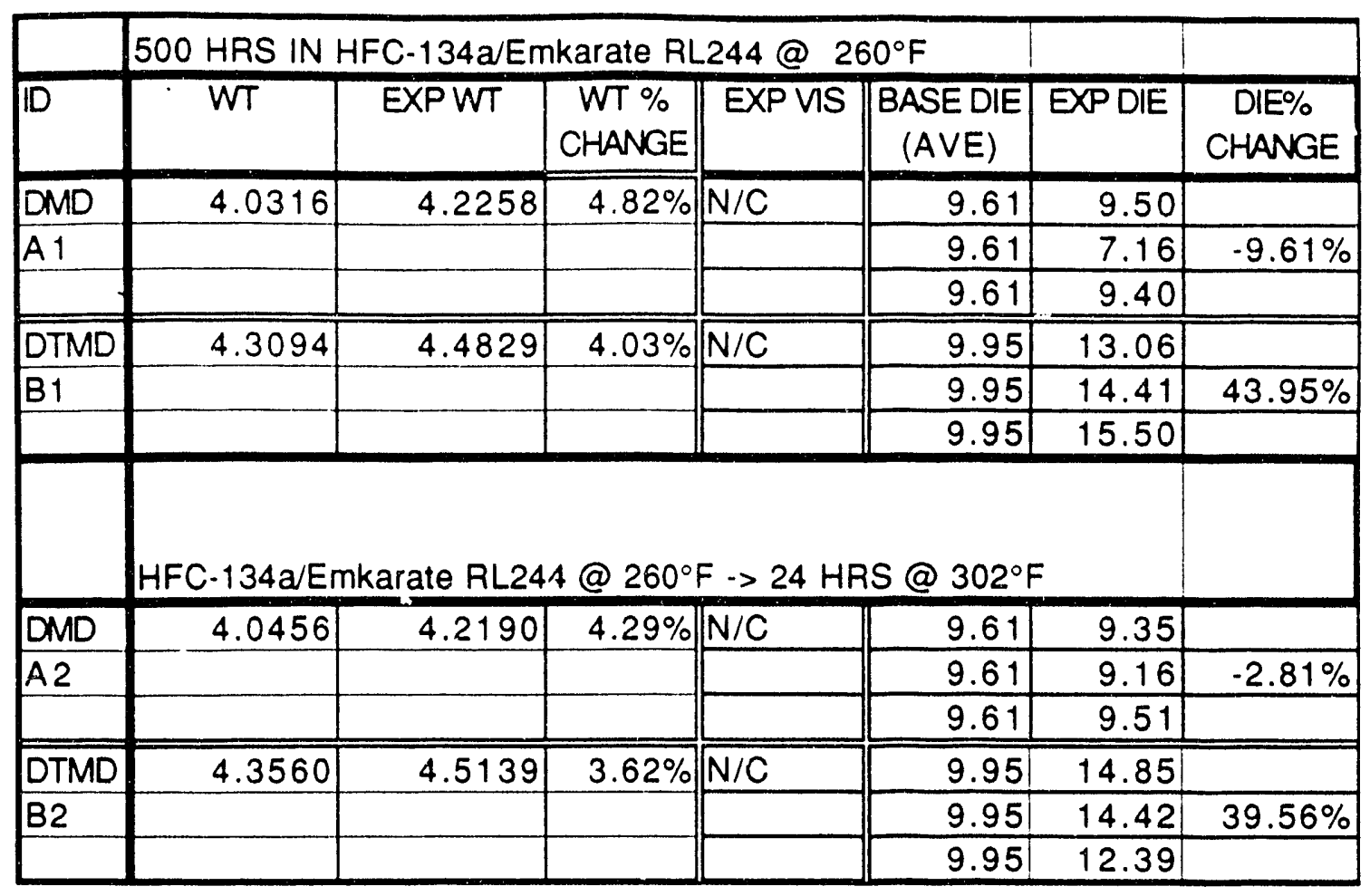




\section{Appendix G}

Experimental Data for HFC-134a/HenkelEmery 2927 ISO-32 Exposure at $126^{\circ} \mathrm{C}\left(260^{\circ} \mathrm{F}\right)$ 


\begin{tabular}{|c|c|c|c|c|c|c|c|c|c|c|}
\hline & 500 hours & HFC-13 & $\mathrm{V} 2927 \mathrm{ISC}$ & 0-32 oil & $1260^{\circ} \mathrm{F}(1$ & $\left.{ }^{\circ} \mathrm{C}\right)$ & & & & \\
\hline ID & WT & EXPWT & $\begin{array}{c}\text { WT \% } \\
\text { CHANGE }\end{array}$ & EXP VS & $\begin{array}{c}\text { BASE BRN } \\
\text { OUT } \\
\text { (AVE) }\end{array}$ & $\begin{array}{c}\text { EXPBRN } \\
\text { OUT }\end{array}$ & $\begin{array}{c}\text { BRNOUT } \\
\% \\
\text { CHANGE }\end{array}$ & $\begin{array}{c}\text { BASE DIE } \\
\text { (AVE) }\end{array}$ & EXP DIE & $\begin{array}{c}\text { DIE \% } \\
\text { CHANGE }\end{array}$ \\
\hline A1 & 25.5908 & 25.6092 & $0.072 \%$ & $N / C$ & 576 & 426 & & 15.80 & 15.09 & \\
\hline & & & & & 576 & 466 & $-16.7 \%$ & 15.80 & 17.59 & $3.0 \%$ \\
\hline & & & & & 576 & 547 & & 15.80 & 16.16 & \\
\hline 81 & 29.3638 & 29.3872 & $0.080 \%$ & $\mathrm{~N} / \mathrm{C}$ & 736 & 731 & & 11.62 & 12.98 & \\
\hline & & & & & 736 & 729 & $-6.8 \%$ & 11.62 & 13.36 & $12.1 \%$ \\
\hline & & & & & 736 & 597 & & 11.62 & 12.73 & \\
\hline $\mathrm{C} 1$ & 27.5387 & 27.5492 & $0.038 \%$ & $\mathrm{~N} / \mathrm{C}$ & 579 & 44 & & 16.58 & 16.90 & \\
\hline & & & & & 579 & 531 & $-22.9 \%$ & 16.58 & 12.67 & $-11.2 \%$ \\
\hline & & & & & 579 & 395 & & 16.58 & 14.59 & \\
\hline & 500 hours & HFC-134 & a/Emkarat & e RL244 & oil at $260^{\circ}$ & $\mathrm{F}\left(127^{\circ} \mathrm{C}\right)$ & plus 24 ho & Durs at 30 & $2^{\circ} \mathrm{F}\left(150^{\circ} \mathrm{C}\right.$ & \\
\hline$A 2$ & 25.3742 & 25.3711 & $-0.012 \%$ & $\mathrm{~N} / \mathrm{C}$ & 576 & 517 & & 15.80 & 13.64 & \\
\hline & & & & & 576 & 499 & $-11.9 \%$ & 15.80 & 15.89 & $-6.9 \%$ \\
\hline & & & & & 576 & 506 & & 15.80 & 14.60 & \\
\hline$B 2$ & 29.0361 & 29.0392 & $0.011 \%$ & $\mathrm{~N} / \mathrm{C}$ & 736 & 735 & & 11.62 & 11.16 & \\
\hline & & & & & 736 & 731 & $-0.5 \%$ & 11.62 & 11.42 & $-1.1 \%$ \\
\hline & & & & & 736 & 732 & & 11.62 & 11.90 & \\
\hline $\mathrm{C2}$ & 27.2952 & 27.2883 & $-0.023 \%$ & $\mathrm{~N} / \mathrm{C}$ & 579 & 441 & & 16.58 & 13.04 & \\
\hline & & & & & 579 & 453 & $-16.5 \%$ & 16.58 & 17.04 & $-4.5 \%$ \\
\hline & & & & & 579 & 557 & & 16.58 & 17.41 & \\
\hline
\end{tabular}


TWISTED PAIRSMIRE AWITH VARNISH-HFC-134a/Emery 2927 Iso-32 oil @260 ${ }^{\circ} \mathrm{F}\left(127^{\circ} \mathrm{C}\right)$

\begin{tabular}{|c|c|c|c|c|c|c|c|c|c|c|c|}
\hline & & 500 hours & in $\mathrm{HFC}-1$ & 34/Emery & 2927 & Iso-32 oi & @260 & $\left(127^{\circ} \mathrm{C}\right)$ & & & \\
\hline & VARN & $\begin{array}{l}\text { TWSTED } \\
\text { PAIR.WT }\end{array}$ & $\begin{array}{l}\text { Exp Pair } \\
\text { weight }\end{array}$ & $\begin{array}{c}\text { WT \% } \\
\text { CHANGE }\end{array}$ & $\begin{array}{l}\text { EXP } \\
\text { VIS }\end{array}$ & BASE DIE & EXP DIE & $\begin{array}{c}\text { DIE \% } \\
\text { CHANGE }\end{array}$ & $\begin{array}{l}\text { BASE } \\
\text { BURN } \\
\text { OUT }\end{array}$ & $\begin{array}{l}\text { EXP } \\
\text { BUPN } \\
\text { OUT }\end{array}$ & $\begin{array}{c}\text { BRNOUT } \\
\% \\
\text { CHANGE }\end{array}$ \\
\hline$P$ & $U-475$ & 22.7747 & 22.8062 & $0.138 \%$ & $\mathrm{~N} / \mathrm{C}$ & 16.24 & 15.50 & & 430 & 529 & \\
\hline 0 & A1 & & & & & 16.24 & 12.52 & $-29.56 \%$ & 430 & 492 & $8.91 \%$ \\
\hline L & & & & & & 16.24 & 6.30 & & 430 & 384 & \\
\hline Y & $Y-390$ & 23.5661 & 23.6164 & $0.213 \%$ & $\mathrm{~N} / \mathrm{C}$ & 18.77 & 19.79 & & 510 & 526 & \\
\hline$E$ & B1 & & & & & 18.77 & cracked & $1.92 \%$ & 510 & 567 & $4.77 \%$ \\
\hline $\mathrm{S}$ & & & & & & 18.77 & 18.47 & & 510 & 510 & \\
\hline$T$ & EA-610 & 23.5019 & 23.5256 & $0.101 \%$ & $\mathrm{~N} / \mathrm{C}$ & 15.57 & 15.75 & & 442 & 391 & \\
\hline$E$ & & & & & & 15.57 & 11.61 & $-11.69 \%$ & 442 & 389 & $.8 .30 \%$ \\
\hline$R$ & & & & & & 15.57 & 13.89 & & 442 & 436 & \\
\hline & $Y-833$ & 23.9209 & 23.9165 & $-0.018 \%$ & $\mathrm{~N} / \mathrm{C}$ & 12.04 & 15.27 & & 578 & 472 & \\
\hline$P$ & D1 & & & & & 12.04 & 14.10 & $21.51 \%$ & 578 & 576 & $-9.34 \%$ \\
\hline 0 & & & & & & 12.04 & 14.52 & & 578 & cracked & \\
\hline$L$ & 923 & 22.6269 & 22.6753 & $0.214 \%$ & $\mathrm{~N} / \mathrm{C}$ & 16.76 & 19.06 & & 606 & 576 & \\
\hline Y & E1 & & & & & 16.76 & 18.71 & $14.16 \%$ & 606 & 583 & $.4 .07 \%$ \\
\hline A & & & & & & 16.76 & 19.63 & & 606 & 585 & \\
\hline$M$ & $150-800$ & 22.8593 & 22.8982 & $0.170 \%$ & $\mathrm{~N} / \mathrm{C}$ & 19.08 & 17.95 & & 580 & 529 & \\
\hline 1 & $\mathrm{~F} 1$ & & & & & 19.08 & 19.19 & $-3.37 \%$ & 580 & 410 & $-13.33 \%$ \\
\hline D & & & & & & 19.08 & 18.17 & & 580 & 569 & \\
\hline$E$ & & 500 hours & in HFC-1 & 34a/Emen & y 292 & 7 Iso-32o & il@260 & $\mathrm{F}\left(127^{\circ} \mathrm{C}\right)$ & olus $24 \mathrm{r}$ & hours@ & $302^{\circ} \mathrm{F}(1$ \\
\hline & U-475 & $22.930 ?$ & 22.9253 & $.0 .021 \%$ & $\mathrm{~N} / \mathrm{C}$ & 16.24 & 12.66 & & 430 & \begin{tabular}{|r|}
419 \\
\end{tabular} & \\
\hline 1 & A2 & & & & & 16.24 & 16.50 & $-12.87 \%$ & 430 & 376 & $-4.81 \%$ \\
\hline$M$ & & & & & & 16.24 & 13.29 & & 430 & 433 & \\
\hline 1 & $Y-390$ & 23.3326 & 23.3564 & $0.102 \%$ & $\mathrm{~N} / \mathrm{C}$ & 18.77 & 19.74 & & 510 & 555 & \\
\hline$D$ & $\mathrm{B2}$ & & & & & 18.77 & 10.26 & $-18.58 \%$ & 510 & 580 & $11.57 \%$ \\
\hline $\mathbf{E}$ & & & & & & 18.77 & 15.85 & & 510 & 572 & \\
\hline & ER-610 & 24.5069 & 24.5182 & $0.046 \%$ & $\mathrm{~N} / \mathrm{C}$ & 15.57 & 14.14 & & 442 & 274 & \\
\hline & C2 & & & & & 15.57 & 12.81 & $-16.53 \%$ & 442 & 314 & $-35.14 \%$ \\
\hline & & & & & & 15.57 & 12.04 & & 442 & 272 & \\
\hline & $Y-833$ & 22.6270 & 22.5196 & $-0.475 \%$ & $\mathrm{~N} / \mathrm{C}$ & 12.04 & 12.33 & & 578 & 444 & \\
\hline & $\mathrm{D} 2$ & & & & & 12.04 & 10.00 & $-3.68 \%$ & 578 & 427 & $-25.55 \%$ \\
\hline & & & & & & 12.04 & 12.46 & & 578 & 420 & \\
\hline & 923 & 22.7473 & 22.7749 & $0.121 \%$ & $\mathrm{~N} / \mathrm{C}$ & 16.76 & 13.08 & & 606 & 4419 & \\
\hline & E2 & & & & & 16.76 & 13.30 & $-10.18 \%$ & 606 & 584 & $.13 .70 \%$ \\
\hline & & & & & & 16.76 & $18.7 i$ & & 606 & 566 & \\
\hline & ISO-800 & 24.7682 & 24.7877 & $0.079 \%$ & $\mathrm{~N} / \mathrm{C}$ & 19.08 & 17.81 & & 580 & 459 & \\
\hline & $\mathrm{F} 2$ & & & & & $\frac{19.08}{1008}$ & $\frac{19.33}{15.23}$ & $-8.51 \%$ & 580 & 438 & $-14.02 \%$ \\
\hline & & & & & & 19.08 & 15.23 & & 580 & 599 & \\
\hline
\end{tabular}


TWISTED PAIRSMIRE BWITH VARNISH -HFC-134a/Emery 2927 Iso-32 oil @260 $\mathrm{F}\left(127^{\circ} \mathrm{C}\right)$

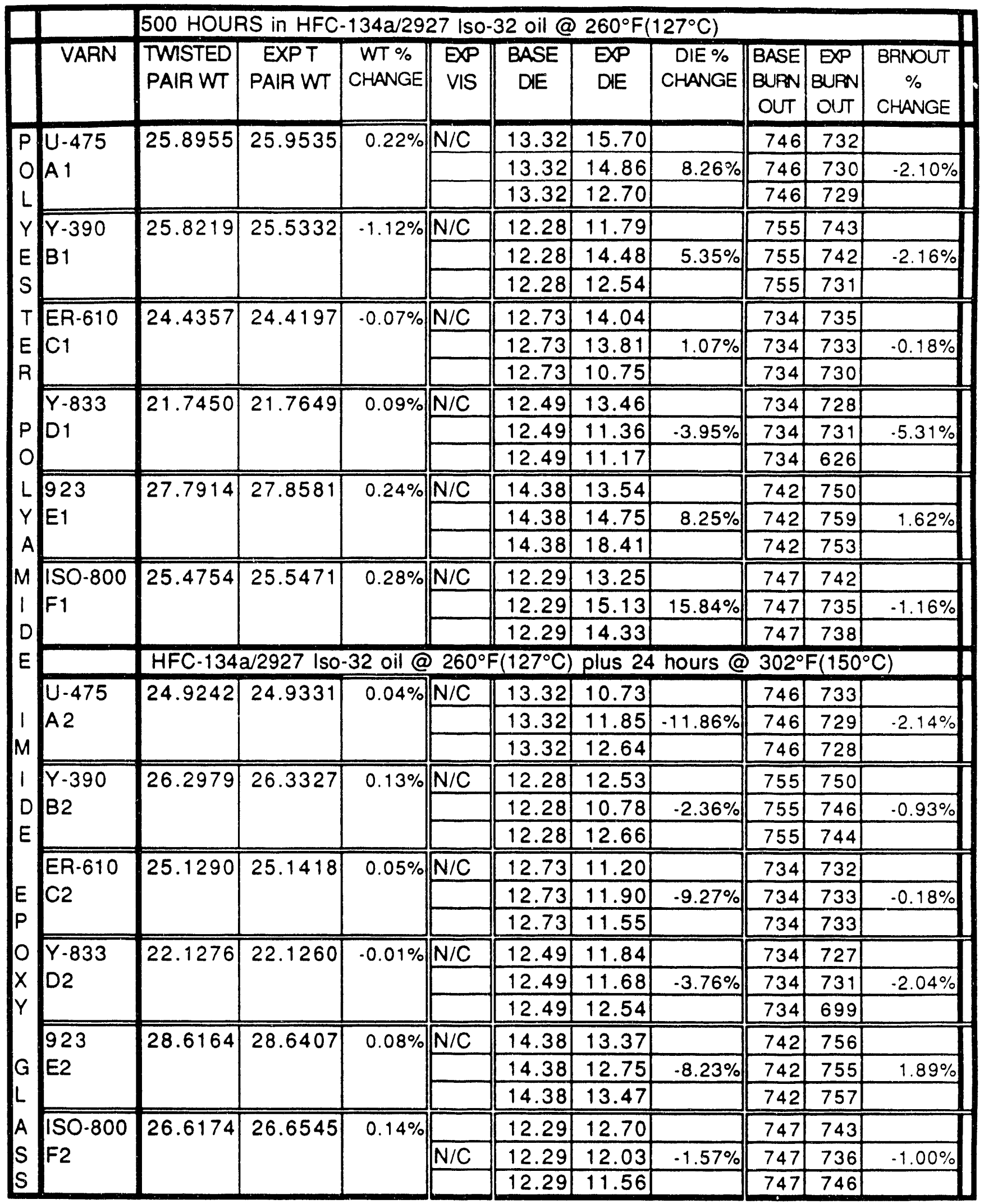


TWISI ED HAIRS/WIRE C/WITH VARNISH-HFC.134a/Emery 2927 Iso-32@ $260^{\circ} \mathrm{F}\left(127^{\circ} \mathrm{C}\right)$

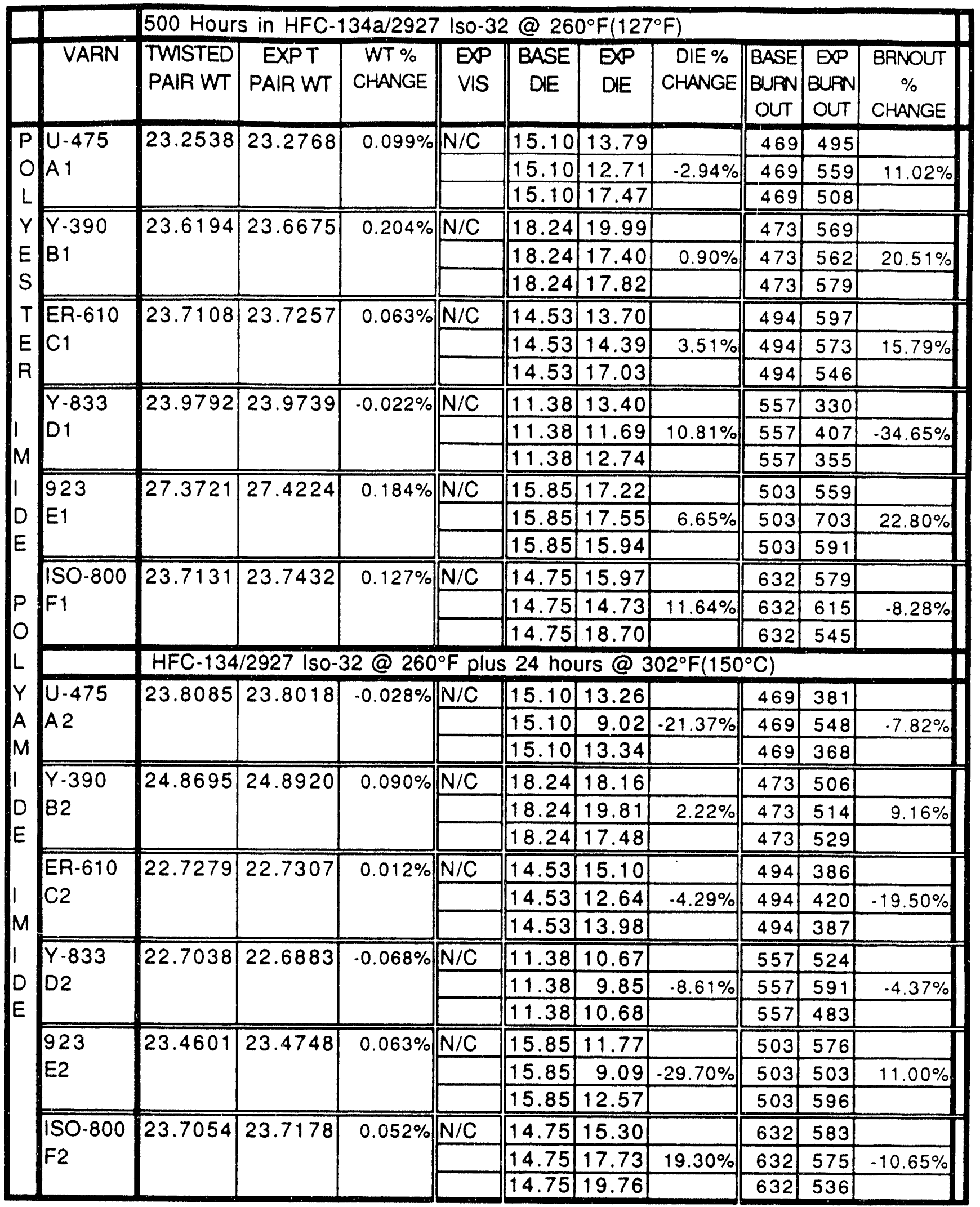


HELICAL COILS/WIRE A...HFC-134a/Emery 2927 Iso-32 @260F(127:C)

\begin{tabular}{|c|c|c|c|c|c|c|c|c|}
\hline & & $500 \mathrm{HRS}$ & in $\mathrm{HFC}$ & 7 & & & & \\
\hline & VARN & $\begin{array}{l}\text { COIL } \\
\text { WT }\end{array}$ & $\begin{array}{c}\text { EXP COIL } \\
W T\end{array}$ & $\begin{array}{c}\text { WT \% } \\
\text { CHANGE }\end{array}$ & EXP VIS & $\begin{array}{c}\text { BASE } \\
\text { BND STR } \\
\text { (AVE) }\end{array}$ & $\begin{array}{c}\text { EXP BND } \\
\text { STR }\end{array}$ & $\begin{array}{c}\text { BND STR } \\
\% \\
\text { CHANGE }\end{array}$ \\
\hline 0 & $\begin{array}{l}\text { U.475 } \\
A 1\end{array}$ & 40.0955 & 40.1711 & $0.189 \%$ & $\mathrm{~N} / \mathrm{C}$ & $\frac{73.73}{73.73}$ & \begin{tabular}{|r|}
$56 . .20$ \\
51.15 \\
66.80 \\
\end{tabular} & $-20.01 \%$ \\
\hline$E$ & $\begin{array}{l}Y-390 \\
B 1\end{array}$ & 38.2069 & 38.3221 & $0.302 \%$ & $\mathrm{~N} / \mathrm{C}$ & $\begin{array}{l}43.78 \\
43.78 \\
43.78 \\
\end{array}$ & \begin{tabular}{|l|}
33.30 \\
22.50 \\
30.65 \\
\end{tabular} & $.34 .18 \%$ \\
\hline$E$ & $\begin{array}{l}\text { ER-610 } \\
C_{1}\end{array}$ & 39.3824 & 39.4532 & $0.180 \%$ & iv/C & \begin{tabular}{|l|}
51.81 \\
51.81 \\
51.81 \\
\end{tabular} & \begin{tabular}{|l|}
55.20 \\
63.35 \\
54.05 \\
\end{tabular} & $11.05 \%$ \\
\hline$P$ & $\begin{array}{l}Y-833 \\
D 1\end{array}$ & 37.1025 & 37.1095 & $0.019 \%$ & $\mathrm{~N} / \mathrm{C}$ & \begin{tabular}{r|}
9.85 \\
9.85 \\
9.85 \\
\end{tabular} & \begin{tabular}{|l|}
14.00 \\
12.50 \\
12.12 \\
\end{tabular} & $30.69 \%$ \\
\hline $\begin{array}{l}\mathrm{L} \\
Y\end{array}$ & $\begin{array}{l}923 \\
\text { E1 }\end{array}$ & 40.2400 & 40.2981 & $0.144 \%$ & $\mathrm{~N} / \mathrm{C}$ & $\begin{array}{l}41.28 \\
41.28 \\
41.28 \\
\end{array}$ & \begin{tabular}{|l|}
32.40 \\
33.85 \\
33.37 \\
\end{tabular} & $.19 .56 \%$ \\
\hline$M$ & $\begin{array}{l}\text { ISO-800 } \\
\text { F1 }\end{array}$ & 37.1134 & 37.1642 & $0.137 \%$ & $\mathrm{~N} / \mathrm{C}$ & $\begin{array}{l}45.01 \\
45.01 \\
45.01\end{array}$ & \begin{tabular}{r|}
27.50 \\
24.47 \\
26.35 \\
\end{tabular} & $-42.27 \%$ \\
\hline E & & $500 \mathrm{HRS}$ & in HFC-134 & a/2927 Iso & -32@26 & $O F \rightarrow 24$ & ARS $302^{\circ} \mathrm{F}$ & \\
\hline M & $\begin{array}{l}U-475 \\
A 2\end{array}$ & 39.5086 & 39.5387 & $0.076 \%$ & $\mathrm{~N} / \mathrm{C}$ & $\begin{array}{l}73.73 \\
73.73 \\
73.73 \\
\end{array}$ & \begin{tabular}{|l|}
67.15 \\
52.07 \\
56.82 \\
\end{tabular} & $-26.16 \%$ \\
\hline D & $\begin{array}{l}Y-390 \\
B 2\end{array}$ & 39.0304 & 39.0729 & $0.109 \%$ & $\mathrm{~N} / \mathrm{C}$ & $\begin{array}{l}43.78 \\
43.78 \\
43.78 \\
\end{array}$ & \begin{tabular}{|l|}
38.82 \\
39.52 \\
32.90 \\
\end{tabular} & $-15.30 \%$ \\
\hline & $\begin{array}{l}\text { ER-610 } \\
\text { C2 }\end{array}$ & 38.1647 & 38.1874 & $0.059 \%$ & $\mathrm{~N} / \mathrm{C}$ & \begin{tabular}{|l|}
51.81 \\
51.81 \\
51.81 \\
\end{tabular} & \begin{tabular}{|l|}
43.42 \\
57.00 \\
39.47 \\
\end{tabular} & $-10.00 \%$ \\
\hline & $\begin{array}{l}Y-833 \\
D 2\end{array}$ & 37.8349 & 37.8058 & $-0.077 \%$ & $\mathrm{~N} / \mathrm{C}$ & $\begin{array}{r}9.85 \\
9.85 \\
9.85 \\
\end{array}$ & $\begin{array}{l}14.20 \\
50.65 \\
17.15 \\
\end{array}$ & $177.50 \%$ \\
\hline & $\begin{array}{l}923 \\
\text { E2 }\end{array}$ & 39.7964 & 39.8247 & $0.071 \%$ & $\mathrm{~N} / \mathrm{C}$ & \begin{tabular}{|l|}
41.28 \\
41.28 \\
41.28 \\
\end{tabular} & \begin{tabular}{|l|}
36.27 \\
38.37 \\
36.70 \\
\end{tabular} & $-10.09 \%$ \\
\hline & $\begin{array}{l}\text { ISO-800 } \\
\text { F2 }\end{array}$ & 36.9206 & 36.9512 & $0.083 \%$ & $\mathrm{~N} / \mathrm{C}$ & $\begin{array}{l}\frac{45.01}{45.01} \\
45.01 \\
\end{array}$ & \begin{tabular}{|l|}
25.77 \\
39.25 \\
27.70 \\
\end{tabular} & $-31.33 \%$ \\
\hline
\end{tabular}


HELICAL COILS/WIRE B.HFC-134a/Emery 2927 Iso-32 Oil @ 260 $\mathrm{F}\left(127^{\circ} \mathrm{C}\right)$

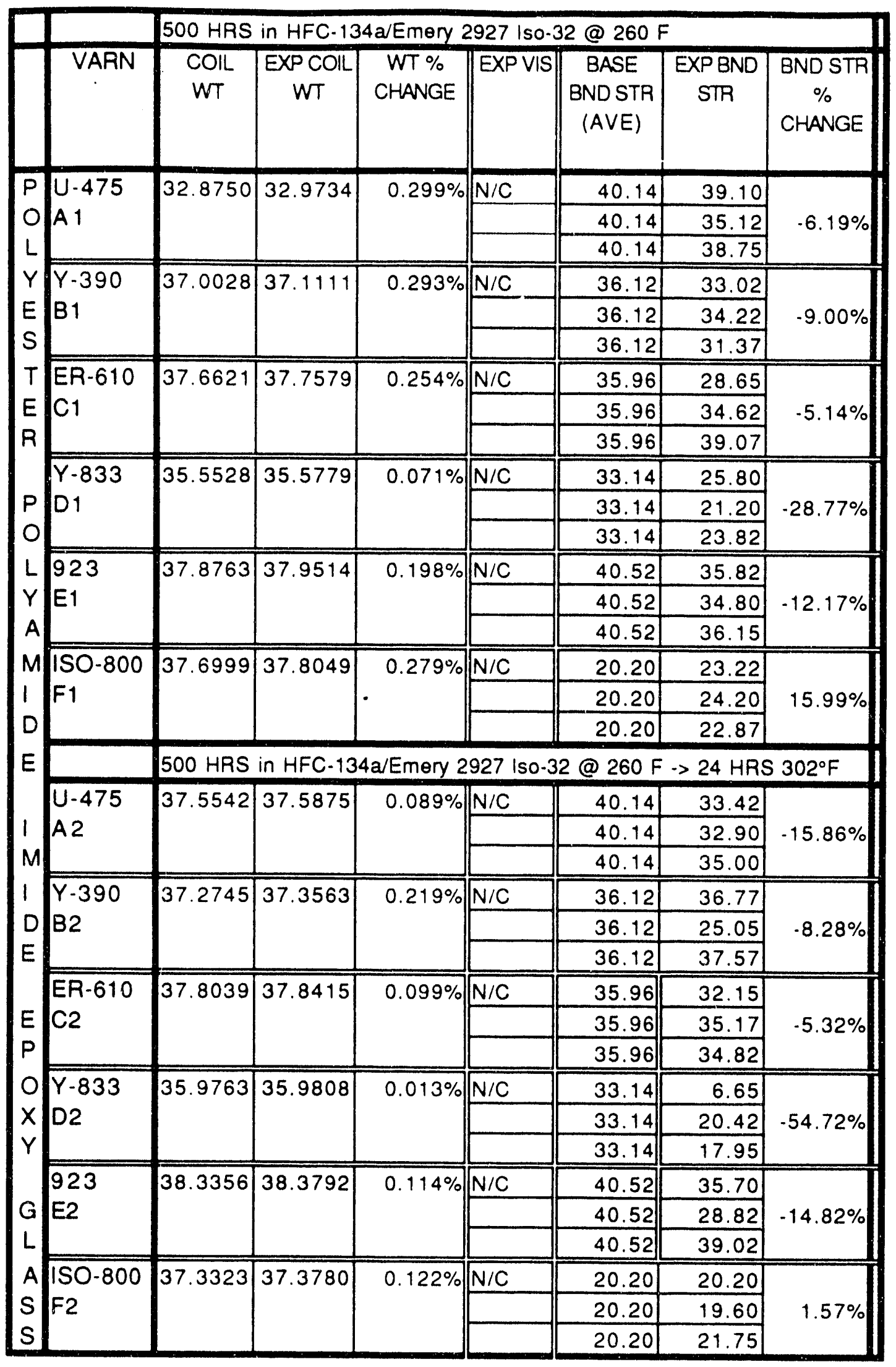


HELICAL COILS/WIRE C.HFC-134a/Emery 2927 Iso-32@ 260.F(127ㅇ $)$

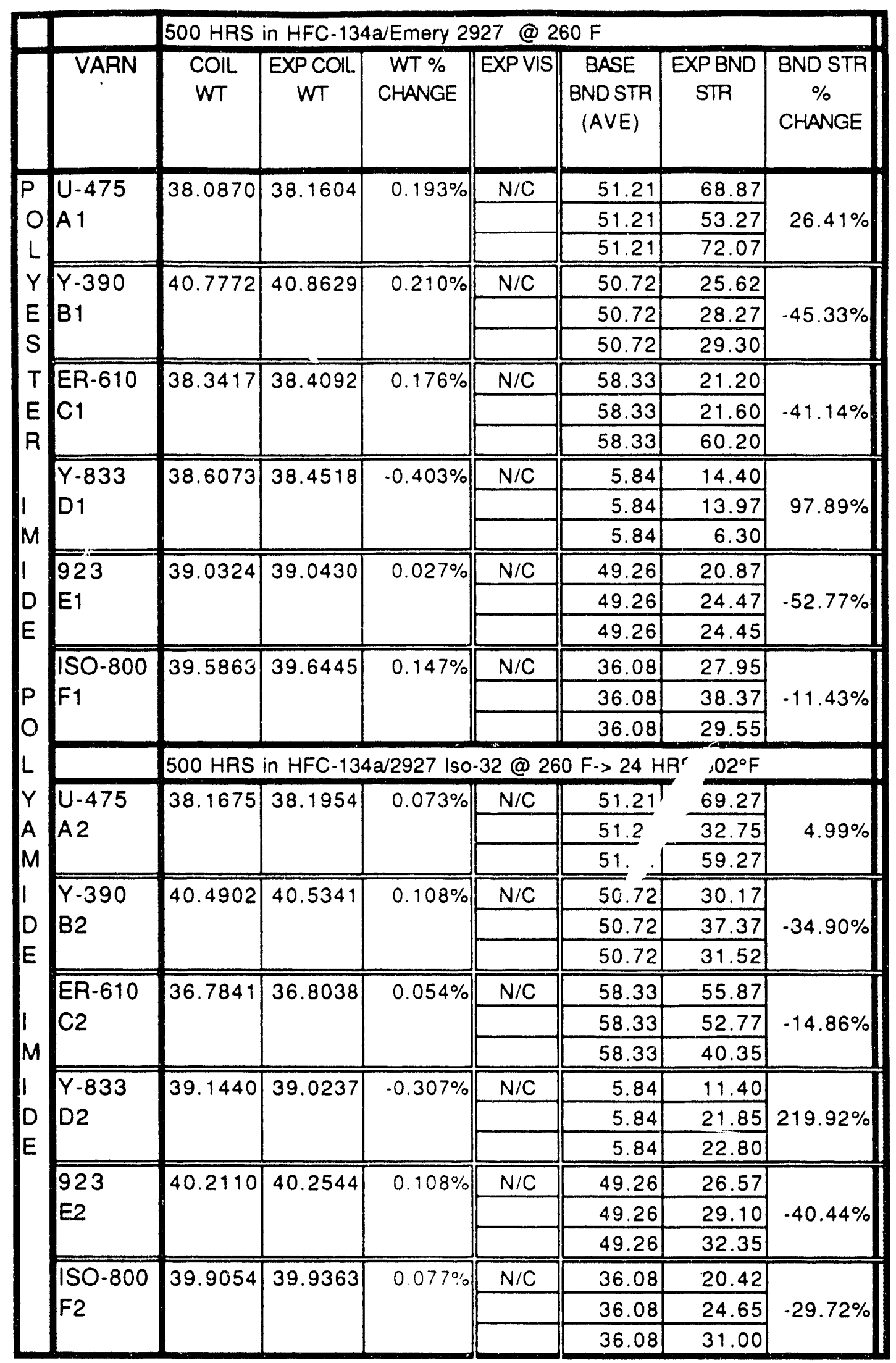


SINGLE MAG WIRE WITHOUT VARNISH.HFC-134a/ICI Emkarate RL244@ $\left.269^{\circ} \mathrm{F}\left(127^{\circ} \mathrm{C}\right)\right)$

\begin{tabular}{|c|c|c|c|c|c|}
\hline 500 & $\mathrm{AS}$ in $\mathrm{HF}$ & $-134 a / 292$ & Iso-32 oil & $@ 260^{\circ} \mathrm{F}$ & $\left.127^{\circ} \mathrm{C}\right)$ \\
\hline $\mathbb{D}$ & WT & EXPWT & $\begin{array}{c}\text { WT \% } \\
\text { CHANGE }\end{array}$ & EXP VIS & EXPFLEX \\
\hline A 1 & 3.7518 & 3.7541 & $0.061 \%$ & $\mathrm{~N} / \mathrm{C}$ & \\
\hline & & & & & YES \\
\hline$B 1$ & 3.4677 & 3.4752 & $0.216 \%$ & $\mathrm{~N} / \mathrm{C}$ & \\
\hline & & & & & YES \\
\hline & & & & & \\
\hline C1 & 3.236 & 3.2378 & $0.056 \%$ & $\mathrm{~N} / \mathrm{C}$ & \\
\hline & & & & & YES \\
\hline & $x^{\prime \prime}$ & 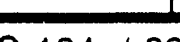 & 7 & & \\
\hline 50 & Ins in HI & $c .134 a / 29$ & $-1150-32->$ & 302 t ior & 24 HRS \\
\hline A2 & 3.6826 & 3.6839 & $0.035 \%$ & $\mathrm{~N} / \mathrm{C}$ & \\
\hline & & & & & YES \\
\hline B2 & 3.4569 & 3.481 & $0.697 \%$ & $\mathrm{~N} / \mathrm{C}$ & \\
\hline & & & & & YES \\
\hline C2 & 3.2563 & 3.2569 & $0.018 \%$ & $\mathrm{~N} / \mathrm{C}$ & \\
\hline & & & & & YES \\
\hline & & & & & \\
\hline
\end{tabular}




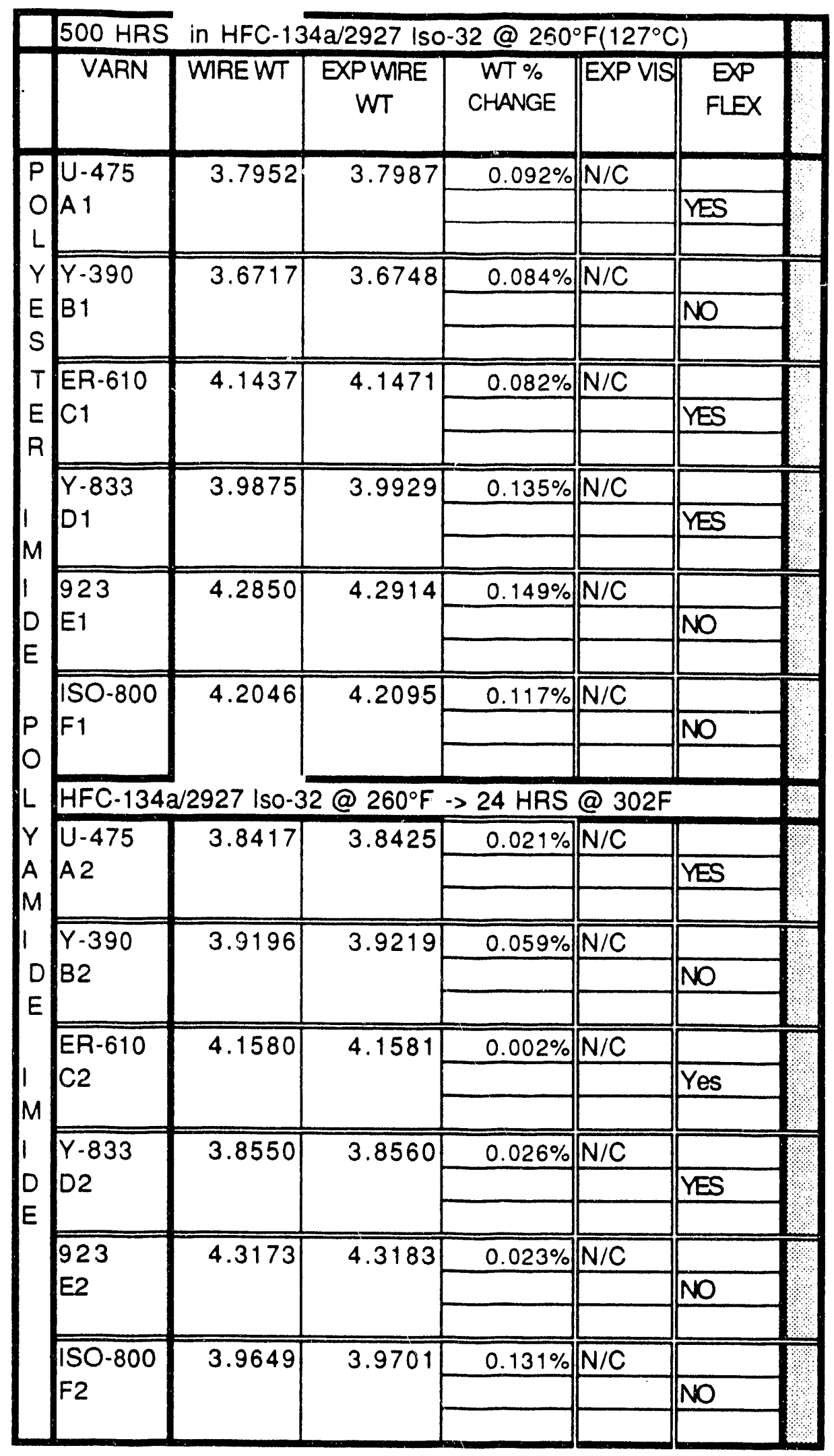




\section{)}

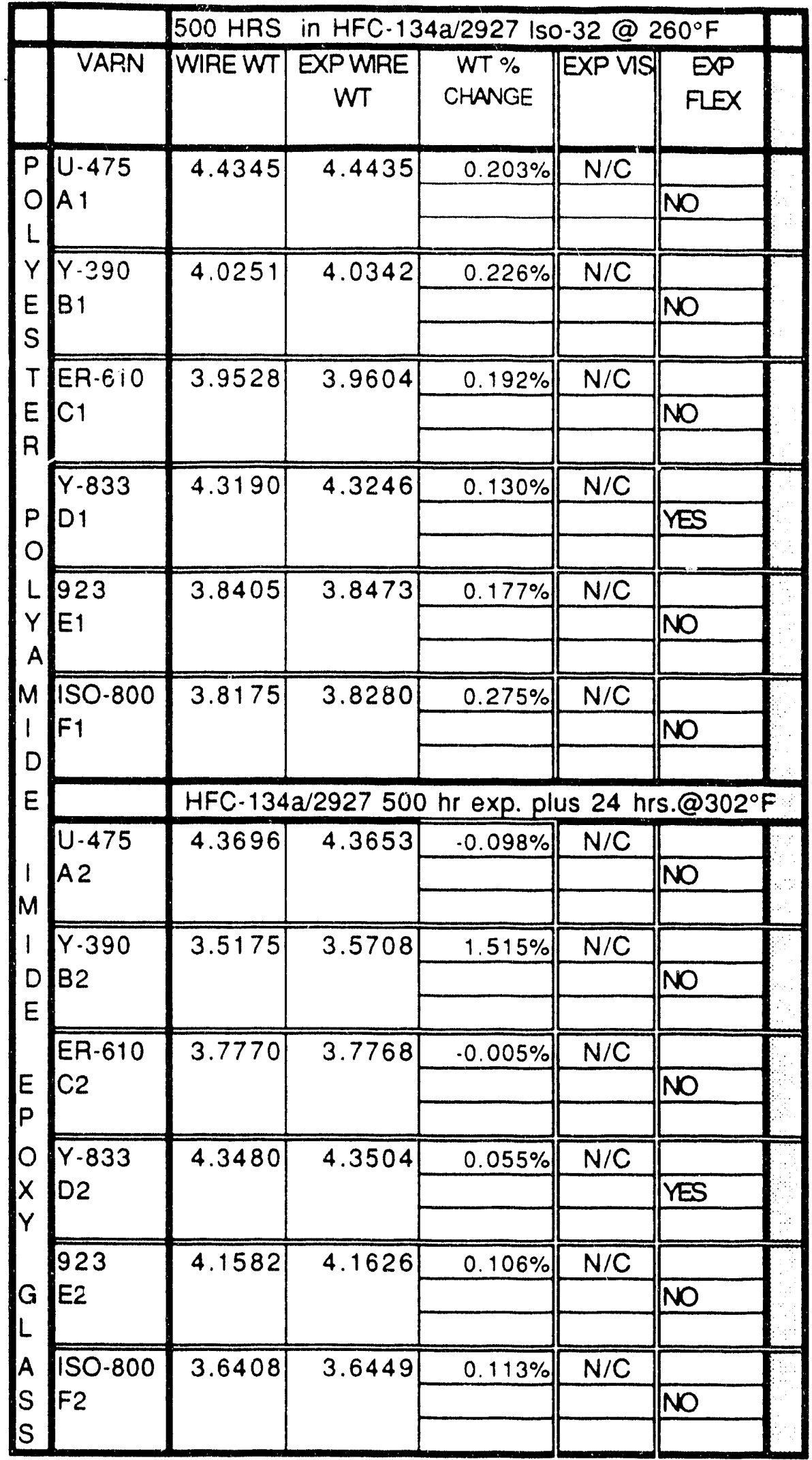

) 


\begin{tabular}{|c|c|c|c|c|c|c|}
\hline & $500 \mathrm{HRS}$ & in HFC-134 & ta/Emery 2 & 927 Iso-32 & $1 @$ & $260^{\circ} \mathrm{F}$ \\
\hline & VARN & WIRE WT & $\begin{array}{c}\text { EXP WIRE } \\
W T\end{array}$ & $\begin{array}{c}\text { WT \% } \\
\text { CHANGE }\end{array}$ & $\begin{array}{l}\text { EPP } \\
\text { VIS }\end{array}$ & $\begin{array}{l}\text { EXP } \\
\text { FLEX }\end{array}$ \\
\hline$P$ & $U-475$ & 4.2894 & 4.2952 & $0.135 \%$ & $\mathrm{~N} / \mathrm{C}$ & \\
\hline O & A1 & & & & & YES \\
\hline L & & & & & & \\
\hline$Y$ & $Y-390$ & 4.1904 & 4.1953 & $0.117 \%$ & $\mathrm{~N} / \mathrm{C}$ & \\
\hline$E$ & B1 & & & & & NO \\
\hline$S$ & & & & & & \\
\hline$T$ & ER-610 & 4.2421 & 4.2424 & $0.007 \%$ & $\mathrm{~N} / \mathrm{C}$ & \\
\hline$E$ & C1 & & & & & YES \\
\hline$R$ & & & & & & \\
\hline & $Y-833$ & 3.9809 & 3.9843 & $0.085 \%$ & $\mathrm{~N} / \mathrm{C}$ & \\
\hline$P$ & D1 & & & & & YES \\
\hline 0 & & & & & & \\
\hline$L$ & $\longdiv { 9 2 3 }$ & 4.3647 & 4.3708 & $0.140 \%$ & $\mathrm{~N} / \mathrm{C}$ & \\
\hline$Y$ & E1 & & & & & $\mathrm{NO}$ \\
\hline A & & & & & & \\
\hline$M$ & ISO-800 & 4.3752 & 4.3823 & $0.162 \%$ & $\mathrm{~N} / \mathrm{C}$ & \\
\hline 1 & F1 & & & & & NO \\
\hline$D$ & & & & & & \\
\hline$E$ & & HFC. $134 a$ & $y / 2927$ Iso- & $32 \rightarrow 24 \mathrm{H}$ & RS @ & $302^{\circ} \mathrm{F}$ \\
\hline & U.475 & 4.3767 & 4.3763 & $-0.009 \%$ & $\mathrm{~N} / \mathrm{C}$ & \\
\hline 1 & A2 & & & & & YES \\
\hline M & & & & & & \\
\hline 1 & $Y-390$ & 3.9793 & 3.9829 & $0.090 \%$ & $\mathrm{~N} / \mathrm{C}$ & \\
\hline D & $B 2$ & & & & & NO \\
\hline E & & & & & & \\
\hline & ER-610 & 4.2380 & 4.2438 & $0.137 \%$ & $\mathrm{~N} / \mathrm{C}$ & \\
\hline & $\mathrm{C} 2$ & & & & & NO \\
\hline & $Y-833$ & 4.3032 & 4.3043 & $0.026 \%$ & $\mathrm{~N} / \mathrm{C}$ & \\
\hline & $\mathrm{D} 2$ & & & & & YES \\
\hline & 923 & 4.3876 & 4.3910 & $0.077 \%$ & $N / C$ & \\
\hline & E2 & & & & & NO \\
\hline & & 42405 & 12430 & $0064 \%$ & $N / C$ & \\
\hline & $\begin{array}{l}150-800 \\
F 2\end{array}$ & 4.2405 & 4.2432 & $0.004 \%$ & iv/ & NO \\
\hline & & & & & & \\
\hline
\end{tabular}


Varnish Disks....HFC-134a/Emery 2927 Iso-32 Oil

500 HRS IN R-134a/Emery 2927 Iso -32@ 260 F

\begin{tabular}{|c|c|c|c|c|c|}
\hline ID & WT & EXPWT & $\begin{array}{l}\text { WT \% } \\
\text { CHANGE }\end{array}$ & EXPVS & EXPFLEX \\
\hline $\begin{array}{l}\text { U-475 } \\
\text { A } 1\end{array}$ & 2.3095 & 2.3381 & $1.24 \%$ & $N / C$ & $\mathrm{~N} / \mathrm{C}$ \\
\hline $\begin{array}{l}Y-390 \\
B 1\end{array}$ & 2.2329 & 2.2163 & $0.74 \%$ & \begin{tabular}{|l|} 
Slightly \\
warped \\
\end{tabular} & $N / C$ \\
\hline $\begin{array}{l}\text { ER-610 } \\
C_{1}\end{array}$ & 1.9911 & 2.0554 & $3.23 \%$ & $N / C$ & $N / C$ \\
\hline $\begin{array}{l}-833 \\
D 1\end{array}$ & 2.6668 & 2.7099 & $1.62 \%$ & $\mathrm{~N} / \mathrm{C}$ & $\mathrm{N} / \mathrm{C}$ \\
\hline $\begin{array}{l}923 \\
\text { E1 }\end{array}$ & 1.7901 & 1.7890 & $-0.06 \%$ & $N / C$ & $\mathrm{~N} / \mathrm{C}$ \\
\hline $\begin{array}{l}\text { ISO-800 } \\
\mathrm{F} 1\end{array}$ & 1.4654 & 1.3866 & $.5 .38 \%$ & \begin{tabular}{|l|} 
Slightly \\
warped \\
\end{tabular} & $\mathrm{N} / \mathrm{C}$ \\
\hline & \multicolumn{5}{|c|}{ R-134a/Emery 2927 at $\cdots>24$ hour at $302^{\circ} \mathrm{F}$. } \\
\hline $\begin{array}{l}\text { U-475 } \\
\text { A2 }\end{array}$ & 2.3525 & 2.3423 & $.0 .43 \%$ & darkened & $\mathrm{N} / \mathrm{C}$ \\
\hline $\begin{array}{l}Y-390 \\
B 2\end{array}$ & 1.9161 & 1.8659 & $-2.62 \%$ & darkened & $\mathrm{N} / \mathrm{C}$ \\
\hline $\begin{array}{l}\text { ER-610 } \\
\mathrm{C} 2\end{array}$ & 2.4518 & 2.4511 & $-0.03 \%$ & darkened & $\mathrm{N} / \mathrm{C}$ \\
\hline $\begin{array}{r}Y-833 \\
\end{array}$ & 2.1826 & 2.1154 & $-3.08 \%$ & $\mathrm{~N} / \mathrm{C}$ & $\mathrm{N} / \mathrm{C}$ \\
\hline $\begin{array}{l}923 \\
\text { E2 }\end{array}$ & 1.8626 & 1.8343 & $-1.52 \%$ & $N / C$ & $\mathrm{~N} / \mathrm{C}$ \\
\hline $\begin{array}{l}\text { SO-800 } \\
\text { F2 }\end{array}$ & 1.7650 & 1.6090 & $.8 .84 \%$ & darkened & \\
\hline & & & & & $\mathrm{N} / \mathrm{C}$ \\
\hline
\end{tabular}




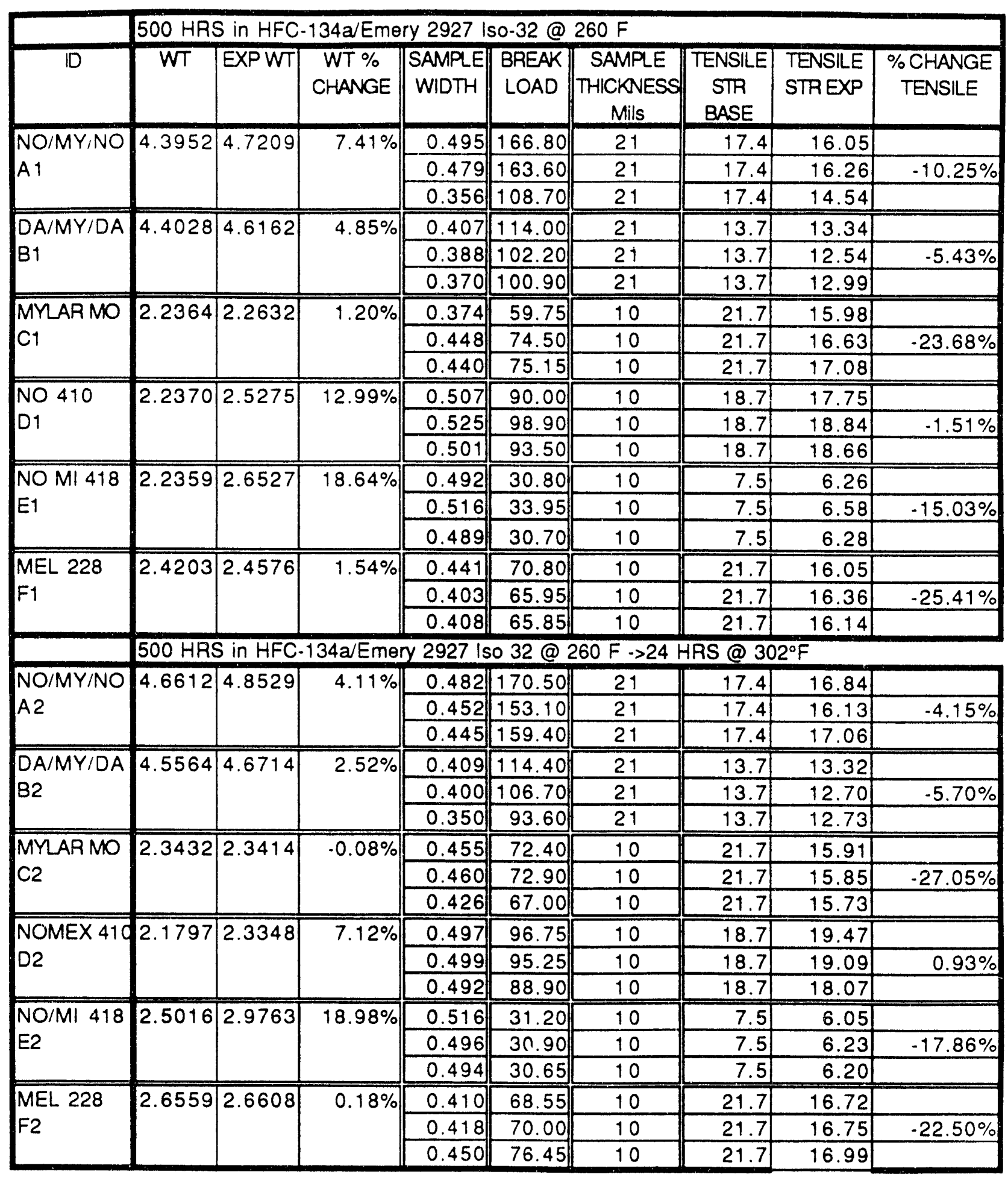




\begin{tabular}{|c|c|c|c|c|c|c|c|c|}
\hline & $00 \mathrm{HRS}$ & $\mathrm{HFC}$ & $34 a / E m$ & 2927 & o-32@ & $260 \mathrm{~F}$ & & \\
\hline ID & $\begin{array}{c}\text { STRETCH } \\
\text { (inch) }\end{array}$ & $\begin{array}{c}\% \\
\text { ELONG }\end{array}$ & $\begin{array}{l}\text { BASE } \\
\text { ELONG } \\
\text { (AVE) }\end{array}$ & $\begin{array}{l}\text { ELONG \% } \\
\text { CHANGE }\end{array}$ & \begin{tabular}{|c} 
BASE \\
DIE \\
$(A \vee E)$
\end{tabular} & EXP DIE & $\begin{array}{c}\text { DIE \% } \\
\text { CHANGE }\end{array}$ & VISUAL EXP \\
\hline NO/MY/NO & 0.50 & $12.5 \%$ & $20.0 \%$ & & $>18.97$ & flash & & 1 piece had 1 \\
\hline A1 & 0.54 & $13.5 \%$ & $20.0 \%$ & $.35 .0 \%$ & $>18.97$ & flash & flash & small bubble \\
\hline & 0.42 & $10.5 \%$ & $20.0 \%$ & & $>18.97$ & flash & & the rest $N / C$ \\
\hline$D A / M Y / D A$ & 0.54 & $27.0 \%$ & $46.0 \%$ & & $>15.27$ & flash & & Yellowed \\
\hline B1 & 0.42 & $21.0 \%$ & $46.0 \%$ & $.47 .5 \%$ & $>15.27$ & flash & flash & slightly \\
\hline & 0.49 & $24.5 \%$ & $46.0 \%$ & & $>15.27$ & flash & & warped \\
\hline MYLAR MO & 1.82 & $91.0 \%$ & $131.0 \%$ & & $>14.91$ & flash & & $\mathrm{N} / \mathrm{C}$ \\
\hline$C_{1}$ & 2.09 & $104.5 \%$ & $131.0 \%$ & $-20.1 \%$ & $>14.91$ & flash & flash & \\
\hline & 2.37 & $118.5 \%$ & $131.0 \%$ & & $>14.91$ & flash & & \\
\hline NO 410 & 0.32 & $16.0 \%$ & $.17 .0 \%$ & & 10.67 & flash & & $N / C$ \\
\hline & 0.44 & $11.0 \%$ & $17.0 \%$ & $.28 .4 \%$ & 10.67 & flash & flash & \\
\hline & 0.38 & $9.5 \%$ & $17.0 \%$ & & 10.67 & flash & & \\
\hline NO MI 418 & 0.04 & $1.0 \%$ & $4.0 \%$ & & 10.23 & 10.79 & & $N / C$ \\
\hline & 0.05 & $1.3 \%$ & $4.0 \%$ & $.72 .9 \%$ & 10.23 & 11.66 & $5.4 \%$ & \\
\hline & 0.04 & $1.0 \%$ & $4.0 \%$ & & 10.23 & 9.89 & & \\
\hline MEL 228 & 2.65 & $132.5 \%$ & $160.0 \%$ & & $>14.22$ & flash & & $\mathrm{N} / \mathrm{C}$ \\
\hline$F 1$ & 3.24 & $162.0 \%$ & $160.0 \%$ & $.11 .5 \%$ & $>14.22$ & flash & flash & \\
\hline & 2.61 & $130.5 \%$ & $160.0 \%$ & & $>14.22$ & flash & & \\
\hline & $500 \mathrm{HRS}$ & in $\mathrm{HFC}$. & 134a/Eme & ry 2927 & so 32@ & $260 \mathrm{~F}$ & 24 HRS & @ $302^{\circ} \mathrm{F}$ \\
\hline NO/MYINO & 0.66 & $16.5 \%$ & $20.0 \%$ & & $>18.97$ & flash & & Delamination \\
\hline A2 & 0.54 & $13.5 \%$ & $20.0 \%$ & $-23.3 \%$ & $>18.97$ & flash & flash & big pockets \\
\hline & 0.64 & $16.0 \%$ & $20.0 \%$ & & $>18.97$ & flash & & \\
\hline$D A / M Y / D A$ & 0.46 & $23.0 \%$ & $46.0 \%$ & & $>15.27$ & flash & & Yellowed \\
\hline B2 & 0.47 & $23.5 \%$ & $46.0 \%$ & $.48 .6 \%$ & $>15.27$ & flash & flash & slightly \\
\hline & 0.49 & $24.5 \%$ & $46.0 \%$ & & 215.27 & flash & & warped \\
\hline MYLAR MO & 2.91 & $145.5 \%$ & $131.0 \%$ & & $>14.91$ & flash & & N/C \\
\hline C2 & 2.64 & $132.0 \%$ & $131.0 \%$ & $-3.2 \%$ & $>14.91$ & flash & flash & \\
\hline & 2.06 & $103.0 \%$ & $131.0 \%$ & & $>14.91$ & flash & & \\
\hline NOMEX 410 & 0.42 & $10.5 \%$ & $17.0 \%$ & & 10.67 & 11.81 & & $\mathrm{~N} / \mathrm{C}$ \\
\hline D2 & 0.42 & $10.5 \%$ & $17.0 \%$ & $.43 .6 \%$ & 10.67 & 12.10 & $6.3 \%$ & 1 piece had 1 \\
\hline & 0.31 & $7.8 \%$ & $17.0 \%$ & & 10.67 & 10.13 & & bubble \\
\hline $\mathrm{NO} / \mathrm{MI} 418$ & 0.05 & $1.3 \%$ & $4.0 \%$ & & 10.23 & 11.76 & & $N / C$ \\
\hline & 0.04 & $1.0 \%$ & $4.0 \%$ & $.70 .8 \%$ & 10.23 & 11.54 & $10.6 \%$ & \\
\hline & 0.05 & $1.3 \%$ & $4.0 \%$ & & 10.23 & 10.63 & & \\
\hline MEL 228 & 1.87 & $93.5 \%$ & $160.0 \%$ & & $>14.22$ & flash & & $N / C$ \\
\hline F2 & 1.66 & $83.0 \%$ & $160.0 \%$ & $-43.9 \%$ & $\geq 14.22$ & flash & flash & \\
\hline & 1.86 & $93.0 \%$ & $160.0 \%$ & & $>14.22$ & flash & & \\
\hline
\end{tabular}




\begin{tabular}{|c|c|c|c|c|}
\hline & \multicolumn{4}{|c|}{500 HRS in HFC-134a/Emery 2927 Iso-32@260 $\mathrm{F}$} \\
\hline & WT & EXPWT & $\begin{array}{c}\% \\
\text { CHANGE }\end{array}$ & EXPVS \\
\hline A1 & 0.5542 & 0.6212 & $12.09 \%$ & $\mathrm{~N} / \mathrm{C}$ \\
\hline $\begin{array}{l}\text { B1 } \\
\text { MYLAR }\end{array}$ & 0.5021 & 0.5120 & $1.97 \%$ & $\mathrm{~N} / \mathrm{C}$ \\
\hline \multirow{2}{*}{$\begin{array}{l}\text { C1 } \\
\text { NO/MY }\end{array}$} & 0.3908 & 0.4087 & $4.58 \%$ & $\mathrm{~N} / \mathrm{C}$ \\
\hline & \multicolumn{4}{|c|}{ HFC-134a/Emery 2927 Iso-32 oil @ 260F plus24 hrs @ 302 ${ }^{\circ} \mathrm{F}$} \\
\hline $\begin{array}{l}\text { A2 } \\
\text { NOMEX }\end{array}$ & 0.5500 & 0.5955 & $8.27 \%$ & $\mathrm{~N} / \mathrm{C}$ \\
\hline $\begin{array}{l}\text { B2 } \\
\text { MYAR }\end{array}$ & 0.4776 & 0.4783 & $0.15 \%$ & $\mathrm{~N} / \mathrm{C}$ \\
\hline \multirow{3}{*}{$\begin{array}{l}\mathrm{C} 2 \\
\mathrm{NO} / \mathrm{MY}\end{array}$} & \multirow[t]{3}{*}{0.4087} & \multirow[t]{3}{*}{0.4189} & \multirow[t]{3}{*}{$2.50 \%$} & $\mathrm{~N} / \mathrm{C}$ \\
\hline & & & & some pockets where mylar \\
\hline & & & & pulled away. \\
\hline
\end{tabular}




\begin{tabular}{|c|c|c|c|c|c|c|c|c|c|}
\hline \multirow[b]{2}{*}{$\overline{I D}$} & \multicolumn{9}{|c|}{500 HRS in HFC-134a/Emery 2927 Iso32 @ 260 F } \\
\hline & WT & EXPWT & $\begin{array}{c}\text { WT \% } \\
\text { CHANGE }\end{array}$ & $\begin{array}{l}\text { BREAK } \\
\text { LOAD } \\
\text { (AVE) }\end{array}$ & $\begin{array}{l}\text { BREAK } \\
\text { LOAD } \\
\text { EP }\end{array}$ & $\begin{array}{c}\% \\
\text { CHANGE } \\
\text { BPK } \\
\text { LOAD }\end{array}$ & $\begin{array}{c}\text { STRETCH } \\
\text { (INCH) }\end{array}$ & \%ELONG| & EXPVIS \\
\hline \multirow{3}{*}{$\begin{array}{l}\text { A } 1 \\
\text { Glass }\end{array}$} & \multirow[t]{3}{*}{1.8596} & \multirow[t]{3}{*}{1.8608} & \multirow[t]{3}{*}{$0.06 \%$} & 39.02 & 46.25 & & 0.04 & $2.00 \%$ & $\mathrm{~N} / \mathrm{C}$ \\
\hline & & & & 39.02 & 52.97 & $28.09 \%$ & 0.05 & $2.50 \%$ & \\
\hline & & & & 39.02 & 50.72 & & 0.05 & $2.50 \%$ & \\
\hline \multirow{3}{*}{$\begin{array}{l}\text { B1 } \\
\text { Polyester }\end{array}$} & \multirow[t]{3}{*}{0.6546} & \multirow[t]{3}{*}{0.6597} & \multirow[t]{3}{*}{$0.78 \%$} & 56.12 & 63.12 & & 0.81 & $40.50 \%$ & $\mathrm{~N} / \mathrm{C}$ \\
\hline & & & & 56.12 & 62.65 & $5.29 \%$ & 0.77 & $38.50 \%$ & \\
\hline & & & & 56.12 & 51.50 & & 0.42 & $21.00 \%$ & \\
\hline \multirow{3}{*}{$\begin{array}{l}1 \\
\text { Permacel }\end{array}$} & \multirow[t]{3}{*}{1.6603} & \multirow[t]{3}{*}{1.7861} & \multirow{3}{*}{$7.58 \%$} & 88.50 & 83.70 & & 0.14 & $7.00 \%$ & Slightly \\
\hline & & & & 88.50 & 93.20 & $.4 .96 \%$ & 0.13 & $6.50 \%$ & darkened \\
\hline & & & & 88.50 & 75.42 & & 0.12 & $6.00 \%$ & \\
\hline & \multicolumn{8}{|c|}{$500 \mathrm{HRS}$ in HFC.134a/Emery 2927 ISO32 @ 260 F.> 302 F $24 \mathrm{HRS}$} & \\
\hline \multirow{3}{*}{$\begin{array}{l}\text { A2 } \\
\text { Glass }\end{array}$} & \multirow[t]{3}{*}{1.7392} & \multirow[t]{3}{*}{1.8018} & \multirow[t]{3}{*}{$3.60 \%$} & 39.02 & 43.15 & & 0.06 & $3.00 \%$ & $\mathrm{~N} / \mathrm{C}$ \\
\hline & & & & 39.02 & 51.55 & $18.70 \%$ & 0.06 & $3.00 \%$ & \\
\hline & & & & 39.02 & 44.25 & & 0.07 & $3.50 \%$ & \\
\hline \multirow{3}{*}{$\begin{array}{l}\text { B2 } \\
\text { Polyester }\end{array}$} & \multirow[t]{3}{*}{0.6804} & \multirow[t]{3}{*}{0.6751} & \multirow[t]{3}{*}{$-0.78 \%$} & 56.12 & 51.75 & & 0.47 & $23.50 \%$ & $\mathrm{~N} / \mathrm{C}$ \\
\hline & & & & 56.12 & 57.25 & $-2.29 \%$ & 0.60 & $30.00 \%$ & \\
\hline & & & & 56.12 & 55.50 & & 0.57 & $28.50 \%$ & \\
\hline \multirow{3}{*}{$\begin{array}{l}\text { C2 } \\
\text { Permacel }\end{array}$} & \multirow[t]{3}{*}{1.5897} & 1.6247 & $2.20 \%$ & 88.50 & 93.85 & & 0.15 & $7.50 \%$ & Slightly \\
\hline & & & & 88.50 & 100.40 & $8.76 \%$ & 0.16 & $8.00 \%$ & Darkened \\
\hline & & & & 88.50 & 94.50 & & 0.15 & $7.50 \%$ & \\
\hline
\end{tabular}


TIE CORD--HFC-134a/Emery 2927 ISO-32 oil @ 260 $\mathrm{F}\left(127^{\circ} \mathrm{C}\right)$

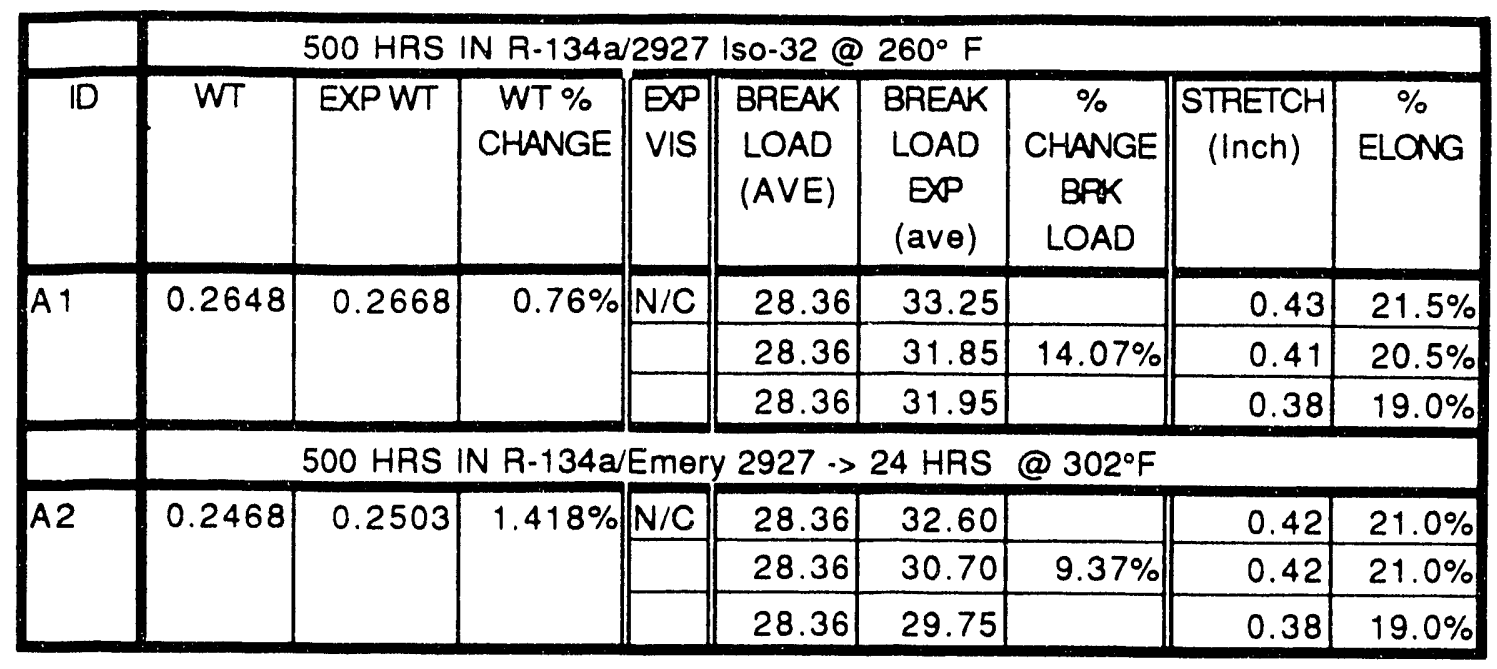


LEAD WIRE INSULATION-HFC-134a/Emery 2927 Iso-32@260F(12דC)

\begin{tabular}{|l|r|r|r||r||r|r|r|}
\hline & 500 HRS IN HFC-134a/Emery 2927 ISO-32 @ 260 & & \\
\hline ID & WT & EXPWT & $\begin{array}{c}\text { WT \% } \\
\text { CHANGE }\end{array}$ & EXPVS & $\begin{array}{r}\text { BASE DIE } \\
\text { (AVE) }\end{array}$ & EXP DIE & $\begin{array}{c}\text { DIE\% } \\
\text { CHANGE }\end{array}$ \\
\hline DMD & 4.0292 & 4.1895 & $3.98 \%$ & N/C & 9.61 & 9.22 & \\
\hline A1 & & & & & 9.61 & 7.92 & $-13.39 \%$ \\
\hline & & & & & 9.61 & 7.83 & \\
\hline DTMD & 4.3703 & 4.5104 & $3.21 \%$ & N/C & 9.95 & 11.43 & \\
\hline B1 & & & & & 9.95 & 12.14 & $22.38 \%$ \\
\hline & & & & & 9.95 & 12.96 & \\
\hline & & & & & & & \\
\hline DMD & 4.0344 & 4.1522 & $2.92 \%$ & N/C & 9.61 & 8.71 & \\
\hline A2 & & & & & 9.61 & 8.50 & $-6.90 \%$ \\
\hline & & & & & 9.61 & 9.63 & \\
\hline \hline DTMD & 4.4165 & 4.5197 & $2.34 \%$ & N/C & 9.95 & 10.51 & \\
\hline B2 & & & & & 9.95 & 12.26 & $12.23 \%$ \\
\hline & & & & & 9.95 & 10.73 & \\
\hline
\end{tabular}




\section{Appendix H}

Experimental Data for HFC-32/HenkelEmery 2927 ISO-32 Exposure at $126^{\circ} \mathrm{C}\left(260^{\circ} \mathrm{F}\right)$ 


\begin{tabular}{|c|c|c|c|c|c|c|c|c|c|c|}
\hline & 0 hours & HFC-32 & 927 Iso. 3 & 2 oil at & $260^{\circ} \mathrm{F}(127$ & & & & & \\
\hline ID & $W T$ & EXPWT & $\begin{array}{c}\text { WT \% } \\
\text { CHANGE }\end{array}$ & EXP VIS & $\begin{array}{c}\text { BASE BRN } \\
\text { OUT } \\
\text { (AVE) }\end{array}$ & $\begin{array}{c}\text { EXP BRN } \\
\text { OUT }\end{array}$ & $\begin{array}{c}\text { BRN OUT } \\
\% \\
\text { CHANGE }\end{array}$ & $\begin{array}{c}\text { BASE DIE } \\
\text { (AVE) }\end{array}$ & EXPDIE & $\begin{array}{c}\text { DIE \% } \\
\text { CHANGE }\end{array}$ \\
\hline$A 1$ & 22.2661 & 22.2758 & $0.044 \%$ & $\mathrm{~N} / \mathrm{C}$ & 576 & 555 & & 15.80 & 17.08 & \\
\hline & & & & & 576 & 427 & $-12.4 \%$ & 15.80 & 15.90 & $3.3 \%$ \\
\hline & & & & & 576 & 532 & & 15.80 & 16.00 & \\
\hline B1 & 24.1414 & 24.1778 & $0.151 \%$ & $N / C$ & 736 & 655 & & 11.62 & 11.53 & \\
\hline & & & & & 736 & 733 & $-4.1 \%$ & 11.62 & 11.56 & $0.4 \%$ \\
\hline & & & & & 736 & 730 & & 11.62 & 11.91 & \\
\hline C1 & 23.9948 & 24.0051 & $0.043 \%$ & $N / C$ & 579 & 437 & & 16.58 & 11.80 & \\
\hline & & & & & 579 & 411 & $-27.9 \%$ & 16.58 & 16.05 & $-10.0 \%$ \\
\hline & & & & & 579 & 404 & & 16.58 & 16.90 & \\
\hline & 500 hours i & HFC-32/E & mkarate & RL244 o & at $260^{\circ} \mathrm{F}$ & $127^{\circ} \mathrm{C}$ ) plu & us 24 hou & rs at $302^{\circ}$ & $\left(150^{\circ} \mathrm{C}\right)$ & \\
\hline$A 2$ & 22.367 & 22.3669 & $0.000 \%$ & $\mathrm{~N} / \mathrm{C}$ & 576 & 535 & & 15.80 & 11.61 & \\
\hline & & & & & 576 & 521 & $-6.4 \%$ & 15.80 & 12.65 & $-18.8 \%$ \\
\hline & & & & & 576 & 561 & & 15.80 & 14.23 & \\
\hline B2 & 23.1092 & 23.1090 & $-0.001 \%$ & $\mathrm{~N} / \mathrm{C}$ & 736 & 732 & & 11.62 & 11.64 & \\
\hline & & & & & 736 & 731 & $-3.5 \%$ & 11.62 & 11.59 & $1.0 \%$ \\
\hline & & & & & 736 & 668 & & 11.62 & 11.99 & \\
\hline $\mathrm{C} 2$ & 22.6258 & 22.6261 & $0.001 \%$ & $\mathrm{~N} / \mathrm{C}$ & 579 & 495 & & 16.58 & 14.23 & \\
\hline & & & & & 579 & 485 & $-14.7 \%$ & 16.58 & 9.81 & $-26.4 \%$ \\
\hline & & & & & 579 & 502 & & 16.58 & 12.58 & \\
\hline
\end{tabular}




\begin{tabular}{|c|c|c|c|c|c|c|c|c|c|c|c|}
\hline & & 500 hours & in $\mathrm{HFC}-3$ & Emery & 2927 & so-32 oil & $@ 260^{\circ}$ & $\left.127^{\circ} \mathrm{C}\right)$ & & & \\
\hline & VARN & $\begin{array}{l}\text { TWISTED } \\
\text { PAIR WT }\end{array}$ & $\begin{array}{l}\text { Exp Pair } \\
\text { weight }\end{array}$ & $\begin{array}{c}\text { WT \% } \\
\text { CHANGE }\end{array}$ & $\begin{array}{l}\text { EXP } \\
\text { VIS }\end{array}$ & BASE DIE & EXP DIE & $\begin{array}{c}\text { DIE \% } \\
\text { CHANGE }\end{array}$ & $\begin{array}{c}\text { BASE } \\
\text { BURN } \\
\text { OUT }\end{array}$ & $\begin{array}{l}\text { EXP } \\
\text { BUPN } \\
\text { OUT }\end{array}$ & $\begin{array}{c}\text { BRNOUT } \\
\% \\
\text { CHANGE }\end{array}$ \\
\hline$P$ & U.475 & 22.8343 & 22.8508 & $0.072 \%$ & $N / C$ & 16.24 & 10.97 & & 430 & 421 & \\
\hline 0 & $A 1$ & & & & & 16.24 & 14.61 & $-16.77 \%$ & 430 & 379 & $-17.44 \%$ \\
\hline L & & & & & & 16.24 & 14.97 & & 430 & 265 & \\
\hline$Y$ & $Y-390$ & 22.8994 & 22.9350 & $0.155 \%$ & $\mathrm{~N} / \mathrm{C}$ & 18.77 & 17.80 & & 510 & 577 & \\
\hline$E$ & B1 & & & & & 18.77 & 15.14 & $-10.12 \%$ & 510 & 568 & $0.46 \%$ \\
\hline $\mathrm{S}$ & & & & & & 18.77 & 17.67 & & 510 & 392 & \\
\hline$T$ & ER-610 & 23.7197 & 23.7382 & $0.078 \%$ & $\mathrm{~N} / \mathrm{C}$ & 15.57 & 15.31 & & 442 & 314 & \\
\hline$E$ & C1 & & & & & 15.57 & 14.33 & $-5.15 \%$ & 442 & 290 & $.28 .96 \%$ \\
\hline$R$ & & & & & & 15.57 & 14.66 & & 442 & 338 & \\
\hline & $Y-833$ & 22.7504 & 22.7342 & $-0.071 \%$ & $N / C$ & 12.04 & 16.60 & & 578 & 565 & \\
\hline$P$ & D1 & & & & & 12.04 & 16.33 & $23.03 \%$ & 578 & 569 & $-6.11 \%$ \\
\hline 0 & & & & & & 12.04 & 11.51 & & 578 & 494 & \\
\hline$L$ & 923 & 22.2736 & 22.3233 & $0.223 \%$ & $\mathrm{~N} / \mathrm{C}$ & 16.76 & 19.66 & & 606 & 449 & \\
\hline$Y$ & E1 & & & & & 16.76 & 19.74 & $10.64 \%$ & 606 & 500 & $.19 .97 \%$ \\
\hline A & & & & & & 16.76 & 16.23 & & 606 & 506 & \\
\hline$M$ & $150-800$ & 21.5768 & 21.6072 & $0.141 \%$ & $\mathrm{~N} / \mathrm{C}$ & 19.08 & 12.71 & & 580 & 470 & \\
\hline 1 & $\mathrm{~F} 1$ & & & & & 19.08 & 17.34 & $-13.64 \%$ & 580 & 395 & $-27.82 \%$ \\
\hline$D$ & & & & & & 19.08 & 19.38 & & 580 & 391 & \\
\hline$E$ & & 500 hours & 3 in HFC-3 & 2/Emery 2 & 2927 & so-32 oil (a) & $2260^{\circ} \mathrm{F}(1$ & $127^{\circ} \mathrm{C}$ ) plu & s 24 hol & rs@3 & $02^{\circ} \mathrm{F}\left(150^{\circ}\right.$ \\
\hline & U-475 & 22.3743 & 22.3718 & $-0.011 \%$ & $N / C$ & 16.24 & 8.44 & & 430 & 367 & \\
\hline 1 & $A 2$ & & & & & 16.24 & 13.77 & $.32 .20 \%$ & 430 & 344 & $-13.02 \%$ \\
\hline$M$ & & & & & & 16.24 & 10.82 & & 430 & 411 & \\
\hline 1 & $Y-390$ & 23.7128 & 23.7186 & $0.024 \%$ & $\mathrm{~N} / \mathrm{C}$ & 18.77 & 19.84 & & 510 & 388 & \\
\hline$D$ & B2 & & & & & 18.77 & 8.06 & $-33.71 \%$ & 510 & 415 & $-17.39 \%$ \\
\hline$E$ & & & & & & 18.77 & 9.43 & & 510 & 461 & \\
\hline & ER-610 & 22.6394 & 22.6372 & $-0.010 \%$ & $\mathrm{~N} / \mathrm{C}$ & 15.57 & 11.47 & & 442 & 363 & \\
\hline & C2 & & & & & 15.57 & 11.95 & $-20.92 \%$ & 442 & 350 & $.17 .80 \%$ \\
\hline & & & & & & 15.57 & 13.52 & & 442 & 377 & \\
\hline & $Y-833$ & 23.0497 & 23.0255 & $-0.105 \%$ & $\mathrm{~N} / \mathrm{C}$ & 12.04 & 11.85 & & 578 & 548 & \\
\hline & D2 & & & & & 12.04 & 10.07 & $.4 .07 \%$ & 578 & 557 & $-5.07 \%$ \\
\hline & & & & & & 12.04 & 12.73 & & 578 & 541 & \\
\hline & 923 & 22.3218 & 22.3268 & $0.022 \%$ & $\mathrm{~N} / \mathrm{C}$ & 16.76 & 13.13 & & 606 & 433 & \\
\hline & $E 2$ & & & & & 16.76 & 11.04 & $.26 .31 \%$ & 606 & 445 & $-27.78 \%$ \\
\hline & & & & & & 16.76 & 12.88 & & 606 & 435 & \\
\hline & $150-800$ & 22.4286 & 22.4379 & $0.041 \%$ & $N / C$ & 19.08 & 19.75 & & 580 & 338 & \\
\hline & F2 & & & & & 19.08 & 14.00 & $-15.55 \%$ & 580 & 441 & $-33.68 \%$ \\
\hline & & & & & & 19.08 & 14.59 & & 580 & 375 & \\
\hline
\end{tabular}


TWISTED PAIRSMIRE BWITH VARNISH -HFC-132/Emery 2927 Iso-32 oil @260 $2\left(127^{\circ} \mathrm{C}\right)$

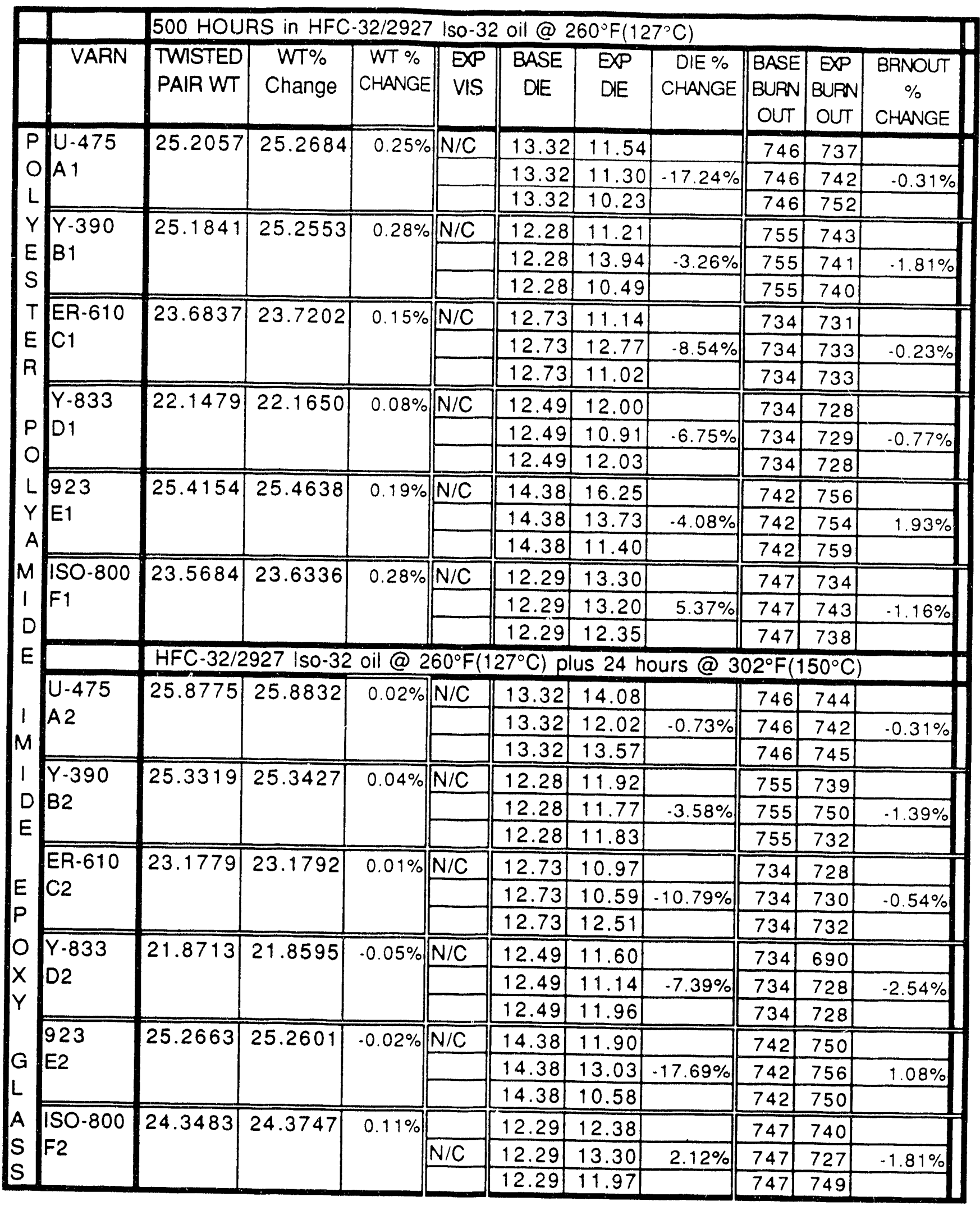


TWISTED PAIRSNIRE ONITH VARNISH-HFC.32/Emery 2927 Iso-32@260 $\mathrm{F}\left(127^{\circ} \mathrm{C}\right)$

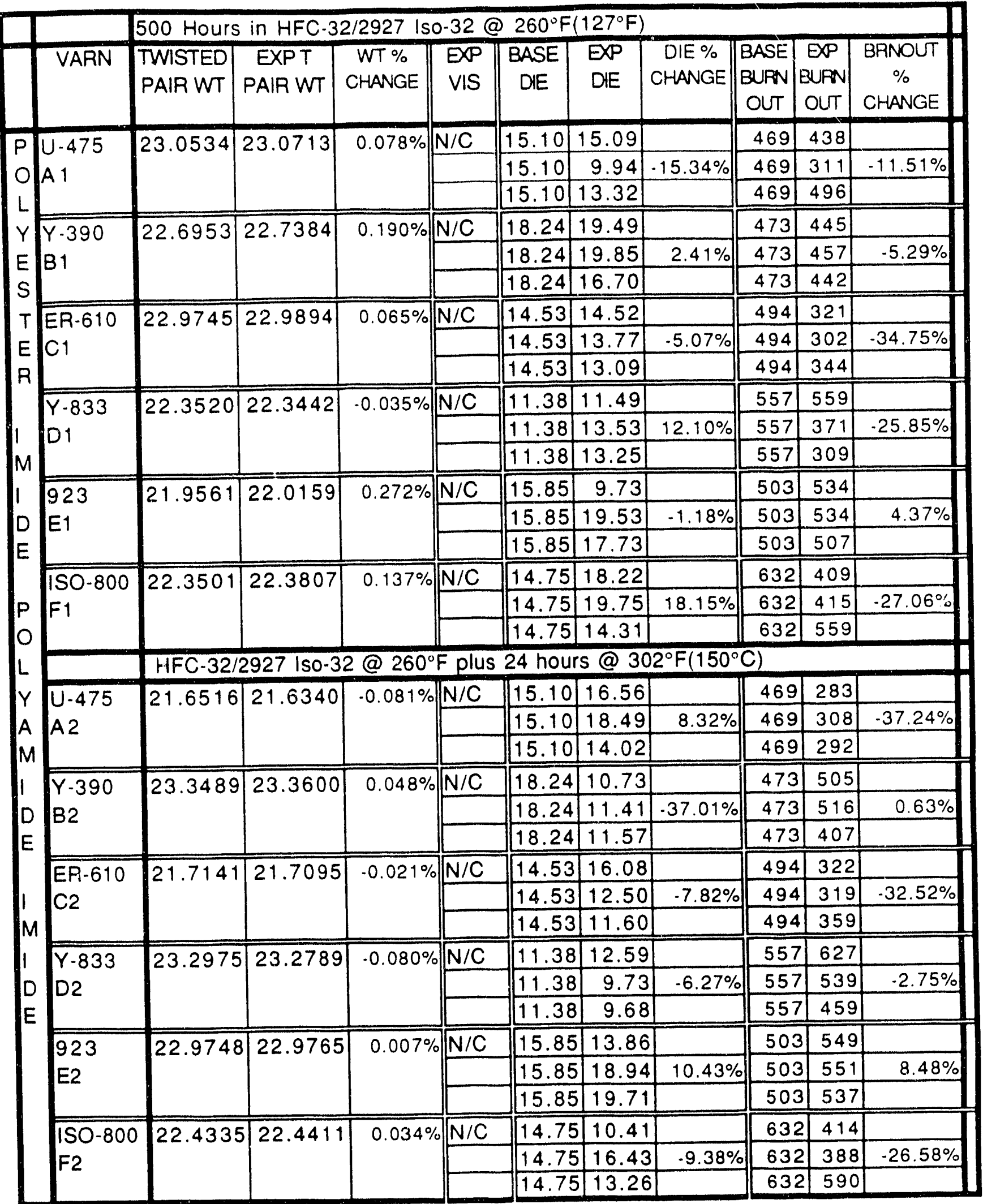


HELICAL COILSMIRE A-.-HFC.32/Emery 2927 Iso-32@260 $\mathrm{F}\left(127^{\circ} \mathrm{C}\right)$

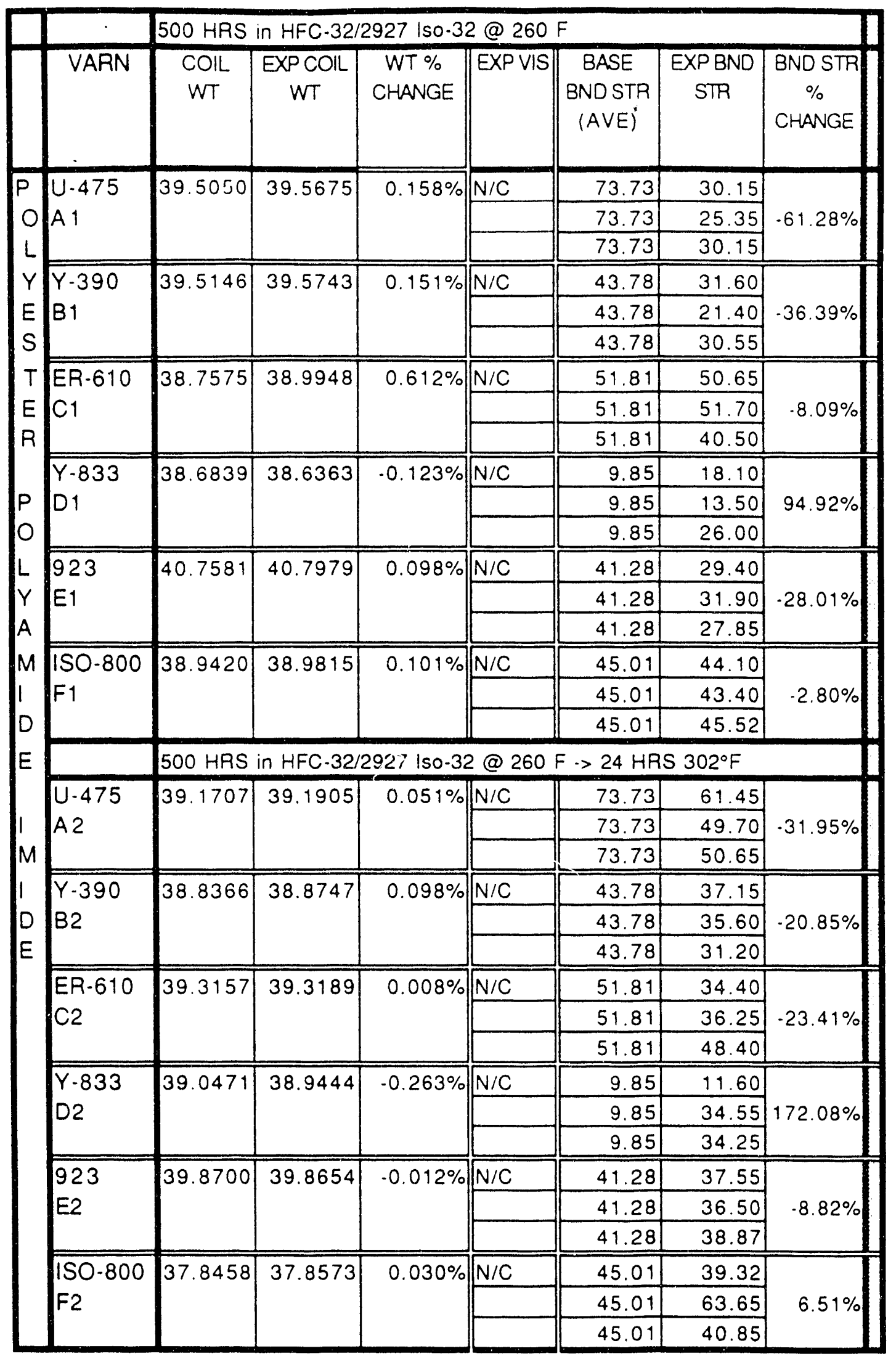


HELICAL COILSMIRE B.HFC-32/Emery 2927 Iso-32 Oil @260 $\mathrm{F}\left(127^{\circ} \mathrm{C}\right)$

\begin{tabular}{|c|c|c|c|c|c|c|c|c|}
\hline & & $500 \mathrm{HRS}$ & in HFC & Emery 2 & so-32 & $260 \mathrm{~F}$ & & \\
\hline & VARN & $\begin{array}{l}\text { COIL } \\
W T\end{array}$ & $\begin{array}{c}\text { EXP COIL } \\
W T\end{array}$ & $\begin{array}{l}\text { WT \% } \\
\text { CHANGE }\end{array}$ & EXPVIS & $\begin{array}{c}\text { BASE } \\
\text { BND STR } \\
\text { (AVE) }\end{array}$ & $\begin{array}{c}\text { EXPBND } \\
\text { STR }\end{array}$ & $\begin{array}{c}\text { BND STR } \\
\% \\
\text { CHANGE }\end{array}$ \\
\hline$P$ & $U .475$ & 37.6020 & 37.6764 & $0.198 \%$ & $N / C$ & 40.14 & 29.87 & \\
\hline 0 & A 1 & & & & & 40.14 & 31.17 & $.25 .60 \%$ \\
\hline$L$ & & & & & & 40.14 & 28.55 & \\
\hline$Y$ & $Y-390$ & 38.4927 & 38.5895 & $0.251 \%$ & $\mathrm{~N} / \mathrm{C}$ & 36.12 & 32.25 & \\
\hline$E$ & $B 1$ & & & & & 36.12 & 27.75 & $.8 .57 \%$ \\
\hline$S$ & & & & & & 36.12 & 39.07 & \\
\hline$T$ & ER-610 & 38.3750 & 38.4481 & $0.190 \%$ & $\mathrm{~N} / \mathrm{C}$ & 35.96 & 26.67 & \\
\hline$E$ & $C_{1}$ & & & & & 35.96 & 24.75 & $.28 .88 \%$ \\
\hline$R$ & & & & & & 35.96 & 25.30 & \\
\hline & $Y \cdot 833$ & 36.7175 & 36.6289 & $-0.241 \%$ & $\mathrm{~N} / \mathrm{C}$ & 33.14 & 17.37 & \\
\hline$P$ & D1 & & & & & 33.14 & broke & $.47 .59 \%$ \\
\hline O & & & & & & 33.14 & broke & \\
\hline L & 923 & 37.9097 & 37.9682 & $0.154 \%$ & $\mathrm{~N} / \mathrm{C}$ & 40.52 & 38.82 & \\
\hline Y & $E 1$ & & & & & 40.52 & 25.95 & $.20 .76 \%$ \\
\hline A & & & & & & 40.52 & 31.55 & \\
\hline$M$ & $150-800$ & 35.8433 & 35.9072 & $0.178 \%$ & $\mathrm{~N} / \mathrm{C}$ & 20.20 & 19.42 & \\
\hline 1 & $\mathrm{~F} 1$ & & & & & 20.20 & broke & $.3 .12 \%$ \\
\hline$D$ & & & & & & 20.20 & 19.72 & \\
\hline$E$ & & $500 \mathrm{HRS}$ & in $\mathrm{HFC}-32$ & Emery 292 & 7 Iso.32 & $@ 260 F->$ & $>24$ HRS 3 & $302^{\circ} \mathrm{F}$ \\
\hline & $U .475$ & 37.7606 & 37.7856 & $0.066 \%$ & $\mathrm{~N} / \mathrm{C}$ & 40.14 & 32.30 & \\
\hline 1 & $A 2$ & & & & & 40.14 & 33.07 & $-22.55 \%$ \\
\hline$M$ & & & & & & 40.14 & 27.90 & \\
\hline 1 & $Y-390$ & 37.8150 & 37.8518 & $0.097 \%$ & $\mathrm{~N} / \mathrm{C}$ & 36.12 & 30.07 & \\
\hline$D$ & B2 & & & & & 36.12 & 26.32 & $-16.68 \%$ \\
\hline$E$ & & & & & & 36.12 & 33.90 & \\
\hline & ER-610 & 38.2920 & 38.3127 & $0.054 \%$ & $\mathrm{~N} / \mathrm{C}$ & 35.96 & 24.80 & \\
\hline$E$ & $\mathrm{C} 2$ & & & & & 35.96 & 22.60 & $.31 .06 \%$ \\
\hline $\mathrm{P}$ & & & & & & 35.96 & 26.97 & \\
\hline 0 & $Y .833$ & 36.2557 & 36.2279 & $-0.077 \%$ & $\mathrm{~N} / \mathrm{C}$ & 33.14 & 1.85 & \\
\hline$x$ & D2 & & & & & 33.14 & 10.90 & $.70 .43 \%$ \\
\hline$Y$ & & & & & & 33.14 & 16.65 & \\
\hline & 923 & 37.7957 & 37.8016 & $0.016 \%$ & $\mathrm{~N} / \mathrm{C}$ & 40.52 & 28.50 & \\
\hline G & E2 & & & & & 40.52 & 27.97 & $-32.94 \%$ \\
\hline$L$ & & & & & & 40.52 & 25.05 & \\
\hline A & $150-800$ & 37.7162 & 37.7297 & $0.036 \%$ & $\mathrm{~N} / \mathrm{C}$ & 20.20 & 15.12 & \\
\hline$s$ & $\mathrm{~F} 2$ & & & & & 20.20 & 14.42 & $-23.37 \%$ \\
\hline$S$ & & & & & & 20.20 & 16.90 & \\
\hline
\end{tabular}


HELICAL COILSMIRE C.HFC-32/Emery 2927 Iso-32@ $260^{\circ} \mathrm{F}\left(127^{\circ} \mathrm{C}\right)$

\begin{tabular}{|c|c|c|c|c|c|c|c|c|}
\hline & & $500 \mathrm{HRS}$ & in $\mathrm{HFC}-32$ & Emery 292 & 7 @ 260 & & & \\
\hline & VARN & $\begin{array}{l}\text { COIL } \\
W T\end{array}$ & $\begin{array}{c}\text { EXP COIL } \\
W T\end{array}$ & $\begin{array}{c}\text { WT \% } \\
\text { CHANGE }\end{array}$ & EXP VIS & $\begin{array}{c}\text { BASE } \\
\text { BND STR } \\
(\text { AVE })\end{array}$ & $\begin{array}{c}\text { EXPBND } \\
\text { STR }\end{array}$ & $\begin{array}{c}\text { BND STR } \\
\% \\
\text { CHANGE }\end{array}$ \\
\hline$P$ & U.475 & 37.8274 & 37.8851 & $0.153 \%$ & $\mathrm{~N} / \mathrm{C}$ & 51.21 & 34.72 & \\
\hline 0 & A 1 & & & & & 51.21 & 63.59 & $3.37 \%$ \\
\hline$L$ & & & & & & 51.21 & 60.50 & \\
\hline Y & $Y-390$ & 40.5103 & 40.5733 & $0.156 \%$ & $\mathrm{~N} / \mathrm{C}$ & 50.72 & 24.05 & \\
\hline$E$ & B1 & & & & & 50.72 & 25.42 & $.50 .43 \%$ \\
\hline S & & & & & & 50.72 & 25.95 & \\
\hline $\mathrm{T}$ & ER-610 & 37.0068 & 37.5080 & $1.354 \%$ & $\mathrm{~N} / \mathrm{C}$ & 58.33 & 22.95 & \\
\hline$E$ & C1 & & & & & 58.33 & 44.82 & $-46.33 \%$ \\
\hline$R$ & & & & & & 58.33 & 26.15 & \\
\hline & $Y-833$ & 38.7483 & 38.6172 & $-0.338 \%$ & $\mathrm{~N} / \mathrm{C}$ & 5.84 & 7.30 & \\
\hline 1 & & & & & & 5.84 & 6.80 & $67.07 \%$ \\
\hline M & & & & & & 5.84 & 15.17 & \\
\hline I & 923 & 39.6056 & 39.6406 & $0.088 \%$ & $\mathrm{~N} / \mathrm{C}$ & 49.26 & 28.72 & \\
\hline D & E1 & & & & & 49.26 & 24.42 & $.46 .79 \%$ \\
\hline$E$ & & & & - & & 49.26 & 25.50 & \\
\hline & $150-800$ & 38.8999 & 38.9368 & $0.095 \%$ & $\mathrm{~N} / \mathrm{C}$ & 36.08 & 30.70 & \\
\hline$P$ & $F 1$ & & & & & 36.08 & 32.67 & $.13 .44 \%$ \\
\hline 0 & & & & & & 36.08 & 30.32 & \\
\hline$L$ & & 500 HRS & in $\mathrm{HFC}-32$ & 2927 Iso.3? & @ 260 & $\therefore 24 \mathrm{HRS}$ & $302^{\circ} \mathrm{F}$ & \\
\hline Y & $U-475$ & 37.9403 & 37.9537 & $0.035 \%$ & $\mathrm{~N} / \mathrm{C}$ & 51.21 & 72.32 & \\
\hline A & $A 2$ & & & & & 51.21 & 65.02 & $18.96 \%$ \\
\hline M & & & & & & 51.21 & 45.42 & \\
\hline I & $Y-390$ & 40.1086 & 40.1297 & $0.053 \%$ & $\mathrm{~N} / \mathrm{C}$ & 50.72 & 34.60 & \\
\hline$D$ & B2 & & & & & 50.72 & 35.62 & $-31.30 \%$ \\
\hline$E$ & & & & & & 50.72 & 34.32 & \\
\hline & ER. 610 & 37.7877 & 37.7910 & $0.009 \%$ & $\mathrm{~N} / \mathrm{C}$ & 58.33 & 29.30 & \\
\hline 1 & C2 & & & & & 58.33 & 64.87 & $.31 .17 \%$ \\
\hline M & & & & & & 58.33 & 26.27 & \\
\hline 1 & $Y-833$ & 38.2443 & 38.0612 & $-0.479 \%$ & $\mathrm{~N} / \mathrm{C}$ & 5.84 & 10.82 & \\
\hline 0 & $\mathrm{D} 2$ & & & & & 5.84 & 14.17 & $116.84 \%$ \\
\hline E & & & & & & 5.84 & 7.00 & \\
\hline & 923 & 39.4140 & 39.4123 & $-0.004 \%$ & $\mathrm{~N} / \mathrm{C}$ & 49.26 & 25.67 & \\
\hline & E2 & & & & & 49.26 & 31.70 & $-41.96 \%$ \\
\hline & & & & & & 49.26 & 28.40 & \\
\hline & ISO-800 & 39.2881 & 39.3053 & $0.044 \%$ & $\mathrm{~N} / \mathrm{C}$ & 36.08 & 41.00 & \\
\hline & F2 & & & & & 36.08 & 35.77 & $21.57 \%$ \\
\hline & & & & & & 36.08 & 54.82 & \\
\hline
\end{tabular}


SINGLE MAG WIRE WITHOUT VARNISH-HFC-32/ICI Emkarate RL244@ 260 $\mathrm{F}\left(127^{\circ} \mathrm{C}\right)$ )

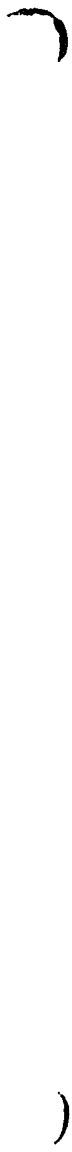

\begin{tabular}{|c|c|c|c|c|c|}
\hline 500 & RS in HF & $-32 / 2927$ & 0-32 oil @ & $260^{\circ} \mathrm{F}(12$ & $\left.7^{\circ} \mathrm{C}\right)$ \\
\hline ID & WT & EXPWT & $\begin{array}{c}\text { WT \% } \\
\text { CHANGE }\end{array}$ & EXP VIS & EXPFLEX \\
\hline$A 1$ & 4.0118 & 4.013 & $0.030 \%$ & $\mathrm{~N} / \mathrm{C}$ & \\
\hline & & & & & YES \\
\hline$\overline{B 1}$ & 35994 & 36035 & $0114 \%$ & $\overline{N / C}$ & \\
\hline & & & & & YES \\
\hline & & & & & \\
\hline C1 & 3.6776 & 3.679 & $0.038 \%$ & $\mathrm{~N} / \mathrm{C}$ & \\
\hline & & & & & YES \\
\hline 50 & $\mathrm{RS}$ in $\mathrm{HF}$ & C-32/ 292 & so-32-> 30 & $2^{\circ} \mathrm{F}$ for 2 & HRS \\
\hline$A 2$ & 4.0097 & 4.0099 & $0.005 \%$ & $\mathrm{~N} / \mathrm{C}$ & \\
\hline & & & & & YES \\
\hline$\overline{B 2}$ & 3.4636 & 3.4631 & $-0.014 \%$ & $\mathrm{~N} / \mathrm{C}$ & \\
\hline & & & & & YES \\
\hline$C_{2}$ & 27032 & 27820 & & $\overline{N / C}$ & \\
\hline $\mathrm{C} 2$ & 3.7833 & 3.1832 & $-0.003 \%$ & N/C & YES \\
\hline & & & & & \\
\hline
\end{tabular}




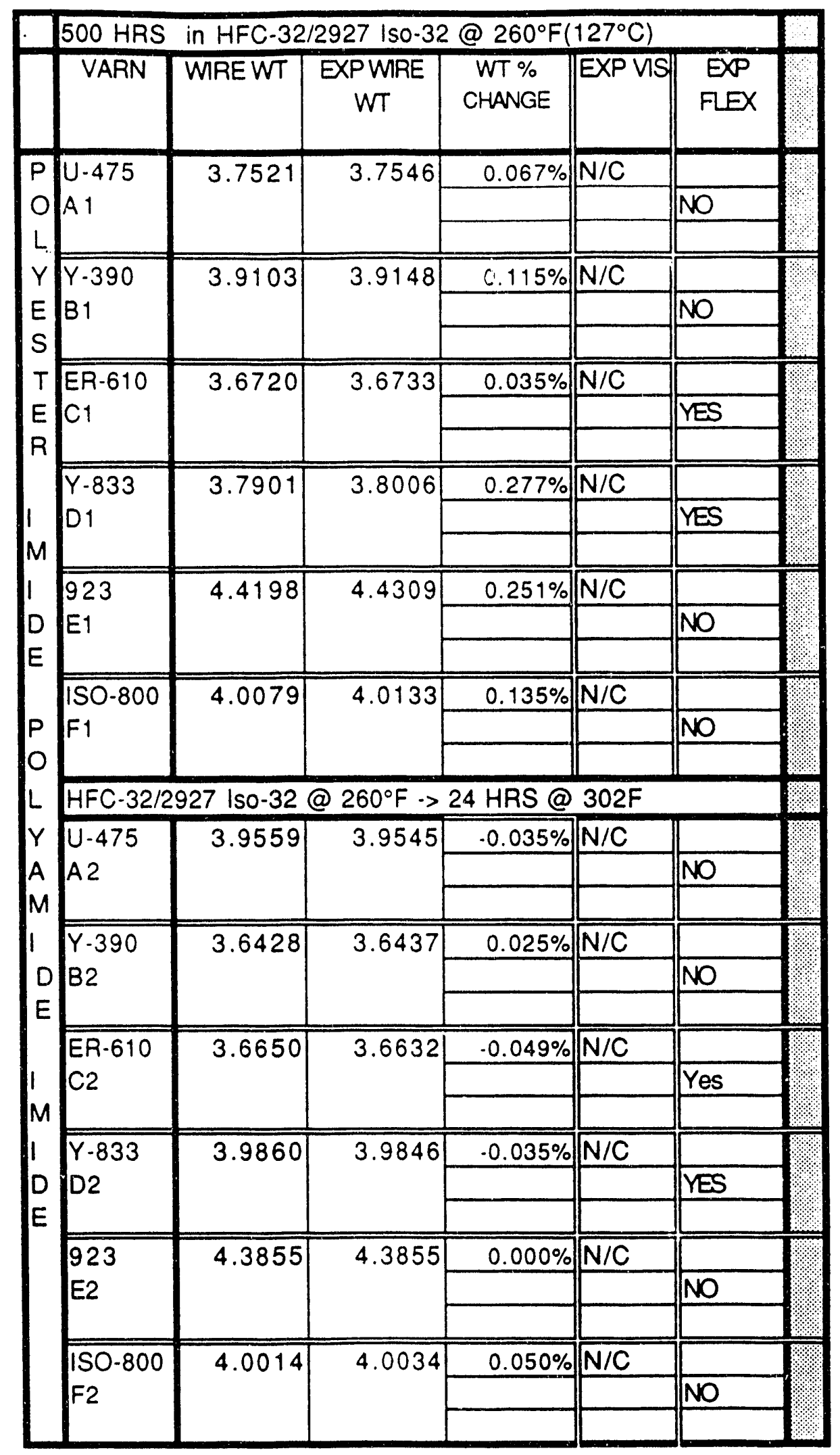




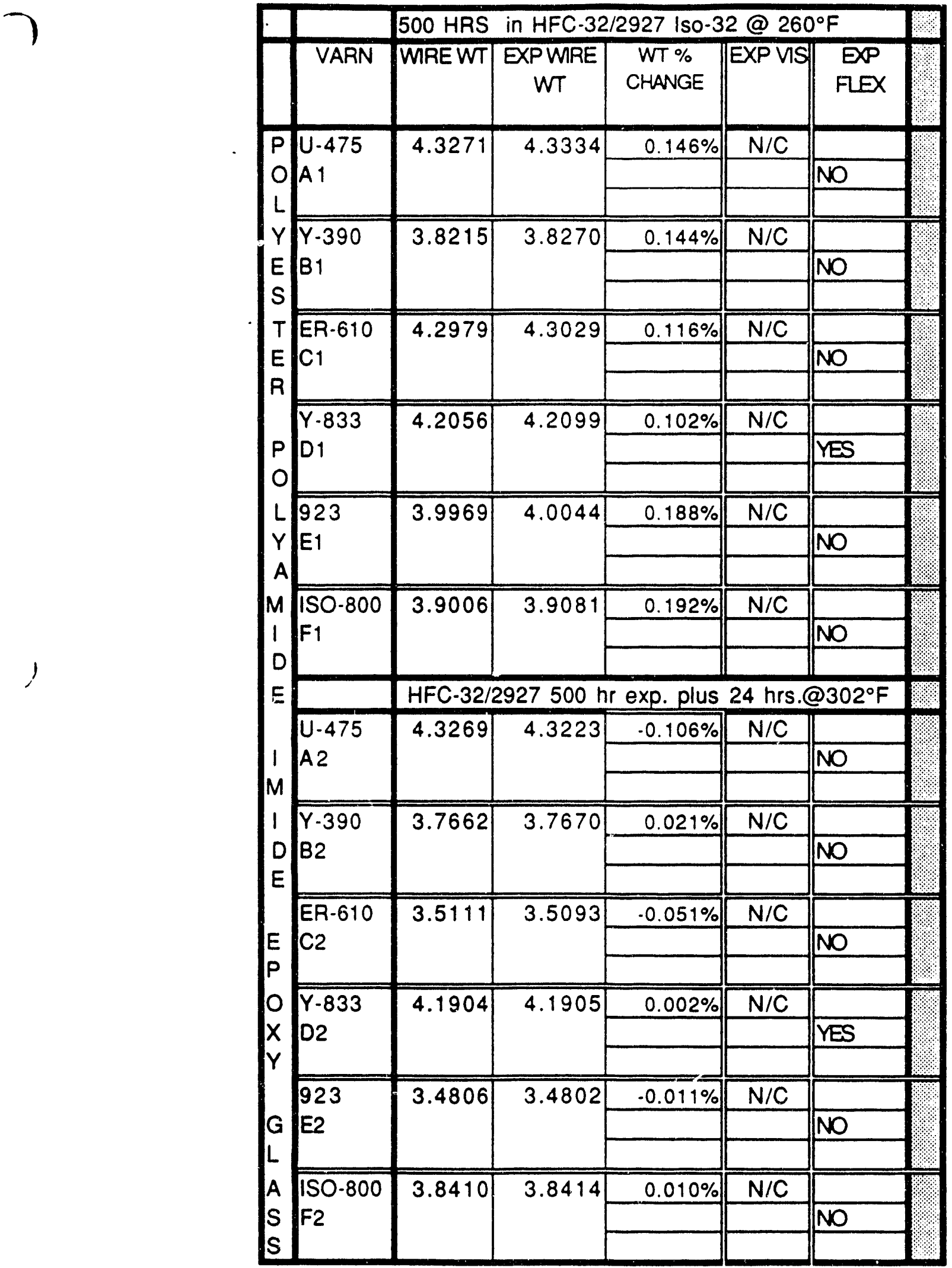




\begin{tabular}{|c|c|c|c|c|c|c|}
\hline $500 \mathrm{HRS}$ & in $\mathrm{HFC}-32 / \mathrm{E}$ & Emery 292 & $7150-320$ & @ 26 & $0^{\circ} \mathrm{F}$ & \\
\hline VARN & WIRE WT & $\begin{array}{c}\text { EXPWRE } \\
W T\end{array}$ & $\begin{array}{l}\text { WT \% } \\
\text { CHANGE }\end{array}$ & $\begin{array}{l}\text { EPP } \\
\text { VIS }\end{array}$ & $\begin{array}{l}\text { EPP } \\
\text { FEX }\end{array}$ & \\
\hline P) U-475 & 4.2468 & 4.2535 & $0.158 \%$ & $\mathrm{~N} / \mathrm{C}$ & & \\
\hline$A 1$ & & & & & NO & \\
\hline L & & & & & & \\
\hline$Y Y-390$ & 4.4631 & 4.4700 & $0.155 \%$ & $\mathrm{~N} / \mathrm{C}$ & & \\
\hline B1 & & & & & NO & \\
\hline s & & & & & & \\
\hline TER-610 & 4.1587 & 4.1612 & $0.060 \%$ & $\mathrm{~N} / \mathrm{C}$ & & \\
\hline C1 & & & & & YES & \\
\hline$Y \cdot 833$ & & 10250 & 003001 & N/C & & \\
\hline $\begin{array}{l}y=833 \\
D 1\end{array}$ & 4.0339 & 4.0352 & $0.032 \%$ & N/C & YES & \\
\hline o & & & & & & \\
\hline L 923 & 4.4912 & 4.4987 & $0.167 \%$ & $\mathrm{~N} / \mathrm{C}$ & & \\
\hline E1 & & & & & NO & \\
\hline A & & & & & & \\
\hline M $150-800$ & 4.2011 & 4.2057 & $0.109 \%$ & $\mathrm{~N} / \mathrm{C}$ & & \\
\hline F1 & & & & & NO & \\
\hline D & & & & & & \\
\hline$E$ & HFC-32/2S & 927 Iso-32 & $\rightarrow 24 \mathrm{HR}$ & @ 3 & $2^{\circ} \mathrm{F}$ & \\
\hline U-475 & 4.1719 & 4.1710 & $-0.022 \%$ & $\mathrm{~N} / \mathrm{C}$ & & \\
\hline A2 & & & & & NO & \\
\hline 80 & & & & & & \\
\hline $\begin{array}{l}Y-390 \\
B 2\end{array}$ & 4.4130 & 4.4145 & $0.034 \%$ & $\mathrm{~N} / \mathrm{C}$ & & \\
\hline B2 & & & & & NO & \\
\hline ER-610 & 4.1049 & 4.1041 & $.0 .019 \%$ & $\mathrm{~N} / \mathrm{C}$ & & \\
\hline & & & & & YES & \\
\hline$Y-833$ & 38998 & 38981 & $.0 .044 \%$ & $N / C$ & & \\
\hline $\mathrm{D} 2$ & & & & & YES & \\
\hline & & & 000001 & N/C & & \\
\hline $\begin{array}{l}923 \\
E 2\end{array}$ & 4.6275 & 4.6274 & & & NNO & \\
\hline & & & & & & \\
\hline ISO-800 & 4.1853 & 4.1868 & $0.036 \%$ & $\mathrm{~N} / \mathrm{C}$ & & \\
\hline & & & & & NO & \\
\hline
\end{tabular}


Varnish Disks-...HFC-32/Emery 2927 Iso-32 Oil

\begin{tabular}{|c|c|c|c|c|c|}
\hline & 500 HRS II & R-32/Emery & 2927 Iso & $-32 @ 260^{\circ}$ & \\
\hline D & WT & EXPWT & $\begin{array}{l}\text { WT \% } \\
\text { CHANGE }\end{array}$ & EXP VS & EXPFLEX \\
\hline$U .475$ & 1.9489 & 1.9639 & $0.77 \%$ & $\mathrm{~N} / \mathrm{C}$ & $\mathrm{N} / \mathrm{C}$ \\
\hline & & & & & \\
\hline$Y-390$ & 2.2456 & 2.2145 & $1.38 \%$ & Slightly & $\mathrm{N} / \mathrm{C}$ \\
\hline B1 & & & & warped & \\
\hline & & & & & \\
\hline $\begin{array}{l}\text { ER-610 } \\
C 1\end{array}$ & 2.4929 & 2.4993 & $0.26 \%$ & $N / C$ & $\mathrm{~N} / \mathrm{C}$ \\
\hline & & & & & \\
\hline $\begin{array}{l}Y-833 \\
D 1\end{array}$ & 2.0526 & 2.0369 & $-0.76 \%$ & $\mathrm{~N} / \mathrm{C}$ & $\mathrm{N} / \mathrm{C}$ \\
\hline & & & & & \\
\hline 923 & 1.7123 & 1.6802 & $-1.87 \%$ & $\mathrm{~N} / \mathrm{C}$ & $\mathrm{N} / \mathrm{C}$ \\
\hline & & & & & \\
\hline $150-800$ & 16437 & 1.5032 & $-8.55 \%$ & Sliahtly & $\sqrt{\mathrm{N} / \mathrm{C}}$ \\
\hline & & & & warped & \\
\hline & & & & lighter in & color \\
\hline & R-32/Emen & 2927 at $\ldots$ & 24 hour a & $1302^{\circ} \mathrm{F}$. & \\
\hline U-475 & 2.3728 & 2.3439 & $-1.22 \%$ & darkened & $\mathrm{N} / \mathrm{C}$ \\
\hline & & & & & \\
\hline Y-390 & 2.0966 & 2.0266 & $-3.34 \%$ & slightly & $\mathrm{N} / \mathrm{C}$ \\
\hline B2 & & & & warped & \\
\hline ER-610 & 2.1913 & 2,1974 & $0.28 \%$ & darkened & $\mathrm{N} / \mathrm{C}$ \\
\hline & & & & & \\
\hline & & & & & \\
\hline$Y-833$ & 2.3819 & 2.2983 & $-3.51 \%$ & $\mathrm{~N} / \mathrm{C}$ & $\mathrm{N} / \mathrm{C}$ \\
\hline D2 & & & & & \\
\hline 923 & 1.8446 & 1.7859 & $-3.18 \%$ & $\mathrm{~N} / \mathrm{C}$ & \\
\hline E2 & & & & & $\sqrt{\mathrm{N} / \mathrm{C}}$ \\
\hline & & & & & \\
\hline $\begin{array}{l}\text { ISO-800 } \\
\text { F2 }\end{array}$ & 1.6317 & 1.4953 & $-8.36 \%$ & $\frac{\text { slightly }}{\text { warped }}$ & $\sqrt{\mathrm{N} / \mathrm{C}}$ \\
\hline & & & & & \\
\hline
\end{tabular}




\begin{tabular}{|c|c|c|c|c|c|c|c|c|c|}
\hline & $500 \mathrm{HRS}$ & $\mathrm{s}$ in $\mathrm{HFC}$. & 32/Emery & 2927 Iso- & $32 @ 260$ & $O F$ & & & \\
\hline ID & WT & EXPWT & $\begin{array}{c}\text { WT \% } \\
\text { CHANGE }\end{array}$ & $\begin{array}{l}\text { SAMPLE } \\
\text { WIDTH }\end{array}$ & $\begin{array}{l}\text { BREAK } \\
\text { LOAD }\end{array}$ & $\begin{array}{c}\text { SAMPLE } \\
\text { THICKNESS } \\
\text { Mils }\end{array}$ & $\begin{array}{c}\text { TENSILE } \\
\text { STR } \\
\text { BASE }\end{array}$ & $\begin{array}{l}\text { TENSILE } \\
\text { STREXP }\end{array}$ & $\begin{array}{c}\% \text { CHANGE } \\
\text { TENSILE }\end{array}$ \\
\hline NO/MY/NO & 7.3929 & 7.9390 & $7.39 \%$ & 0.360 & 115.90 & 21 & 17.4 & 15.33 & \\
\hline A1 & & & & 0.436 & 136.50 & 21 & 17.4 & 14.91 & $-11.31 \%$ \\
\hline & & & & 0.465 & 156.80 & 21 & 17.4 & 16.06 & \\
\hline$D A / M Y / D A$ & 6.7978 & 6.7273 & $-1.04 \%$ & 0.421 & 115.70 & 21 & 13.7 & 13.09 & \\
\hline 81 & & & & 0.375 & 91.10 & 21 & 13.7 & 11.57 & $-8.53 \%$ \\
\hline & & & & 0.355 & 96.45 & 21 & 13.7 & 12.94 & \\
\hline MYLAR MO & 4.0289 & 4.0738 & $1.11 \%$ & 0.433 & 70.50 & 10 & 21.7 & 16.28 & \\
\hline $\mathrm{Cl}$ & & & & 0.519 & 83.40 & 10 & 21.7 & 16.07 & $.24 .82 \%$ \\
\hline & & & & 0.481 & 79.80 & 10 & 21.7 & 16.59 & \\
\hline NO 410 & 3.3988 & 3.8298 & $12.68 \%$ & 0.499 & 92.75 & 10 & 18.7 & 18.59 & \\
\hline D1 & & & & 0.451 & 77.90 & 10 & 18.7 & 17.27 & $-3.16 \%$ \\
\hline & & & & 0.495 & 91.40 & 10 & 18.7 & 18.46 & \\
\hline NO MI 418 & 3.7879 & 4.5382 & $19.81 \%$ & 0.480 & 28.85 & 10 & 7.5 & 6.01 & ' \\
\hline E1 & & & & 0.504 & 31.65 & 10 & 7.5 & 6.28 & $-18.46 \%$ \\
\hline & & & & 0.492 & 29.80 & 10 & 7.5 & 6.06 & \\
\hline MEL 228 & 4.1540 & 4.2240 & $1.69 \%$ & 0.463 & 69.15 & 10 & 21.7 & 14.94 & \\
\hline $\mathrm{F}_{1}$ & & & & 0.443 & 67.80 & 10 & 21.7 & 15.30 & $-27.67 \%$ \\
\hline & & & & 0.420 & 70.75 & 10 & 21.7 & 16.85 & \\
\hline & 500 HRS & $\mathrm{S}$ in HFC- & 32/Emery? & 2927 Iso & $32 @ 260$ & $F->24 H R$ & S@302\% & & \\
\hline NO/MY/NO & 6.9871 & 7.2587 & $3.89 \%$ & 0.378 & 108.90 & 21 & 17.4 & 13.72 & \\
\hline A2 & & & & 0.365 & 127.50 & 21 & 17.4 & 16.63 & $-9.09 \%$ \\
\hline & & & & 0.389 & 139.70 & 21 & 17.4 & 17.10 & \\
\hline$D A / M Y / D A$ & 7.4979 & 7.4215 & $-1.02 \%$ & 0.387 & 105.10 & 21 & 13.7 & 12.93 & \\
\hline $\mathrm{B2}$ & & & & 0.490 & 129.50 & 21 & 13.7 & 12.59 & $-6.15 \%$ \\
\hline & & & & 0.460 & 126.10 & 21 & 13.7 & 13.05 & \\
\hline MYLAR MO & 3.7276 & 3.7159 & $-0.31 \%$ & 0.469 & 74.70 & 10 & 21.7 & 15.93 & \\
\hline C2 & & & & 0.381 & 60.60 & 10 & 21.7 & 15.91 & $.24 .82 \%$ \\
\hline & & & & 0.412 & 70.50 & 10 & 21.7 & 17.11 & \\
\hline NOMEX 410 & 3.5330 & 3.8297 & $8.40 \%$ & 0.496 & 97.95 & 10 & 18.7 & 19.75 & \\
\hline D2 & & & & 0.463 & 88.60 & 10 & 18.7 & 19.14 & $3.33 \%$ \\
\hline & & & & 0.427 & 81.50 & 10 & 18.7 & 19.09 & \\
\hline NO/MI 418 & 3.3990 & 4.0009 & $17.71 \%$ & 0.510 & 30.90 & 10 & 7.5 & 6.06 & \\
\hline E2 & & & & 0.506 & 32.75 & 10 & 7.5 & 6.47 & $.16 .90 \%$ \\
\hline & & & & 0.484 & 29.85 & 10 & 7.5 & 6.17 & \\
\hline MEL 228 & 4.3911 & 4.4073 & $0.37 \%$ & 0.501 & 84.30 & 10 & 21.7 & 16.83 & \\
\hline $\mathrm{F} 2$ & & & & 0.508 & 85.70 & 10 & 21.7 & 16.87 & $-22.18 \%$ \\
\hline & & & & 0.461 & 78.20 & 10 & 21.7 & 16.96 & \\
\hline
\end{tabular}




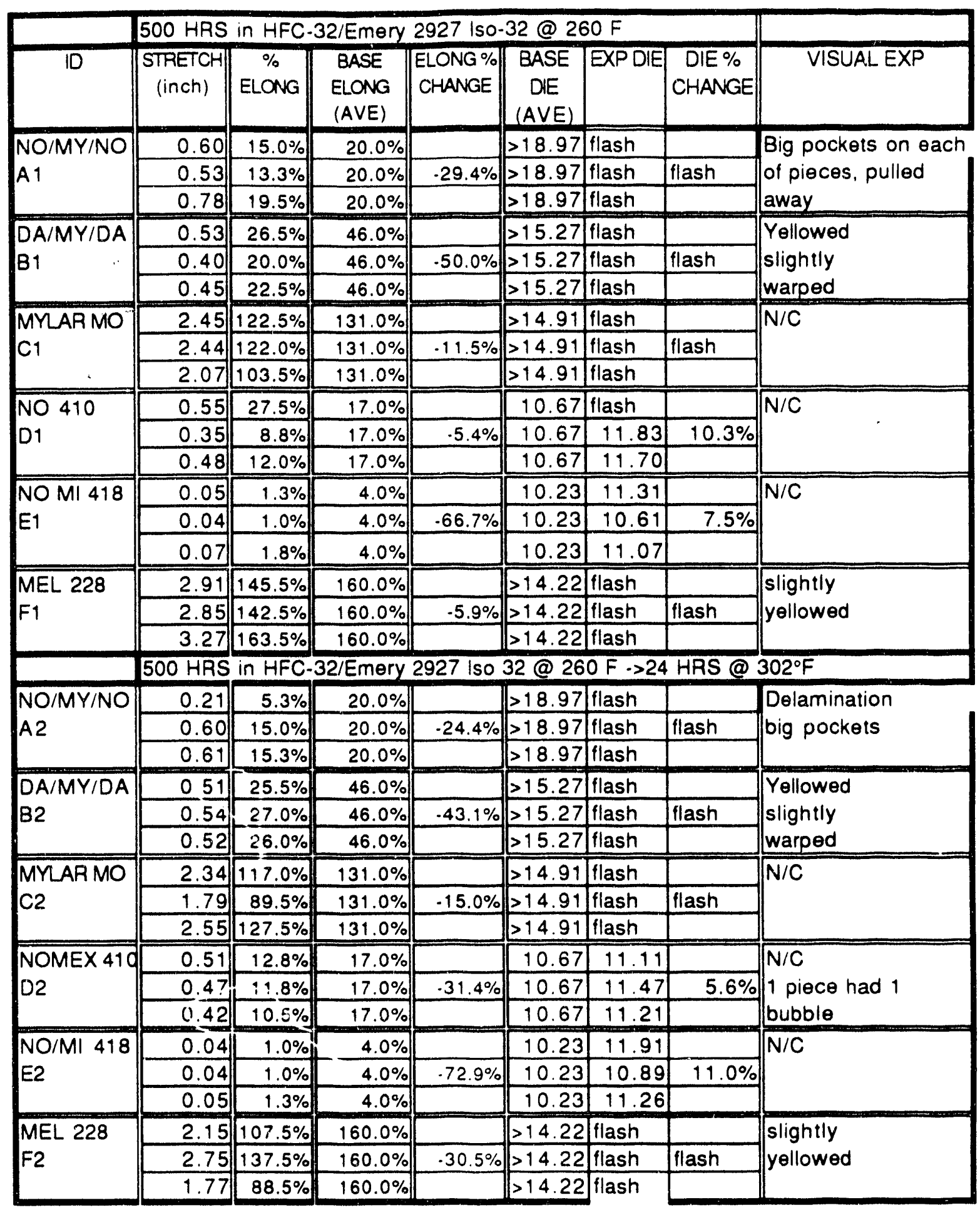




\begin{tabular}{|c|c|c|c|c|}
\hline & \multicolumn{4}{|c|}{500 HRS in HFC-32/Emery 2927 Iso. $32 @ 260^{\circ} \mathrm{F}$} \\
\hline & WT & EXPWT & $\begin{array}{c}\% \\
\text { CHANGE }\end{array}$ & EXPVS \\
\hline $\begin{array}{l}\text { A1 } \\
\text { NOMEX }\end{array}$ & 0.5445 & 0.6158 & $13.09 \%$ & $\mathrm{~N} / \mathrm{C}$ \\
\hline $\begin{array}{l}\text { B1 } \\
\text { MYLAR }\end{array}$ & 0.4589 & 0.4678 & $1.94 \%$ & $\mathrm{~N} / \mathrm{C}$ \\
\hline \multirow{2}{*}{$\begin{array}{l}\text { C1 } \\
\text { NO/MY }\end{array}$} & 0.4021 & 0.4214 & $4.80 \%$ & $\mathrm{~N} / \mathrm{C}$ \\
\hline & \multicolumn{4}{|c|}{ HFC-32/Emery Iso-32 oil @ 260 $\mathrm{F}$ plus24 hrs @ 302 $\mathrm{F}$} \\
\hline A2 & 0.5699 & 0.6109 & $7.19 \%$ & $\mathrm{~N} / \mathrm{C}$ \\
\hline $\begin{array}{l}\text { B2 } \\
\text { MYAR }\end{array}$ & 0.4529 & 0.4549 & $0.44 \%$ & $\frac{\mathrm{N} / \mathrm{C}}{\text { some pockets }}$ \\
\hline & & & \multirow{4}{*}{$2.29 \%$} & \\
\hline \multirow{3}{*}{\begin{tabular}{|l|}
$\mathrm{C} 2$ \\
$\mathrm{NO} / \mathrm{MY}$
\end{tabular}} & \multirow[t]{3}{*}{0.3969} & \multirow[t]{3}{*}{0.4060} & & $\mathrm{~N} / \mathrm{C}$ \\
\hline & & & & some pockets where mylar \\
\hline & & & & pulled away. \\
\hline
\end{tabular}




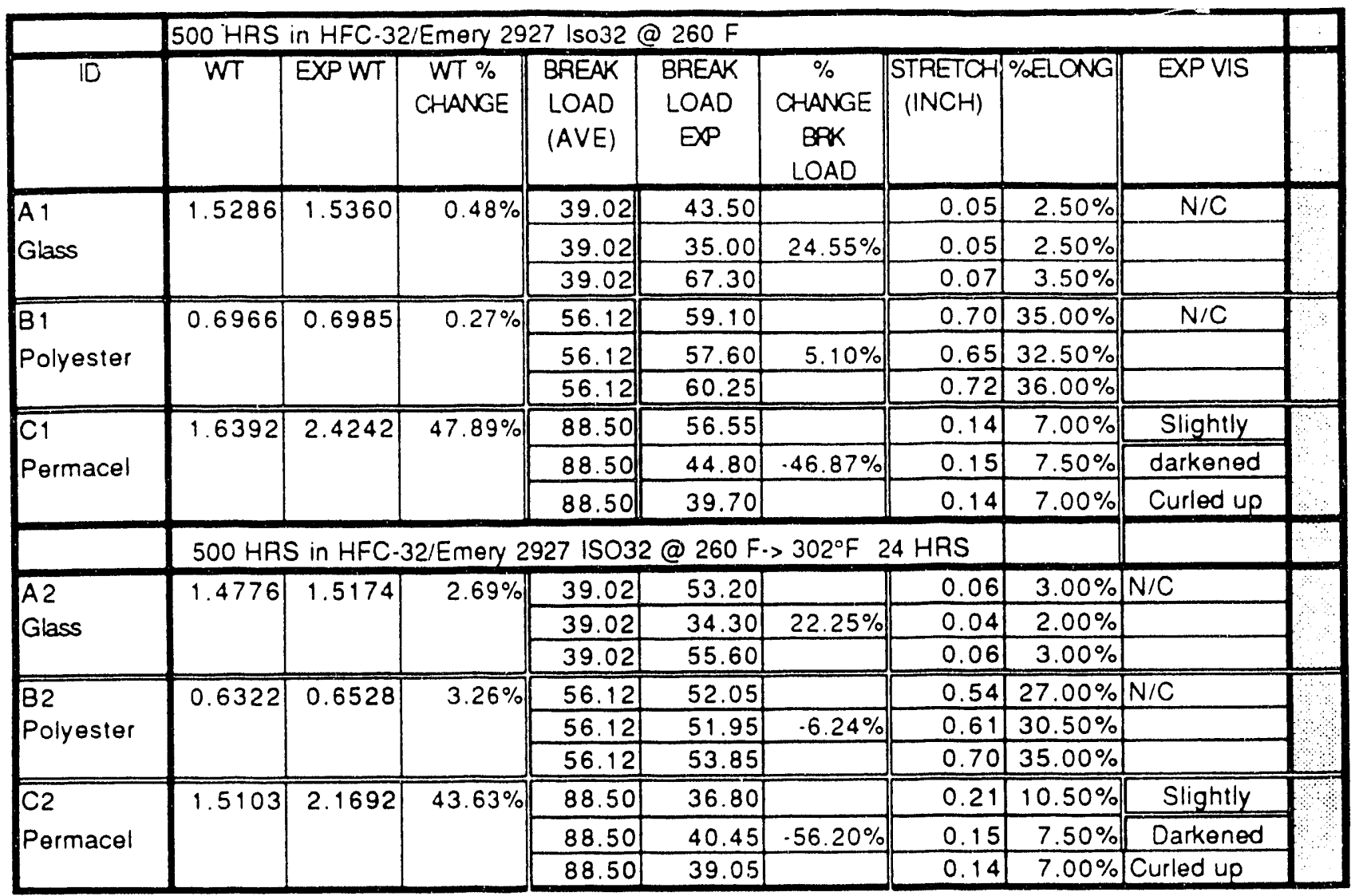


TIE CORD--HFC-32/Emery 2927 ISO-32 oil @ 260 $\mathrm{F}\left(127^{\circ} \mathrm{C}\right)$

\begin{tabular}{|c|c|c|c|c|c|c|c|c|c|}
\hline \multicolumn{10}{|c|}{500 HRS IN R-32/2927 Iso-32@ 260\% F } \\
\hline ID & WT & EXPWT & $\begin{array}{l}\text { WT \% } \\
\text { CHANGE }\end{array}$ & $\begin{array}{l}E P \\
\text { VIS }\end{array}$ & $\begin{array}{l}\text { BREAK } \\
\text { LOAD } \\
\text { (AVE) }\end{array}$ & $\begin{array}{l}\text { BREAK } \\
\text { LOAD } \\
\text { EXP } \\
(\text { ave })\end{array}$ & $\begin{array}{c}\% \\
\text { CHANGE } \\
\text { BPK } \\
\text { LOAD }\end{array}$ & $\begin{array}{c}\text { STRETCH } \\
\text { (Inch) }\end{array}$ & $\begin{array}{c}\% \\
\text { ELONG }\end{array}$ \\
\hline \multirow{4}{*}{ A 1} & \multirow[t]{3}{*}{0.3683} & \multirow[t]{3}{*}{0.3679} & \multirow[t]{3}{*}{$-0.11 \%$} & $\mathrm{~N} / \mathrm{C}$ & 28.36 & 32.10 & & 0.45 & $22.5 \%$ \\
\hline & & & & & 28.36 & 31.50 & \multirow[t]{2}{*}{$15.19 \%$} & 0.42 & $21.0 \%$ \\
\hline & & & & & 28.36 & 34.40 & & 0.44 & $22.0 \%$ \\
\hline & \multicolumn{9}{|c|}{500 HRS IN R-32/Emery 2927 -> 24 HRS @ 302 $\mathrm{F}$} \\
\hline \multirow[t]{3}{*}{ A2 } & \multirow[t]{3}{*}{0.4393} & \multirow[t]{3}{*}{0.439} & \multirow[t]{3}{*}{$-0.068 \%$} & $\mathrm{~N} / \mathrm{C}$ & 28.36 & 30.55 & & 0.46 & $23.0 \%$ \\
\hline & & & & & 28.36 & 33.30 & $15.24 \%$ & 0.45 & $22.5 \%$ \\
\hline & & & & & 28.36 & 34.20 & & 0.49 & $24.5 \%$ \\
\hline
\end{tabular}


LEAD WIRE INSULATION-HFC-32/Emery 2927 Iso-32@ $260^{\circ} \mathrm{F}\left(127^{\circ} \mathrm{C}\right)$

\begin{tabular}{|c|c|c|c|c|c|c|c|}
\hline & 500 HRS IN I & FC-32/Emer & y 2927 Is & $0-32 @ 2$ & $0^{\circ} \mathrm{F}$ & & \\
\hline ID & WT & EXPWT & $\begin{array}{c}\text { WT \% } \\
\text { CHANGE }\end{array}$ & EXP VIS & $\begin{array}{c}\text { BASE DIE } \\
(\text { A } V E)\end{array}$ & EXP DIE & $\begin{array}{c}\text { DIE\% } \\
\text { CHANGE }\end{array}$ \\
\hline DMD & 3.9348 & 4.0656 & $3.32 \%$ & $\mathrm{~N} / \mathrm{C}$ & 9.61 & 8.99 & \\
\hline A 1 & & & & & 9.61 & 8.70 & $-6.94 \%$ \\
\hline & & & & & 9.61 & 9.14 & \\
\hline DTMD & 4.1988 & 4.3136 & $2.73 \%$ & $N / C$ & 9.95 & 12.25 & \\
\hline$B 1$ & & & & & 9.95 & 11.63 & $21.17 \%$ \\
\hline & & & & & 9.95 & 12.29 & \\
\hline & HFC-32/Emer & 2927 Iso-3 & $2 @ 260^{\circ}$ & $F \rightarrow 24 \mathrm{HF}$ & IS@302\% $\mathrm{F}$ & & \\
\hline DMD & 3.9344 & 4.0072 & $1.85 \%$ & $\mathrm{~N} / \mathrm{C}$ & 9.61 & 9.32 & \\
\hline$A 2$ & & & & & 9.61 & 9.89 & $-17.59 \%$ \\
\hline & & & & & 9.61 & 4.55 & \\
\hline DTMD & 4.2128 & 4.3172 & $2.48 \%$ & $\mathrm{~N} / \mathrm{C}$ & 9.95 & 14.15 & \\
\hline 82 & & & & & 9.95 & 13.50 & $40.64 \%$ \\
\hline & & & & & 9.95 & 14.33 & \\
\hline
\end{tabular}




\section{Appendix I}

Experimental Data for HFC-125/HenkelEmery 2927 ISO-32 Exposure at $126^{\circ} \mathrm{C}\left(260^{\circ} \mathrm{F}\right)$ 


\begin{tabular}{|c|c|c|c|c|c|c|c|c|c|c|}
\hline & 0 hours & $\mathrm{HFC}-12$ & 927 Iso- & oil & & & & & & \\
\hline ID & $W T$ & EXPWT & $\begin{array}{c}\text { WT \% } \\
\text { CHANGE }\end{array}$ & EXP VS & $\begin{array}{c}\text { BASE BRN } \\
\text { OUT } \\
\text { (AVE) }\end{array}$ & $\begin{array}{c}\text { EXP BRN } \\
\text { OUT }\end{array}$ & $\begin{array}{c}\text { BRN OUT } \\
\% \\
\text { CHANGE }\end{array}$ & $\begin{array}{c}\text { BASE DIE } \\
\text { (AVE) }\end{array}$ & EXP DIE & $\begin{array}{c}\text { DIE \% } \\
\text { CHANGE }\end{array}$ \\
\hline A1 & 21.9868 & 21.9926 & $0.026 \%$ & $\mathrm{~N} / \mathrm{C}$ & 576 & 542 & & 15.80 & 15.11 & \\
\hline & & & & & 576 & 433 & $-17.6 \%$ & 15.80 & 16.10 & $-5.0 \%$ \\
\hline & & & & & 576 & 449 & & 15.80 & 13.83 & \\
\hline B1 & 24.4029 & 24.4357 & $0.134 \%$ & $\mathrm{~N} / \mathrm{C}$ & 736 & 728 & & 11.62 & 10.87 & \\
\hline & & & & & 736 & 735 & $-7.0 \%$ & 11.62 & 12.39 & $2.5 \%$ \\
\hline & & & & & 736 & 591 & & 11.62 & 12.46 & \\
\hline C1 & 22.7713 & 22.8330 & $0.271 \%$ & $\mathrm{~N} / \mathrm{C}$ & 579 & 548 & & 16.58 & 13.90 & \\
\hline & & & & & 579 & 410 & $-12.4 \%$ & 16.58 & 16.18 & $-4.0 \%$ \\
\hline & & & & & 579 & 564 & & 16.58 & 17.65 & \\
\hline & 500 hours i & HFC-125 & Emery 29 & 27 Iso-3 & 2 oil at 26 & ${ }^{\circ} \mathrm{F}\left(127^{\circ} \mathrm{C}\right)$ & plus 24 & hours at 3 & $02^{\circ} \mathrm{F}\left(150^{\circ}\right.$ & $\left.{ }^{\circ} \mathrm{C}\right)$ \\
\hline A2 & 22.8158 & 22.8188 & $0.013 \%$ & $\mathrm{~N} / \mathrm{C}$ & 576 & 350 & & 15.80 & 14.75 & \\
\hline & & & & & 576 & 473 & $-24.7 \%$ & 15.80 & 14.22 & $-10.2 \%$ \\
\hline & & & & & 576 & 478 & & 15.80 & 13.58 & \\
\hline B2 & 25.225 & 25.2291 & $0.016 \%$ & $\mathrm{~N} / \mathrm{C}$ & 736 & 728 & & 11.62 & 11.00 & \\
\hline & & & & & 736 & 582 & $-7.6 \%$ & 11.62 & 11.26 & $-3.7 \%$ \\
\hline & & & & & 736 & 730 & & 11.62 & 11.32 & \\
\hline $\mathrm{C} 2$ & 22.8455 & 22.8489 & $0.015 \%$ & $\mathrm{~N} / \mathrm{C}$ & 579 & 447 & & 16.58 & 15.13 & \\
\hline & & & & & 579 & 414 & $-20.8 \%$ & 16.58 & 12.24 & $-22.0 \%$ \\
\hline & & & & & 579 & 514 & & 16.58 & 11.44 & \\
\hline
\end{tabular}


TWISTED PAIRSMIRE AWITH VARNISH.HFC-125/Emery 2927 Iso-32 oil @260 $\mathrm{F}\left(127^{\circ} \mathrm{C}\right)$

\begin{tabular}{|c|c|c|c|c|c|c|c|c|c|c|c|}
\hline & \multirow{2}{*}{ VARN } & \multicolumn{10}{|c|}{500 hours in HFC-125/Emery 2927 Iso-32 oil @ 260 $\mathrm{F}\left(127^{\circ} \mathrm{C}\right)$} \\
\hline & & $\begin{array}{l}\text { TWISTED } \\
\text { PAIR WT }\end{array}$ & $\begin{array}{l}\text { Exp Pair } \\
\text { weight }\end{array}$ & $\begin{array}{c}\text { WT \% } \\
\text { CHANGE }\end{array}$ & $\begin{array}{l}\text { EXP } \\
\text { VIS }\end{array}$ & BASE DIE & EXPDIE & $\begin{array}{c}\text { DIE \% } \\
\text { CHANGE }\end{array}$ & $\begin{array}{l}\text { BASE } \\
\text { BUPN } \\
\text { OUT }\end{array}$ & $\begin{array}{l}\text { EXP } \\
\text { BUPN } \\
\text { OUT }\end{array}$ & $\begin{array}{c}\text { BRNOUT } \\
\% \\
\text { CHANGE }\end{array}$ \\
\hline \multirow{3}{*}{$\begin{array}{l}P \\
O \\
L\end{array}$} & \multirow{3}{*}{$\begin{array}{l}\text { U. } 475 \\
\text { A } 1\end{array}$} & \multirow[t]{3}{*}{22.4530} & \multirow[t]{3}{*}{22.4590} & \multirow[t]{3}{*}{$0.027 \%$} & $\mathrm{~N} / \mathrm{C}$ & 16.24 & 13.32 & & 430 & 290 & \\
\hline & & & & & & 16.24 & 15.38 & $-14.80 \%$ & 430 & 329 & $-23.57 \%$ \\
\hline & & & & & & 16.24 & 12.81 & & 430 & 367 & \\
\hline \multirow{3}{*}{$\begin{array}{l}Y \\
E \\
S\end{array}$} & $Y-390$ & \multirow[t]{3}{*}{23.9087} & \multirow[t]{3}{*}{23.9291} & \multirow[t]{3}{*}{$0.085 \%$} & $\mathrm{~N} / \mathrm{C}$ & 18.77 & 19.81 & & 510 & 395 & \\
\hline & \multirow{2}{*}{ B1 } & & & & & 18.77 & 19.87 & $5.82 \%$ & 510 & 394. & $-21.37 \%$ \\
\hline & & & & & & 18.77 & 19.91 & & 510 & 414 & \\
\hline$T$ & ER-610 & 22.4630 & 22.4705 & $0.033 \%$ & $\mathrm{~N} / \mathrm{C}$ & 15.57 & 11.47 & & 442 & 403 & \\
\hline$E$ & & & & & & 15.57 & 12.58 & $-22.44 \%$ & 442 & 380 & $.14 .10 \%$ \\
\hline$R$ & & & & & & 15.57 & 12.18 & & 442 & 356 & \\
\hline & $Y-833$ & 23.8725 & 23.8610 & $.0 .048 \%$ & $\mathrm{~N} / \mathrm{C}$ & 12.04 & 12.65 & & 578 & 461 & \\
\hline$P$ & D1 & & & & & 12.04 & 13.45 & $10.33 \%$ & 578 & 457 & $-20.93 \%$ \\
\hline 0 & & & & & & 12.04 & 13.75 & & 578 & 453 & \\
\hline$L$ & 923 & 22.7707 & 22.7991 & $0.125 \%$ & $\mathrm{~N} / \mathrm{C}$ & 16.76 & 13.24 & & 606 & 551 & \\
\hline$Y$ & E1 & & & & & 16.76 & 19.10 & $-11.18 \%$ & 606 & 484 & $-17.88 \%$ \\
\hline$A$ & & & & & & 16.76 & 12.32 & & 606 & 458 & \\
\hline$M$ & $150-800$ & 23.7819 & 23.7958 & $0.058 \%$ & $\mathrm{~N} / \mathrm{C}$ & 19.08 & 17.40 & & 580 & 572 & \\
\hline 1 & & & & & & 19.08 & 18.24 & $-5.19 \%$ & 580 & 565 & $\therefore 43 \%$ \\
\hline$D$ & & & & & & 19.08 & 18.63 & & 580 & 526 & \\
\hline$E$ & & 500 hours & in HFC-1 & 25/Emery & 2927 & Iso-32 oil & @ 260 $\mathrm{F}$ & $\left(127^{\circ} \mathrm{C}\right)$ & Slus $24 \mathrm{~h}$ & ours@ & $302^{\circ} \mathrm{F}($ \\
\hline & $U-475$ & 23.2361 & 23.2368 & $0.003 \%$ & $\mathrm{~N} / \mathrm{C}$ & 16.24 & \begin{tabular}{|l|}
10.89 \\
\end{tabular} & & 430 & 367 & \\
\hline 1 & $A 2$ & & & & & 16.24 & 11.37 & $-34.48 \%$ & 430 & 373 & $-12.79 \%$ \\
\hline$M$ & & & & & & 16.24 & 9.66 & & 430 & 385 & \\
\hline 1 & $Y \cdot 390$ & 23.8013 & 23.8185 & $0.072 \%$ & $\mathrm{~N} / \mathrm{C}$ & 18.77 & 18.24 & & 510 & 393 & \\
\hline$D$ & 82 & & & & & 18.77 & 17.41 & $-1.19 \%$ & 510 & 383 & $-23.73 \%$ \\
\hline$E$ & & & & & & 18.77 & 19.99 & & 510 & 391 & \\
\hline & ER-610 & 22.3660 & 22.3682 & $0.010 \%$ & $\mathrm{~N} / \mathrm{C}$ & 15.57 & 8.77 & & 442 & 378 & \\
\hline & & & & & & 15.57 & 12.21 & $-27.66 \%$ & 442 & 370 & $-12.07 \%$ \\
\hline & & & & & & 15.57 & 12.81 & & 442 & 418 & \\
\hline & $Y-833$ & 23.5070 & 23.4905 & $-0.070 \%$ & $\mathrm{~N} / \mathrm{C}$ & 12.04 & 10.85 & & 578 & 457 & \\
\hline & D2 & & & & & 12.04 & 11.22 & $-7.75 \%$ & 578 & 489 & $-19.09 \%$ \\
\hline & & & & & & 12.04 & 11.25 & & 578 & 457 & \\
\hline & 923 & 22.9660 & 22.9776 & $0.051 \%$ & $\mathrm{~N} / \mathrm{C}$ & 16.76 & 19.60 & & 606 & 532 & \\
\hline & E2 & & & & & 16.76 & 15.30 & $8.91 \%$ & 606 & 573 & $-11.33 \%$ \\
\hline & & & & & & 16.76 & 19.86 & & 606 & 507 & \\
\hline & ISO-800 & 23.5627 & 23.5685 & $0.025 \%$ & $\mathrm{~N} / \mathrm{C}$ & 19.08 & 13.48 & & 580 & 578 & \\
\hline & F2 & & & & & 19.08 & $\frac{11.74}{15.27}$ & $-29.26 \%$ & 580 & 462 & $.6 .95 \%$ \\
\hline & & & & & & 19.08 & 15.27 & & 580 & 579 & \\
\hline
\end{tabular}


TWISTED PAIRSWIRE BWITH VARNISH -HFC-125/Emery 2927 Iso-32 oil @260 $\mathrm{F}\left(127^{\circ} \mathrm{C}\right)$

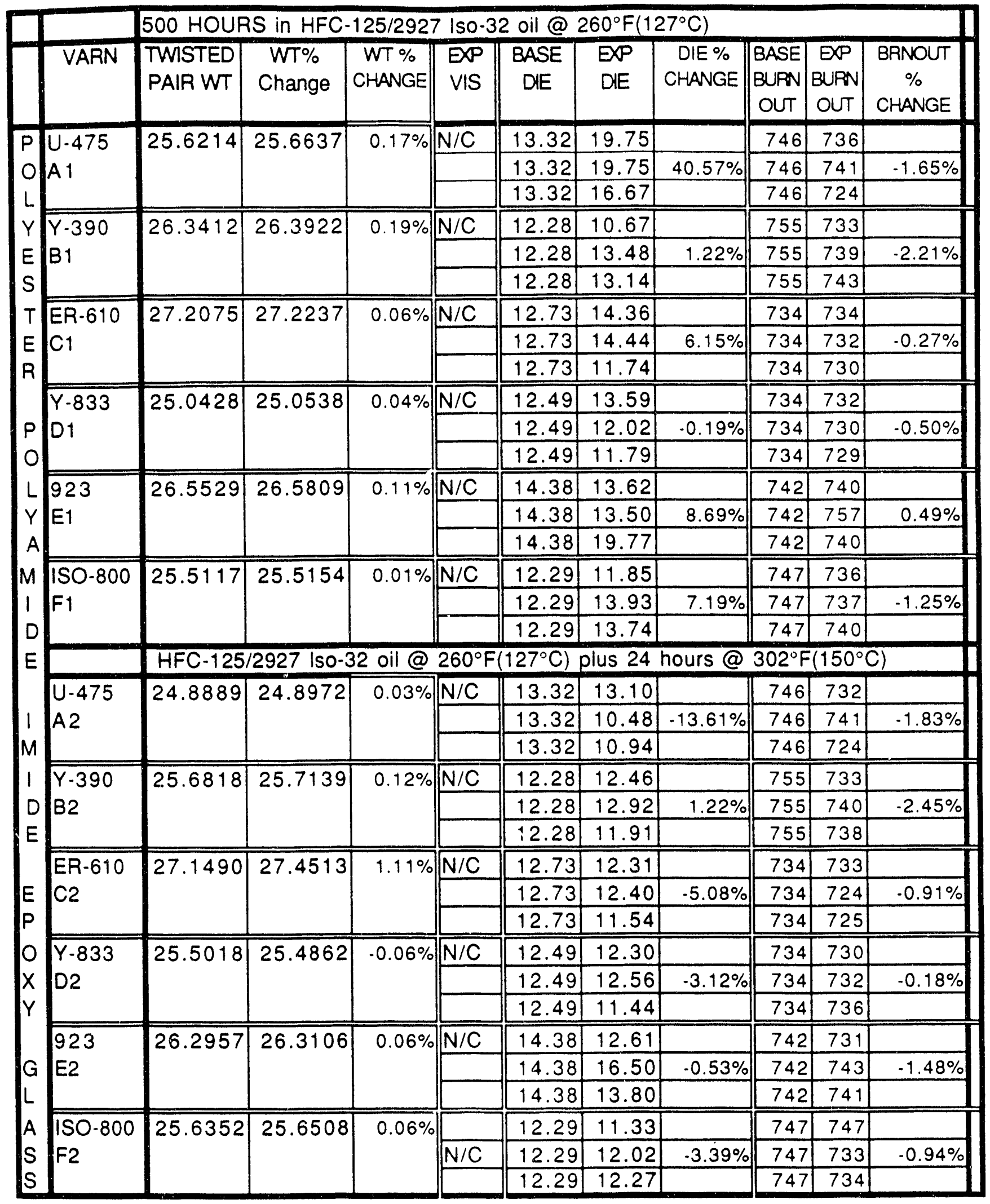


TWISTED PAIRS/WIRE CNITH VARNISH-HFC-125/Emery 2927 Iso-32@ $260^{\circ} \mathrm{F}\left(127^{\circ} \mathrm{C}\right)$

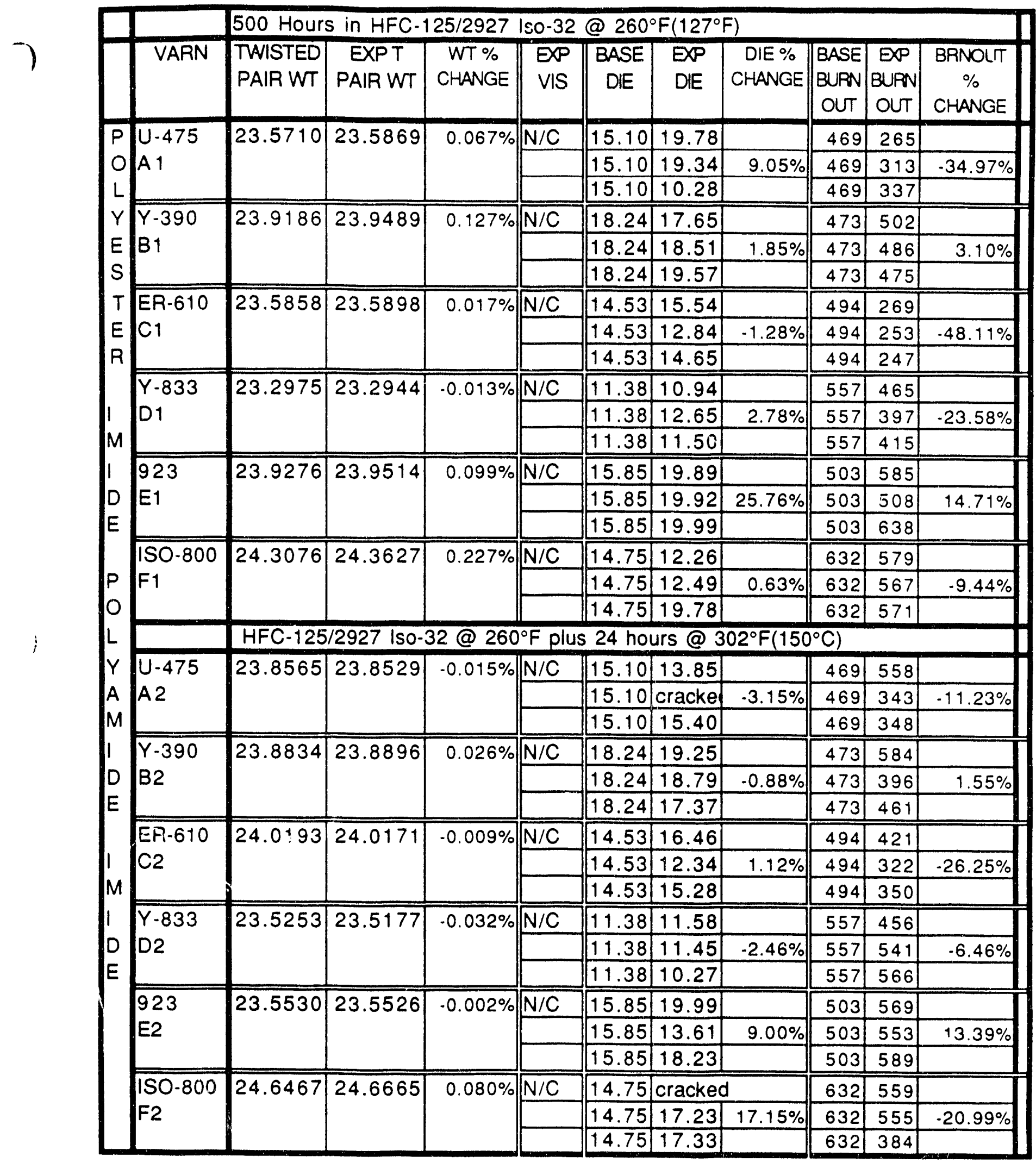




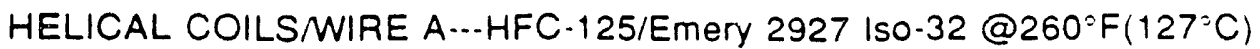

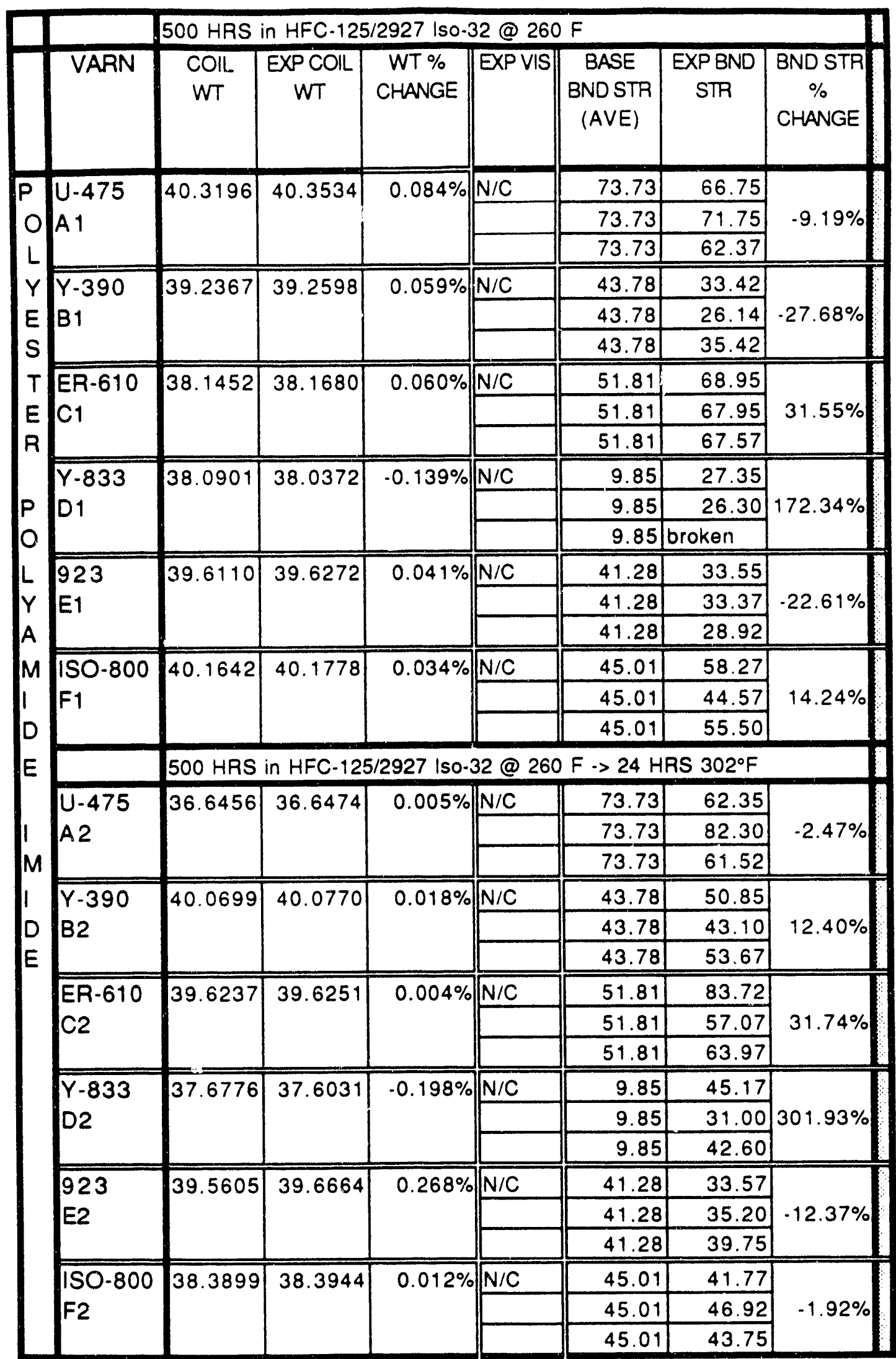




\begin{tabular}{|c|c|c|c|c|c|c|c|c|}
\hline & & $500 \mathrm{HRS}$ & in $\mathrm{HFC}-1$ & 5/Emery ? & 27 Iso-: & $60 \mathrm{~F}$ & & \\
\hline & VARN & $\begin{array}{l}\text { COIL } \\
W T\end{array}$ & $\begin{array}{c}\text { EXP COIL } \\
W T\end{array}$ & $\begin{array}{c}\text { WT \% } \\
\text { CHANGE }\end{array}$ & EXPVIS & \begin{tabular}{|c|} 
BASE \\
BND STR \\
(AVE)
\end{tabular} & $\begin{array}{c}\text { EXPBND } \\
\text { STR }\end{array}$ & $\begin{array}{c}\text { BND STR } \\
\% \\
\text { CHANGE }\end{array}$ \\
\hline$P$ & U-475 & 37.0686 & 37.1048 & $0.098 \%$ & $\mathrm{~N} / \mathrm{C}$ & 40.14 & 31.55 & \\
\hline 0 & A 1 & & & & & 40.14 & 28.85 & $-25.12 \%$ \\
\hline L & & & & & & 40.14 & 29.77 & \\
\hline Y & $Y-390$ & 37.9747 & 38.0096 & $0.092 \%$ & $\mathrm{~N} / \mathrm{C}$ & 36.12 & 40.27 & \\
\hline$E$ & B1 & & & & & 36.12 & 41.32 & $14.40 \%$ \\
\hline$S$ & & & & & & 36.12 & 42.37 & \\
\hline$T$ & ER-610 & 36.7359 & 36.7681 & $0.088 \%$ & $\mathrm{~N} / \mathrm{C}$ & 35.96 & 27.67 & \\
\hline$E$ & C1 & & & & & 35.96 & 30.25 & $.20 .87 \%$ \\
\hline R & & & & & & 35.96 & 27.45 & \\
\hline & $Y-833$ & 35.6944 & 35.6795 & $.0 .042 \%$ & $\mathrm{~N} / \mathrm{C}$ & 33.14 & 48.87 & \\
\hline$P$ & D1 & & & & & 33.14 & 25.02 & $20.14 \%$ \\
\hline 0 & & & & & & 33.14 & 45.55 & \\
\hline$L$ & 923 & $36.9907 \mid$ & 37.0113 & $0.056 \%$ & $\mathrm{~N} / \mathrm{C}$ & 40.52 & 37.70 & \\
\hline$Y$ & E1 & & & & & 40.52 & 37.97 & $-8.75 \%$ \\
\hline A & & & & & & 40.52 & 35.25 & \\
\hline$M$ & | $150-800$ & 36.2408 & 36.2695 & $0.079 \%$ & $N / C$ & 20.20 & 10.95 & \\
\hline 1 & F1 & & & & & 20.20 & 11.95 & $-26.73 \%$ \\
\hline D & & & & & & 20.20 & 21.50 & \\
\hline$E$ & & $500 \mathrm{HRS}$ & in HFC- 12 & 5/Emery 29 & 27 Iso-3? & @ $260 \mathrm{~F}$ & $>24$ HRS & $302^{\circ} \mathrm{F}$ \\
\hline & $U-475$ & 36.4628 & 36.4722 & $0.026 \%$ & $N / C$ & 40.14 & 28.50 & \\
\hline I & $A 2$ & & & & & 40.14 & 36.47 & $-16.43 \%$ \\
\hline$M$ & & & & & & 40.14 & 35.67 & \\
\hline 1 & $Y-390$ & 37.3953 & 37.4210 & $0.069 \%$ & $\mathrm{~N} / \mathrm{C}$ & 36.12 & 43.17 & \\
\hline$D$ & B2 & & & & & 36.12 & 44.05 & $15.40 \%$ \\
\hline$E$ & & & & & & 36.12 & 37.82 & \\
\hline & ER-610 & 37.2815 & 36.9642 & $.0 .851 \%$ & $N / C$ & 35.96 & 28.82 & \\
\hline$E$ & C2 & & & & & 35.96 & 29.45 & $-15.15 \%$ \\
\hline$P$ & & & & & & 35.96 & 33.27 & \\
\hline 0 & $Y-833$ & 37.0231 & 37.2908 & $0.723 \%$ & $\mathrm{~N} / \mathrm{C}$ & 33.14 & 40.87 & \\
\hline$x$ & D2 & & & & & 33.14 & 28.20 & $7.87 \%$ \\
\hline$Y$ & & & & & & 33.14 & 38.17 & \\
\hline & 923 & 37.2594 & 37.2690 & $0.026 \%$ & $\mathrm{~N} / \mathrm{C}$ & 40.52 & 30.02 & \\
\hline G & E2 & & & & & 40.52 & 32.42 & $-19.46 \%$ \\
\hline$L$ & & & & & & 40.52 & 35.47 & \\
\hline A & ISO-800 & 36.7570 & 36.7698 & $0.035 \%$ & $\mathrm{~N} / \mathrm{C}$ & 20.20 & 17.52 & \\
\hline$S$ & F2 & & & & & 20.20 & 20.87 & $-3.20 \%$ \\
\hline S & & & & & & 20.20 & 20.27 & \\
\hline
\end{tabular}




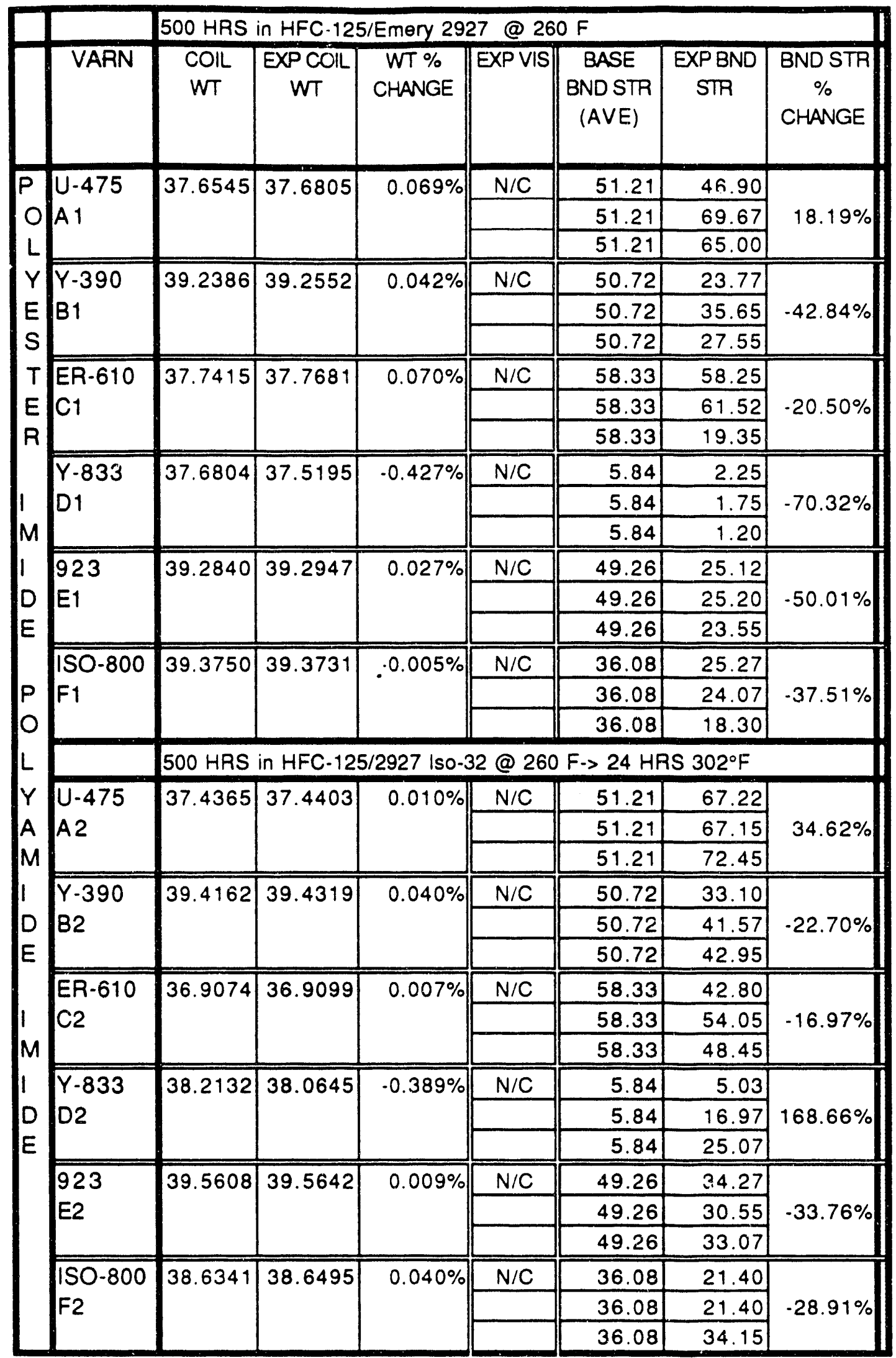


SINGLE MAG WIRE WITHOUT VARNISH-HFC-125/Emery 2927 Iso-32@260 $\mathrm{F}\left(127^{\circ} \mathrm{C}\right)$ )

\begin{tabular}{|c|c|c|c|c|c|}
\hline \multicolumn{6}{|c|}{500 HRS in HFC-125/2927 Iso-32 oil @ 260 $\mathrm{F}\left(127^{\circ} \mathrm{C}\right)$} \\
\hline ID & WT & EXPWT & $\begin{array}{c}\text { WT \% } \\
\text { CHANGE }\end{array}$ & EXP VS & EXPFLEX \\
\hline \multirow[t]{2}{*}{ A1 } & \multirow[t]{2}{*}{3.0322} & \multirow[t]{2}{*}{3.0329} & \multirow[t]{2}{*}{$0.023 \%$} & $\mathrm{~N} / \mathrm{C}$ & \\
\hline & & & & & YES \\
\hline \multirow{2}{*}{$\overline{B 1}$} & \multirow{2}{*}{3.3354} & \multirow{2}{*}{3.3400} & \multirow{2}{*}{$0.138 \%$} & $\mathrm{~N} / \mathrm{C}$ & \\
\hline & & & & & YES \\
\hline \multirow{2}{*}{$\overline{\mathrm{C} 1}$} & \multirow{2}{*}{3.3243} & \multirow{2}{*}{3.3244} & \multirow{2}{*}{$0.003 \%$} & $\mathrm{~N} / \mathrm{C}$ & \\
\hline & & & & & YES \\
\hline \multicolumn{6}{|c|}{$500 \mathrm{HRS}$ in HFC-125/ 2927 Iso-32-> $302^{\circ} \mathrm{F}$ for $24 \mathrm{HRS}$} \\
\hline \multirow{2}{*}{ A2 } & \multirow{2}{*}{3.1630} & \multirow[t]{2}{*}{3.1623} & \multirow[t]{2}{*}{$-0.022 \%$} & $N / C$ & \\
\hline & & & & & YES \\
\hline \multirow[t]{2}{*}{$\mathrm{B2}$} & \multirow[t]{2}{*}{3.6183} & \multirow[t]{2}{*}{3.6187} & \multirow[t]{2}{*}{$0.011 \%$} & $\mathrm{~N} / \mathrm{C}$ & \\
\hline & & & & & YES \\
\hline \multirow[t]{3}{*}{$\mathrm{C} 2$} & \multirow[t]{3}{*}{3.2230} & \multirow[t]{3}{*}{3.2228} & \multirow[t]{3}{*}{$-0.006 \%$} & $N / C$ & \\
\hline & & & & & YES \\
\hline & & & & & \\
\hline
\end{tabular}




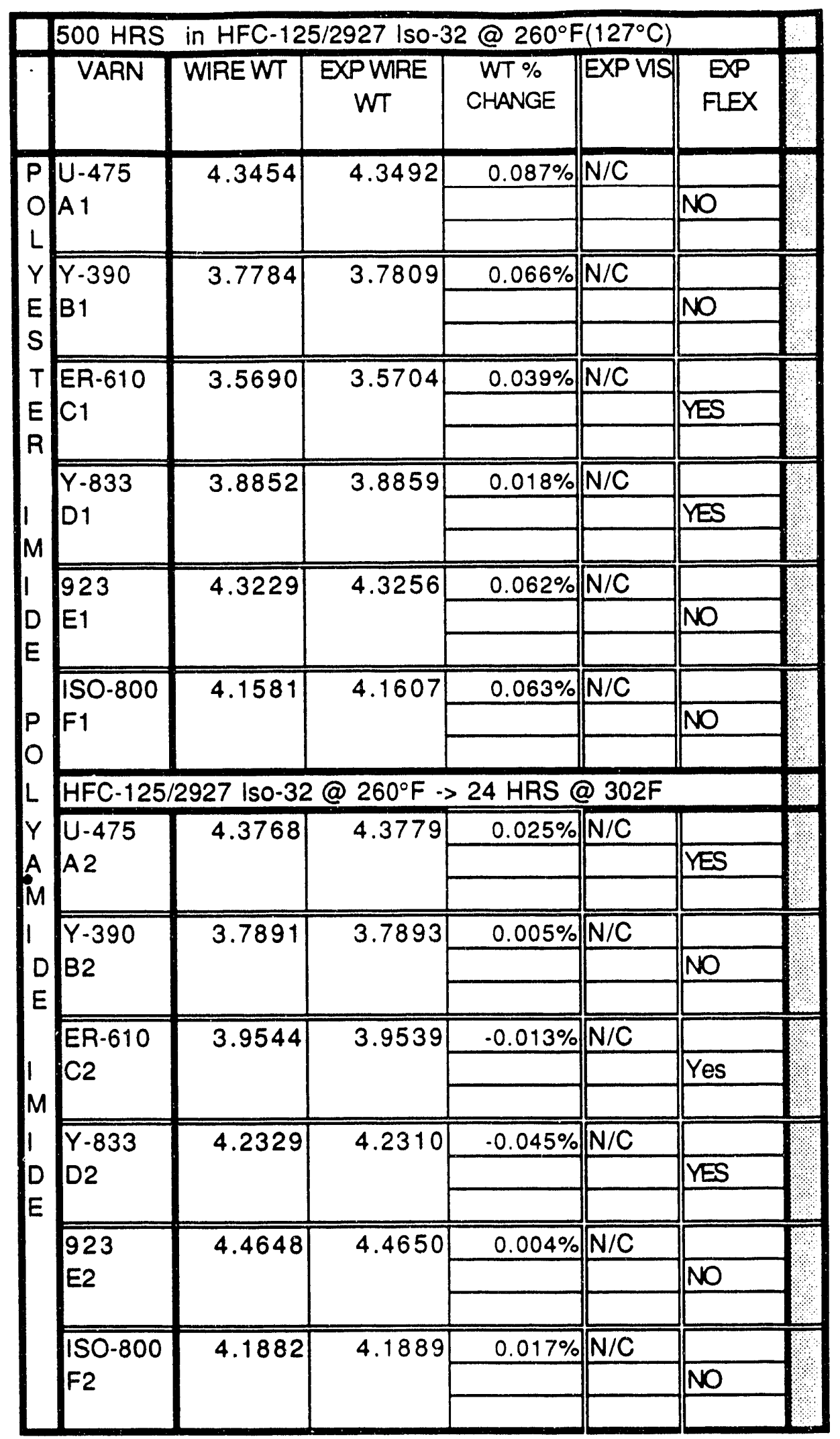




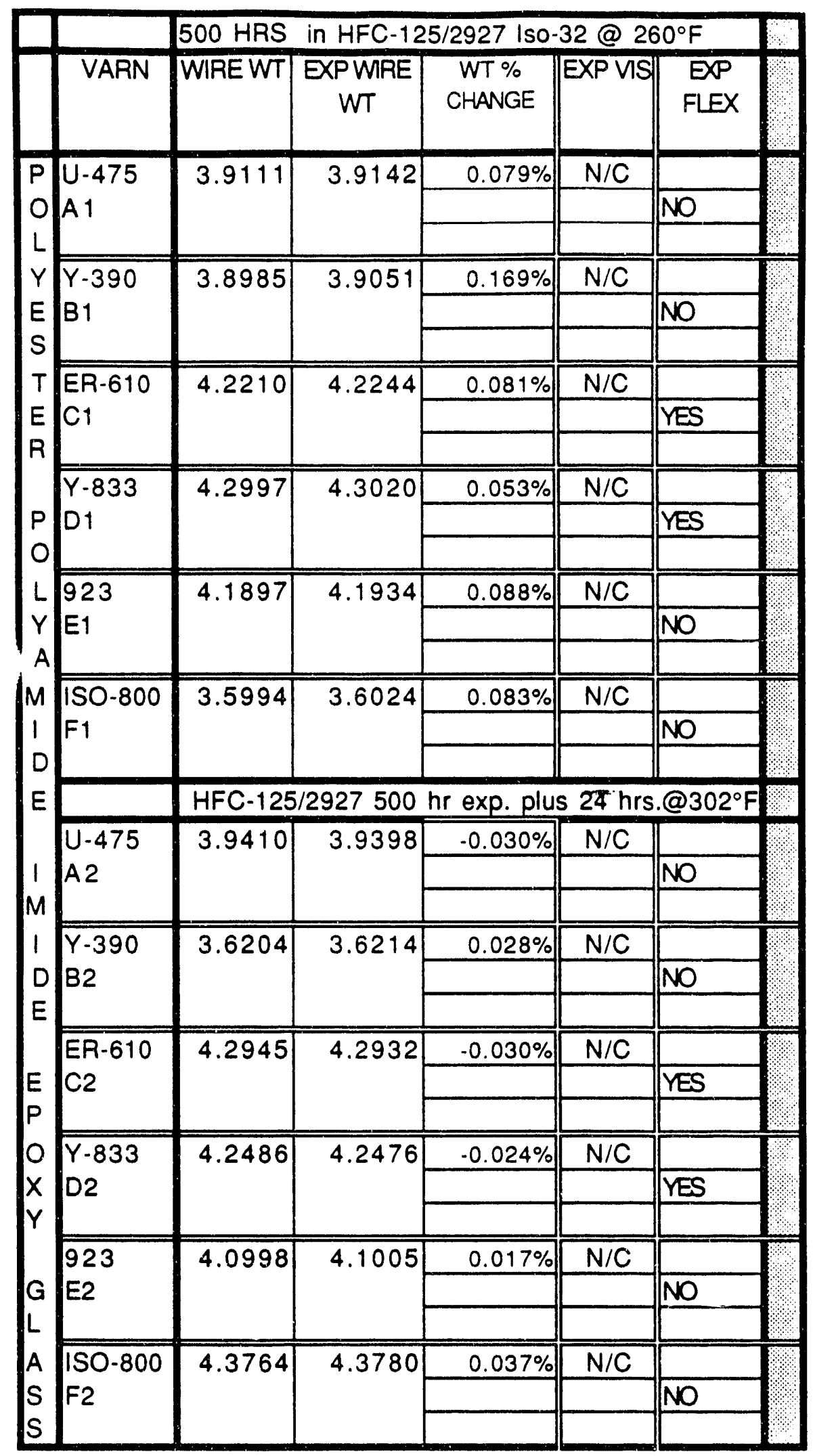

J 


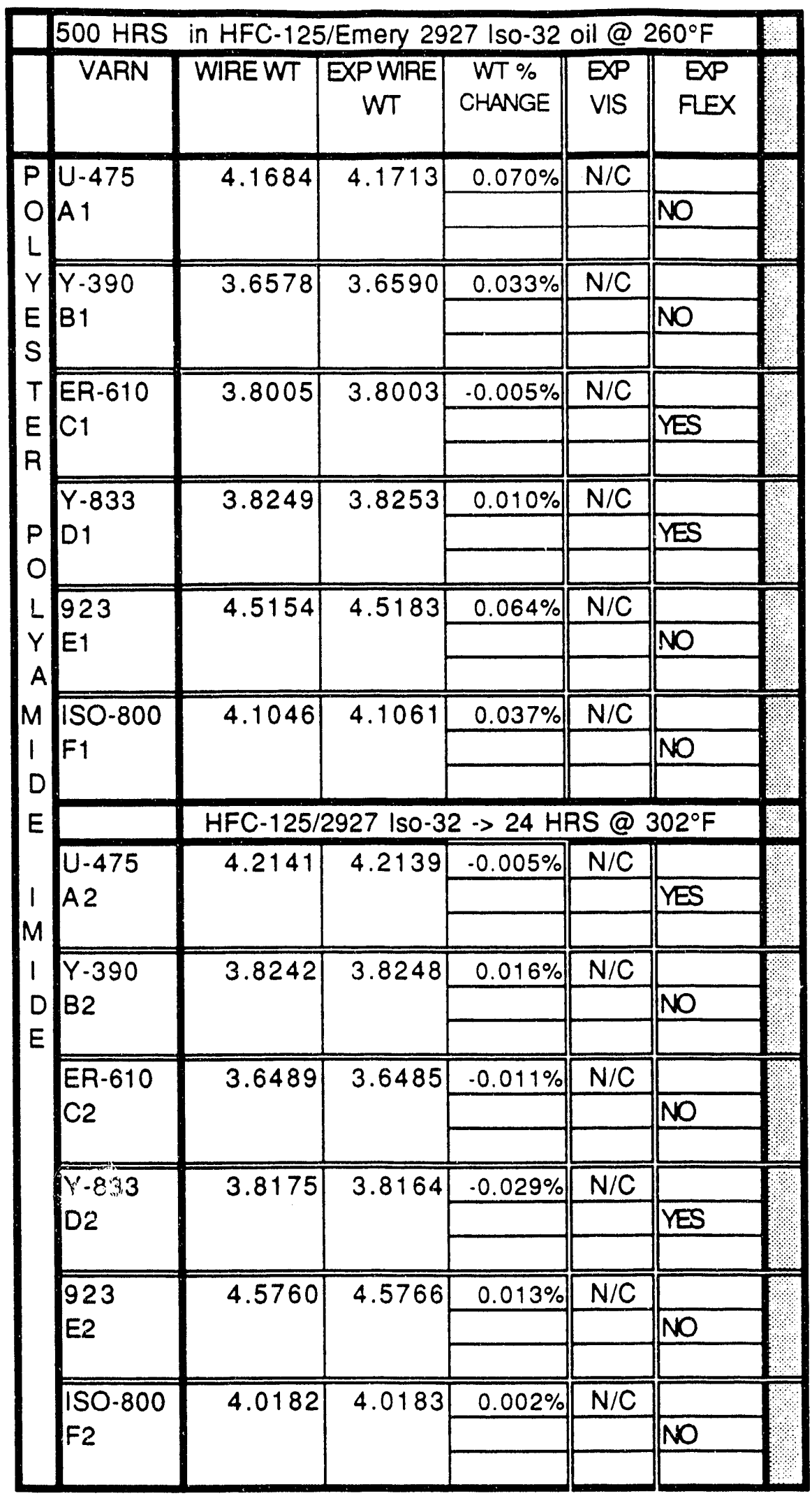


Varnish Disks-...-HFC-125/Emery 2927 Iso-32 Oil

\begin{tabular}{|c|c|c|c|c|c|}
\hline \multicolumn{6}{|c|}{500 HRS IN R-125/Emery 2927 Iso -32@ 260F } \\
\hline ID & WT & EXPWT & $\begin{array}{c}\text { WT \% } \\
\text { CHANGE }\end{array}$ & EXP VIS & EXP FLEX \\
\hline $\begin{array}{l}U-475 \\
A 1\end{array}$ & 2.6493 & 2.5922 & $-2.16 \%$ & $\mathrm{~N} / \mathrm{C}$ & $\mathrm{N} / \mathrm{C}$ \\
\hline $\begin{array}{l}Y-390 \\
B 1\end{array}$ & 2.1438 & 2.1044 & $1.84 \%$ & \begin{tabular}{|l} 
Slightly \\
warped \\
\end{tabular} & $\mathrm{N} / \mathrm{C}$ \\
\hline $\begin{array}{l}\text { ER-610 } \\
\text { C1 }\end{array}$ & 2.4810 & 2.5116 & $1.23 \%$ & $N / C$ & $\mathrm{~N} / \mathrm{C}$ \\
\hline $\begin{array}{l}Y-833 \\
D 1\end{array}$ & 2.6583 & 2.6801 & $0.82 \%$ & \begin{tabular}{|l|l|l|l|l|} 
greened \\
\end{tabular} & $\mathrm{N} / \mathrm{C}$ \\
\hline $\begin{array}{l}923 \\
\text { E1 }\end{array}$ & 2.2984 & 2.2518 & $-2.03 \%$ & \begin{tabular}{|l} 
slightly \\
warped
\end{tabular} & $\mathrm{N} / \mathrm{C}$ \\
\hline $\begin{array}{l}\mathrm{ISO}-800 \\
\mathrm{~F} 1\end{array}$ & 2.1103 & 2.0774 & $-1.56 \%$ & \begin{tabular}{|l|} 
Slightly \\
warped \\
lighter in
\end{tabular} & \begin{tabular}{|l} 
N/C \\
color \\
\end{tabular} \\
\hline & R-125/Eme & 2927 at -. & $>24$ hour & at $302^{\circ} \mathrm{F}$. & \\
\hline $\begin{array}{l}U-475 \\
A 2\end{array}$ & 2.3801 & 2.3084 & $-3.01 \%$ & darkened & $\mathrm{N} / \mathrm{C}$ \\
\hline $\begin{array}{l}Y-390 \\
B 2\end{array}$ & 1.9410 & 1.8872 & $-2.77 \%$ & \begin{tabular}{|l} 
darkened \\
slightly \\
warped \\
\end{tabular} & $\mathrm{N} / \mathrm{C}$ \\
\hline $\begin{array}{l}\text { ER-610 } \\
\text { C2 }\end{array}$ & 2.9145 & 2.9043 & $-0.35 \%$ & darkened & $N / C$ \\
\hline $\begin{array}{l}Y-833 \\
D 2\end{array}$ & 4.0109 & $4 . C 258$ & $0.37 \%$ & $N / C$ & $\mathrm{~N} / \mathrm{C}$ \\
\hline $\begin{array}{l}923 \\
E 2\end{array}$ & 2.4654 & 2.4104 & $-2.23 \%$ & \begin{tabular}{|l} 
slightly \\
warped
\end{tabular} & $\mathrm{N} / \mathrm{C}$ \\
\hline $\begin{array}{l}\text { ISO-800 } \\
\text { F2 }\end{array}$ & 2.5525 & 2.4927 & $-2.34 \%$ & \begin{tabular}{|l} 
slightly \\
warped \\
darkened \\
\end{tabular} & $N / C$ \\
\hline
\end{tabular}




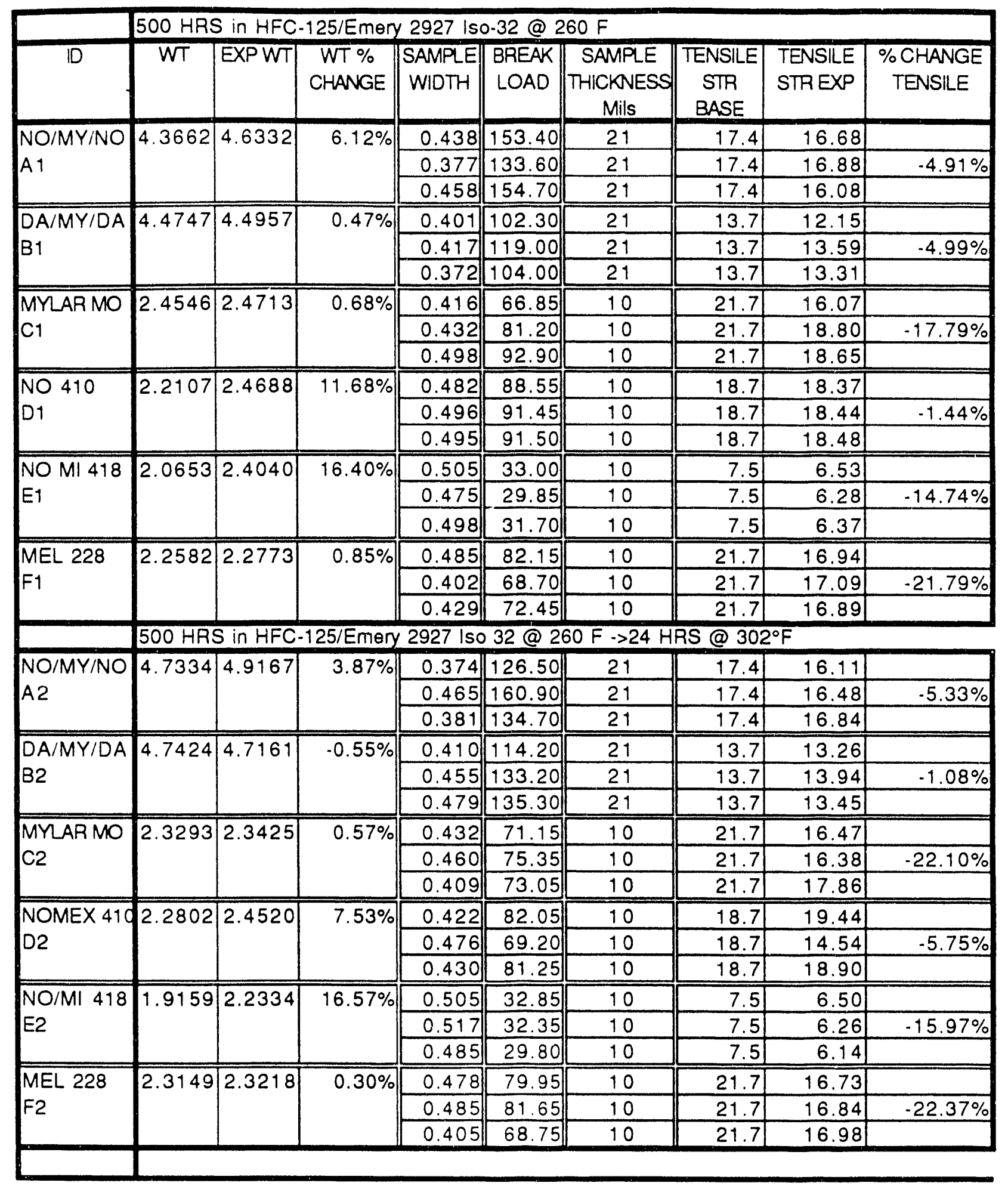




\begin{tabular}{|c|c|c|c|c|c|c|c|c|}
\hline & \multicolumn{7}{|c|}{500 HRS in HFC-125/Emery 2927 Iso-32 @ 260 F } & \multirow[b]{2}{*}{ VISUAL EXP } \\
\hline ID & $\begin{array}{c}\text { STRETCH } \\
\text { (inch) }\end{array}$ & $\begin{array}{c}\% \\
\text { ELONG }\end{array}$ & $\begin{array}{l}\text { BASE } \\
\text { ELONG } \\
\text { (AVE) }\end{array}$ & $\begin{array}{l}\text { ELONG \% } \\
\text { CHANGE }\end{array}$ & $\begin{array}{c}\text { BASE } \\
\text { DIE } \\
(A \vee E)\end{array}$ & EXPDIE & $\begin{array}{c}\text { DIE \% } \\
\text { CHANGE }\end{array}$ & \\
\hline \multirow{3}{*}{$\begin{array}{l}\mathrm{NO} / \mathrm{MY} / \mathrm{NO} \\
\mathrm{A} 1\end{array}$} & 0.64 & $16.0 \%$ & $20.0 \%$ & & $>18.97$ & flash & & \multirow[t]{3}{*}{$\mathrm{N} / \mathrm{C}$} \\
\hline & 0.55 & $13.8 \%$ & $20.0 \%$ & $.25 .6 \%$ & $>18.97$ & flash & flash & \\
\hline & 0.47 & $11.8 \%$ & $20.0 \%$ & & $>18.97$ & flash & & \\
\hline \multirow{3}{*}{$\begin{array}{l}D A / M Y / D A \\
B 1\end{array}$} & 0.48 & $24.0 \%$ & $46.0 \%$ & & $\geq 15.27$ & flash & & \multirow{3}{*}{$\begin{array}{l}\text { slightly yellowed } \\
\text { slightly } \\
\text { warped }\end{array}$} \\
\hline & 0.53 & $26.5 \%$ & $46.0 \%$ & $-47.5 \%$ & $>15.27$ & flash & flash & \\
\hline & 0.44 & $22.0 \%$ & $46.0 \%$ & & $>15.27$ & flash & & \\
\hline \multirow{3}{*}{$\begin{array}{l}\text { MYLAR MO } \\
C_{1}\end{array}$} & 2.44 & $122.0 \%$ & $131.0 \%$ & & $>14.91$ & flash & & \multirow[t]{3}{*}{$\mathrm{N} / \mathrm{C}$} \\
\hline & 2.82 & $141.0 \%$ & $131.0 \%$ & $5.3 \%$ & $>14.91$ & flash & flash & \\
\hline & 3.02 & $151.0 \%$ & $131.0 \%$ & & $>14.91$ & flash & & \\
\hline \multirow{3}{*}{$\begin{array}{l}\text { NO } 410 \\
\text { D1 }\end{array}$} & 0.39 & $19.5 \%$ & $17.0 \%$ & & 10.67 & flash & & \multirow[t]{3}{*}{$\mathrm{N} / \mathrm{C}$} \\
\hline & 0.36 & $9.0 \%$ & $17.0 \%$ & $.27 .9 \%$ & 10.67 & flash & flash & \\
\hline & 0.33 & $8.3 \%$ & $17.0 \%$ & & 10.67 & flash & & \\
\hline \multirow{3}{*}{$\begin{array}{l}\text { NO MI } 418 \\
\text { E1 }\end{array}$} & 0.04 & $1.0 \%$ & $4.0 \%$ & & 10.23 & 8.81 & & \multirow{3}{*}{$N / C$} \\
\hline & 0.05 & $1.3 \%$ & $4.0 \%$ & $.72 .9 \%$ & 10.23 & 9.01 & $-14.2 \%$ & \\
\hline & 0.04 & $1.0 \%$ & $4.0 \%$ & & 10.23 & 8.51 & & \\
\hline \multirow{3}{*}{$\begin{array}{l}\text { MEL } 228 \\
F 1\end{array}$} & 2.85 & $142.5 \%$ & $160.0 \%$ & & $>14.22$ & flash & & \multirow{3}{*}{$\begin{array}{l}\text { very slightly } \\
\text { yellowed }\end{array}$} \\
\hline & 2.29 & $114.5 \%$ & $160.0 \%$ & $.17 .6 \%$ & $>14.22$ & flash & flash & \\
\hline & 2.77 & $138.5 \%$ & $160.0 \%$ & & $>14.22$ & flash & & \\
\hline & 500 HRS & in $\mathrm{HFC}-1$ & 25/Emery & 2927 Iso & 32@26 & $60 \mathrm{~F} \rightarrow 2$ & 24 & $302^{\circ} \mathrm{F}$ \\
\hline \multirow{3}{*}{$\begin{array}{l}\text { NO/MY/NO } \\
\text { A2 }\end{array}$} & 0.45 & $11.3 \%$ & $20.0 \%$ & & $>18.97$ & flash & & \multirow{3}{*}{$\begin{array}{l}\text { delamination } \\
\text { big pockets }\end{array}$} \\
\hline & 0.51 & $12.8 \%$ & $20.0 \%$ & $-24.4 \%$ & $>18.97$ & flash & flash & \\
\hline & 0.70 & $17.5 \%$ & $20.0 \%$ & & $>18.97$ & flash & & \\
\hline \multirow{3}{*}{$\begin{array}{l}\mathrm{DA} / \mathrm{MY} / \mathrm{DA} \\
\mathrm{B} 2\end{array}$} & 0.49 & $24.5 \%$ & $46.0 \%$ & & $\geq 15.27$ & flash & & \multirow{3}{*}{$\begin{array}{l}\text { Yellowed/Browned } \\
\text { slightly } \\
\text { warped }\end{array}$} \\
\hline & 0.49 & $24.5 \%$ & $46.0 \%$ & $.46 .4 \%$ & $>15.27$ & flash & flash & \\
\hline & 0.50 & $25.0 \%$ & $46.0 \%$ & & $>15.27$ & flash & & \\
\hline \multirow{3}{*}{$\begin{array}{l}\text { MYLAR MO } \\
\text { C2 }\end{array}$} & 2.19 & $109.5 \%$ & $131.0 \%$ & & $>14.91$ & flash & & \multirow[t]{3}{*}{$\mathrm{N} / \mathrm{C}$} \\
\hline & 2.12 & $106.0 \%$ & $131.0 \%$ & $-9.7 \%$ & $>14.91$ & flash & flash & \\
\hline & 2.79 & $139.5 \%$ & $131.0 \%$ & & $>14.91$ & flash & & \\
\hline \multirow{3}{*}{$\begin{array}{l}\text { NOMEX } 410 \\
\text { D2 }\end{array}$} & 0.40 & $10.0 \%$ & $17.0 \%$ & & 10.67 & 11.69 & & \multirow{3}{*}{$\begin{array}{l}\text { N/C } \\
1 \text { piece had } 1 \\
\text { bubble }\end{array}$} \\
\hline & 0.17 & $4.3 \%$ & $17.0 \%$ & $-52.9 \%$ & 10.67 & 11.48 & $8.9 \%$ & \\
\hline & 0.39 & $9.8 \%$ & $17.0 \%$ & & 10.67 & 11.69 & & \\
\hline \multirow{3}{*}{$\begin{array}{l}\mathrm{NO} / \mathrm{MI} \\
\mathrm{E} 2\end{array}$} & 0.05 & $1.3 \%$ & $4.0 \%$ & & 10.23 & 9.71 & & \multirow{3}{*}{$N / C$} \\
\hline & 0.05 & $1.3 \%$ & $4.0 \%$ & $-68.8 \%$ & 10.23 & 10.40 & $1.2 \%$ & \\
\hline & 0.05 & $1.3 \%$ & $4.0 \%$ & & 10.23 & 10.96 & & \\
\hline \multirow{3}{*}{$\begin{array}{l}\text { MEL } 228 \\
\text { F2 }\end{array}$} & 2.80 & $140.0 \%$ & $160.0 \%$ & & $>14.22$ & flash & & very slightly \\
\hline & 2.77 & $138.5 \%$ & $160.0 \%$ & $.5 .9 \%$ & $>14.22$ & flash & flash & yellowed \\
\hline & 3.46 & $173.0 \%$ & $160.0 \%$ & & $>14.22$ & flash & & \\
\hline
\end{tabular}


SLEEVING-.HFC-125/Emery 2927 Iso-32@260 $\mathrm{F}\left(127^{\circ} \mathrm{C}\right)$

\begin{tabular}{|c|c|c|c|c|}
\hline & \multicolumn{4}{|c|}{500 HRS in HFC-125/Emery 2927 Iso-32@260 9} \\
\hline & WT & EXPWT & $\begin{array}{c}\% \\
\text { CHANGE }\end{array}$ & EXPVIS \\
\hline NOMEX & 0.5415 & 0.6130 & $13.20 \%$ & $\mathrm{~N} / \mathrm{C}$ \\
\hline $\begin{array}{l}\text { B1 } \\
\text { MYLAR }\end{array}$ & 0.4713 & 0.4787 & $1.57 \%$ & $\begin{array}{l}\text { If rolled bewteen fingers } \\
\text { would become cloudy }\end{array}$ \\
\hline \multirow{2}{*}{$\begin{array}{l}\mathrm{C1} \\
\mathrm{NO} / \mathrm{MY}\end{array}$} & 0.3926 & 0.4112 & $4.74 \%$ & $\mathrm{~N} / \mathrm{C}$ \\
\hline & \multicolumn{4}{|c|}{ HFC-125/Emery Iso 32 oil @ 260F plus24 hrs @ 302 F } \\
\hline \multirow{2}{*}{ A2 } & \multirow[t]{2}{*}{0.5365} & \multirow[t]{2}{*}{0.5772} & \multirow[t]{2}{*}{$7.59 \%$} & Slightly \\
\hline & & & & Darkened \\
\hline \multirow{2}{*}{$\begin{array}{l}\text { B2 } \\
\text { MYLAR }\end{array}$} & \multirow[t]{2}{*}{0.4859} & \multirow[t]{2}{*}{0.4904} & \multirow[t]{2}{*}{$0.93 \%$} & $\mathrm{~N} / \mathrm{C}$ \\
\hline & & & & some pockets \\
\hline \multirow{3}{*}{$\begin{array}{l}\mathrm{C} 2 \\
\mathrm{NO} / \mathrm{MY}\end{array}$} & \multirow[t]{3}{*}{0.3984} & \multirow[t]{3}{*}{0.4055} & \multirow[t]{3}{*}{$1.78 \%$} & $\mathrm{~N} / \mathrm{C}$ \\
\hline & & & & some pockets where mylar \\
\hline & & & & pulled away. \\
\hline
\end{tabular}




\begin{tabular}{|c|c|c|c|c|c|c|c|c|c|c|}
\hline \multirow[b]{2}{*}{ ID } & \multicolumn{9}{|c|}{500 HRS in HFC-125/Emery 2927 Iso32@ @260 F } & \\
\hline & WT & EXPWT & $\begin{array}{c}\text { WT \% } \\
\text { CHANGE }\end{array}$ & $\begin{array}{l}\text { BREAK } \\
\text { LOAD } \\
\text { (AVE) }\end{array}$ & $\begin{array}{l}\text { BREAK } \\
\text { LOAD } \\
\text { EP }\end{array}$ & $\begin{array}{c}\% \\
\text { CHANGE } \\
\text { BRK } \\
\text { LOAD }\end{array}$ & $\begin{array}{l}\text { STRETCH } \\
(\text { INCH) }\end{array}$ & \%ELONG & EXPVIS & \\
\hline \multirow{3}{*}{$\begin{array}{l}\text { A1 } \\
\text { Glass }\end{array}$} & \multirow[t]{3}{*}{1.7033} & \multirow[t]{3}{*}{1.8260} & \multirow[t]{3}{*}{$7.20 \%$} & 39.02 & 61.17 & & 0.06 & $3.00 \%$ & $\mathrm{~N} / \mathrm{C}$ & \\
\hline & & & & 39.02 & 45.95 & $10.06 \%$ & 0.05 & $2.50 \%$ & & \\
\hline & & & & 39.02 & 21.72 & & 0.03 & $1.50 \%$ & & \\
\hline \multirow{3}{*}{$\begin{array}{l}\text { B1 } \\
\text { Polyester }\end{array}$} & \multirow[t]{3}{*}{0.7162} & \multirow[t]{3}{*}{0.7240} & \multirow[t]{3}{*}{$1.09 \%$} & 56.12 & 51.05 & & 0.40 & $20.00 \%$ & $\mathrm{~N} / \mathrm{C}$ & \\
\hline & & & & 56.12 & 59.15 & $-1.48 \%$ & 0.60 & $30.00 \%$ & & \\
\hline & & & & 56.12 & 55.67 & & 0.50 & $25.00 \%$ & & \\
\hline \multirow{4}{*}{$\begin{array}{l}\text { C1 } \\
\text { Permacel }\end{array}$} & \multirow[t]{3}{*}{1.4730} & \multirow[t]{3}{*}{2.1331} & \multirow[t]{3}{*}{$44.81 \%$} & 88.50 & 51.15 & & 0.12 & $6.00 \%$ & Slightly & \\
\hline & & & & 88.50 & 27.80 & $.57 .43 \%$ & 0.11 & $5.50 \%$ & darkened & \\
\hline & & & & 88.50 & 34.07 & & 0.08 & $4.00 \%$ & Curled up & \\
\hline & \multicolumn{8}{|c|}{$500 \mathrm{HRS}$ in HFC-125/Emery 2927 ISO32 @ 260 F $>302^{\circ} \mathrm{F} 24 \mathrm{HRS}$} & & \\
\hline \multirow{3}{*}{$\begin{array}{l}\text { A2 } \\
\text { Glass }\end{array}$} & \multirow[t]{3}{*}{1.8214} & \multirow[t]{3}{*}{1.7039} & \multirow[t]{3}{*}{$-6.45 \%$} & 39.02 & 47.90 & & $\begin{array}{l}0.07 \\
\end{array}$ & $3.50 \%$ & $\mathrm{~N} / \mathrm{C}$ & \\
\hline & & & & 39.02 & 64.80 & $51.72 \%$ & 0.06 & $3.00 \%$ & & \\
\hline & & & & 39.02 & 64.90 & & 0.06 & $3.00 \%$ & & \\
\hline \multirow{3}{*}{$\begin{array}{l}\text { B2 } \\
\text { Polyester }\end{array}$} & \multirow[t]{3}{*}{0.5782} & \multirow[t]{3}{*}{0.5797} & \multirow[t]{3}{*}{$0.26 \%$} & 56.12 & 54.95 & & 0.55 & $27.50 \%$ & $\mathrm{~N} / \mathrm{C}$ & \\
\hline & & & & 56.12 & 60.00 & $3.71 \%$ & 0.74 & $37.00 \%$ & & \\
\hline & & & & 56.12 & 59.65 & & 0.69 & $34.50 \%$ & & \\
\hline \multirow{3}{*}{$\begin{array}{l}\text { C2 } \\
\text { Permacel }\end{array}$} & 1.3434 & 1.7317 & $28.90 \%$ & 88.50 & 68.40 & & 0.17 & $8.50 \%$ & Slightly & \\
\hline & & & & 88.50 & 75.00 & $-16.08 \%$ & 0.16 & $8.00 \%$ & Darkened & \\
\hline & & & & 88.50 & 79.40 & & 0.15 & $7.50 \%$ & Curled up & \\
\hline
\end{tabular}


TIE CORD--HFC-125/Emery 2927 ISO-32 oil @ 260 $\mathrm{F}\left(127^{\circ} \mathrm{C}\right)$

\begin{tabular}{|c|c|c|c|c|c|c|c|c|c|}
\hline \multicolumn{10}{|c|}{500 HRS IN R-125/2927 Iso-32@260\% F } \\
\hline ID & WT & EXPWT & $\begin{array}{c}\text { WT \% } \\
\text { CHANGE }\end{array}$ & $\begin{array}{l}E X P \\
V I S\end{array}$ & $\begin{array}{l}\text { BREAK } \\
\text { LOAD } \\
\text { (AVE) }\end{array}$ & $\begin{array}{c}\text { BREAK } \\
\text { LOAD } \\
\text { EXP } \\
(\operatorname{av\theta })\end{array}$ & $\begin{array}{c}\% \\
\text { CHANGE } \\
\text { BAK } \\
\text { LOAD }\end{array}$ & $\begin{array}{c}\text { STRETCH } \\
\text { (Inch) }\end{array}$ & $\begin{array}{c}\% \\
\text { ELONG }\end{array}$ \\
\hline \multirow[t]{4}{*}{ A1 } & \multirow[t]{3}{*}{0.3245} & \multirow[t]{3}{*}{0.3268} & \multirow[t]{3}{*}{$0.71 \%$} & \multirow[t]{3}{*}{$N / C$} & 28.36 & 35.32 & & 0.40 & $20.0 \%$ \\
\hline & & & & & 28.36 & 34.25 & \multirow[t]{2}{*}{$21.79 \%$} & 0.41 & $20.5 \%$ \\
\hline & & & & & 28.36 & 34.05 & & 0.38 & $19.0 \%$ \\
\hline & \multicolumn{9}{|c|}{500 HRS IN R-125/Emery 2927 -> 24 HRS @ $302^{\circ} \mathrm{F}$} \\
\hline \multirow[t]{3}{*}{ A2 } & \multirow[t]{3}{*}{0.2956} & \multirow[t]{3}{*}{0.295} & \multirow[t]{3}{*}{$-0.203 \%$} & $\mathrm{~N} / \mathrm{C}$ & 28.36 & 32.20 & & 0.41 & $20.5 \%$ \\
\hline & & & & & 28.36 & 33.75 & $18.71 \%$ & 0.48 & $24.0 \%$ \\
\hline & & & & & 28.36 & 35.05 & & 0.39 & $19.5 \%$ \\
\hline
\end{tabular}


LEAD WIRE INSULATION-HFC-125/Emery 2927 Iso-32 @ 260 $\mathrm{F}\left(127^{\circ} \mathrm{C}\right)$

\begin{tabular}{|c|c|c|c|c|c|c|c|}
\hline & 500 HRS IN & $\mathrm{FC}-125 / \mathrm{Em}$ & ery 29271 & so-32@ & $260^{\circ} \mathrm{F}$ & & \\
\hline ID & WT & EXPWT & $\begin{array}{c}\text { WT \% } \\
\text { CHANGE }\end{array}$ & EXP VIS & $\begin{array}{c}\text { BASEDIE } \\
\text { (AVE) }\end{array}$ & EXPDIE & $\begin{array}{c}\text { DIE\% } \\
\text { CHANGE }\end{array}$ \\
\hline DMD & 4.1351 & 4.3079 & $4.18 \%$ & $\mathrm{~N} / \mathrm{C}$ & 9.61 & 7.71 & \\
\hline A1 & & & & & 9.61 & 8.34 & $-16.55 \%$ \\
\hline & & & & & 9.61 & 8.01 & \\
\hline DTMD & 4.4821 & 4.6290 & $3.28 \%$ & $\mathrm{~N} / \mathrm{C}$ & 9.95 & 12.17 & \\
\hline$B 1$ & & & & & 9.95 & 13.01 & $31.09 \%$ \\
\hline & & & & & 9.95 & 13.95 & \\
\hline & HFC-125/Em & y 2927 Iso- & $32 @ 260$ & ${ }^{\circ} \mathrm{F} \cdot>24 \mathrm{H}$ & IRS@ @302 & & \\
\hline DMD & 4.0035 & 4.1464 & $3.57 \%$ & $\mathrm{~N} / \mathrm{C}$ & 9.61 & 9.50 & \\
\hline$A 2$ & & & & & 9.61 & 9.73 & $1.39 \%$ \\
\hline & & & & & 9.61 & 10.00 & \\
\hline DTMD & 4.3964 & 4.5274 & $2.98 \%$ & $\mathrm{~N} / \mathrm{C}$ & 9.95 & $\mid 12.24$ & \\
\hline $\mathrm{B} 2$ & & & & & 9.95 & 12.51 & $26.43 \%$ \\
\hline & & & & & 9.95 & 12.99 & \\
\hline
\end{tabular}




\section{Appendix $\mathbf{J}$}

Experimental Data for HFC-143a/HenkelEmery 2927 ISO-32 Exposure at $126^{\circ} \mathrm{C}\left(260^{\circ} \mathrm{F}\right)$ 


\begin{tabular}{|c|c|c|c|c|c|c|c|c|c|c|}
\hline & 500 hours & HFC- 143 & $\mathrm{a} / 2927 \mathrm{Iso}$ & -32 oil a & at $260^{\circ} \mathrm{F}(12$ & & & & & \\
\hline ID & $W T$ & EXPWT & $\begin{array}{c}\text { WT \% } \\
\text { CHANGE }\end{array}$ & EXP VIS & $\begin{array}{c}\text { BASE BRN } \\
\text { OUT } \\
\text { (AVE) }\end{array}$ & $\begin{array}{c}\text { EXP BRN } \\
\text { OUT }\end{array}$ & $\begin{array}{c}\text { BRN OUT } \\
\% \\
\text { CHANGE }\end{array}$ & $\begin{array}{c}\text { BASE DIE } \\
\text { (AVE) }\end{array}$ & EXPDIE & $\begin{array}{c}\text { DIE \% } \\
\text { CHANGE }\end{array}$ \\
\hline A1 & 23.8199 & 23.8242 & $0.018 \%$ & $\mathrm{~N} / \mathrm{C}$ & 576 & 566 & & 15.80 & 10.70 & \\
\hline & & & & & 576 & 398 & $-22.6 \%$ & 15.80 & 13.73 & $-18.5 \%$ \\
\hline & & & & & 576 & 373 & & 15.80 & 14.22 & \\
\hline$B 1$ & 26.9424 & 26.9826 & $0.149 \%$ & $N / C$ & 736 & 734 & & 11.62 & 14.30 & \\
\hline & & & & & 736 & 638 & $-10.8 \%$ & 11.62 & 12.42 & $11.8 \%$ \\
\hline & & & & & 736 & 598 & & 11.62 & 12.25 & \\
\hline$\overline{C 1}$ & 23.5798 & 23.5850 & $0.022 \%$ & $\mathrm{~N} / \mathrm{C}$ & 579 & 528 & & 16.58 & 16.42 & \\
\hline & & & & & 579 & 583 & $-3.6 \%$ & 16.58 & 16.25 & $-9.5 \%$ \\
\hline & & & & & 579 & 564 & & 16.58 & 12.36 & \\
\hline & 500 hours i & HFC- 143 & J'Emery 2 & 927 oil a & $260^{\circ} \mathrm{F}(12$ & $\left.7^{\circ} \mathrm{C}\right)$ plus & 24 hours & at $302^{\circ} \mathrm{F}($ & $\left.150^{\circ} \mathrm{C}\right)$ & \\
\hline A2 & 24.7001 & 24.6989 & $-0.005 \%$ & $\mathrm{~N} / \mathrm{C}$ & 576 & 450 & & 15.80 & 12.78 & \\
\hline & & & & & 576 & 439 & $-22.7 \%$ & 15.80 & 11.25 & $-13.1 \%$ \\
\hline & & & & & 576 & 446 & & 15.80 & 17.14 & \\
\hline $\mathrm{B2}$ & 27.3281 & 27.3177 & $-0.038 \%$ & $\mathrm{~N} / \mathrm{C}$ & 736 & 671 & & 11.62 & 10.46 & \\
\hline & & & & & 736 & 729 & $-3.5 \%$ & 11.62 & 12.04 & $-2.1 \%$ \\
\hline & & & & & 736 & 730 & & 11.62 & 11.62 & \\
\hline $\mathrm{C2}$ & 24.0234 & 24.0229 & $.0 .002 \%$ & $\mathrm{~N} / \mathrm{C}$ & 579 & 569 & & 16.58 & 12.99 & \\
\hline & & & & & 579 & 440 & $-16.9 \%$ & 16.58 & 14.66 & $-14.7 \%$ \\
\hline & & & & & 579 & 435 & & 16.58 & 14.76 & \\
\hline
\end{tabular}


TWISTED PAIRSMIRE AWITH VARNISH-HFC-143a/Emery 2927 Iso-32 oil @260 $0^{\circ} \mathrm{F}\left(127^{\circ} \mathrm{C}\right)$

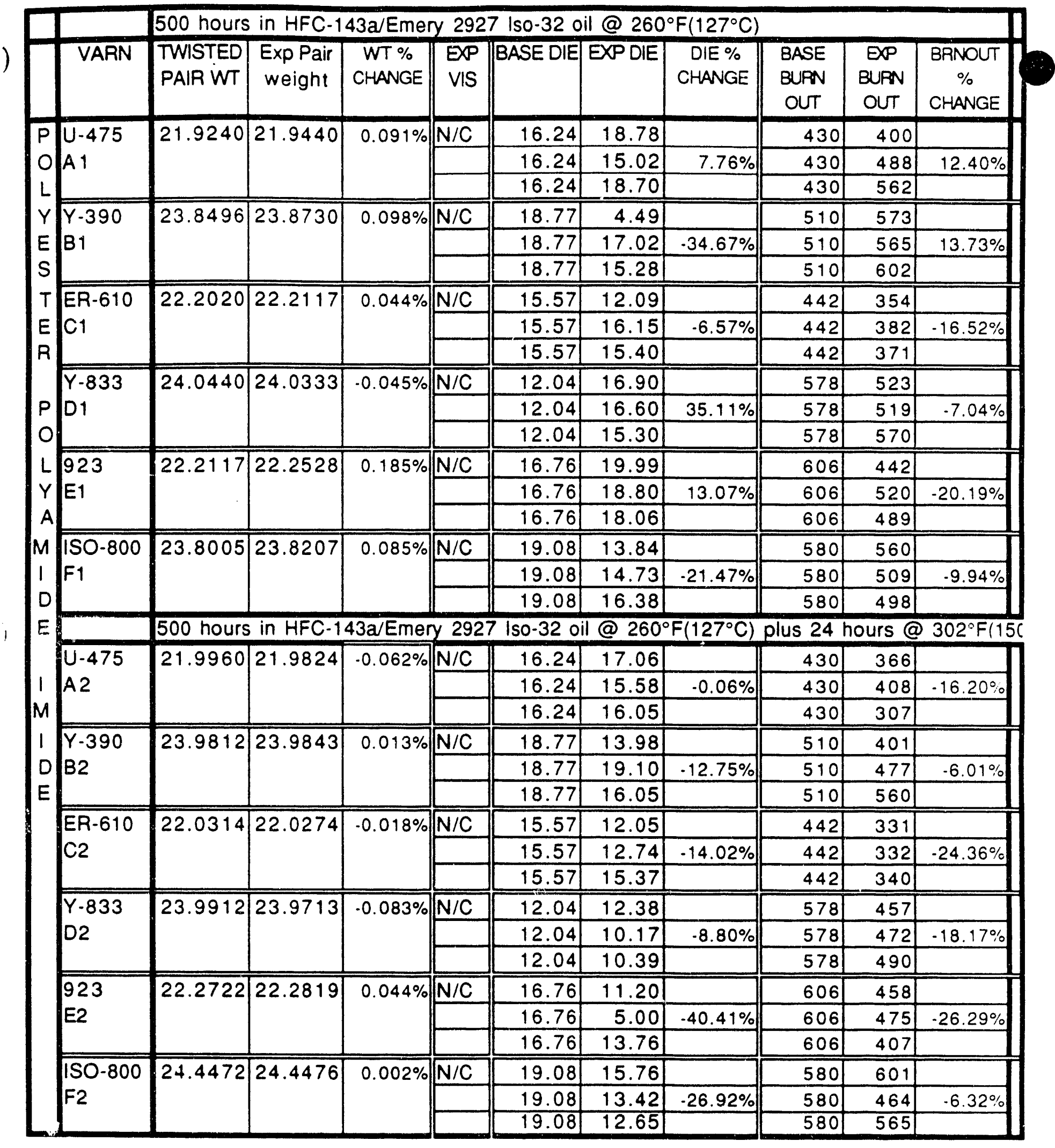


TWISTED PAIRSIWIRE BNWITH VARNISH -HFC-143a/Emery 2927 Iso-32 oil @260F(127ㅇ)

\begin{tabular}{|c|c|c|c|c|c|c|c|c|c|c|c|}
\hline & & \multicolumn{10}{|c|}{500 HOURS in HFC- $143 a / 2927$ Iso-32 oil @ $260^{\circ} \mathrm{F}\left(127^{\circ} \mathrm{C}\right)$} \\
\hline & VARN & $\begin{array}{l}\text { TWISTED } \\
\text { PAIR WT }\end{array}$ & $\begin{array}{c}\text { WT\% } \\
\text { Change }\end{array}$ & $\begin{array}{c}\text { WT \% } \\
\text { CHANGE }\end{array}$ & $\begin{array}{l}\text { EXP } \\
\text { VIS }\end{array}$ & $\begin{array}{c}\text { BASE } \\
\text { DIE }\end{array}$ & $\begin{array}{l}\text { EPP } \\
\text { DIE }\end{array}$ & $\begin{array}{c}\text { DIE \% } \\
\text { CHANGE }\end{array}$ & \begin{tabular}{|l|} 
BASE \\
BUPN \\
OUT
\end{tabular} & \begin{tabular}{|c|} 
EP \\
BUPN \\
OUT
\end{tabular} & $\begin{array}{c}\text { BRNOUT } \\
\% \\
\text { CHANGE }\end{array}$ \\
\hline \multirow{4}{*}{$\begin{array}{l}P \\
O \\
L\end{array}$} & \multirow{3}{*}{$\begin{array}{l}\text { U-475 } \\
\text { A } 1\end{array}$} & \multirow[t]{3}{*}{26.7364} & \multirow[t]{3}{*}{26.7882} & \multirow[t]{3}{*}{$0.19 \%$} & $\mathrm{~N} / \mathrm{C}$ & 13.32 & 16.33 & & 746 & 747 & \\
\hline & & & & & & 13.32 & 16.95 & $28.85 \%$ & 746 & 729 & $-1.07 \%$ \\
\hline & & & & & & 13.32 & 18.21 & & 746 & 738 & \\
\hline \multirow{3}{*}{\multicolumn{2}{|c|}{$\begin{array}{l}Y .390 \\
E \\
\text { S }\end{array}$}} & \multirow[t]{3}{*}{26.9630} & \multirow[t]{3}{*}{27.0373} & \multirow[t]{3}{*}{$0.28 \%$} & $\mathrm{~N} / \mathrm{C}$ & 12.28 & 13.70 & & 755 & 739 & \\
\hline & & & & & & 12.28 & 8.26 & $.5 .89 \%$ & 755 & 741 & $-1.99 \%$ \\
\hline & & & & & & 12.28 & 12.71 & & 755 & 740 & \\
\hline \multirow{4}{*}{$\begin{array}{l}T \\
E \\
R\end{array}$} & \multirow{3}{*}{$\begin{array}{l}\text { ER-610 } \\
\text { C1 }\end{array}$} & \multirow[t]{3}{*}{25.8041} & \multirow[t]{3}{*}{25.8375} & \multirow[t]{3}{*}{$0.13 \%$} & $\mathrm{~N} / \mathrm{C}$ & 12.73 & 12.91 & & 734 & 737 & \\
\hline & & & & & & 12.73 & 14.52 & $0.71 \%$ & 734 & 738 & $0.23 \%$ \\
\hline & & & & & & 12.73 & 11.03 & & 734 & 732 & \\
\hline & \multirow{3}{*}{$\begin{array}{l}Y \cdot 833 \\
D 1\end{array}$} & \multirow[t]{3}{*}{25.8621} & \multirow[t]{3}{*}{25.8671} & \multirow[t]{3}{*}{$0.02 \%$} & $\mathrm{~N} / \mathrm{C}$ & 12.49 & 11.98 & & 734 & 731 & \\
\hline$P$ & & & & & & 12.49 & 13.27 & $0.91 \%$ & 734 & 734 & $.0 .14 \%$ \\
\hline 0 & & & & & & 12.49 & 12.56 & & 734 & 734 & \\
\hline \multirow{3}{*}{$\begin{array}{l}\mathrm{L} \\
Y \\
A\end{array}$} & \multirow{3}{*}{$\begin{array}{l}923 \\
E 1 \\
\end{array}$} & \multirow[t]{3}{*}{26.3069} & \multirow[t]{3}{*}{26.3802} & $0.28 \%$ & $\mathrm{~N} / \mathrm{C}$ & 14.38 & 13.32 & & 742 & 750 & \\
\hline & & & & & & 14.38 & 10.41 & $-15.16 \%$ & 742 & 748 & $0.85 \%$ \\
\hline & & & & & & 14.38 & 12.87 & & 742 & 747 & \\
\hline$M$ & ISO-800 & 30.7829 & 30.8480 & $0.21 \%$ & $\mathrm{~N} / \mathrm{C}$ & 12.29 & 13.61 & & 747 & 748 & \\
\hline 1 & & & & & & 12.29 & 11.10 & $4.88 \%$ & 747 & 736 & $-0.58 \%$ \\
\hline$D$ & & & & & & 12.29 & 13.96 & & 747 & 744 & \\
\hline$E$ & & HFC-143 & $\mathrm{a} / 2927$ Iso & -32 oil@ & $260^{\circ}$ & $\left(127^{\circ} \mathrm{C}\right)$ & plus 24 & hours@ & $302^{\circ}$ & $F\left(150^{\circ}\right.$ & C) \\
\hline & $U .475$ & 25.8841 & 25.8754 & $-0.03 \%$ & $N / C$ & 13.32 & 12.97 & & 746 & 743 & \\
\hline 1 & $A 2$ & & & & & 13.32 & 12.61 & $.7 .46 \%$ & 746 & 746 & $-0.18 \%$ \\
\hline$M$ & & & & & & 13.32 & 11.40 & & 746 & 745 & \\
\hline 1 & $Y-390$ & 27.0235 & 27.0359 & $0.05 \%$ & $\mathrm{~N} / \mathrm{C}$ & 12.28 & 12.59 & & 755 & 747 & \\
\hline$D$ & B2 & & & & & 12.28 & 12.58 & $2.44 \%$ & 755 & 743 & $.1 .32 \%$ \\
\hline$E$ & & & & & & 12.28 & 12.57 & & 755 & 740 & \\
\hline & ER-610 & 30.7630 & 30.7563 & $-0.02 \%$ & $\mathrm{~N} / \mathrm{C}$ & 12.73 & 13.25 & & 734 & 730 & \\
\hline$E$ & $\mathrm{C} 2$ & & & & & 12.73 & 10.67 & $-5.73 \%$ & 734 & 731 & $.0 .50 \%$ \\
\hline$P$ & & & & & & 12.73 & 12.08 & & 734 & 730 & \\
\hline 0 & $Y-833$ & 26.1481 & 26.1191 & $.0 .11 \%$ & $\mathrm{~N} / \mathrm{C}$ & 12.49 & 13.46 & & 734 & 734 & \\
\hline$x$ & D2 & & & & & 12.49 & 13.08 & $4.46 \%$ & 734 & 729 & $-0.36 \%$ \\
\hline$Y$ & & & & & & 12.49 & 12.60 & & 734 & 731 & \\
\hline & 923 & 26.1041 & 26.1136 & $0.04 \%$ & $\mathrm{~N} / \mathrm{C}$ & 14.38 & 13.75 & & 742 & 750 & \\
\hline$G$ & E2 & & & & & 14.38 & 10.79 & $-15.37 \%$ & 742 & 752 & $1.08 \%$ \\
\hline$L$ & & & & & & 14.38 & 11.97 & & 742 & 744 & \\
\hline A & $150-800$ & 26.4135 & 26.4217 & $0.03 \%$ & & 12.29 & 11.87 & & 747 & 745 & \\
\hline S & $\mathrm{F} 2$ & & & & $N / C$ & 12.29 & 12.16 & $-3.31 \%$ & 747 & 739 & $-0.67 \%$ \\
\hline$S$ & & & & & & 12.29 & 11.62 & & 747 & 747 & \\
\hline
\end{tabular}




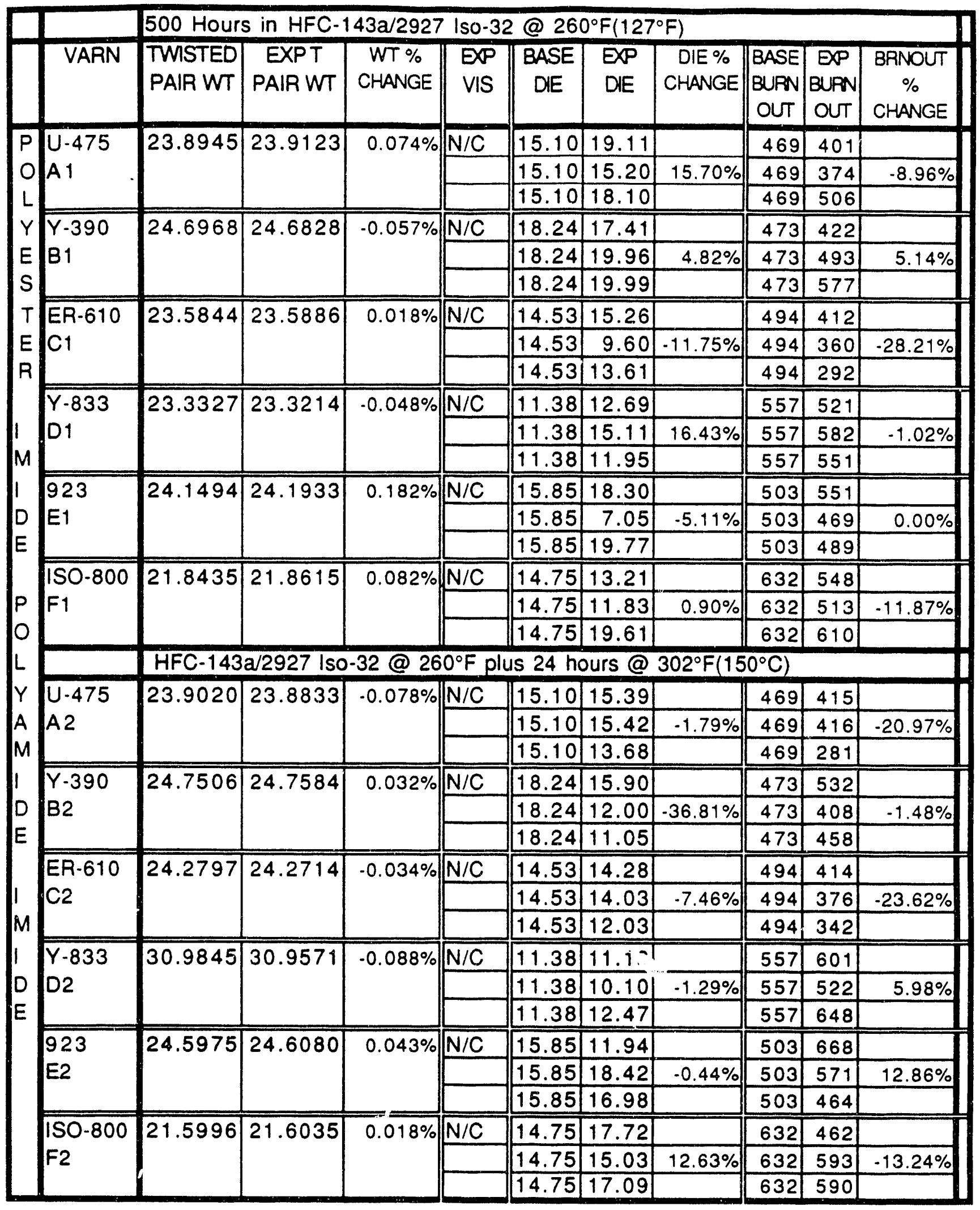


HELICAL COILS/WIRE A-..HFC-143a/Emery 29?7 Iso-32@260 $\mathrm{F}\left(127^{\circ} \mathrm{C}\right)$

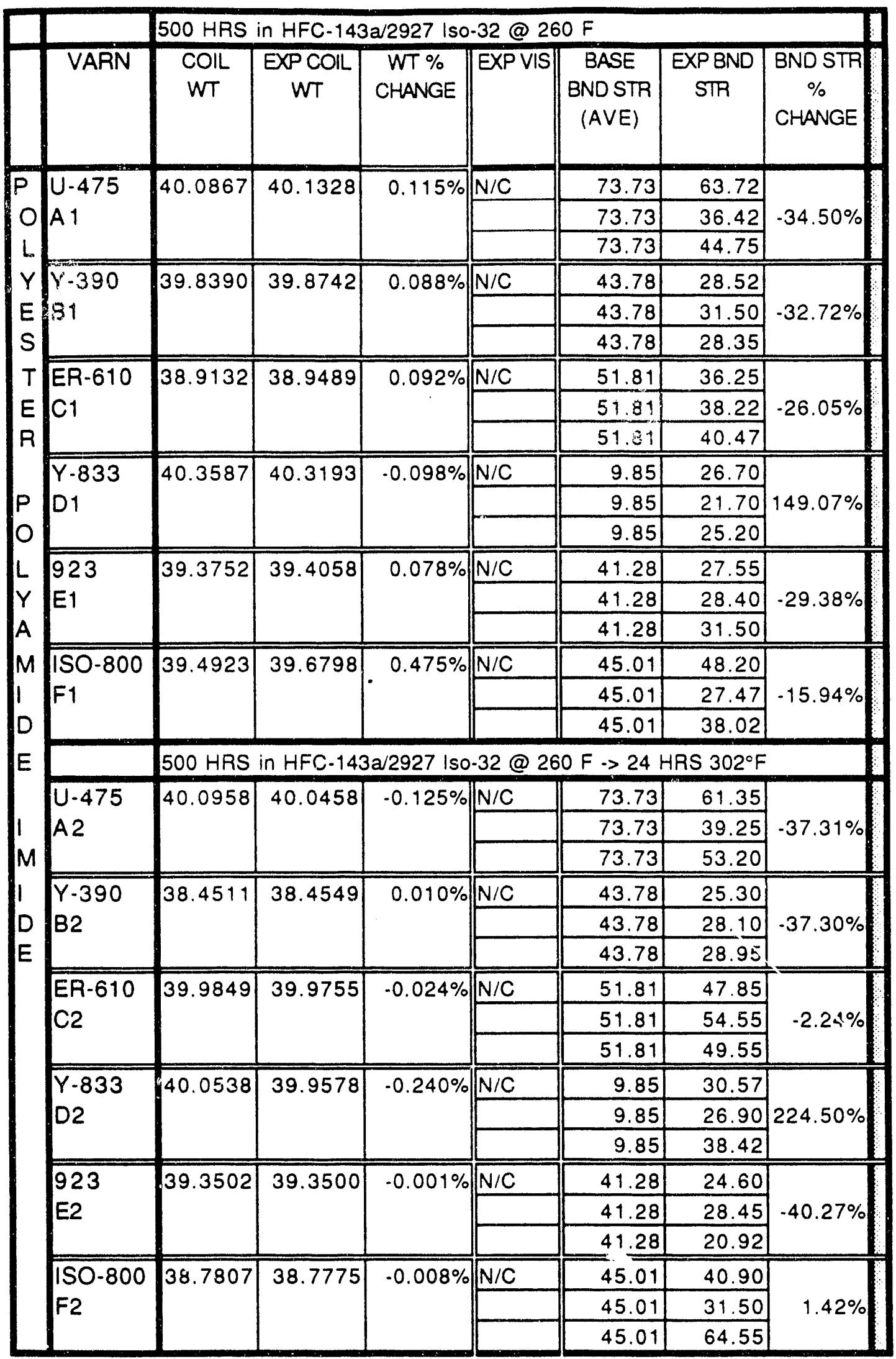


HELICAL COILS/WIRE B-HFC-143a/Emery 2927 Iso-32 Oil @ 260 $\mathrm{F}\left(127^{\circ} \mathrm{C}\right)$

\begin{tabular}{|c|c|c|c|c|c|c|c|c|}
\hline & \multirow{2}{*}{ VARN } & \multicolumn{7}{|c|}{500 HRS in HFC-143a/Emery 2927 Iso-32 @ 260 F } \\
\hline & & $\begin{array}{l}\text { COIL } \\
\text { WT }\end{array}$ & $\begin{array}{c}\text { EXP COIL } \\
W T\end{array}$ & $\begin{array}{c}\text { WT \% } \\
\text { CHANGE }\end{array}$ & EXP VIS & $\begin{array}{c}\text { BASE } \\
\text { BND STR } \\
\text { (AVE) }\end{array}$ & $\begin{array}{c}\text { EXP BND } \\
\text { STR }\end{array}$ & $\begin{array}{c}\text { BND STR } \\
\% \\
\text { CHANGE }\end{array}$ \\
\hline \multirow{5}{*}{$\begin{array}{l}P \\
O \\
L \\
Y \\
E \\
S\end{array}$} & \multirow{3}{*}{$\begin{array}{l}\text { U. } 475 \\
\text { A } 1\end{array}$} & \multirow[t]{3}{*}{36.9717} & \multirow[t]{3}{*}{37.0321} & \multirow[t]{3}{*}{$0.163 \%$} & $\mathrm{~N} / \mathrm{C}$ & 40.14 & 30.57 & \multirow{3}{*}{$-25.75 \%$} \\
\hline & & & & & & 40.14 & 32.77 & \\
\hline & & & & & & 40.14 & 26.07 & \\
\hline & \multirow{3}{*}{$\begin{array}{l}Y-390 \\
B 1\end{array}$} & \multirow[t]{3}{*}{36.8758} & \multirow[t]{3}{*}{36.9689} & \multirow[t]{3}{*}{$0.252 \%$} & $\mathrm{~N} / \mathrm{C}$ & 36.12 & 40.15 & \multirow{3}{*}{$-0.31 \%$} \\
\hline & & & & & & 36.12 & 33.90 & \\
\hline S & & & & & & 36.12 & 33.97 & \\
\hline \multirow{4}{*}{$\begin{array}{l}T \\
E \\
R\end{array}$} & \multirow{3}{*}{$\begin{array}{l}\text { ER-610 } \\
\text { C1 }\end{array}$} & \multirow[t]{3}{*}{37.2169} & \multirow[t]{3}{*}{37.2741} & \multirow[t]{3}{*}{$0.154 \%$} & $\mathrm{~N} / \mathrm{C}$ & 35.96 & 35.50 & \\
\hline & & & & & & 35.96 & 28.97 & $-10.85 \%$ \\
\hline & & & & & & 35.96 & 31.70 & \\
\hline & $Y-833$ & 36.0573 & 36.0418 & $-0.043 \%$ & $\mathrm{~N} / \mathrm{C}$ & 33.14 & broke & \\
\hline$P$ & & & & & & 33.14 & 19.15 & $-41.66 \%$ \\
\hline 0 & & & & & & 33.14 & 19.52 & \\
\hline$L$ & 923 & 36.8274 & 36.8749 & $0.129 \%$ & $\mathrm{~N} / \mathrm{C}$ & 40.52 & 31.57 & \\
\hline$Y$ & E1 & & & & & 40.52 & 39.20 & $-13.80 \%$ \\
\hline A & & & & & & 40.52 & 34.02 & \\
\hline$M$ & $150-800$ & 37.3820 & 37.4356 & $0.143 \%$ & $\mathrm{~N} / \mathrm{C}$ & 20.20 & 17.50 & \\
\hline 1 & $F 1$ & & & & & 20.20 & 19.52 & $-3.76 \%$ \\
\hline$D$ & & & & & & 20.20 & 21.30 & \\
\hline$E$ & & 500 HRS & in $\mathrm{HFC}-14$ & 3a/Emery 2 & 927 Iso-3 & $2 @ 260 \mathrm{~F}$ & $->24 \mathrm{HRS}$ & $302^{\circ} \mathrm{F}$ \\
\hline & U-475 & 37.9488 & 37.9471 & $-0.004 \%$ & $\mathrm{~N} / \mathrm{C}$ & 40.14 & 33.47 & \\
\hline 1 & $A 2$ & & & & & 40.14 & 31.12 & $-23.53 \%$ \\
\hline$M$ & & & & & & 40.14 & 27.50 & \\
\hline 1 & $\overline{Y-390}$ & 37.0463 & 37.0626 & $0.044 \%$ & $\mathrm{~N} / \mathrm{C}$ & 36.12 & 24.00 & \\
\hline$D$ & B2 & & & & & 36.12 & 27.42 & $-21.77 \%$ \\
\hline$E$ & & & & & & 36.12 & 33.35 & \\
\hline & ER-610 & 37.2936 & 37.2873 & $-0.017 \%$ & $\mathrm{~N} / \mathrm{C}$ & 35.96 & 23.90 & \\
\hline$E$ & $\mathrm{C} 2$ & & & & & 35.96 & 24.37 & $-29.25 \%$ \\
\hline$P$ & & & & & & 35.96 & 28.05 & \\
\hline 0 & $Y-833$ & 36.5244 & 36.4538 & $-0.193 \%$ & $\mathrm{~N} / \mathrm{C}$ & 33.14 & 22.15 & \\
\hline$x$ & $\mathrm{D} 2$ & & & & & 33.14 & 23.60 & $.35 .78 \%$ \\
\hline$Y$ & & & & & & 33.14 & 18.10 & \\
\hline & 923 & 38.0268 & 38.0265 & $-0.001 \%$ & $\mathrm{~N} / \mathrm{C}$ & 40.52 & 25.90 & \\
\hline G & E2 & & & & & 40.52 & 32.40 & $-29.98 \%$ \\
\hline$L$ & & & & & & 40.52 & 26.82 & \\
\hline$n$ & ISO-800 & 37.2620 & 37.2664 & $0.012 \%$ & $\mathrm{~N} / \mathrm{C}$ & 20.20 & 18.87 & \\
\hline$s$ & $\mathrm{~F} 2$ & & & & & 20.20 & 22.12 & $-26.09 \%$ \\
\hline$S$ & & & & & & 20.20 & 3.80 & \\
\hline
\end{tabular}


HELICAL COILSNWIRE C-HFC-143a/Emery 2927 Iso-32@ 260 $0^{\circ} \mathrm{F}\left(127^{\circ} \mathrm{C}\right)$

\begin{tabular}{|c|c|c|c|c|c|c|c|c|}
\hline & & 500 HRS in & in $\mathrm{HFC} \cdot 143$ & a/Emery 29 & $27 @ 2$ & $60 \mathrm{~F}$ & & \\
\hline & VARN & $\begin{array}{l}\text { COIL } \\
\text { WT }\end{array}$ & $\begin{array}{c}\text { EXP COIL } \\
W T\end{array}$ & $\begin{array}{c}\text { WT \% } \\
\text { CHANGE }\end{array}$ & EXPVIS & $\begin{array}{c}\text { BASE } \\
\text { BND STR } \\
\text { (AVE) }\end{array}$ & $\begin{array}{c}\text { EXP BND } \\
\text { STR }\end{array}$ & $\begin{array}{c}\text { BND STR } \\
\% \\
\text { CHANGE }\end{array}$ \\
\hline$P$ & $U-475$ & 38.1615 & 38.2060 & $0.117 \%$ & $\mathrm{~N} / \mathrm{C}$ & 51.21 & 24.02 & \\
\hline 0 & $A 1$ & & & & & 51.21 & 39.90 & $-32.41 \%$ \\
\hline$L$ & & & & & & 51.21 & 39.92 & \\
\hline$Y$ & $Y-390$ & 40.7515 & 40.8386 & $0.214 \%$ & $\mathrm{~N} / \mathrm{C}$ & 50.72 & 25.07 & \\
\hline$E$ & $B 1$ & & & & & 50.72 & 27.50 & $-46.28 \%$ \\
\hline$S$ & & & & & & 50.72 & 29.17 & \\
\hline$T$ & ER-610 & 37.9729 & 38.0087 & $0.094 \%$ & $\mathrm{~N} / \mathrm{C}$ & 58.33 & 21.77 & \\
\hline$E$ & C1 & & & & & 58.33 & 45.10 & $.51 .41 \%$ \\
\hline$R$ & & & & & & 58.33 & 18.15 & \\
\hline & $Y-833$ & 38.9045 & 38.7517 & $-0.393 \%$ & $\mathrm{~N} / \mathrm{C}$ & 5.84 & $\begin{array}{r}7.00 \\
\end{array}$ & \\
\hline । & D1 & & & & & 5.84 & broke & $56.68 \%$ \\
\hline$M$ & & & & & & 5.84 & \begin{tabular}{|l|}
11.30 \\
\end{tabular} & \\
\hline 1 & 923 & 40.6574 & 40.6848 & $0.067 \%$ & $\mathrm{~N} / \mathrm{C}$ & 49.26 & 24.67 & \\
\hline$D$ & E1 & & & & & 49.26 & 23.87 & $-50.97 \%$ \\
\hline$E$ & & & & & & 49.26 & 23.92 & \\
\hline & $150-800$ & 39.5886 & 39.6062 & $0.044 \%$ & $\mathrm{~N} / \mathrm{C}$ & 36.08 & 34.87 & \\
\hline$P$ & $F 1$ & & & & & 36.08 & 24.15 & $.17 .78 \%$ \\
\hline 0 & & & & & & 36.08 & 29.97 & \\
\hline$L$ & & 500 HRS & in HFC-143 & $32 / 2927$ Iso- & $32 @ 26$ & $0 \mathrm{~F}->24 \mathrm{H}$ & tRS $302^{\circ} \mathrm{F}$ & \\
\hline$Y$ & U.475 & 38.1255 & 38.1210 & $-0.012 \%$ & $\mathrm{~N} / \mathrm{C}$ & \begin{tabular}{|r|}
51.21 \\
\end{tabular} & \begin{tabular}{|r|}
60.22 \\
\end{tabular} & \\
\hline A & A2 & & & & & 51.21 & 55.45 & $-5.77 \%$ \\
\hline$M$ & & & & & & 51.21 & 29.10 & \\
\hline 1 & $Y-390$ & 41.3271 & 41.3607 & $0.081 \%$ & $\mathrm{~N} / \mathrm{C}$ & 50.72 & 24.55 & \\
\hline$D$ & B2 & & & & & 50.72 & 30.90 & $.46 .00 \%$ \\
\hline$E$ & & & & & & 50.72 & 26.72 & \\
\hline & ER-610 & 37.0130 & 37.0094 & $-0.010 \%$ & $\mathrm{~N} / \mathrm{C}$ & 58.33 & 25.50 & \\
\hline 1 & $\mathrm{C} 2$ & & & & & 58.33 & 56.27 & $-22.01 \%$ \\
\hline$M$ & & & & & & 58.33 & 54.70 & \\
\hline 1 & $Y-833$ & 38.7091 & 38.5439 & $-0.427 \%$ & $\mathrm{~N} / \mathrm{C}$ & 5.84 & 5.10 & \\
\hline$D$ & $\mathrm{D} 2$ & & & & & 5.84 & 20.92 & $152.11 \%$ \\
\hline$E$ & & & & & & 5.84 & 18.15 & \\
\hline & 923 & 40.0347 & 40.0337 & $-0.002 \%$ & $\mathrm{~N} / \mathrm{C}$ & 49.26 & 25.95 & \\
\hline & E2 & & & & & 49.26 & 27.25 & $-44.31 \%$ \\
\hline & & & & & & 49.26 & 29.10 & \\
\hline & $150-800$ & 40.4949 & 40.4978 & $0.007 \%$ & $\mathrm{~N} / \mathrm{C}$ & 36.08 & 26.40 & \\
\hline & $\mathrm{F} 2$ & & & & & 36.08 & 43.80 & $-12.58 \%$ \\
\hline & & & & & & 36.08 & 24.42 & \\
\hline
\end{tabular}


SINGLE MAG WIRE WITHOUT VARNISH-HFC-143a/Emery 2927 Iso-32@ @ $60^{\circ} \mathrm{F}\left(127^{\circ} \mathrm{C}\right)$ )

\begin{tabular}{|c|c|c|c|c|c|}
\hline 500 & $\mathrm{RS}$ in $\mathrm{HF}$ & $-143 a / 292$ & Iso-32 oil & $@ 260^{\circ} \mathrm{F}$ & $\left.127^{\circ} \mathrm{C}\right)$ \\
\hline ID & $W T$ & EXPWT & $\begin{array}{c}\text { WT \% } \\
\text { CHANGE }\end{array}$ & EXP VIS & EXP FLEX \\
\hline A 1 & 3.1397 & 3.1392 & $-0.016 \%$ & $\mathrm{~N} / \mathrm{C}$ & \\
\hline & & & & & YES \\
\hline B1 & 3.4415 & 3.445 & $0.102 \%$ & $\mathrm{~N} / \mathrm{C}$ & \\
\hline & & & & & YES \\
\hline C1 & 3.8875 & 3.888 & $0.013 \%$ & $N / C$ & \\
\hline & & & & & YES \\
\hline 50 & RS in $H$ & $C-143 a / 20$ & 27 Iso-32-> & $302^{\circ} \mathrm{F}$ fo & $24+R S$ \\
\hline A2 & 2.9437 & 2.943 & $-0.024 \%$ & $\mathrm{~N} / \mathrm{C}$ & \\
\hline & & & & & YES \\
\hline B2 & 3.3795 & 3.3764 & $-0.092 \%$ & $\mathrm{~N} / \mathrm{C}$ & \\
\hline & & & & & YES \\
\hline C2 & 3.1789 & 3.1789 & $0.000 \%$ & $\mathrm{~N} / \mathrm{C}$ & \\
\hline & & & & & YES \\
\hline & & & & & \\
\hline
\end{tabular}




\begin{tabular}{|c|c|c|c|c|c|c|}
\hline & $\begin{array}{l}500 \text { HRS } \\
\text { VARN }\end{array}$ & $\frac{\text { in HFC-14 }}{\text { WIREWT }}$ & 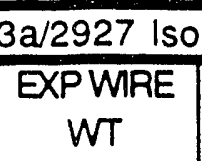 & 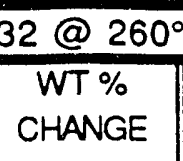 & $\frac{F\left(127^{\circ} \mathrm{C}\right.}{\text { EXPVIS }}$ & $\begin{array}{l}\text { EXP } \\
\text { FEX }\end{array}$ \\
\hline PI & U.475 & 3.8467 & 3.8487 & $0.052 \%$ & $\mathrm{~N} / \mathrm{C}$ & \\
\hline 이 & $A 1$ & & & & & NO \\
\hline L & & & & & & \\
\hline YI & $Y-390$ & 3.8472 & 3.8479 & $0.018 \%$ & $\mathrm{~N} / \mathrm{C}$ & \\
\hline $\mathrm{E}$ & B1 & & & & & NO \\
\hline s! & & & & & & \\
\hline$T$ & ER-610 & 3.8410 & 3.8409 & $-0.003 \%$ & $\mathrm{~N} / \mathrm{C}$ & \\
\hline$E$ & c1 & & & & & YES \\
\hline$R$ & & & & & & \\
\hline & Y-833 & 4.0479 & 4.0483 & $0.010 \%$ & $\mathrm{~N} / \mathrm{C}$ & \\
\hline 1 & D1 & & & & & YES \\
\hline$M$ & & & & & & \\
\hline 1 & 923 & 4.6385 & 4.6424 & $0.084 \%$ & $\mathrm{~N} / \mathrm{C}$ & \\
\hline D & E1 & & & & & NO \\
\hline$E$ & & & & & & \\
\hline & ISO-800 & 3.9671 & 3.9697 & $0.066 \%$ & $\mathrm{~N} / \mathrm{C}$ & \\
\hline$P$ & & & & & & $\mathrm{NO}$ \\
\hline 0 & & & & & & \\
\hline L & HFC-143a & $/ 2927$ Iso-3 & $2 @ 260^{\circ} \mathrm{F}$ & $>24 \mathrm{HRS}$ & @ 302F & \\
\hline Y & U-475 & 3.9156 & 3.9131 & $-0.064 \%$ & $\mathrm{~N} / \mathrm{C}$ & \\
\hline A & A2 & & & & & NO \\
\hline$M$ & & & & & & \\
\hline 1 & $Y-390$ & 3.9300 & 3.9293 & $.0 .018 \%$ & $\mathrm{~N} / \mathrm{C}$ & \\
\hline D & B2 & & & & & NO \\
\hline$E$ & & & & & & \\
\hline & ER-610 & 3.8529 & 3.8511 & $.0 .047 \%$ & $\mathrm{~N} / \mathrm{C}$ & \\
\hline 1 & $\mathrm{C} 2$ & & & & & Yes \\
\hline M & & & & & & \\
\hline 1 & Y-833 & 4.0648 & 4.0643 & $-0.012 \%$ & $\mathrm{~N} / \mathrm{C}$ & \\
\hline D & & & & & & YES \\
\hline E & & 19000 & & & $\| N / C$ & 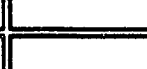 \\
\hline & $E 2$ & 4.0000 & 4.0000 & & & NO \\
\hline & & & & & & \\
\hline & $150-800$ & 4.1658 & 4.1654 & $-0.010 \%$ & $\mathrm{~N} / \mathrm{C}$ & \\
\hline & F2 & & & & & NO \\
\hline & & & & & & \\
\hline
\end{tabular}




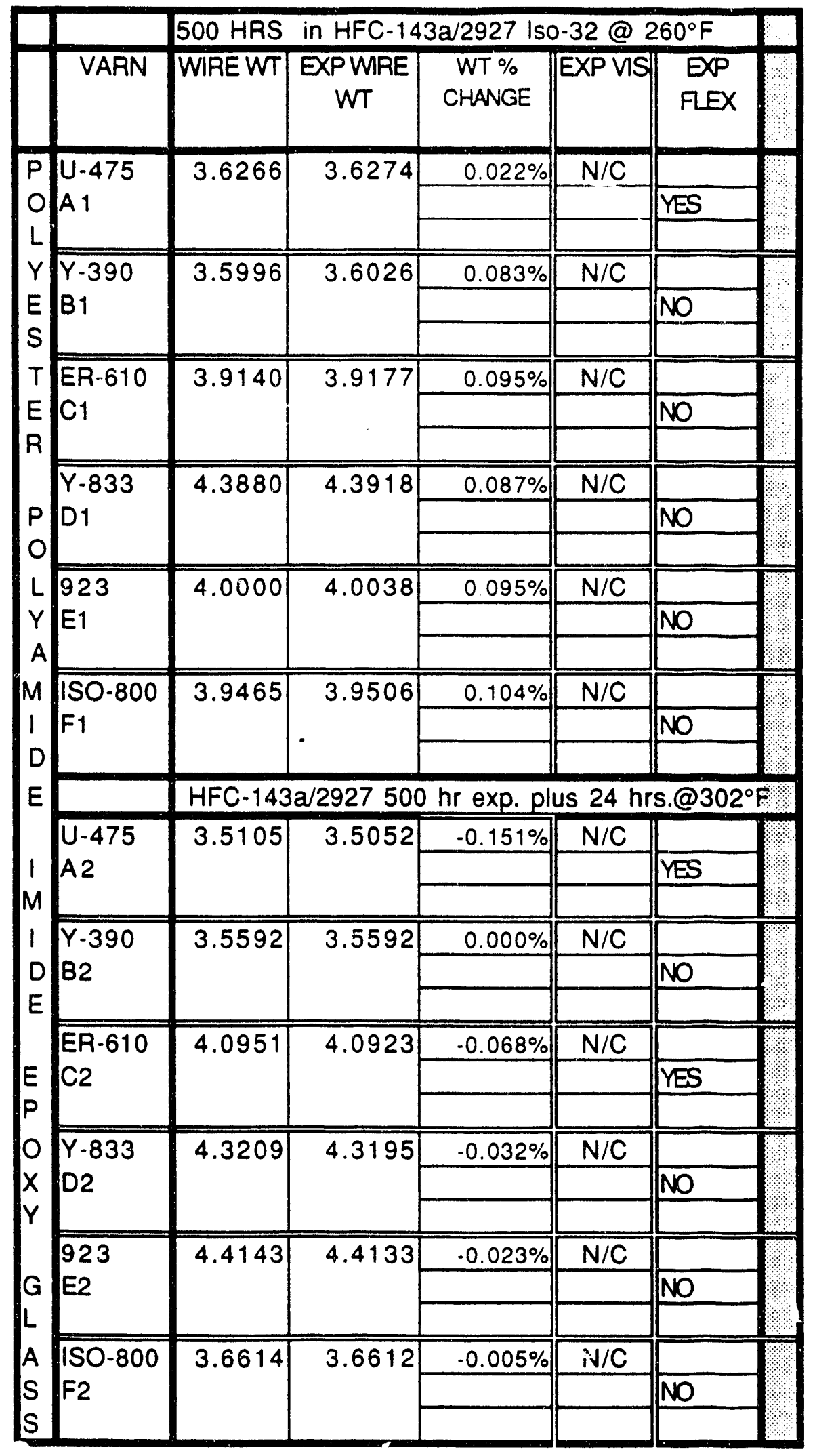

) 


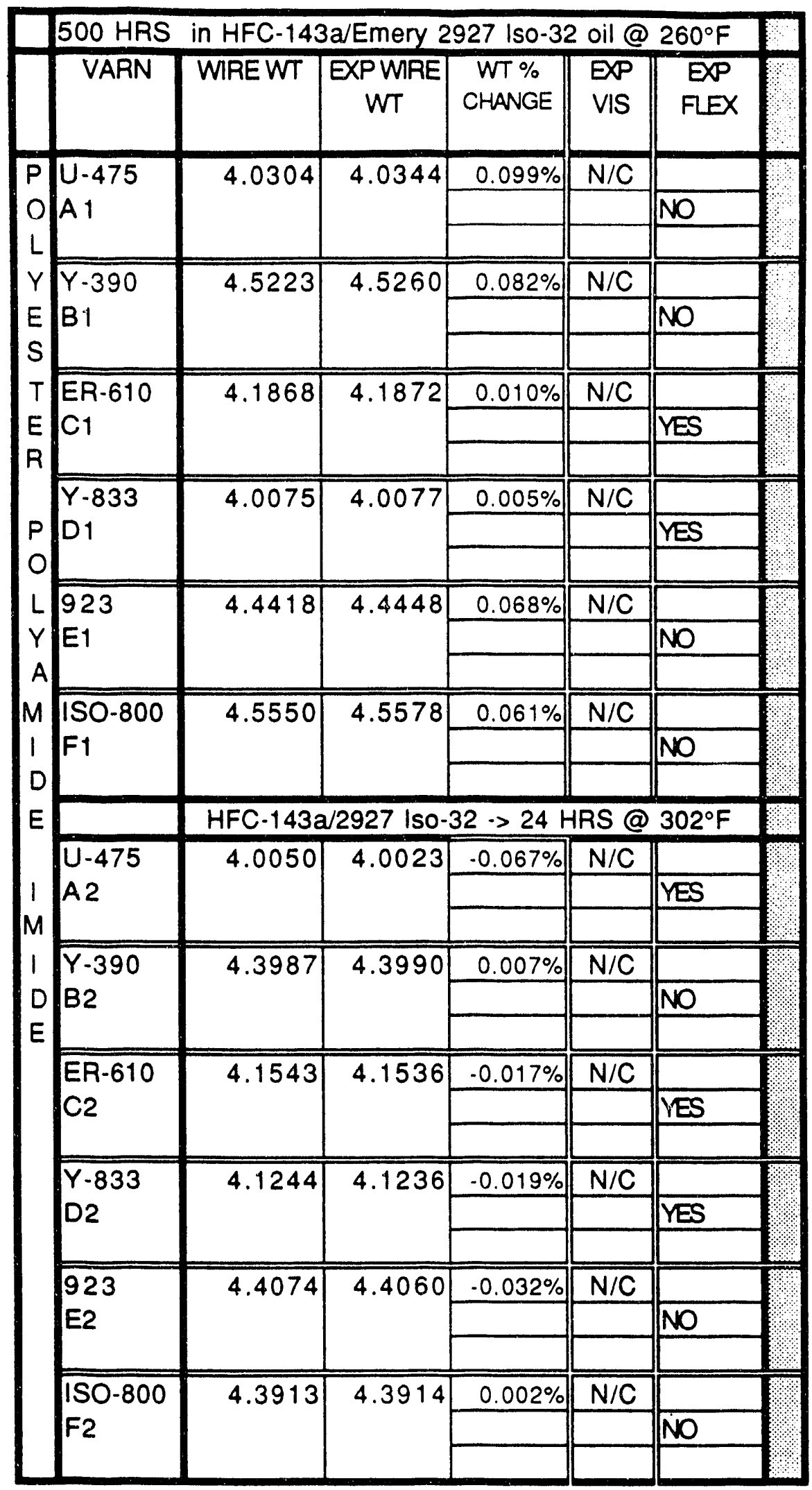




\begin{tabular}{|c|c|c|c|c|c|}
\hline \multicolumn{6}{|c|}{500 HRS IN R-143a/Emery 2927 Iso -32@ 260 F } \\
\hline ID & WT & EXPWT & $\begin{array}{l}\text { WT \% } \\
\text { CHANGE }\end{array}$ & EXPVIS & EXPFLEX \\
\hline $\begin{array}{l}\text { U-475 } \\
\text { A } 1\end{array}$ & 2.4280 & $2.4090_{1}$ & $-0.78 \%$ & N/C & $\mathrm{N} / \mathrm{C}$ \\
\hline$Y-390$ & 2.2646 & 2.1947 & $3.09 \%$ & $\mathrm{~N} / \mathrm{C}$ & $\mathrm{N} / \mathrm{C}$ \\
\hline & & & & & \\
\hline ER-610 & 2.5289 & 2.5588 & $1.18 \%$ & $\mathrm{~N} / \mathrm{C}$ & $\mathrm{N} / \mathrm{C}$ \\
\hline & & & & & \\
\hline Y-833 & 2.6216 & 2.6212 & $-0.02 \%$ & $\mathrm{~N} / \mathrm{C}$ & $\mathrm{N} / \mathrm{C}$ \\
\hline & & & & & \\
\hline 923 & 1.4781 & 1.4488 & $.1 .98 \%$ & $N / C$ & $\mathrm{~N} / \mathrm{C}$ \\
\hline E1 & & & & & \\
\hline $150-800$ & 1.6443 & 1.6003 & $-2.68 \%$ & $\mathrm{~N} / \mathrm{C}$ & $\mathrm{N} / \mathrm{C}$ \\
\hline & & & & & \\
\hline & & & & lighter in & color \\
\hline & $\mathrm{R}-143 a / \mathrm{Em}$ & y 2927 al. & $\rightarrow>24$ hou & at $302^{\circ} \mathrm{F}$ & \\
\hline$U-475$ & 2.2079 & 2.1568 & $-2.31 \%$ & darkened & $\mathrm{N} / \mathrm{C}$ \\
\hline & & & & some & \\
\hline$Y-390$ & 1.7525 & $\mid 1.6546$ & $-5.59 \%$ & slightly & $\mathrm{N} / \mathrm{C}$ \\
\hline B2 & & & & darkened & \\
\hline ER-610 & 2.1702 & 2.1441 & $-1.20 \%$ & darkened & $\mathrm{N} / \mathrm{C}$ \\
\hline & & & & & \\
\hline Y.833 & 2.3114 & 2.2724 & $-1.69 \%$ & $\sqrt{N / C}$ & $N / C$ \\
\hline D2 & & & & & \\
\hline 923 & 19701 & 19147 & $-281 \%$ & sliahtlv & \\
\hline E2 & & & & darkened & $N / C$ \\
\hline & & & & & \\
\hline ISO-800 & 1.8043 & 1.7389 & $-3.62 \%$ & slightly & \\
\hline F2 & & & & warped & $N / C$ \\
\hline & & & & darkened & \\
\hline
\end{tabular}




\begin{tabular}{|c|c|c|c|c|c|c|c|c|c|}
\hline & $500 \mathrm{HR}$ & in $\mathrm{HFC}$ & $.143 \mathrm{a} / \mathrm{Em}$ & ry 2927 & $-32 @$ & $260 \mathrm{~F}$ & & & \\
\hline ID & WT & EXPWT & $\begin{array}{l}\text { WT \% } \\
\text { CHANGE }\end{array}$ & $\begin{array}{l}\text { SAMPLE } \\
\text { WIDTH }\end{array}$ & $\begin{array}{l}\text { BREAK } \\
\text { LOAD }\end{array}$ & \begin{tabular}{|c|c} 
SAMPLE \\
THICKNESS \\
Mils
\end{tabular} & $\begin{array}{c}\text { TENSILE } \\
\text { STR } \\
\text { BASE }\end{array}$ & $\begin{array}{l}\text { TENSILE } \\
\text { STREXP }\end{array}$ & $\begin{array}{l}\text { \% CHANGE } \\
\text { TENSILE }\end{array}$ \\
\hline NO/MYINO & 7.1251 & 7.5123 & $5.43 \%$ & 0.468 & 155.30 & 21 & 17.4 & 15.80 & \\
\hline A1 & & & & 0.325 & 112.40 & 21 & 17.4 & 16.47 & $.7 .06 \%$ \\
\hline & & & & 0.396 & 135.10 & 21 & 17.4 & 16.25 & \\
\hline$D A / M Y / D A$ & 7.2354 & 7.3359 & $1.39 \%$ & 0.397 & 110.80 & 21 & 13.7 & 13.29 & \\
\hline & & & & 0.408 & 112.80 & 21 & 13.7 & 13.17 & $-2.63 \%$ \\
\hline & & & & 0.423 & 120.50 & 21 & 13.7 & 13.57 & \\
\hline MYAR MO & 3.7590 & 3.7849 & $0.69 \%$ & 0.418 & 68.85 & 10 & 21.7 & 16.47 & \\
\hline C1 & & & & 0.491 & 81.95 & 10 & 21.7 & 16.69 & $-22.25 \%$ \\
\hline & & & & 0.340 & 59.35 & 10 & 21.7 & 17.46 & \\
\hline NO 410 & 3.5082 & 3.9127 & $11.53 \%$ & 0.520 & 97.65 & 10 & 18.7 & 18.78 & \\
\hline D1 & & & & 0.455 & 85.10 & 10 & 18.7 & 18.70 & $0.43 \%$ \\
\hline & & & & 0.503 & 94.85 & 10 & 18.7 & 18.86 & \\
\hline NO MI 418 & 3.6766 & 4.2713 & $16.18 \%$ & 0.514 & 29.95 & 10 & 7.5 & 5.83 & \\
\hline & & & & 0.486 & 28.40 & 10 & 7.5 & 5.84 & $-23.04 \%$ \\
\hline & & & & 0.480 & 27.10 & 10 & 7.5 & 5.65 & \\
\hline MEL 228 & 4.3058 & 4.3475 & $0.97 \%$ & 0.467 & 77.65 & 10 & 21.7 & 16.63 & \\
\hline & & & & 0.425 & 69.45 & 10 & 21.7 & 16.34 & $-24.23 \%$ \\
\hline & & & & 0.493 & 80.65 & 10 & 21.7 & 16.36 & \\
\hline & $500 \mathrm{HRS}$ & $S$ in $H F C$ & -143a/Emer & ry 2927 is & $032 @$ & $260 \mathrm{~F}->24 \mathrm{H}$ & IRS @ 30 & $2^{\circ} \mathrm{F}$ & \\
\hline NO/MY/NO & 7.4362 & 7.6394 & $2.73 \%$ & 0.395 & 135.70 & 21 & 17.4 & 16.36 & \\
\hline A2 & & & & 0.425 & 154.50 & 21 & 17.4 & 17.31 & $-2.90 \%$ \\
\hline & & & & 0.387 & 138.30 & 21 & 17.4 & 17.02 & \\
\hline$D A / M Y / D A$ & 7.5410 & 7.4617 & $-1.05 \%$ & 0.385 & 104.20 & 21 & 13.7 & 12.89 & \\
\hline & & & & 0.461 & 126.90 & 21 & 13.7 & 13.11 & $-4.89 \%$ \\
\hline & & & & 0.468 & 128.70 & 21 & 13.7 & 13.10 & \\
\hline MYAR MO & 4.1074 & 4.1039 & $-0.09 \%$ & 0.410 & 69.60 & 10 & 21.7 & 16.98 & \\
\hline C2 & & & & 0.485 & 79.80 & 10 & 21.7 & 16.45 & $-23.02 \%$ \\
\hline & & & & 0.478 & 79.75 & 10 & 21.7 & 16.68 & \\
\hline NOMEX 410 & 3.6405 & 3.8461 & $5.65 \%$ & 0.474 & 93.55 & 10 & 18.7 & 19.74 & \\
\hline D2 & & & & 0.490 & 95.25 & 10 & 18.7 & 19.44 & $2.79 \%$ \\
\hline & & & & 0.418 & 77.30 & 10 & 18.7 & 18.49 & \\
\hline $\mathrm{NO} / \mathrm{MI} 418$ & 3.5418 & 3.9607 & $11.83 \%$ & 0.504 & 32.10 & 10 & 7.5 & 6.37 & \\
\hline & & & & 0.502 & 31.00 & 10 & 7.5 & 6.18 & $-17.47 \%$ \\
\hline & & & & 0.502 & 30.25 & 10 & 7.5 & 6.03 & \\
\hline MEL 228 & 4.1519 & 4.1568 & $0.12 \%$ & 0.484 & 82.05 & 10 & 21.7 & 16.95 & \\
\hline F2 & & & & 0.432 & 73.45 & 10 & 21.7 & 17.00 & $-21.63 \%$ \\
\hline & & & & 0.443 & 75.60 & 10 & 21.7 & 17.07 & \\
\hline
\end{tabular}




\begin{tabular}{|c|c|c|c|c|c|c|c|c|}
\hline & $10 \mathrm{HR}$ & in HFC & $3 a / E n$ & y 2927 is & so-32 @ & $260 \mathrm{~F}$ & & \\
\hline ID & $\begin{array}{c}\text { STRETCH } \\
\text { (inch) }\end{array}$ & $\begin{array}{c}\% \\
\text { ELONG }\end{array}$ & $\begin{array}{l}\text { BASE } \\
\text { ELONG } \\
\text { (AVE) }\end{array}$ & $\begin{array}{l}\text { ELONG \% } \\
\text { CHANGE }\end{array}$ & $\begin{array}{c}\text { BASE } \\
\text { DE } \\
(A \vee E)\end{array}$ & EXPDIE & $\begin{array}{c}\text { DIE \% } \\
\text { CHANGE }\end{array}$ & VISUAL EXP \\
\hline NO/MY/NO & 0.50 & $12.5 \%$ & $20.0 \%$ & & $>18.97$ & flash & & Small pockets on eac \\
\hline & 0.59 & $14.8 \%$ & $20.0 \%$ & $.31 .9 \%$ & $>18.97$ & flash & flash & of pieces, pulled \\
\hline & 0.50 & $12.5 \%$ & $20.0 \%$ & & $>18.97$ & flash & & \\
\hline DA/MY/DA & 0.50 & $25.0 \%$ & $46.0 \%$ & & $>15.27$ & flash & & Yellowed \\
\hline & 0.52 & $26.0 \%$ & $46.0 \%$ & $.44 .2 \%$ & $>15.27$ & flash & flash & slightly \\
\hline & 0.52 & $26.0 \%$ & $46.0 \%$ & & $>15.27$ & flash & & warped \\
\hline MYLAR MO & 2.29 & $114.5 \%$ & $131.0 \%$ & & $>14.91$ & flash & & $\mathrm{N} / \mathrm{C}$ \\
\hline & 1.89 & $94.5 \%$ & $131.0 \%$ & $.17 .7 \%$ & $>14.91$ & flash & flash & \\
\hline & 2.29 & $114.5 \%$ & $131.0 \%$ & & 214.91 & flash & & \\
\hline NO 410 & 0.40 & $20.0 \%$ & $17.0 \%$ & & 10.67 & flash & & $N / C$ \\
\hline D1 & 0.41 & $10.3 \%$ & $17.0 \%$ & $-18.6 \%$ & 10.67 & flash & flash & \\
\hline & 0.45 & $11.3 \%$ & $17.0 \%$ & & 10.67 & flash & & \\
\hline NO MI 418 & 0.06 & $1.5 \%$ & $4.0 \%$ & & 10.23 & 11.73 & & $N / C$ \\
\hline E1 & 0.06 & $1.5 \%$ & $4.0 \%$ & $.62 .5 \%$ & 10.23 & 9.91 & $2.8 \%$ & \\
\hline & 0.06 & $1.5 \%$ & $4.0 \%$ & & 10.23 & 9.92 & & \\
\hline MEL 228 & 3.07 & $153.5 \%$ & $160.0 \%$ & & $>14.22$ & flash & & slightly \\
\hline & 1.46 & $73.0 \%$ & $160.0 \%$ & $.21 .4 \%$ & $>14.22$ & flash & flash & yellowed \\
\hline & 3.02 & $151.0 \%$ & $160.0 \%$ & & $>14.22$ & flash & & \\
\hline & $500 \mathrm{HRS}$ & in HFC. & $43 a / E m \theta$ & ry 2927 is & so32@ & $260 \mathrm{~F} \cdot>$ & 24 HRS & (9 $302^{\circ} \mathrm{F}$ \\
\hline NO/MY/NO & 0.48 & $12.0 \%$ & $20.0 \%$ & & $>18.97$ & flash & & Delamination \\
\hline & 0.60 & $15.0 \%$ & $20.0 \%$ & $.29 .2 \%$ & $>18.97$ & flash & flash & \\
\hline & 0.62 & $15.5 \%$ & $20.0 \%$ & & $>18.97$ & flash & & \\
\hline$D A / M Y / D A$ & 0.35 & $17.5 \%$ & $46.0 \%$ & & $>15.27$ & fiash & & Yellowed \\
\hline & 0.45 & $22.5 \%$ & $46.0 \%$ & $.52 .9 \%$ & $>15.27$ & flash & flash & slightly \\
\hline & 0.50 & $25.0 \%$ & $46.0 \%$ & & $>15.27$ & flash & & warped \\
\hline MYARMO & 2.34 & $117.0 \%$ & $131.0 \%$ & & $>14.91$ & flash & & $\mathrm{N} / \mathrm{C}$ \\
\hline & 2.45 & $122.5 \%$ & $131.0 \%$ & $-14.9 \%$ & $>14.91$ & flash & flash & \\
\hline & 1.90 & $95.0 \%$ & $131.0 \%$ & & $>14.91$ & flash & & \\
\hline NOMEX 410 & 0.43 & $10.8 \%$ & $17.0 \%$ & & 10.67 & 12.29 & & $\mathrm{~N} / \mathrm{C}$ \\
\hline $\mathrm{D} 2$ & 0.38 & $9.5 \%$ & $17.0 \%$ & $.45 .6 \%$ & 10.67 & 11.56 & $10.8 \%$ & 1 piece had 1 \\
\hline & 0.30 & $7.5 \%$ & $17.0 \%$ & & 10.67 & 11.62 & & bubble \\
\hline NO/MI 418 & 0.05 & $1.3 \%$ & $4.0 \%$ & & 10.23 & 10.10 & & $N / C$ \\
\hline & 0.05 & $1.3 \%$ & $4.0 \%$ & $.68 .8 \%$ & 10.23 & 10.97 & $-1.0 \%$ & \\
\hline & 0.05 & $1.3 \%$ & $4.0 \%$ & & 10.23 & 9.31 & & \\
\hline MEL 228 & 2.27 & $113.5 \%$ & $160.0 \%$ & & $>14.22$ & flash & & slightly \\
\hline & 2.42 & $121.0 \%$ & $160.0 \%$ & $.16 .6 \%$ & $>14.22$ & flash & flash & yellowed \\
\hline & 3.32 & $166.0 \%$ & $160.0 \%$ & & $>14.22$ & flash & & \\
\hline
\end{tabular}


SLEEVING--HFC-143a/Emery 2927 Iso-32@260 $\mathrm{F}\left(127^{\circ} \mathrm{C}\right)$

\begin{tabular}{|c|c|c|c|c|}
\hline & \multicolumn{4}{|c|}{500 HRS in HFC-143a/Emery 2927 Iso.32@ $260^{\circ} \mathrm{F}$} \\
\hline & WT & EXPWT & $\begin{array}{c}\% \\
\text { CHANGE } \\
\end{array}$ & EXP VIS \\
\hline A1 & 0.5741 & 0.6394 & $11.37 \%$ & $\mathrm{~N} / \mathrm{C}$ \\
\hline & 0.5050 & 0.5120 & $1.39 \%$ & Pockets \\
\hline \multirow{2}{*}{$\begin{array}{l}\text { C1 } \\
\text { NO/MY }\end{array}$} & 0.3988 & 0.4146 & $3.96 \%$ & some pockets \\
\hline & \multicolumn{4}{|c|}{ HFC-143a/Emkarate RL244 oil @ 260 F plus24 hrs @ 302 ${ }^{\circ} \mathrm{F}$} \\
\hline $\begin{array}{l}\text { A2 } \\
\text { NOMEX }\end{array}$ & 0.5559 & 0.5903 & $6.19 \%$ & $\mathrm{~N} / \mathrm{C}$ \\
\hline \multirow{2}{*}{$\begin{array}{l}\text { B2 } \\
\text { MYAAR }\end{array}$} & 0.4790 & 0.4783 & $-0.15 \%$ & $\mathrm{~N} / \mathrm{C}$ \\
\hline & & & & some pockets \\
\hline \multirow{3}{*}{$\begin{array}{l}\mathrm{C} 2 \\
\mathrm{NO} / \mathrm{MY}\end{array}$} & 0.3963 & 0.4000 & $0.93 \%$ & $\mathrm{~N} / \mathrm{C}$ \\
\hline & & - & & some pockets where mylar \\
\hline & & & & pulled away. \\
\hline
\end{tabular}




\begin{tabular}{|c|c|c|c|c|c|c|c|c|c|c|}
\hline & 0 HRS & In HFC-1 & 3a/Emery & 927 Iso? & $\omega$ & & & & & \\
\hline ID & WT & EXPWT & $\begin{array}{c}\text { WT \% } \\
\text { CHANGE }\end{array}$ & $\begin{array}{l}\text { BREAK } \\
\text { LOAD } \\
\text { (AVE) }\end{array}$ & $\begin{array}{l}\text { BREAK } \\
\text { LOAD } \\
\text { EPP }\end{array}$ & $\begin{array}{c}\% \\
\text { CHANGE } \\
\text { BPK } \\
\text { LOAD }\end{array}$ & $\begin{array}{l}\text { STRETCH } \\
\text { (INCH) }\end{array}$ & \%ELONG & EXPVIS & \\
\hline A 1 & 1.7175 & 1.7169 & $-0.03 \%$ & 39.02 & 53.50 & & 0.06 & $3.00 \%$ & $\mathrm{~N} / \mathrm{C}$ & \\
\hline Glass & & & & 39.02 & 52.80 & $41.64 \%$ & 0.06 & $3.00 \%$ & & \\
\hline & & & & 39.02 & 59.50 & & 0.06 & $3.00 \%$ & & \\
\hline$B 1$ & 0.7559 & 0.7620 & $0.81 \%$ & 56.12 & 53.60 & & 0.51 & $25.50 \%$ & $\mathrm{~N} / \mathrm{C}$ & \\
\hline Polyester & & & & 56.12 & 57.95 & $-4.82 \%$ & 0.60 & $30.00 \%$ & & \\
\hline & & & & 56.12 & 48.70 & & 0.38 & $19.00 \%$ & & \\
\hline$C_{1}$ & 1.6088 & 1.6001 & $-0.54 \%$ & 88.50 & 86.60 & & 0.17 & $8.50 \%$ & Slightly & \\
\hline Permacel & & & & 88.50 & 60.65 & $-18.23 \%$ & 0.13 & $6.50 \%$ & darkened & \\
\hline & & & & 88.50 & 69.85 & & 0.13 & $6.50 \%$ & Curled up & \\
\hline & $500 \mathrm{HR}$ & $S$ in HFC. & 43a/Emer & 2927 ISC & $32 @ 260$ & $F->302^{\circ} \mathrm{F}$ & 24 HRS & & & \\
\hline A2 & 1.5224 & 1.5207 & $-0.11 \%$ & 39.02 & 45.25 & & 0.05 & $2.50 \%$ & $\mathrm{~N} / \mathrm{C}$ & \\
\hline Glass & & & & 39.02 & 54.85 & $41.94 \%$ & 0.06 & $3.00 \%$ & & \\
\hline & & & & 39.02 & 66.05 & & 0.07 & $3.50 \%$ & & \\
\hline & 0.6862 & 0.6822 & $-0.58 \%$ & 56.12 & 52.15 & & 0.51 & $25.50 \%$ & $\mathrm{~N} / \mathrm{C}$ & \\
\hline Polyester & & & & 56.12 & 51.75 & $.6 .93 \%$ & 0.46 & $23.00 \%$ & & \\
\hline & & & & 56.12 & 52.80 & & 0.50 & $25.00 \%$ & & \\
\hline $\mathrm{C2}$ & 1.4177 & 1.2292 & $-13.30 \%$ & 88.50 & 73.50 & & 0.09 & $4.50 \%$ & Slightly & \\
\hline Permacel & & & & 88.50 & 106.20 & $2.22 \%$ & 0.13 & $6.50 \%$ & Darkened & \\
\hline & & & & 88.50 & 91.70 & & 0.10 & $5.00 \%$ & Curled up & \\
\hline
\end{tabular}


TIE CORD--HFC-143a/Emery 2927 ISO-32 oil @ $260^{\circ} \mathrm{F}\left(127^{\circ} \mathrm{C}\right)$

\begin{tabular}{|c|c|c|c|c|c|c|c|c|c|}
\hline \multicolumn{10}{|c|}{500 HRS IN R-143a/2927 Iso-32@260\% F } \\
\hline ID & WT & EXPWT & $\begin{array}{c}\text { WT \% } \\
\text { CHANGE }\end{array}$ & $\begin{array}{l}\text { EXP } \\
\text { VIS }\end{array}$ & $\begin{array}{l}\text { BREAK } \\
\text { LOAD } \\
\text { (AVE) }\end{array}$ & $\begin{array}{l}\text { BREAK } \\
\text { LOAD } \\
\text { EXP } \\
(\operatorname{av\theta })\end{array}$ & $\begin{array}{c}\% \\
\text { CHANGE } \\
\text { BPK } \\
\text { LOAD }\end{array}$ & $\begin{array}{c}\text { STRETCH } \\
\text { (lnch) }\end{array}$ & $\begin{array}{c}\% \\
\text { ELONG }\end{array}$ \\
\hline \multirow[t]{4}{*}{ A1 } & \multirow[t]{3}{*}{0.2839} & \multirow[t]{3}{*}{0.2838} & \multirow[t]{3}{*}{$-0.04 \%$} & $\mathrm{~N} / \mathrm{C}$ & 28.36 & 29.30 & & 0.38 & $19.0 \%$ \\
\hline & & & & & 28.36 & 37.30 & \multirow[t]{2}{*}{$18.07 \%$} & 0.45 & $22.5 \%$ \\
\hline & & & & & 28.36 & 33.85 & & 0.42 & $21.0 \%$ \\
\hline & \multicolumn{9}{|c|}{500 HRS IN R-143a/Emery 2927 -> 24 HRS @ 302 ${ }^{\circ} \mathrm{F}$} \\
\hline \multirow[t]{3}{*}{ A2 } & \multirow[t]{3}{*}{0.3217} & \multirow[t]{3}{*}{0.3188} & \multirow[t]{3}{*}{$-0.901 \%$} & $\mathrm{~N} / \mathrm{C}$ & 28.36 & 25.50 & & 0.46 & $23.0 \%$ \\
\hline & & & & & 28.36 & 37.55 & $12.31 \%$ & 0.47 & $23.5 \%$ \\
\hline & & & & & 28.36 & 32.50 & & 0.42 & $21.0 \%$ \\
\hline
\end{tabular}




\begin{tabular}{|c|c|c|c|c|c|c|c|}
\hline & $500 \mathrm{HRS}$ IN & $\mathrm{FC}-143 \mathrm{a} / \mathrm{En}$ & hery 2927 & Iso-32.@ & $260^{\circ} \mathrm{F}$ & & \\
\hline ID & $W T$ & EXPWT & $\begin{array}{c}\text { WT \% } \\
\text { CHANGE }\end{array}$ & EXP VIS & $\begin{array}{l}\text { BASE DIE } \\
\text { (AVE) }\end{array}$ & EXP DIE & $\begin{array}{c}\text { DIE\% } \\
\text { CHANGE }\end{array}$ \\
\hline DMD & 4.1503 & 4.2912 & $3.39 \%$ & $\mathrm{~N} / \mathrm{C}$ & 9.61 & 9.37 & \\
\hline A1 & & & & & 9.61 & 8.59 & $-18.66 \%$ \\
\hline & & & & & 9.61 & 5.49 & \\
\hline DTMD & 4.4449 & 4.5720 & $2.86 \%$ & $\mathrm{~N} / \mathrm{C}$ & 9.95 & flash & \\
\hline $\mathrm{B1}$ & & & & & 9.95 & 12.91 & $29.75 \%$ \\
\hline & & & & & 9.95 & flash & \\
\hline & HFC-143a/En & ery 2927 Is & -32@26 & $0^{\circ} \mathrm{F}->2$ & HRS@30 & $2^{\circ} \mathrm{F}$ & \\
\hline DMD & 4.0071 & 4.0820 & $1.87 \%$ & $\mathrm{~N} / \mathrm{C}$ & 9.61 & 9.25 & \\
\hline$A 2$ & & & & & 9.61 & 9.79 & $0.38 \%$ \\
\hline & & & & & 9.61 & 9.90 & \\
\hline DTMD & 4.3087 & 4.3560 & $1.10 \%$ & $\mathrm{~N} / \mathrm{C}$ & 9.95 & 10.40 & \\
\hline $\mathrm{B} 2$ & & & & & 9.95 & 11.54 & $8.14 \%$ \\
\hline & & & & & 9.95 & 10.34 & \\
\hline
\end{tabular}

) 


\section{Appendix K}

Experimental Data for HFC-134/HenkelEmery 2927 ISO-32 Exposure at $126^{\circ} \mathrm{C}\left(260^{\circ} \mathrm{F}\right)$ 


\begin{tabular}{|c|c|c|c|c|c|c|c|c|c|c|}
\hline & 500 hours & $7 \mathrm{HFC}-13$ & 2927 Iso- & 32 oil a & It $260^{\circ} \mathrm{F}(12$ & & & & & \\
\hline ID & WT & EXPWT & $\begin{array}{c}\text { WT \% } \\
\text { CHANGE }\end{array}$ & EXP VIS & $\begin{array}{c}\text { BASE BRN } \\
\text { OUT } \\
(A \vee E)\end{array}$ & $\begin{array}{c}\text { EXP BRN } \\
\text { OUT }\end{array}$ & $\begin{array}{c}\text { BRN OUTT } \\
\% \\
\text { CHANGE }\end{array}$ & $\begin{array}{c}\text { BASE DIE } \\
\text { (AVE) }\end{array}$ & EXP DIE & $\begin{array}{c}\text { DIE \% } \\
\text { CHANGE }\end{array}$ \\
\hline A1 & 24.3676 & 24.3803 & $0.052 \%$ & $N / C$ & 576 & 553 & & 15.80 & 15.27 & \\
\hline & & & & & 576 & 464 & $-9.1 \%$ & 15.80 & 16.22 & $-2.6 \%$ \\
\hline & & & & & 576 & 553 & & 15.80 & 14.66 & \\
\hline $\mathrm{B1}$ & 26.3649 & 26.4054 & $0.154 \%$ & $\mathrm{~N} / \mathrm{C}$ & 736 & 734 & & 11.62 & 12.86 & \\
\hline & & & & & 736 & 734 & $-0.3 \%$ & 11.62 & 11.81 & $9.0 \%$ \\
\hline & & & & & 736 & 734 & & 11.62 & 13.31 & \\
\hline C1 & 25.4219 & 25.4334 & $0.045 \%$ & $N / C$ & 579 & 567 & & 16.58 & 11.74 & \\
\hline & & & & & 579 & 418 & $-10.4 \%$ & 16.58 & 12.14 & $-18.9 \%$ \\
\hline & & & & & 579 & 572 & & 16.58 & 16.46 & \\
\hline & 500 hours i & HFC-134 & /Emery 29 & 27 oil at & $260^{\circ} \mathrm{F}(127$ & $\left.{ }^{\circ} \mathrm{C}\right)$ plus 2 & 24 hours a & $302^{\circ} \mathrm{F}(1$ & $\left.50^{\circ} \mathrm{C}\right)$ & \\
\hline A2 & 23.8171 & 23.8118 & $-0.022 \%$ & $N / C$ & 576 & 541 & & 15.80 & 15.07 & \\
\hline & & & & & 576 & 567 & $-4.6 \%$ & 15.80 & 10.49 & $-13.0 \%$ \\
\hline & & & & & 576 & 540 & & 15.80 & 15.66 & \\
\hline$\overline{\mathrm{B2}}$ & 25.8679 & 25.8489 & $-0.073 \%$ & $\mathrm{~N} / \mathrm{C}$ & 736 & 729 & & 11.62 & 11.89 & \\
\hline & & & & & 736 & 729 & $-0.9 \%$ & 11.62 & 11.43 & $-2.0 \%$ \\
\hline & & & & & 736 & 730 & & 11.62 & 10.86 & \\
\hline$\overline{\mathrm{C} 2}$ & 28.3845 & 28.3830 & $.0 .005 \%$ & $\mathrm{~N} / \mathrm{C}$ & 579 & 565 & & 16.58 & 15.57 & \\
\hline & & & & & 579 & 479 & $-7.4 \%$ & 16.58 & 12.61 & $-16.6 \%$ \\
\hline & & & & & 579 & 565 & & 16.58 & 13.29 & \\
\hline
\end{tabular}


TWISTED PAIRSIWIRE AJWITH VARNISH-HFC-134/Emery 2927 Iso-32 oil @260 $\mathrm{F}\left(127^{\circ} \mathrm{C}\right)$

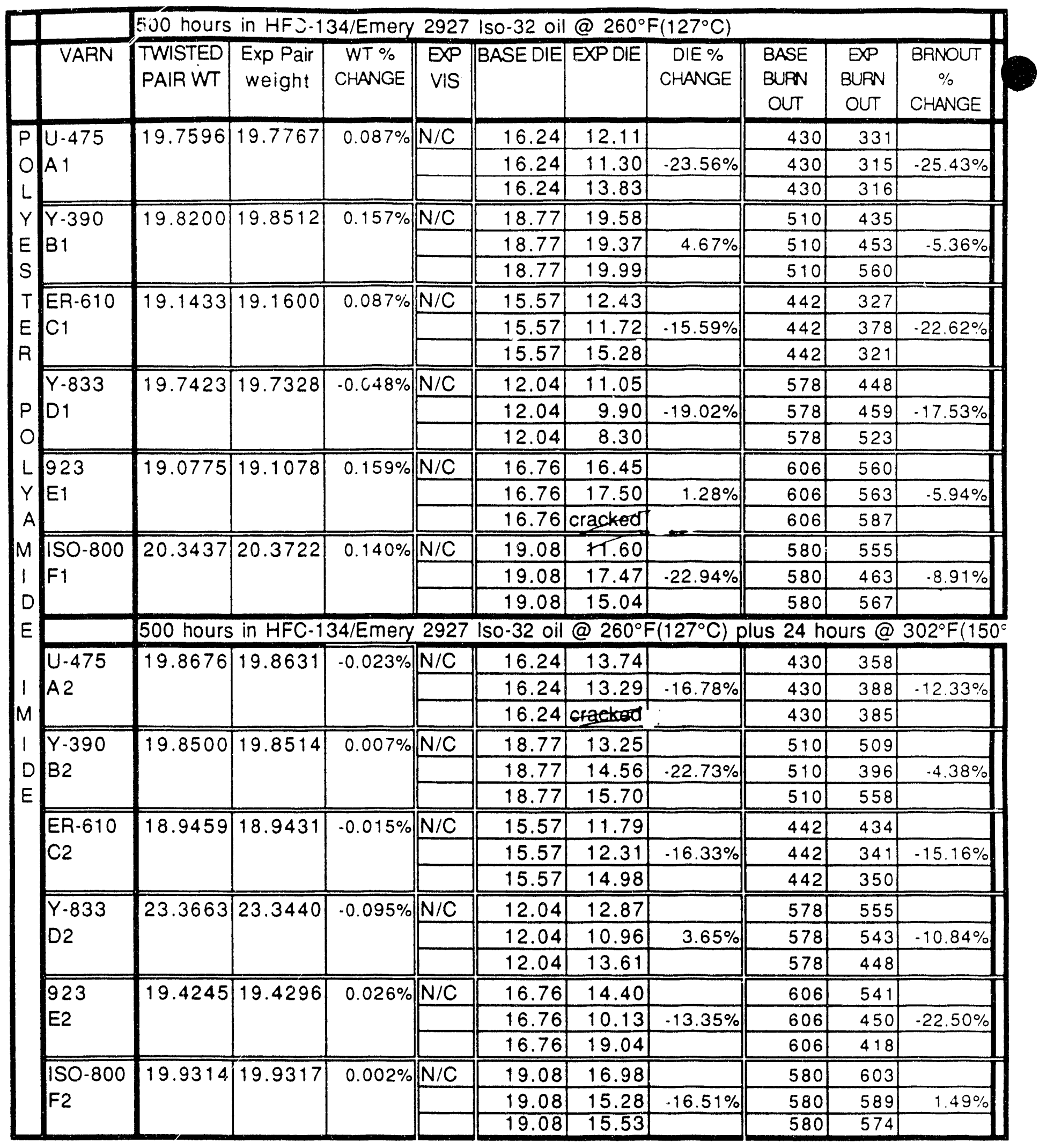


TWISTED PAIRS/WIRE BNITH VARNISH -HFC-134/Emery 2927 Iso-32 oil @260 $2\left(127^{\circ} \mathrm{C}\right)$

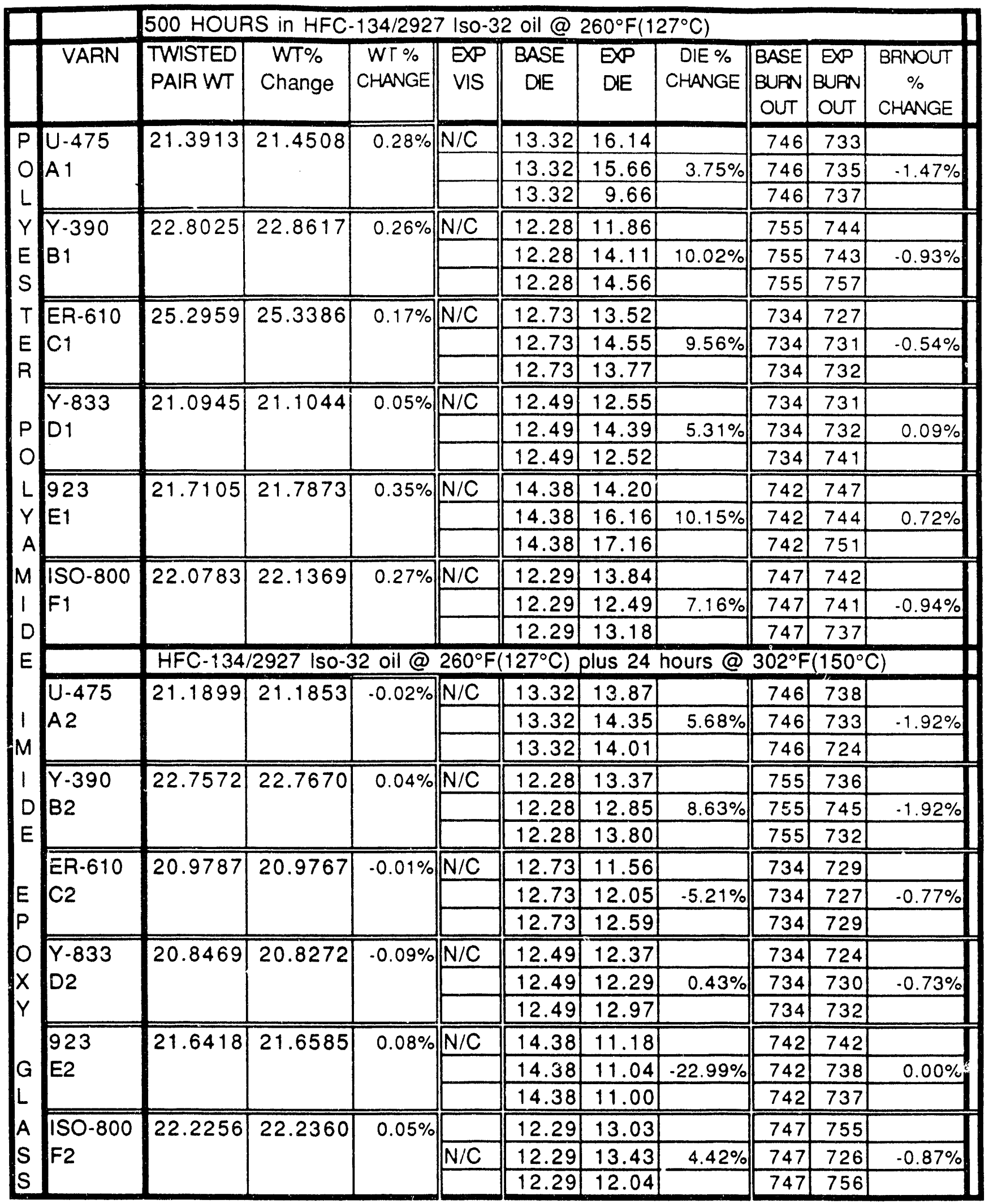


TWISTED PAIRSNIRE CNITH VARNISH-HFC-134/Emery 2927 Iso-32@ $260^{\circ} \mathrm{F}\left(127^{\circ} \mathrm{C}\right)$

\begin{tabular}{|c|c|c|c|c|c|c|c|c|c|c|c|}
\hline & & \multicolumn{10}{|c|}{ 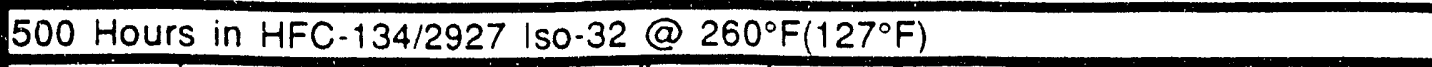 } \\
\hline & VARN & $\begin{array}{l}\text { TWISTED } \\
\text { PAIR WT }\end{array}$ & $\begin{array}{c}\text { EXP T } \\
\text { PAIR WT }\end{array}$ & $\begin{array}{c}\text { WT \% } \\
\text { CHANGE }\end{array}$ & $\begin{array}{l}\text { EXP } \\
\text { VIS }\end{array}$ & \begin{tabular}{|c|} 
BASE \\
DIE
\end{tabular} & \begin{tabular}{|l|} 
EXP \\
$D I E$
\end{tabular} & $\begin{array}{c}\text { DIE \% } \\
\text { CHANGE }\end{array}$ & $\begin{array}{l}\text { BASE } \\
\text { BUPN } \\
\text { OUT }\end{array}$ & $\begin{array}{l}\text { EXP } \\
\text { BUPN } \\
\text { OUT }\end{array}$ & $\begin{array}{c}\text { BRNOUT } \\
\% \\
\text { CHANGE }\end{array}$ \\
\hline \multirow{3}{*}{$\begin{array}{l}P \\
O \\
L\end{array}$} & \multirow{3}{*}{$\begin{array}{l}\text { U-475 } \\
\text { A } 1\end{array}$} & \multirow[t]{3}{*}{19.9950} & \multirow[t]{3}{*}{20.0244} & \multirow[t]{3}{*}{$0.147 \%$} & $\mathrm{~N} / \mathrm{C}$ & 15.10 & 15.83 & & 469 & 387 & \\
\hline & & & & & & 15.10 & 15.00 & $4.48 \%$ & 469 & 492 & $-11.44 \%$ \\
\hline & & & & & & 15.10 & 16.50 & & 469 & 367 & \\
\hline \multirow{3}{*}{$\begin{array}{l}Y \\
E \\
S\end{array}$} & \multirow{3}{*}{$\begin{array}{l}Y-390 \\
B 1\end{array}$} & \multirow[t]{3}{*}{19.6595} & \multirow[t]{3}{*}{19.6997} & \multirow[t]{3}{*}{$0.204 \%$} & $\mathrm{~N} / \mathrm{C}$ & 18.24 & 19.75 & & 473 & 559 & \\
\hline & & & & & & 18.24 & 18.86 & $-1.44 \%$ & 473 & 647 & $24.88 \%$ \\
\hline & & & & & & 18.24 & 15.32 & & 473 & 566 & \\
\hline \multirow{4}{*}{$\begin{array}{l}T \\
E \\
R\end{array}$} & \multirow{3}{*}{$\begin{array}{l}\text { ER-610 } \\
\text { C1 }\end{array}$} & \multirow[t]{3}{*}{19.7168} & \multirow[t]{3}{*}{19.7311} & \multirow[t]{3}{*}{$0.073 \%$} & $N / C$ & 14.53 & 14.50 & & 494 & 334 & \\
\hline & & & & & & 14.53 & 12.46 & $-1.22 \%$ & 494 & 413 & $-22.13 \%$ \\
\hline & & & & & & 14.53 & 16.10 & & 494 & 407 & \\
\hline & \multirow{3}{*}{$\begin{array}{l}\mathrm{Y}-833 \\
\mathrm{D} 1\end{array}$} & \multirow[t]{3}{*}{20.0809} & \multirow[t]{3}{*}{20.0781} & \multirow[t]{3}{*}{$-0.014 \%$} & $\mathrm{~N} / \mathrm{C}$ & 11.38 & 10.36 & & 557 & 442 & \\
\hline 1 & & & & & & 11.38 & 9.89 & $-9.20 \%$ & 557 & 603 & $-4.55 \%$ \\
\hline$M$ & & & & & & 11.38 & 10.75 & & 557 & 550 & \\
\hline & \multirow{3}{*}{$\begin{array}{l}923 \\
E 1\end{array}$} & \multirow[t]{3}{*}{21.0105} & \multirow[t]{3}{*}{21.0441} & $0.160 \%$ & $\mathrm{~N} / \mathrm{C}$ & 15.85 & 17.79 & & 503 & 583 & \\
\hline$D$ & & & & & & 15.85 & 18.23 & $15.10 \%$ & 503 & 553 & $12.99 \%$ \\
\hline$E$ & & & & & & 15.85 & 18.71 & & 503 & 569 & \\
\hline & $150-800$ & 19.5118 & 19.5342 & $0.115 \%$ & $\mathrm{~N} / \mathrm{C}$ & 14.75 & 7.99 & & 632 & 583 & \\
\hline$P$ & $F 1$ & & & & & 14.75 & 16.05 & $-6.89 \%$ & 632 & 588 & $-8.33 \%$ \\
\hline 0 & & & & & & 14.75 & 17.16 & & 632 & 567 & \\
\hline L & & HFC-134 & 12927 Iso- & $32 @ 260$ & ${ }^{\circ} \mathrm{F}$ plu & 24 hol & urs @ 3 & $302^{\circ} \mathrm{F}(150$ & $\left.{ }^{\circ} \mathrm{C}\right)$ & & \\
\hline$Y$ & $U-475$ & 19.5759 & 19.5623 & $-0.069 \%$ & $\mathrm{~N} / \mathrm{C}$ & 15.10 & 13.40 & & 469 & 348 & \\
\hline A & & & & & & 15.10 & 13.69 & $.3 .73 \%$ & 469 & 475 & $-16.63 \%$ \\
\hline$M$ & & & & & & 15.10 & 16.52 & & 469 & 350 & \\
\hline I & $Y-390$ & 19.5051 & 19.5124 & $0.037 \%$ & $N / C$ & 18.24 & 14.66 & & 473 & 574 & \\
\hline$D$ & $\mathrm{~B} 2$ & & & & & 18.24 & 18.53 & $-1.15 \%$ & 473 & 568 & $21.56 \%$ \\
\hline$E$ & & & & & & 18.24 & 17.53 & & 473 & 583 & \\
\hline & ER-610 & 19.3095 & 19.3053 & $.0 .022 \%$ & $N / C$ & 14.53 & 13.38 & & 494 & 374 & \\
\hline 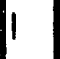 & $\mathrm{C} 2$ & & & & & 14.53 & 14.36 & $-8.47 \%$ & 494 & 412 & $.20 .45 \%$ \\
\hline$M$ & & & & & & 14.53 & 12.16 & & 494 & lost & \\
\hline 1 & $Y-833$ & 20.2166 & 20.2027 & $.0 .069 \%$ & $\mathrm{~N} / \mathrm{C}$ & 11.38 & 9.03 & & 557 & 602 & \\
\hline$D$ & D2 & & & & & 11.38 & 6.77 & $.25 .81 \%$ & 557 & 592 & $4.37 \%$ \\
\hline$E$ & & & & & & 11.38 & 9.53 & & 557 & 550 & \\
\hline & 923 & 20.2816 & 20.2841 & $0.012 \%$ & $\mathrm{~N} / \mathrm{C}$ & 15.85 & 14.42 & & 503 & 677 & \\
\hline & E2 & & & & & 15.85 & 15.22 & $.0 .59 \%$ & 503 & 615 & $17.16 \%$ \\
\hline & & & & & & 15.85 & 17.63 & & 503 & 476 & \\
\hline & $150-800$ & 19.3812 & 19.3834 & $0.011 \%$ & $\mathrm{~N} / \mathrm{C}$ & 14.75 & 15.09 & & 632 & 608 & \\
\hline & $\mathrm{F} 2$ & & & & & 14.75 & 15.90 & $0.34 \%$ & 632 & 682 & $-0.63 \%$ \\
\hline & & & & & & 14.75 & 13.41 & & 632 & $\overline{394}$ & \\
\hline
\end{tabular}


HELICAL COILSMIRE A...HFC-134/Emery 2927 Iso-32@260 $\mathrm{F}\left(127^{\circ} \mathrm{C}\right)$

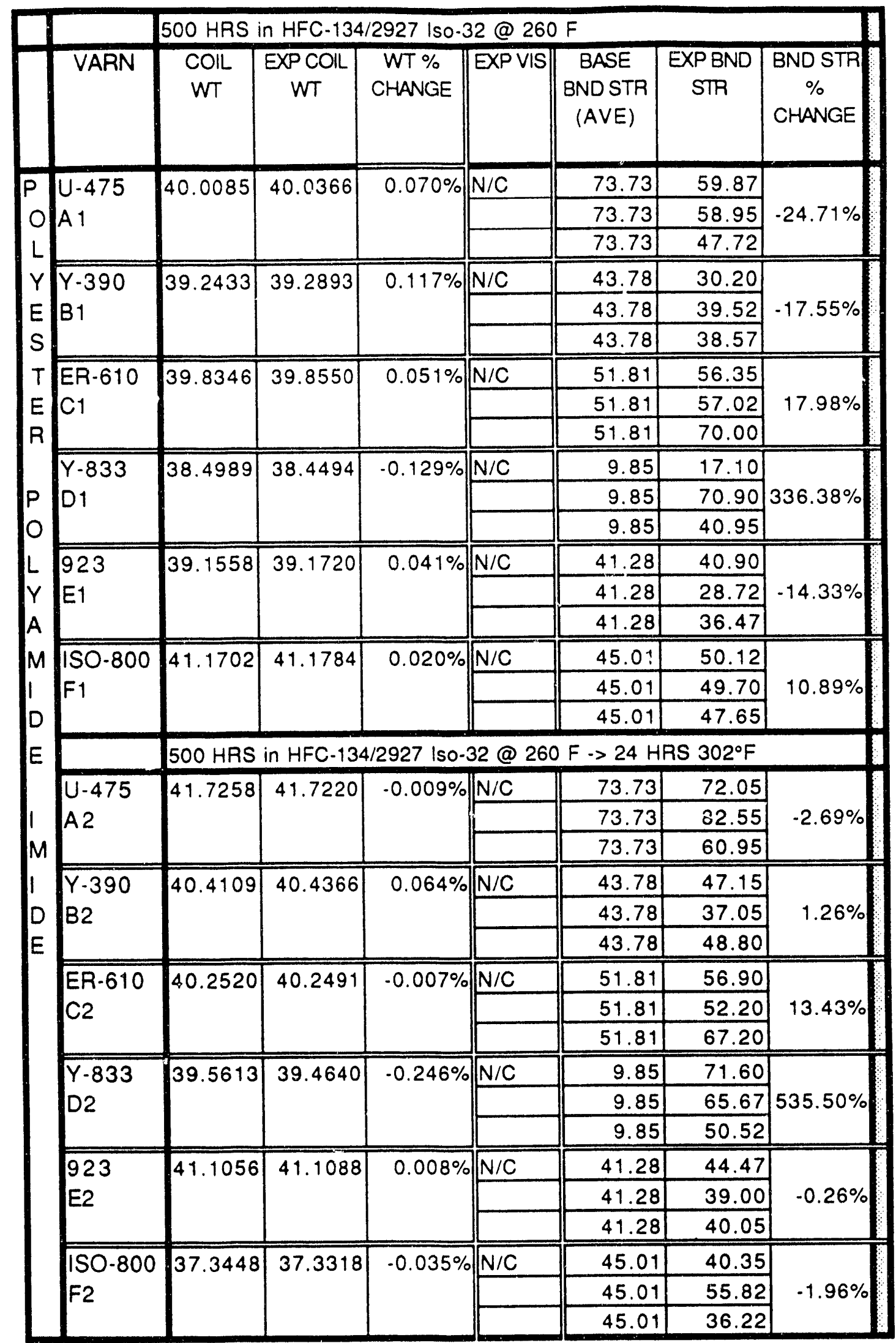




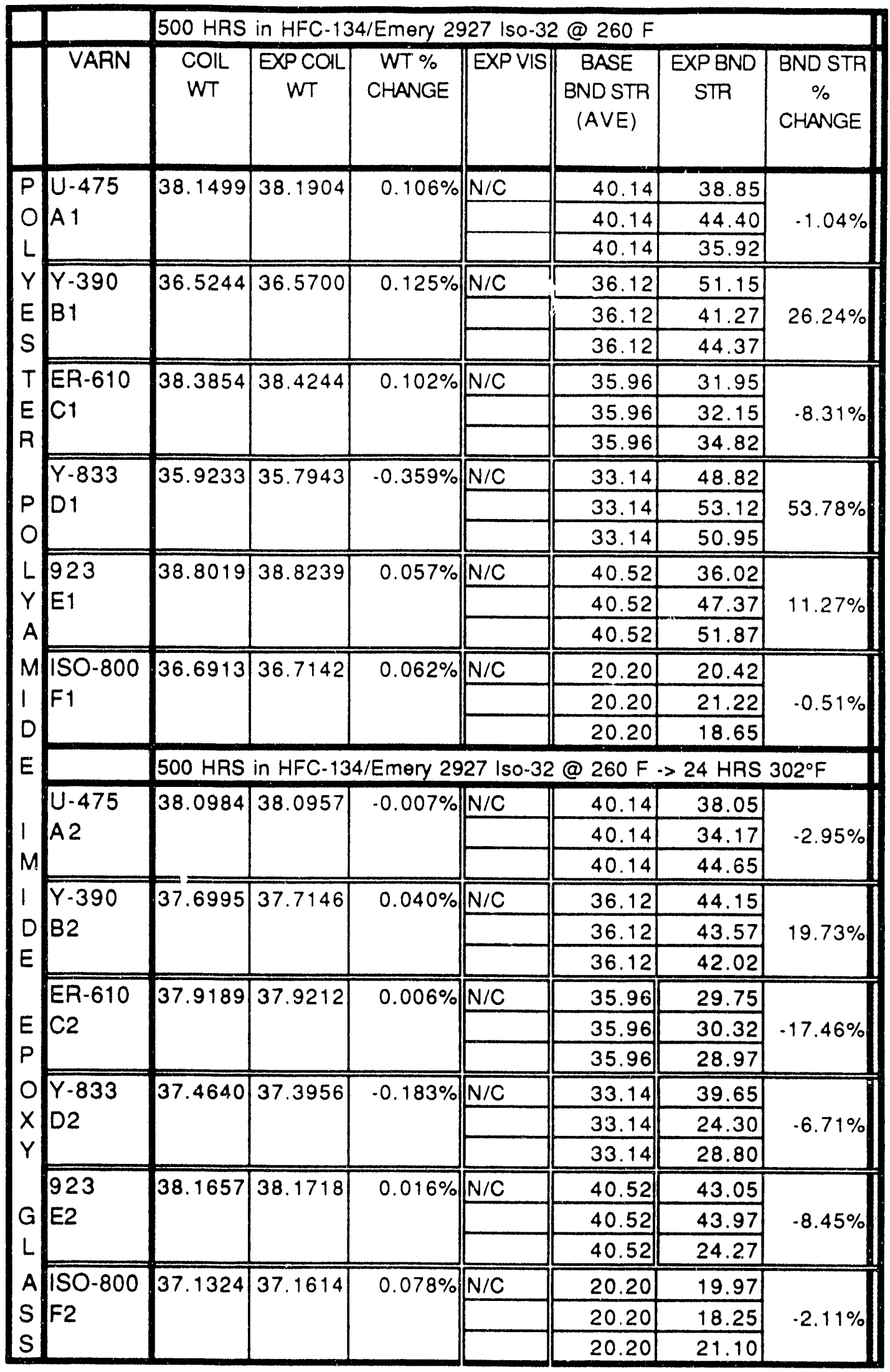


HELICAL COILSMIRE C.HFC.134/Emery 2927 Iso-32@260 $\mathrm{F}\left(127^{\circ} \mathrm{C}\right)$

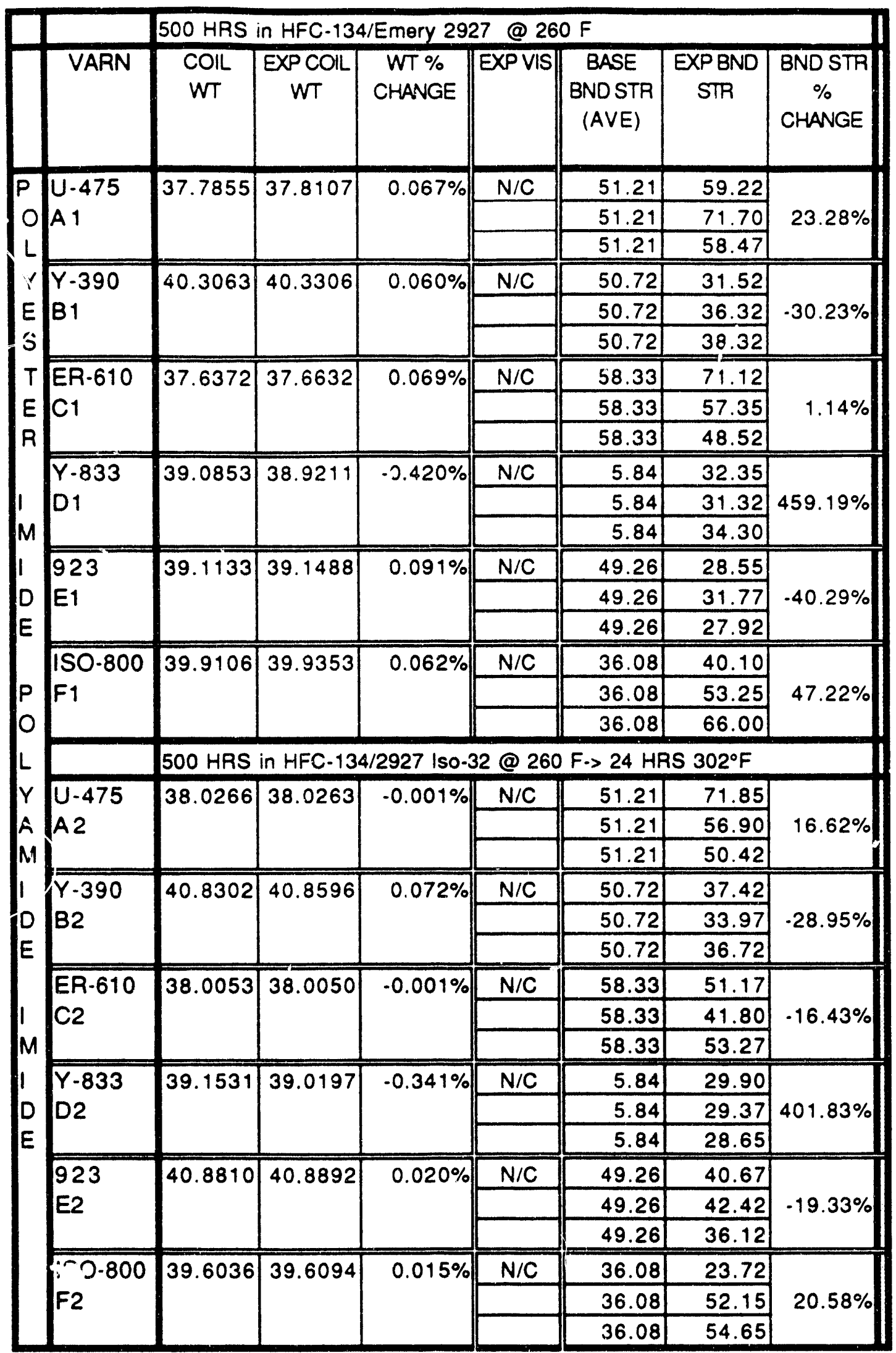




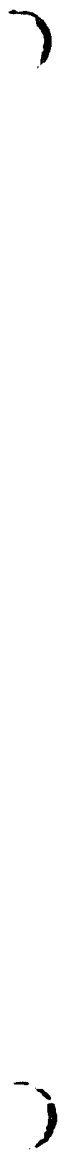

\begin{tabular}{|c|c|c|c|c|c|}
\hline 500 & $3 \mathrm{~S}$ in $\mathrm{HF}$ & $-134 / 2927$ & Iso-32 oil c & $260^{\circ} F(1$ & $\left.27^{\circ} \mathrm{C}\right)$ \\
\hline ID & WT & EXPWT & $\begin{array}{l}\text { WT \% } \\
\text { CHANGE }\end{array}$ & EXP VS & EXPFLEX \\
\hline A 1 & 3.2576 & 3.315 & $1.762 \%$ & $\mathrm{~N} / \mathrm{C}$ & \\
\hline & & & & & YES \\
\hline B1 & 4.0809 & 4.0857 & $0.118 \%$ & $N / C$ & \\
\hline & & & & & YES \\
\hline C1 & 3,3887 & 3.3874 & $-0.038 \%$ & $N / C$ & \\
\hline & & & & & YES \\
\hline 50 & IRS in $\mathrm{H}$ & $c-134 / 29$ & Iso-32-> 3 & $02^{\circ} \mathrm{F}$ for & 24 HRS \\
\hline A2 & 3.4983 & 3.4973 & $-0.029 \%$ & $\mathrm{~N} / \mathrm{C}$ & \\
\hline & & & & & YES \\
\hline B2 & 3.9575 & 3.9542 & $-0.083 \%$ & $N / C$ & \\
\hline & & & & & YES \\
\hline $\mathrm{C} 2$ & 3.6412 & 3.6405 & $-0.019 \%$ & $N / C$ & \\
\hline & & & & & YES \\
\hline & & & & & \\
\hline
\end{tabular}




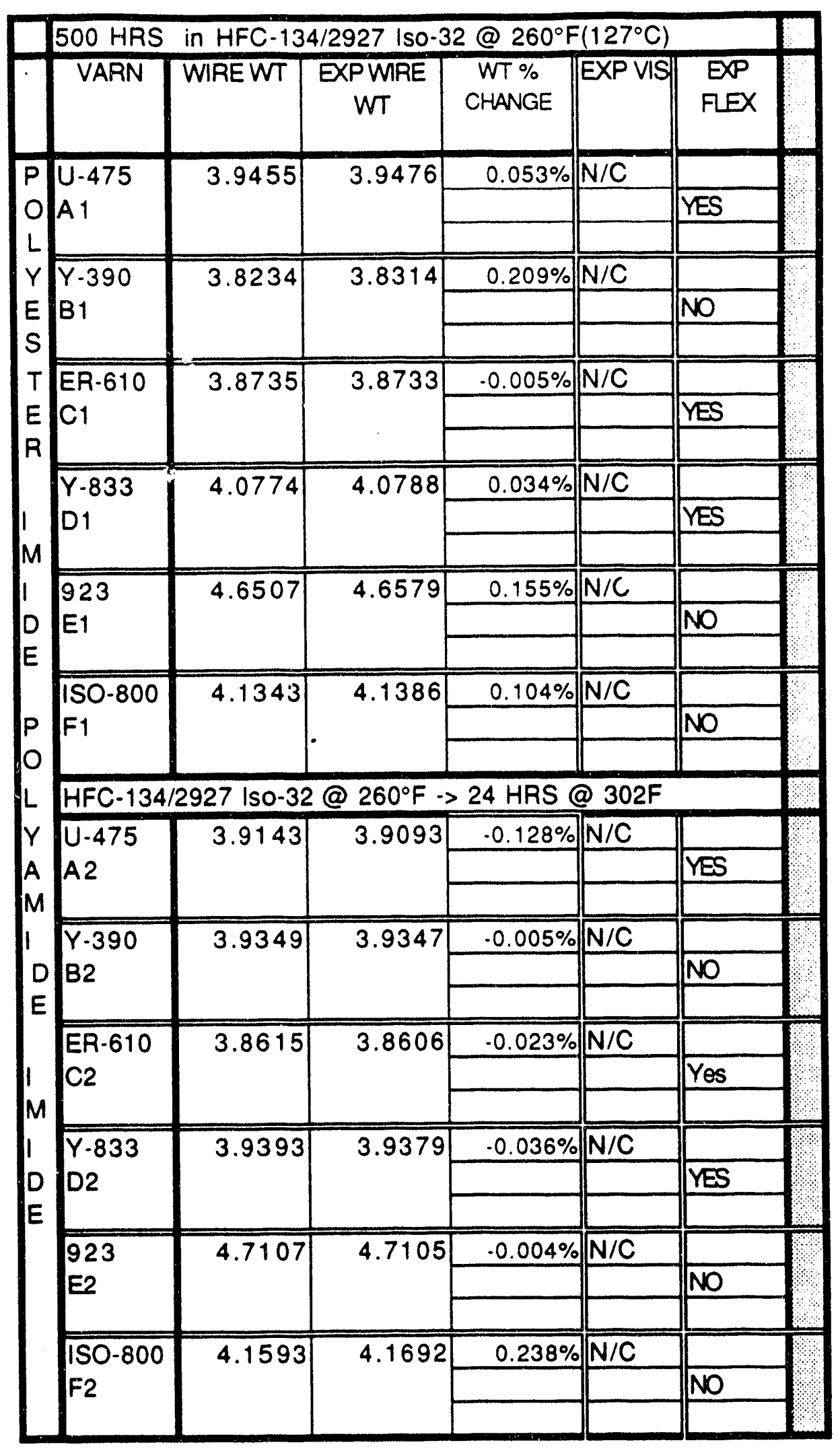



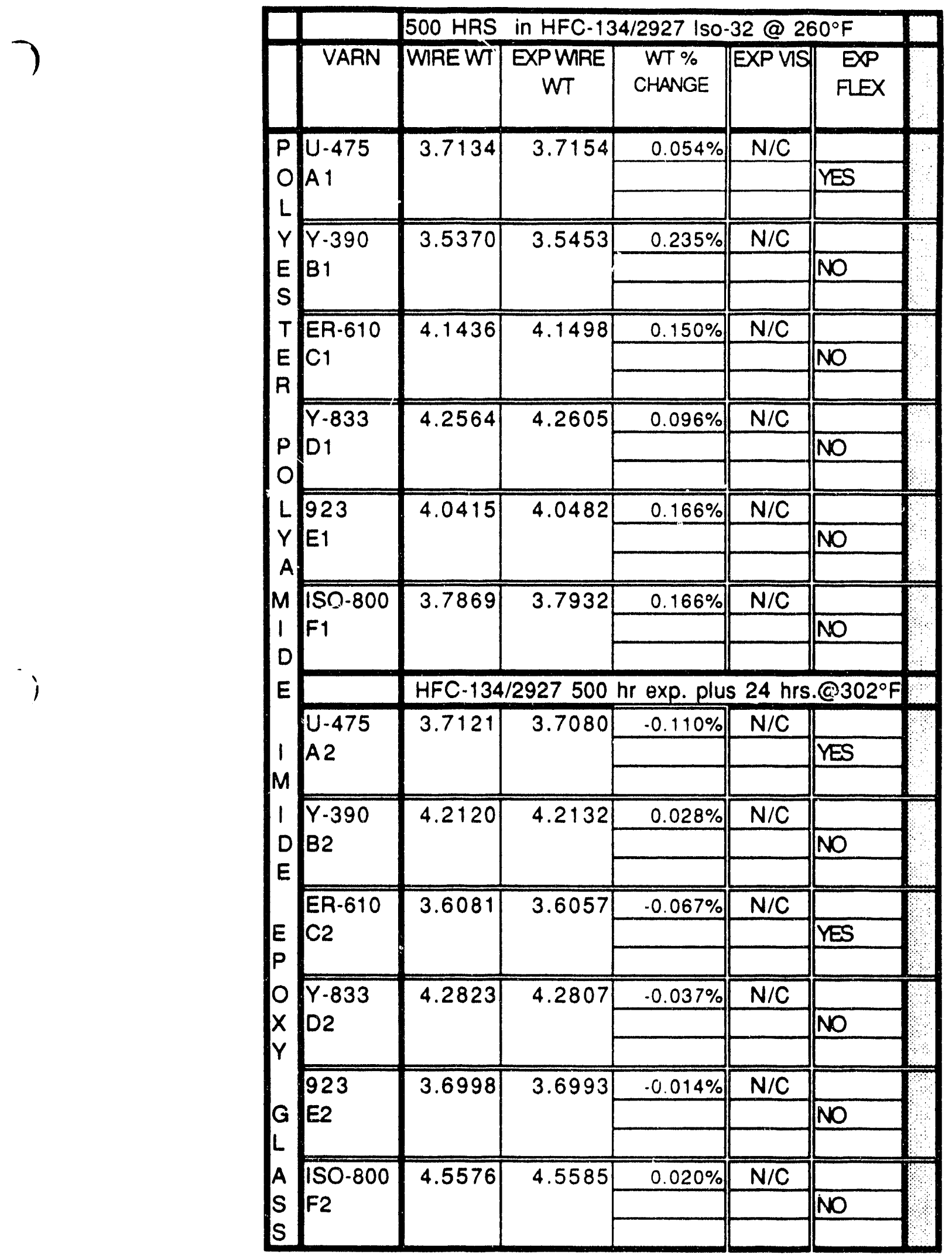


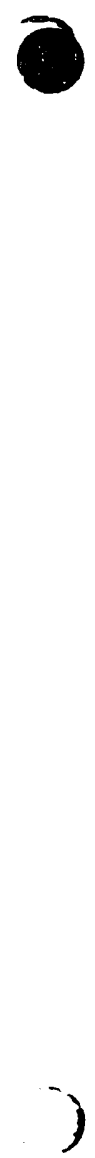

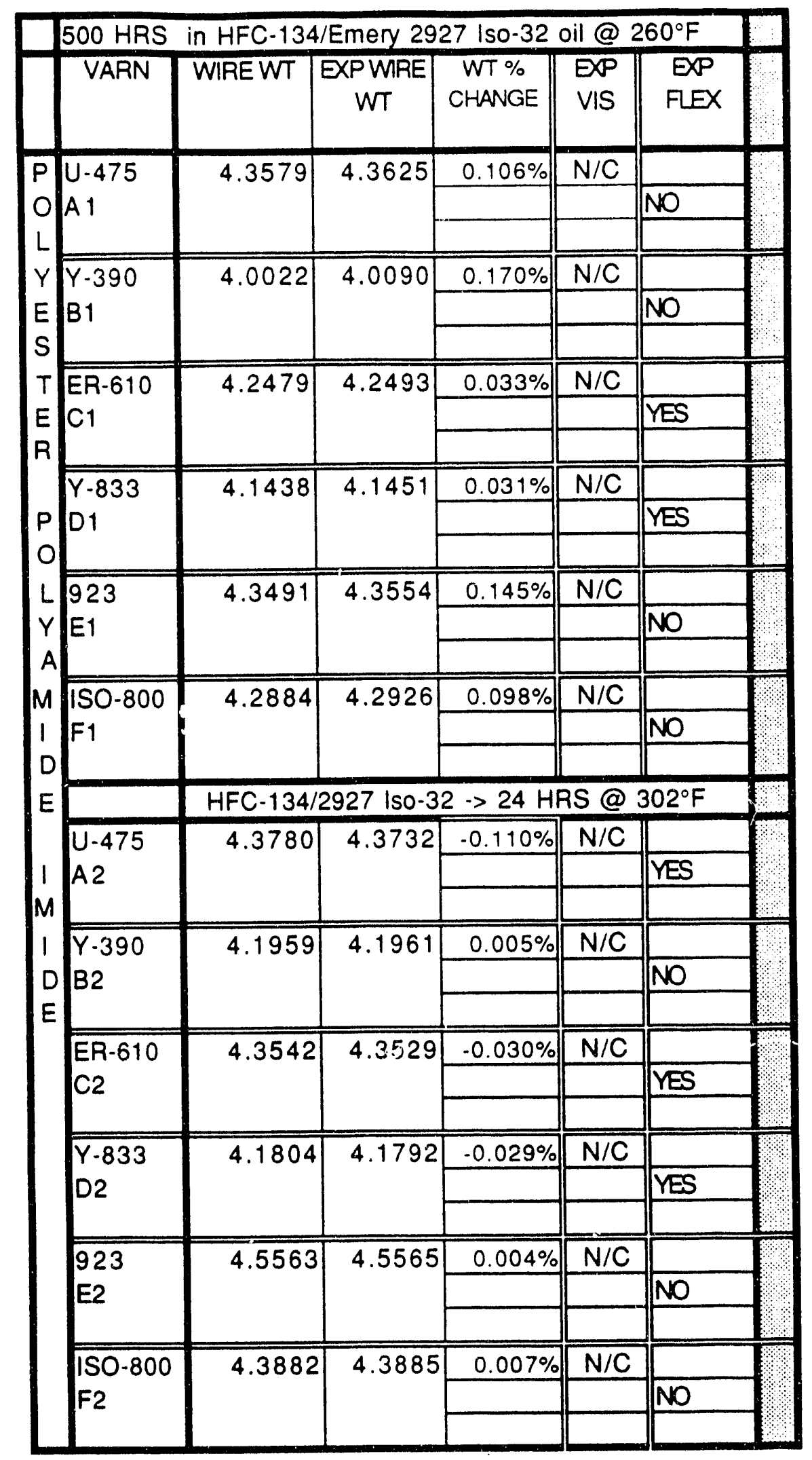


Varnish Disks-..-HFC-134/Emery 2927 Iso-32 Oil

\begin{tabular}{|c|c|c|c|c|c|}
\hline \multicolumn{6}{|c|}{500 HRS IN R-134/Emery 2927 Iso -32@ 260 F } \\
\hline ID & WT & EXPWT & $\begin{array}{c}\text { WT \% } \\
\text { CHANGE }\end{array}$ & EXP VIS & EXPFLEX \\
\hline $\begin{array}{l}\text { U-475 } \\
\text { A } 1\end{array}$ & 2.9688 & 2.9704 & $0.05 \%$ & $\begin{array}{l}\text { Surface } \\
\text { Cloudy } \\
\end{array}$ & $\mathrm{N} / \mathrm{C}$ \\
\hline $\begin{array}{l}Y-390 \\
B 1\end{array}$ & 2.1557 & 2.1116 & $2.05 \%$ & \begin{tabular}{|l|} 
Slightly \\
warped \\
lightened \\
\end{tabular} & $N / C$ \\
\hline $\begin{array}{l}\text { ER-610 } \\
\text { C1 }\end{array}$ & 2.5972 & 2.6685 & $2.75 \%$ & \begin{tabular}{|l|} 
Slightly \\
Darkened \\
\end{tabular} & $N / C$ \\
\hline $\begin{array}{l}Y-833 \\
D 1\end{array}$ & 3.9723 & 4.0394 & $1.69 \%$ & $\begin{array}{l}\text { Light } \\
\text { green in } \\
\text { color }\end{array}$ & $\mathrm{N} / \mathrm{C}$ \\
\hline $\begin{array}{l}923 \\
E 1\end{array}$ & 2.5062 & 2.4724 & $-1.35 \%$ & \begin{tabular}{|l|} 
Lightened \\
Warped \\
\end{tabular} & $N / C$ \\
\hline $\begin{array}{l}\text { ISO-800 } \\
\text { F1 }\end{array}$ & 2.2163 & 2.1957 & $-0.93 \%$ & \begin{tabular}{|l|} 
Slightly \\
warped \\
lighter in \\
\end{tabular} & \begin{tabular}{|l|} 
N/C \\
color \\
\end{tabular} \\
\hline & R-134/Eme & 2927 at $\ldots$ & $>24$ hour & at $302^{\circ} \mathrm{F}$. & \\
\hline $\begin{array}{l}\text { U-475 } \\
\text { A2 }\end{array}$ & 2.4905 & 2.4055 & $-3.41 \%$ & \begin{tabular}{|l|} 
Surface \\
Cloudy \\
Darkened \\
\end{tabular} & $N / C$ \\
\hline $\begin{array}{l}Y-390 \\
B 2\end{array}$ & 2.0581 & 2.0051 & $-2.58 \%$ & \begin{tabular}{|l|} 
Slightly \\
warped \\
Darkened \\
\end{tabular} & $\mathrm{N} / \mathrm{C}$ \\
\hline $\begin{array}{l}\text { ER-610 } \\
\text { C2 }\end{array}$ & 2.8314 & 2.8199 & $-0.41 \%$ & Darkened & $N / C$ \\
\hline $\begin{array}{l}Y-833 \\
D 2\end{array}$ & 3.3365 & 3.3227 & $-0.41 \%$ & $\mathrm{~N} / \mathrm{C}$ & $\mathrm{N} / \mathrm{C}$ \\
\hline $\begin{array}{l}923 \\
E 2\end{array}$ & 2.2994 & 2.2283 & $-3.09 \%$ & Warped & $N / C$ \\
\hline $\begin{array}{l}\text { ISO-800 } \\
\mathrm{F} 2\end{array}$ & 2.2531 & 2.1796 & $-3.26 \%$ & $\begin{array}{l}\text { slightly } \\
\text { warped } \\
\end{array}$ & $\mathrm{N} / \mathrm{C}$ \\
\hline
\end{tabular}




\begin{tabular}{|c|c|c|c|c|c|c|c|c|c|}
\hline \multicolumn{10}{|c|}{500 HRS in HFC-134/Emery 2927 Iso-32 @ 260 F } \\
\hline $\mathrm{D}$ & WT & EXP WT & $\begin{array}{c}\text { WT \% } \\
\text { CHANGE }\end{array}$ & $\begin{array}{l}\text { SAMPLE } \\
\text { WIDTH }\end{array}$ & $\begin{array}{l}\text { BREAK } \\
\text { LOAD }\end{array}$ & $\begin{array}{c}\text { SAMPLE } \\
\text { THICKNESS } \\
\text { Mils }\end{array}$ & $\begin{array}{c}\text { TENSILE } \\
\text { STR } \\
\text { BASE }\end{array}$ & $\begin{array}{l}\text { TENSILE } \\
\text { STREXP }\end{array}$ & $\begin{array}{c}\% \text { CHANGE } \\
\text { TENSILE }\end{array}$ \\
\hline \multirow{3}{*}{$\begin{array}{l}\text { NO/MY/NO } \\
\text { A1 }\end{array}$} & \multirow[t]{3}{*}{7.6901} & \multirow[t]{3}{*}{8.3478} & \multirow[t]{3}{*}{$8.55 \%$} & 0.430 & 150.10 & 21 & 17.4 & 16.62 & \\
\hline & & & & 0.457 & 159.40 & 21 & 17.4 & 16.61 & $.3 .66 \%$ \\
\hline & & & & 0.462 & $165.50 \mathrm{i}$ & 21 & 17.4 & 17.06 & \\
\hline \multirow{3}{*}{$\begin{array}{l}D A / M Y / D A \\
B 1\end{array}$} & \multirow{3}{*}{7.4090} & \multirow[t]{3}{*}{7.5004} & \multirow[t]{3}{*}{$1.23 \%$} & 0.372 & 109.70 & 21 & 13.7 & 14.04 & \\
\hline & & & & 0.410 & 124.80 & 21 & 13.7 & 14.49 & $4.31 \%$ \\
\hline & & & & 0.380 & 114.40 & 21 & 13.7 & 14.34 & \\
\hline \multirow{3}{*}{$\begin{array}{l}\text { MYLARMO } \\
\text { C1 }\end{array}$} & \multirow[t]{3}{*}{3.9631} & \multirow[t]{3}{*}{4.0206} & \multirow[t]{3}{*}{$1.45 \%$} & 0.460 & 94.90 & 10 & 21.7 & 20.03 & \\
\hline & & & & 0.494 & 93.90 & 10 & 21.7 & 19.01 & $.11 .17 \%$ \\
\hline & & & & 0.403 & 73.30 & 10 & 21.7 & 18.19 & \\
\hline \multirow{3}{*}{$\begin{array}{l}\text { NO } 410 \\
\text { D1 }\end{array}$} & \multirow[t]{3}{*}{3.4816} & \multirow[t]{3}{*}{3.9993} & \multirow[t]{3}{*}{$14.87 \%$} & 0.511 & 98.00 & 10 & 18.7 & 19.18 & \\
\hline & & & & 0.437 & 80.90 & 10 & 18.7 & 18.51 & $.0 .44 \%$ \\
\hline & & & & 0.495 & 89.90 & 10 & 18.7 & 18.16 & \\
\hline \multirow{3}{*}{$\begin{array}{l}\text { NO MI } 418 \\
\text { E1 }\end{array}$} & \multirow[t]{3}{*}{3.9442} & \multirow[t]{3}{*}{5.2101} & \multirow[t]{3}{*}{$32.10 \%$} & 0.501 & 37.15 & 10 & 7.5 & 7.42 & \\
\hline & & & & $0 . \overline{489}$ & 34.70 & 10 & 7.5 & 7.10 & $-1.94 \%$ \\
\hline & & & & 0.486 & 36.70 & 10 & 7.5 & 7.55 & \\
\hline \multirow{3}{*}{$\begin{array}{l}\text { MEL } 228 \\
\text { F1 }\end{array}$} & \multirow[t]{3}{*}{4.2508} & \multirow[t]{3}{*}{4.3221} & \multirow[t]{3}{*}{$1.68 \%$} & 0.396 & 75.15 & 10 & 21.7 & 18.98 & \\
\hline & & & & 0.508 & 93.75 & 10 & 21.7 & 18.45 & $-13.65 \%$ \\
\hline & & & & 0.460 & 86.40 & 10 & \begin{tabular}{|l|}
21.7 \\
\end{tabular} & 18.78 & \\
\hline & $500 \mathrm{HRS}$ & $S$ in $\mathrm{HFC}$ & 134/Emery & Y2927 Iso & 32@26 & $60 \mathrm{~F}->24 \mathrm{HP}$ & RS@302 & ${ }^{\circ} \mathrm{F}$ & \\
\hline NO/MY/NO & 7.4454 & 7.6985 & $3.40 \%$ & 0.420 & 148.80 & 21 & 17.4 & 16.87 & \\
\hline$A 2$ & & & & 0.500 & 174.80 & 21 & 17.4 & 16.65 & $-3.98 \%$ \\
\hline & & & & 0.343 & 119.60 & 21 & 17.4 & 16.60 & \\
\hline$D A / M Y / D A$ & 7.6135 & 7.5755 & $.0 .50 \%$ & 0.365 & 107.40 & 21 & 13.7 & 14.01 & \\
\hline 82 & & & & 0.365 & 102.60 & 21 & 13.7 & 13.39 & $-1.17 \%$ \\
\hline & & & & 0.380 & 105.50 & 21 & 13.7 & 13.22 & \\
\hline MYLAR MO & 3.7889 & 3.9858 & $5.20 \%$ & 0.426 & 77.75 & 10 & 21.7 & 18.25 & \\
\hline $\mathrm{C} 2$ & & & & 0.425 & 77.10 & 10 & 21.7 & 18.14 & $-16.64 \%$ \\
\hline & & & & 0.440 & 78.65 & 10 & 21.7 & 17.88 & \\
\hline NOMEX 410 & 3.7257 & 4.0421 & $8.49 \%$ & 0.450 & 88.65 & 10 & 18.7 & 19.70 & \\
\hline D2 & & & & 0.478 & 86.80 & 10 & 18.7 & 18.16 & $2.04 \%$ \\
\hline & & & & 0.490 & 95.00 & 10 & 18.7 & 19.39 & \\
\hline $\mathrm{NO} / \mathrm{MI} 418$ & 4.6669 & 5.7738 & $23.72 \%$ & 0.492 & 31.20 & 10 & 7.5 & 6.34 & \\
\hline E2 & & & & 0.508 & 33.60 & 10 & 7.5 & 6.61 & $.12 .74 \%$ \\
\hline & & & & 0.480 & 32.05 & 10 & 7.5 & 6.68 & \\
\hline MEL 228 & 4.3370 & 4.3502 & $0.30 \%$ & 0.513 & 83.35 & 10 & 21.7 & 16.25 & \\
\hline $\mathrm{F} 2$ & & & & 0.461 & 77.15 & 10 & 21.7 & 16.74 & $-24.17 \%$ \\
\hline & & & & 0.435 & 71.25 & 10 & 21.7 & 16.38 & \\
\hline
\end{tabular}




\begin{tabular}{|c|c|c|c|c|c|c|c|c|}
\hline & 500 HRS & in HFC. & 34/Emer & y 2927 Iso & o.32@2 & $260 \mathrm{~F}$ & & \\
\hline ID & $\begin{array}{c}\text { STRETCH } \\
\text { (inch) }\end{array}$ & $\begin{array}{c}\% \\
\text { ELONG }\end{array}$ & $\begin{array}{l}\text { BASE } \\
\text { ELONG } \\
(A \vee E)\end{array}$ & $\begin{array}{l}\text { ELONG \% } \\
\text { CHANGE }\end{array}$ & $\begin{array}{c}\text { BASE } \\
\text { DIE } \\
\text { (AVE) }\end{array}$ & EXPDIE & $\begin{array}{c}\text { DIE } \% \\
\text { CHANGE }\end{array}$ & VISUAL EXP \\
\hline $\mathrm{NO} / \mathrm{MY} / \mathrm{NO}$ & 0.55 & $13.8 \%$ & $20.0 \%$ & & $>18.97$ & flash & & $\mathrm{N} / \mathrm{C}$ \\
\hline A 1 & 0.63 & $15.8 \%$ & $20.0 \%$ & $-26.3 \%$ & $>18.97$ & flash & flash & \\
\hline & 0.63 & $15.8 \%$ & $20.0 \%$ & & $>18.97$ & flash & & \\
\hline$D A / M Y / D A$ & 0.57 & $28.5 \%$ & $46.0 \%$ & & $>15.27$ & flash & & Yellowed \\
\hline B1 & 0.57 & $28.5 \%$ & $46.0 \%$ & $.39 .1 \%$ & $\geq 15.27$ & flash & flash & slightly \\
\hline & 0.54 & $27.0 \%$ & $46.0 \%$ & & $>15.27$ & flash & & warped \\
\hline MYLAR MO & 2.69 & $134.5 \%$ & $131.0 \%$ & & $>14.91$ & flash & & $\sqrt{N / C}$ \\
\hline C1 & 2.74 & $137.0 \%$ & $131.0 \%$ & $.3 .1 \%$ & $>14.91$ & flash & flash & \\
\hline & 2.19 & $109.5 \%$ & $131.0 \%$ & & $>14.91$ & flash & & \\
\hline NO 410 & 0.37 & $18.5 \%$ & $17.0 \%$ & & 10.67 & 12.89 & & $\mathrm{~N} / \mathrm{C}$ \\
\hline D1 & 0.30 & $7.5 \%$ & $17.0 \%$ & $.34 .3 \%$ & 10.67 & flash & $20.8 \%$ & \\
\hline & 0.30 & $7.5 \%$ & $17.0 \%$ & & 10.67 & flash & & \\
\hline NO MI 418 & 0.05 & $1.3 \%$ & $4.0 \%$ & & 10.23 & flash & & $\mathrm{N} / \mathrm{C}$ \\
\hline E1 & 0.04 & $1.0 \%$ & $4.0 \%$ & $.72 .9 \%$ & 10.23 & flash & FLASH & \\
\hline & 0.04 & $1.0 \%$ & $4.0 \%$ & & 10.23 & flash & & \\
\hline MEL 228 & 3.32 & $166.0 \%$ & $160.0 \%$ & & $>14.22$ & flash & & slightly \\
\hline $\mathrm{Fi}$ & 2.89 & $144.5 \%$ & $160.0 \%$ & $.4 .2 \%$ & $>14.22$ & flash & flash & yellowed \\
\hline & 2.99 & $149.5 \%$ & $160.0 \%$ & & $>14.22$ & flash & & \\
\hline & 500 HRS & in HFC. & 34/Emery & y 2927 Iso & $32 @ 2$ & $60 \mathrm{~F} \rightarrow 2$ & 4 HRS@ & $302^{\circ} \mathrm{F}$ \\
\hline NO/MY/NO & 0.58 & $14.5 \%$ & $20.0 \%$ & & $>18.97$ & flash & & Delamination \\
\hline$A 2$ & 0.52 & $13.0 \%$ & $20.0 \%$ & $-35.4 \%$ & $>18.97$ & flash & flash & big pockets \\
\hline & 0.45 & $11.3 \%$ & $20.0 \%$ & & $>18.97$ & flash & & \\
\hline$D A / M Y / D A$ & 0.54 & $27.0 \%$ & $46.0 \%$ & & $\geq 15.27$ & flash & & Yellowed \\
\hline $\mathrm{B2}$ & 0.52 & $26.0 \%$ & $46.0 \%$ & $.43 .8 \%$ & $>15.27$ & flash & flash & slightly \\
\hline & 0.49 & $24.5 \%$ & $46.0 \%$ & & $>15.27$ & flash & & warped \\
\hline MYLAR MO & 2.81 & $140.5 \%$ & $131.0 \%$ & & $>14.91$ & flash & & $\mathrm{N} / \mathrm{C}$ \\
\hline $\mathrm{C} 2$ & 2.70 & $135.0 \%$ & $131.0 \%$ & $4.3 \%$ & $>14.91$ & flash & flash & \\
\hline & 2.69 & $134.5 \%$ & $131.0 \%$ & & $>14.91$ & flash & & \\
\hline NOMEX 410 & 0.41 & $10.3 \%$ & $17.0 \%$ & & 10.67 & 11.20 & & $\mathrm{~N} / \mathrm{C}$ \\
\hline D2 & 0.31 & $7.8 \%$ & $17.0 \%$ & $.46 .1 \%$ & 10.67 & 11.45 & $7.7 \%$ & \\
\hline & 0.38 & $9.5 \%$ & $17.0 \%$ & & 10.67 & 11.84 & & \\
\hline $\mathrm{NO} / \mathrm{MI} 418$ & 0.04 & $1.0 \%$ & $4.0 \%$ & & 10.23 & 12.50 & & $\mathrm{~N} / \mathrm{C}$ \\
\hline E2 & 0.04 & $1.0 \%$ & $4.0 \%$ & $.75 .0 \%$ & 10.23 & 11.79 & $17.2 \%$ & \\
\hline & 0.04 & $1.0 \%$ & $4.0 \%$ & & 10.23 & 11.67 & & \\
\hline MEL 228 & 3.30 & $165.0 \%$ & $160.0 \%$ & & $\geq 14.22$ & flash & & slightly \\
\hline $\mathrm{F} 2$ & 3.42 & $171.0 \%$ & $160.0 \%$ & $2.2 \%$ & $>14.22$ & flash & flash & yellowed \\
\hline & 3.09 & $154.5 \%$ & $160.0 \%$ & & $>14.22$ & flash & & \\
\hline
\end{tabular}


TIE CORD--HFC-134/Emery 2927 ISO-32 oil @ 260 $0^{\circ} \mathrm{F}\left(127^{\circ} \mathrm{C}\right)$

\begin{tabular}{|c|c|c|c|c|c|c|c|c|c|}
\hline \multicolumn{10}{|c|}{\begin{tabular}{|c|c|}
500 HRS IN R-134/2927 Iso-32 @ 260 $\mathrm{F}$ \\
EXPWT & WT \%
\end{tabular}} \\
\hline ID & $W T$ & EXPWT & $\begin{array}{c}\text { WT \% } \\
\text { CHANGE }\end{array}$ & $\begin{array}{l}\text { EXP } \\
\text { VIS }\end{array}$ & $\begin{array}{l}\text { BREAK } \\
\text { LOAD } \\
\text { (AVE) }\end{array}$ & $\begin{array}{c}\text { BREAK } \\
\text { LOAD } \\
\text { EXP } \\
(\operatorname{ave})\end{array}$ & $\begin{array}{c}\% \\
\text { CHANGE } \\
\text { BAK } \\
\text { LOAD }\end{array}$ & $\mid \begin{array}{c}\text { STRETCH } \\
\text { (Inch) }\end{array}$ & $\begin{array}{c}\% \\
\text { ELONG }\end{array}$ \\
\hline$A 1$ & 0.2852 & 0.2875 & $0.81 \%$ & $\mathrm{~N} / \mathrm{C}$ & 28.36 & 37.42 & & 0.46 & $23.0 \%$ \\
\hline & & & & & 28.36 & 34.97 & $26.46 \%$ & 0.41 & $20.5 \%$ \\
\hline & & & & & 28.36 & 35.20 & & 0.42 & $21.0 \%$ \\
\hline & & $500 \mathrm{HRS}$ & IN R-134/E & Emery & $2927->2$ & 4 HRS & $302^{\circ} \mathrm{F}$ & & \\
\hline$A 2$ & 0.2731 & 0.2727 & $-0.146 \%$ & $\mathrm{~N} / \mathrm{C}$ & 28.36 & 36.20 & & 0.46 & $23.0 \%$ \\
\hline & & & & & 28.36 & 30.50 & $14.97 \%$ & 0.48 & $24.0 \%$ \\
\hline & & & & & 28.36 & 31.12 & & 0.43 & $21.5 \%$ \\
\hline
\end{tabular}


LEAD WIRE INSULATION-HFC-134/Emery 2927 ISO-32@ $260^{\circ} \mathrm{F}\left(127^{\circ} \mathrm{C}\right)$

7

\begin{tabular}{|c|c|c|c|c|c|c|c|}
\hline \multirow[b]{2}{*}{ ID } & \multicolumn{7}{|c|}{\begin{tabular}{|l|l|l|}
500 HRS IN HFC-134/Emery 2927 Iso-32 @ 260 $\mathrm{F}$ & \\
\end{tabular}} \\
\hline & \begin{tabular}{|l|}
$\mathrm{WT}$ \\
\end{tabular} & EXPWT & \begin{tabular}{|c|} 
WT \% \\
CHANGE \\
\end{tabular} & EXPVIS & $\begin{array}{c}\text { BASE DIE } \\
(\text { AVE })\end{array}$ & EXPDIE & $\begin{array}{c}\text { DIE\% } \\
\text { CHANGE }\end{array}$ \\
\hline DMC) & 4.1489 & 4.3403 & $4.61 \%$ & $N / C$ & 9.61 & 8.50 & \\
\hline \multirow[t]{2}{*}{ A 1} & & & & & 9.61 & 7.00 & $-20.22 \%$ \\
\hline & & & & & 9.61 & 7.50 & \\
\hline DTMD & 4.5354 & 4.7338 & $4.37 \%$ & $\mathrm{~N} / \mathrm{C}$ & 9.95 & 12.00 & \\
\hline \multirow[t]{3}{*}{ B1 } & & & & & 9.95 & 12.23 & $27.00 \%$ \\
\hline & & & & & 9.95 & 13.68 & \\
\hline & \multicolumn{6}{|c|}{ 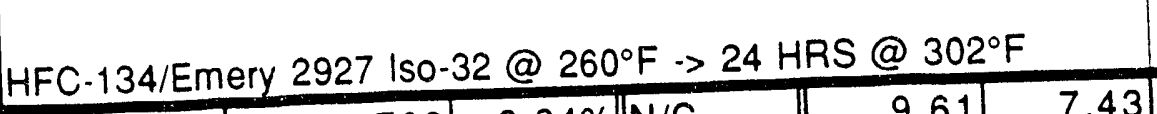 } & \\
\hline DMD & $\begin{array}{r}4.2144 \\
\end{array}$ & 4.3762 & \begin{tabular}{|l|}
$3.84 \%$ \\
\end{tabular} & $\mathrm{~N} / \mathrm{C}$ & 9.61 & 7.43 & \\
\hline \multirow[t]{2}{*}{$A 2$} & & & & & 9.61 & 9.99 & $-14.98 \%$ \\
\hline & & & & & 9.61 & 7.09 & \\
\hline \multirow{3}{*}{ DTMD } & 4.2914 & 4.3821 & $2.11 \%$ & $\mathrm{~N} / \mathrm{C}$ & 9.95 & 11.79 & \\
\hline & & & & & 9.95 & flash & $22.51 \%$ \\
\hline & & & & & 9.95 & 12.59 & \\
\hline
\end{tabular}




\section{Appendix L}

Experimental Data for HFC-245ca/Henkel-

Emery 2927 ISO-32 Exposure at

$126^{\circ} \mathrm{C}\left(260^{\circ} \mathrm{F}\right)$ 


\begin{tabular}{|c|c|c|c|c|c|c|c|c|c|c|}
\hline & 500 HRS IN & $\mathrm{R}-245 \mathrm{ca} \mathrm{a}$ & Id ISO 32 & @ 250 $\mathrm{F}$ & $=\left(121^{\circ} \mathrm{C}\right)$ & & & & & \\
\hline ID & WT & EXPWT & $\begin{array}{c}\text { WT \% } \\
\text { CHANGE }\end{array}$ & EXP VS & $\begin{array}{c}\text { BASE BRN } \\
\text { OUT } \\
\text { (AVE) }\end{array}$ & $\begin{array}{c}\text { EXP BRN } \\
\text { OUT }\end{array}$ & $\begin{array}{c}\text { BRN OUTT } \\
\% \\
\text { CHANGE }\end{array}$ & EXPDIE & EXPDIE & $\begin{array}{c}\text { DIE \% } \\
\text { CHANGE }\end{array}$ \\
\hline$A 1$ & 22.9986 & 23.0050 & $0.028 \%$ & $\mathrm{~N} / \mathrm{C}$ & 576 & 578 & & 15.80 & 13.49 & \\
\hline & & & & & 576 & 503 & $-9.5 \%$ & 15.80 & 14.04 & $-11.8 \%$ \\
\hline & & & & & 576 & 483 & & 15.80 & 14.26 & \\
\hline B1 & 22.2954 & 22.3486 & $0.239 \%$ & $\mathrm{~N} / \mathrm{C}$ & 736 & 733 & & 11.60 & 11.63 & \\
\hline & & & & & 736 & 729 & $-4.2 \%$ & 11.60 & 12.55 & $3.8 \%$ \\
\hline & & & & & 736 & 653 & & 11.60 & 11.93 & \\
\hline $\mathrm{C1}$ & 23.7815 & 23.7855 & $0.017 \%$ & $\mathrm{~N} / \mathrm{C}$ & 579 & 490 & & 16.40 & 11.70 & \\
\hline & & & & & 579 & 539 & $.7 .6 \%$ & 16.40 & 12.49 & $-23.4 \%$ \\
\hline & & & & & 579 & 576 & & 16.40 & 13.50 & \\
\hline & $500 \mathrm{hr}$ in $\mathrm{F}$ & $245 \mathrm{ca}$ and & ISO $32 \mathrm{pl}$ & us $24 \mathrm{hr}$ & at $302 \mathrm{~F}$ & & & & & \\
\hline$A 2$ & 22.6116 & 22.6125 & $0.004 \%$ & $N / C$ & 576 & 577 & & 15.80 & 9.87 & \\
\hline & & & & & 576 & 560 & $-1.3 \%$ & 15.80 & 16.29 & $-18.2 \%$ \\
\hline & & & & & 576 & 569 & & 15.80 & 12.63 & \\
\hline $\mathrm{B2}$ & 23.1963 & 23.2014 & $0.022 \%$ & $\mathrm{~N} / \mathrm{C}$ & 736 & 644 & & 11.60 & 11.67 & \\
\hline & & & & & 736 & 727 & $-4.9 \%$ & 11.60 & 10.76 & $-5.6 \%$ \\
\hline & & & & & 736 & 728 & & 11.60 & 10.43 & \\
\hline $\mathrm{C} 2$ & 23.6962 & 23.6811 & $.0 .064 \%$ & $\mathrm{~N} / \mathrm{C}$ & 579 & 502 & & 16.60 & 14.34 & \\
\hline & & & & & 579 & 581 & $-4.7 \%$ & 16.60 & 15.33 & $-15.8 \%$ \\
\hline & & & & & 579 & 573 & & 16.60 & 12.27 & \\
\hline
\end{tabular}




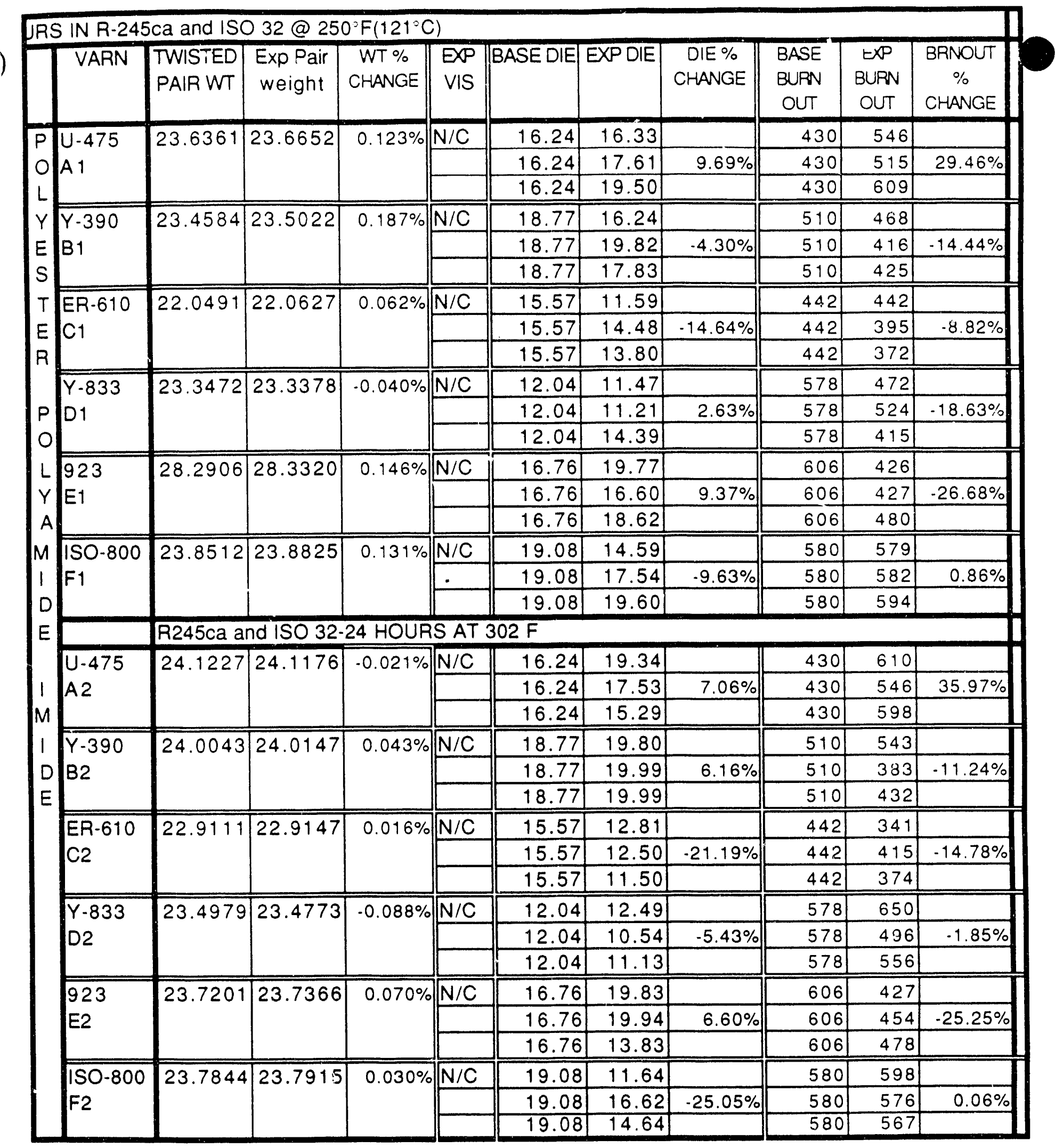




\begin{tabular}{|c|c|c|c|c|c|c|c|c|c|c|c|}
\hline \multicolumn{12}{|c|}{ JRS IN R-245ca and ISO $32 @ 250^{\circ} \mathrm{F}\left(121^{\circ} \mathrm{C}\right)$} \\
\hline & VARN & $\begin{array}{l}\text { TWISTED } \\
\text { PAIR WT }\end{array}$ & $\begin{array}{c}\text { EXPT } \\
\text { PAIR WT }\end{array}$ & $\begin{array}{c}\text { WT \% } \\
\text { CHANGE }\end{array}$ & $\begin{array}{l}\text { EXP } \\
\text { VIS }\end{array}$ & $\begin{array}{l}\text { BASE } \\
\text { DIE }\end{array}$ & $\begin{array}{l}\text { EXP } \\
\text { DIE }\end{array}$ & $\begin{array}{c}\text { DIE \% } \\
\text { CHANGE }\end{array}$ & $\begin{array}{l}\text { BASE } \\
\text { BUPN } \\
\text { OUT }\end{array}$ & \begin{tabular}{|l|} 
EXP \\
BURN \\
OUT \\
\end{tabular} & $\begin{array}{c}\text { BRNOUT } \\
\% \\
\text { CHANGE }\end{array}$ \\
\hline \multirow{3}{*}{$\begin{array}{l}P \\
O \\
L\end{array}$} & \multirow{3}{*}{$\begin{array}{l}\text { U-475 } \\
\text { A } 1 \\
\end{array}$} & \multirow[t]{3}{*}{26.0295} & \multirow[t]{3}{*}{26.0955} & \multirow[t]{3}{*}{$0.25 \%$} & $\mathrm{~N} / \mathrm{C}$ & 13.32 & 10.78 & & 746 & 732 & \\
\hline & & & & & & 13.32 & 15.56 & $5.53 \%$ & 746 & 732 & $-1.39 \%$ \\
\hline & & & & & & 13.32 & 15.83 & & 746 & 743 & \\
\hline \multirow{3}{*}{$\begin{array}{l}Y \\
E \\
S\end{array}$} & \multirow{3}{*}{$\begin{array}{l}Y-390 \\
B 1\end{array}$} & \multirow[t]{3}{*}{26.6030} & \multirow[t]{3}{*}{26.6669} & \multirow[t]{3}{*}{$0.24 \%$} & $\mathrm{~N} / \mathrm{C}$ & 12.28 & 6.82 & & 755 & 756 & \\
\hline & & & & & & 12.28 & 12.82 & $-13.52 \%$ & 755 & 745 & $-0.93 \%$ \\
\hline & & & & & & 12.28 & 12.22 & & 755 & 743 & \\
\hline \multirow{4}{*}{$\begin{array}{l}T \\
E \\
R\end{array}$} & \multirow{3}{*}{$\begin{array}{l}\text { ER-610 } \\
\text { C1 }\end{array}$} & \multirow[t]{3}{*}{25.5703} & \multirow[t]{3}{*}{25.6090} & \multirow[t]{3}{*}{$0.15 \%$} & $\mathrm{~N} / \mathrm{C}$ & 12.73 & 12.89 & & 734 & 734 & \\
\hline & & & & & & 12.73 & 13.58 & $3.56 \%$ & 734 & 731 & $-0.36 \%$ \\
\hline & & & & & & 12.73 & 13.08 & & 734 & 729 & \\
\hline & \multirow{3}{*}{$\begin{array}{l}Y-833 \\
D 1\end{array}$} & \multirow[t]{3}{*}{21.9947} & \multirow[t]{3}{*}{21.0126} & \multirow[t]{3}{*}{$-4.47 \%$} & $\mathrm{~N} / \mathrm{C}$ & 12.49 & 13.03 & & 734 & 706 & \\
\hline$P$ & & & & & & 12.49 & 11.82 & $-0.93 \%$ & 734 & 727 & $-1.86 \%$ \\
\hline 0 & & & & & & 12.49 & 12.27 & & 734 & 728 & \\
\hline \multirow{3}{*}{$\begin{array}{l}L \\
Y \\
A\end{array}$} & \multirow{3}{*}{$\begin{array}{l}923 \\
E 1 \\
\end{array}$} & \multirow[t]{3}{*}{27.5576} & 27.6023 & $0.16 \%$ & $\mathrm{~N} / \mathrm{C}$ & 14.38 & 16.95 & & 742. & 751 & \\
\hline & & & & & & 14.38 & 11.16 & $-8.95 \%$ & 742 & 746 & $0.76 \%$ \\
\hline & & & & & & 14.38 & 11.17 & & 742 & 746 & \\
\hline$M$ & $150-800$ & 25.8444 & 25.9078 & $0.25 \%$ & $\mathrm{~N} / \mathrm{C}$ & 12.29 & 9.96 & & 747 & 738 & \\
\hline 1 & $F 1$ & & & & & 12.29 & 13.00 & $-3.82 \%$ & 747 & 743 & $-0.94 \%$ \\
\hline$D$ & & & & & & 12.29 & 12.50 & & 747 & 739 & \\
\hline$E$ & & $\mathrm{R}-245 \mathrm{ca}$ a & nd $1 S O 32$ & $\because>24 \mathrm{hc}$ & ours (c & $302 \mathrm{~F}$ & & & & & \\
\hline & U-475 & 25.3928 & 25.3935 & $0.00 \%$ & $\mathrm{~N} / \mathrm{C}$ & 13.32 & 11.06 & & 746 & 727 & \\
\hline 1 & $\mathrm{~A} 2$ & & & & & 13.32 & 13.13 & $-10.54 \%$ & 746 & 735 & $-1.39 \%$ \\
\hline$M$ & & & & & & 13.32 & 11.56 & & 746 & 745 & \\
\hline 1 & $Y-390$ & 26.5283 & 26.5639 & $0.13 \%$ & $\mathrm{~N} / \mathrm{C}$ & 12.28 & 11.23 & & 755 & 744 & \\
\hline$D$ & $\mathrm{~B} 2$ & & & & & 12.28 & 13.54 & $-1.93 \%$ & 755 & 747 & $-1.26 \%$ \\
\hline$E$ & & & & & & 12.28 & 11.36 & & 755 & 750 & \\
\hline & ER-610 & 26.2690 & 26.2704 & $0.01 \%$ & $\mathrm{~N} / \mathrm{C}$ & 12.73 & 13.42 & & 734 & 727 & \\
\hline$E$ & & & & & & 12.73 & 12.73 & $0.31 \%$ & 734 & 696 & $-3.13 \%$ \\
\hline$P$ & & & & & & 12.73 & 12.16 & & 734 & 710 & \\
\hline 0 & $Y-833$ & 23.2361 & 23.2192 & $-0.07 \%$ & $\mathrm{~N} / \mathrm{C}$ & 12.49 & 11.00 & & 734 & 690 & \\
\hline$x$ & D2 & & & & & 12.49 & 11.65 & $-5.12 \%$ & 734 & 729 & $-2.50 \%$ \\
\hline$Y$ & & & & & & 12.49 & 12.90 & & 734 & 728 & \\
\hline & 923 & 26.7176 & 26.7350 & $0.07 \%$ & $\mathrm{~N} / \mathrm{C}$ & 14.38 & 14.31 & & 742 & 730 & \\
\hline $\mathrm{G}$ & E2 & & & & & 14.38 & 13.04 & $1.46 \%$ & 742 & 752 & $-1.62 \%$ \\
\hline L & & & & & & 14.38 & 16.42 & & 742 & 728 & \\
\hline A & ISO-800 & 26.2920 & 26.3103 & $0.07 \%$ & & 12.29 & 12.10 & & 747 & 741 & \\
\hline$S$ & $\mathrm{~F} 2$ & & & & $\mathrm{~N} / \mathrm{C}$ & 12.29 & 13.20 & $-1.57 \%$ & 747 & 741 & $-0.80 \%$ \\
\hline$S$ & & & & & & 12.29 & 10.99 & & 747 & 736 & \\
\hline
\end{tabular}




\begin{tabular}{|c|c|c|c|c|c|c|c|c|c|c|c|}
\hline \multicolumn{12}{|c|}{ JRS IN R-245ca and ISO $32 @ 250^{\circ} \mathrm{F}\left(121^{\circ} \mathrm{C}\right)$} \\
\hline & VARN & $\begin{array}{l}\text { TWISTED } \\
\text { PAIR WT }\end{array}$ & $\begin{array}{c}\text { EXPT } \\
\text { PAIR WT }\end{array}$ & $\begin{array}{c}\text { WT \% } \\
\text { CHANGE }\end{array}$ & $\begin{array}{l}\text { EXP } \\
\text { VIS }\end{array}$ & $\begin{array}{l}\text { BASE } \\
\text { DIE }\end{array}$ & $\begin{array}{l}\text { EXP } \\
\text { DIE }\end{array}$ & $\begin{array}{c}\text { DIE \% } \\
\text { CHANGE }\end{array}$ & $\begin{array}{c}\text { BASE } \\
\text { BURN } \\
\text { OUT }\end{array}$ & $\begin{array}{c}\text { EXP } \\
\text { BURN } \\
\text { OUT } \\
\end{array}$ & $\begin{array}{c}\text { BRNOUT } \\
\% \\
\text { CHANGE }\end{array}$ \\
\hline \multirow{3}{*}{$\begin{array}{l}P \\
O \\
L\end{array}$} & \multirow{3}{*}{$\begin{array}{l}U-475 \\
\text { A1 }\end{array}$} & \multirow[t]{3}{*}{24.4353} & \multirow[t]{3}{*}{24.4649} & \multirow[t]{3}{*}{$0.121 \%$} & $\mathrm{~N} / \mathrm{C}$ & 15.10 & 18.90 & & 469 & 585 & \\
\hline & & & & & & 15.10 & 19.41 & $27.26 \%$ & 469 & 553 & $24.31 \%$ \\
\hline & & & & & & 15.10 & 19.34 & & 469 & 611 & \\
\hline \multirow{3}{*}{$\begin{array}{l}Y \\
E\end{array}$} & \multirow{3}{*}{$\begin{array}{l}\text { Y.390 } \\
\text { B1 }\end{array}$} & \multirow[t]{3}{*}{23.1067} & \multirow[t]{3}{*}{23.1395} & \multirow[t]{3}{*}{$0.142 \%$} & $\mathrm{~N} / \mathrm{C}$ & 18.24 & 19.94 & & 473 & 569 & \\
\hline & & & & & & 18.24 & 19.98 & $9.32 \%$ & 473 & 585 & $22.97 \%$ \\
\hline & & & & & & 18.24 & 19.90 & & 473 & 591 & \\
\hline \multirow{4}{*}{$\begin{array}{l}T \\
E \\
R\end{array}$} & \multirow{3}{*}{$\begin{array}{l}\text { ER-610 } \\
\text { C1 }\end{array}$} & \multirow[t]{3}{*}{25.6318} & \multirow[t]{3}{*}{25.6465} & \multirow[t]{3}{*}{$0.057 \%$} & $\mathrm{~N} / \mathrm{C}$ & 14.53 & 15.61 & & 494 & 337 & \\
\hline & & & & & & 14.53 & 17.74 & $5.16 \%$ & 494 & 352 & $.28 .48 \%$ \\
\hline & & & & & & 14.53 & 12.49 & & 494 & 371 & \\
\hline & \multirow{3}{*}{$\begin{array}{l}Y-833 \\
D 1\end{array}$} & \multirow[t]{3}{*}{21.7789} & \multirow[t]{3}{*}{21.7726} & \multirow[t]{3}{*}{$-0.029 \%$} & $\mathrm{~N} / \mathrm{C}$ & 11.38 & 16.16 & & 557 & 475 & \\
\hline 1 & & & & & & 11.38 & 16.57 & $37.11 \%$ & 557 & 478 & $-13.23 \%$ \\
\hline$M$ & & & & & & 11.38 & 14.08 & & 557 & 497 & \\
\hline \multirow{4}{*}{$\begin{array}{l}\mathrm{I} \\
\mathrm{D} \\
\mathrm{E}\end{array}$} & \multirow{3}{*}{$\begin{array}{l}923 \\
E 1\end{array}$} & \multirow[t]{3}{*}{23.8526} & \multirow[t]{3}{*}{23.8852} & $0.137 \%$ & $N / C$ & 15.85 & 17.93 & & 503 & 495 & \\
\hline & & & & & & 15.85 & 13.18 & $7.32 \%$ & 503 & 539 & $11.60 \%$ \\
\hline & & & & & & 15.85 & 19.92 & & 503 & 650 & \\
\hline & $150-800$ & 23.2874 & 23.3173 & $0.128 \%$ & $\mathrm{~N} / \mathrm{C}$ & 14.75 & 18.10 & & 632 & 580 & \\
\hline$p$ & $F 1$ & & & & & 14.75 & 15.83 & $16.52 \%$ & 632 & 594 & $-4.69 \%$ \\
\hline 0 & & & & & & 14.75 & 17.63 & & 632 & 633 & \\
\hline$L$ & & $\mathrm{R}-245 \mathrm{ca} a$ & and ISO 32 & $-\because>24$ ho & ours@ & $302 \mathrm{~F}$ & & & & & \\
\hline$Y$ & $U-4 / 5$ & 24.7625 & 24.7518 & $-0.043 \%$ & $N / C$ & 15.10 & 19.37 & & 469 & 645 & \\
\hline$A$ & $A 2$ & & & & & 15.10 & 13.52 & $13.91 \%$ & 469 & 600 & $28.36 \%$ \\
\hline$M$ & & & & & & 15.10 & 18.71 & & 469 & 561 & \\
\hline 1 & $Y-390$ & 24.0038 & 24.0151 & $0.047 \%$ & $N / C$ & 18.24 & 16.93 & & 473 & 553 & \\
\hline 0 & $B 2$ & & & & & 18.24 & 19.77 & $-2.49 \%$ & 473 & 548 & $8.74 \%$ \\
\hline$E$ & & & & & & 18.24 & 15.80 & & 473 & 442 & \\
\hline & ER-510 & 21.3030 & 2.1 .3036 & $0.003 \%$ & $\mathrm{~N} / \mathrm{C}$ & 14.53 & 12.73 & & 494 & 375 & \\
\hline 1 & C2 & & & & & 14.53 & 8.24 & $-20.83 \%$ & 494 & 385 & $-23.14 \%$ \\
\hline$M$ & & & & & & 14.53 & 13.54 & & 494 & 379 & \\
\hline 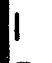 & 1.633 & 23.1073 & 23.0949 & $.0 .054 \%$ & $\mathrm{~N} / \mathrm{C}$ & 11.38 & 10.39 & & 557 & 555 & \\
\hline$D$ & D2 & & & & & 11.38 & 10.07 & $-1.99 \%$ & 557 & 503 & $.4 .13 \%$ \\
\hline$E$ & & & & & & 11.38 & 13.00 & & 557 & 544 & \\
\hline & 923 & 24.4082 & 24.4205 & $0.050 \%$ & $\mathrm{~N} / \mathrm{C}$ & 15.85 & 16.31 & & 503 & 646 & \\
\hline & E2 & & & & & 15.85 & 18.29 & $3.13 \%$ & 503 & 550 & $17.43 \%$ \\
\hline & & & & & & 15.85 & 14.44 & & 503 & 576 & \\
\hline & $150-800$ & 23.4258 & 23.4330 & $0.031 \%$ & $\mathrm{~N} / \mathrm{C}$ & 14.75 & 12.34 & & 632 & 573 & \\
\hline & $F 2$ & & & & & 14.75 & 18.86 & $12.56 \%$ & 632 & 590 & $-11.08 \%$ \\
\hline & & & & & & 14.75 & 18.61 & & 632 & 523 & \\
\hline
\end{tabular}


HELICAL COILS/WIRE A-.-HFC-245ca/Emery 2927 Iso-32@260 $\mathrm{F}\left(127^{\circ} \mathrm{C}\right)$

\begin{tabular}{|c|c|c|c|c|c|c|c|c|}
\hline & & 500 HRS & in $\mathrm{HFC}-245$ & $\mathrm{ca} / 2927$ Isc & $0.32 @$ & $60 \mathrm{~F}$ & & \\
\hline & VARN & $\begin{array}{l}\text { COIL } \\
W T\end{array}$ & $\begin{array}{c}\text { EXP COIL } \\
W T\end{array}$ & $\begin{array}{c}\text { WT \% } \\
\text { CHANGE }\end{array}$ & EXP VIS & $\begin{array}{c}\text { BASE } \\
\text { BND STR } \\
\text { (AVE) }\end{array}$ & $\begin{array}{c}\text { EXPBND } \\
\text { STR }\end{array}$ & $\begin{array}{c}\text { BND STR } \\
\% \\
\text { CHANGE }\end{array}$ \\
\hline $\mathbf{P}$ & $U-475$ & 38.2276 & 38.2586 & $0.081 \%$ & $\mathrm{~N} / \mathrm{C}$ & 73.73 & 47.37 & \\
\hline o & A 1 & & & & & 73.73 & 52.00 & $.21 .45 \%$ \\
\hline$L$ & & & & & & 73.73 & 74.37 & \\
\hline$Y$ & $Y-390$ & 38.9996 & 39.0238 & $0.062 \%$ & $\mathrm{~N} / \mathrm{C}$ & 43.78 & 31.25 & \\
\hline$E$ & B1 & & & & & 43.78 & 33.80 & $-29.79 \%$ \\
\hline$S$ & & & & & & 43.78 & 27.17 & \\
\hline$T$ & ER-610 & 38.8697 & 38.8989 & $0.075 \%$ & $\mathrm{~N} / \mathrm{C}$ & 51.81 & 63.85 & \\
\hline$E$ & $\mathrm{Cl}$ & & & & & 51.81 & 48.35 & $6.74 \%$ \\
\hline$R$ & & & & & & 51.81 & 53.70 & \\
\hline & $Y-833$ & 38.9208 & 38.8727 & $-0.124 \%$ & $\mathrm{~N} / \mathrm{C}$ & 9.85 & 14.87 & \\
\hline$P$ & D1 & & & & & 9.85 & 23.67 & $102.50 \%$ \\
\hline 0 & & & & & & 9.85 & 21.30 & \\
\hline$L$ & 923 & 38.9798 & 38.9938 & $0.036 \%$ & $\mathrm{~N} / \mathrm{C}$ & 41.28 & 28.47 & \\
\hline$Y$ & E1 & & & & & 41.28 & 34.30 & $-20.73 \%$ \\
\hline$A$ & & & & & & 41.28 & 35.40 & \\
\hline$M$ & 150.800 & 38.4800 & 38.5001 & $0.052 \%$ & $\mathrm{~N} / \mathrm{C}$ & 45.01 & 34.90 & \\
\hline 1 & $F 1$ & & & & & 45.01 & 35.50 & $-21.80 \%$ \\
\hline$D$ & & & & & & 45.01 & 37.62 & \\
\hline$E$ & & $500 \mathrm{HRS}$ & in HFC.245 & $\mathrm{ca} / 2927$ Is & 0.32@2 & $60 \mathrm{~F}>24$ & HRS $302^{\circ} \mathrm{F}$ & \\
\hline & $U-475$ & 38.5043 & 38.5140 & $0.025 \%$ & $\mathrm{~N} / \mathrm{C}$ & 73.73 & 67.27 & \\
\hline 1 & $\mathrm{~A} 2$ & & & & & 73.73 & 76.66 & $3.63 \%$ \\
\hline$M$ & & & & & & 73.73 & 76.15 & \\
\hline 1 & $\overline{Y-390}$ & 38.1401 & 38.1545 & $0.038 \%$ & $\mathrm{~N} / \mathrm{C}$ & 43.78 & 44.15 & \\
\hline$D$ & B2 & & & & & 43.78 & 42.05 & $9.73 \%$ \\
\hline$E$ & & & & & & 43.78 & 57.92 & \\
\hline & ER-610 & 36.8086 & 36.8175 & $0.024 \%$ & $\mathrm{~N} / \mathrm{C}$ & 51.81 & 51.72 & \\
\hline & $\mathrm{C} 2$ & & & & & 51.81 & 53.12 & $2.77 \%$ \\
\hline & & & & & & 51.81 & 54.90 & \\
\hline & $Y-833$ & 37.1936 & 36.9296 & $.0 .710 \%$ & $\mathrm{~N} / \mathrm{C}$ & 9.85 & 13.95 & \\
\hline & D2 & & & & & 9.85 & 27.22 & $82.13 \%$ \\
\hline & & & & & & 9.85 & 12.65 & \\
\hline & 923 & 39.8052 & 39.8091 & $0.010 \%$ & $\mathrm{~N} / \mathrm{C}$ & 41.28 & 31.10 & \\
\hline & E2 & & & & & 41.28 & 37.70 & $-16.37 \%$ \\
\hline & & & & & & 41.28 & 34.77 & \\
\hline & $150-800$ & 37.9994 & 38.0012 & $0.005 \%$ & $\mathrm{~N} / \mathrm{C}$ & 45.01 & 45.55 & \\
\hline & $\mathrm{F} 2$ & & & & & 45.01 & 50.72 & $5.27 \%$ \\
\hline & & & & & & 45.01 & 45.87 & \\
\hline
\end{tabular}


HELICAL COILS/WIRE B.HFC.245ca/Emery 2927 Iso-32 Oil @260 F $\left(127^{\circ} \mathrm{C}\right)$

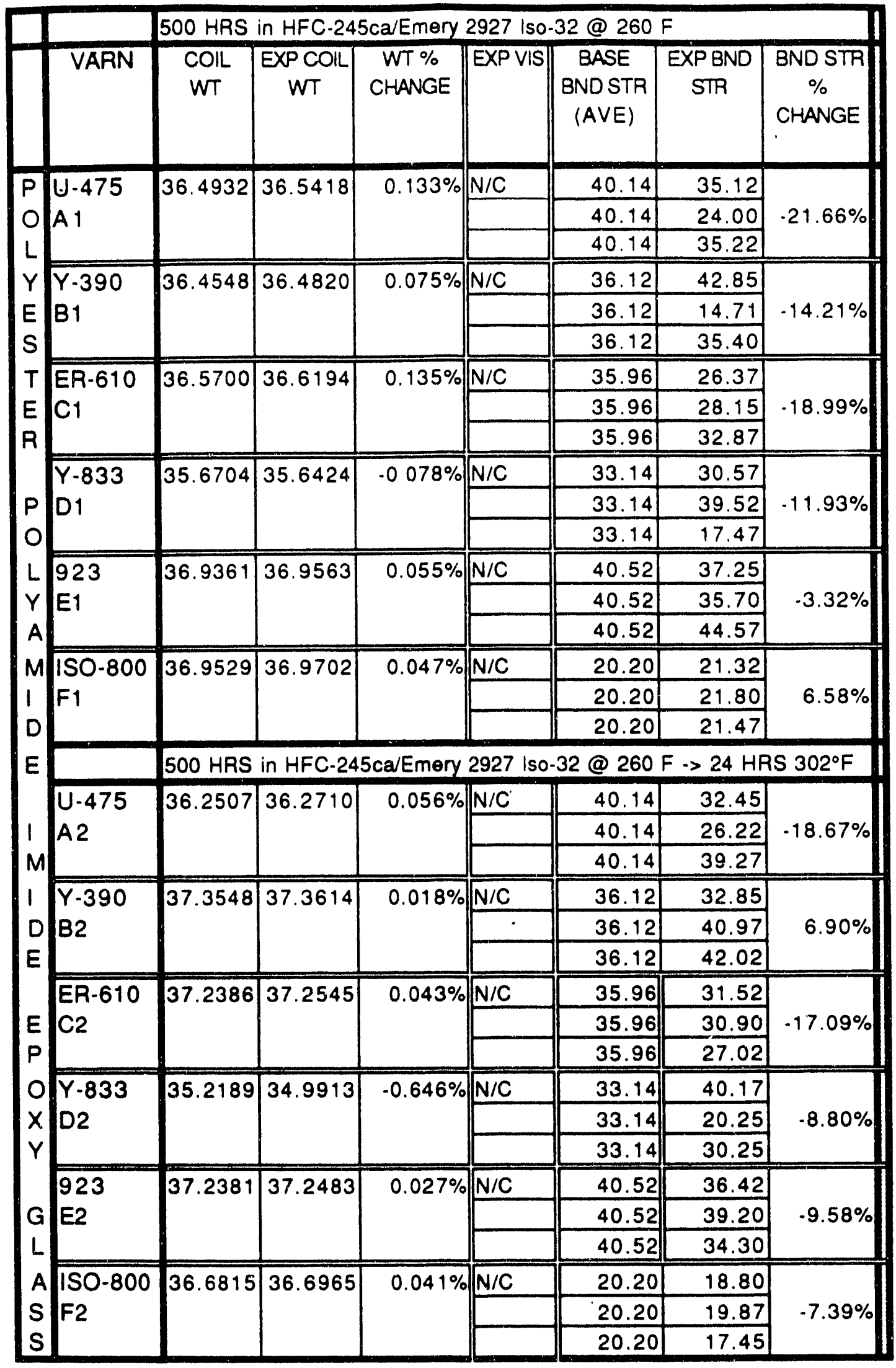


HELICAL COILS/WIRE C.HFC.R245ca/Emery 2927 Iso-32@260 $\mathrm{F}\left(127^{\circ} \mathrm{C}\right)$

\begin{tabular}{|c|c|c|c|c|c|c|c|c|}
\hline & & $500 \mathrm{HRS}$ & in $\mathrm{HFC}-24$ & jca/Emery & 1927 @ & $O F$ & & \\
\hline & VARN & $\begin{array}{l}\text { COIL } \\
\text { WT }\end{array}$ & $\begin{array}{c}\text { EXP COIL } \\
W T\end{array}$ & $\begin{array}{l}\text { WT \% } \\
\text { CHANGE }\end{array}$ & EXP VIS & $\begin{array}{c}\text { BASE } \\
\text { BND STR } \\
\text { (AVE) }\end{array}$ & $\begin{array}{c}\text { EXP BND } \\
\text { STR }\end{array}$ & $\begin{array}{c}\text { BND STR } \\
\% \\
\text { CHANGE }\end{array}$ \\
\hline$P$ & U. 475 & 42.0674 & 42.1125 & $0.107 \%$ & $\mathrm{~N} / \mathrm{C}$ & 51.21 & 52.77 & \\
\hline 0 & $A 1$ & & & & & 51.21 & 51.42 & $2.95 \%$ \\
\hline$L$ & & & & & & 51.21 & 53.97 & \\
\hline Y & $Y-390$ & 40.3415 & 40.3656 & $0.060 \%$ & $\mathrm{~N} / \mathrm{C}$ & 50.72 & 45.95 & \\
\hline$E$ & B1 & & & & & 50.72 & 26.60 & $-34.56 \%$ \\
\hline$S$ & & & & & & 50.72 & 27.02 & \\
\hline $\mathrm{T}$ & ER-610 & 38.6515 & 38.7005 & $0.12 .7 \%$ & $\mathrm{~N} / \mathrm{C}$ & 58.33 & 57.97 & \\
\hline$E$ & C1 & & & & & 58.33 & 50.20 & $-11.10 \%$ \\
\hline$R$ & & & & & & 58.33 & 47.40 & \\
\hline & $Y-833$ & 38.5670 & 38.4861 & $-0.210 \%$ & $\mathrm{~N} / \mathrm{C}$ & 5.84 & 22.47 & \\
\hline & D1 & & & & & 5.84 & 22.25 & $274.09 \%$ \\
\hline$M$ & & & & & & 5.84 & 20.82 & \\
\hline 1 & 923 & 38.4840 & 38.5033 & $0.050 \%$ & $\mathrm{~N} / \mathrm{C}$ & 49.26 & 27.15 & \\
\hline$D$ & E1 & & & & & 49.26 & 23.10 & $.49 .81 \%$ \\
\hline$E$ & & & & & & 49.26 & 23.92 & \\
\hline & ISO-800 & 39.6351 & 39.5900 & $\cdot-0.114 \%$ & $\mathrm{~N} / \mathrm{C}$ & 36.08 & 22.70 & \\
\hline$P$ & F1 & & & & & 36.08 & 19.97 & $-38.39 \%$ \\
\hline 0 & & & & & & 36.08 & 24.02 & \\
\hline$L$ & & 500 HRS & in $\mathrm{HFC}-24$ & $5 \mathrm{ca} / 2927$ /s & -32 @2 & $60 \mathrm{~F} \cdot>24$ & ARS $302^{\circ} \mathrm{F}$ & \\
\hline$Y$ & $U-475$ & 40.2249 & 40.2395 & $0.036 \%$ & $\mathrm{~N} / \mathrm{C}$ & 51.21 & 63.12 & \\
\hline A & A 2 & & & & & 51.21 & 60.62 & $14.68 \%$ \\
\hline$M$ & & & & & & 51.21 & 52.45 & \\
\hline 1 & $Y-390$ & 40.2627 & 40.2694 & $0.017 \%$ & $\mathrm{~N} / \mathrm{C}$ & 50.72 & 50.27 & \\
\hline$D$ & B2 & & & & & 50.72 & 45.45 & $-4.94 \%$ \\
\hline$E$ & & & & & & 50.72 & 48.92 & \\
\hline & ER-610 & 37.1413 & 37.1562 & $0.040 \%$ & $\mathrm{~N} / \mathrm{C}$ & 58.33 & 26.90 & \\
\hline I & $\mathrm{C} 2$ & & & & & 58.33 & 79.65 & $1.69 \%$ \\
\hline$M$ & & & & & & 58.33 & 71.40 & \\
\hline I & $Y-833$ & 37.6643 & 37.5740 & $-0.240 \%$ & $\mathrm{~N} / \mathrm{C}$ & 5.84 & 34.17 & \\
\hline D & D2 & & & & & 5.84 & 26.82 & $442.47 \%$ \\
\hline$E$ & & & & & & 5.84 & 34.05 & \\
\hline & 923 & 39.3709 & 39.3742 & $0.008 \%$ & $\mathrm{~N} / \mathrm{C}$ & 49.26 & 39.65 & \\
\hline & E2 & & & & & 49.26 & 38.35 & $-25.36 \%$ \\
\hline & & & & & & 49.26 & 32.30 & \\
\hline & ISO-800 & 38.0345 & 37.9402 & $-0.248 \%$ & $\mathrm{~N} / \mathrm{C}$ & 36.08 & 28.85 & \\
\hline & F2 & & & & & 36.08 & 25.40 & $-26.09 \%$ \\
\hline & & & & & & 36.08 & 25.75 & \\
\hline
\end{tabular}


SINGLE MAG WIRE IN $245 \mathrm{ca}$ and ISO 32 @250 $\mathrm{F}\left(121^{\circ} \mathrm{C}\right)$

\begin{tabular}{|c|c|c|c|c|c|}
\hline & 500 HRS IN & R-245@2 & $0^{\circ} \mathrm{F}\left(121^{\circ} \mathrm{C}\right)$ & & \\
\hline ID & WT & EXPWT & $\begin{array}{l}\text { WT \% } \\
\text { CHANGE }\end{array}$ & EXP VIS & EXPFLEX \\
\hline A1 & 3.3419 & 3.3433 & $0.042 \%$ & $\mathrm{~N} / \mathrm{C}$ & \\
\hline & & & & & YES \\
\hline B1 & 3.7053 & 37106 & $143 \%$ & $N / C$ & \\
\hline & & & & & YES \\
\hline C1 & 3.38 & 3.3811 & $0.033 \%$ & $N / C$ & \\
\hline & & & & & YES \\
\hline & $R-245 \rightarrow 3$ & $2^{\circ} \mathrm{F}$ for 24 & IRS & & \\
\hline$A 2$ & 3.6396 & 3.6393 & $-0.008 \%$ & $\mathrm{~N} / \mathrm{C}$ & \\
\hline & & & & & YES \\
\hline B2 & 3.7723 & 37717 & $-0016 \%$ & $N / C$ & \\
\hline & & & & & YES \\
\hline $\mathrm{C} 2$ & 3.2839 & 3.2835 & $-0.012 \%$ & $N / C$ & \\
\hline & & & & & YES \\
\hline
\end{tabular}




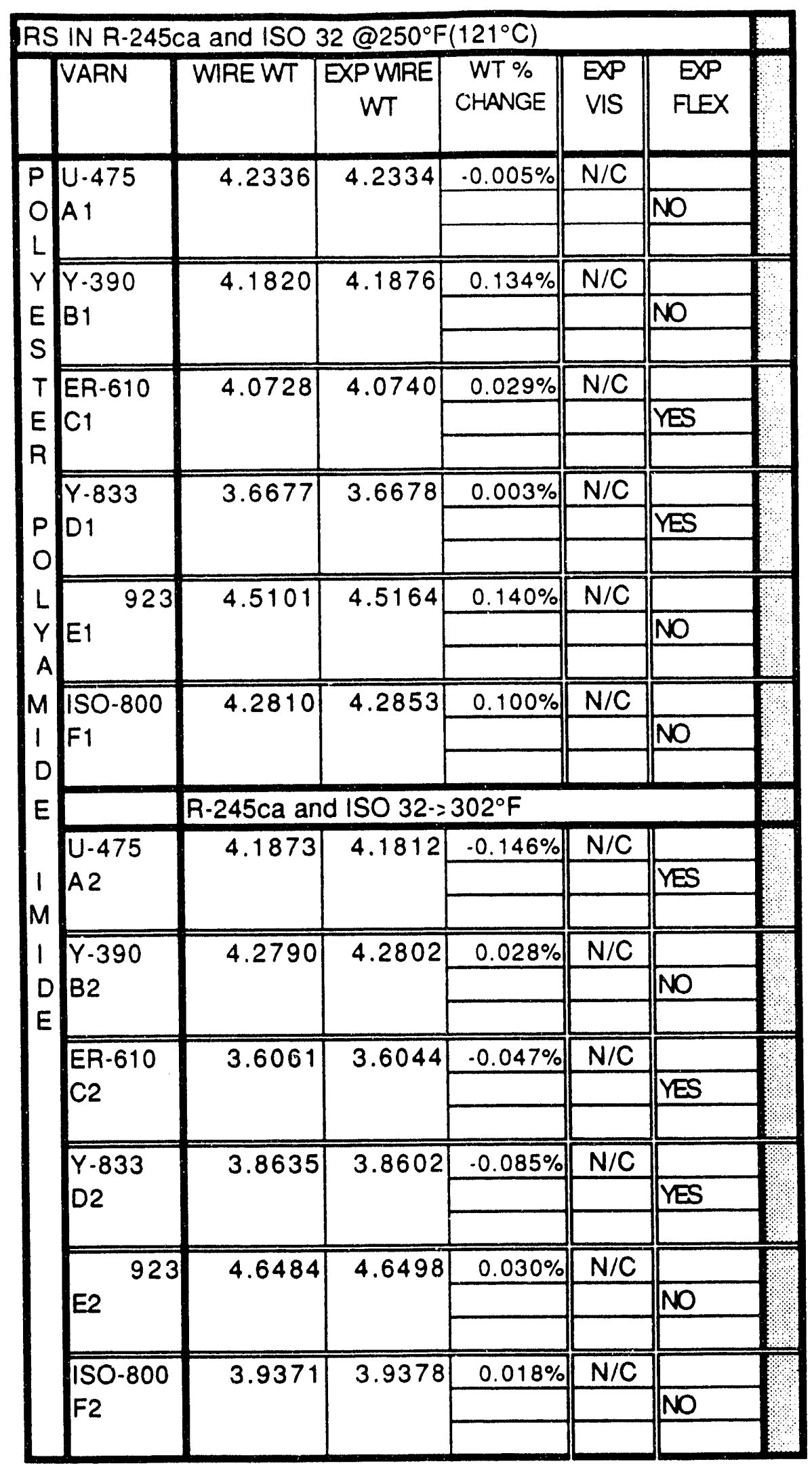




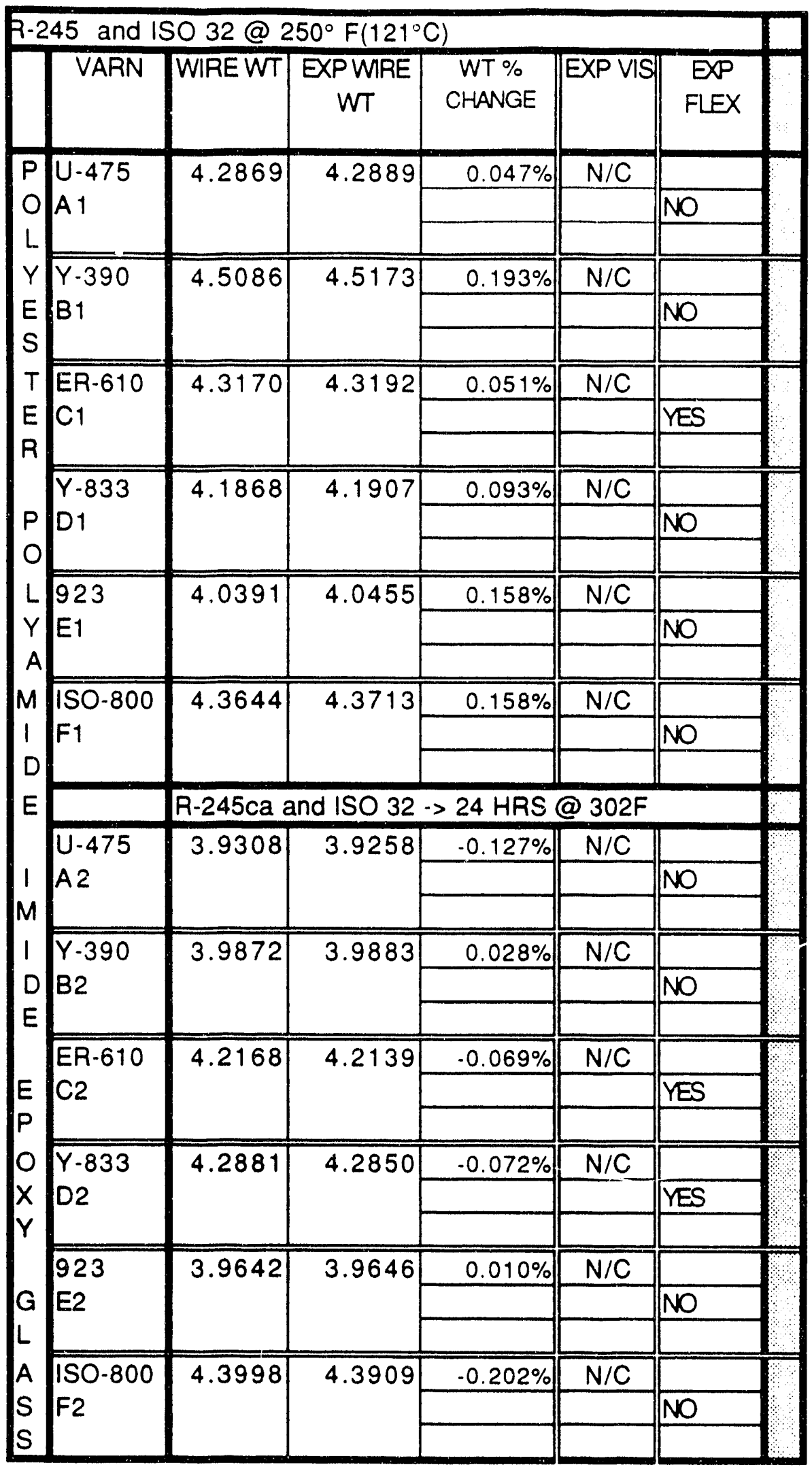




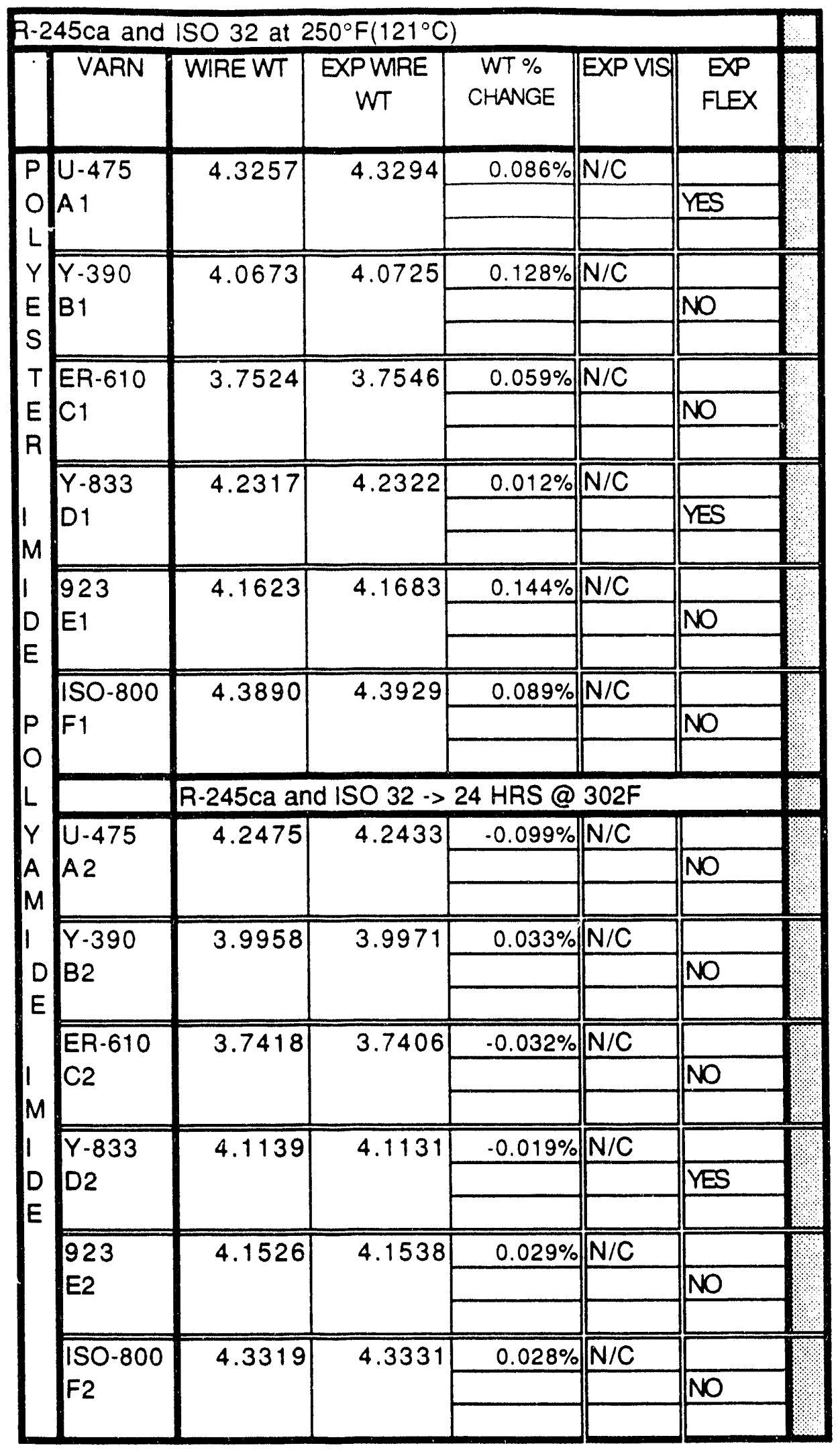




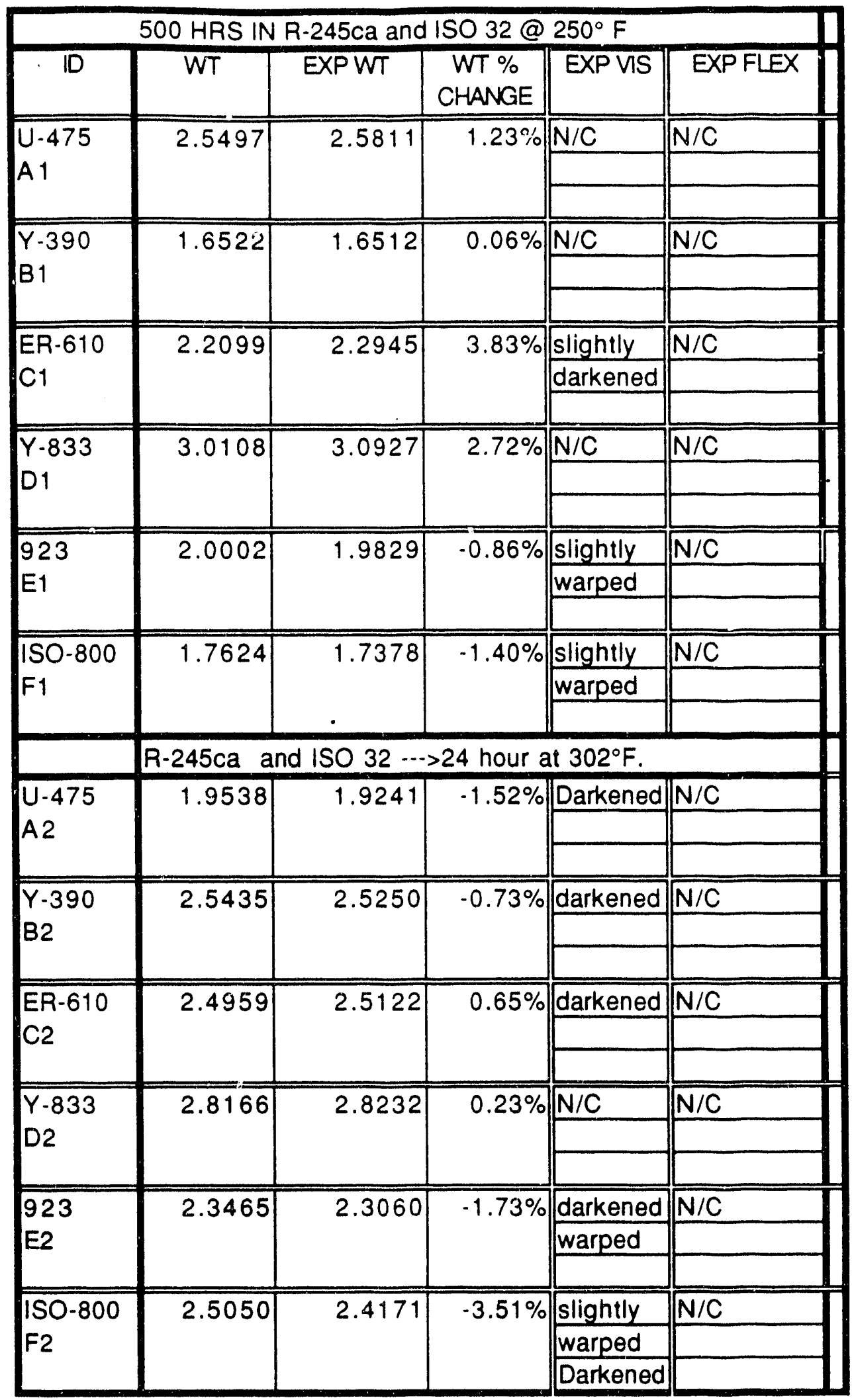




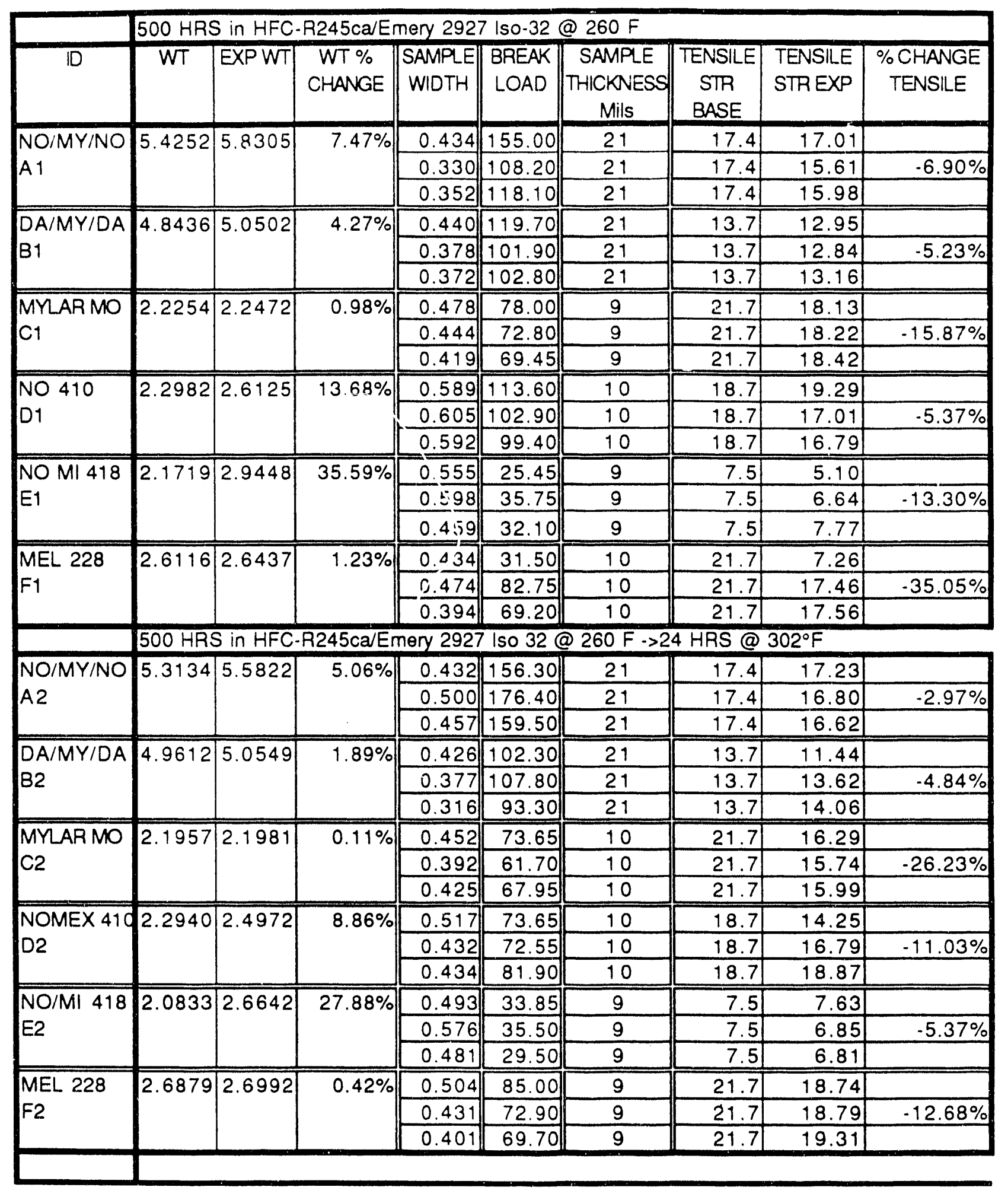




\begin{tabular}{|c|c|c|c|c|c|c|c|c|}
\hline & \multicolumn{7}{|c|}{500 HRS in HFC-R245ca/Emery 2927 Iso-32 @ 260 F } & \multirow[b]{2}{*}{ VISUAL EXP } \\
\hline ID & $\begin{array}{c}\text { STRETCH } \\
\text { (inch) }\end{array}$ & $\begin{array}{c}\% \\
\text { ELONG }\end{array}$ & $\begin{array}{l}\text { BASE } \\
\text { ELONG } \\
\text { (AVE) }\end{array}$ & $\begin{array}{l}\text { ELONG \% } \\
\text { CHANGE }\end{array}$ & \begin{tabular}{|c|} 
BASE \\
DIE \\
$(A V E)$
\end{tabular} & EXP DIE & $\begin{array}{c}\text { DIE \% } \\
\text { CHANGE }\end{array}$ & \\
\hline \multirow{3}{*}{$\begin{array}{l}\text { NO/MY/NO } \\
\text { A1 }\end{array}$} & 0.54 & $13.5 \%$ & $20.0 \%$ & & $\geq 18.97$ & flash & & \multirow[t]{3}{*}{$\mathrm{N} / \mathrm{C}$} \\
\hline & 0.26 & $6.5 \%$ & $20.0 \%$ & $.50 .0 \%$ & $>18.97$ & flash & flash & \\
\hline & 0.35 & $8.8 \%$ & $20.0 \%$ & & $>18.97$ & flash & & \\
\hline \multirow{3}{*}{$\begin{array}{l}\mathrm{DA} / \mathrm{MY} / \mathrm{DA} \\
\mathrm{B} 1\end{array}$} & 0.42 & $21.0 \%$ & $46.0 \%$ & & $>15.27$ & flash & & \multirow{3}{*}{$\begin{array}{l}\text { slightly yellowed } \\
\text { warped }\end{array}$} \\
\hline & 0.40 & $20.0 \%$ & $46.0 \%$ & $.54 .3 \%$ & $>15.2 .7$ & flash & flash & \\
\hline & 0.44 & $22.0 \%$ & $46.0 \%$ & & $>15.27$ & flash & & \\
\hline \multirow{3}{*}{$\begin{array}{l}\text { MYLAR NOO } \\
\text { C1 }\end{array}$} & 1.13 & $56.5 \%$ & $131.0 \%$ & & $>14.91$ & flash & & \multirow[t]{3}{*}{$\mathrm{N} / \mathrm{C}$} \\
\hline & 1.77 & $88.5 \%$ & $131.0 \%$ & $-42.0 \%$ & $>14.91$ & flash & flash & \\
\hline & 1.66 & $83.0 \%$ & $131.0 \%$ & & $>14.91$ & flash & & \\
\hline \multirow{3}{*}{$\begin{array}{l}\text { NO } 410 \\
\text { D1 }\end{array}$} & 0.35 & $17.5 \%$ & $17.0 \%$ & & 10.67 & flash & & \multirow[t]{3}{*}{$\mathrm{N} / \mathrm{C}$} \\
\hline & 0.22 & $5.5 \%$ & $17.0 \%$ & $-44.6 \%$ & 10.67 & flash & flash & \\
\hline & 0.21 & $5.3 \%$ & $17.0 \%$ & & 10.67 & flash & & \\
\hline \multirow{3}{*}{$\begin{array}{l}\text { NO MI } 418 \\
\text { E1 }\end{array}$} & 0.03 & $0.8 \%$ & $4.0 \%$ & & 10.23 & flash & & \multirow[t]{3}{*}{$\mathrm{N} / \mathrm{C}$} \\
\hline & 0.04 & $1.0 \%$ & $4.0 \%$ & $.79 .2 \%$ & 10.23 & flash & flash & \\
\hline & 0.03 & $0.8 \%$ & $4.0 \%$ & & 10.23 & flash & & \\
\hline \multirow{4}{*}{$\begin{array}{l}\text { MEL } 228 \\
\text { F1 }\end{array}$} & 0.99 & $49.5 \%$ & $160.0 \%$ & & $>14.22$ & flash & & \multirow[t]{3}{*}{$N / C$} \\
\hline & 1.54 & $77.0 \%$ & $160.0 \%$ & $-63.9 \%$ & $>14.22$ & flash & flash & \\
\hline & 0.94 & $47.0 \%$ & $160.0 \%$ & & $>14.22$ & flash & & \\
\hline & \multicolumn{7}{|c|}{500 HRS in HFC-R245calEmery 2927 Iso $32 @ 260 \mathrm{~F}}$. & $\mathrm{S} @ 302^{\circ} \mathrm{F}$ \\
\hline \multirow{3}{*}{$\begin{array}{l}\text { NO/MY/NO } \\
\text { A2 }\end{array}$} & 0.53 & $13.3 \%$ & $20.0 \%$ & & $>18.97$ & flash & & \multirow{3}{*}{$N / C$} \\
\hline & 0.54 & $13.5 \%$ & $20.0 \%$ & $-39.4 \%$ & $>18.97$ & flash & flash & \\
\hline & 0.43 & $10.8 \%$ & $20.0 \%$ & & $>18.97$ & flash & & \\
\hline \multirow{3}{*}{$\begin{array}{l}\text { DA/MY/DA } \\
\text { B2 }\end{array}$} & 0.47 & $23.5 \%$ & $46.0 \%$ & & $>15.27$ & flash & & \multirow{3}{*}{$\begin{array}{l}\text { slightly } \\
\text { darkened }\end{array}$} \\
\hline & 0.47 & $23.5 \%$ & $46.0 \%$ & $.49 .3 \%$ & $>15.27$ & flash & flash & \\
\hline & 0.46 & $23.0 \%$ & $46.0 \%$ & & $>15.27$ & flash & & \\
\hline \multirow{3}{*}{$\begin{array}{l}\text { MYLAR MO } \\
\text { C2 }\end{array}$} & 1.09 & $54.5 \%$ & $131.0 \%$ & & $>14.91$ & flash & & \multirow[t]{3}{*}{$N / C$} \\
\hline & 1.31 & $65.5 \%$ & $131.0 \%$ & $-47.5 \%$ & $>14.91$ & flash & flash & \\
\hline & 1.73 & $86.5 \%$ & $131.0 \%$ & & $>14.91$ & flash & & \\
\hline \multirow{3}{*}{$\begin{array}{l}\text { NOMEX 410 } \\
\text { D2 }\end{array}$} & 0.16 & $4.0 \%$ & $17.0 \%$ & & 10.67 & 11.65 & & \multirow{3}{*}{$\mathrm{N} / \mathrm{C}$} \\
\hline & 0.25 & $6.3 \%$ & $17.0 \%$ & $-62.7 \%$ & 10.67 & 11.25 & $7.0 \%$ & \\
\hline & 0.35 & $8.8 \%$ & $17.0 \%$ & & 10.67 & 11.36 & & \\
\hline \multirow{3}{*}{$\begin{array}{l}\text { NO/MI } 418 \\
\text { E2 }\end{array}$} & 0.05 & $1.3 \%$ & $4.0 \%$ & & 10.23 & flash & & \multirow[t]{3}{*}{$\mathrm{N} / \mathrm{C}$} \\
\hline & 0.04 & $1.0 \%$ & $4.0 \%$ & $-72.9 \%$ & 10.23 & flash & flash & \\
\hline & 0.04 & $1.0 \%$ & $4.0 \%$ & & 10.23 & flash & & \\
\hline MEL 228 & 1.29 & $64.5 \%$ & $160.0 \%$ & & $>14.22$ & flash & & $\mathrm{N} / \mathrm{C}$ \\
\hline $\mathrm{F} 2$ & 2.53 & $126.5 \%$ & $160.0 \%$ & $.49 .2 \%$ & $>14.22$ & flash & flash & \\
\hline & 1.06 & $53.0 \%$ & $160.0 \%$ & & $\|>14.22$ & flash & & \\
\hline
\end{tabular}


SLEEVING-HFC-R245ca/Emery 2927 Iso-32@ $260^{\circ} \mathrm{F}\left(127^{\circ} \mathrm{C}\right)$

\begin{tabular}{|c|c|c|c|c|}
\hline & \multicolumn{4}{|c|}{$500 \mathrm{HRS}$ in HFC-R245ca/Emery 2927 Iso-32@260 $\mathrm{F}$} \\
\hline & WT & EXPWT & $\begin{array}{c}\% \\
\text { CHANGE }\end{array}$ & EXPVIS \\
\hline $\begin{array}{l}\text { A1 } \\
\text { NOMEX }\end{array}$ & 0.5595 & 0.6419 & $14.73 \%$ & $\mathrm{~N} / \mathrm{C}$ \\
\hline $\begin{array}{l}\text { B1 } \\
\text { MYLAR }\end{array}$ & 0.5164 & 0.5287 & $2.38 \%$ & $\mathrm{~N} / \mathrm{C}$ \\
\hline \multirow{2}{*}{$\begin{array}{l}\text { C1 } \\
\text { NO/MY }\end{array}$} & 0.3983 & 0.4204 & $5.55 \%$ & $\mathrm{~N} / \mathrm{C}$ \\
\hline & \multicolumn{4}{|c|}{ 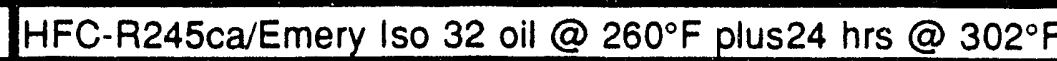 } \\
\hline A2 & 0.5303 & 0.5800 & $9.37 \%$ & $\mathrm{~N} / \mathrm{C}$ \\
\hline $\begin{array}{l}2 \\
\text { MYLAR }\end{array}$ & 0.3972 & 0.3912 & $-1.51 \%$ & $\mathrm{~N} / \mathrm{C}$ \\
\hline & 0.4531 & 0.4090 & $-9.73 \%$ & $\mathrm{~N} / \mathrm{C}$ \\
\hline NO/MY & & & & \\
\hline
\end{tabular}




\begin{tabular}{|c|c|c|c|c|c|c|c|c|c|}
\hline & 500 HRS & in $\mathrm{HFC}-2$ & 45ca/Emery & 2927 iso & @@260 & & & & \\
\hline 10 & WT & EXPWT & $\begin{array}{c}\text { WT \% } \\
\text { CHANGE }\end{array}$ & $\begin{array}{l}\text { BREAK } \\
\text { LOAD } \\
\text { (AVE) }\end{array}$ & $\begin{array}{c}\text { BREAK } \\
\text { LOAD } \\
\text { EPP }\end{array}$ & $\begin{array}{c}\% \\
\text { CHANGE } \\
\text { BPK } \\
\text { LOAD }\end{array}$ & $\begin{array}{c}\text { STRETCH } \\
\text { (INCH) }\end{array}$ & \%ELNG & EXPVIS \\
\hline A 1 & 1.8444 & 1.8549 & $0.57 \%$ & 39.02 & 60.72 & & 0.05 & $2.50 \%$ & $\mathrm{~N} / \mathrm{C}$ \\
\hline Glass & & & & 39.02 & 78.17 & $73.77 \%$ & 0.07 & $3.50 \%$ & \\
\hline & & & & 39.02 & 64.52 & & 0.07 & $3.50 \%$ & \\
\hline 81 & 0.6923 & 0.7539 & $8.90 \%$ & 56.12 & 59.95 & & 0.61 & $30.50 \%$ & $\mathrm{~N} / \mathrm{C}$ \\
\hline Polyester & & & & 56.12 & 55.05 & $1.81 \%$ & 0.64 & $32.00 \%$ & \\
\hline & & & & 56.12 & 56.40 & & 1.16 & $58.00 \%$ & \\
\hline$C_{1}$ & 1.6023 & 2.1784 & $35.95 \%$ & 88.50 & 102.30 & & 0.17 & $8.50 \%$ & Slightly \\
\hline Permacel & & & & 88.50 & 103.90 & $14.99 \%$ & 0.16 & $8.00 \%$ & curled up \\
\hline & & & & 88.50 & 99.10 & & 0.17 & $8.50 \%$ & \\
\hline & $500 \mathrm{HRS}$ & $S$ in HFC- & $\mathrm{R} 245 \mathrm{ca} / \mathrm{Em}$ & ery 29271 & 5032@ @ 2 & $60 \mathrm{~F} \cdot>302$ & ${ }^{\circ} \mathrm{F} 24 \mathrm{HR}$ & & \\
\hline A2 & 1.4282 & 1.4745 & $3.24 \%$ & 39.02 & 51.07 & & 0.04 & $2.00 \%$ & $\mathrm{~N} / \mathrm{C}$ \\
\hline Glass & & & & 39.02 & 48.17 & $26.57 \%$ & 0.08 & $4.00 \%$ & \\
\hline & & & & 39.02 & 48.92 & & 0.07 & $3.50 \%$ & \\
\hline $\mathrm{B2}$ & 0.7453 & 0.7331 & $-1.64 \%$ & 56.12 & 54.85 & & 0.71 & $35.50 \%$ & $\mathrm{~N} / \mathrm{C}$ \\
\hline Polyester & & & & 56.12 & 53.27 & $-3.40 \%$ & 0.48 & $24.00 \%$ & \\
\hline & & & & 56.12 & 54.52 & & 0.50 & $25.00 \%$ & \\
\hline $\mathrm{C} 2$ & 1.3903 & 1.6978 & $22.12 \%$ & 88.50 & 88.30 & & 0.14 & $7.00 \%$ & $\mathrm{~N} / \mathrm{C}$ \\
\hline Permacel & & & & 88.50 & 84.20 & $-2.69 \%$ & 0.15 & $7.50 \%$ & \\
\hline & & & & 88.50 & 85.85 & & 0.15 & $7.50 \%$ & \\
\hline
\end{tabular}


TIE CORD-.HFC-245ca/Emery 2927 ISO-32 oil @ 260F(127ㅇ)

\begin{tabular}{|c|c|c|c|c|c|c|c|c|c|}
\hline \multicolumn{10}{|c|}{500 HRS IN R-245ca/2927 Iso-32@260\% F } \\
\hline ID & WT & EXPWT & $\begin{array}{c}\text { WT \% } \\
\text { CHANGE }\end{array}$ & \begin{tabular}{|l|}
$E X P$ \\
VIS
\end{tabular} & $\begin{array}{l}\text { BREAK } \\
\text { LOAD } \\
\text { (AVE) }\end{array}$ & $\begin{array}{c}\text { BREAK } \\
\text { LOAD } \\
\text { EPP } \\
(\operatorname{av\theta })\end{array}$ & $\begin{array}{c}\% \\
\text { CHANGE } \\
\text { BRK } \\
\text { LOAD }\end{array}$ & $\mid \begin{array}{c}\text { STRETCH } \\
\text { (Inch) }\end{array}$ & $\begin{array}{c}\% \\
\text { ELONG }\end{array}$ \\
\hline \multirow[t]{4}{*}{$A 1$} & \multirow[t]{3}{*}{0.2907} & \multirow[t]{3}{*}{0.2933} & \multirow[t]{3}{*}{$0.89 \%$} & $\mathrm{~N} / \mathrm{C}$ & 28.36 & 29.52 & & C.34 & $17.0 \%$ \\
\hline & & & & & 28.36 & 31.75 & \multirow[t]{2}{*}{$7.71 \%$} & 0.34 & $17.0 \%$ \\
\hline & & & & & 28.36 & 30.37 & & 0.32 & $16.0 \%$ \\
\hline & \multicolumn{9}{|c|}{500 HRS IN R-245ca/Emery 2927 > 24 HRS @ 302 $\mathrm{F}$} \\
\hline \multirow[t]{3}{*}{$A 2$} & \multirow[t]{3}{*}{0.3252} & \multirow[t]{3}{*}{0.3258} & \multirow[t]{3}{*}{$0.185 \%$} & $\mathrm{~N} / \mathrm{C}$ & 28.36 & 27.32 & & 0.44 & $22.0 \%$ \\
\hline & & & & & 28.36 & 31.87 & $15.82 \%$ & 0.45 & $22.5 \%$ \\
\hline & & & & & 28.36 & 39.35 & & 0.49 & $24.5 \%$ \\
\hline
\end{tabular}


LEAD WIRE INSULATION-HFC-R245ca/Emery 2927 Iso-32@ $260^{\circ} \mathrm{F}\left(127^{\circ} \mathrm{C}\right)$

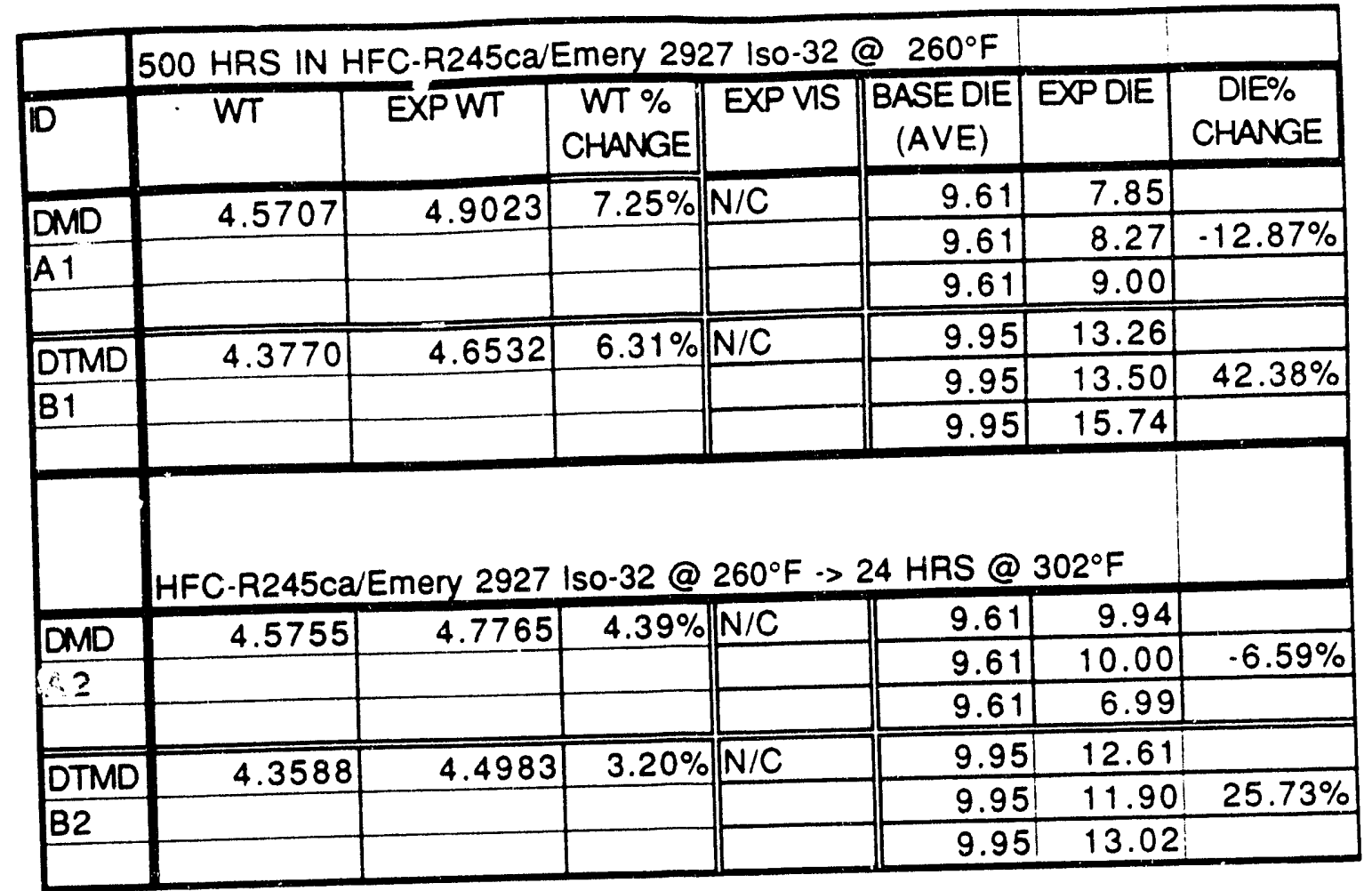




\section{Appendix M}

\section{Experimental Data for $\mathrm{HFC}-134 \mathrm{a} / \mathrm{ICI}$ Emkarox VG32 Exposure at $126^{\circ} \mathrm{C}\left(260^{\circ} \mathrm{F}\right)$}


500 hours in HFC-134a/lCl Emkarox VG32 oil at $260^{\circ} \mathrm{F}\left(127^{\circ} \mathrm{C}\right)$

\begin{tabular}{|c|c|c|c|c|c|c|c|c|c|c|}
\hline ID & $W T$ & EXPWT & $\begin{array}{l}\text { WT \% } \\
\text { CHANGE }\end{array}$ & EXP VIS & $\begin{array}{c}\text { BASE BRN } \\
\text { OUT } \\
\text { (AVE) }\end{array}$ & $\begin{array}{c}\text { EXP BRN } \\
\text { OUT }\end{array}$ & $\begin{array}{c}\text { BRN OUT } \\
\% \\
\text { CHANGE }\end{array}$ & $\begin{array}{c}\text { BASE DIE } \\
\text { (AVE) }\end{array}$ & EXPDIE & $\begin{array}{c}\text { DIE \% } \\
\text { CHANGE }\end{array}$ \\
\hline \multirow[t]{3}{*}{ A1 1} & 23.2957 & 23.3087 & $0.056 \%$ & $N / C$ & 576 & 579 & & 15.80 & 17.61 & \\
\hline & & & & & 576 & 521 & $-4.1 \%$ & 15.80 & 17.83 & $3.2 \%$ \\
\hline & & & & & 576 & 557 & & 15.80 & 13.49 & \\
\hline \multirow[t]{3}{*}{ B1 } & 23.4946 & 23.5413 & $0.199 \%$ & $N / C$ & 736 & 732 & & 11.62 & 11.24 & \\
\hline & & & & & 736 & 731 & $-0.6 \%$ & 11.62 & 12.29 & $5.6 \%$ \\
\hline & & & & & 736 & 732 & & 11.62 & 13.28 & \\
\hline \multirow[t]{4}{*}{$C_{1}$} & 20.6632 & 20.6721 & $0.043 \%$ & $N / C$ & 579 & 556 & & 16.58 & 16.21 & \\
\hline & & & & & 579 & 584 & $-7.6 \%$ & 16.58 & 15.02 & $-8.4 \%$ \\
\hline & & & & & 579 & 465 & & 16.58 & 14.35 & \\
\hline & 500 hours & HFC-134 & $\mathrm{J} / \mathrm{Cl}$ Emk & rox VG & oil at 26 & $\mathrm{~F}\left(127^{\circ} \mathrm{C}\right.$ & . & ours at 3 & $02^{\circ} \mathrm{F}\left(150^{\circ}\right.$ & \\
\hline \multirow[t]{3}{*}{ A2 } & 23.8079 & 23.8098 & $0.008 \%$ & $\mathrm{~N} / \mathrm{C}$ & 576 & 455 & & 15.80 & 14.80 & \\
\hline & & & & & 576 & 455 & $-21.4 \%$ & 15.80 & 10.32 & $-16.6 \%$ \\
\hline & & & & & 576 & 448 & & 15.80 & 14.43 & \\
\hline \multirow[t]{3}{*}{ B2 } & 22.977 & 22.9822 & $0.023 \%$ & $N / C$ & 736 & 717 & & 11.62 & 11.53 & \\
\hline & & & & & 736 & 731 & $-3.4 \%$ & 11.62 & 12.06 & $1.3 \%$ \\
\hline & & & & & 736 & 684 & & 11.62 & 11.74 & \\
\hline \multirow[t]{3}{*}{$\mathrm{C} 2$} & 21.4172 & 21.4189 & $0.008 \%$ & $N / C$ & 579 & 546 & & 16.58 & 10.52 & \\
\hline & & & & & 579 & 560 & $-12.0 \%$ & 16.58 & 11.31 & $-29.6 \%$ \\
\hline & & & & & 579 & 422 & & 16.58 & 13.17 & \\
\hline
\end{tabular}




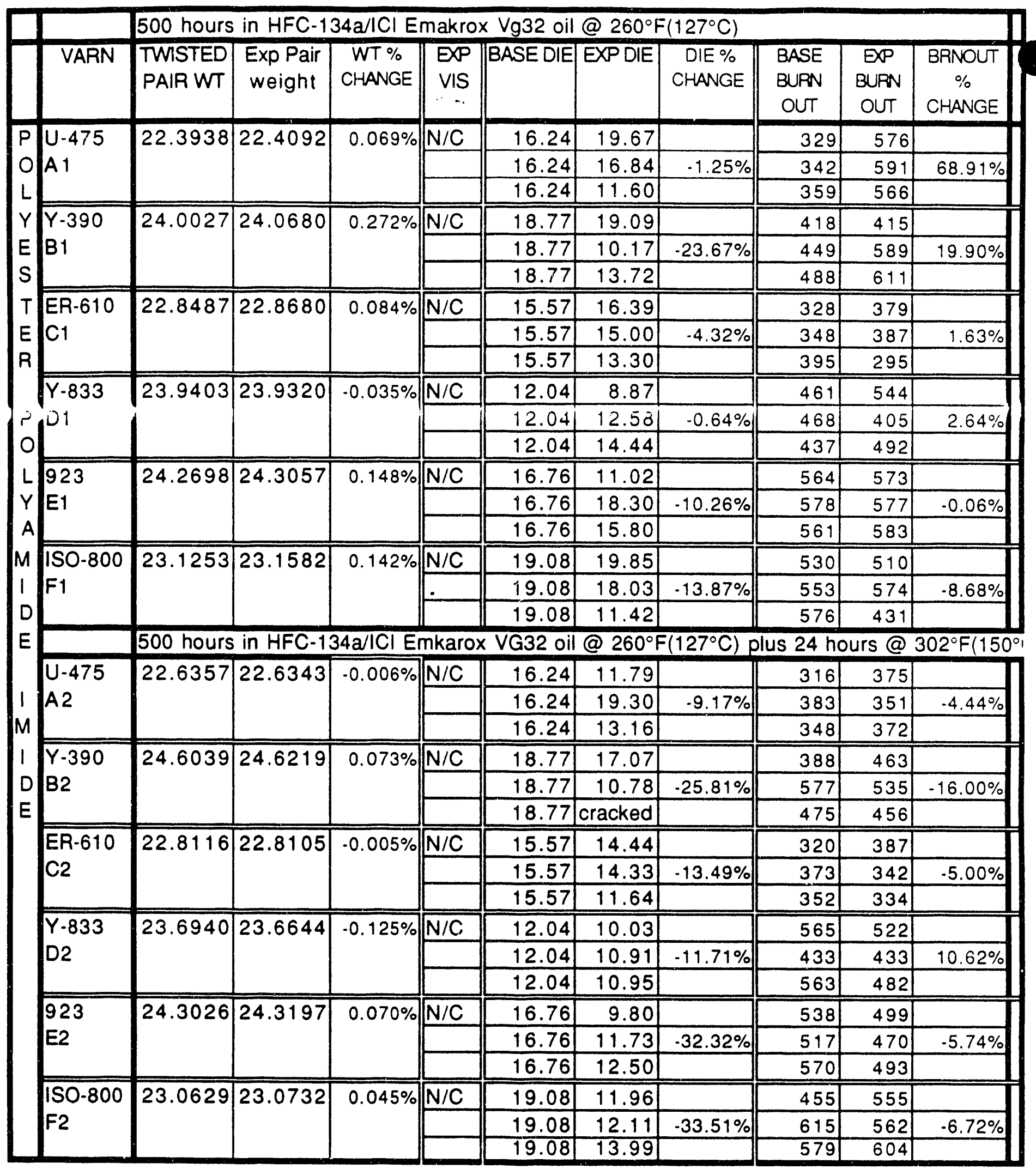


TWISTED PAIRS/WIRE BNITH VARNISH ·HFC-134a/lCI Emkarox VG32 oil @260 $\mathrm{F}\left(127^{\circ} \mathrm{C}\right)$

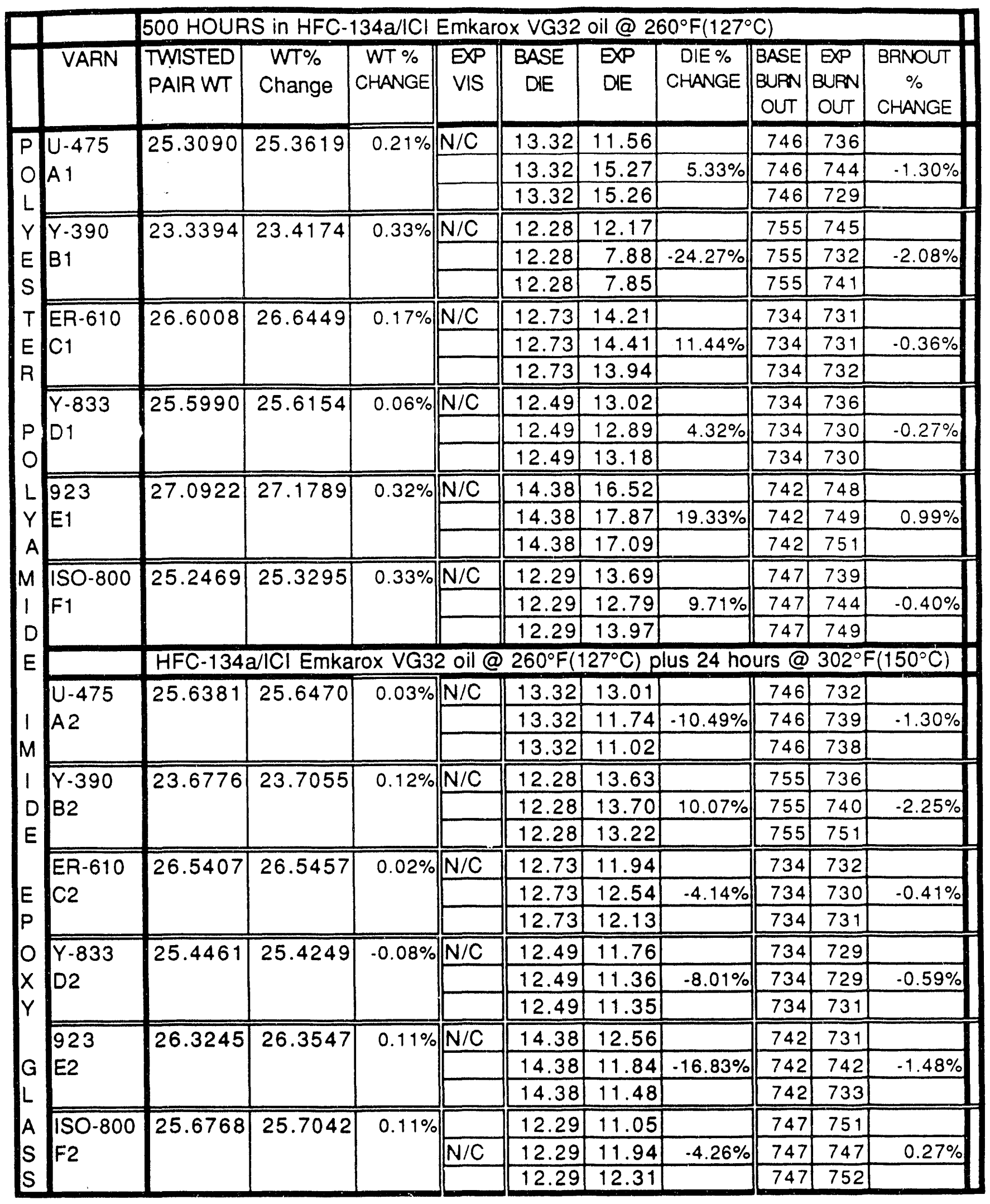


TWISTED PAIRSMIRE CWITH VARNISH.HFC-134alCI Emkarox VG $32 @ 260^{\circ} \mathrm{F}\left(127^{\circ} \mathrm{C}\right)$

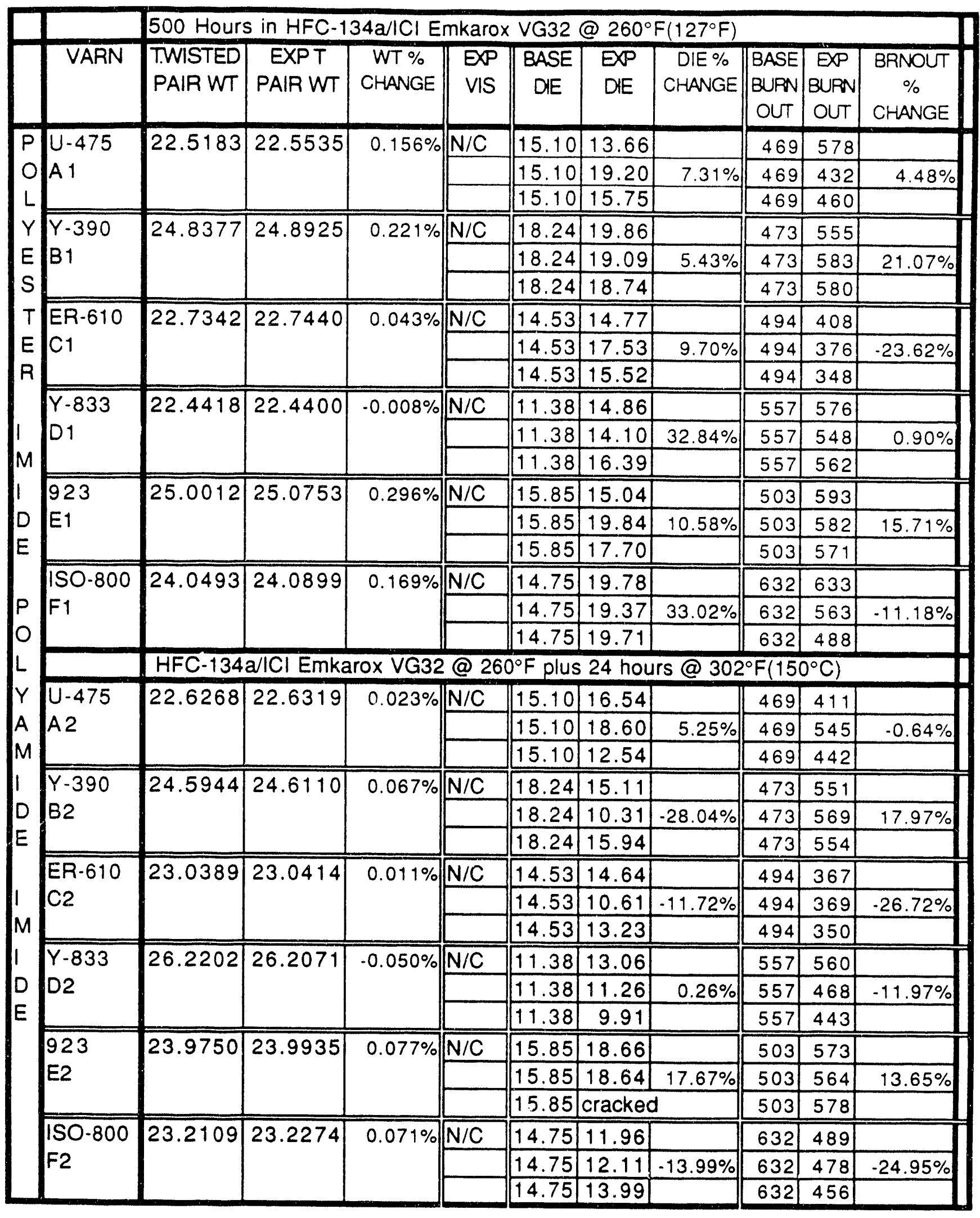


HELICAL COILS/WIRE A...HFC-134a/ICI Emkarox VG32@260 $\mathrm{F}\left(127^{\circ} \mathrm{C}\right)$

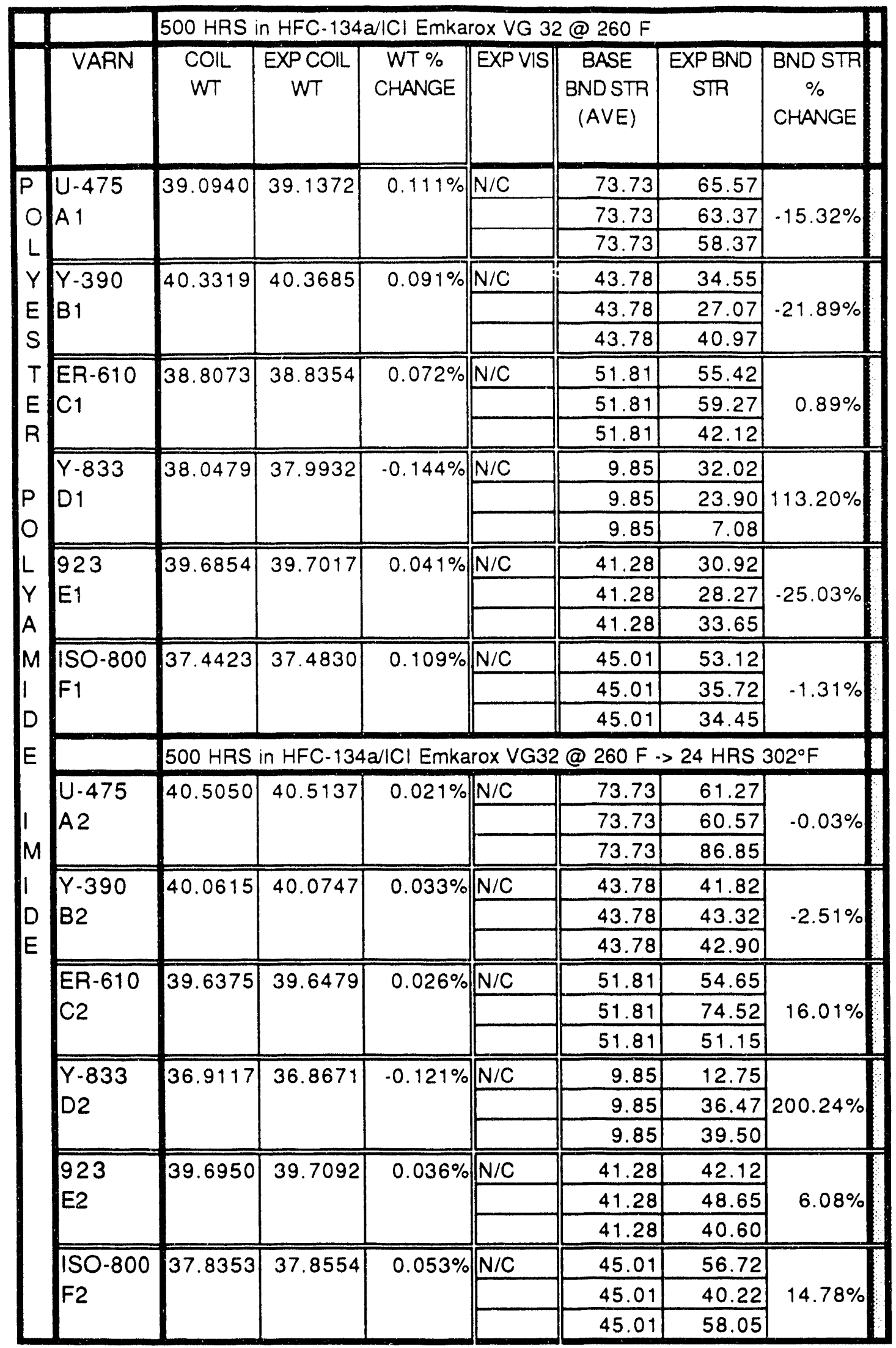




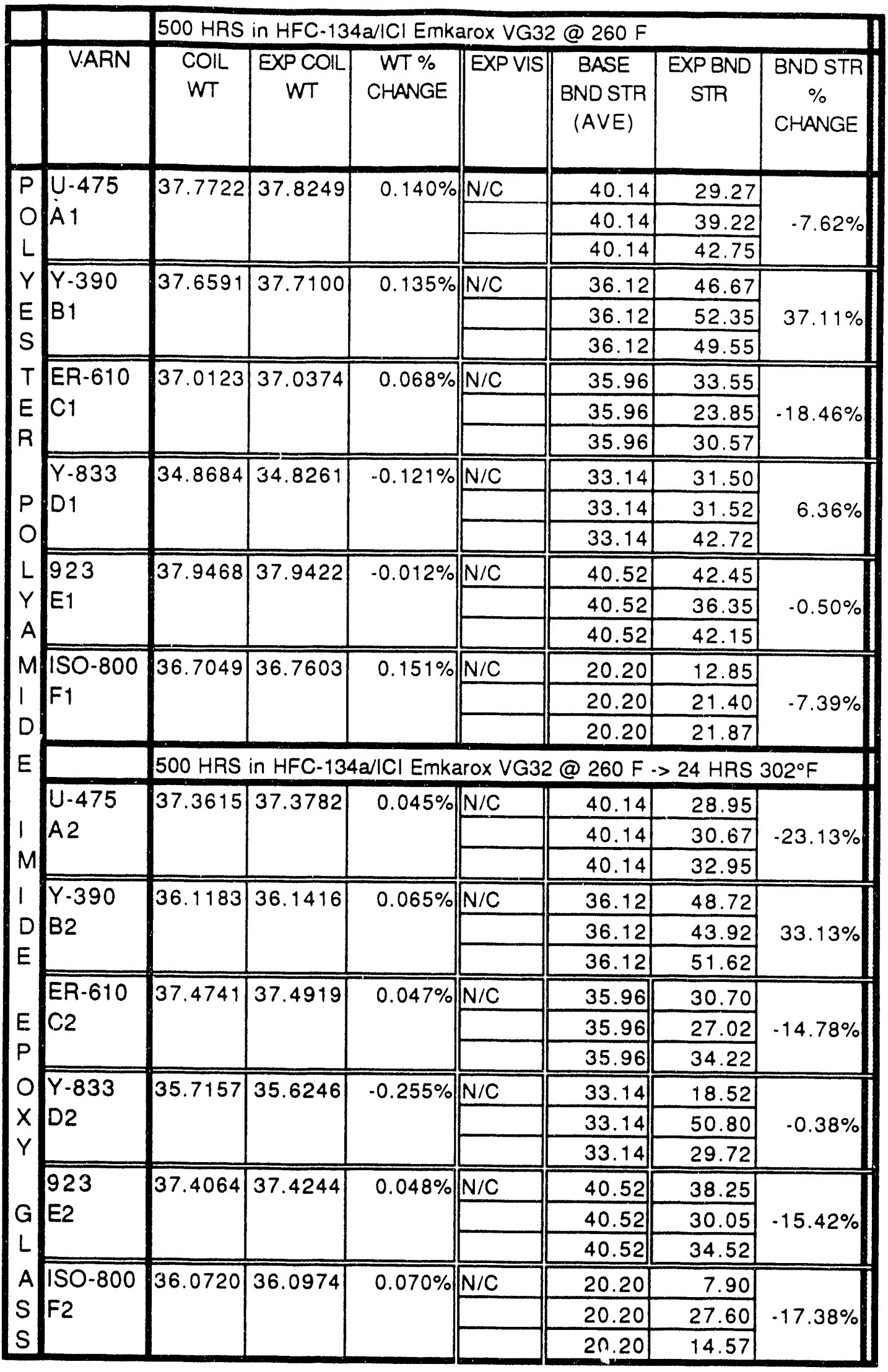




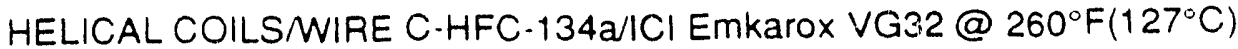

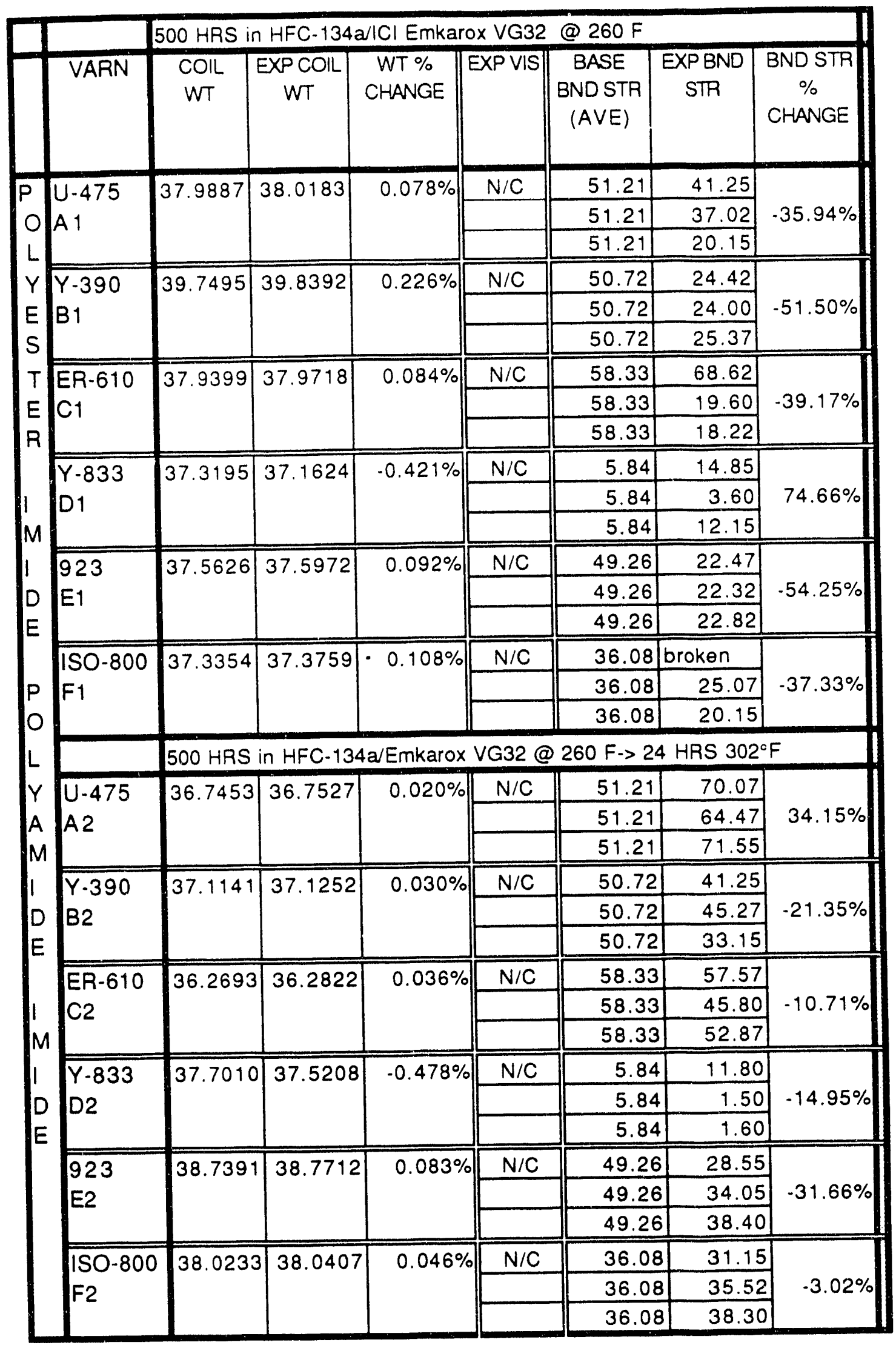




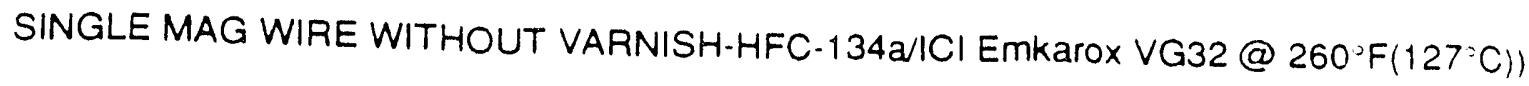

\begin{tabular}{|c|c|c|c|c|c|}
\hline 500 & $\mathrm{AS}$ in $\mathrm{HF}$ & $-134 a / / C l$ & nkarox VG? & 2 oil @2 & $60^{\circ} \mathrm{F}\left(127^{\circ} \mathrm{C}\right)$ \\
\hline ID & $W T$ & EXPWT & $\begin{array}{c}\text { WT \% } \\
\text { CHANGE }\end{array}$ & EXP VIS & EXPFLEX \\
\hline$A 1$ & 3.6219 & 3.6233 & $0.039 \%$ & $\mathrm{~N} / \mathrm{C}$ & \\
\hline & & & & & YES \\
\hline B1 & 3.3462 & 3.3513 & $0.152 \%$ & $N / C$ & \\
\hline & & & & & YES \\
\hline & & & & & \\
\hline C1 & 3.2042 & 3.2058 & $0.050 \%$ & $\mathrm{~N} / \mathrm{C}$ & \\
\hline & & & & & YES \\
\hline & & & & & \\
\hline 500 & $\mathrm{RS}$ in $\mathrm{HF}$ & $-134 a / E m$ & arox oil-> 3 & $2^{\circ} \mathrm{F}$ for 2 & 4 HRS \\
\hline$A 2$ & 3.2982 & 3.298 & $-0.006 \%$ & $\mathrm{~N} / \mathrm{C}$ & \\
\hline & & & & & YES \\
\hline & & & & & \\
\hline$B 2$ & 3.2914 & 3.2918 & $0.012 \%$ & $\mathrm{~N} / \mathrm{C}$ & \\
\hline & & & & & YES \\
\hline $\mathrm{C} 2$ & 34076 & 34078 & & & \\
\hline $\boldsymbol{U} \boldsymbol{L}$ & 3.4976 & $3.49 / 8$ & $0.006 \%$ & $\mathrm{~N} / \mathrm{C}$ & \begin{tabular}{|l} 
\\
YES
\end{tabular} \\
\hline & & & & & \\
\hline
\end{tabular}




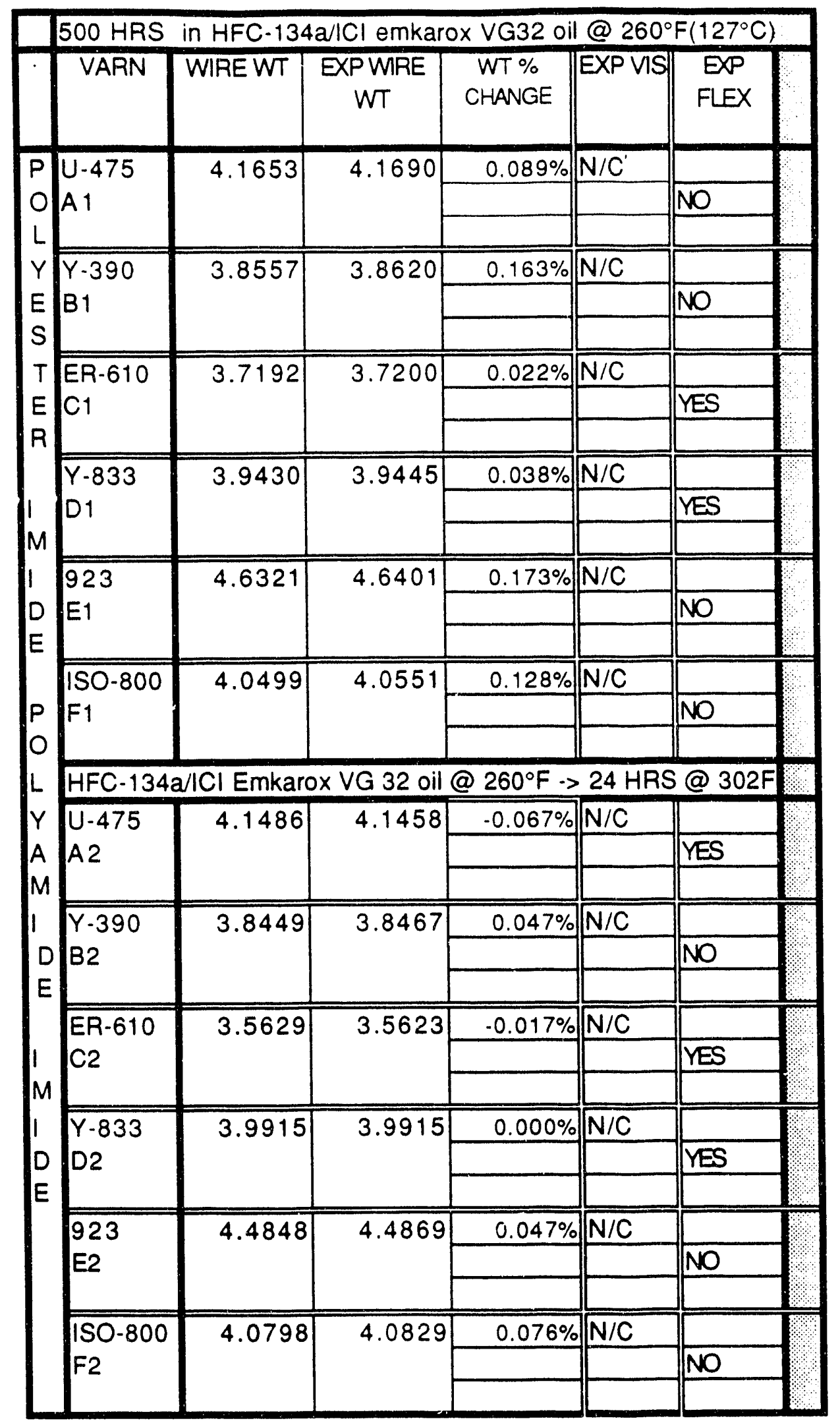




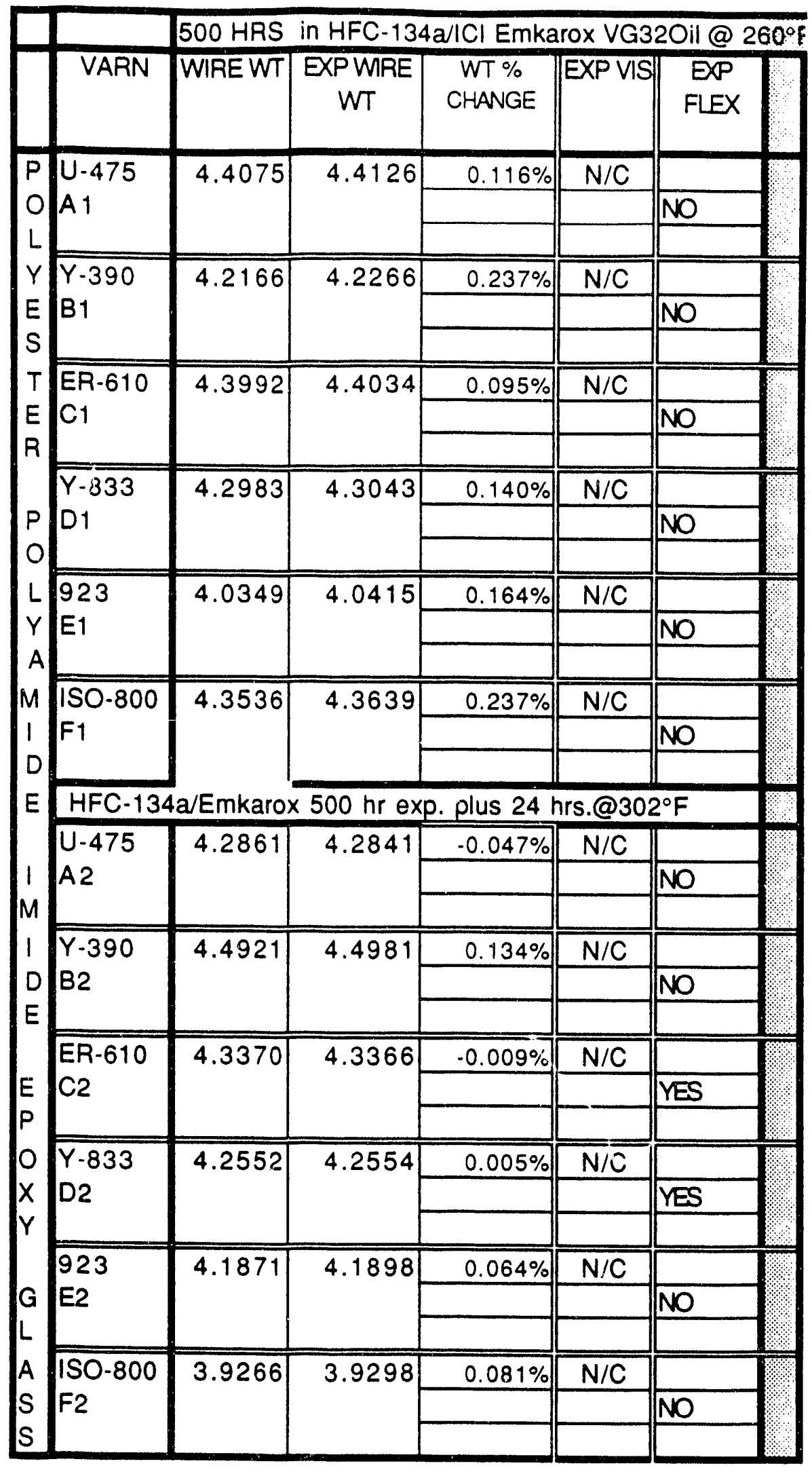




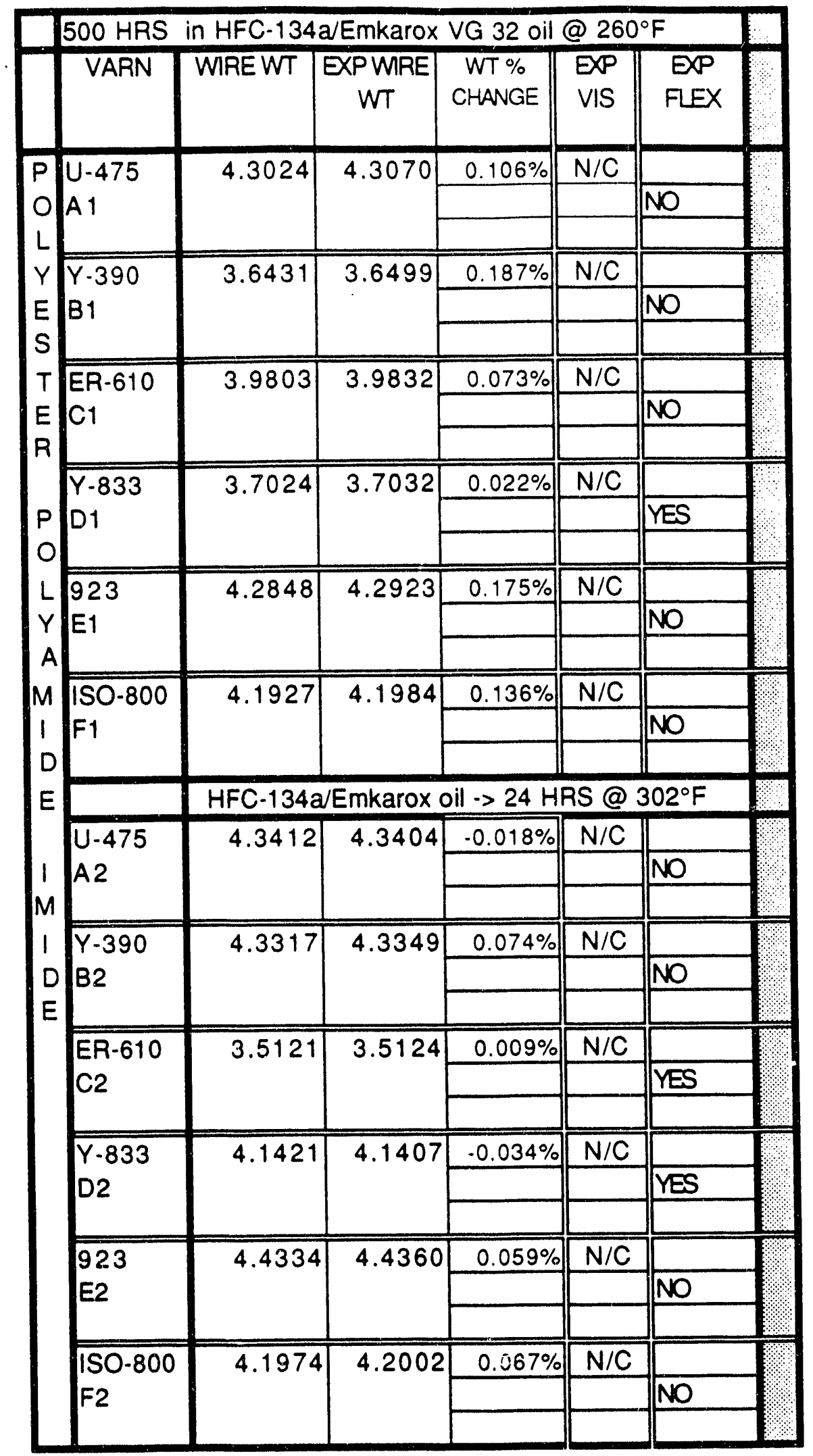


Varnish Disks-...HFC-134a/lCI Emkarox VG 32 Oil @ 260'F(127\% F)

\begin{tabular}{|c|c|c|c|c|c|}
\hline \multicolumn{6}{|c|}{500 HRS IN R-134a/ICI Emkarox VG32@ 260 } \\
\hline ID & WT & EXPWT & $\begin{array}{l}\text { WT \% } \\
\text { CHANGE }\end{array}$ & ||EXPVIS & EXPFLEX \\
\hline $\begin{array}{l}\text { U.475 } \\
\text { A } 1\end{array}$ & 2.2979 & 2.3144 & $0.72 \%$ & \begin{tabular}{|l} 
Slightly \\
warped
\end{tabular} & $\mathrm{N} / \mathrm{C}$ \\
\hline \multirow{2}{*}{$\begin{array}{l}\text { Y.390 } \\
B 1\end{array}$} & \multirow[t]{2}{*}{1.7789} & \multirow[t]{2}{*}{1.7719} & \multirow[t]{2}{*}{$0.39 \%$} & $N / C$ & $\mathrm{~N} / \mathrm{C}$ \\
\hline & & & & & \\
\hline \multirow{2}{*}{$\begin{array}{l}\text { ER-610 } \\
\mathrm{C} 1\end{array}$} & \multirow{2}{*}{2.1911} & \multirow{2}{*}{2.2599} & \multirow{2}{*}{$3.14 \%$} & $N / C$ & $N / G$ \\
\hline & & & & & \\
\hline \multirow{2}{*}{$\begin{array}{l}Y \cdot 833 \\
D 1\end{array}$} & \multirow[t]{2}{*}{3.5244} & \multirow{2}{*}{3.6102} & \multirow[t]{3}{*}{$2.43 \%$} & $\mathrm{~N} / \mathrm{C}$ & $N / C$ \\
\hline & & & & & \\
\hline & \multirow{3}{*}{2.4599} & \multirow{3}{*}{2.4139} & & & \\
\hline \multirow{3}{*}{$\begin{array}{l}923 \\
\text { E1 }\end{array}$} & & & \multirow[t]{2}{*}{$-1.87 \%$} & & $\mathrm{~N} / \mathrm{C}$ \\
\hline & & & & Warped & \\
\hline & \multirow{2}{*}{2.0935} & \multirow{3}{*}{2.0523} & \multirow{3}{*}{$-1.97 \%$} & & \\
\hline \multirow{3}{*}{$\begin{array}{l}\text { ISO-800 } \\
\mathrm{F} 1\end{array}$} & & & & $\mathrm{~N} / \mathrm{C}$ & $\mathrm{N} / \mathrm{C}$ \\
\hline & \multirow{2}{*}{\multicolumn{5}{|c|}{ R-134a/lCl Emkarox VG 32 at $\cdots>24$ hour at $302^{\circ} \mathrm{F}$. }} \\
\hline & & & & & \\
\hline \multirow{3}{*}{$\begin{array}{l}\text { U-475 } \\
\text { A2 }\end{array}$} & \multirow[t]{3}{*}{2.6905} & \multirow[t]{3}{*}{2.6300} & \multirow[t]{3}{*}{$-2.25 \%$} & Darkened & $\mathrm{N} / \mathrm{C}$ \\
\hline & & & & Slightly & \\
\hline & & & & warped & \\
\hline \multirow{2}{*}{$\begin{array}{l}Y-390 \\
B 2\end{array}$} & 1.9718 & 1.9195 & $-2.65 \%$ & $\mathrm{~N} / \mathrm{C}$ & $\mathrm{N} / \mathrm{C}$ \\
\hline & & & & & \\
\hline ER-610 & 2.3770 & 2.3779 & $0.04 \%$ & Darkened & $\mathrm{N} / \mathrm{C}$ \\
\hline $\mathrm{C} 2$ & & & & & \\
\hline $\begin{array}{c}Y-833 \\
-8\end{array}$ & 31030 & 30855 & 05701 & Ongtrent & ब10 \\
\hline $\mathrm{D} 2$ & 3.1032 & 3.0855 & $-0.57 \%$ & Darkened & N/C \\
\hline$\overline{923}$ & 2.0671 & 2.0134 & $-2.60 \%$ & Warped & \\
\hline E2 & & & & Darkened & $\mathrm{N} / \mathrm{C}$ \\
\hline ISO-800 & 2.3994 & 2.3371 & $-260 \%$ & darkened & \\
\hline $\mathrm{F} 2$ & & & & & $\mathrm{~N} / \mathrm{C}$ \\
\hline & & & & & \\
\hline
\end{tabular}




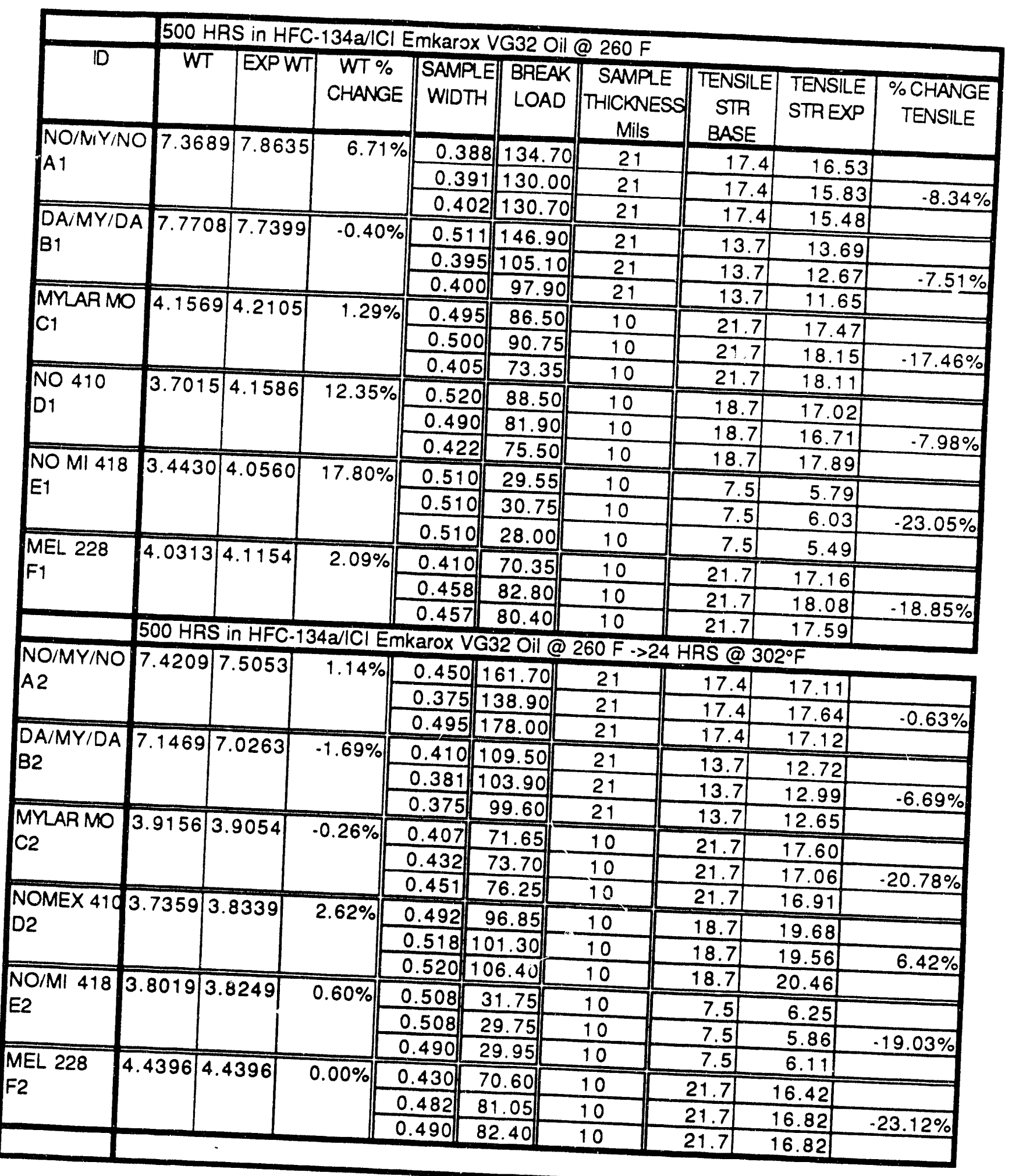




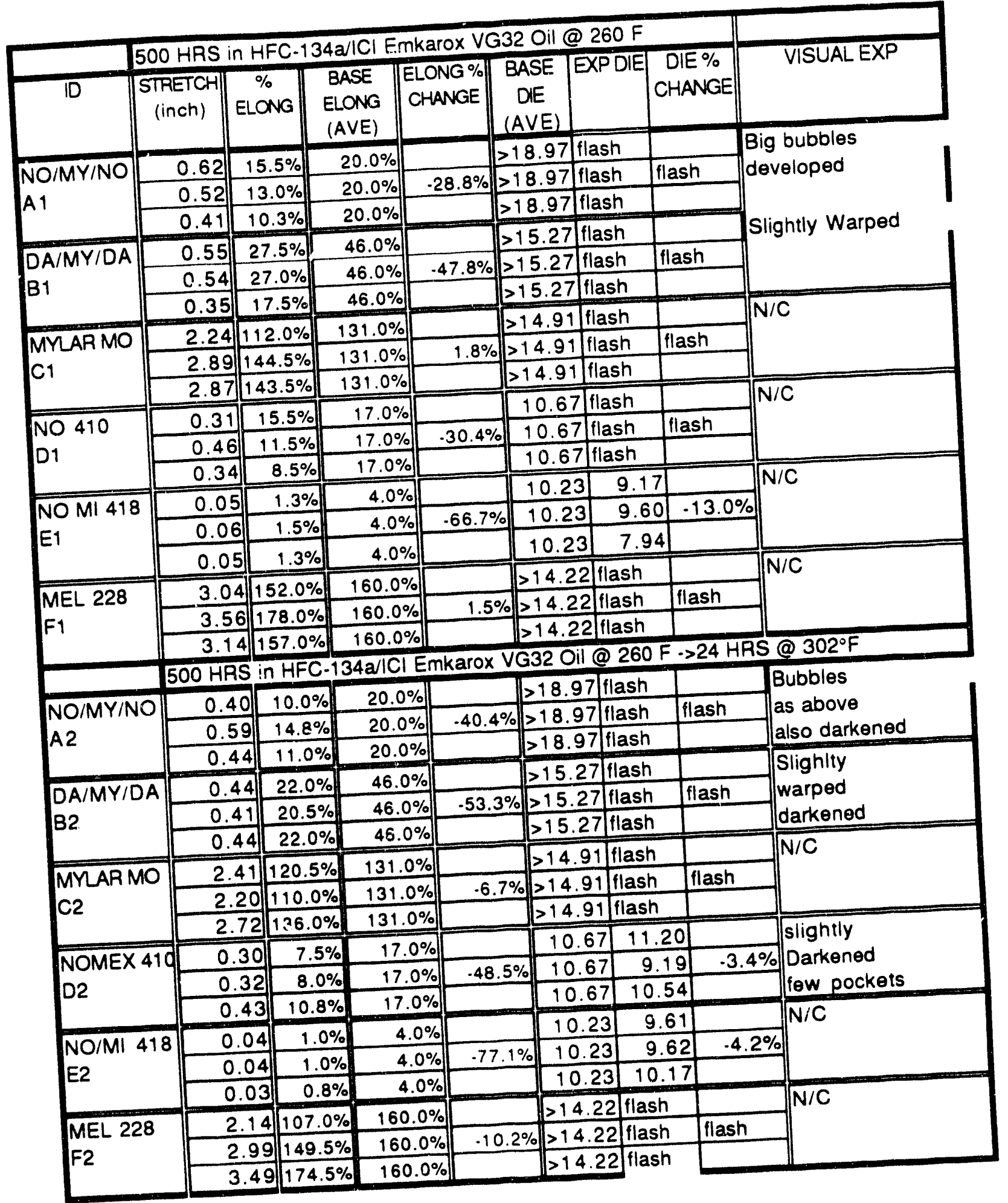


SLEEVING--HFC-134a/ICI Emkarox VG 32 Oil @ 260 $F\left(127^{\circ} \mathrm{C}\right)$

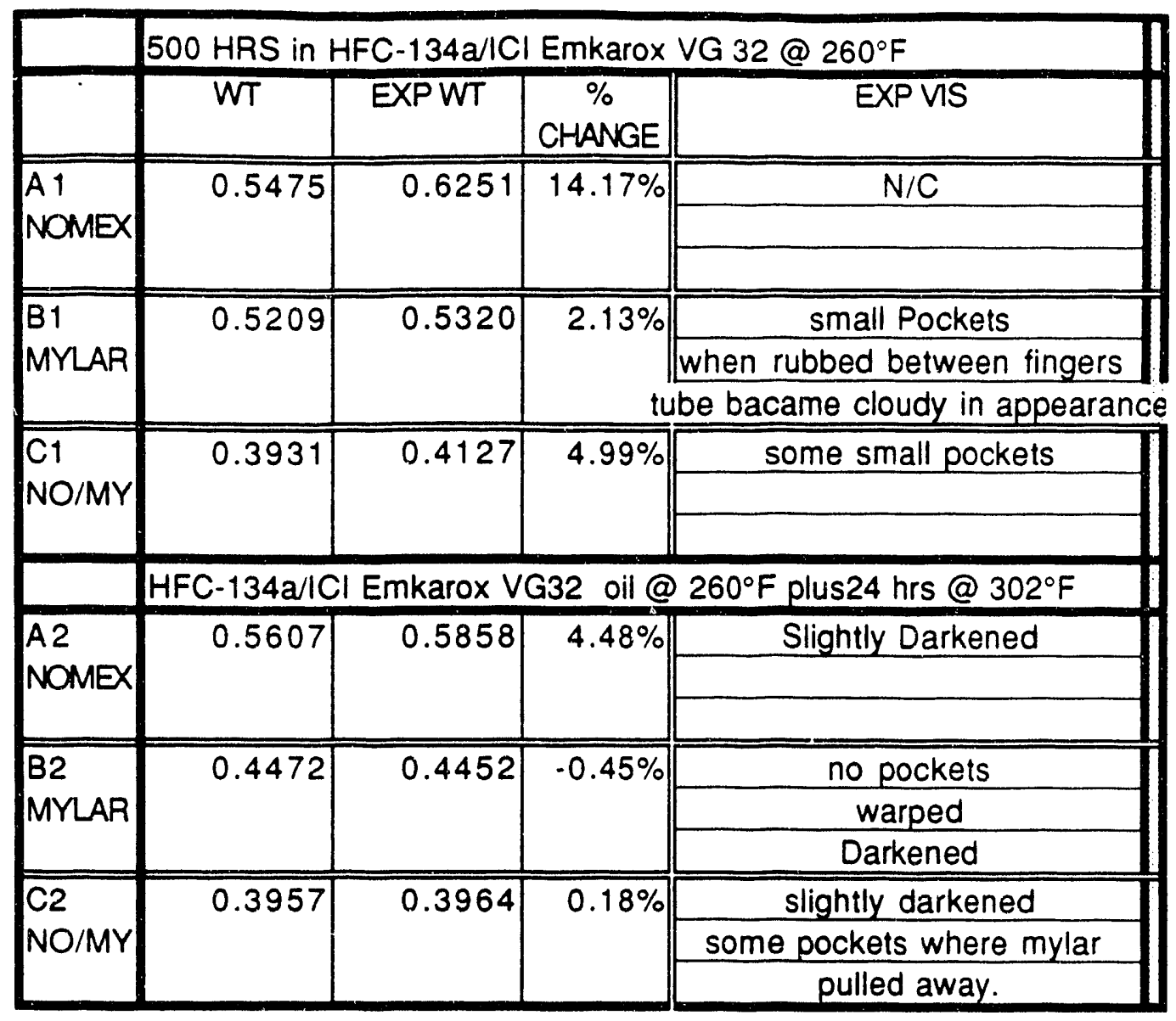




\begin{tabular}{|c|c|c|c|c|c|c|c|c|c|}
\hline \multirow[b]{2}{*}{ ID } & \multicolumn{9}{|c|}{500 HRS in HFC-134a/lCl Emkarox VG32 @ $260 \mathrm{~F}$} \\
\hline & WT & EXP WT & $\begin{array}{c}\text { WT \% } \\
\text { CHANGE }\end{array}$ & $\begin{array}{l}\text { BREAK } \\
\text { LOAD } \\
\text { (AVE) }\end{array}$ & $\begin{array}{c}\text { BREAK } \\
\text { LOAD } \\
\text { EXP }\end{array}$ & $\begin{array}{c}\% \\
\text { CHANGE } \\
\text { BRK } \\
\text { LOAD }\end{array}$ & $\begin{array}{l}\text { STRETCH } \\
(\mathrm{INCH})\end{array}$ & $\%$ ELNG & EXPVIS \\
\hline \multirow{3}{*}{$\begin{array}{l}\text { A } 1 \\
\text { Glass }\end{array}$} & \multirow[t]{3}{*}{1.7612} & \multirow[t]{3}{*}{1.7637} & \multirow[t]{3}{*}{$0.14 \%$} & 39.02 & 49.50 & & 0.05 & $2.50 \%$ & $\mathrm{~N} / \mathrm{C}$ \\
\hline & & & & 39.02 & 52.60 & $37.24 \%$ & 0.05 & $2.50 \%$ & \\
\hline & & & & 39.02 & 58.55 & & 0.06 & $3.00 \%$ & \\
\hline \multirow{3}{*}{$\begin{array}{l}\text { B1 } \\
\text { Polyester }\end{array}$} & \multirow[t]{3}{*}{0.7222} & \multirow[t]{3}{*}{0.7332} & \multirow[t]{3}{*}{$1.52 \%$} & 56.12 & 59.10 & & 0.67 & $33.50 \%$ & $\mathrm{~N} / \mathrm{C}$ \\
\hline & & & & 56.12 & 58.85 & $5.87 \%$ & 0.70 & $35.00 \%$ & \\
\hline & & & & 56.12 & 60.30 & & 0.70 & $35.00 \%$ & \\
\hline \multirow{3}{*}{$\begin{array}{l}\text { C1 } \\
\text { Permacel }\end{array}$} & \multirow[t]{3}{*}{1.6011} & \multirow[t]{3}{*}{1.8334} & \multirow[t]{3}{*}{$14.51 \%$} & 88.50 & 45.45 & & 0.16 & $8.00 \%$ & Slightly \\
\hline & & & & 88.50 & 46.15 & $.47 .93 \%$ & 0.12 & $6.00 \%$ & darkened \\
\hline & & & & 88.50 & 46.65 & & 0.14 & $7.00 \%$ & Curled up \\
\hline & \multicolumn{8}{|c|}{$500 \mathrm{HRS}$ in HFC-134a/lCl Emkarox VG32@260 F $>302^{\circ} \mathrm{F} 24 \mathrm{HRS}$} & \\
\hline \multirow{3}{*}{$\begin{array}{l}\text { A2 } \\
\text { Glass }\end{array}$} & \multirow[t]{3}{*}{1.6269} & \multirow[t]{3}{*}{1.6257} & \multirow[t]{3}{*}{$.0 .07 \%$} & 39.02 & 66.20 & & 0.07 & $3.50 \%$ & $\mathrm{~N} / \mathrm{C}$ \\
\hline & & & & 39.02 & 55.60 & $59.15 \%$ & 0.06 & $3.00 \%$ & \\
\hline & & & & 39.02 & 64.50 & & 0.06 & $3.00 \%$ & \\
\hline \multirow{3}{*}{$\begin{array}{l}\text { B2 } \\
\text { Polyester }\end{array}$} & \multirow[t]{3}{*}{0.6570} & \multirow[t]{3}{*}{0.6566} & \multirow[t]{3}{*}{$.0 .06 \%$} & 56.12 & 51.20 & & 0.59 & $29.50 \%$ & $\mathrm{~N} / \mathrm{C}$ \\
\hline & & & & 56.12 & 54.38 & $-2.51 \%$ & 0.58 & $29.00 \%$ & \\
\hline & & & & 56.12 & 58.55 & & 0.74 & $37.00 \%$ & \\
\hline \multirow{3}{*}{$\begin{array}{l}\text { C2 } \\
\text { Permacel }\end{array}$} & \multirow[t]{3}{*}{1.6392} & \multirow[t]{3}{*}{1.4265} & \multirow[t]{3}{*}{$-12.98 \%$} & 88.50 & 111.30 & & 0.13 & $6.50 \%$ & Slightly \\
\hline & & & & 88.50 & 112.30 & $12.11 \%$ & 0.14 & $7.00 \%$ & Darkened \\
\hline & & & & 88.50 & 74.05 & & 0.10 & $5.00 \%$ & Curled up \\
\hline
\end{tabular}


TIE CORD--HFC-134a/ICl Emkarox VG32 oil @ 260 $\mathrm{F}\left(127^{\circ} \mathrm{C}\right)$

\begin{tabular}{|c|c|c|c|c|c|c|c|c|c|}
\hline \multicolumn{10}{|c|}{500 HRS IN R-134a/ICI Emkarox VG32 @ 260 F } \\
\hline ID & WT & EXPWT & $\begin{array}{c}\text { WT \% } \\
\text { CHANGE }\end{array}$ & $\begin{array}{l}\text { EXP } \\
\text { VIS }\end{array}$ & $\begin{array}{l}\text { BREAK } \\
\text { LOAD } \\
\text { (AVE) }\end{array}$ & $\begin{array}{l}\text { BREAK } \\
\text { LOAD } \\
\text { EXP } \\
(\text { ave } \theta\end{array}$ & $\begin{array}{c}\% \\
\text { CHANGE } \\
\text { BPK } \\
\text { LOAD }\end{array}$ & $\begin{array}{c}\text { STRETCH } \\
\text { (Inch) }\end{array}$ & $\begin{array}{c}\% \\
\text { ELONG }\end{array}$ \\
\hline \multirow[t]{4}{*}{ A 1} & \multirow[t]{3}{*}{0.3073} & \multirow[t]{3}{*}{0.3097} & \multirow[t]{3}{*}{$0.78 \%$} & $\mathrm{~N} / \mathrm{C}$ & 28.36 & 32.05 & & 0.42 & $21.0 \%$ \\
\hline & & & & & 28.36 & 33.75 & \multirow[t]{2}{*}{$15.71 \%$} & 0.44 & $22.0 \%$ \\
\hline & & & & & 28.36 & 32.65 & & 0.44 & $22.0 \%$ \\
\hline & \multicolumn{9}{|c|}{500 HRS IN R-134a/ICI Emkarox VG32 -> 24 HRS @ 302 ${ }^{\circ} \mathrm{F}$} \\
\hline \multirow[t]{3}{*}{ A2 } & \multirow[t]{3}{*}{0.3308} & \multirow[t]{3}{*}{0.3271} & \multirow[t]{3}{*}{$.1 .119 \%$} & $\mathrm{~N} / \mathrm{C}$ & 28.36 & 35.20 & & 0.52 & $26.0 \%$ \\
\hline & & & & & 28.36 & 32.00 & $12.60 \%$ & 0.47 & $23.5 \%$ \\
\hline & & & & & 28.36 & 28.60 & & 0.40 & $20.0 \%$ \\
\hline
\end{tabular}


LEAD WIRE INSULATION-HFC-134a/ICI Emkarox VG32@260 $\mathrm{F}\left(127^{\circ} \mathrm{C}\right)$

\begin{tabular}{|c|c|c|c|c|c|c|c|}
\hline & \multicolumn{5}{|c|}{500 HRS IN HFC-134a/ICI Emkarox VG32@260 F } & \multirow[b]{2}{*}{ EXP DIE } & \\
\hline $\mathbb{D}$ & WT & EXPWT & $\begin{array}{c}\text { WT \% } \\
\text { CHANGE }\end{array}$ & EXP VIS & $\begin{array}{c}\text { BASE DIE } \\
\text { (AVE) }\end{array}$ & & $\begin{array}{c}\text { DIE\% } \\
\text { CHANGE }\end{array}$ \\
\hline DMD & 3.9868 & 4.1276 & $3.53 \%$ & $\mathrm{~N} / \mathrm{C}$ & 9.61 & 7.54 & \\
\hline A 1 & & & & & 9.61 & 7.84 & $-14.01 \%$ \\
\hline & & & & & 9.61 & 9.41 & \\
\hline DTMD & 4.2939 & 4.4234 & $3.02 \%$ & $N / C$ & 9.95 & 9.55 & \\
\hline B1 & & & & & 9.95 & 13.97 & $23.89 \%$ \\
\hline & & & & & 9.95 & 13.46 & \\
\hline & HFC.134a/IC & Emkarox VG & i32@260 & ${ }^{\circ} \mathrm{F}->24 \mathrm{H}$ & RS@ @302 & & \\
\hline DMD & 4.0105 & 4.0530 & $1.06 \%$ & $\mathrm{~N} / \mathrm{C}$ & 9.61 & 9.34 & \\
\hline A2 & & & & & 9.61 & 9.52 & $1.46 \%$ \\
\hline & & & & & 9.61 & 10.39 & \\
\hline DTMD & 4.2991 & 4.3301 & $0.72 \%$ & $\mathrm{~N} / \mathrm{C}$ & 9.95 & 9.06 & \\
\hline$B 2$ & & & & & 9.95 & 11.20 & $5.46 \%$ \\
\hline & & & & & 9.95 & 11.22 & \\
\hline
\end{tabular}




\section{Appendix $\mathbf{N}$}

Experimental Data for HFC-32/ICI Emkarox VG32 Exposure at $126^{\circ} \mathrm{C}\left(260^{\circ} \mathrm{F}\right)$ 


\begin{tabular}{|c|c|c|c|c|c|c|c|c|c|c|}
\hline & 500 hours in & HFC-32/lC & l Emkaro) & $x$ VG32 & oil at $260^{\circ}$ & $\left.27^{\circ} \mathrm{C}\right)$ & & & & \\
\hline ID & WT & EXPWT & $\begin{array}{c}\text { WT \% } \\
\text { CHANGE }\end{array}$ & EXP VIS & $\begin{array}{c}\text { BASE BRN } \\
\text { OUT } \\
\text { (AVE) }\end{array}$ & $\begin{array}{c}\text { EXP BRN } \\
\text { OUT }\end{array}$ & $\begin{array}{c}\text { BRN OUT } \\
\% \\
\text { CHANGE }\end{array}$ & $\begin{array}{c}\text { BASE DIE } \\
\text { (AVE) }\end{array}$ & EXPDIE & $\begin{array}{c}\text { DIE \% } \\
\text { CHANGE }\end{array}$ \\
\hline$A 1$ & 23.2679 & 23.2837 & $0.068 \%$ & $\mathrm{~N} / \mathrm{C}$ & 576 & 387 & & 15.80 & 15.20 & \\
\hline & & & & & 576 & 411 & $-30.2 \%$ & 15.80 & 16.69 & $-9.3 \%$ \\
\hline & & & & & 576 & 409 & & 15.80 & 11.08 & \\
\hline$\overline{B 1}$ & 24.0092 & 24.0629 & $0.224 \%$ & $\mathrm{~N} / \mathrm{C}$ & 736 & 731 & & 11.62 & 15.60 & \\
\hline & & & & & 736 & 626 & $-5.4 \%$ & 11.62 & 15.59 & $27.1 \%$ \\
\hline & & & & & 736 & 731 & & 11.62 & 13.10 & \\
\hline $\mathrm{C}_{1}$ & 23.4613 & 23.4800 & $0.080 \%$ & $\mathrm{~N} / \mathrm{C}$ & 579 & 411 & & 16.58 & 9.71 & \\
\hline & & & & & 579 & 568 & $-20.8 \%$ & 16.58 & 14.19 & $-16.6 \%$ \\
\hline & & & & & 579 & 397 & & 16.58 & 17.57 & \\
\hline & 500 hours i & $\mathrm{HFC}-32 / 1$ & Cl Emkaro & $x$ VG32 & oil at $260^{\circ} \mathrm{F}$ & $=\left(127^{\circ} \mathrm{C}\right) \mathrm{p}$ & olus 24 ho & urs at 302 & $\mathrm{~F}\left(150^{\circ} \mathrm{C}\right)$ & \\
\hline$A 2$ & 26.1094 & 26.1107 & $0.005 \%$ & $\mathrm{~N} / \mathrm{C}$ & \begin{tabular}{|l|}
576 \\
\end{tabular} & 532 & & 15.80 & 12.94 & \\
\hline & & & & & 576 & 533 & $-8.3 \%$ & 15.80 & 13.26 & $-14.6 \%$ \\
\hline & & & & & 576 & 519 & & 15.80 & 14.26 & \\
\hline B2 & 24.1791 & 24.1929 & $0.057 \%$ & $\mathrm{~N} / \mathrm{C}$ & 736 & 730 & & 11.62 & 11.09 & \\
\hline & & & & & 736 & 586 & $-7.4 \%$ & 11.62 & 12.78 & $3.1 \%$ \\
\hline & & & & & 736 & 729 & & 11.62 & 12.06 & \\
\hline$\overline{\mathrm{C} 2}$ & 23.0145 & 23.0150 & $0.002 \%$ & $\mathrm{~N} / \mathrm{C}$ & 579 & 505 & & 16.58 & 11.71 & \\
\hline & & & & & 579 & 560 & $-5.6 \%$ & 16.58 & 15.69 & $-13.8 \%$ \\
\hline & & & & & 579 & 574 & & 16.58 & 15.49 & \\
\hline
\end{tabular}




\begin{tabular}{|c|c|c|c|c|c|c|c|c|c|c|c|}
\hline & \multirow{2}{*}{ VARN } & \multicolumn{10}{|c|}{500 hours in HFC-32/ICl Emakrox Vg32 oil @ $260^{\circ} \mathrm{F}\left(127^{\circ} \mathrm{C}\right)$} \\
\hline & & $\begin{array}{l}\text { TWISTED } \\
\text { PAIR WT }\end{array}$ & $\begin{array}{l}\text { Exp Pair } \\
\text { weight }\end{array}$ & $\begin{array}{c}\text { WT \% } \\
\text { CHANGE }\end{array}$ & \begin{tabular}{|l} 
EXP \\
VIS
\end{tabular} & BASE DIE & EXP DIE & $\begin{array}{c}\text { DIE \% } \\
\text { CHANGE }\end{array}$ & $\begin{array}{l}\text { BASE } \\
\text { BUPN } \\
\text { OUT }\end{array}$ & $\begin{array}{c}\text { EXP } \\
\text { BURN } \\
\text { OUT }\end{array}$ & $\begin{array}{c}\text { BRNOUT } \\
\% \\
\text { CHANGE }\end{array}$ \\
\hline \multirow{3}{*}{$\begin{array}{l}P \\
O \\
L\end{array}$} & \multirow{3}{*}{$\begin{array}{l}\text { U-475 } \\
\text { A } 1\end{array}$} & \multirow[t]{3}{*}{22.2075} & \multirow[t]{3}{*}{22.2262} & \multirow[t]{3}{*}{$0.084 \%$} & $\mathrm{~N} / \mathrm{C}$ & 16.24 & 18.60 & & 329 & 311 & \\
\hline & & & & & & 16.24 & 16.13 & $4.15 \%$ & 342 & 369 & $0.19 \%$ \\
\hline & & & & & & 16.24 & 16.01 & & 359 & 348 & \\
\hline \multirow{3}{*}{$\begin{array}{l}Y \\
E \\
S\end{array}$} & \multirow{3}{*}{$\begin{array}{l}Y-390 \\
B 1\end{array}$} & \multirow[t]{3}{*}{23.8225} & \multirow[t]{3}{*}{23.8834} & \multirow[t]{3}{*}{$0.256 \%$} & $\mathrm{~N} / \mathrm{C}$ & 18.77 & 19.00 & & 418 & 473 & \\
\hline & & & & & & 18.77 & 17.00 & $-1.92 \%$ & 449 & 430 & $-2.90 \%$ \\
\hline & & & & & & 18.77 & 19.23 & & 488 & 405 & \\
\hline \multirow{4}{*}{$\begin{array}{l}T \\
E \\
R\end{array}$} & ER-610 & 22.5570 & 22.5790 & $0.098 \%$ & $\mathrm{~N} / \mathrm{C}$ & 15.57 & 19.79 & & 328 & 346 & \\
\hline & C1 & & & & & 15.57 & 11.66 & $-2.06 \%$ & 348 & 361 & $2.39 \%$ \\
\hline & & & & & & 15.57 & 14.30 & & 395 & 362 & \\
\hline & $Y-833$ & 23.5932 & 23.5815 & $.0 .050 \%$ & $\mathrm{~N} / \mathrm{C}$ & 12.04 & 16.84 & & 461 & 498 & \\
\hline$P$ & D1 & & & & & 12.04 & 12.87 & $25.69 \%$ & 468 & 442 & $0.57 \%$ \\
\hline O & & & & & & 12.04 & 15.69 & & 437 & 472 & \\
\hline$L$ & 923 & 24.0456 & 24.1240 & $0.326 \%$ & $\mathrm{~N} / \mathrm{C}$ & 16.76 & 19.99 & & 564 & 4454 & \\
\hline$Y$ & E1 & & & & & 16.76 & 19.97 & $5.31 \%$ & 578 & 421 & $-23.82 \%$ \\
\hline A & & & & & & 16.76 & 12.99 & & 561 & 446 & \\
\hline$M$ & 150.800 & 23.7970 & 23.8377 & $0.171 \%$ & $\mathrm{~N} / \mathrm{C}$ & 19.08 & 18.89 & & 530 & 560 & \\
\hline 1 & $F 1$ & & & & & 19.08 & 19.81 & $-2.25 \%$ & 553 & 376 & $-8.74 \%$ \\
\hline$D$ & & & & & & 19.08 & 17.25 & & 576 & 578 & \\
\hline$E$ & & 500 hours & in HFC-3 & ?/Cl Emk & $\operatorname{arox}$ & G32 oil @ & $260^{\circ} \mathrm{F}(1$ & $27^{\circ} \mathrm{C}$ ) plus & 24 houl & @ 30 & $\mathrm{D}^{\circ} \mathrm{F}\left(150^{\circ} \mathrm{C}\right.$ \\
\hline & U-475 & 22.4562 & 22.4515 & $-0.021 \%$ & $\mathrm{~N} / \mathrm{C}$ & $\begin{array}{l}16.24 \\
\end{array}$ & 15.97 & & 316 & 345 & \\
\hline 1 & A2 & & & & & 16.24 & 16.09 & $-10.71 \%$ & 383 & 344 & $-16.88 \%$ \\
\hline M & & & & & & 16.24 & 11.44 & & 348 & 266 & \\
\hline 1 & $Y-390$ & 24.8404 & 24.8440 & $0.014 \%$ & $\mathrm{~N} / \mathrm{C}$ & 18.77 & 16.84 & & 388 & 543 & \\
\hline$D$ & $\mathrm{~B} 2$ & & & & & 18.77 & 16.08 & $-6.04 \%$ & 577 & 534 & $-9.76 \%$ \\
\hline$E$ & & & & & & 18.77 & 19.99 & & 475 & 485 & \\
\hline & ER-610 & 22.9406 & 22.9398 & $-0.003 \%$ & $\mathrm{~N} / \mathrm{C}$ & 15.57 & 10.44 & & 320 & 380 & \\
\hline & C2 & & & & & 15.57 & 14.00 & $-26.31 \%$ & 373 & 416 & $4.11 \%$ \\
\hline & & & & & & 15.57 & 9.98 & & 352 & 369 & \\
\hline & $Y-833$ & 24.2168 & 24.1752 & $-0.172 \%$ & $\mathrm{~N} / \mathrm{C}$ & 12.04 & 11.65 & & 565 & 554 & \\
\hline & D2 & & & & & 12.04 & 11.63 & $-5.98 \%$ & 433 & 565 & $32.10 \%$ \\
\hline & & & & & & 12.04 & 10.68 & & 563 & 597 & \\
\hline & 923 & 23.8721 & 23.8785 & $0.027 \%$ & $\mathrm{~N} / \mathrm{C}$ & 16.76 & 16.62 & & 538 & 545 & \\
\hline & E2 & & & & & 16.76 & 16.72 & $5.67 \%$ & 517 & 466 & $-8.58 \%$ \\
\hline & & & & & & 16.76 & 19.79 & & 570 & 407 & \\
\hline & $150-800$ & 23.2770 & 23.2963 & $0.083 \%$ & $\mathrm{~N} / \mathrm{C}$ & 19.08 & 16.30 & & 455 & 581 & \\
\hline & $\mathrm{F} 2$ & & & & & 19.08 & 15.61 & $-11.50 \%$ & 615 & 550 & $-9.70 \%$ \\
\hline & & & & & & 19.08 & 18.75 & & 579 & 535 & \\
\hline
\end{tabular}


TWISTED PAIRS/WIRE BWITH VARNISH -HFC-32/ICI Emkarox VG32 oil @260:F(127C)

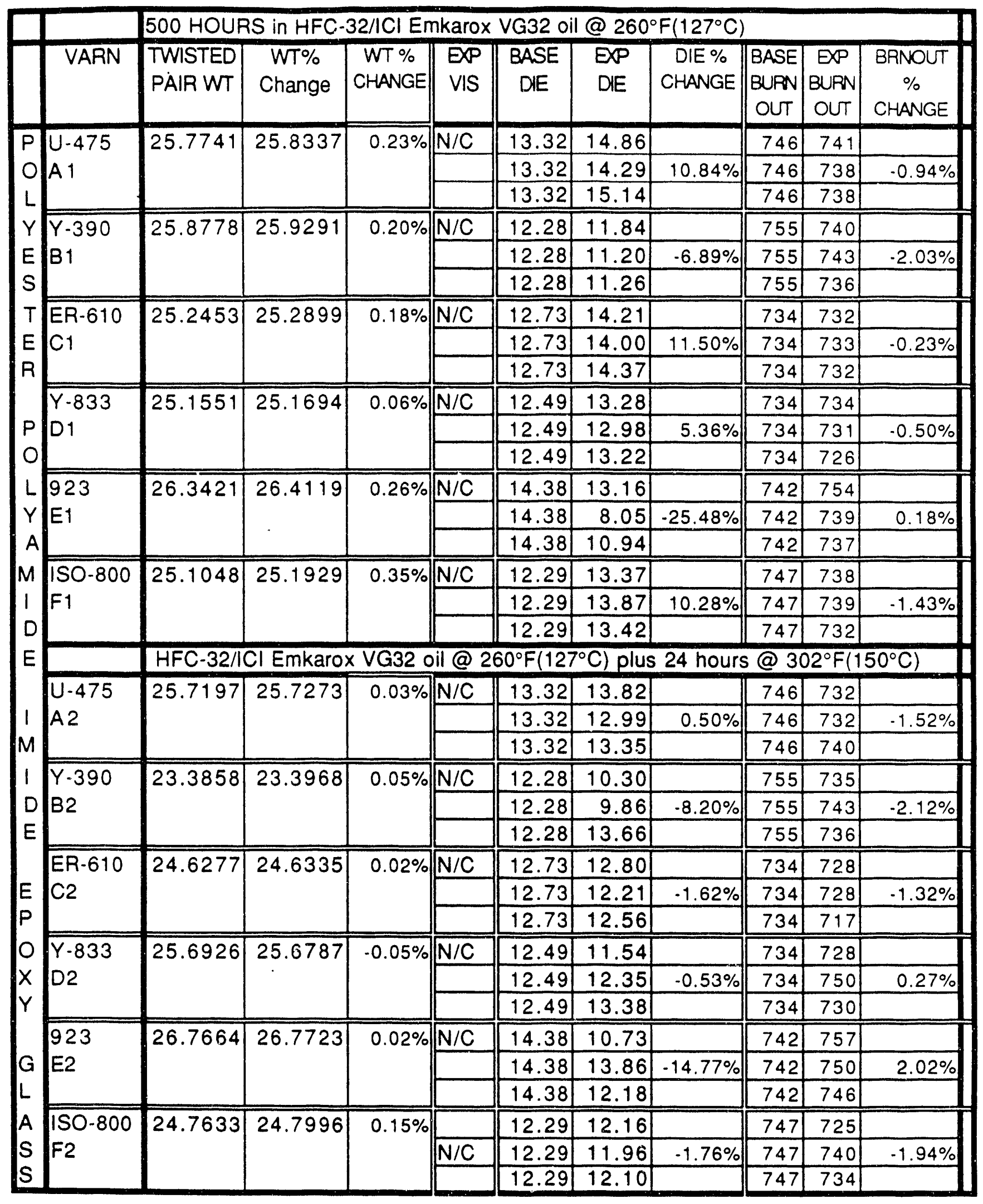


TWISTED PAIRS WIRE C,WITH VARNISH.HFC-32/ICI Emkarox VG $32 @ 260^{\circ} \mathrm{F}\left(127^{\circ} \mathrm{C}\right)$

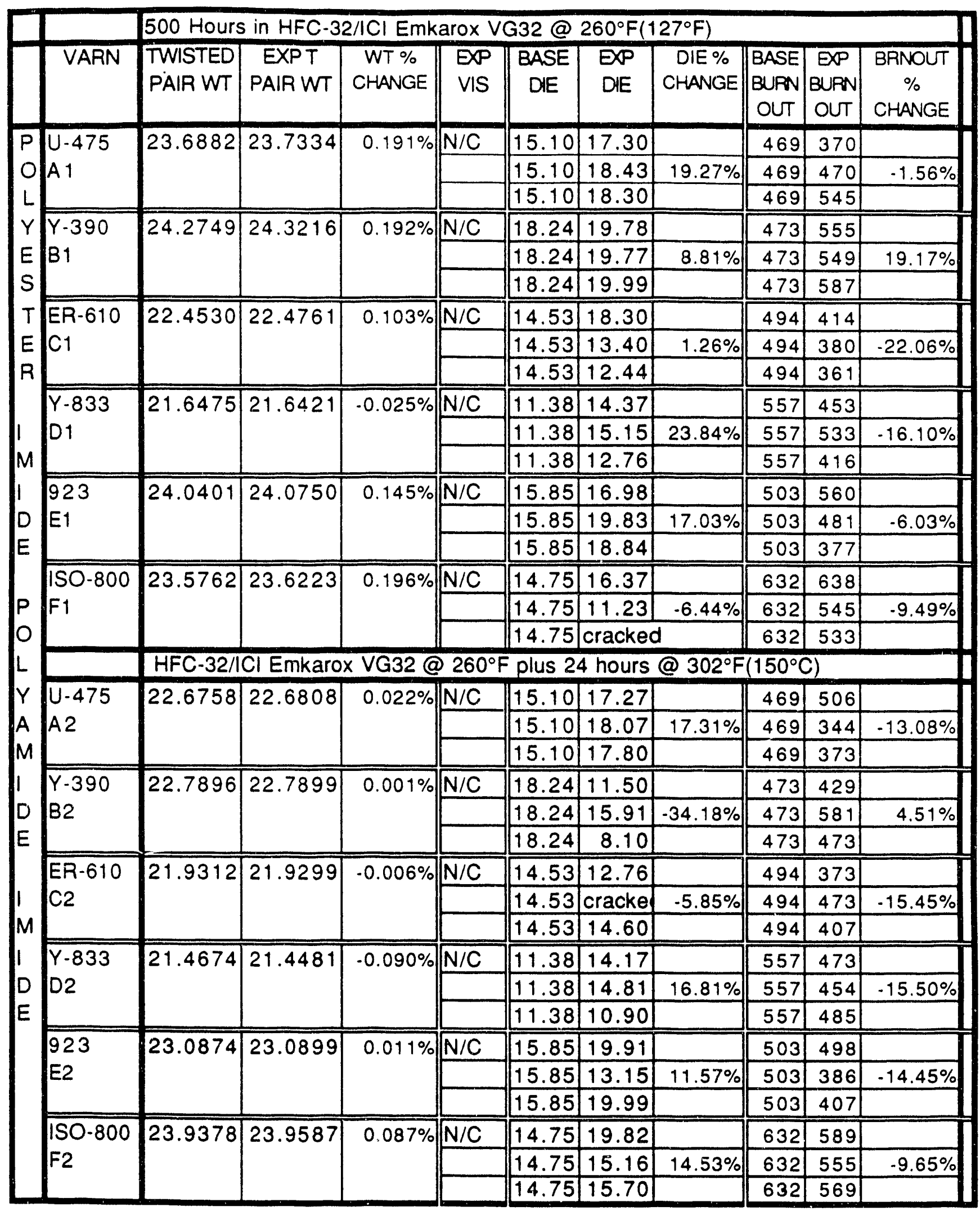




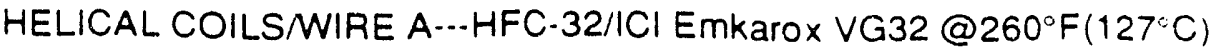

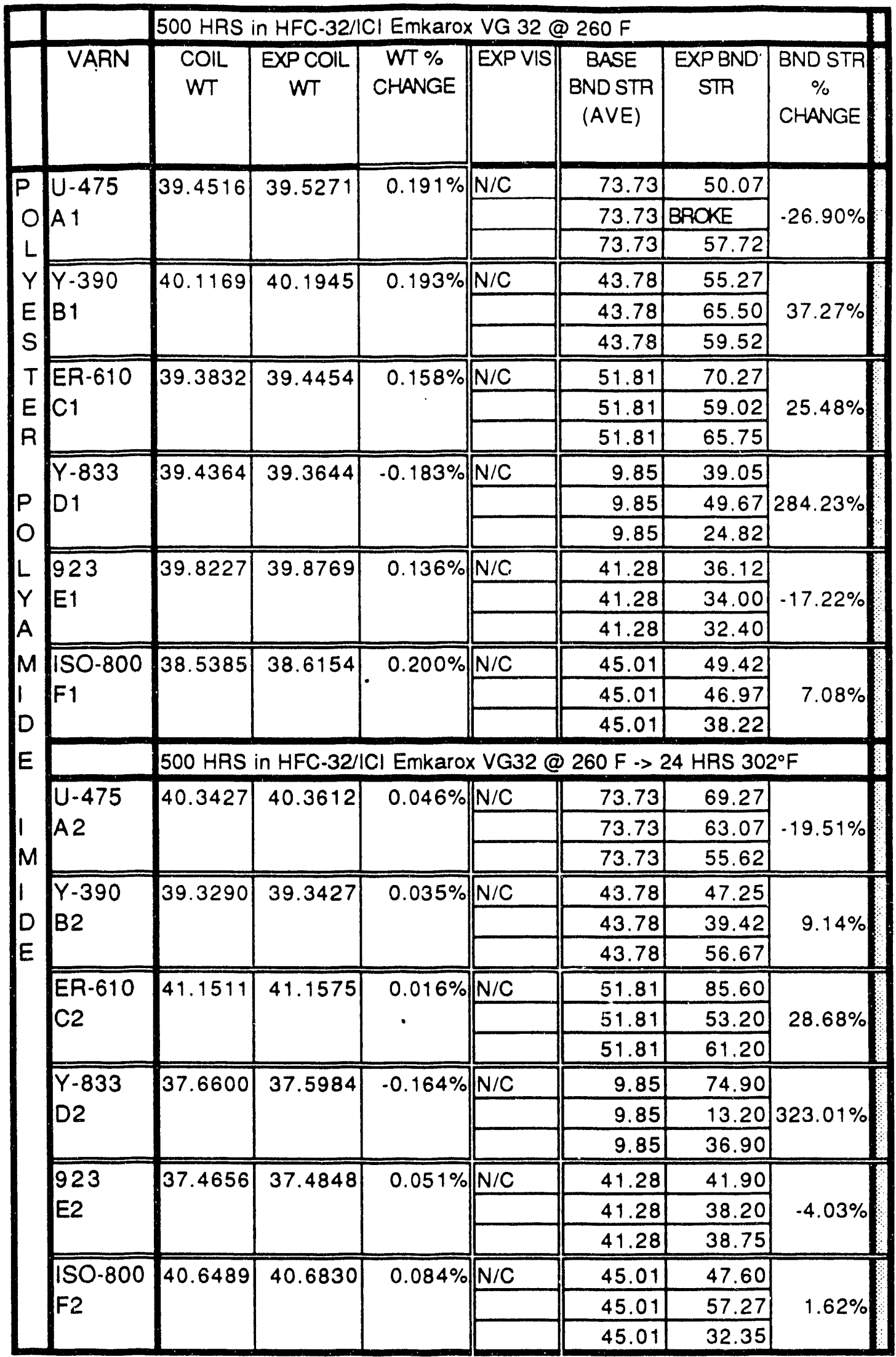


HELICAL COILSNIRE B-HFC-32/ICI Emkarox VG32 Oil @ 260 $0^{\circ} \mathrm{F}\left(127^{\circ} \mathrm{C}\right)$

\begin{tabular}{|c|c|c|c|c|c|c|c|c|}
\hline & & 500 & & Emke & 32 & $50 \mathrm{~F}$ & & \\
\hline & VARN & $\begin{array}{l}\text { COIL } \\
W T\end{array}$ & $\begin{array}{c}\text { EXP COIL } \\
W T\end{array}$ & $\begin{array}{c}\text { WT \% } \\
\text { CHANGE }\end{array}$ & EXPVIS & $\begin{array}{l}\text { BASE } \\
\text { BND STR } \\
\text { (AVE) }\end{array}$ & $\begin{array}{c}\text { EXPBND } \\
\text { STR }\end{array}$ & $\begin{array}{c}\text { BND STR } \\
\% \\
\text { CHANGE }\end{array}$ \\
\hline$P$ & U-475 & 37.4549 & 37.5233 & $0.183 \%$ & $\mathrm{~N} / \mathrm{C}$ & 40.14 & 49.87 & \\
\hline 0 & A 1 & & & & & 40.14 & 46.72 & $22.56 \%$ \\
\hline$L$ & & & & & & 40.14 & 51.00 & \\
\hline Y & $Y-390$ & 36.7117 & 36.8251 & $0.309 \%$ & $\mathrm{~N} / \mathrm{C}$ & 36.12 & 54.57 & \\
\hline$E$ & B1 & & & & & 36.12 & 52.60 & $48.64 \%$ \\
\hline S & & & & & & 36.12 & 53.90 & \\
\hline$T$ & ER-610 & 37.8198 & 37.9058 & $0.227 \%$ & $N / C$ & 35.96 & 35.25 & \\
\hline$E$ & C1 & & & & & 35.96 & 33.02 & $-4.16 \%$ \\
\hline $\mathrm{R}$ & & & & & & 35.96 & 35.12 & \\
\hline & $Y-833$ & 35.0460 & 34.9982 & $-0.136 \%$ & $N / C$ & 33.14 & 36.02 & \\
\hline$P$ & D1 & & & & & 33.14 & 28.82 & $-10.47 \%$ \\
\hline 0 & & & & & & 33.14 & 24.17 & \\
\hline$L$ & 923 & 36.3769 & 36.4733 & $0.265 \%$ & $\mathrm{~N} / \mathrm{C}$ & 40.52 & 46.17 & \\
\hline$Y$ & E1 & & & & & 40.52 & 45.12 & $8.87 \%$ \\
\hline A & & & & & & 40.52 & 41.05 & \\
\hline M & 1SO-800 & 36.9607 & 37.0612 & $0.272 \%$ & $N / C$ & 20.20 & 18.87 & \\
\hline 1 & $F 1$ & & & & & 20.20 & 22.50 & $10.05 \%$ \\
\hline D & & & & & & 20.20 & 25.32 & \\
\hline$E$ & & $500 \mathrm{HRS}$ & in HFC.32 & ICl Emkaro & $x$ VG32 C & $260 \mathrm{~F} \rightarrow$ & 24 HRS 30 & \\
\hline & $U \cdot 475$ & 37.9453 & 37.9538 & $0.022 \%$ & $\mathrm{~N} / \mathrm{C}$ & 40.14 & 34.65 & \\
\hline 1 & A2 & & & & & 40.14 & 29.82 & $-12.50 \%$ \\
\hline$M$ & & & & & & 40.14 & 40.90 & \\
\hline 1 & $Y-390$ & 37.3597 & 37.3764 & $0.045 \%$ & $N / C$ & 36.12 & 41.20 & \\
\hline D & B2 & & & & & 36.12 & 41.42 & $13.58 \%$ \\
\hline $\mathrm{E}$ & & & & & & 36.12 & 40.45 & \\
\hline & ER-610 & 37.0202 & 37.0334 & $0.036 \%$ & $\mathrm{~N} / \mathrm{C}$ & 35.96 & 32.27 & \\
\hline$E$ & $\mathrm{C} 2$ & & & & & 35.96 & 28.62 & $-16.35 \%$ \\
\hline$P$ & & & & & & 35.96 & 29.35 & \\
\hline 0 & $Y-833$ & 34.9830 & 34.8787 & $-0.298 \%$ & $\mathrm{~N} / \mathrm{C}$ & 33.14 & 27.90 & \\
\hline $\begin{array}{l}x \\
y\end{array}$ & D2 & & & & & 33.14 & 43.17 & $16.14 \%$ \\
\hline Y & & & & & & 33.14 & 44.40 & \\
\hline & 923 & 37.5785 & 37.5923 & $0.037 \%$ & $\mathrm{~N} / \mathrm{C}$ & 40.52 & 42.75 & \\
\hline G & E2 & & & & & 40.52 & 43.20 & $3.26 \%$ \\
\hline$L$ & & & & & & 40.52 & 39.57 & \\
\hline A & $1 \mathrm{SO}-800$ & 37.1318 & 37.1656 & $0.091 \%$ & $\mathrm{~N} / \mathrm{C}$ & 20.20 & 18.62 & \\
\hline$S$ & $\mathrm{~F} 2$ & & & & & 20.20 & 21.60 & $2.59 \%$ \\
\hline S & & & & & & 20.20 & 21.95 & \\
\hline
\end{tabular}


HELICAL COILSMIRE C-HFC.32/ICI Emkarox VG32@ $260^{\circ} \mathrm{F}\left(127^{\circ} \mathrm{C}\right)$

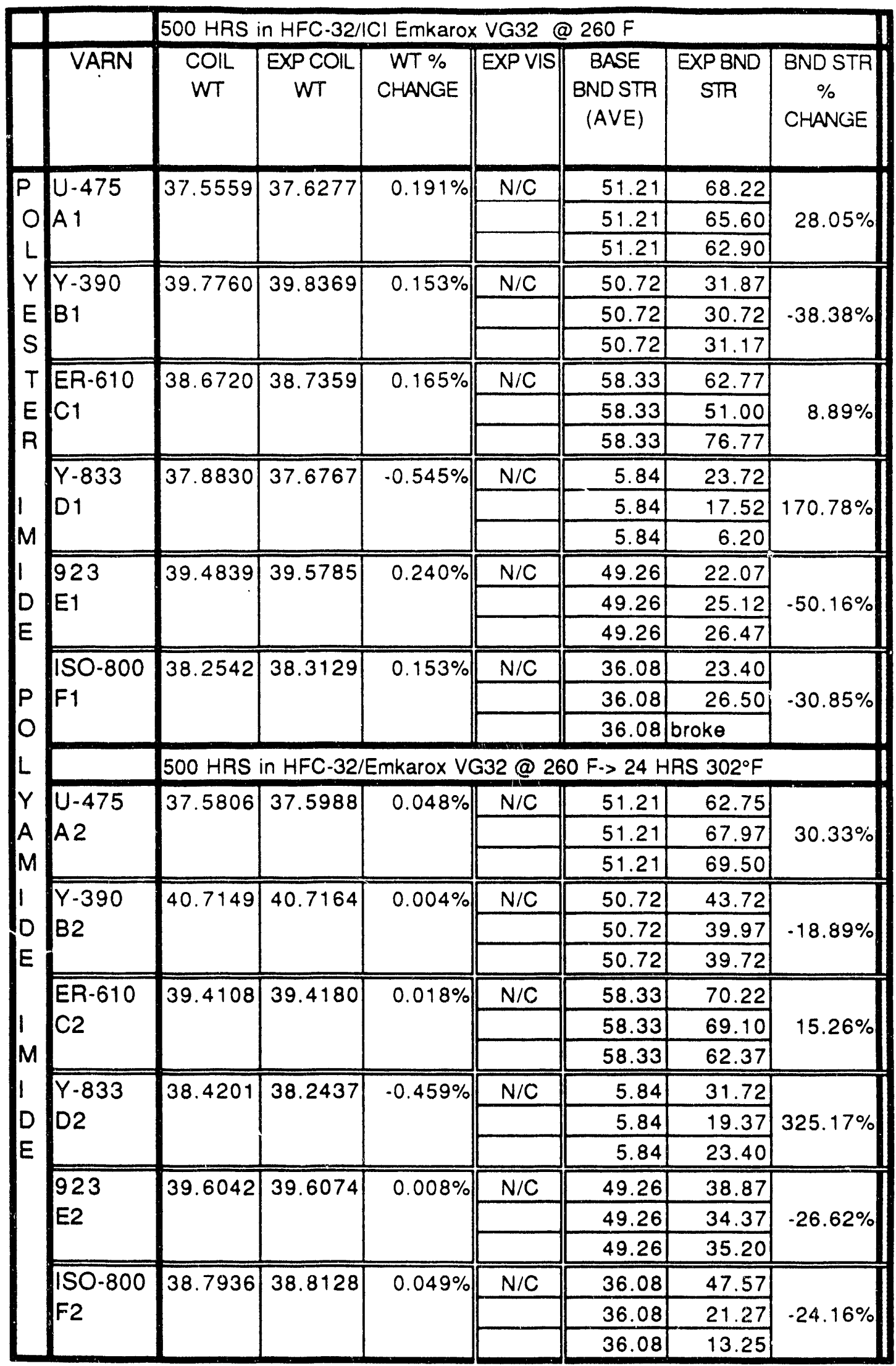


SINGLE MAG WIRE WITHOUT VARNISH-HFC-32/ICI Emkarox VG32@ 260 $\left.\mathrm{F}\left(127^{\circ} \mathrm{C}\right)\right)$

\begin{tabular}{|c|c|c|c|c|c|}
\hline \multicolumn{6}{|c|}{$500 \mathrm{HRS}$ in HFC-32/ICl Emkarox VG32 oil @ 260 $\mathrm{F}\left(127^{\circ} \mathrm{C}\right)$} \\
\hline ID & WT & EXPWT & $\begin{array}{c}\text { WT \% } \\
\text { CHANGE }\end{array}$ & EXP VS & EXPFLEX \\
\hline \multirow[t]{2}{*}{ A1 } & \multirow[t]{2}{*}{3.3121} & \multirow[t]{2}{*}{3.314} & \multirow[t]{2}{*}{$0.057 \%$} & $\mathrm{~N} / \mathrm{C}$ & \\
\hline & & & & & NO \\
\hline \multirow[t]{2}{*}{$B 1$} & \multirow[t]{2}{*}{3.4008} & \multirow[t]{2}{*}{3.4073} & \multirow{2}{*}{$0.191 \%$} & $\mathrm{~N} / \mathrm{C}$ & \\
\hline & & & & & NO \\
\hline \multirow[t]{2}{*}{ C1 } & \multirow[t]{2}{*}{3.5312} & \multirow{2}{*}{3.5339} & \multirow{2}{*}{$0.076 \%$} & $\mathrm{~N} / \mathrm{C}$ & \\
\hline & & & & & NO \\
\hline \multicolumn{6}{|c|}{$500 \mathrm{HRS}$ in HFC-32/Emkarox oil-> $302^{\circ} \mathrm{F}$ for $24 \mathrm{HRS}$} \\
\hline \multirow[t]{2}{*}{$\overline{A 2}$} & \multirow[t]{2}{*}{3.6291} & \multirow[t]{2}{*}{3.63} & \multirow[t]{2}{*}{$0.025 \%$} & $\mathrm{~N} / \mathrm{C}$ & \\
\hline & & & & & YES \\
\hline \multirow[t]{2}{*}{$\overline{\mathrm{B2}}$} & \multirow[t]{2}{*}{3.335} & \multirow[t]{2}{*}{3.3377} & \multirow[t]{2}{*}{$0.081 \%$} & $\mathrm{~N} / \mathrm{C}$ & \\
\hline & & & & & YES \\
\hline \multirow[t]{3}{*}{$\mathrm{C2}$} & \multirow[t]{3}{*}{3.4362} & \multirow[t]{3}{*}{3.4374} & \multirow[t]{3}{*}{$0.035 \%$} & $\mathrm{~N} / \mathrm{C}$ & \\
\hline & & & & & YES \\
\hline & & & & & \\
\hline
\end{tabular}




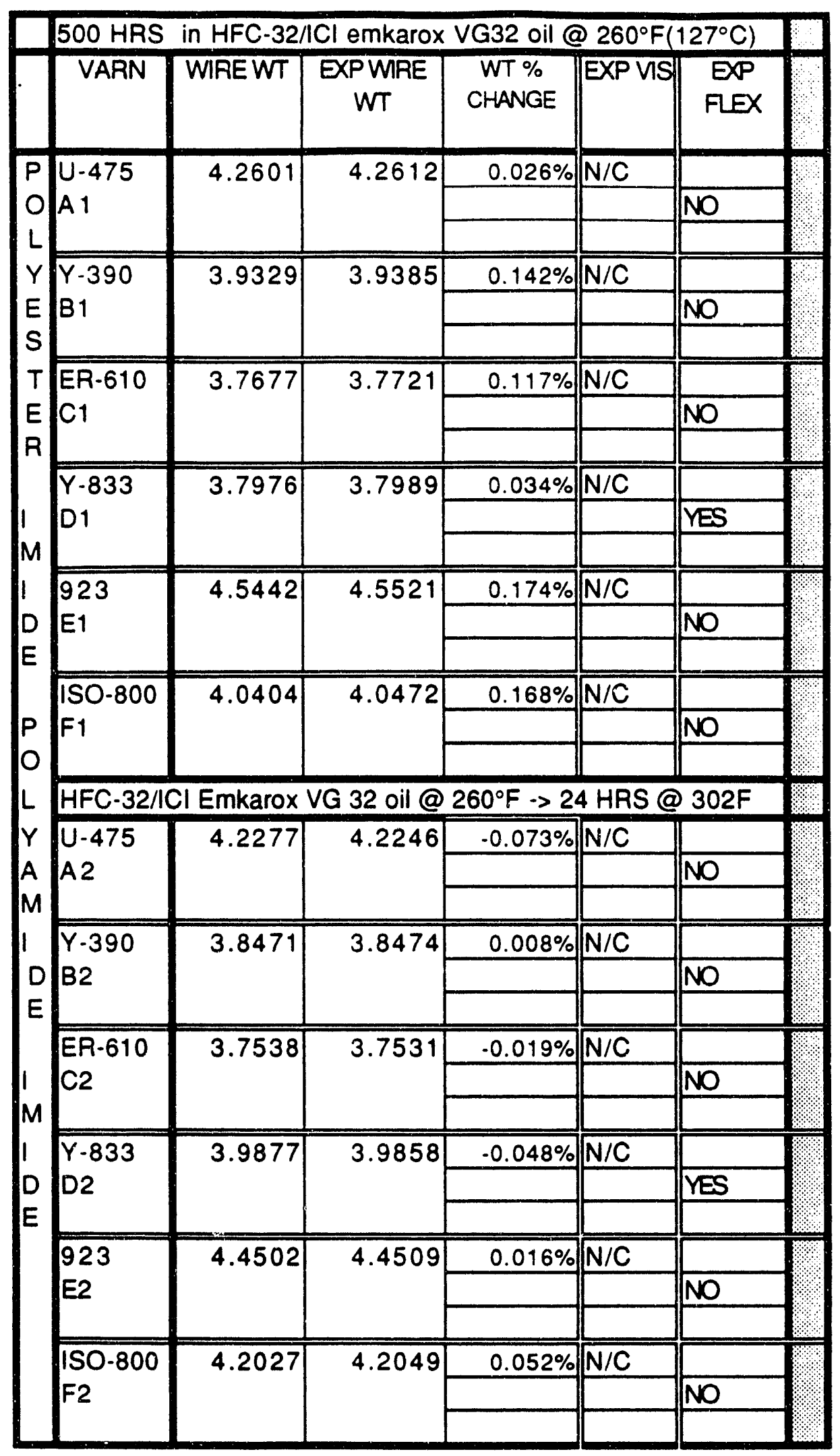




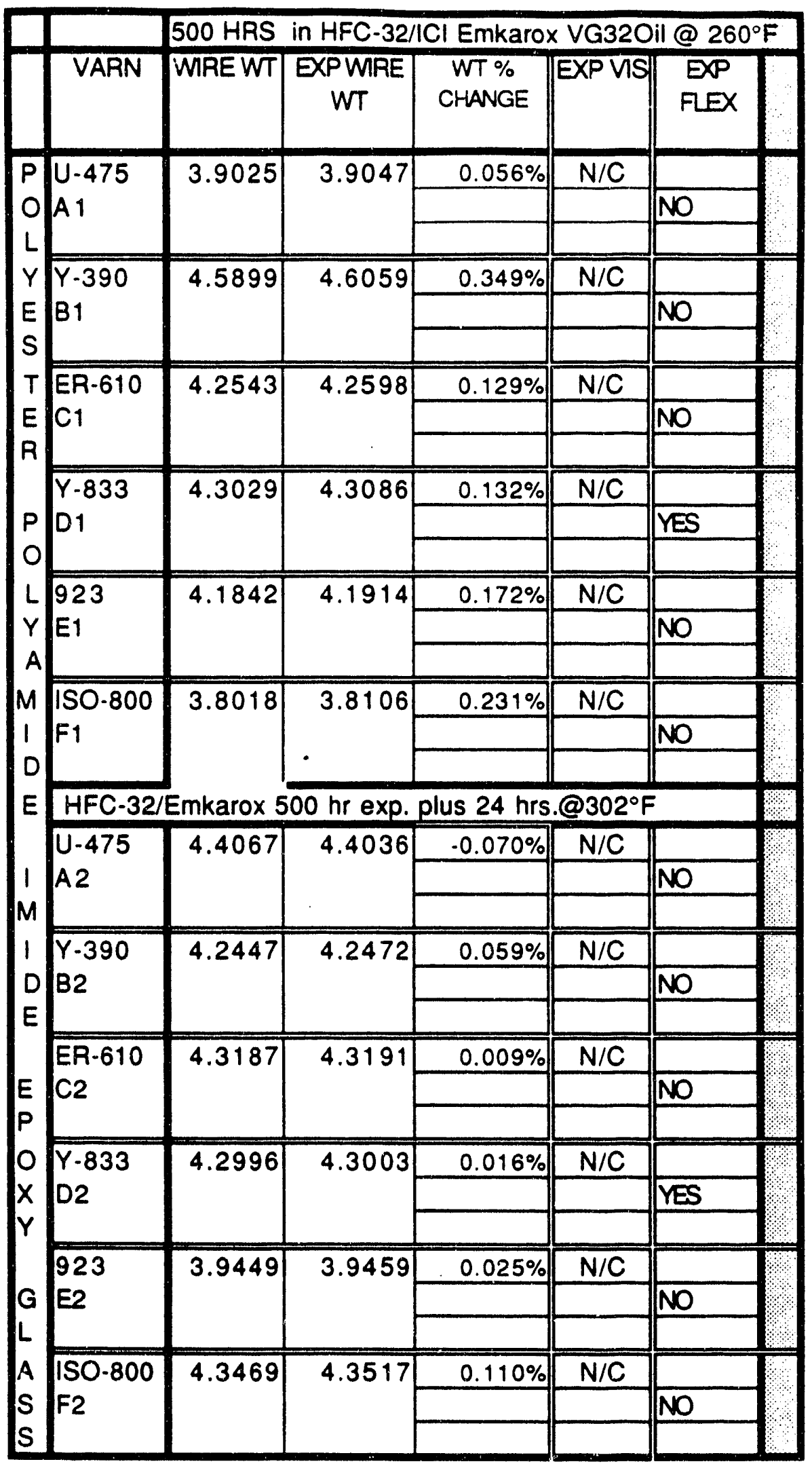




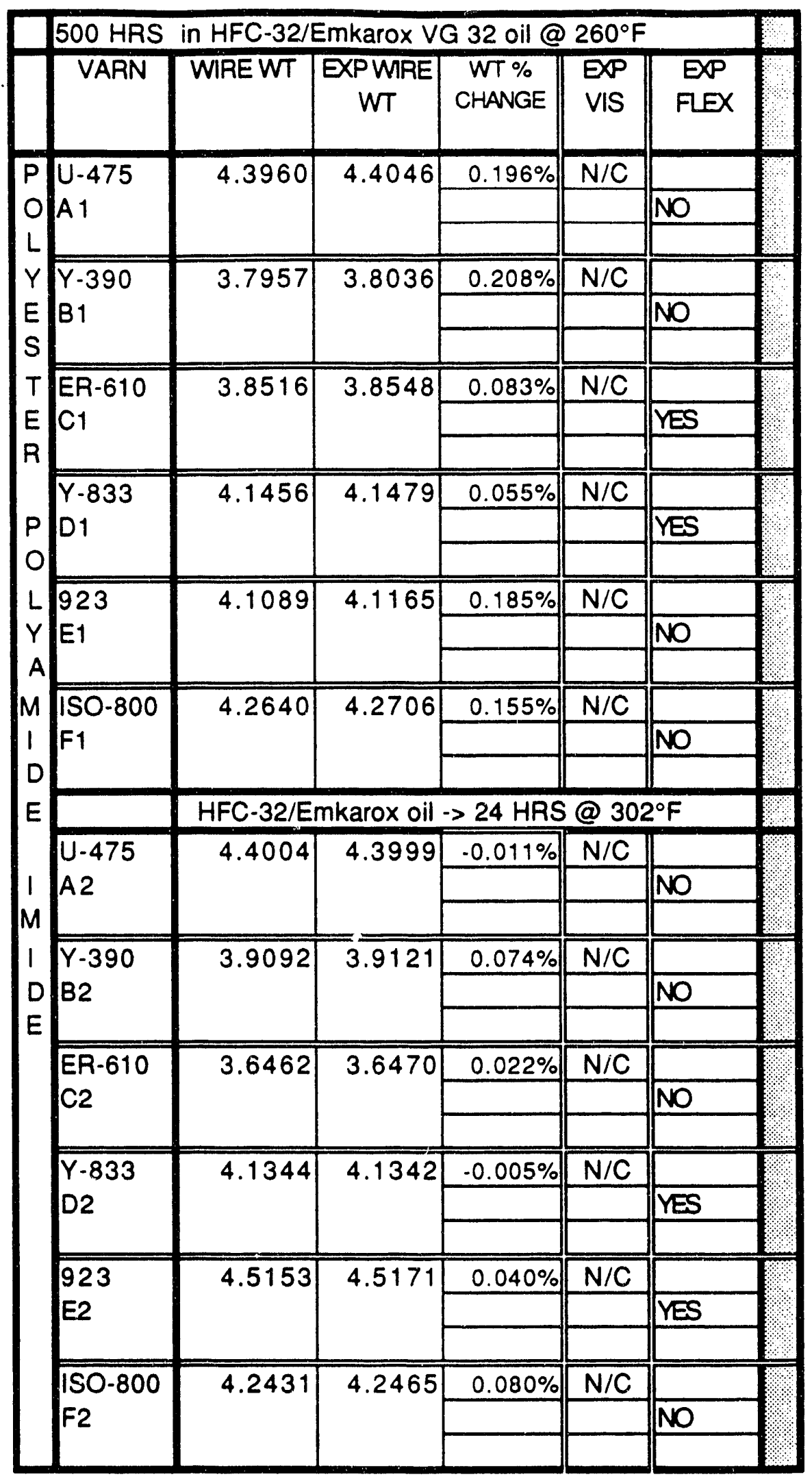


Varnish Disks-...-HFC-32/ICl Emkarox VG 32 Oil @ 260 $\mathrm{F}\left(127^{\circ} \mathrm{F}\right)$

\begin{tabular}{|c|c|c|c|c|c|}
\hline \multicolumn{6}{|c|}{500 HRS IN R-32/ICI Emkarox VG32 @ 260 F } \\
\hline ID & WT & EXPWT & $\begin{array}{c}\text { WT } \% \\
\text { CHANGE }\end{array}$ & EXPVIS & EXPFLEX \\
\hline $\begin{array}{l}\text { U-475 } \\
\text { A } 1\end{array}$ & 2.9860 & 2.9802 & $-0.19 \%$ & $\mathrm{~N} / \mathrm{C}$ & $\mathrm{N} / \mathrm{C}$ \\
\hline$Y \cdot 390$ & 1.9188 & 1.9243 & $0.29 \%$ & $N / C$ & $\mathrm{~N} / \mathrm{C}$ \\
\hline $\begin{array}{l}\text { ER-610 } \\
\text { C1 }\end{array}$ & 2.6391 & 2.7146 & $2.86 \%$ & $\frac{\text { Slightly }}{\text { Darkened }}$ & $N / C$ \\
\hline & & & & & \\
\hline $\begin{array}{l}\text { Y-833 } \\
D 1\end{array}$ & 3.2833 & 3.3054 & $0.67 \%$ & $N / C$ & $\mathrm{~N} / \mathrm{C}$ \\
\hline 923 & 2.7264 & 2.6456 & $-2.96 \%$ & & lighteried \\
\hline E1 & & & & Warped & \\
\hline ISO-800 & 1.7445 & 1.7253 & $-1.10 \%$ & $\mathrm{~N} / \mathrm{C}$ & $\mathrm{N} / \mathrm{C}$ \\
\hline & & & & & \\
\hline & $\mathrm{R}-32 / 1 \mathrm{Cl} \mathrm{En}$ & rarox VG 32 & at $\cdots>24$ & hour at $3 c$ & $2^{\circ} \mathrm{F}$. \\
\hline$U-475$ & 3.0373 & 2.9512 & $-2.83 \%$ & Darkened & Cloudy \\
\hline A2 & & & & Slightly & appearance \\
\hline & & & & warped & on surface \\
\hline $\begin{array}{l}Y-390 \\
B 2\end{array}$ & 2.0302 & 1.9775 & $-2.60 \%$ & \begin{tabular}{|l} 
slightly \\
warped
\end{tabular} & $\mathrm{N} / \mathrm{C}$ \\
\hline ER-610 & 2.7027 & 2.7062 & $0.13 \%$ & slightly & $\mathrm{N} / \mathrm{C}$ \\
\hline & & & & darkened & \\
\hline Y-833 & 4.0676 & 3.9079 & $-3.93 \%$ & $\mathrm{~N} / \mathrm{C}$ & $\mathrm{N} / \mathrm{C}$ \\
\hline D2 & & & & & \\
\hline 923 & 2.0522 & 1.9633 & $-4.33 \%$ & Warped & \\
\hline E2 & & & & lightened & $\mathrm{N} / \mathrm{C}$ \\
\hline $150-800$ & 2.0326 & 1.9764 & $-2.76 \%$ & slightly & \\
\hline & & & & warped & $\mathrm{N} / \mathrm{C}$ \\
\hline & & & & & \\
\hline
\end{tabular}




\begin{tabular}{|c|c|c|c|c|c|c|c|c|c|}
\hline \multirow{2}{*}{ ID } & \multicolumn{9}{|c|}{500 HRS in HFC-32/ICl Emkarox VG32 Oil @ 260 F } \\
\hline & WT & EXPWT & $\begin{array}{c}\text { WT \% } \\
\text { CHANGE }\end{array}$ & $\begin{array}{l}\text { SAMPLE } \\
\text { WIDTH }\end{array}$ & $\begin{array}{l}\text { BREAK } \\
\text { LOAD }\end{array}$ & $\begin{array}{c}\text { SAMPLE } \\
\text { THICKNESS } \\
\text { Mils }\end{array}$ & $\begin{array}{c}\text { TENSILE } \\
\text { STR } \\
\text { BASE }\end{array}$ & $\begin{array}{l}\text { TENSILE } \\
\text { STREXP }\end{array}$ & $\begin{array}{c}\% \text { CHANGE } \\
\text { TENSILE }\end{array}$ \\
\hline \multirow{3}{*}{$\begin{array}{l}\text { NO/MY/NO } \\
\text { A1 }\end{array}$} & \multirow[t]{3}{*}{4.2830} & \multirow[t]{3}{*}{4.6089} & \multirow[t]{3}{*}{$7.61 \%$} & 0.387 & 125.90 & 21 & 17.4 & 15.49 & \\
\hline & & & & 0.446 & 150.10 & 21 & 17.4 & 16.03 & $-8.80 \%$ \\
\hline & & & & 0.375 & 126.70 & 21 & 17.4 & 16.09 & \\
\hline \multirow{3}{*}{$\begin{array}{l}\mathrm{DA} / \mathrm{MY} / \mathrm{DA} \\
\mathrm{B} 1\end{array}$} & \multirow{3}{*}{4.3871} & \multirow[t]{3}{*}{4.4495} & \multirow[t]{3}{*}{$1.42 \%$} & 0.370 & 100.80 & 21 & 13.7 & 12.97 & \\
\hline & & & & 0.464 & 130.50 & 21 & 13.7 & 13.39 & $.6 .81 \%$ \\
\hline & & & & 0.391 & 98.00 & 21 & 13.7 & 11.94 & \\
\hline \multirow{3}{*}{$\begin{array}{l}\text { MYLARMO } \\
C 1\end{array}$} & \multirow[t]{3}{*}{2.2271} & \multirow[t]{3}{*}{2.2505} & \multirow[t]{3}{*}{$1.05 \%$} & 0.420 & 76.75 & 10 & 21.7 & 18.27 & \\
\hline & & & & 0.415 & 70.75 & 10 & 21.7 & 17.05 & $-18.82 \%$ \\
\hline & & & & 0.410 & 71.85 & 10 & 21.7 & 17.52 & \\
\hline \multirow{3}{*}{$\begin{array}{l}\text { NO } 410 \\
\text { D1 }\end{array}$} & \multirow[t]{3}{*}{2.2068} & 2.5156 & $13.99 \%$ & 0.490 & 88.35 & 10 & 18.7 & 18.03 & \\
\hline & & & & 0.492 & 89.05 & 10 & 18.7 & 18.10 & $-5.56 \%$ \\
\hline & & & & 0.492 & 82.90 & 10 & 18.7 & 16.85 & \\
\hline NO MI 418 & 2.2645 & 2.7773 & $22.65 \%$ & 0.482 & 30.00 & 10 & 7.5 & 6.22 & \\
\hline E1 & & & & 0.509 & 31.75 & 10 & 7.5 & 6.24 & $.16 .11 \%$ \\
\hline & & & & 0.492 & 31.55 & 10 & 7.5 & 6.41 & \\
\hline MEL 228 & 2.3586 & 2.3969 & $1.62 \%$ & 0.469 & 79.70 & 10 & 21.7 & 16.99 & \\
\hline$F 1$ & & & & 0.423 & 75.30 & 10 & 21.7 & 17.80 & $-17.54 \%$ \\
\hline & & & & 0.494 & 93.30 & 10 & 21.7 & 18.89 & \\
\hline & $500 \mathrm{HRS}$ & 3 in HFC-? & 32//Cl Emka & arox VG3 & 20il@2 & $60 \mathrm{~F}->24 \mathrm{H}$ & RS@302 & ${ }^{\circ} \mathrm{F}$ & \\
\hline NO/MY/NO & 4.2187 & 4.3160 & $2.31 \%$ & 0.410 & 141.20 & 21 & 17.4 & 16.40 & \\
\hline$A 2$ & & & & 0.380 & 134.90 & 21 & 17.4 & 16.90 & $-3.47 \%$ \\
\hline & & & & 0.383 & 137.40 & 21 & 17.4 & 17.08 & \\
\hline$D A / M Y / D A$ & 4.6832 & 4.6681 & $-0.32 \%$ & 0.397 & 109.60 & 21 & 13.7 & 13.15 & \\
\hline $\mathrm{B2}$ & & & & 0.422 & 117.70 & 21 & 13.7 & 13.28 & $-4.49 \%$ \\
\hline & & & & 0.375 & 101.00 & 21 & 13.7 & 12.83 & \\
\hline MYLAR MO & 2.3691 & 2.3631 & $-0.25 \%$ & 0.444 & 70.50 & 10 & 21.7 & 15.88 & \\
\hline $\mathrm{C} 2$ & & & & 0.406 & 66.15 & 10 & 21.7 & 16.29 & $-26.22 \%$ \\
\hline & & & & 0.435 & 69.00 & 10 & 21.7 & 15.86 & \\
\hline NOMEX 410 & 2.3429 & 2.4302 & $3.73 \%$ & 0.480 & 92.00 & 10 & 18.7 & 19.17 & \\
\hline D2 & & & & 0.492 & 94.45 & 10 & 18.7 & 19.20 & $1.83 \%$ \\
\hline & & & & 0.484 & 90.80 & 10 & 18.7 & 18.76 & \\
\hline $\mathrm{NO} / \mathrm{MI} 418$ & 2.2381 & 2.2608 & $1.01 \%$ & 0.515 & 32.85 & 10 & 7.5 & 6.38 & \\
\hline E2 & & & & 0.500 & 31.20 & 10 & 7.5 & 6.24 & $-17.51 \%$ \\
\hline & & & & 0.515 & 30.60 & 10 & 7.5 & 5.94 & \\
\hline MEL 228 & 2.3657 & 2.3701 & $0.19 \%$ & 0.523 & 89.70 & 10 & 21.7 & 17.15 & \\
\hline F2 & & & & 0.501 & 84.85 & 10 & 21.7 & 16.94 & $-21.84 \%$ \\
\hline & & & & 0.420 & 70.55 & 10 & 21.7 & 16.80 & \\
\hline
\end{tabular}




\begin{tabular}{|c|c|c|c|c|c|c|c|c|}
\hline & $500 \mathrm{HRS}$ & in $\mathrm{HFC}-1$ & $2 / 1 \mathrm{ClEmk}$ & karox VG3 & 32 Oil @ & $260 \mathrm{~F}$ & & \\
\hline ID & $\begin{array}{c}\text { STRETCH } \\
\text { (inch) }\end{array}$ & $\begin{array}{c}\% \\
\text { ELONG }\end{array}$ & $\begin{array}{l}\text { BASE } \\
\text { ELONG } \\
\text { (AVE) }\end{array}$ & $\begin{array}{l}\text { ELONG \% } \\
\text { CHANGE }\end{array}$ & \begin{tabular}{|c} 
BASE \\
DIE \\
$(A V E)$
\end{tabular} & EXP DIE & $\begin{array}{c}\text { DIE \% } \\
\text { CHANGE }\end{array}$ & VISUAL EXP \\
\hline NO/MY/NO & 0.64 & $16.0 \%$ & $20.0 \%$ & & $>18.97$ & flash & & small bubbles \\
\hline & 0.69 & $17.3 \%$ & $20.0 \%$ & $-16.9 \%$ & $>18.97$ & flash & flash & developed \\
\hline & 0.68 & $17.0 \%$ & $20.0 \%$ & & $>18.97$ & flash & & pockets \\
\hline$D A / M Y / D A$ & 0.48 & $24.0 \%$ & $46.0 \%$ & & $>15.27$ & flash & & Slightly Warped \\
\hline B1 & 0.54 & $27.0 \%$ & $46.0 \%$ & $.47 .8 \%$ & $>15.27$ & flash & flash & \\
\hline & 0.42 & $21.0 \%$ & $46.0 \%$ & & $>15.27$ & flash & & \\
\hline MYARMO & 2.94 & $147.0 \%$ & $131.0 \%$ & & $>14.91$ & flash & & $N / C$ \\
\hline$c_{1}$ & 2.62 & $131.0 \%$ & $131.0 \%$ & $5.2 \%$ & $>14.91$ & flash & flash & \\
\hline & 2.71 & $135.5 \%$ & $131.0 \%$ & & $>14.91$ & flash & & \\
\hline NO 410 & 0.41 & $20.5 \%$ & $17.0 \%$ & & 10.67 & 12.27 & & $\mathrm{~N} / \mathrm{C}$ \\
\hline & 0.46 & $11.5 \%$ & $17.0 \%$ & $-22.5 \%$ & 10.67 & 12.92 & $19.3 \%$ & \\
\hline & 0.30 & $7.5 \%$ & $17.0 \%$ & & 10.67 & 12.99 & & \\
\hline NO MI 418 & 0.05 & $1.3 \%$ & $4.0 \%$ & & 10.23 & 9.71 & & $N / C$ \\
\hline & 0.04 & $1.0 \%$ & $4.0 \%$ & $.70 .8 \%$ & 10.23 & 8.66 & $-5.9 \%$ & \\
\hline & 0.05 & $1.3 \%$ & $4.0 \%$ & & 10.23 & 10.51 & & \\
\hline MEL 228 & 3.11 & $155.5 \%$ & $160.0 \%$ & & $>14.22$ & flash & & slightly \\
\hline & 3.30 & $165.0 \%$ & $160.0 \%$ & $-1.8 \%$ & $>14.22$ & flash & flash & yellowed \\
\hline & 3.02 & $151.0 \%$ & $160.0 \%$ & & $>14.22$ & flash & & \\
\hline & $500 \mathrm{HRS}$ & in $\mathrm{HFC}$ - & $32 / 1 \mathrm{Cl} E \mathrm{ml}$ & karox VG3 & 32 Oil @ & $260 \mathrm{~F} \cdot \mathrm{P}$ & 24 HRS & $302^{\circ} \mathrm{F}$ \\
\hline NO/MY/NO & 0.36 & $9.0 \%$ & $20.0 \%$ & & $>18.97$ & flash & & Bubbles \\
\hline$A 2$ & 0.52 & $13.0 \%$ & $20.0 \%$ & $-41.3 \%$ & $>18.97$ & flash & flash & pockets as above \\
\hline & 0.53 & $13.3 \%$ & $20.0 \%$ & & $>18.97$ & flash & & but bigger \\
\hline DA/MY/DA & 0.46 & $23.0 \%$ & $46.0 \%$ & & $>15.27$ & flash & & Slighlty \\
\hline & 0.46 & $23.0 \%$ & $46.0 \%$ & $.53 .3 \%$ & $>15.27$ & flash & flash & warped \\
\hline & 0.37 & $18.5 \%$ & $46.0 \%$ & & $>15.27$ & flash & & darkened \\
\hline MYLARMO & 2.59 & $129.5 \%$ & $131.0 \%$ & & $>14.91$ & flash & & $\mathrm{N} / \mathrm{C}$ \\
\hline $\mathrm{C} 2$ & 2.41 & $120.5 \%$ & $131.0 \%$ & $-0.6 \%$ & $>14.91$ & flash & flash & \\
\hline & 2.81 & $140.5 \%$ & $131.0 \%$ & & $>14.91$ & flash & & \\
\hline NOMEX 410 & 0.37 & $9.3 \%$ & $17.0 \%$ & & 10.67 & 10.51 & & \\
\hline $\mathrm{D} 2$ & 0.34 & $8.5 \%$ & $17.0 \%$ & $-50.0 \%$ & 10.67 & 10.84 & $1.4 \%$ & \\
\hline & 0.31 & $7.8 \%$ & $17.0 \%$ & & 10.67 & 11.11 & & fow pockets \\
\hline $\mathrm{NO} / \mathrm{Ml} 418$ & 0.04 & $1.0 \%$ & $4.0 \%$ & & 10.23 & 9.72 & & $N / C$ \\
\hline & 0.04 & $1.0 \%$ & $4.0 \%$ & $.75 .0 \%$ & 10.23 & 10.69 & $0.7 \%$ & \\
\hline & 0.04 & $1.0 \%$ & $4.0 \%$ & & 10.23 & 10.50 & & \\
\hline MEL 228 & 2.79 & $139.5 \%$ & $160.0 \%$ & & $>14.22$ & flash & & slightly \\
\hline & 2.90 & $144.8 \%$ & $160.0 \%$ & $.8 .6 \%$ & $>14.22$ & flash & flash & yellowed \\
\hline & 3.09 & $154.5 \%$ & $160.0 \%$ & & $>14.22$ & flash & & \\
\hline
\end{tabular}


SLEEVING--HFC-32/ICI Emkarox VG 32 Oil @ 260 $\mathrm{F}\left(127^{\circ} \mathrm{C}\right)$

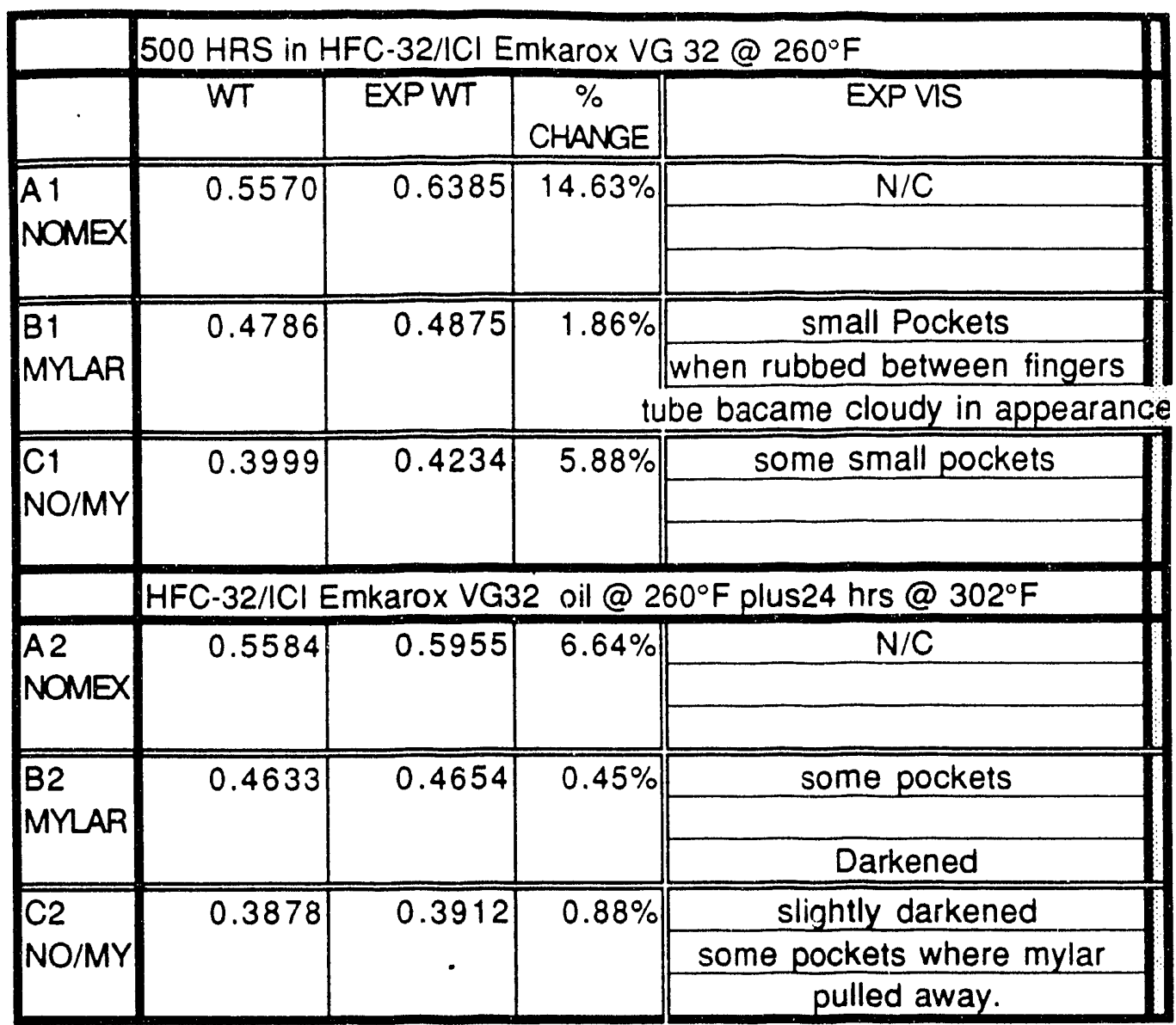




\begin{tabular}{|c|c|c|c|c|c|c|c|c|c|c|}
\hline & 500 HRS & in $\mathrm{HFC}-3$ & |CI Emkal & $x \vee G 32$ & $260 \mathrm{~F}$ & & & & & \\
\hline ID & WT & EXPWT & $\begin{array}{l}\text { WT \% } \\
\text { CHANGE }\end{array}$ & $\begin{array}{l}\text { BREAK } \\
\text { LOAD } \\
\text { (AVE) }\end{array}$ & $\begin{array}{l}\text { BREAK } \\
\text { LOAD } \\
\text { EXP }\end{array}$ & $\begin{array}{c}\% \\
\text { CHANGE } \\
\text { BRK } \\
\text { LOAD }\end{array}$ & & $\%$ EONG & EXP VIS & \\
\hline$A_{1}$ & 1.8604 & 1.8727 & $0.66 \%$ & 39.02 & 70.60 & & 0.06 & $3.00 \%$ & $\mathrm{~N} / \mathrm{C}$ & \\
\hline Glass & & & & 39.02 & 71.65 & $79.22 \%$ & 0.07 & $3.50 \%$ & & \\
\hline & & & & 39.02 & 67.55 & & 0.06 & $3.00 \%$ & & \\
\hline$\overline{B 1}$ & 0.7228 & 0.7357 & $1.78 \%$ & 56.12 & 60.30 & & 0.70 & $35.00 \%$ & $\mathrm{~N} / \mathrm{C}$ & \\
\hline Polyester & & & & 56.12 & 57.00 & $6.02 \%$ & 0.59 & $29.50 \%$ & & \\
\hline & & & & 56.12 & 61.20 & & 0.76 & $38.00 \%$ & & \\
\hline$C_{1}$ & 1.6518 & 1.7254 & $4.46 \%$ & 88.50 & 39.00 & & 0.14 & $7.00 \%$ & & \\
\hline Permacel & & & & 88.50 & 40.60 & $-55.08 \%$ & 0.15 & $7.50 \%$ & & \\
\hline & & & & 88.50 & 39.65 & & 0.15 & $7.50 \%$ & Curled up & \\
\hline & $500 \mathrm{HRS}$ & $S$ in HFC. & 32/ ICl Emka & rox VG32 & @ 260 F- & $302^{\circ} \mathrm{F} 24$ & & & & \\
\hline$A 2$ & 1.6875 & 1.6875 & $0.00 \%$ & 39.02 & 49.90 & & 0.05 & $2.50 \%$ & $\mathrm{~N} / \mathrm{C}$ & \\
\hline Glass & & & & 39.02 & 51.10 & $29.25 \%$ & 0.05 & $2.50 \%$ & & \\
\hline & & & & 39.02 & 50.30 & & 0.04 & $2.00 \%$ & & \\
\hline & 0.7281 & 0.7251 & $-0.41 \%$ & 56.12 & 48.60 & & 0.39 & $19.50 \%$ & $\mathrm{~N} / \mathrm{C}$ & \\
\hline Polyester & & & & 56.12 & 57.80 & $-1.52 \%$ & 0.68 & $34.00 \%$ & & \\
\hline & & & & 56.12 & 59.40 & & 0.67 & $33.50 \%$ & & \\
\hline $\mathrm{C} 2$ & 1.5229 & 1.3850 & $.9 .06 \%$ & 88.50 & 97.90 & & 0.13 & $6.50 \%$ & Slightly & \\
\hline Permacel & & & & 88.50 & 68.55 & $.5 .05 \%$ & 0.09 & $4.50 \%$ & & \\
\hline & & & & 88.50 & 85.65 & & 0.11 & $5.50 \%$ & Curled up & \\
\hline
\end{tabular}




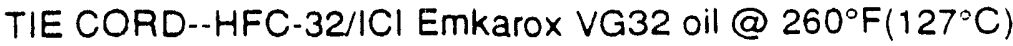

\begin{tabular}{|c|c|c|c|c|c|c|c|c|c|}
\hline \multicolumn{10}{|c|}{500 HRS IN R-32/ICI Emkarox VG32 @ 260 F } \\
\hline ID & WT & EXPWT & $\begin{array}{c}\text { WT \% } \\
\text { CHANGE }\end{array}$ & $\begin{array}{l}\text { EXP } \\
V I S\end{array}$ & $\begin{array}{l}\text { BREAK } \\
\text { LOAD } \\
\text { (AVE) }\end{array}$ & $\begin{array}{l}\text { BREAK } \\
\text { LOAD } \\
\text { EXP } \\
(\operatorname{av} \theta)\end{array}$ & $\begin{array}{c}\% \\
\text { CHANGE } \\
\text { BRK } \\
\text { LOAD }\end{array}$ & $\begin{array}{c}\text { STRETCH } \\
\text { (Inch) }\end{array}$ & $\begin{array}{c}\% \\
\text { ELONG }\end{array}$ \\
\hline \multirow[t]{4}{*}{ A1 } & \multirow[t]{3}{*}{0.3081} & \multirow[t]{3}{*}{0.3116} & \multirow[t]{3}{*}{$1.14 \%$} & $\mathrm{~N} / \mathrm{C}$ & 28.36 & 32.95 & & 0.44 & $22.0 \%$ \\
\hline & & & & & 28.36 & 32.15 & \multirow[t]{2}{*}{$16.13 \%$} & 0.44 & $22.0 \%$ \\
\hline & & & & & 28.36 & 33.70 & & 0.42 & $21.0 \%$ \\
\hline & \multicolumn{9}{|c|}{500 HRS IN R.32/ICl Emkarox VG32 > 24 HRS @ $302^{\circ} \mathrm{F}$} \\
\hline \multirow[t]{3}{*}{$A 2$} & \multirow{3}{*}{0.3091} & \multirow[t]{3}{*}{0.3073} & \multirow[t]{3}{*}{$-0.582 \%$} & $\mathrm{~N} / \mathrm{C}$ & 28.36 & 35.10 & & 0.46 & $23.0 \%$ \\
\hline & & & & & 28.36 & 34.20 & $25.82 \%$ & 0.47 & $23.5 \%$ \\
\hline & & & & & 28.36 & 37.75 & & 0.53 & $26.5 \%$ \\
\hline
\end{tabular}


LEAD WIRE INSULATION-HFC-32/ICI Emkarox VG32@260 $\mathrm{F}\left(127^{\circ} \mathrm{C}\right)$

\begin{tabular}{|c|c|c|c|c|c|c|c|}
\hline & $500 \mathrm{HRS}$ IN & $=\mathrm{C}-32 / \mathrm{ICl} \mathrm{Er}$ & nkarox VC & $32 @ 260$ & & & \\
\hline ID & WT & EXPWT & $\begin{array}{l}\text { WT \% } \\
\text { CHANGE }\end{array}$ & EXP VIS & $\begin{array}{c}\text { BASE DIE } \\
\text { (AVE) }\end{array}$ & EXPDIE & $\begin{array}{c}\text { DIE\% } \\
\text { CHANGE }\end{array}$ \\
\hline DMD & 4.0632 & 4.2218 & $3.90 \%$ & $\mathrm{~N} / \mathrm{C}$ & 9.61 & 7.56 & \\
\hline A 1 & & & & & 9.61 & 8.89 & $-14.36 \%$ \\
\hline & & & & & 9.61 & 8.24 & \\
\hline DTMD & 4.2893 & 4.4205 & $3.06 \%$ & $\mathrm{~N} / \mathrm{C}$ & 9.95 & 12.40 & \\
\hline B1 & & & & & 9.95 & 13.31 & $30.62 \%$ \\
\hline & & & & & 9.95 & 13.28 & \\
\hline & $\mathrm{HFC}-32 / \mathrm{Cl} \mathrm{E}$ & karox VG32 & @ $260^{\circ} \mathrm{F}$ & $\rightarrow 24 \mathrm{HRS}$ & @ $302^{\circ} \mathrm{F}$ & & \\
\hline DMD & 4.0809 & 4.1333 & $1.28 \%$ & $\mathrm{~N} / \mathrm{C}$ & 9.61 & 8.79 & \\
\hline$A 2$ & & & & & 9.61 & 7.69 & $-11.20 \%$ \\
\hline & & & & & 9.61 & 9.12 & \\
\hline DTMD & 4.3318 & 4.3724 & $0.94 \%$ & $\mathrm{~N} / \mathrm{C}$ & 9.95 & 11.79 & \\
\hline $\mathrm{B} 2$ & & & & & 9.95 & 12.23 & $18.39 \%$ \\
\hline & & & & & 9.95 & 11.32 & \\
\hline
\end{tabular}




\section{Appendix 0}

\section{Experimental Data for HFC-125/ICI Emkarox VG32 Exposure at $126^{\circ} \mathrm{C}\left(260^{\circ} \mathrm{F}\right)$}




\begin{tabular}{|c|c|c|c|c|c|c|c|c|c|c|}
\hline \multirow{2}{*}{ ID } & \multicolumn{10}{|c|}{500 hours in $\mathrm{HFC}=125 / \mathrm{ICl}$ Emkarox VG32 oil at $260^{\circ} \mathrm{F}\left(127^{\circ} \mathrm{C}\right)$} \\
\hline & $\mathrm{WT}$ & EXPWT & $\begin{array}{c}\text { WT \% } \\
\text { CHANGE }\end{array}$ & EXP VIS & $\begin{array}{c}\text { BASE BRN } \\
\text { OUT } \\
\text { (AVE) }\end{array}$ & $\begin{array}{c}\text { EXP BRN } \\
\text { CUT }\end{array}$ & $\begin{array}{c}\text { BRN OUT } \\
\% \\
\text { CHANGE }\end{array}$ & $\begin{array}{c}\text { BASE DIE } \\
\text { (AVE) }\end{array}$ & EXPDIE & $\begin{array}{c}\text { DIE \% } \\
\text { CHANGE }\end{array}$ \\
\hline \multirow[t]{3}{*}{ A1 } & \multirow[t]{3}{*}{23.0548} & \multirow[t]{3}{*}{23.0555} & \multirow[t]{3}{*}{$0.003 \%$} & $\mathrm{~N} / \mathrm{C}$ & 576 & 564 & & 15.80 & 16.54 & \\
\hline & & & & & 576 & 556 & $-3.0 \%$ & 15.80 & 11.70 & $-4.6 \%$ \\
\hline & & & & & 576 & 557 & & 15.80 & 16.99 & \\
\hline \multirow[t]{3}{*}{$B 1$} & \multirow[t]{3}{*}{25.3615} & \multirow[t]{3}{*}{25.3932} & \multirow[t]{3}{*}{$0.125 \%$} & $\mathrm{~N} / \mathrm{C}$ & 736 & 729 & & 11.62 & 14.50 & \\
\hline & & & & & 736 & 730 & $-2.7 \%$ & 11.62 & 14.64 & $22.7 \%$ \\
\hline & & & & & 736 & 689 & & 11.62 & 13.63 & \\
\hline \multirow[t]{4}{*}{$\mathrm{C1}$} & \multirow[t]{3}{*}{22.2454} & \multirow[t]{3}{*}{22.2468} & \multirow[t]{3}{*}{$0.006 \%$} & $\mathrm{~N} / \mathrm{C}$ & 579 & 577 & & 16.58 & 12.56 & \\
\hline & & & & & 579 & 588 & $0.6 \%$ & 16.58 & 9.26 & $-30.9 \%$ \\
\hline & & & & & 579 & 583 & & 16.58 & 12.53 & \\
\hline & \multicolumn{10}{|c|}{500 hours in $\mathrm{HFC}-125 / \mathrm{ICl}$ Emkarox VG32 oil at $260^{\circ} \mathrm{F}\left(127^{\circ} \mathrm{C}\right)$ plus 24 hours at $302^{\circ} \mathrm{F}\left(150^{\circ} \mathrm{C}\right)$} \\
\hline \multirow[t]{3}{*}{ A2 } & \multirow[t]{3}{*}{22.72} & \multirow[t]{3}{*}{22.7202} & \multirow[t]{3}{*}{$0.001 \%$} & $\mathrm{~N} / \mathrm{C}$ & 576 & 541 & & 15.80 & 11.94 & \\
\hline & & & & & 576 & 473 & $-12.0 \%$ & 15.80 & 14.16 & $-14.6 \%$ \\
\hline & & & & & 576 & 507 & & 15.80 & 14.39 & \\
\hline \multirow[t]{3}{*}{ B2 } & \multirow[t]{3}{*}{24.4631} & \multirow[t]{3}{*}{24.4686} & \multirow[t]{3}{*}{$0.022 \%$} & $\mathrm{~N} / \mathrm{C}$ & 736 & 730 & & 11.62 & 12.00 & \\
\hline & & & & & 736 & 724 & $-1.1 \%$ & 11.62 & 11.59 & $2.2 \%$ \\
\hline & & & & & 736 & 729 & & 11.62 & 12.04 & \\
\hline \multirow[t]{3}{*}{$\mathrm{C} 2$} & \multirow[t]{3}{*}{22.4597} & \multirow[t]{3}{*}{22.4606} & \multirow[t]{3}{*}{$0.004 \%$} & $\mathrm{~N} / \mathrm{C}$ & 579 & 573 & & 16.58 & 15.85 & \\
\hline & & & & & 579 & 434 & $-8.9 \%$ & 16.58 & 15.45 & $-12.4 \%$ \\
\hline & & & & & 579 & 576 & & 16.58 & 12.29 & \\
\hline
\end{tabular}


TWISTED PAIRS/WIRE AWITH VARNISH-HFC-125/ICI Emkarox VG32 oil @260 $\mathrm{F}\left(127^{\circ} \mathrm{C}\right)$

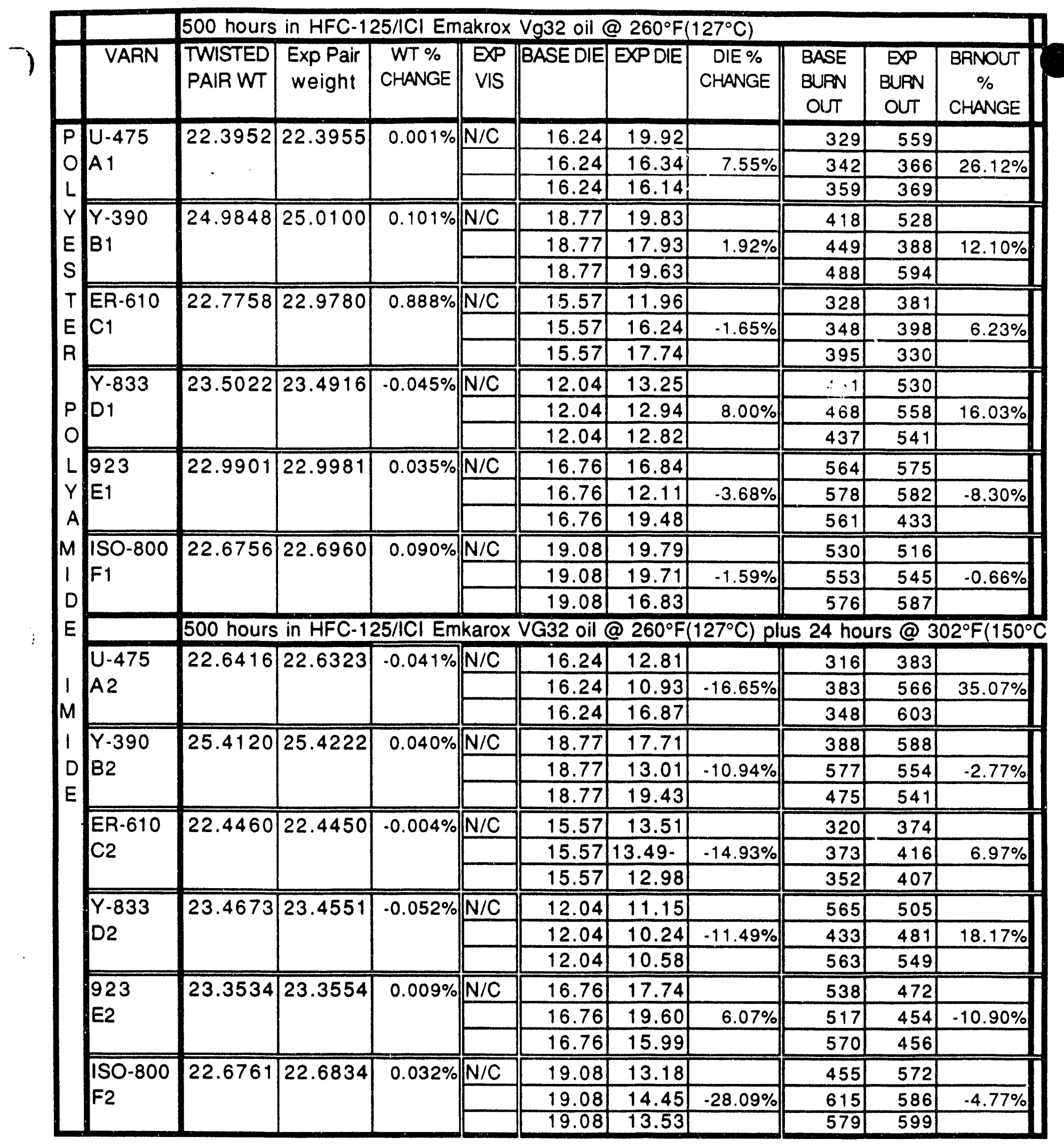


TWISTED PAIRS/WIRE BMITH VARNISH -HFC-125/ICI Emkärox VG32 oil @260*F(127*C)

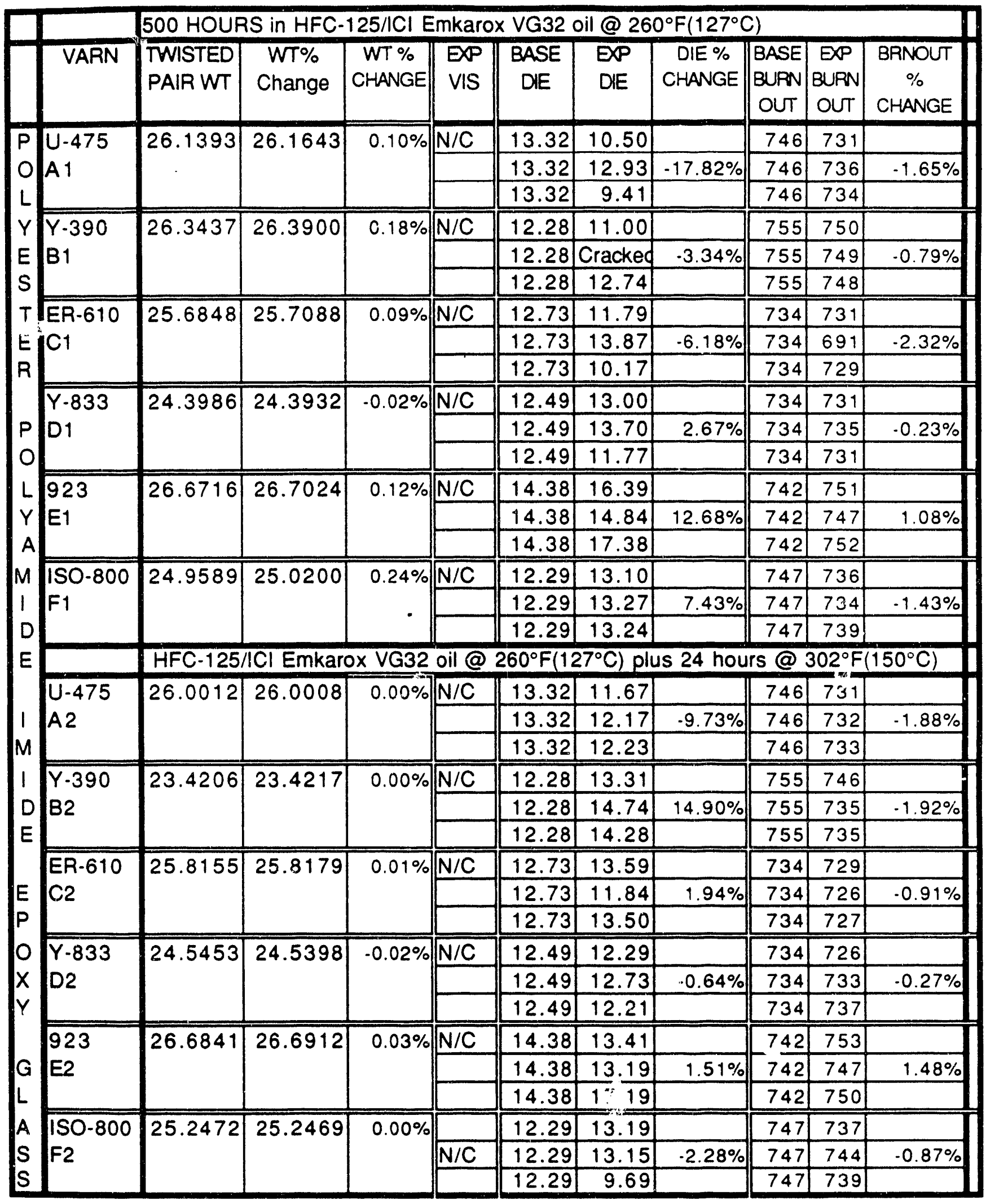


TWISTED PAIRS/WIRE CMITH VARNISH-HFC-125/ICI EmIkarox VG 32 @ 260 $\mathrm{F}\left(127^{\circ} \mathrm{C}\right)$

\begin{tabular}{|c|c|c|c|c|c|c|c|c|c|c|c|}
\hline & & \multicolumn{10}{|c|}{500 Hours in HFC-125/ICl Emkarox VG32 @ 260 $\mathrm{F}\left(127^{\circ} \mathrm{F}\right)$} \\
\hline & VARN & $\begin{array}{l}\text { TWSTED } \\
\text { PAIR WT }\end{array}$ & $\begin{array}{c}\text { EXPT } \\
\text { PAIR WT }\end{array}$ & $\begin{array}{c}\text { WT \% } \\
\text { CHANGE }\end{array}$ & $\begin{array}{l}\text { EXP } \\
\text { VIS }\end{array}$ & $\begin{array}{c}\text { BASE } \\
\text { DIE }\end{array}$ & $\begin{array}{l}\text { EXP } \\
D I E\end{array}$ & $\begin{array}{c}\text { DIE \% } \\
\text { CHANGE }\end{array}$ & $\begin{array}{l}\text { BASE } \\
\text { BURN } \\
\text { OUT }\end{array}$ & \begin{tabular}{|c|} 
EXP \\
BURN \\
OUT
\end{tabular} & $\begin{array}{c}\text { BRNOUT } \\
\% \\
\text { CHANGE }\end{array}$ \\
\hline \multirow{3}{*}{$\begin{array}{l}P \\
O \\
L\end{array}$} & \multirow{3}{*}{$\begin{array}{l}\text { U. } 475 \\
\text { A } 1\end{array}$} & 23.0745 & 23.0780 & $0.015 \%$ & $N / C$ & 15.10 & 14.79 & & 469 & 331 & \\
\hline & & & & & & 15.10 & 18.30 & $11.21 \%$ & 469 & 348 & $-26.44 \%$ \\
\hline & & & & & & 15.10 & 17.29 & & 469 & 356 & \\
\hline \multirow{3}{*}{$\begin{array}{l}Y \\
E\end{array}$} & \multirow{3}{*}{$\begin{array}{l}-390 \\
B 1\end{array}$} & 23.9563 & 23.9753 & $0.079 \%$ & $\mathrm{~N} / \mathrm{C}$ & 18.24 & 16.23 & & 473 & 507 & \\
\hline & & & & & & 18.24 & 19.83 & $-7.64 \%$ & 473 & 549 & $14.52 \%$ \\
\hline & & & & & & 18.24 & 14.48 & & 473 & 569 & \\
\hline \multirow{3}{*}{$\begin{array}{l}T \\
E \\
R\end{array}$} & \multirow{3}{*}{$\begin{array}{l}\text { ER-610 } \\
\text { C1 }\end{array}$} & 21.9102 & 21.9128 & $0.012 \%$ & $\mathrm{~N} / \mathrm{C}$ & 14.53 & 14.70 & & 494 & 315 & \\
\hline & & & & & & 14.53 & 13.76 & $4.15 \%$ & 494 & 352 & $-29.89 \%$ \\
\hline & & & & & & 14.53 & 16.94 & & 494 & 372 & \\
\hline & \multirow{3}{*}{$\begin{array}{l}Y-833 \\
D 1\end{array}$} & 21.2447 & 21.2329 & $-0.056 \%$ & $N / C$ & 11.38 & 15.09 & & 557 & 512 & \\
\hline 1 & & & & & & 11.38 & 14.95 & $31.20 \%$ & 557 & 561 & $-1.86 \%$ \\
\hline$M$ & & & & & & 11.38 & 14.75 & & 557 & 567 & \\
\hline \multirow{3}{*}{$\begin{array}{l}I \\
D \\
E\end{array}$} & \multirow{3}{*}{$\begin{array}{l}923 \\
\text { E1 }\end{array}$} & 23.2926 & 23.2958 & $0.014 \%$ & $\mathrm{~N} / \mathrm{C}$ & 15.85 & 18.33 & & 503 & 636 & \\
\hline & & & & & & 15.85 & 19.76 & $17.83 \%$ & 503 & 521 & $2.65 \%$ \\
\hline & & & & & & 15.85 & 17.94 & & 503 & 392 & \\
\hline & \multirow{4}{*}{$\begin{array}{l}\text { ISO-800 } \\
\text { F1 }\end{array}$} & 23.732 .2 & 23.7587 & $0.112 \%$ & $\mathrm{~N} / \mathrm{C}$ & 14.75 & 16.76 & & 632 & 585 & \\
\hline$P$ & & & & & & 14.75 & 19.99 & $27.77 \%$ & 632 & 634 & $-2.22 \%$ \\
\hline 0 & & & & & & 14.75 & 19.79 & & 632 & 635 & \\
\hline$L$ & & HFC-125 & ICI Emkar & ox VG32 & @ 26c & F plus & 24 hour & $s @ 302^{\circ}$ & $F(150$ & $\left.{ }^{\circ} \mathrm{C}\right)$ & \\
\hline \multirow{3}{*}{$\begin{array}{l}Y \\
A \\
M\end{array}$} & \multirow{3}{*}{$\begin{array}{l}U-475 \\
A 2\end{array}$} & 22.9789 & 22.9803 & $0.006 \%$ & $\mathrm{~N} / \mathrm{C}$ & 15.10 & 19.33 & & 469 & 571 & \\
\hline & & & & & & 15.10 & 19.95 & $29.76 \%$ & 469 & 520 & $15.57 \%$ \\
\hline & & & & & & 15.10 & 19.50 & & 469 & 535 & \\
\hline \multirow{3}{*}{$\begin{array}{l}I \\
D \\
E\end{array}$} & \multirow{3}{*}{$\begin{array}{l}Y-390 \\
B 2\end{array}$} & 24.1247 & 24.1303 & $0.023 \%$ & $\mathrm{~N} / \mathrm{C}$ & 18.24 & 19.89 & & 473 & 493 & \\
\hline & & & & & & 18.24 & 19.89 & $6.52 \%$ & 473 & 402 & $0.70 \%$ \\
\hline & & & & & & 18.24 & 18.97 & & 473 & 534 & \\
\hline & \multirow{3}{*}{$\begin{array}{l}\text { ER-610 } \\
\text { C2 }\end{array}$} & 22.0432 & 22.0424 & $-0.004 \%$ & $\mathrm{~N} / \mathrm{C}$ & 14.53 & 15.20 & & 494 & 414 & \\
\hline 1 & & & & & & 14.53 & 13.74 & $-3.79 \%$ & 494 & 376 & $-22.47 \%$ \\
\hline$M$ & & & & & & 14.53 & 13.00 & & 494 & 359 & \\
\hline \multirow{9}{*}{$\begin{array}{l}I \\
D \\
E\end{array}$} & \multirow{3}{*}{$\begin{array}{l}\mathrm{Y}-833 \\
D 2\end{array}$} & 21.1846 & 21.1733 & $-0.053 \%$ & $N / C$ & 11.38 & 14.45 & & 557 & 570 & \\
\hline & & & & & & 11.38 & 11.82 & $13.09 \%$ & 557 & 472 & $-5.27 \%$ \\
\hline & & & & & & 11.38 & 12.34 & & 557 & 541 & \\
\hline & \multirow{3}{*}{$\begin{array}{l}923 \\
E 2\end{array}$} & 23.4410 & 23.4429 & $0.008 \%$ & $\mathrm{~N} / \mathrm{C}$ & 15.85 & 19.80 & & 503 & 475 & \\
\hline & & & & & & 15.85 & 16.69 & $14.55 \%$ & 503 & 487 & $.0 .27 \%$ \\
\hline & & & & & & 15.85 & 17.98 & & 503 & 543 & \\
\hline & \multirow{3}{*}{$\begin{array}{l}150-800 \\
F 2\end{array}$} & 23.6657 & 23.6852 & $0.082 \%$ & $N / C$ & 14.75 & 18.46 & & 632 & 615 & \\
\hline & & & & & & 14.75 & 19.81 & $25.81 \%$ & 632 & 649 & $-1.64 \%$ \\
\hline & & & & & & 14.75 & 17.40 & & 632 & 601 & \\
\hline
\end{tabular}




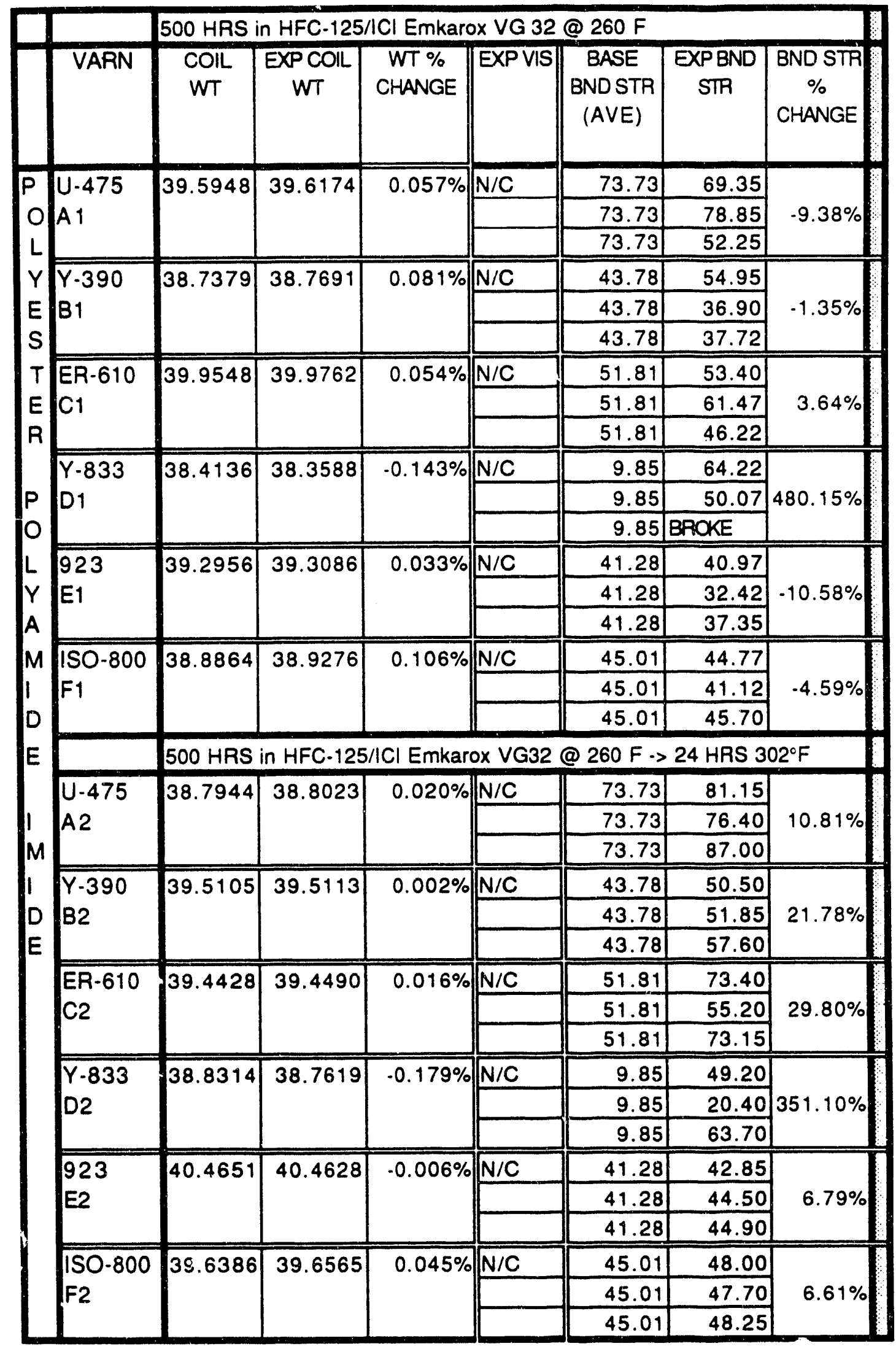


HELICAL COILSMIRE B-HFC-125/ICI Emkarox VGł́2 Oil @ 260 $\mathrm{F}\left(127^{\circ} \mathrm{C}\right)$

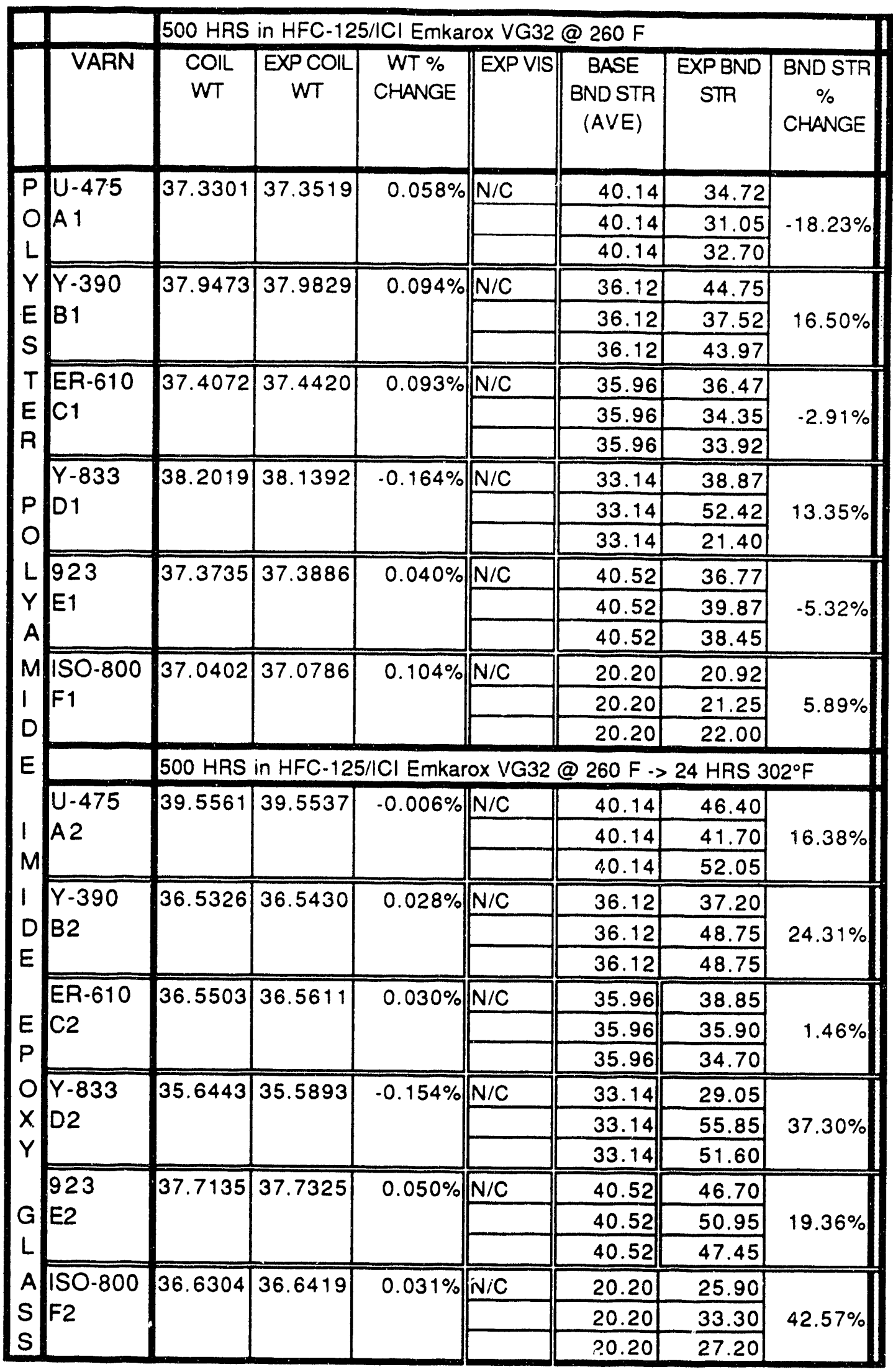


HELICAL COILSWIRE C.HFC-125/ICI Emkarox VG32 @ 260 $\mathrm{F}\left(127^{\circ} \mathrm{C}\right)$

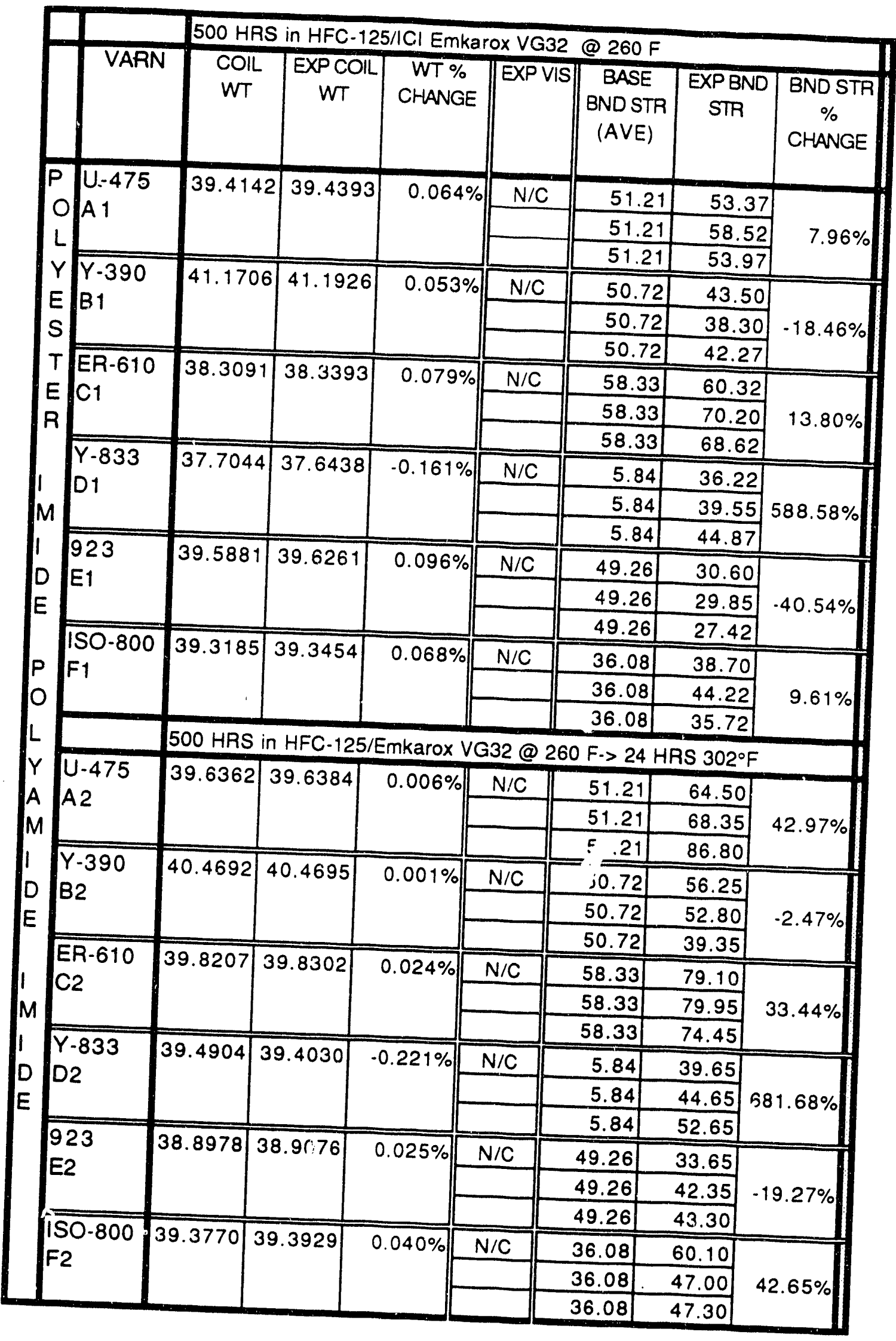




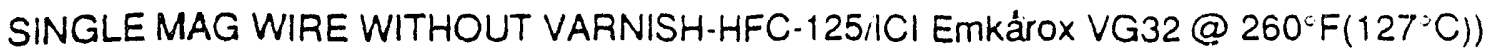

\begin{tabular}{|c|c|c|c|c|c|}
\hline 500 & IS in $\mathrm{HF}$ & $-125 / \mathrm{Cl} \mathrm{E}$ & karox VG32 & oil @26 & ${ }^{\circ} \mathrm{F}\left(127^{\circ} \mathrm{C}\right)$ \\
\hline D & $\overline{W T}$ & EXPWT & $\begin{array}{c}\text { WT \% } \\
\text { CHANGE }\end{array}$ & EXPVIS & EXPFLEX \\
\hline A1 & 3.3289 & 3.3301 & $0.036 \%$ & $\mathrm{~N} / \mathrm{C}$ & \\
\hline & & & & & YES \\
\hline B1 & 308531 & 30886 & 0 1070 & N/C & \\
\hline & & & & & NO \\
\hline & & & & & \\
\hline C1 & 3.6696 & 3.6704 & $0.022 \%$ & $\mathrm{~N} / \mathrm{C}$ & \\
\hline & & & & & YES \\
\hline & & & & & \\
\hline 50 & $\mathrm{RS}$ in $\mathrm{HF}$ & - -125/Emk & rox oil-> 30 & ${ }^{\circ} \mathrm{F}$ for 24 & HRS \\
\hline A2 & 3.5204 & 3.521 & $0.017 \%$ & $\mathrm{~N} / \mathrm{C}$ & \\
\hline & & & & & NO \\
\hline & & & & & \\
\hline B2 & 3.5074 & 3.5085 & $0.031 \%$ & $\mathrm{~N} / \mathrm{C}$ & \\
\hline & & & & & NO \\
\hline $\mathrm{C} 2$ & 3.4683 & 3.4688 & $0.014 \%$ & $\mathrm{~N} / \mathrm{C}$ & \\
\hline & & & & & NO \\
\hline & & & & & \\
\hline
\end{tabular}




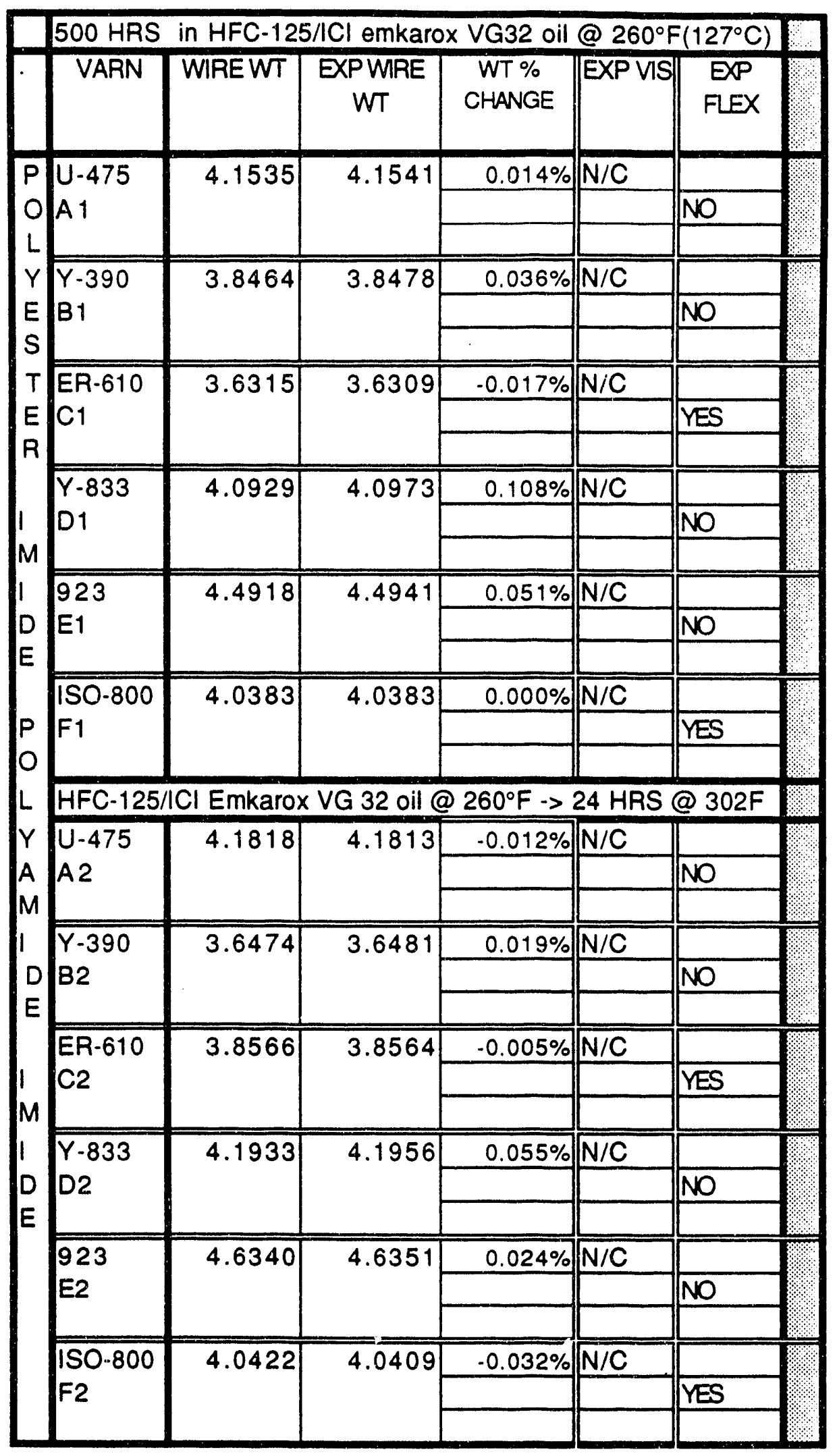




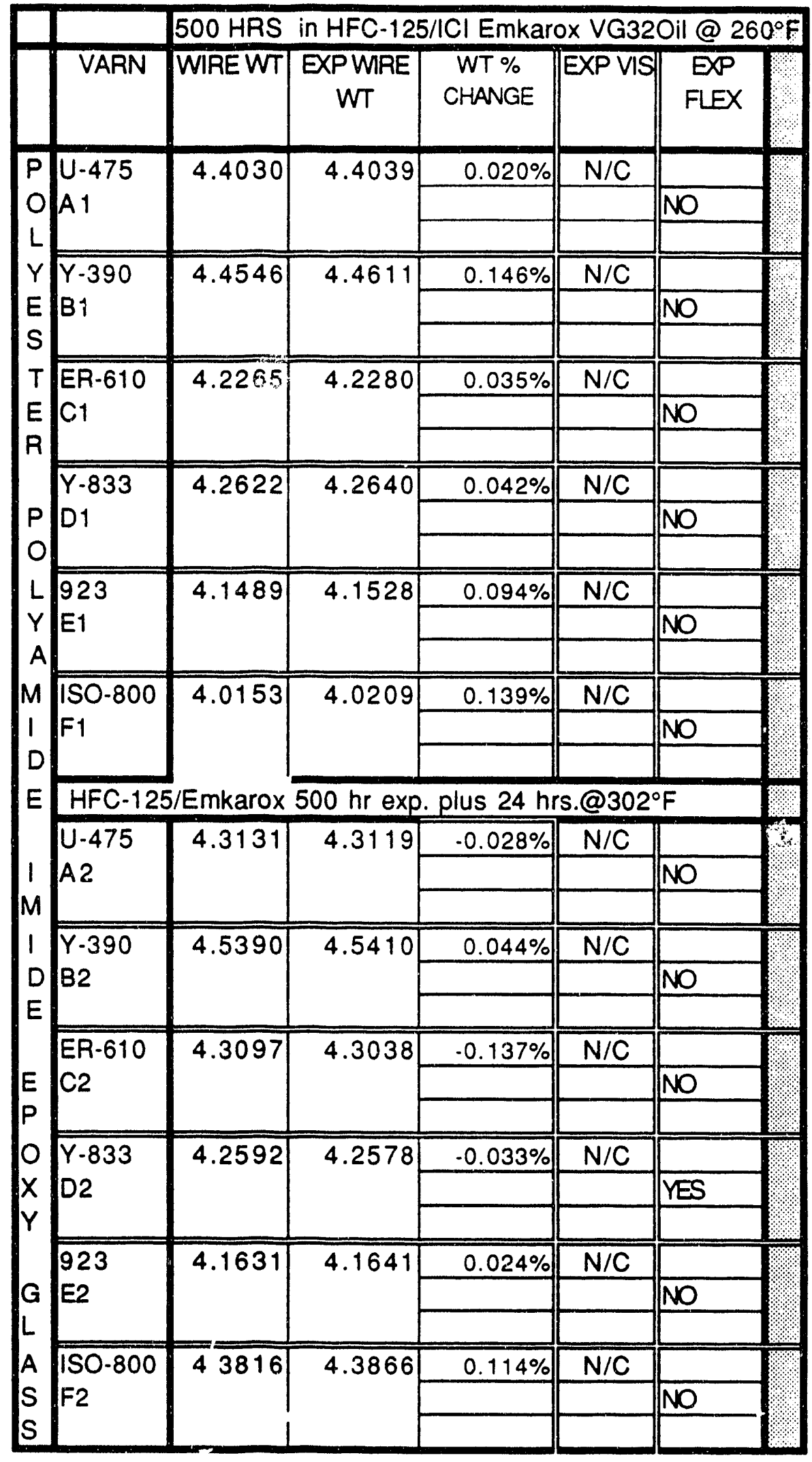




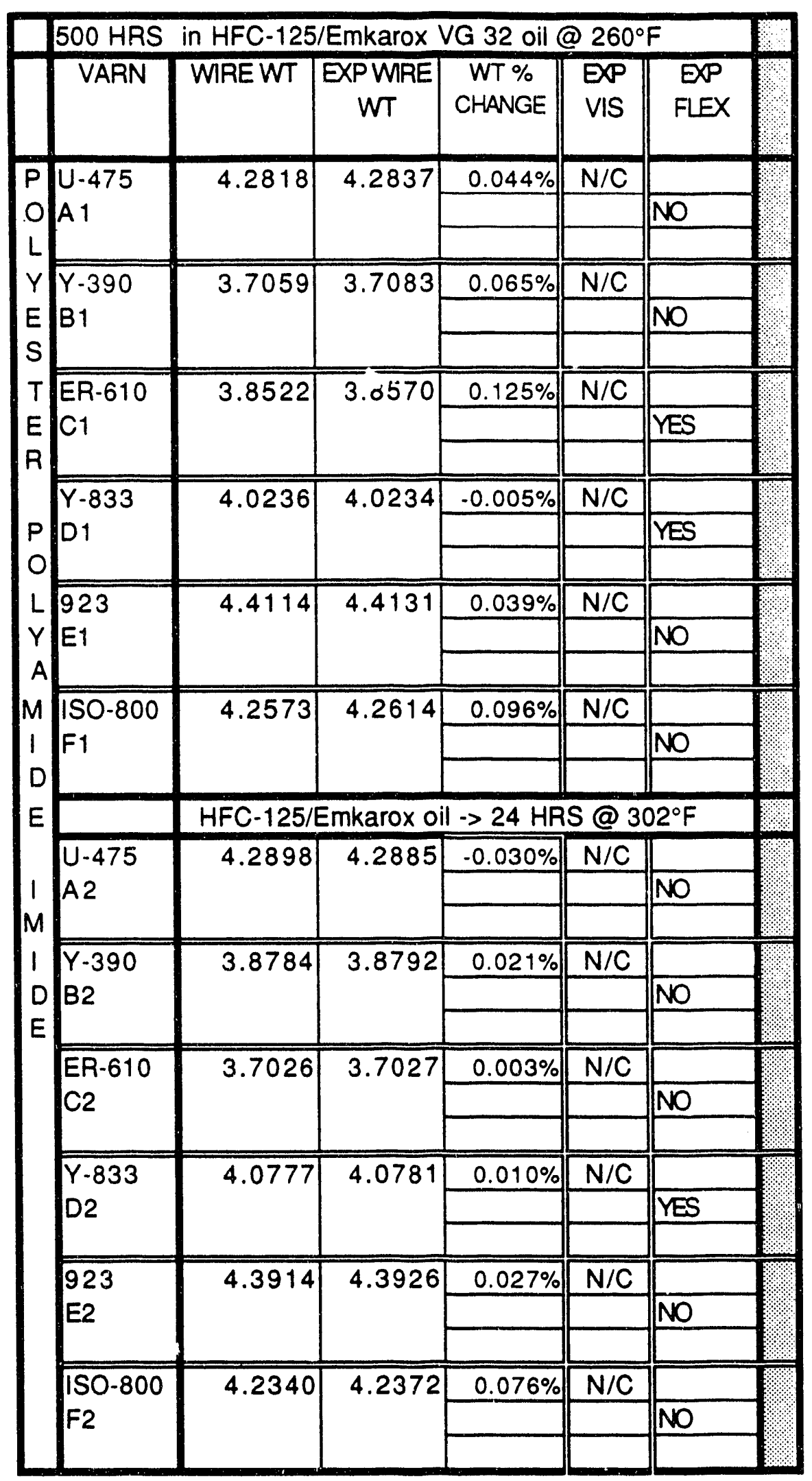




\begin{tabular}{|c|c|c|c|c|c|}
\hline \multicolumn{6}{|c|}{500 HRS IN R-125/ICl Emkarox VG32 @ 260 F } \\
\hline ID & WT & EXPWT & $\begin{array}{c}\text { WT \% } \\
\text { CHANGE }\end{array}$ & EXP VS & EXPFLEX \\
\hline $\begin{array}{l}\text { U-475 } \\
\text { A1 }\end{array}$ & 2.3778 & 2.3400 & $-1.59 \%$ & $\mathrm{~N} / \mathrm{C}$ & $\mathrm{N} / \mathrm{C}$ \\
\hline $\begin{array}{l}\text { Y } 390 \\
B 1\end{array}$ & 1.9172 & 1.8785 & $2.02 \%$ & $\mathrm{~N} / \mathrm{C}$ & $\mathrm{N} / \mathrm{C}$ \\
\hline $\begin{array}{l}\text { ER-610 } \\
\text { C1 }\end{array}$ & 2.8180 & 2.8379 & $0.71 \%$ & \begin{tabular}{|l|} 
Slightly \\
Darkened
\end{tabular} & $\mathrm{N} / \mathrm{C}$ \\
\hline $\begin{array}{l}\text { Y.833 } \\
\text { D1 }\end{array}$ & 2.9165 & 2.8723 & $-1.52 \%$ & $\mathrm{~N} / \mathrm{C}$ & $\mathrm{N} / \mathrm{C}$ \\
\hline $\begin{array}{l}923 \\
E 1\end{array}$ & 2.2818 & 2.2420 & $-1.74 \%$ & Warped & $\mathrm{N} / \mathrm{C}$ \\
\hline $\begin{array}{l}\text { ISO-800 } \\
\text { F1 }\end{array}$ & 2.1471 & 2.1308 & $-0.76 \%$ & $\begin{array}{l}\text { slightly } \\
\text { warped }\end{array}$ & $\mathrm{N} / \mathrm{C}$ \\
\hline & $R-125 / \mathrm{ICI} E$ & Ikarox VG 3 & 2 at $\cdots>24$ & hour at 3 & $02^{\circ} \mathrm{F}$. \\
\hline $\begin{array}{l}\text { U-475 } \\
\text { A2 }\end{array}$ & 2.3172 & 2.2553 & $-2.67 \%$ & Darkened & \begin{tabular}{|l} 
Cloudy \\
appearance
\end{tabular} \\
\hline & & & & & on surface \\
\hline $\begin{array}{l}Y-390 \\
B 2\end{array}$ & 1.7275 & 1.6955 & $-1.85 \%$ & $\mathrm{~N} / \mathrm{C}$ & $\mathrm{N} / \mathrm{C}$ \\
\hline $\begin{array}{l}\text { ER-610 } \\
\text { C2 }\end{array}$ & 1.8194 & 1.8198 & $0.02 \%$ & darkened & $\mathrm{N} / \mathrm{C}$ \\
\hline $\begin{array}{l}Y-833 \\
D 2\end{array}$ & 3.5958 & 3.6027 & $0.19 \%$ & $\mathrm{~N} / \mathrm{C}$ & $\mathrm{N} / \mathrm{C}$ \\
\hline $\begin{array}{l}923 \\
E 2\end{array}$ & 2.3593 & 2.3088 & $-2.14 \%$ & Warped & $\overline{N / C}$ \\
\hline $\begin{array}{l}\text { ISO-800 } \\
\text { F2 }\end{array}$ & 1.9143 & 1.8840 & $-1.58 \%$ & $\begin{array}{l}\text { slightly } \\
\text { warped }\end{array}$ & $\mathrm{N} / \mathrm{C}$ \\
\hline
\end{tabular}




\begin{tabular}{|c|c|c|c|c|c|c|c|c|c|}
\hline \multirow{2}{*}{ ID } & \multicolumn{9}{|c|}{500 HRS in HFC-125/lCl Emkarox VG32 Oil @260 F } \\
\hline & WT & EXPWT & $\begin{array}{c}\text { WT \% } \\
\text { CHANGE }\end{array}$ & $\begin{array}{l}\text { SAMPLE } \\
\text { WIDTH }\end{array}$ & $\begin{array}{l}\text { BREAK } \\
\text { LOAD }\end{array}$ & $\begin{array}{c}\text { SAMPLE } \\
\text { THICKNESS } \\
\text { Mils }\end{array}$ & $\begin{array}{c}\text { TENSILE } \\
\text { STR } \\
\text { BASE }\end{array}$ & $\begin{array}{l}\text { TENSILE } \\
\text { STREXP }\end{array}$ & $\begin{array}{c}\% \text { CHANGE } \\
\text { TENSILE }\end{array}$ \\
\hline \multirow{3}{*}{$\begin{array}{l}\mathrm{NO} / \mathrm{MY} / \mathrm{NO} \\
\mathrm{A} 1\end{array}$} & \multirow{3}{*}{4.3484} & \multirow[t]{3}{*}{4.6446} & \multirow[t]{3}{*}{$6.81 \%$} & 0.451 & 162.40 & 21 & 17.4 & 17.15 & \\
\hline & & & & 0.410 & 143.80 & 21 & 17.4 & 16.70 & $-0.90 \%$ \\
\hline & & & & 0.489 & 183.60 & 21 & 17.4 & 17.88 & \\
\hline \multirow{3}{*}{$\begin{array}{l}\mathrm{DA} / \mathrm{MY} / \mathrm{DA} \\
\mathrm{B} 1\end{array}$} & \multirow[t]{3}{*}{4.5114} & \multirow[t]{3}{*}{4.5190} & \multirow[t]{3}{*}{$0.17 \%$} & 0.401 & 111.50 & 21 & 13.7 & 13.24 & \\
\hline & & & & 0.390 & 109.90 & 21 & 13.7 & 13.42 & $0.18 \%$ \\
\hline & & & & 0.415 & 126.50 & 21 & 13.7 & 14.52 & \\
\hline \multirow{3}{*}{$\begin{array}{l}\text { MYLAR MO } \\
\mathrm{C} 1\end{array}$} & \multirow[t]{3}{*}{2.1159} & \multirow[t]{3}{*}{2.1237} & \multirow[t]{3}{*}{$0.37 \%$} & 0.500 & 94.95 & 10 & 21.7 & 18.99 & \\
\hline & & & & 0.450 & 85.05 & 10 & 21.7 & 18.90 & $-13.94 \%$ \\
\hline & & & & 0.410 & 74.35 & 10 & 21.7 & 18.13 & \\
\hline \multirow{3}{*}{$\begin{array}{l}\text { NO } 410 \\
\text { D1 }\end{array}$} & \multirow[t]{3}{*}{2.4443} & \multirow[t]{3}{*}{2.7771} & \multirow[t]{3}{*}{$13.62 \%$} & 0.451 & 85.15 & 10 & 18.7 & 18.88 & \\
\hline & & & & 0.502 & 97.40 & 10 & 18.7 & 19.40 & $0.30 \%$ \\
\hline & & & & 0.491 & 88.30 & 10 & 18.7 & 17.98 & \\
\hline \multirow{3}{*}{$\begin{array}{l}\text { NO Ml } 418 \\
\text { E1 }\end{array}$} & \multirow[t]{3}{*}{2.1762} & \multirow[t]{3}{*}{2.7255} & \multirow[t]{3}{*}{$25.24 \%$} & 0.494 & 32.75 & 10 & 7.5 & 6.63 & \\
\hline & & & & 0.510 & 31.95 & 10 & 7.5 & 6.26 & $-14.80 \%$ \\
\hline & & & & 0.474 & 29.75 & 10 & 7.5 & 6.28 & \\
\hline \multirow{3}{*}{$\begin{array}{l}\text { MEL } 228 \\
\text { F1 }\end{array}$} & \multirow{3}{*}{2.4962} & \multirow{3}{*}{2.5150} & $0.75 \%$ & .0 .521 & 87.65 & 10 & 21.7 & 16.82 & \\
\hline & & & & 0.464 & 78.25 & 10 & 21.7 & 16.86 & $-22.03 \%$ \\
\hline & & & & 0.490 & 83.65 & 10 & 21.7 & 17.07 & \\
\hline & 500 HRS & 5 in HFC. & 125/lCl Em & karox VG & 1320il@ & $260 \mathrm{~F} \cdot>24$ & tRS@3C & $2^{\circ} \mathrm{F}$ & \\
\hline NO/MY/NO & 4.3484 & 4.4336 & $1.96 \%$ & 0.375 & 127.40 & 21 & 17.4 & 16.18 & \\
\hline A2 & & & & 0.365 & 128.50 & 21 & 17.4 & 16.76 & $-4.37 \%$ \\
\hline & & & & 0.400 & 142.60 & 21 & 17.4 & 16.98 & \\
\hline$D A / M Y / D A$ & 4.3766 & 4.3461 & $-0.70 \%$ & 0.489 & 135.30 & 21 & 13.7 & 13.18 & \\
\hline$B 2$ & & & & 0.380 & 110.00 & 21 & 13.7 & 13.78 & $-0.85 \%$ \\
\hline & & & & 0.403 & 116.70 & 21 & 13.7 & 13.79 & \\
\hline MYLAR MO & 2.1783 & 2.1762 & $-0.10 \%$ & 0.482 & 87.35 & 10 & 21.7 & 18.12 & \\
\hline $\mathrm{C} 2$ & & & & 0.500 & 93.70 & 10 & 21.7 & 18.74 & $-17.65 \%$ \\
\hline & & & & 0.418 & 70.00 & 10 & 21.7 & 16.75 & \\
\hline NOMEX 410 & 2.2316 & 2.3118 & $3.59 \%$ & 0.522 & 104.90 & 9 & 18.7 & 22.33 & \\
\hline D2 & & & & 0.495 & 79.40 & 9 & 18.7 & 17.82 & $7.50 \%$ \\
\hline & & & & 0.498 & 90.35 & 9 & 18.7 & 20.16 & \\
\hline $\mathrm{NO} / \mathrm{MI} 418$ & 2.4320 & 2.4516 & $0.81 \%$ & 0.515 & 31.00 & 10 & 7.5 & 6.02 & \\
\hline E2 & & & & 0.495 & 29.25 & 10 & 7.5 & 5.91 & $-21.88 \%$ \\
\hline & & & & 0.455 & 25.70 & 10 & 7.5 & 5.65 & \\
\hline MEL 228 & 2.2831 & 2.2860 & $0.13 \%$ & 0.498 & 81.25 & 10 & 21.7 & 16.32 & \\
\hline F2 & & & & 0.522 & 89.05 & 10 & 21.7 & 17.06 & $-23.90 \%$ \\
\hline & & & & 0.464 & 75.00 & 10 & 21.7 & 16.16 & \\
\hline
\end{tabular}




\begin{tabular}{|c|c|c|c|c|c|c|c|c|}
\hline & 500 HRS & in $\mathrm{HFC}$ & $25 / \mathrm{lCl} \mathrm{Em}$ & nkarox VGi & ¿32 Oil @ & $260 \mathrm{~F}$ & & \\
\hline ID & $\begin{array}{l}\text { STRETCH } \\
\text { (inch) }\end{array}$ & $\begin{array}{c}\% \\
\text { ELONG }\end{array}$ & $\begin{array}{l}\text { BASE } \\
\text { ELONG } \\
\text { (AVE) }\end{array}$ & $\begin{array}{l}\text { ELONG \% } \\
\text { CHANGE }\end{array}$ & $\begin{array}{c}\text { BASE } \\
\text { DIE } \\
(A V E)\end{array}$ & EXPDIE & $\begin{array}{c}\text { DIE \% } \\
\text { CHANGE }\end{array}$ & VISUAL EXP \\
\hline NO/MY/NO & 0.67 & $16.8 \%$ & $20.0 \%$ & & $\triangle 18.97$ & flash & & N/C \\
\hline A1 & 0.47 & $11.8 \%$ & $20.0 \%$ & $-28.8 \%$ & $>18.97$ & flash & flash & \\
\hline & 0.71 & $17.8 \%$ & $20.0 \%$ & & $>18.97$ & flash & & \\
\hline $\mathrm{DA} / \mathrm{MY} / \mathrm{DA}$ & 0.49 & $24.5 \%$ & $46.0 \%$ & & $\geq 15.27$ & flash & & Slightly Warped \\
\hline B1 & 0.56 & $28.0 \%$ & $46.0 \%$ & $-43.1 \%$ & $>15.27$ & flash & flash & \\
\hline & 0.52 & $26.0 \%$ & $46.0 \%$ & & $\mid>15.27$ & flash & & \\
\hline MYLAR MO & 2.88 & $144.0 \%$ & $131.0 \%$ & & $\geq 14.91$ & flash & & $\mathrm{N} / \mathrm{C}$ \\
\hline C1 & 2.98 & $149.0 \%$ & $131.0 \%$ & $6.5 \%$ & $>14.91$ & flash & flash & \\
\hline & 2.51 & $125.5 \%$ & $131.0 \%$ & & $>14.91$ & flash & & \\
\hline NO 410 & 0.37 & $18.5 \%$ & $17.0 \%$ & & 10.67 & flash & & $N / C$ \\
\hline D1 & 0.39 & $9.8 \%$ & $17.0 \%$ & $-28.4 \%$ & 10.67 & flash & flash & \\
\hline & 0.33 & $8.3 \%$ & $17.0 \%$ & & 10.67 & flash & & \\
\hline NO MI 418 & 0.04 & $1.0 \%$ & $4.0 \%$ & & 10.23 & 11.85 & & $\mathrm{~N} / \mathrm{C}$ \\
\hline & 0.04 & $1.0 \%$ & $4.0 \%$ & $.75 .0 \%$ & 10.23 & 11.76 & $15.1 \%$ & \\
\hline & 0.04 & $1.0 \%$ & $4.0 \%$ & & 10.23 & 11.70 & & \\
\hline MEL 228 & 3.01 & $150.5 \%$ & $160.0 \%$ & & $>14.22$ & flash & & slightly \\
\hline & 3.27 & $16: 5 \%$ & $160.0 \%$ & $2.3 \%$ & $>14.22$ & flash & flash & yellowed \\
\hline & 3.54 & $177.0 \%$ & $160.0 \%$ & & $\mid>14.22$ & flash & & \\
\hline & 500 HAS & in HFC- & 25/IClEm & nkarox VG & 332 Oil@ & $260 \mathrm{~F}$ & $>24$ HRS & @ $302^{\circ} \mathrm{F}$ \\
\hline NO/MY/NO & 0.51 & $12.8 \%$ & $20.0 \%$ & & $>18.97$ & flash & & Bubbles \\
\hline$A 2$ & 0.51 & $12.8 \%$ & $20.0 \%$ & $-37.1 \%$ & $>18.97$ & flash & flash & pockets \\
\hline & 0.49 & $12.3 \%$ & $20.0 \%$ & & $>18.97$ & flash & & \\
\hline $\mathrm{DA} / \mathrm{MY} / \mathrm{DA}$ & 0.49 & $24.5 \%$ & $46.0 \%$ & & $>15.27$ & flash & & Slighlty \\
\hline B2 & 0.56 & $28.0 \%$ & $46.0 \%$ & $-42.4 \%$ & $>15.27$ & flash & flash & warped \\
\hline & 0.54 & $27.0 \%$ & $46.0 \%$ & & $>15.27$ & flash & & darkened \\
\hline MYLAR MO & 2.87 & $143.5 \%$ & $131.0 \%$ & & $>14.91$ & flash & & $\mathrm{N} / \mathrm{C}$ \\
\hline $\mathrm{C}_{2}$ & 3.12 & $156.0 \%$ & $131.0 \%$ & $2.8 \%$ & $>14.91$ & flash & flash & \\
\hline & 2.09 & $104.5 \%$ & $131.0 \%$ & & $>14.91$ & flash & & \\
\hline NOMEX 410 & 0.46 & $11.5 \%$ & $17.0 \%$ & & 10.67 & 10.91 & & $\mathrm{~N} / \mathrm{C}$ \\
\hline D2 & 0.20 & $5.0 \%$ & $17.0 \%$ & $-52.0 \%$ & 10.67 & 6.15 & $-12.3 \%$ & \\
\hline & 0.32 & $8.0 \%$ & $17.0 \%$ & & 10.67 & 11.02 & & \\
\hline $\mathrm{NO} / \mathrm{MI} 418$ & 0.04 & $1.0 \%$ & $4.0 \%$ & & 10.23 & 9.43 & & $N / C$ \\
\hline & 0.04 & $1.0 \%$ & $4.0 \%$ & $-77.1 \%$ & 10.23 & 10.41 & $-2.5 \%$ & \\
\hline & 0.03 & $0.8 \%$ & $4.0 \%$ & & 10.23 & 10.07 & & \\
\hline MEL 228 & 2.39 & $119.5 \%$ & $160.0 \%$ & & $>14.22$ & flash & & slightly \\
\hline F2 & 2.94 & $147.0 \%$ & $160.0 \%$ & $-27.1 \%$ & $>14.22$ & flash & flash & yellowed \\
\hline & 1.67 & $83.5 \%$ & $160.0 \%$ & & $\mid>14.22$ & flash & & \\
\hline
\end{tabular}


SLEEVING--HFC-125//CI Emkarox VG 32 Oil @ 260 $\mathrm{F}\left(127^{\circ} \mathrm{C}\right)$

\begin{tabular}{|c|c|c|c|c|}
\hline & \multicolumn{4}{|c|}{$500 \mathrm{HRS}$ in HFC-125/ICl -mkarox VG 32 @ 260 $\mathrm{F}$} \\
\hline & WT & EXPWT & $\begin{array}{c}\% \\
\text { CHANGE }\end{array}$ & EXPVIS \\
\hline $\begin{array}{l}\text { A1 } \\
\text { NOMEX }\end{array}$ & 0.5457 & 0.6185 & $13.34 \%$ & $\mathrm{~N} / \mathrm{C}$ \\
\hline $\begin{array}{l}\text { B1 } \\
\text { MYLAR }\end{array}$ & 0.4748 & 0.4812 & $1.35 \%$ & \begin{tabular}{|c|} 
small Pockets \\
vhen rubbed between fingers \\
e bacame cloudy in appearance
\end{tabular} \\
\hline $\begin{array}{l}\text { C1 } \\
\text { NO/MY }\end{array}$ & 0.3935 & 0.4124 & $4.80 \%$ & $\mathrm{~N} / \mathrm{C}$ \\
\hline & $\mathrm{HFC}-125 / \mathrm{ICl}$ & Emkarox VG & 32 oil @ & $60^{\circ} \mathrm{F}$ plus24 hrs@302\% F \\
\hline $\begin{array}{l}\text { A2 } \\
\text { NOMEX }\end{array}$ & 0.5432 & 0.5745 & $5.76 \%$ & $\mathrm{~N} / \mathrm{C}$ \\
\hline $\mathrm{B2}$ & 0.4432 & 0.4451 & $0.43 \%$ & some pockets \\
\hline & & & & Darkened \\
\hline & 0.3956 & $0.398 i]$ & $0.61 \%$ & slightly darkened \\
\hline $\mathrm{NO} / \mathrm{MY}$ & & & & $\frac{\text { some pockets where mylar }}{\text { pulled away. }}$ \\
\hline
\end{tabular}




\begin{tabular}{|c|c|c|c|c|c|c|c|c|c|}
\hline & 500 HRS & in HFC-12 & 5/lCl Emka & rox VG32 & @260 F & & & & \\
\hline $\mathrm{ID}$ & WT & EXPWT & $\begin{array}{c}\text { WT \% } \\
\text { CHANGE }\end{array}$ & $\begin{array}{l}\text { BREAK } \\
\text { LOAD } \\
\text { (AVE) }\end{array}$ & $\begin{array}{c}\text { BREAK } \\
\text { LOAD } \\
\text { EPP }\end{array}$ & $\begin{array}{c}\% \\
\text { CHANGE } \\
\text { BPK } \\
\text { LOAD }\end{array}$ & & $\%$ EONG & EXPVIS \\
\hline A 1 & 1.7216 & 1.7184 & $.0 .19 \%$ & 39.02 & 52.85 & & 0.05 & $2.50 \%$ & $\mathrm{~N} / \mathrm{C}$ \\
\hline Glass & & & & 39.02 & 53.20 & $30.62 \%$ & 0.04 & $2.00 \%$ & \\
\hline & & & & 39.02 & 46.85 & & 0.07 & $3.50 \%$ & \\
\hline B1 & 0.7252 & 0.7318 & $0.91 \%$ & 56.12 & 50.70 & & 0.44 & $22.00 \%$ & $\mathrm{~N} / \mathrm{C}$ \\
\hline Polyester & & & & 56.12 & 64.15 & $3.14 \%$ & 0.84 & $42.00 \%$ & \\
\hline & & & & 56.12 & 58.80 & & 0.61 & $30.50 \%$ & \\
\hline C1 & 1.4849 & 1.7065 & $14.92 \%$ & 88.50 & 47.15 & & 0.16 & $8.00 \%$ & curled up \\
\hline Permacel & & & & 88.50 & 43.80 & $-46.85 \%$ & 0.13 & $6.50 \%$ & \\
\hline & & & & 88.50 & 50.15 & & 0.16 & $8.00 \%$ & \\
\hline & $500 \mathrm{HR}$ & $S$ in HFC- & 125/lCl Eml & arox VG3 & @ @260 F & $>302^{\circ} \mathrm{F} 2$ & ARS & & \\
\hline$A 2$ & 1.6798 & 1.6769 & $-0.17 \%$ & 39.02 & 59.90 & & 0.04 & $2.00 \%$ & $\mathrm{~N} / \mathrm{C}$ \\
\hline Glass & & & & 39.02 & 50.75 & $26.39 \%$ & 0.06 & $3.00 \%$ & \\
\hline & & & & 39.02 & 37.30 & & 0.04 & $2.00 \%$ & \\
\hline$B 2$ & 0.6955 & 0.6939 & $-0.23 \%$ & 56.12 & 52.55 & & 0.55 & $27.50 \%$ & $\mathrm{~N} / \mathrm{C}$ \\
\hline Polyester & & & & 56.12 & 53.60 & $-0.45 \%$ & 0.48 & $24.00 \%$ & \\
\hline & & & & 56.12 & 61.45 & & 0.73 & $36.50 \%$ & \\
\hline $\mathrm{C} 2$ & 1.4702 & 1.2551 & $-14.63 \%$ & 88.50 & 86.05 & & 0.10 & $5.00 \%$ & Slightly \\
\hline Permacel & & & & 88.50 & 83.35 & $-2.49 \%$ & 0.10 & $5.00 \%$ & darkened \\
\hline & & & & 88.50 & 89.50 & & 0.09 & $4.50 \%$ & Curled up \\
\hline
\end{tabular}




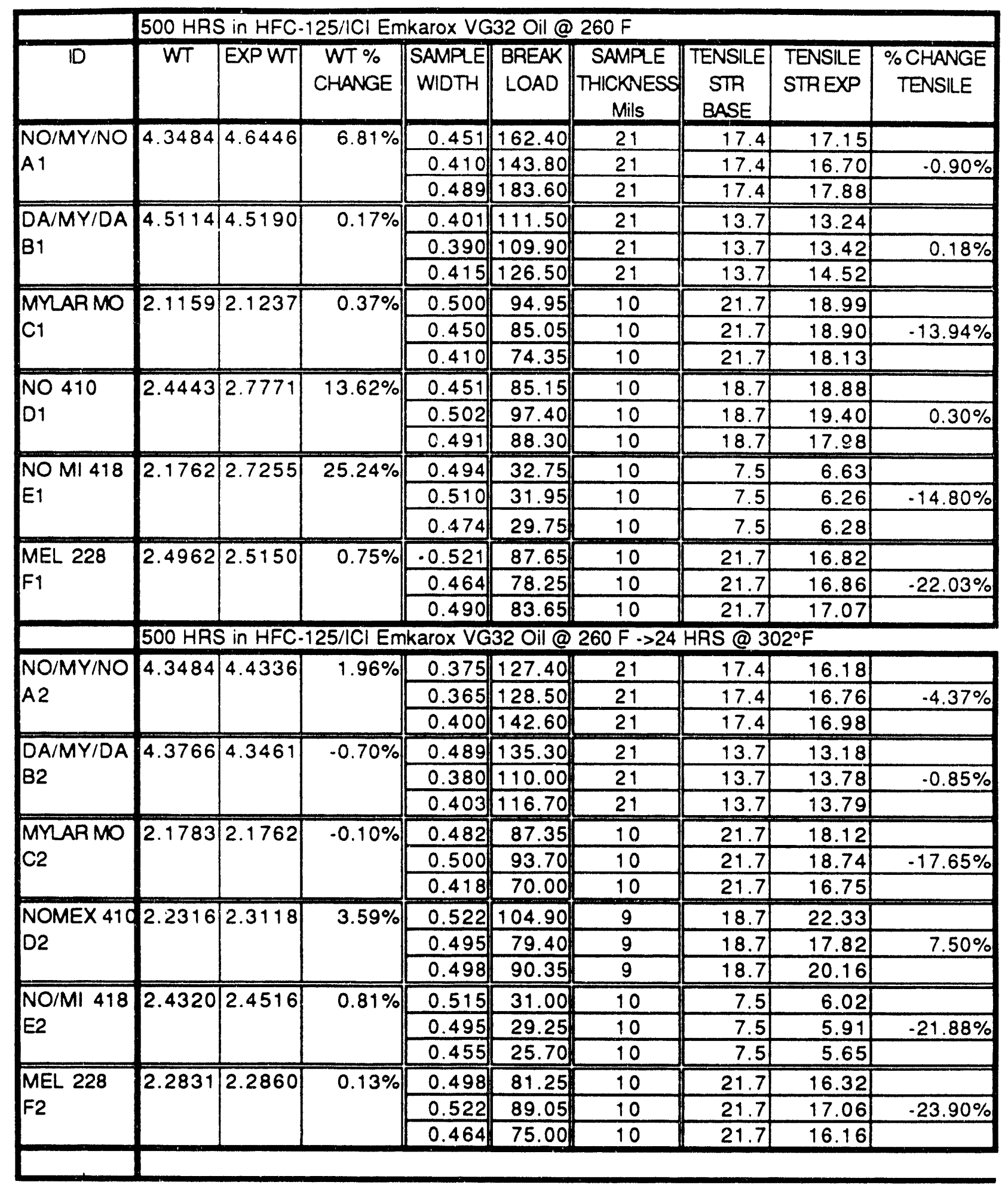




\begin{tabular}{|c|c|c|c|c|c|c|c|c|}
\hline & $500 \mathrm{HRS}$ & in $\mathrm{HFC}$ & $25 / \mid \mathrm{Cl} E \mathrm{~m}$ & nkarox VG: & 32 Oil@ @ & $260 \mathrm{~F}$ & & \\
\hline ID & $\begin{array}{c}\text { STRETCH } \\
\text { (inch) }\end{array}$ & $\begin{array}{c}\% \\
\text { ELONG }\end{array}$ & $\begin{array}{l}\text { BASE } \\
\text { ELONG } \\
\text { (AVE) }\end{array}$ & $\begin{array}{l}\text { ELONG \% } \\
\text { CHANGE }\end{array}$ & \begin{tabular}{|c|} 
BASE \\
DEE \\
$(\mathrm{AVE})$ \\
\end{tabular} & EXPDIE & $\begin{array}{c}\text { DIE \% } \\
\text { CHANGE }\end{array}$ & VISUAL EXP \\
\hline NO/MY/NO & 0.67 & $16.8 \%$ & $20.0 \%$ & & $>18.97$ & flash & & $\mathrm{N} / \mathrm{C}$ \\
\hline & 0.47 & $11.8 \%$ & $20.0 \%$ & $.28 .8 \%$ & $>18.97$ & flash & flash & \\
\hline & 0.71 & $17.8 \%$ & $20.0 \%$ & & $>18.97$ & flash & & \\
\hline $\mathrm{DA} / \mathrm{MY} / \mathrm{DA}$ & 0.49 & $24.5 \%$ & $46.0 \%$ & & $>15.27$ & flash & & Slightly Warped \\
\hline & 0.56 & $28.0 \%$ & $46.0 \%$ & $.43 .1 \%$ & $>15.27$ & flash & flash & \\
\hline & 0.52 & $26.0 \%$ & $46.0 \%$ & & $>15.27$ & flash & & \\
\hline MYLAR MO & 2.88 & $144.0 \%$ & $131.0 \%$ & & $>14.91$ & flash & & $\mathrm{N} / \mathrm{C}$ \\
\hline $\mathrm{C}_{1}$ & 2.98 & $149.0 \%$ & $131.0 \%$ & $6.5 \%$ & $\geq 14.91$ & flash & flash & \\
\hline & 2.51 & $125.5 \%$ & $131.0 \%$ & & $>14.91$ & flash & & \\
\hline NO 410 & 0.37 & $18.5 \%$ & $17.0 \%$ & & \begin{tabular}{|l|}
10.67 \\
\end{tabular} & flash & & $\mathrm{N} / \mathrm{C}$ \\
\hline D1 & 0.39 & $9.8 \%$ & $17.0 \%$ & $.28 .4 \%$ & 10.67 & flash & flash & \\
\hline & 0.33 & $8.3 \%$ & $17.0 \%$ & & 10.67 & flash & & \\
\hline NO MI 418 & 0.04 & $1.0 \%$ & $4.0 \%$ & & 10.23 & 11.85 & & $\mathrm{~N} / \mathrm{C}$ \\
\hline E1 & 0.04 & $1.0 \%$ & $4.0 \%$ & $.75 .0 \%$ & 10.23 & 11.76 & $15.1 \%$ & \\
\hline & 0.04 & $1.0 \%$ & $4.0 \%$ & & 10.23 & 11.70 & & \\
\hline MEL 22:8 & 3.01 & $150.5 \%$ & $160.0 \%$ & & $>14.22$ & flash & & slightly \\
\hline & 3.27 & $16 \div 5 \%$ & $160.0 \%$ & $2.3 \%$ & $\geq 14.22$ & flash & flash & yellowed \\
\hline & 3.54 & $177.0 \%$ & $160.0 \%$ & & $>14.22$ & flash & & \\
\hline & 500 HRS & in HFC- & $25 / 1 \mathrm{Cl} E \mathrm{~m}$ & nkarox VG & $3320 i l @$ & $260 \mathrm{~F}$. & $>24$ HRS & @ $302^{\circ} \mathrm{F}$ \\
\hline NO/MY/NO & 0.51 & $12.8 \%$ & $20.0 \%$ & & $>18.97$ & flash & & Bubbles \\
\hline A2 & 0.51 & $12.8 \%$ & $20.0 \%$ & $.37 .1 \%$ & $\geq 18.97$ & flash & flash & pockets \\
\hline & 0.49 & $12.3 \%$ & $20.0 \%$ & & $>18.97$ & flash & & \\
\hline$D A / M Y / D A$ & 0.49 & $24.5 \%$ & $46.0 \%$ & & $>15.27$ & flash & & Slighlty \\
\hline & 0.56 & $28.0 \%$ & $46.0 \%$ & $-42.4 \%$ & $>15.27$ & flash & flash & warped \\
\hline & 0.54 & $27.0 \%$ & $46.0 \%$ & & $>15.27$ & flash & & darkened \\
\hline MYLAR MO & 2.87 & $143.5 \%$ & $131.0 \%$ & & $>14.91$ & flash & & $\mathrm{N} / \mathrm{C}$ \\
\hline & 3.12 & $\mid 156.0 \%$ & $131.0 \%$ & $2.8 \%$ & $>14.9 i$ & fflash & flash & \\
\hline & 2.09 & $104.5 \%$ & $131.0 \%$ & & $>14.91$ & flash & & \\
\hline NOMEX 410 & 0.46 & $11.5 \%$ & $17.0 \%$ & & 10.67 & 10.91 & & $N / C$ \\
\hline D2 & 0.20 & $5.0 \%$ & $17.0 \%$ & $-52.0 \%$ & 10.67 & 6.15 & $-12.3 \%$ & \\
\hline & 0.32 & $8.0 \%$ & $17.0 \%$ & & 10.67 & 11.02 & & \\
\hline $\mathrm{NO} / \mathrm{MI} 418$ & 0.04 & $1.0 \%$ & $4.0 \%$ & & 10.23 & 9.43 & & $N / C$ \\
\hline E2 & 0.04 & $1.0 \%$ & $4.0 \%$ & $.77 .1 \%$ & 10.23 & 10.41 & $-2.5 \%$ & \\
\hline & 0.03 & $0.8 \%$ & $4.0 \%$ & & 10.23 & 10.07 & & \\
\hline MEL 228 & 2.39 & $119.5 \%$ & $160.0 \%$ & & $>14.22$ & flash & & slightly \\
\hline F2 & 2.94 & $147.0 \%$ & $160.0 \%$ & $.27 .1 \%$ & $>14.22$ & flash & flash & yellowed \\
\hline & 1.67 & $83.5 \%$ & $160.0 \%$ & & $>14.22$ & fflash & & \\
\hline
\end{tabular}


SLEEVING-.HFC-125/ICI Emkarox VG 32 Oil @ 260 $\mathrm{F}\left(127^{\circ} \mathrm{C}\right)$

$500 \mathrm{HRS}$ in HFC-125/lCl Emkarox VG 32 @260 $\mathrm{F}$

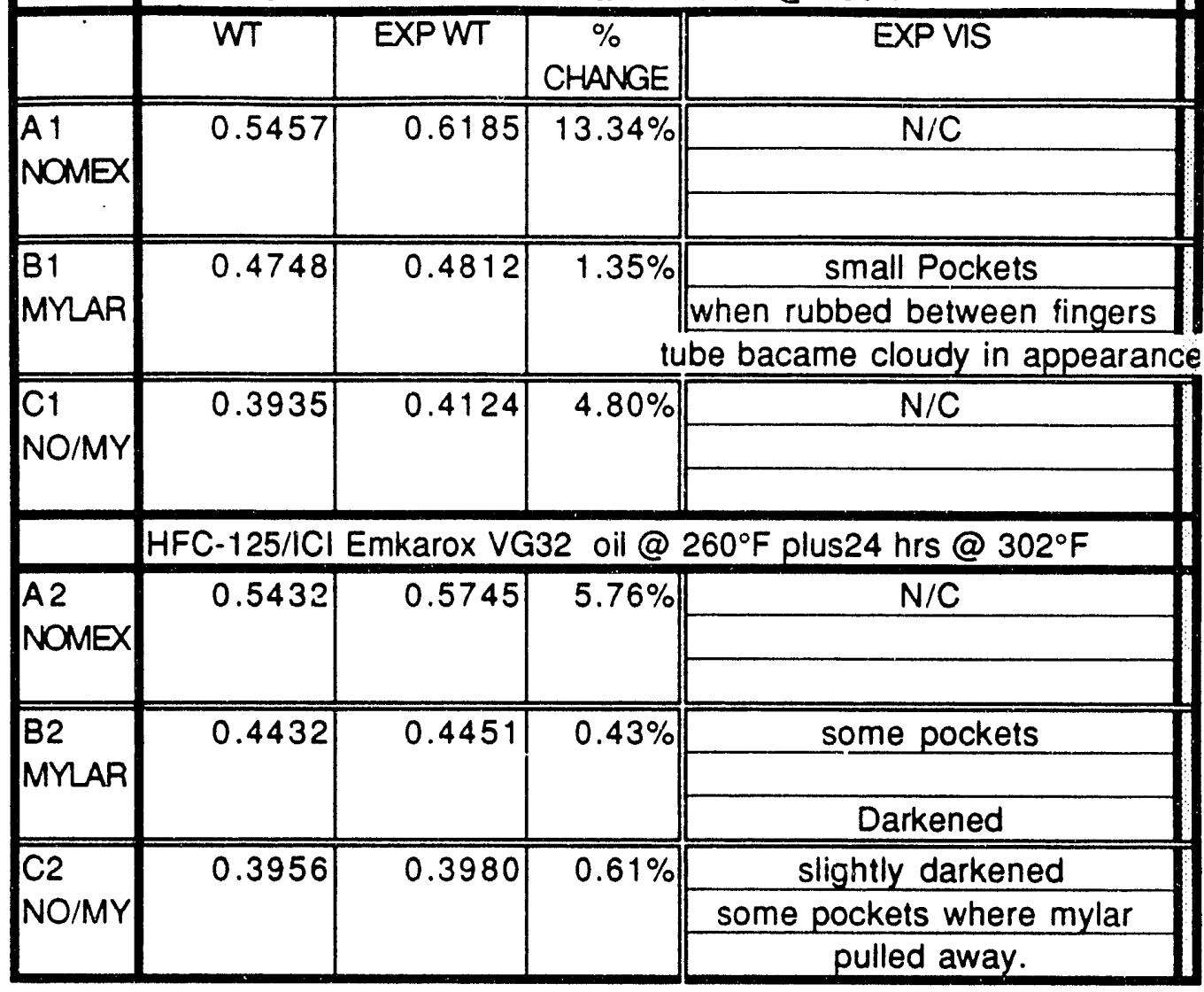




\begin{tabular}{|c|c|c|c|c|c|c|c|c|c|}
\hline & 500 HRS & in $\mathrm{HFC}-12$ & 5/lCl Emka & ox VG32 & @ $260 \mathrm{~F}$ & & & & \\
\hline ID & WT & EXPWT & $\begin{array}{c}\text { WT } \% \\
\text { CHANGE }\end{array}$ & $\begin{array}{l}\text { BREAK } \\
\text { LOAD } \\
\text { (AVE) }\end{array}$ & $\begin{array}{c}\text { BREAK } \\
\text { LOAD } \\
\text { EPP }\end{array}$ & $\begin{array}{c}\% \\
\text { CHANGE } \\
\text { BRK } \\
\text { LOAD }\end{array}$ & & \%ELONG & EXP VIS \\
\hline$A 1$ & 1.7216 & 1.7184 & $-0.19 \%$ & 39.02 & 52.85 & & 0.05 & $2.50 \%$ & $\mathrm{~N} / \mathrm{C}$ \\
\hline Glass & & & & 39.02 & 53.20 & $30.62 \%$ & 0.04 & $2.00 \%$ & \\
\hline & & & & 39.02 & 46.85 & & 0.07 & $3.50 \%$ & \\
\hline$B 1$ & 0.7252 & 0.7318 & $0.91 \%$ & 56.12 & 50.70 & & 0.44 & $22.00 \%$ & $\mathrm{~N} / \mathrm{C}$ \\
\hline Polyester & & & & 56.12 & 64.15 & $3.14 \%$ & 0.84 & $42.00 \%$ & \\
\hline & & & & 56.12 & 58.80 & & 0.61 & $30.50 \%$ & \\
\hline $\mathrm{C}_{1}$ & 1.4849 & 1.7065 & $14.92 \%$ & 88.50 & 47.15 & & 0.16 & $8.00 \%$ & curled up \\
\hline Permacel & & & & 88.50 & 43.80 & $-46.85 \%$ & 0.13 & $6.50 \%$ & \\
\hline & & & & 88.50 & 50.15 & & 0.16 & $8.00 \%$ & \\
\hline & $500 \mathrm{HRS}$ & $S$ in HFC. & 25//Cl Emk & arox VG3 & @ 260 F & $>302^{\circ} \mathrm{F} 2$ & ARS & & \\
\hline $\mathrm{A} 2$ & 1.6798 & 1.6769 & $-0.17 \%$ & 39.02 & 59.90 & & 0.04 & $2.00 \%$ & $\mathrm{~N} / \mathrm{C}$ \\
\hline Glass & & & & 39.02 & 50.75 & $26.39 \%$ & 0.06 & $3.00 \%$ & \\
\hline & & & & 39.02 & 37.30 & & 0.04 & $2.00 \%$ & \\
\hline $\mathrm{B2}$ & 0.6955 & 0.6939 & $-0.23 \%$ & 56.12 & 52.55 & & 0.55 & $27.50 \%$ & $\mathrm{~N} / \mathrm{C}$ \\
\hline Polyester & & & & 56.12 & 53.60 & $-0.45 \%$ & 0.48 & $24.00 \%$ & \\
\hline & & & & 56.12 & 61.45 & & 0.73 & $36.50 \%$ & \\
\hline $\mathrm{C2}$ & 1.4702 & 1.2551 & $-14.63 \%$ & 88.50 & 86.05 & & 0.10 & $5.00 \%$ & Slightly \\
\hline Permacel & & & & 88.50 & 83.35 & $-2.49 \%$ & 0.10 & $5.00 \%$ & darkened \\
\hline & & & & 88.50 & 89.50 & & 0.09 & $4.50 \%$ & Curled up \\
\hline
\end{tabular}


TIE CORD--HFC-125/ICI Emkarox VG32 oil @ $260^{\circ} \mathrm{F}\left(127^{\circ} \mathrm{C}\right)$

\begin{tabular}{|c|c|c|c|c|c|c|c|c|c|}
\hline \multicolumn{10}{|c|}{500 HRS IN R-125/ICI Emkarox VG32 @ 260 F } \\
\hline ID & WT & EXPWT & $\begin{array}{c}\text { WT \% } \\
\text { CHANGE }\end{array}$ & $\begin{array}{l}\text { EXP } \\
\text { VIS }\end{array}$ & $\begin{array}{l}\text { BREAK } \\
\text { LOAD } \\
\text { (AVE) }\end{array}$ & $\begin{array}{c}\text { BREAK } \\
\text { LOAD } \\
\text { EXP } \\
(\text { ave })\end{array}$ & $\begin{array}{c}\% \\
\text { CHANGE } \\
\text { BRK } \\
\text { LOAD }\end{array}$ & $\begin{array}{c}\text { STRETCH } \\
\text { (Inch) }\end{array}$ & $\begin{array}{c}\% \\
\text { ELONG }\end{array}$ \\
\hline \multirow[t]{4}{*}{ A 1} & \multirow[t]{3}{*}{0.3560} & \multirow[t]{3}{*}{0.3569} & \multirow[t]{3}{*}{$0.25 \%$} & $\mathrm{~N} / \mathrm{C}$ & 28.36 & 31.10 & & 0.37 & $18.5 \%$ \\
\hline & & & & & 28.36 & 32.00 & $10.90 \%$ & 0.39 & $19.5 \%$ \\
\hline & & & & & 28.36 & 31.25 & & 0.40 & $20.0 \%$ \\
\hline & \multicolumn{9}{|c|}{500 HRS IN R-125/ICl Emkarox VG32 -> 24 HRS @ 302 $\mathrm{F}$} \\
\hline \multirow[t]{3}{*}{$A 2$} & \multirow[t]{3}{*}{0.3250} & \multirow[t]{3}{*}{0.3222} & \multirow[t]{3}{*}{$-0.862 \%$} & $\mathrm{~N} / \mathrm{C}$ & 28.36 & 30.65 & & 0.38 & $19.0 \%$ \\
\hline & & & & & 28.36 & 30.65 & $10.72 \%$ & 0.39 & $19.5 \%$ \\
\hline & & & & & 28.36 & 32.90 & & 0.41 & $20.5 \%$ \\
\hline
\end{tabular}




\begin{tabular}{|l|r|r|r||r||r|r|r|}
\hline & 500 HRS IN HFC-125/ICI Emkarox VG32 @ 260 F & & \\
\hline ID & WT & EXPWT & $\begin{array}{c}\text { WT \% } \\
\text { CHANGE }\end{array}$ & EXP VIS & $\begin{array}{r}\text { BASE DIE } \\
\text { (AVE) }\end{array}$ & EXP DIE & $\begin{array}{c}\text { DIE\% } \\
\text { CHANGE }\end{array}$ \\
\hline DMD & 3.9326 & 4.0754 & $3.63 \%$ & N/C & 9.61 & 8.39 & \\
\hline A1 & & & & & 9.61 & 8.11 & $-11.17 \%$ \\
\hline \hline DTMD & 4.2722 & 4.4021 & $3.04 \%$ & N/C & 9.95 & 12.31 & \\
\hline B1 & & & & & 9.95 & 13.96 & $26.16 \%$ \\
\hline & & & & & 9.95 & 11.39 & \\
\hline & & & & & & & \\
\hline DMD & 4.0440 & 4.1207 & $1.90 \%$ & N/C & 9.61 & 9.17 & \\
\hline A2 & & & & & 9.61 & 8.74 & $-5.52 \%$ \\
\hline & & & & & 9.61 & 9.33 & \\
\hline DTMD & 4.2830 & 4.3076 & $0.57 \%$ & N/C & 9.95 & 9.89 & \\
\hline B2 & & & & & 9.95 & 7.74 & $-9.82 \%$ \\
\hline & & & & & 9.95 & 9.29 & \\
\hline
\end{tabular}




\section{Appendix P}

Experimental Data for HFC-134a/Allied Signal BRL-150 Exposure at $126^{\circ} \mathrm{C}\left(260^{\circ} \mathrm{F}\right)$ 


\begin{tabular}{|c|c|c|c|c|c|c|c|c|c|c|}
\hline & 500 hours & HFC-13 & I/BRL-15 & oil at & $0^{\circ} \mathrm{F}\left(127^{\circ} \mathrm{C}\right.$ & & & & & \\
\hline ID & WT & EXP WT & $\begin{array}{c}\text { WT \% } \\
\text { CHANGE }\end{array}$ & EXP VIS & $\begin{array}{c}\text { BASE BRN } \\
\text { OUT } \\
\text { (AVE) }\end{array}$ & $\begin{array}{c}\text { EXP BRN } \\
\text { OUT }\end{array}$ & $\begin{array}{c}\text { BRN OUT } \\
\% \\
\text { CHANGE }\end{array}$ & $\begin{array}{c}\text { BASE DIE } \\
\text { (AVE) }\end{array}$ & EXPDIE & $\begin{array}{c}\text { DIE \% } \\
\text { CHANGE }\end{array}$ \\
\hline$A 1$ & 23.4786 & 23.4927 & $0.060 \%$ & $\mathrm{~N} / \mathrm{C}$ & 576 & 472 & & 15.80 & 11.66 & \\
\hline & & & & & 576 & 540 & $-9.9 \%$ & 15.80 & 16.44 & $-14.4 \%$ \\
\hline & & & & & 576 & 545 & & 15.80 & 12.46 & \\
\hline 81 & 24.0141 & 24.0714 & $0.239 \%$ & $\mathrm{~N} / \mathrm{C}$ & 736 & 736 & & 11.62 & 14.61 & \\
\hline & & & & & 736 & 728 & $-0.6 \%$ & 11.62 & 13.95 & $23.6 \%$ \\
\hline & & & & & 736 & 730 & & 11.62 & 14.51 & \\
\hline C1 & 22.0007 & 22.0146 & $0.063 \%$ & $\mathrm{~N} / \mathrm{C}$ & 579 & 593 & & 16.58 & 12.97 & \\
\hline & & & & & 579 & 572 & $-9.6 \%$ & 16.58 & 15.91 & $-12.6 \%$ \\
\hline & & & & & 579 & 406 & & 16.58 & 14.61 & \\
\hline & 500 hours i & HFC-134 & /BRL-150 & oil at 2 & $60^{\circ} \mathrm{F}\left(127^{\circ} \mathrm{C}\right.$ & plus 24 & hours at 3 & $302^{\circ} \mathrm{F}(150$ & & \\
\hline$A 2$ & 23.9117 & 23.9149 & $0.013 \%$ & $\mathrm{~N} / \mathrm{C}$ & 576 & 538 & & 15.80 & 14.86 & \\
\hline & & & & & 576 & 531 & $-7.8 \%$ & 15.80 & 12.90 & $-8.5 \%$ \\
\hline & & & & & 576 & 524 & & 15.80 & 15.60 & \\
\hline$B 2$ & 24.0572 & 24.0693 & $0.050 \%$ & $\mathrm{~N} / \mathrm{C}$ & 736 & 734 & & 11.62 & 11.39 & \\
\hline & & & & & 736 & 731 & $-0.9 \%$ & 11.62 & 11.10 & $1.1 \%$ \\
\hline & & & & & 736 & 724 & & 11.62 & 12.76 & \\
\hline $\mathrm{C2}$ & 22.9357 & 22.9411 & $0.024 \%$ & $\mathrm{~N} / \mathrm{C}$ & 579 & 555 & & 16.58 & 13.06 & \\
\hline & & & & & 579 & 579 & $-5.6 \%$ & 16.58 & 16.33 & $-14.9 \%$ \\
\hline & & & & & 579 & 505 & & 16.58 & 12.92 & \\
\hline
\end{tabular}




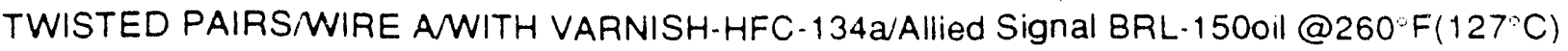

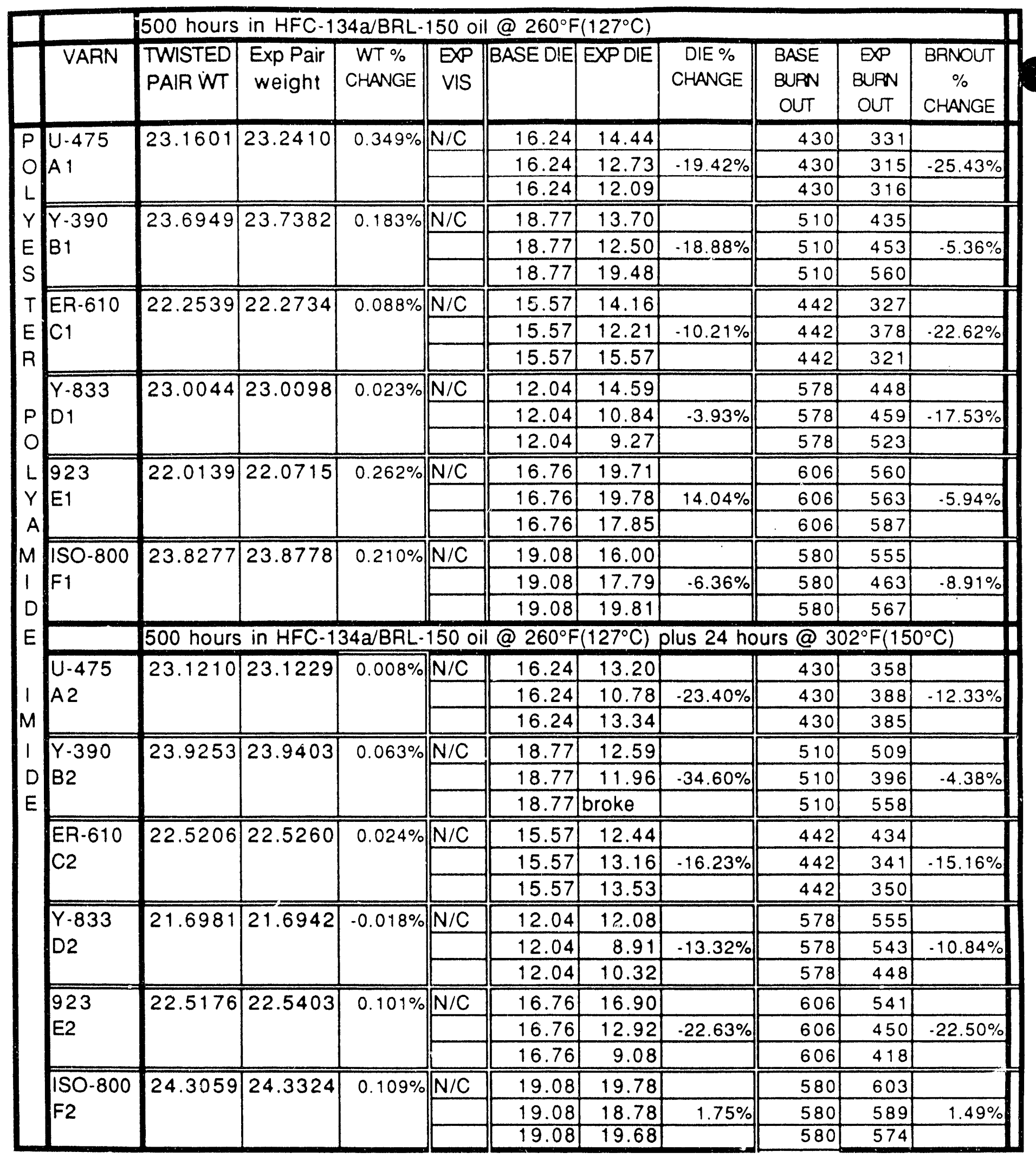


TWISTED PAIRSMIRE BMITH VARNISH -HFC-134a/Allied Signal BRL-150 oil @260 $\mathrm{F}\left(127^{\circ} \mathrm{C}\right)$

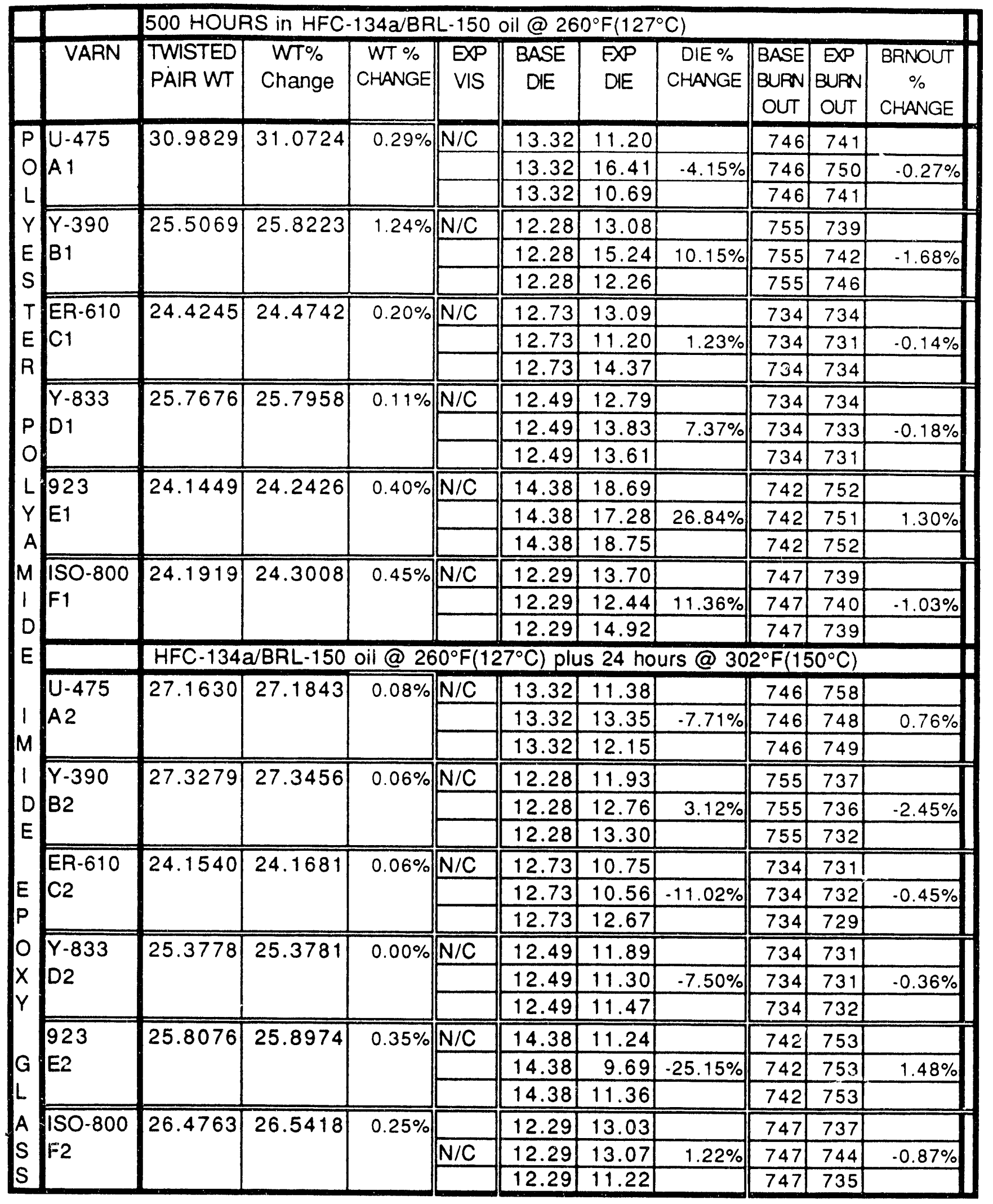




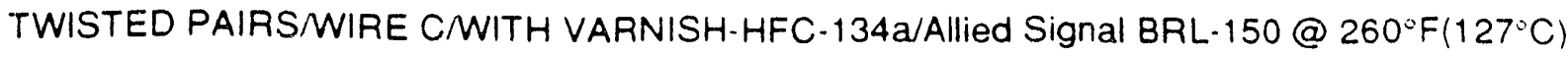

\begin{tabular}{|c|c|c|c|c|c|c|c|c|c|c|c|}
\hline & & 500 Hours & $S$ in $H F C-1$ & $34 \mathrm{a} / \mathrm{BRL}-$ & $150 @$ & $260^{\circ} \mathrm{F}(1$ & $127^{\circ} \mathrm{F}$ ) & & & & \\
\hline & VARN & $\begin{array}{l}\text { TWSTED } \\
\text { PAIR WT }\end{array}$ & $\begin{array}{c}\text { EXPT } \\
\text { PAIR WT }\end{array}$ & $\begin{array}{c}\text { WT \% } \\
\text { CHANGE }\end{array}$ & $\begin{array}{l}\text { EXP } \\
\text { VIS }\end{array}$ & $\begin{array}{l}\text { BASE } \\
\text { DIE }\end{array}$ & $\begin{array}{l}\text { EXP } \\
\text { DIE }\end{array}$ & $\begin{array}{c}\text { DIE \% } \\
\text { CHANGE }\end{array}$ & \begin{tabular}{|l|} 
BASE \\
BUPN \\
OUT
\end{tabular} & \begin{tabular}{|} 
EXP \\
BUPN \\
OUT
\end{tabular} & $\begin{array}{c}\text { BRNOUT } \\
\% \\
\text { CHANGE }\end{array}$ \\
\hline$P$ & U-475 & 24.0458 & 24.0830 & $0.155 \%$ & $\mathrm{~N} / \mathrm{C}$ & 15.10 & 17.07 & & 469 & 507 & \\
\hline 0 & A 1 & & & & & 15.10 & 15.86 & $14.61 \%$ & 469 & 428 & $-2.13 \%$ \\
\hline$L$ & & & & & & 15.10 & 18.99 & & 469 & 442 & \\
\hline Y & $Y-390$ & 23.5241 & 23.5929 & $0.292 \%$ & $\mathrm{~N} / \mathrm{C}$ & 18.24 & 19.99 & & 473 & 493 & \\
\hline$E$ & 81 & & & & & 18.24 & 19.77 & $1.48 \%$ & 473 & 568 & $13.46 \%$ \\
\hline $\mathrm{S}$ & & & & & & 18.24 & 15.77 & & 473 & 549 & \\
\hline $\mathrm{T}$ & ER-610 & 22.6079 & 22.6238 & $0.070 \%$ & $\mathrm{~N} / \mathrm{C}$ & 14.53 & 14.26 & & 494 & 304 & \\
\hline$E$ & C1 & & & & & 14.53 & 12.60 & $-5.39 \%$ & 494 & 347 & $-36.98 \%$ \\
\hline$R$ & & & & & & \begin{tabular}{|l|}
4.53 \\
\end{tabular} & 14.38 & & 494 & 283 & \\
\hline & $Y-833$ & 23.4045 & 23.4003 & $-0.018 \%$ & $\mathrm{~N} / \mathrm{C}$ & 11.38 & 8.63 & & 557 & 553 & \\
\hline 1 & D1 & & & & & 11.38 & 11.39 & $-23.73 \%$ & 557 & 532 & $-1.08 \%$ \\
\hline$M$ & & & & & & 11.38 & 6.02 & & 557 & 568 & \\
\hline 1 & 923 & 23.1189 & 23.1823 & $0.274 \%$ & $\mathrm{~N} / \mathrm{C}$ & 15.85 & 11.12 & & 503 & 574 & \\
\hline$D$ & $\mathrm{E} 1$ & & & & & 15.85 & 18.10 & $-25.09 \%$ & 503 & 553 & $14.84 \%$ \\
\hline$E$ & & & & & & 15.85 & 6.40 & & 503 & 606 & \\
\hline & $150-800$ & 23.5525 & 23.6103 & $0.245 \%$ & $\mathrm{~N} / \mathrm{C}$ & 14.75 & 16.97 & & 632 & 585 & \\
\hline$P$ & $F 1$ & & & & & 14.75 & 19.87 & $27.71 \%$ & 632 & 586 & $-5.33 \%$ \\
\hline 0 & & & & & & 14.75 & 19.67 & & 632 & 624 & \\
\hline$L$ & & HFC-134 & a/BRL-15 & @ 260 $\mathrm{F}$ & plus : & 4 hours & @ 302 & ${ }^{\circ} \mathrm{F}\left(150^{\circ} \mathrm{C}\right.$ & & & \\
\hline Y & $U-475$ & 24.3352 & 24.3418 & $0.027 \%$ & $\mathrm{~N} / \mathrm{C}$ & 15.10 & 11.25 & & 469 & 559 & \\
\hline A & A 2 & & & & & 15.10 & 15.00 & $-11.59 \%$ & 469 & 520 & $-0.78 \%$ \\
\hline$M$ & & & & & & 15.10 & 13.80 & & 469 & 317 & \\
\hline 1 & $Y-390$ & 24.5380 & 24.5534 & $0.063 \%$ & $\mathrm{~N} / \mathrm{C}$ & 18.24 & 17.53 & & 473 & 420 & \\
\hline$D$ & B2 & & & & & 18.21 & 15.00 & $-4.77 \%$ & 473 & 529 & $9.44 \%$ \\
\hline$E$ & & & & & & 18.24 & 19.74 & & 473 & 604 & \\
\hline & ER-610 & 23.2040 & 23.2079 & $0.017 \%$ & $\mathrm{~N} / \mathrm{C}$ & 14.53 & 15.83 & & 494 & 372 & \\
\hline 1 & C2 & & & & & 14.53 & 15.63 & $1.95 \%$ & 494 & 365 & $-33.40 \%$ \\
\hline$M$ & & & & & & 14.53 & 12.98 & & 494 & 250 & \\
\hline 1 & $Y-833$ & 21.9467 & 21.9446 & $-0.010 \%$ & $\mathrm{~N} / \mathrm{C}$ & 11.38 & 10.13 & & 557 & 551 & \\
\hline D & D2 & & & & & 11.38 & 9.51 & $-9.46 \%$ & 557 & 561 & $0.42 \%$ \\
\hline$E$ & & & & & & 11.38 & 11.27 & & 557 & 566 & \\
\hline & 923 & 23.4690 & 23.4922 & $0.099 \%$ & $\mathrm{~N} / \mathrm{C}$ & 15.85 & cracked & & 503 & 577 & \\
\hline & E2 & & & & & 15.85 & 17.49 & $-30.57 \%$ & 503 & 570 & $14.65 \%$ \\
\hline & & & & & & 15.85 & 4.52 & & 503 & 583 & \\
\hline & $150-800$ & 23.2748 & 23.3535 & $0.338 \%$ & $\mathrm{~N} / \mathrm{C}$ & 14.75 & 16.92 & & 632 & 586 & \\
\hline & $\mathrm{F} 2$ & & & & & 14.75 & 19.90 & $25.99 \%$ & 632 & 564 & $-8.07 \%$ \\
\hline & & & & & & 14.75 & 18.93 & & 632 & 593 & \\
\hline
\end{tabular}




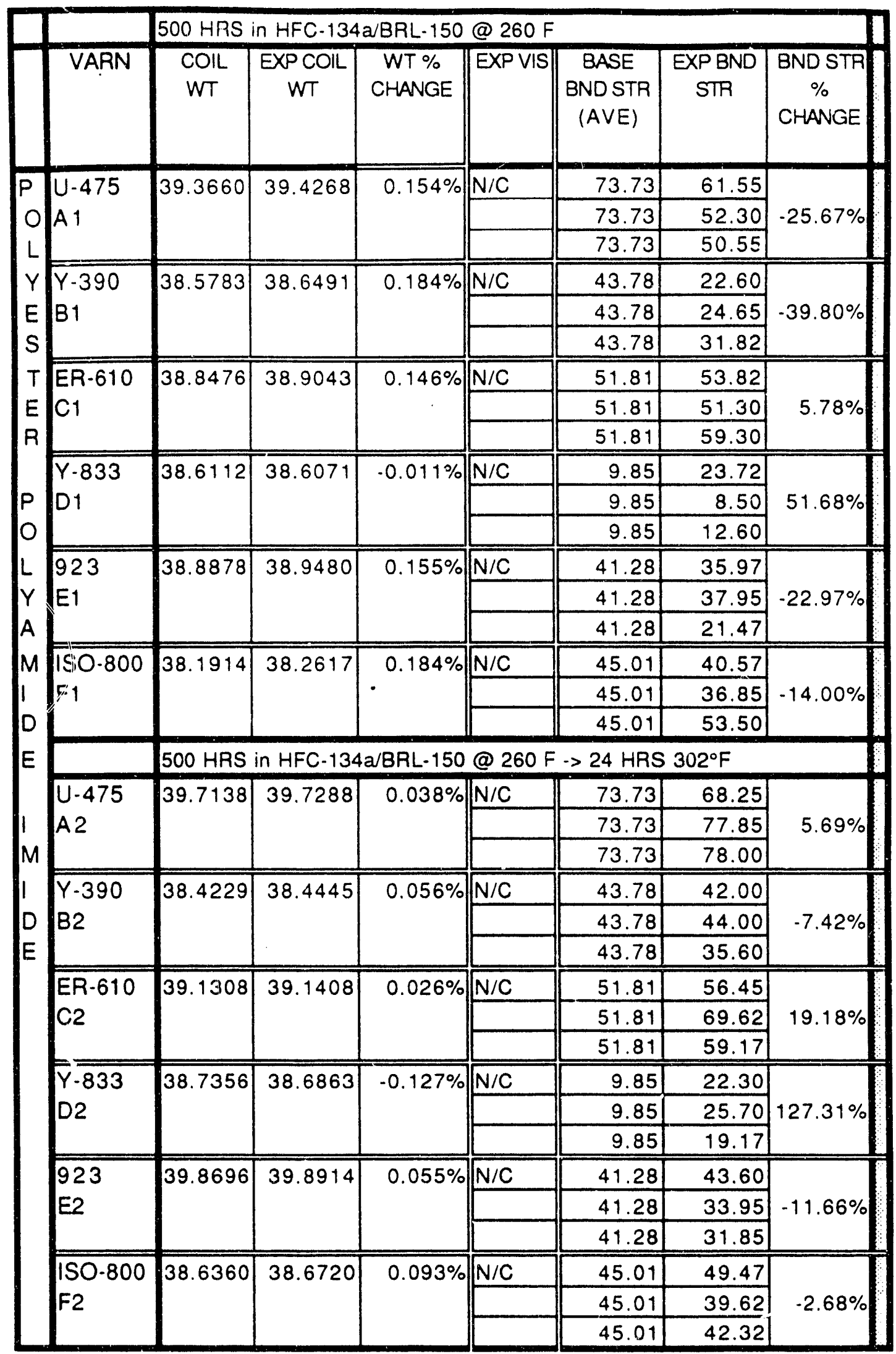


HELICAL COILSMIRE B-HFC-134a/BRL-150 Oil@260 $9\left(1,7^{\circ} \mathrm{C}\right)$

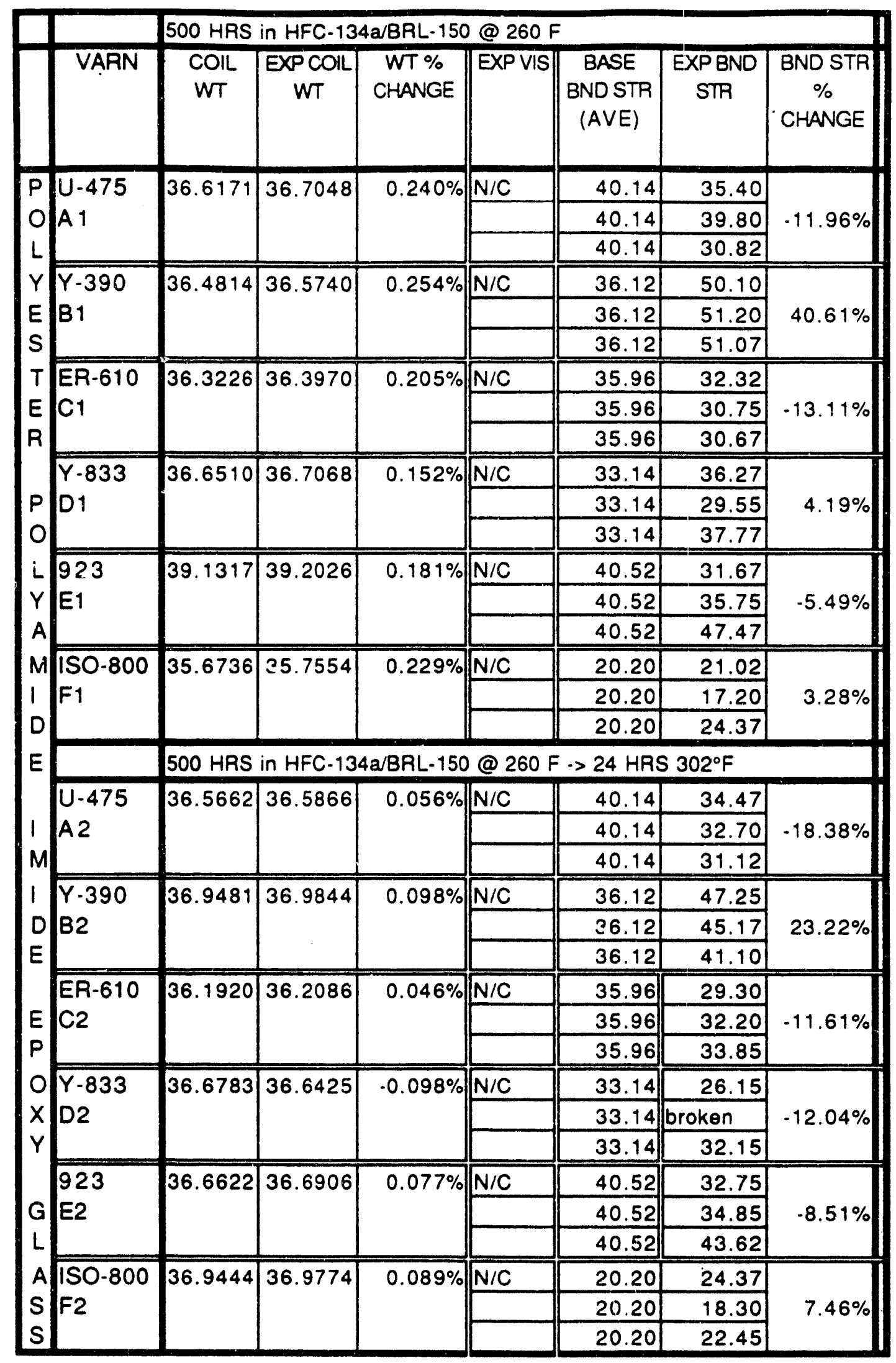


HELICAL COILSMIRE C.HFC-134a/Allied Signal BRL-150@260 $\mathrm{F}\left(127^{\circ} \mathrm{C}\right)$

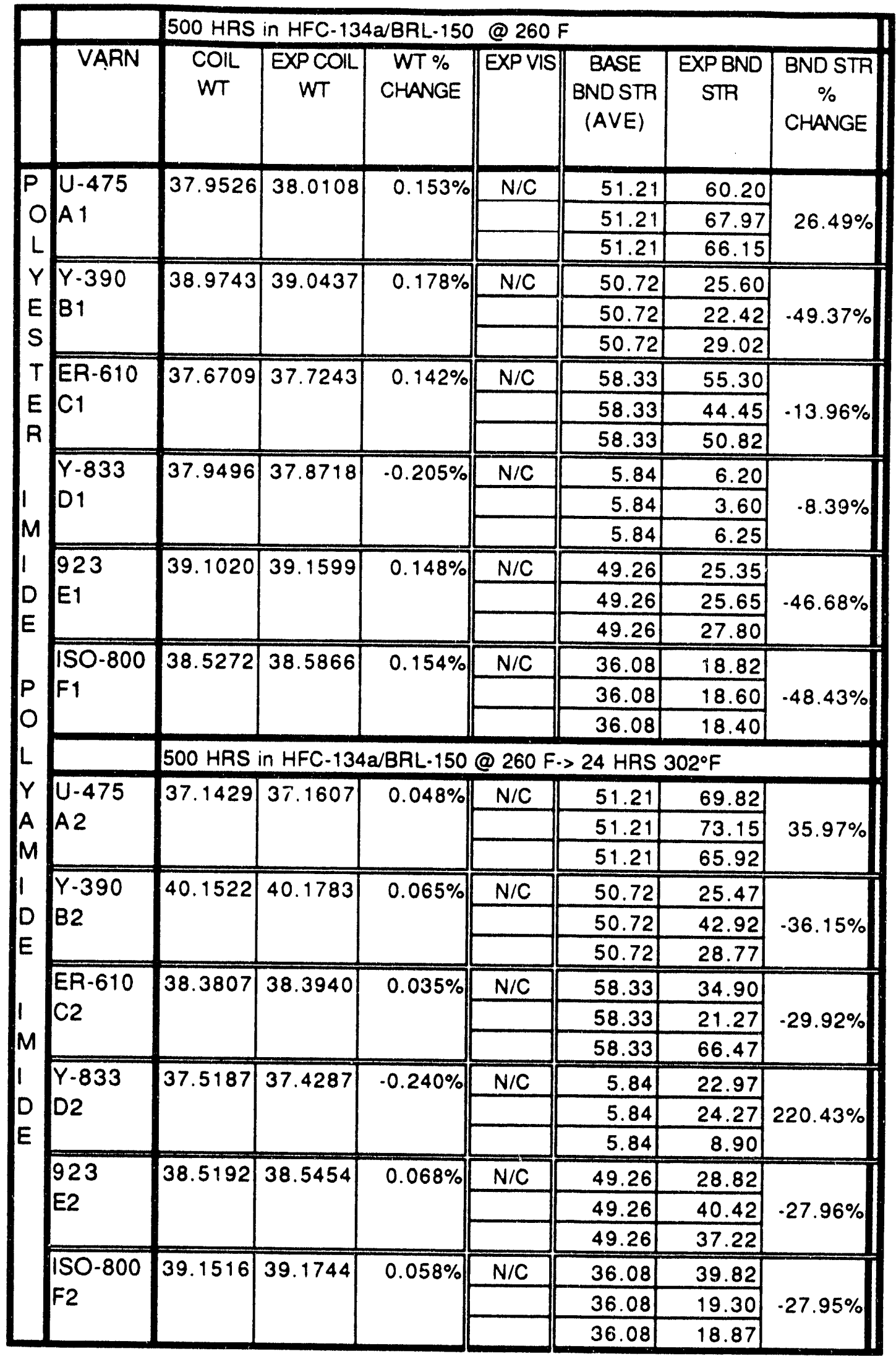




\begin{tabular}{|c|c|c|c|c|c|}
\hline \multicolumn{6}{|c|}{$500 \mathrm{HRS}$ in HFC-134a/BRL-150 oil @ 260 $0^{\circ} \mathrm{F}\left(127^{\circ} \mathrm{C}\right)$} \\
\hline ID & WT & EXPWT & $\begin{array}{c}\text { WT \% } \\
\text { CHANGE }\end{array}$ & EXP VS & EXPFLEX \\
\hline \multirow{2}{*}{ A1 } & \multirow{2}{*}{3.8951} & \multirow{2}{*}{3.8969} & \multirow[t]{2}{*}{$0.046 \%$} & $\mathrm{~N} / \mathrm{C}$ & \\
\hline & & & & & YES \\
\hline \multirow[t]{2}{*}{ B1 } & \multirow[t]{2}{*}{4.1159} & \multirow[t]{2}{*}{4.1232} & \multirow[t]{2}{*}{$0.177 \%$} & $\mathrm{~N} / \mathrm{C}$ & \\
\hline & & & & & YES \\
\hline \multirow{2}{*}{$\mathrm{C1}$} & \multirow{2}{*}{3.8271} & \multirow[t]{2}{*}{3.8289} & \multirow{2}{*}{$0.047 \%$} & $\mathrm{~N} / \mathrm{C}$ & \\
\hline & & & & & YES \\
\hline \multicolumn{6}{|c|}{ 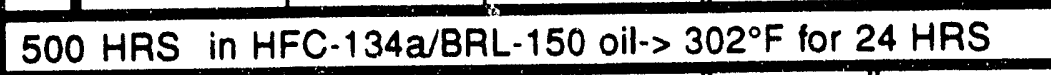 } \\
\hline \multirow[t]{2}{*}{ A2 } & \multirow[t]{2}{*}{3.8069} & \multirow[t]{2}{*}{3.8069} & \multirow[t]{2}{*}{$0.000 \%$} & $\mathrm{~N} / \mathrm{C}$ & \\
\hline & & & & & YES \\
\hline \multirow[t]{2}{*}{$\mathrm{B2}$} & \multirow[t]{2}{*}{3.9457} & \multirow[t]{2}{*}{3.9477} & \multirow[t]{2}{*}{$0.051 \%$} & $\mathrm{~N} / \mathrm{C}$ & \\
\hline & & & & & YES \\
\hline \multirow[t]{3}{*}{$\mathrm{C2}$} & \multirow[t]{3}{*}{3.754} & \multirow[t]{3}{*}{3.7545} & \multirow[t]{3}{*}{$0.013 \%$} & $\mathrm{~N} / \mathrm{C}$ & \\
\hline & & & & & YES \\
\hline & & & & & \\
\hline
\end{tabular}




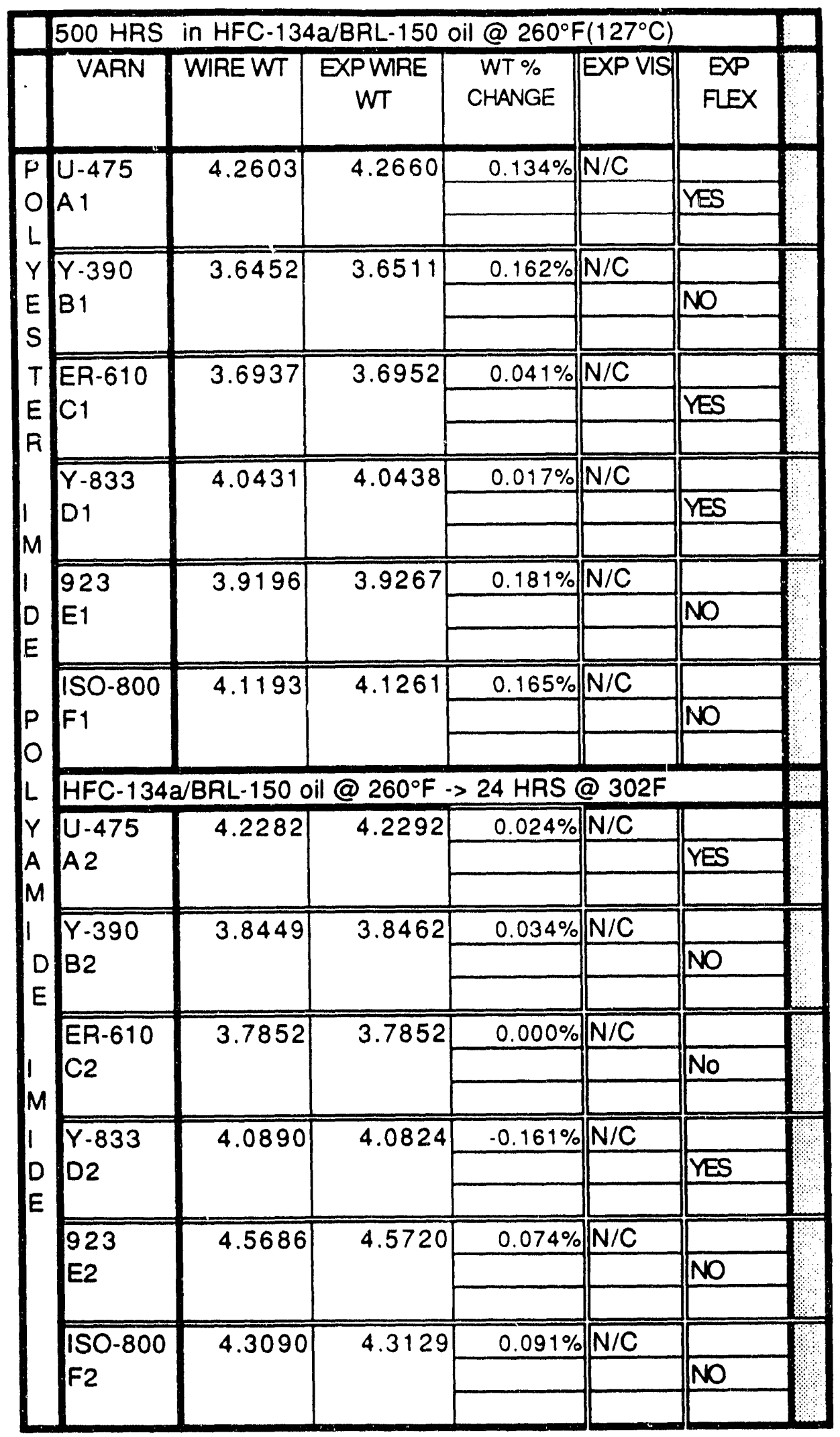




\begin{tabular}{|c|c|c|c|c|c|c|c|}
\hline & & 500 HRS & in HFC -134 & a/BRL-150 & Oil @2 & $60^{\circ} \mathrm{F}$ & \\
\hline & VARN & WIREWT & $\begin{array}{c}\text { EXPWRE } \\
\text { WT }\end{array}$ & $\begin{array}{l}\text { WT \% } \\
\text { CHANGE }\end{array}$ & EXX VIS & $\begin{array}{l}\text { EXP } \\
\text { FEXX }\end{array}$ & \\
\hline P & $U-475$ & 4.4450 & 4.4540 & $0.202 \%$ & $\mathrm{~N} / \mathrm{C}$ & & \\
\hline 이 & & & & & & NO & \\
\hline$y$ & $\overline{Y-390}$ & 37892 & 37965 & $0193 \%$ & $N / C$ & & \\
\hline E & $B 1$ & & & & & NO & \\
\hline$s$ & & & & & & & \\
\hline$T$ & ER-610 & 3.6409 & 3.6466 & $0.157 \%$ & $\mathrm{~N} / \mathrm{C}$ & & \\
\hline E & & & & & & NO & \\
\hline 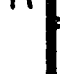 & $Y-833$ & 4.2758 & 4.2806 & $0.112 \%$ & $N / C$ & & \\
\hline $\mathrm{P}$ & & & & & & YES & \\
\hline o & & & & & & & \\
\hline L & 923 & 4.1238 & 4.1315 & $0.187 \%$ & $\mathrm{~N} / \mathrm{C}$ & & \\
\hline Y & E1 & & & & & NO & \\
\hline A & & & & & & & \\
\hline M & ISO-800 & 3.9181 & 3.9264 & $0.212 \%$ & $\mathrm{~N} / \mathrm{C}$ & & \\
\hline 1 & & & & & & No & \\
\hline$D$ & & & & & & & \\
\hline$E$ & HFC-136 & 4a/BRL-15 & $0500 \mathrm{hr}$ ex & D. plus 24 & hrs.@3c & $2^{\circ} \mathrm{F}$ & \\
\hline & U-475 & 4.4640 & 4.4650 & $0.022 \%$ & $\mathrm{~N} / \mathrm{C}$ & & \\
\hline 1 & A2 & & & & & NO & \\
\hline$M$ & & & & & & & \\
\hline 1 & $Y-390$ & 3.9887 & 3.9906 & $0.048 \%$ & $\mathrm{~N} / \mathrm{C}$ & & \\
\hline D & B2 & & & & & NO & \\
\hline E & & & & & & & \\
\hline$E$ & $\begin{array}{l}\text { ER-610 } \\
\text { C2 }\end{array}$ & 3.9139 & 3.9145 & $0.015 \%$ & $\mathrm{~N} / \mathrm{C}$ & VES & \\
\hline$P$ & & & & & & & \\
\hline 0 & $Y-833$ & 4.2772 & 4.2788 & $0.037 \%$ & $\mathrm{~N} / \mathrm{C}$ & & \\
\hline$x$ & D2 & & & & & YES & \\
\hline & 923 & 3.6178 & 3.6193 & $0.041 \%$ & $N / C$ & & \\
\hline G & E2 & & & & & NO & \\
\hline L & & & & & & & \\
\hline$A_{1}$ & ISO-800 & 4.0105 & 4.0140 & $0.087 \%$ & $\mathrm{~N} / \mathrm{C}$ & & \\
\hline$s$ & F2 & & & & & NO & \\
\hline$s$ & & & & & & & \\
\hline
\end{tabular}




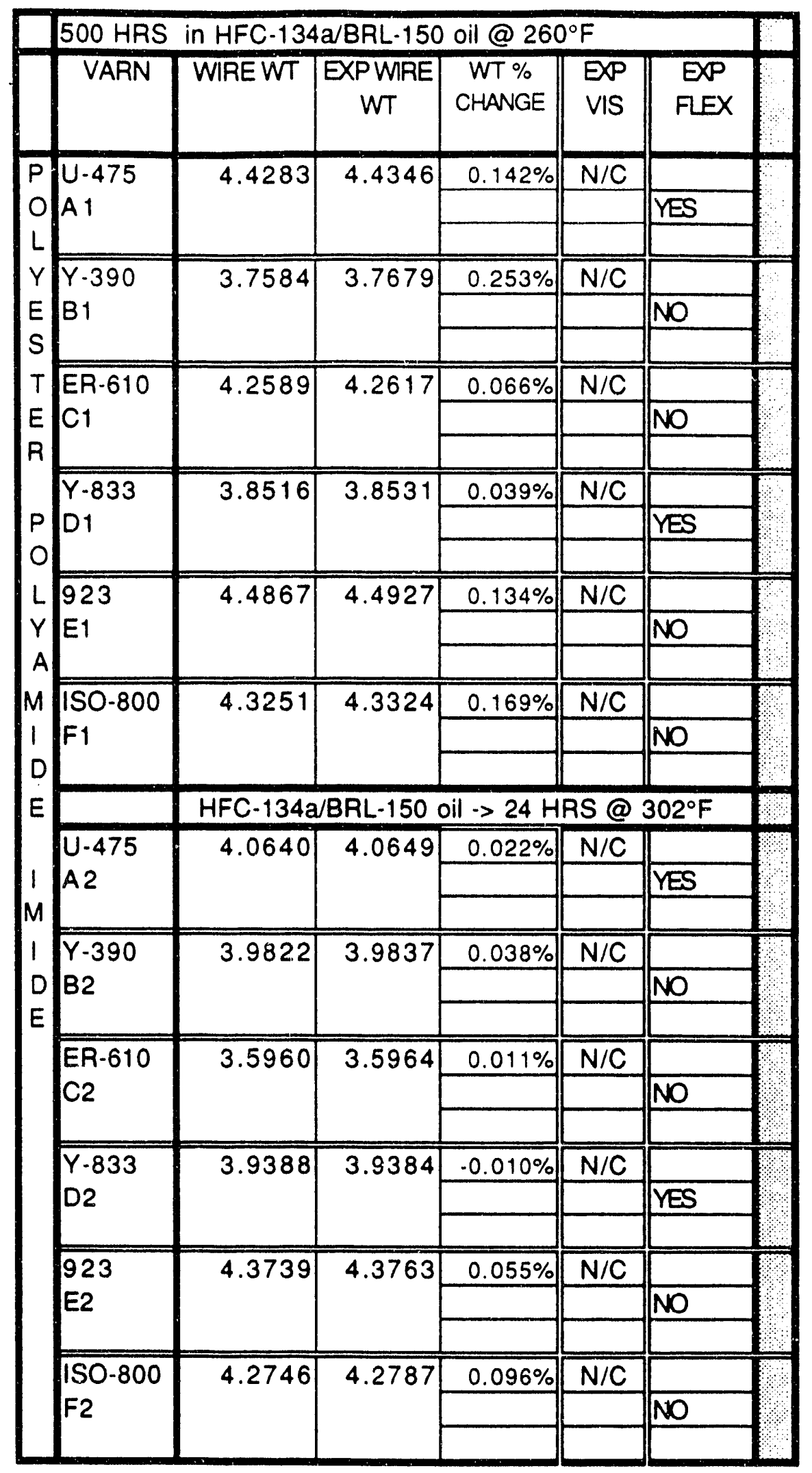


Varnish Disks-....HFC-134a/Allied Signal BRL-150 Oil

\begin{tabular}{|c|c|c|c|c|c|}
\hline & 500 HRS IN & R-134a/BRL & 150@26 & $0^{\circ} \mathrm{F}$ & \\
\hline ID & $\overline{W T}$ & EXP WT & $\begin{array}{c}\text { WT \% } \\
\text { CHANGE }\end{array}$ & EXP VIS & EXP FLEX \\
\hline $\begin{array}{l}\text { U-475 } \\
\text { A } 1\end{array}$ & 2.8320 & 2.7994 & $-1.15 \%$ & \begin{tabular}{|l|} 
Surface \\
Cloudy \\
\end{tabular} & $\mathrm{N} / \mathrm{C}$ \\
\hline & & & & S. Warped & \\
\hline$Y-390$ & 1.7486 & 1.7225 & $1.49 \%$ & $N / C$ & $\mathrm{~N} / \mathrm{C}$ \\
\hline 81 & & & & & \\
\hline ER-610 & 2.7223 & 2.7812 & $2.16 \%$ & $\mathrm{~N} / \mathrm{C}$ & $\mathrm{N} / \mathrm{C}$ \\
\hline & & & & & \\
\hline$Y .833$ & 3.0798 & 3.1426 & $2.04 \%$ & $N / C$ & $N / C$ \\
\hline D1 & & & & & \\
\hline 923 & 2.0957 & 2.0746 & $-1.01 \%$ & & $\mathrm{~N} / \mathrm{C}$ \\
\hline $\mathrm{E} 1$ & & & & Warped & \\
\hline ISO-800 & 2.1930 & 2.1822 & $-0.49 \%$ & Slightly & $\mathrm{N} / \mathrm{C}$ \\
\hline$F 1$ & & & & warped & \\
\hline & $R-134 a / B R$ & 150 at $\cdots>$ & 24 hour at & $302^{\circ} \mathrm{F}$. & \\
\hline$U-475$ & 2.7819 & 2.7020 & $-2.87 \%$ & Surface & $\mathrm{N} / \mathrm{C}$ \\
\hline & & & & Cloudy & \\
\hline & & & & Darkened & \\
\hline$Y-390$ & 2.2997 & 2.2288 & $-3.08 \%$ & Slightly & $\mathrm{N} / \mathrm{C}$ \\
\hline B2 & & & & warped & \\
\hline & & & & Darkened & \\
\hline ER-610 & 3.0567 & 3.0368 & $-0.65 \%$ & Darkened & $\mathrm{N} / \mathrm{C}$ \\
\hline & & & & & \\
\hline$Y-833$ & 3.3808 & 3.3904 & $0.28 \%$ & Darkened & $\mathrm{N} / \mathrm{C}$ \\
\hline D2 & & & & & \\
\hline 923 & 2.1348 & 2.0711 & $-2.98 \%$ & Warped & \\
\hline E2 & & & & Darkened & $\mathrm{N} / \mathrm{C}$ \\
\hline ISO-800 & 2.0461 & 1.9896 & $-2.76 \%$ & |slightly & \\
\hline & & & & warped & $N / C$ \\
\hline & & & & Darkened & \\
\hline
\end{tabular}




\begin{tabular}{|c|c|c|c|c|c|c|c|c|c|}
\hline & $500 \mathrm{HRS}$ & $S$ in HFC. & $134 a / B R L$. & -150 Oil & $260 \mathrm{~F}$ & & & & \\
\hline $1 D$ & WT & EXPWT & $\begin{array}{c}\text { WT \% } \\
\text { CHANGE }\end{array}$ & $\begin{array}{l}\text { SAMPLE } \\
\text { WIDTH }\end{array}$ & $\begin{array}{l}\text { BREAK } \\
\text { LOAD }\end{array}$ & \begin{tabular}{|c} 
SAMPLE \\
THICKNESS \\
Mils
\end{tabular} & $\begin{array}{c}\text { TENSILE } \\
\text { STR } \\
\text { BASE }\end{array}$ & $\begin{array}{l}\text { TENSILE } \\
\text { STREXP }\end{array}$ & $\begin{array}{l}\text { \% CHANGE } \\
\text { TENSILE }\end{array}$ \\
\hline NO/MY/NO & 4.2565 & 4.5722 & $7.42 \%$ & 0.512 & 178.10 & 21 & 17.4 & 16.56 & \\
\hline A 1 & & & & 0.402 & 132.70 & 21 & 17.4 & 15.72 & $-10.60 \%$ \\
\hline & & & & 0.400 & 120.80 & 21 & 17.4 & 14.38 & \\
\hline$D A / M Y / D A$ & 4.6712 & 4.6765 & $0.11 \%$ & 0.390 & 112.40 & 21 & 13.7 & 13.72 & \\
\hline & & & & 0.378 & 105.10 & 21 & 13.7 & 13.24 & $-2.09 \%$ \\
\hline & & & & 0.402 & 112.10 & 21 & 13.7 & 13.28 & \\
\hline MYLAR MO & 2.2688 & 2.2914 & $1.00 \%$ & 0.407 & 65.65 & 10 & 21.7 & 16.13 & \\
\hline & & & & 0.336 & 51.10 & 10 & 21.7 & 15.21 & $.27 .34 \%$ \\
\hline & & & & 0.425 & 67.85 & 10 & 21.7 & 15.96 & \\
\hline NO 410 & 2.0265 & 2.3099 & $13.98 \%$ & 0.480 & 85.40 & 10 & 18.7 & 17.79 & \\
\hline D1 & & & & 0.504 & 93.65 & 10 & 18.7 & 18.58 & $-2.35 \%$ \\
\hline & & & & 0.518 & 95.35 & 10 & 18.7 & 18.41 & \\
\hline NO MI 418 & 2.3971 & 2.8668 & $19.59 \%$ & 0.516 & 31.95 & 10 & 7.5 & 6.19 & \\
\hline & & & & 0.490 & 31.75 & 10 & 7.5 & 6.48 & $-16.95 \%$ \\
\hline & & & & 0.493 & 29.65 & 10 & 7.5 & 6.01 & \\
\hline MEL 228 & 2.3656 & 2.3997 & $1.44 \%$ & 0.460 & 76.10 & 10 & 21.7 & 16.54 & \\
\hline F1 & & & & 0.418 & 69.40 & 10 & 21.7 & 16.60 & $-23.89 \%$ \\
\hline & & & & 0.404 & 66.25 & 10 & 21.7 & 16.40 & \\
\hline & $500 \mathrm{HRS}$ & $S$ in $H F C$. & $134 \mathrm{a} / \mathrm{BRL}$ & 150 Oil@ & $260 \mathrm{~F}$ & $>24$ HRS@ & $302^{\circ} \mathrm{F}$ & & \\
\hline NO/MY/NO & 4.3750 & 4.4194 & $1.01 \%$ & 0.393 & 127.30 & 21 & 17.4 & 15.42 & \\
\hline A2 & & & & 0.416 & 155.10 & 21 & 17.4 & 17.75 & $-6.53 \%$ \\
\hline & & & & 0.366 & 120.00 & 21 & 17.4 & 15.61 & \\
\hline$D A / M Y / D A$ & 4.7905 & 4.7346 & $-1.17 \%$ & 0.455 & 127.60 & 21 & 13.7 & 13.35 & \\
\hline B2 & & & & 0.400 & 115.10 & 21 & 13.7 & 13.70 & $-0.94 \%$ \\
\hline & & & & 0.356 & 102.10 & 21 & 13.7 & 13.66 & \\
\hline MYLAR MO & 2.1513 & 2.1512 & $0.00 \%$ & 0.470 & 81.25 & 10 & 21.7 & 17.29 & \\
\hline & & & & 0.400 & 67.20 & 10 & 21.7 & 16.80 & $-22.42 \%$ \\
\hline & & & & 0.456 & 74.85 & 10 & 21.7 & 16.41 & \\
\hline NOMEX 410 & 2.2885 & 2.3406 & $2.28 \%$ & 0.566 & 112.40 & 10 & 18.7 & 19.86 & \\
\hline D2 & & & & 0.508 & 103.80 & 10 & 18.7 & 20.43 & $7.18 \%$ \\
\hline & & & & 0.555 & 110.10 & 10 & 18.7 & 19.84 & \\
\hline NO/MI 418 & 2.3585 & 2.3762 & $0.75 \%$ & 0.481 & 25.10 & 10 & 7.5 & 5.22 & \\
\hline E2 & & & & 0.495 & 30.25 & 10 & 7.5 & 6.11 & $-22.41 \%$ \\
\hline & & & & 0.496 & 30.40 & 10 & 7.5 & 6.13 & \\
\hline MEL 228 & 2.3245 & 2.3259 & $0.06 \%$ & 0.462 & 77.05 & 10 & 21.7 & 16.68 & \\
\hline F2 & & & & 0.393 & 66.40 & 10 & 21.7 & 16.90 & $-22.70 \%$ \\
\hline & & & & 0.461 & 77.20 & 10 & 21.7 & 16.75 & \\
\hline
\end{tabular}




\begin{tabular}{|c|c|c|c|c|c|c|c|c|}
\hline & 500 HRS & in HFC-1 & 34a/BRL. & -150 Oil@ & $2260 \mathrm{~F}$ & & & \\
\hline ID & $\begin{array}{c}\text { STRETCH } \\
\text { (inch) }\end{array}$ & $\begin{array}{c}\% \\
\text { ELONG }\end{array}$ & $\begin{array}{l}\text { BASE } \\
\text { ELONG } \\
(A \vee E)\end{array}$ & $\begin{array}{c}\text { ELONG \% } \\
\text { CHANGE }\end{array}$ & $\begin{array}{c}\text { BASE } \\
\text { DIE } \\
\text { (AVE) }\end{array}$ & EXPDIE & $\begin{array}{c}\text { DIE \% } \\
\text { CHANGE }\end{array}$ & VISUAL EXP \\
\hline NO/MY/NO & 0.56 & $14.0 \%$ & $20.0 \%$ & & $>18.97$ & flash & & $\mathrm{N} / \mathrm{C}$ \\
\hline A 1 & 0.40 & $10.0 \%$ & $20.0 \%$ & $-40.0 \%$ & $>18.97$ & flash & flash & \\
\hline & 0.37 & $9.3 \%$ & $20.0 \%$ & & $>18.97$ & flash & & \\
\hline$D A / M Y / D A$ & 0.50 & $25.0 \%$ & $46.0 \%$ & & $>15.27$ & flash & & Yellowed \\
\hline $\mathrm{B1}$ & 0.48 & $24.0 \%$ & $46.0 \%$ & $-48.9 \%$ & $>15.27$ & flash & flash & slightly \\
\hline & 0.43 & $21.5 \%$ & $46.0 \%$ & & $>15.27$ & flash & & warped \\
\hline MYAR MO & 0.89 & $44.5 \%$ & $131.0 \%$ & & $>14.91$ & flash & & \\
\hline C1 & 0.68 & $34.0 \%$ & $131.0 \%$ & $-69.0 \%$ & $>14.91$ & flash & flash & lightened \\
\hline & 0.87 & $43.5 \%$ & $131.0 \%$ & & $>14.91$ & flash & & \\
\hline NO 410 & 0.29 & $14.5 \%$ & $17.0 \%$ & & 10.67 & flash & & $N / C$ \\
\hline 01 & 0.35 & $8.8 \%$ & $17.0 \%$ & $-37.7 \%$ & 10.67 & flash & flash & \\
\hline & 0.34 & $8.5 \%$ & $17.0 \%$ & & 10.67 & flash & & \\
\hline NO MI 418 & 0.04 & $1.0 \%$ & $4.0 \%$ & & 10.23 & 9.32 & & $\mathrm{~N} / \mathrm{C}$ \\
\hline E1 & 0.05 & $1.3 \%$ & $4.0 \%$ & $.72 .9 \%$ & 10.23 & 10.00 & $-1.9 \%$ & \\
\hline & 0.04 & $1.0 \%$ & $4.0 \%$ & & 10.23 & 10.80 & & \\
\hline MEL 228 & 2.75 & $137.5 \%$ & $160.0 \%$ & & $>14.22$ & flash & & slightly \\
\hline $\mathrm{F} 1$ & 3.07 & $153.5 \%$ & $160.0 \%$ & $.13 .3 \%$ & $>14.22$ & flash & flash & darkened \\
\hline & 2.50 & $125.0 \%$ & $160.0 \%$ & & $\|>14.22$ & flash & & \\
\hline & 500 HAS & in HFC. & 134a/BRL. & -1500il@ & $260 \mathrm{~F}$. & $>24$ HRS & $5 @ 302^{\circ} \mathrm{F}$ & \\
\hline NO/MY/NO & 0.55 & $13.8 \%$ & $20.0 \%$ & & $\geq 18.97$ & flash & & Delamination \\
\hline$A 2$ & 0.62 & $15.5 \%$ & $20.0 \%$ & $-39.6 \%$ & $>18.97$ & flash & flash & big pockets \\
\hline & 0.28 & $7.0 \%$ & $20.0 \%$ & & $>18.97$ & flash & & \\
\hline$D A / M Y / D A$ & 0.38 & $19.0 \%$ & $46.0 \%$ & & $>15.27$ & flash & & Browned \\
\hline B2 & 0.45 & $22.5 \%$ & $46.0 \%$ & $-54.3 \%$ & $>15.27$ & flash & flash & slightly \\
\hline & 0.43 & $21.5 \%$ & $46.0 \%$ & & $>15.27$ & flash & & warped \\
\hline MYLAR MO & 0.60 & $30.0 \%$ & $131.0 \%$ & & $\geq 14.91$ & flash & & $\mathrm{N} / \mathrm{C}$ \\
\hline C2 & 0.95 & $47.5 \%$ & $131.0 \%$ & $.63 .1 \%$ & $>14.91$ & flash & flash & \\
\hline & 1.35 & $67.5 \%$ & $131.0 \%$ & & $>14.91$ & flash & & \\
\hline NOMEX 410 & 0.33 & $8.3 \%$ & $17.0 \%$ & & 10.67 & $\begin{array}{r}9.79 \\
\end{array}$ & & slightly \\
\hline D2 & 0.47 & $11.8 \%$ & $17.0 \%$ & $-39.7 \%$ & 10.67 & 10.13 & $-4.6 \%$ & Darkened \\
\hline & 0.43 & $10.8 \%$ & $17.0 \%$ & & 10.67 & 10.62 & & \\
\hline $\mathrm{NO} / \mathrm{MI} 418$ & 0.03 & $0.8 \%$ & $4.0 \%$ & & 10.23 & 9.36 & & $N / C$ \\
\hline E2 & 0.04 & $1.0 \%$ & $4.0 \%$ & $.77 .1 \%$ & 10.23 & 9.61 & $-3.7 \%$ & \\
\hline & 0.04 & $1.0 \%$ & $4.0 \%$ & & 10.23 & 10.57 & & \\
\hline MEL 228 & 2.82 & $141.0 \%$ & $160.0 \%$ & & $\geq 14.22$ & flash & & Browned \\
\hline $\mathrm{F} 2$ & 3.40 & $170.0 \%$ & $160.0 \%$ & $-4.1 \%$ & $>14.22$ & flash & flash & darkened \\
\hline & 2.99 & $149.5 \%$ & $160.0 \%$ & & $\|>14.22$ & flash & & \\
\hline
\end{tabular}


SLEEVING--HFC-134a/Allied Signal BRL-150@260 $\mathrm{F}\left(127^{\circ} \mathrm{C}\right)$

\begin{tabular}{|c|c|c|c|c|}
\hline & \multicolumn{4}{|c|}{$500 \mathrm{HRS}$ in HFC-134a/BRL-150@260 $\mathrm{F}$} \\
\hline & WT & EXPWT & $\begin{array}{c}\% \\
\text { CHANGE }\end{array}$ & EXP VIS \\
\hline $\begin{array}{l}\text { A1 } \\
\text { NOMEX }\end{array}$ & 0.5235 & 0.5991 & $14.44 \%$ & $\mathrm{~N} / \mathrm{C}$ \\
\hline $\begin{array}{l}\text { B1 } \\
\text { MYLAR }\end{array}$ & 0.4937 & 0.5031 & $\begin{array}{r}1.90 \% \\
t \\
\end{array}$ & \begin{tabular}{|c|} 
small Pockets \\
when rubbed between fingers \\
dbe bacame cloudy in appearanc
\end{tabular} \\
\hline $\begin{array}{l}\mathrm{C} 1 \\
\mathrm{NO} / \mathrm{MY}\end{array}$ & 0.3833 & 0.4043 & $5.48 \%$ & some small pockets \\
\hline & HFC-134a/B & $L-150$ oil $C$ & $260^{\circ} \mathrm{Fp}$ & lus24 hrs@302F \\
\hline $\begin{array}{l}\text { A2 } \\
\text { NOMEX }\end{array}$ & 0.5267 & 0.5440 & $3.28 \%$ & \begin{tabular}{|l} 
Slightly Darkened \\
\end{tabular} \\
\hline $\begin{array}{l}\text { B2 } \\
\text { MYLAR }\end{array}$ & 0.4601 & 0.4605 & $0.09 \%$ & $\frac{\text { Big pockets }}{\text { warped }}$ \\
\hline $\begin{array}{l}\mathrm{C} 2 \\
\mathrm{NO} / \mathrm{MY}\end{array}$ & 0.3929 & $\begin{array}{c}0.3939 \\
.\end{array}$ & $0.25 \%$ & \begin{tabular}{|c|} 
Nomex delaminated from mylar \\
some pockets where mylar \\
pulled away.
\end{tabular} \\
\hline
\end{tabular}




\begin{tabular}{|c|c|c|c|c|c|c|c|c|c|}
\hline & 500 HRS & in $\mathrm{HFC}-13$ & $4 \mathrm{a} / \mathrm{BRL}-15$ & @260 & & & & & \\
\hline ID & WT & EXPWT & $\begin{array}{c}\text { WT \% } \\
\text { CHANGE }\end{array}$ & $\begin{array}{l}\text { BREAK } \\
\text { LOAD } \\
\text { (AVE) }\end{array}$ & $\begin{array}{l}\text { BREAK } \\
\text { LOAD } \\
\text { EPP }\end{array}$ & $\begin{array}{c}\% \\
\text { CHANGE } \\
\text { BRK } \\
\text { LOAD }\end{array}$ & $\begin{array}{c}\text { STRETCH } \\
(\mathrm{INCH})\end{array}$ & $\%$ ELONG & EXPVIS \\
\hline $\mathrm{A} 1_{1}$ & 1.4811 & 1.4832 & $0.14 \%$ & 39.02 & 66.57 & & 0.08 & $4.00 \%$ & $\mathrm{~N} / \mathrm{C}$ \\
\hline Glass & & & & 39.02 & 51.65 & $38.28 \%$ & 0.06 & $3.00 \%$ & \\
\hline & & & & 39.02 & 43.65 & & 0.06 & $3.00 \%$ & \\
\hline B1 & 0.7040 & 0.7083 & $0.61 \%$ & 56.12 & 57.70 & & 0.62 & $31.00 \%$ & $\mathrm{~N} / \mathrm{C}$ \\
\hline Polyester & & & & 56.12 & 58.15 & $3.65 \%$ & 0.65 & $32.50 \%$ & \\
\hline & & & & 56.12 & 58.65 & & 0.70 & $35.00 \%$ & \\
\hline $\mathrm{C} 1$ & 1.5619 & 1.9732 & $26.33 \%$ & 83.50 & 63.30 & & 0.14 & $7.00 \%$ & Slightly \\
\hline Permacel & & & & 88.50 & 85.20 & $-21.68 \%$ & 0.14 & $7.00 \%$ & darkened \\
\hline & & & & 88.50 & 59.45 & & 0.12 & $6.00 \%$ & Curled up \\
\hline & $500 \mathrm{HR}$ & $\mathrm{S}$ in HFC. & $34 a / B R L-1$ & $50 @ 260$ & $\mathrm{~F}->302^{\circ} \mathrm{F}$ & 24 HRS & & & \\
\hline$A 2$ & 1.4762 & 1.4758 & $-0.03 \%$ & 39.02 & 66.60 & & 0.07 & $3.50 \%$ & $\mathrm{~N} / \mathrm{C}$ \\
\hline Glass & & & & 39.02 & 58.20 & $65.51 \%$ & 0.06 & $3.00 \%$ & \\
\hline & & & & 39.02 & 68.95 & & 0.07 & $3.50 \%$ & \\
\hline & 0.6662 & 0.6668 & $0.09 \%$ & 56.12 & 56.80 & & 0.45 & $22.50 \%$ & $\mathrm{~N} / \mathrm{C}$ \\
\hline Polyester & & & & 56.12 & 56.55 & $1.36 \%$ & 0.53 & $26.50 \%$ & \\
\hline & & & & 56.12 & 57.30 & & 0.58 & $29.00 \%$ & \\
\hline $\mathrm{C} 2$ & 1.3299 & 1.2823 & $-3.58 \%$ & 88.50 & 66.35 & & 0.07 & $3.50 \%$ & Slightly \\
\hline Permacel & & & & 88.50 & 87.75 & $-2.37 \%$ & 0.10 & $5.00 \%$ & Darkened \\
\hline & & & & 88.50 & 105.10 & & 0.12 & $6.00 \%$ & \\
\hline
\end{tabular}


TIE CORD-.HFC-134a/Allied Signal BRL-150 oil @ $260^{\circ} \mathrm{F}\left(127^{\circ} \mathrm{C}\right)$

\begin{tabular}{|c|c|c|c|c|c|c|c|c|c|}
\hline \multicolumn{10}{|c|}{500 HRS IN R-134a/BRL-150@ $260^{\circ} \mathrm{F}$} \\
\hline ID & WT & EXPWT & $\begin{array}{c}\text { WT \% } \\
\text { CHANGE }\end{array}$ & $\begin{array}{l}E X P \\
V I S\end{array}$ & $\begin{array}{l}\text { BREAK } \\
\text { LOAD } \\
(A \vee E)\end{array}$ & $\begin{array}{l}\text { BREAK } \\
\text { LOAD } \\
\text { EXP } \\
(\operatorname{av\theta })\end{array}$ & $\begin{array}{c}\% \\
\text { CHANGE } \\
\text { BPK } \\
\text { LOAD }\end{array}$ & $\begin{array}{c}\text { STRETCH } \\
\text { (Inch) }\end{array}$ & $\begin{array}{c}\% \\
\text { ELONG }\end{array}$ \\
\hline \multirow[t]{4}{*}{ A1 } & \multirow[t]{3}{*}{0.2935} & \multirow[t]{3}{*}{0.2948} & \multirow[t]{3}{*}{$0.44 \%$} & $\mathrm{~N} / \mathrm{C}$ & 28.36 & 36.15 & & 0.17 & $8.5 \%$ \\
\hline & & & & & 28.36 & 36.75 & \multirow{2}{*}{\begin{tabular}{|l}
$29.55 \%$ \\
\end{tabular}} & 0.16 & $8.0 \%$ \\
\hline & & & & & 28.36 & 37.32 & & 0.19 & $9.5 \%$ \\
\hline & \multicolumn{9}{|c|}{500 HRS IN R-134a/BRL-150 -> 24 HRS@ @ $302^{\circ} \mathrm{F}$} \\
\hline \multirow[t]{3}{*}{$A 2$} & \multirow[t]{3}{*}{0.2940} & \multirow[t]{3}{*}{0.2924} & \multirow[t]{3}{*}{$-0.544 \%$} & $\mathrm{~N} / \mathrm{C}$ & 28.36 & 40.05 & & 0.48 & $24.0 \%$ \\
\hline & & & & & 28.36 & 39.00 & $37.75 \%$ & 0.42 & $21.0 \%$ \\
\hline & & & & & 28.36 & 38.15 & & 0.44 & $22.0 \%$ \\
\hline
\end{tabular}


LEAD WIRE INSULATION-HFC-134a/Allied Signal BRL-150@260 $\mathrm{F}\left(127^{\circ} \mathrm{C}\right)$

\begin{tabular}{|c|c|c|c|c|c|c|c|}
\hline & 500 HRS IN & $=C-134 a / B F$ & L-150@ & $260^{\circ} \mathrm{F}$ & & & \\
\hline$\overline{I D}$ & WT & EXPWT & $\begin{array}{c}\text { WT \% } \\
\text { CHANGE }\end{array}$ & EXP VIS & $\begin{array}{c}\text { BASE DIE } \\
\text { (AVE) }\end{array}$ & EXPDIE & $\begin{array}{c}\text { DIE\% } \\
\text { CHANGE }\end{array}$ \\
\hline DMD & 4.1841 & 4.3643 & $4.31 \%$ & $\mathrm{~N} / \mathrm{C}$ & 9.61 & 9.03 & \\
\hline A1 & & & & & 9.61 & 10.10 & $0.80 \%$ \\
\hline & & & & & 9.61 & 9.93 & \\
\hline DTMD & 4.4807 & 4.6371 & $3.49 \%$ & $\mathrm{~N} / \mathrm{C}$ & 9.95 & 12.63 & \\
\hline B1 & & & & & 9.95 & 12.74 & $29.51 \%$ \\
\hline & & & & & 9.95 & 13.29 & \\
\hline & HFC-134a/BF & $-150 @ 26$ & )$^{\circ} \mathrm{F} \rightarrow 24$ & HRS@30 & $2^{\circ} \mathrm{F}$ & & \\
\hline DMD & 4.0540 & 4.1041 & $1.24 \%$ & $\mathrm{~N} / \mathrm{C}$ & 9.61 & 8.59 & \\
\hline $\mathrm{A} 2$ & & & & & 9.61 & 9.54 & $-5.62 \%$ \\
\hline & & & & & 9.61 & 9.08 & \\
\hline DTMD & 4.3707 & 4.3899 & $0.44 \%$ & $N / C$ & 9.95 & 11.22 & \\
\hline $\mathrm{B} 2$ & & & & & 9.95 & 9.70 & $2.95 \%$ \\
\hline & & & & & 9.95 & 9.81 & \\
\hline
\end{tabular}




\section{Appendix $\mathbf{Q}$}

Experimental Data for $H F C-125 /$ Allied
Signal BRL-150 Exposure at $126^{\circ} \mathrm{C}\left(260^{\circ} \mathrm{F}\right)$ 


\begin{tabular}{|c|c|c|c|c|c|c|c|c|c|c|}
\hline & rs & $F C$ & & al & $127^{\circ} \mathrm{C}$ & & & & & \\
\hline ID & WT & EXPWT & $\begin{array}{l}\text { WT \% } \\
\text { CHANGE }\end{array}$ & EXP VS & $\begin{array}{c}\text { BASE BRN } \\
\text { OUT } \\
\text { (AVE) }\end{array}$ & $\begin{array}{c}\text { EXP BRN } \\
\text { OUT }\end{array}$ & $\begin{array}{c}\text { BRNOUT } \\
\% \\
\text { CHANGE }\end{array}$ & $\begin{array}{c}\text { BASE DIE } \\
\text { (AVE) }\end{array}$ & EXPDIE & $\begin{array}{c}\text { DIE \% } \\
\text { CHANGE }\end{array}$ \\
\hline$\overline{A 1}$ & 22.8116 & 22.8259 & $0.063 \%$ & $\mathrm{~N} / \mathrm{C}$ & 576 & 553 & & 15.80 & 14.61 & \\
\hline & & & & & 576 & 408 & $-11.6 \%$ & 15.80 & 14.50 & $-8.6 \%$ \\
\hline & & & & & 576 & 567 & & 15.80 & 14.20 & \\
\hline$\overline{B 1}$ & 21.8079 & 21.8574 & $0.227 \%$ & $\mathrm{~N} / \mathrm{C}$ & 736 & 736 & & 11.62 & 11.09 & \\
\hline & & & & & 736 & 642 & $-4.4 \%$ & 11.62 & 11.45 & $-7.0 \%$ \\
\hline & & & & & 736 & 732 & & 11.62 & 9.87 & \\
\hline C1 & 22.8212 & 22.8329 & $0.051 \%$ & $\mathrm{~N} / \mathrm{C}$ & 579 & 572 & & 16.58 & 16.91 & \\
\hline & & & & & 579 & 566 & $-10.8 \%$ & 16.58 & 12.81 & $-17.8 \%$ \\
\hline & & & & & 579 & 412 & & 16.58 & 11.16 & \\
\hline & 500 hours & HFC-R12 & 5/BRL-15C & oil at 2 & $60^{\circ} \mathrm{F}\left(127^{\circ} \mathrm{C}\right.$ & i) plus 24 & hours at & $302^{\circ} \mathrm{F}(150$ & $\left.{ }^{\circ} \mathrm{C}\right)$ & \\
\hline$\overline{A 2}$ & 22.7902 & 22.8012 & $0.048 \%$ & $\mathrm{~N} / \mathrm{C}$ & 576 & 481 & & 15.80 & 15.90 & \\
\hline & & & & & 576 & 496 & $-16.7 \%$ & 15.80 & 13.49 & $-11.2 \%$ \\
\hline & & & & & 576 & 462 & & 15.80 & 12.69 & \\
\hline 82 & 25.1673 & 25.1925 & $0.100 \%$ & $\mathrm{~N} / \mathrm{C}$ & 736 & 731 & & 11.62 & 11.31 & \\
\hline & & & & & 736 & 724 & $-1.1 \%$ & 11.62 & 11.71 & $0.0 \%$ \\
\hline & & & & & 736 & 728 & & 11.62 & 11.84 & \\
\hline$\overline{C 2}$ & 22.7738 & 22.7808 & $0.031 \%$ & $\mathrm{~N} / \mathrm{C}$ & 579 & 561 & & 16.58 & 8.48 & \\
\hline & & & & & 579 & 449 & $-17.0 \%$ & 16.58 & 14.42 & $-26.5 \%$ \\
\hline & & & & & 579 & 432 & & 16.58 & 13.64 & \\
\hline
\end{tabular}


TWISTED PAIRSMIRE AWITH VARNISH-HFC-R125/Allied Signal BRL-1500il @260 F $\left(127^{\circ} \mathrm{C}\right)$

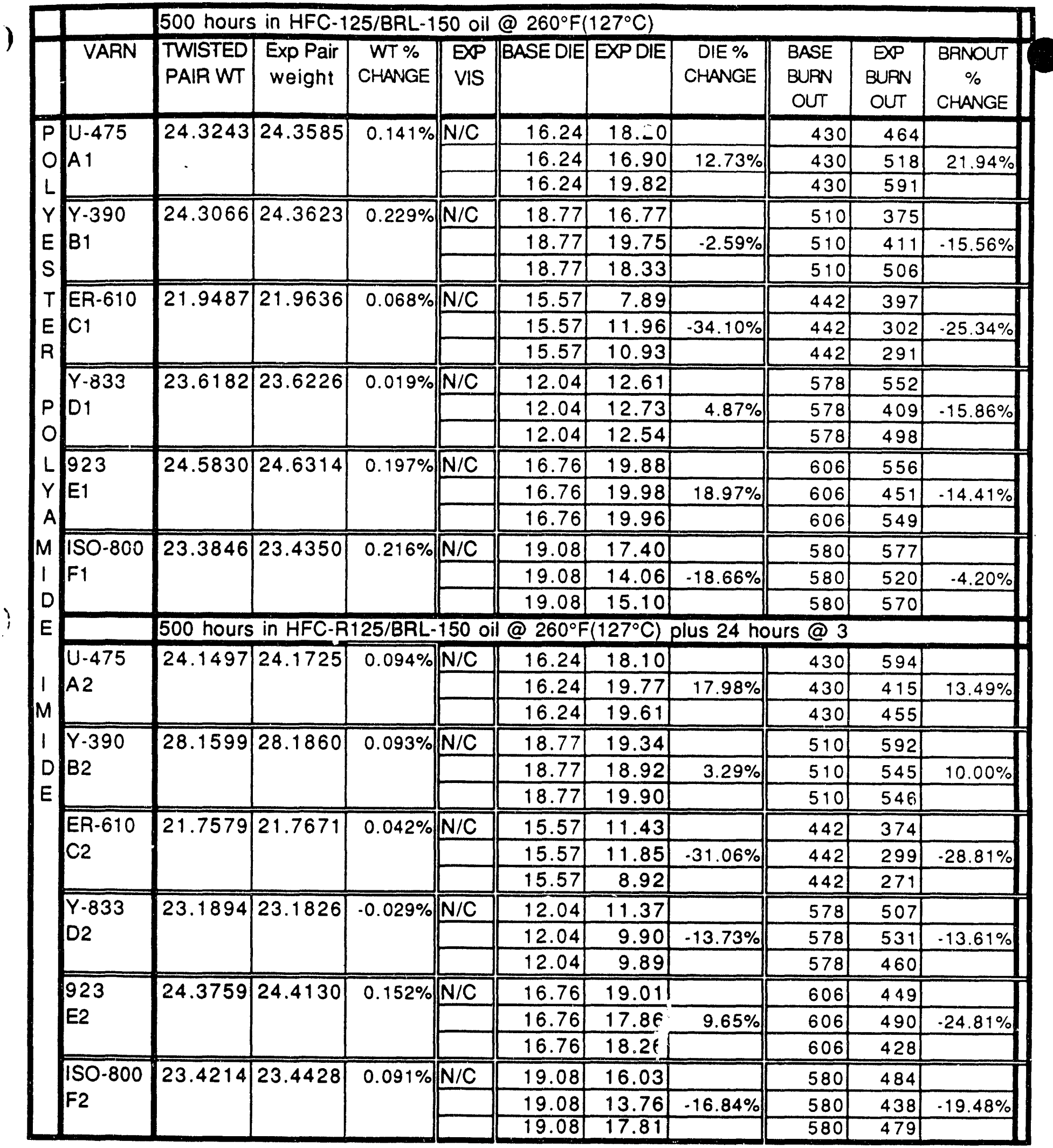




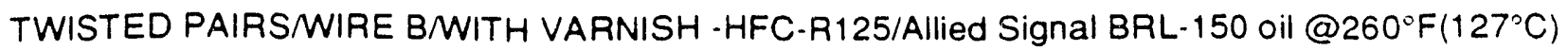

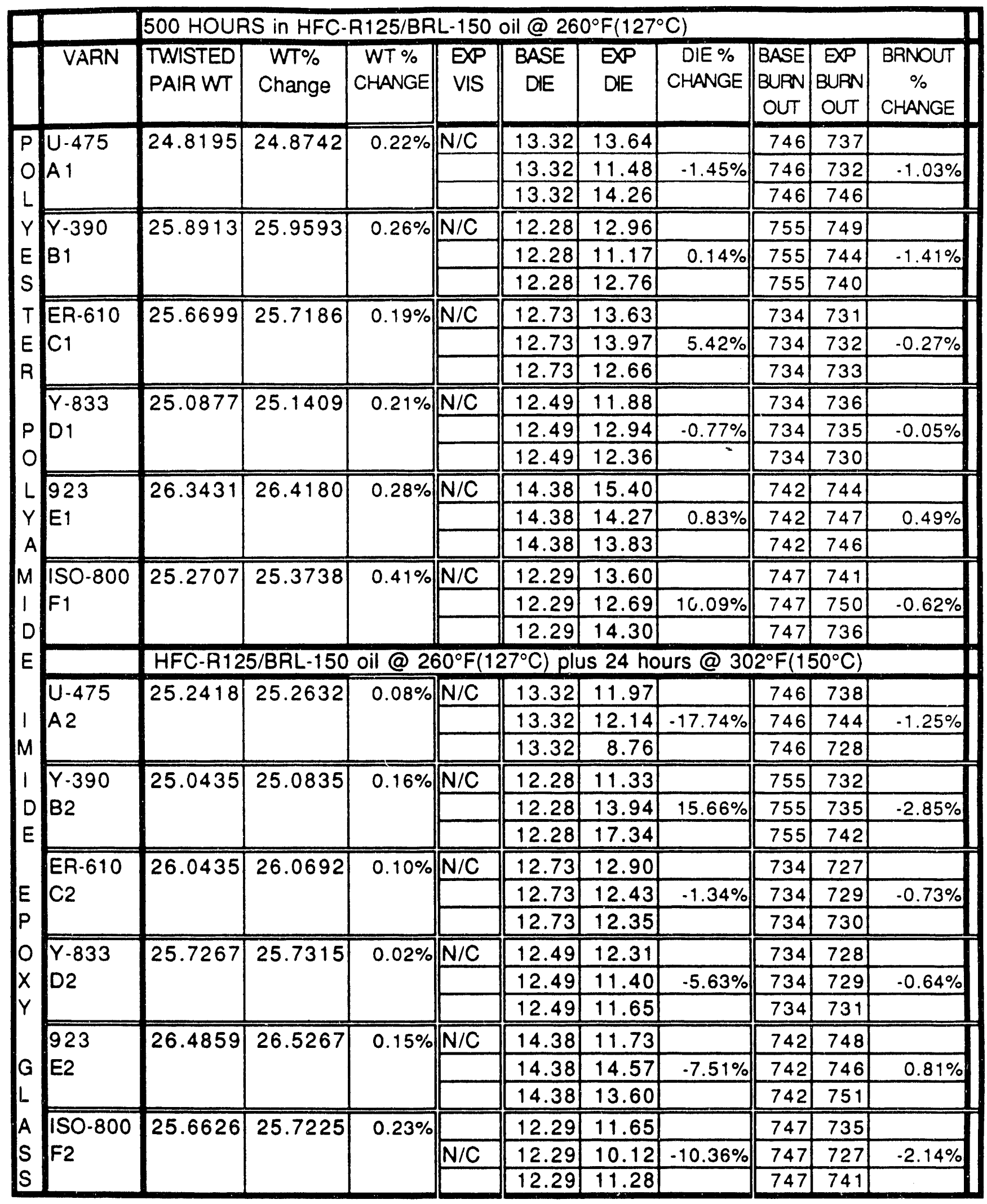


TWISTED PAIRSNIRE CNITH VARNISH-HFC-R125/Allied Signal BRL-150@ $260^{\circ} \mathrm{F}\left(127^{\circ} \mathrm{C}\right)$

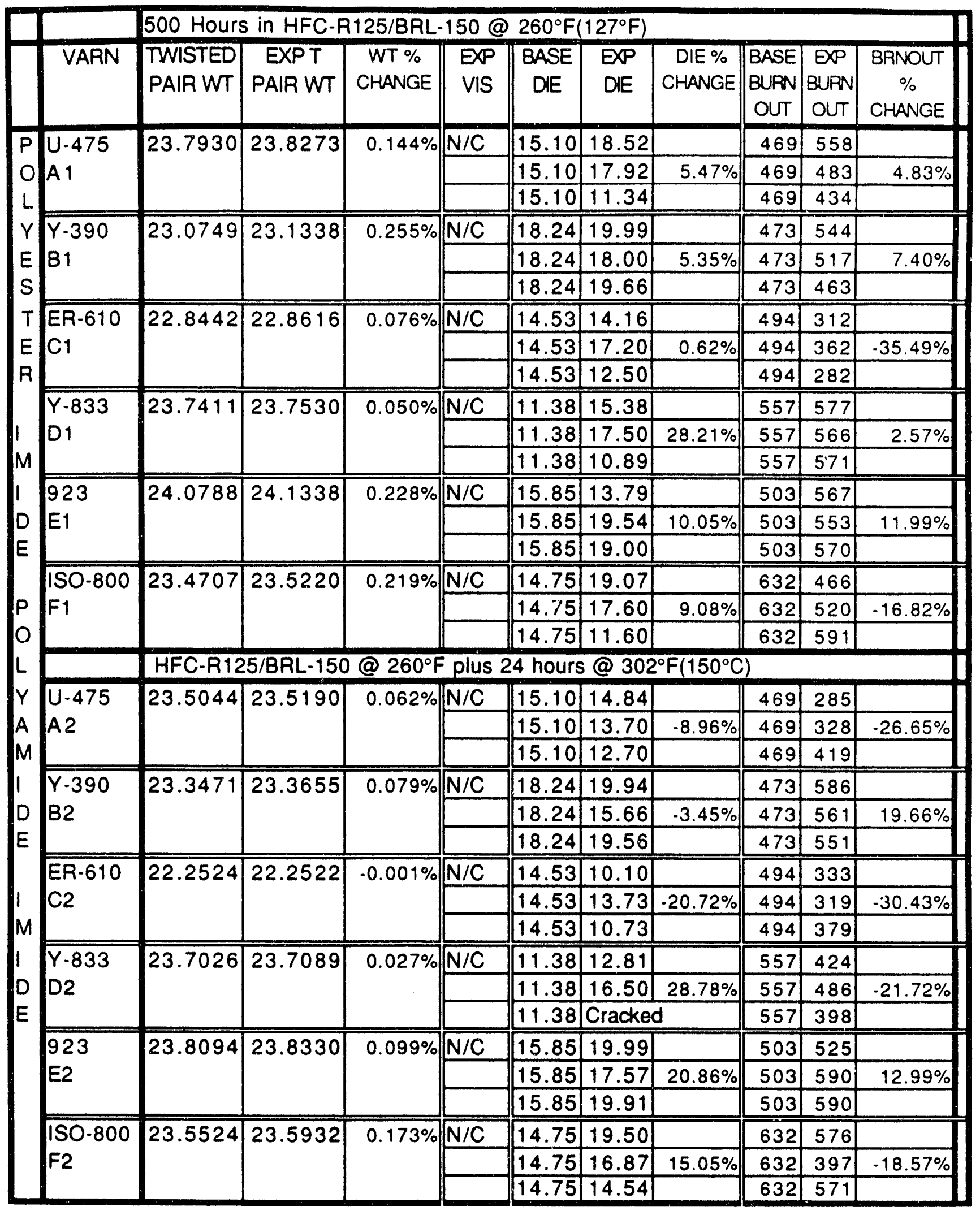


HELICAL COILSMIRE A-..-HFC-125/Allied Signal BRL-150@260 $\mathrm{F}\left(i 27^{\circ} \mathrm{C}\right)$

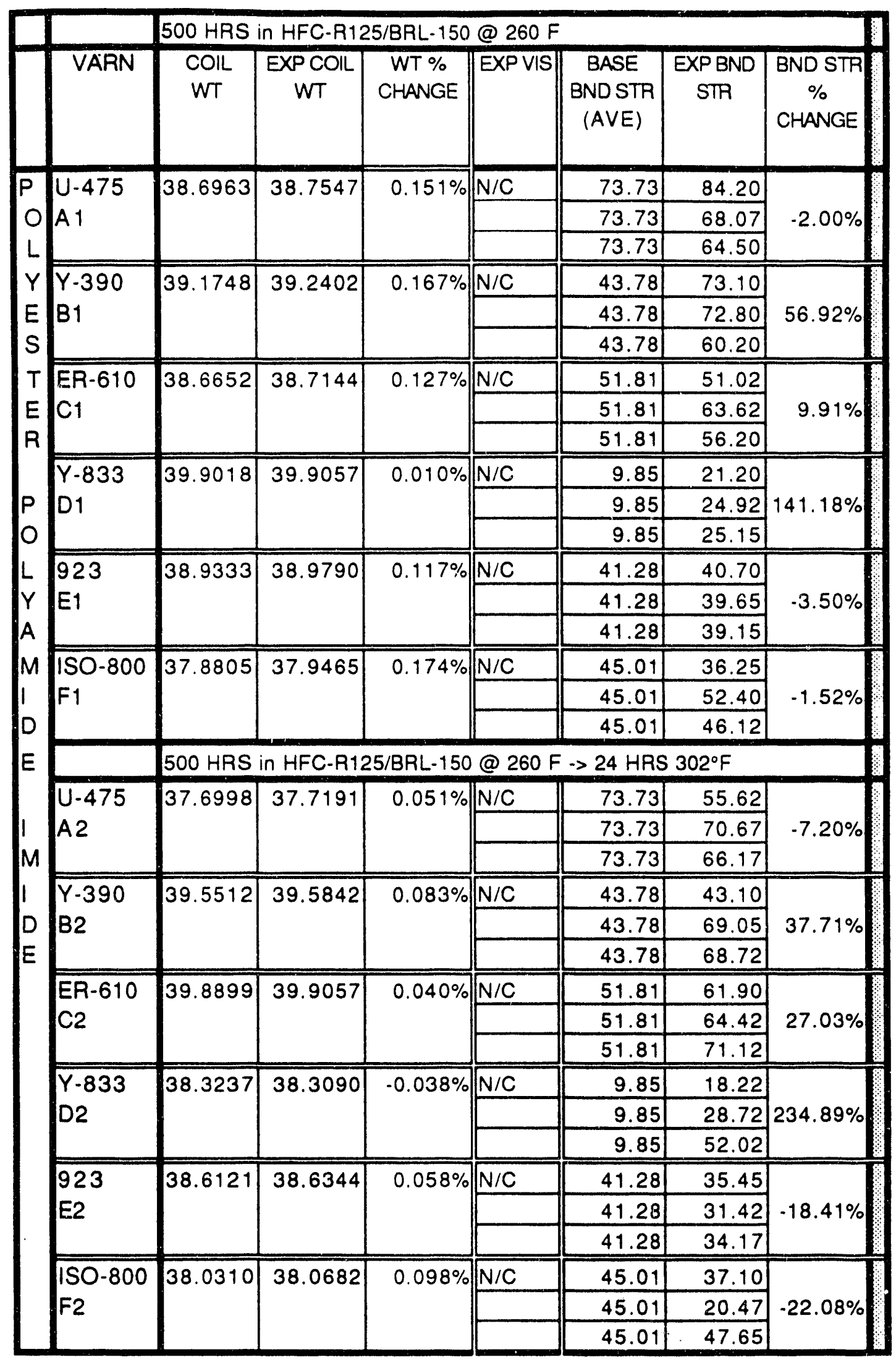




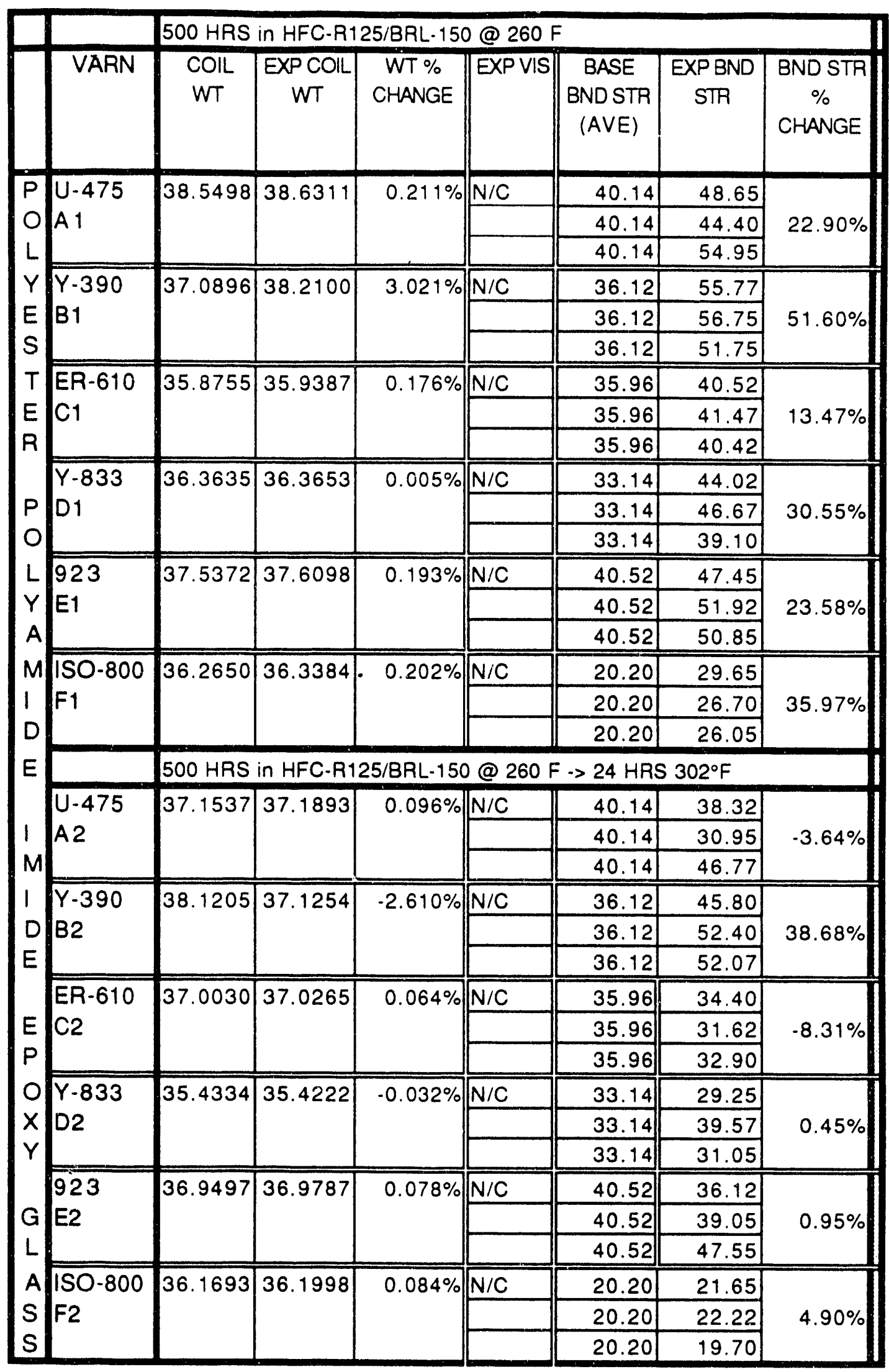


HELICAL COILSMIRE C.HFC.R125/Allied Signal BRL-150@260 $\mathrm{F}\left(127^{\circ} \mathrm{C}\right)$

\begin{tabular}{|c|c|c|c|c|c|c|c|c|}
\hline & & $500 \mathrm{HRS}$ & in HFC.R1 & $5 / B R L-150$ & @260 & & & \\
\hline & VARN & $\begin{array}{l}\text { COIL } \\
W T\end{array}$ & $\begin{array}{c}\text { EXP COIL } \\
W T\end{array}$ & $\begin{array}{c}\text { WT \% } \\
\text { CHANGE }\end{array}$ & EXP VIS & $\begin{array}{c}\text { BASE } \\
\text { BND STR } \\
(\text { AVE })\end{array}$ & $\begin{array}{c}\text { EXPBND } \\
\text { STR }\end{array}$ & $\begin{array}{c}\text { BND STR } \\
\% \\
\text { CHANGE }\end{array}$ \\
\hline$P$ & U.475 & 38.8294 & 38.8911 & $0.159 \%$ & $\mathrm{~N} / \mathrm{C}$ & 51.21 & 68.32 & \\
\hline 0 & A 1 & & & & & 51.21 & 73.35 & $31.76 \%$ \\
\hline$L$ & & & & & & 51.21 & 60.75 & \\
\hline$Y$ & $Y-390$ & 40.2661 & 40.3406 & $0.185 \%$ & $\mathrm{~N} / \mathrm{C}$ & 50.72 & 54.52 & \\
\hline$E$ & & & & & & 50.72 & 43.35 & $-6.26 \%$ \\
\hline S & & & & & & 50.72 & 44.77 & \\
\hline$T$ & ER-610 & 38.5631 & 38.6155 & $0.136 \%$ & $\mathrm{~N} / \mathrm{C}$ & 58.33 & 76.22 & \\
\hline$E$ & $\mathrm{C} 1$ & & & & & 58.33 & 73.92 & $21.29 \%$ \\
\hline$R$ & & & & & & 58.33 & 62.10 & \\
\hline & $Y-833$ & 37.2576 & 37.2791 & $0.058 \%$ & $\mathrm{~N} / \mathrm{C}$ & 5.84 & 26.22 & \\
\hline & D1 & & & & & 5.84 & 38.65 & $432.19 \%$ \\
\hline M & & & & & & 5.84 & 28.37 & \\
\hline 1 & 923 & 39.0369 & 39.0866 & $0.127 \%$ & $\mathrm{~N} / \mathrm{C}$ & 49.26 & 27.75 & \\
\hline$D$ & E1 & & & & & 49.26 & 28.90 & $-43.21 \%$ \\
\hline E & & & & & & 49.26 & 27.27 & \\
\hline & $1 S O-800$ & 39.2795 & 39.3536 & $0.189 \%$ & $\mathrm{~N} / \mathrm{C}$ & 36.08 & 59.70 & \\
\hline$P$ & F1 & & & & & 36.08 & 61.00 & $54.54 \%$ \\
\hline 0 & & & & & & 36.08 & 46.57 & \\
\hline$L$ & & 500 HRS & in $\mathrm{HFC}-\mathrm{R} 1$ & 5/ERL-150 & @ 260 F & $\rightarrow 24 \mathrm{HRS}$ & $302^{\circ} \mathrm{F}$ & \\
\hline$Y$ & U.475 & 35.9312 & 35.9583 & $0.075 \%$ & $\mathrm{~N} / \mathrm{C}$ & 51.21 & 61.67 & \\
\hline A & A2 & & & & & 51.21 & 63.15 & $20.37 \%$ \\
\hline M & & & & & & 51.21 & 60.10 & \\
\hline 1 & $Y-390$ & 38.3756 & 38.4103 & $0.090 \%$ & $\mathrm{~N} / \mathrm{C}$ & 50.72 & 57.12 & \\
\hline$D$ & & & & & & 50.72 & 50.67 & $-2.00 \%$ \\
\hline$E$ & & & & & & 50.72 & 41.32 & \\
\hline & ER-610 & 40.0733 & 40.0964 & $0.058 \%$ & $\mathrm{~N} / \mathrm{C}$ & 58.33 & 75.80 & \\
\hline 1 & $\mathrm{C} 2$ & & & & & 58.33 & 78.05 & $17.04 \%$ \\
\hline$M$ & & & & & & 58.33 & 50.95 & \\
\hline 1 & $Y-833$ & 37.4496 & 37.4640 & $0.038 \%$ & $\mathrm{~N} / \mathrm{C}$ & 5.84 & 24.72 & \\
\hline$D$ & D2 & & & & & 5.84 & 35.35 & $366.27 \%$ \\
\hline E & & & & & & 5.84 & 21.62 & \\
\hline & 923 & 38.2843 & 38.3075 & $0.061 \%$ & $\mathrm{~N} / \mathrm{C}$ & 49.26 & 29.15 & \\
\hline & E2 & & & & & 49.26 & 30.90 & $-39.83 \%$ \\
\hline & & & & & & 49.26 & 28.87 & \\
\hline & ISO-800 & 37.1101 & 37.1396 & $0.079 \%$ & $\mathrm{~N} / \mathrm{C}$ & 36.08 & 42.65 & \\
\hline & F2 & & & & & 36.08 & 20.90 & $10.70 \%$ \\
\hline & & & & & & 36.08 & 56.27 & \\
\hline
\end{tabular}


SINGLE MAG WIRE WITHOUT VARNISH-HFC-R125/Allied Signal BRL-150@260 $\mathrm{F}\left(127^{\circ} \mathrm{C}\right)$ )

\begin{tabular}{|c|c|c|c|c|c|}
\hline \multicolumn{6}{|c|}{$500 \mathrm{HRS}$ in HFC-R125/BRL-150 oil @ 260 $\mathrm{F}\left(127^{\circ} \mathrm{C}\right)$} \\
\hline ID & $W T$ & EXPWT & $\begin{array}{c}\text { WT \% } \\
\text { CHANGE }\end{array}$ & EXP VIS & EXPFLEX \\
\hline \multirow{2}{*}{ A1 } & \multirow[t]{2}{*}{3.7172} & \multirow[t]{2}{*}{3.719} & \multirow[t]{2}{*}{$0.048 \%$} & $\mathrm{~N} / \mathrm{C}$ & \\
\hline & & & & & YES \\
\hline \multirow{2}{*}{$B 1$} & \multirow{2}{*}{3.7608} & \multirow{2}{*}{3.7671} & \multirow{2}{*}{$0.168 \%$} & $\mathrm{~N} / \mathrm{C}$ & \\
\hline & & & & & YES \\
\hline \multirow[t]{2}{*}{ C1 } & \multirow[t]{2}{*}{3.5924} & \multirow[t]{2}{*}{3.5941} & \multirow[t]{2}{*}{$0.047 \%$} & $\mathrm{~N} / \mathrm{C}$ & \\
\hline & & & & & YES \\
\hline \multicolumn{6}{|c|}{$500 \mathrm{HRS}$ in HFC-R125/BRL-150 oil-> $302^{\circ} \mathrm{F}$ for $24 \mathrm{HRS}$} \\
\hline \multirow[t]{2}{*}{ A2 } & \multirow[t]{2}{*}{3.6589} & \multirow[t]{2}{*}{3.6597} & \multirow[t]{2}{*}{$0.022 \%$} & $\mathrm{~N} / \mathrm{C}$ & \\
\hline & & & & & YES \\
\hline \multirow[t]{2}{*}{$\mathrm{B2}$} & \multirow[t]{2}{*}{3.4408} & \multirow[t]{2}{*}{3.444} & \multirow[t]{2}{*}{$0.093 \%$} & $\mathrm{~N} / \mathrm{C}$ & \\
\hline & & & & & YES \\
\hline \multirow[t]{3}{*}{$\mathrm{C} 2$} & \multirow[t]{3}{*}{3.3865} & \multirow[t]{3}{*}{3.3876} & \multirow[t]{3}{*}{$0.032 \%$} & $\mathrm{~N} / \mathrm{C}$ & \\
\hline & & & & & YES \\
\hline & & & & & \\
\hline
\end{tabular}




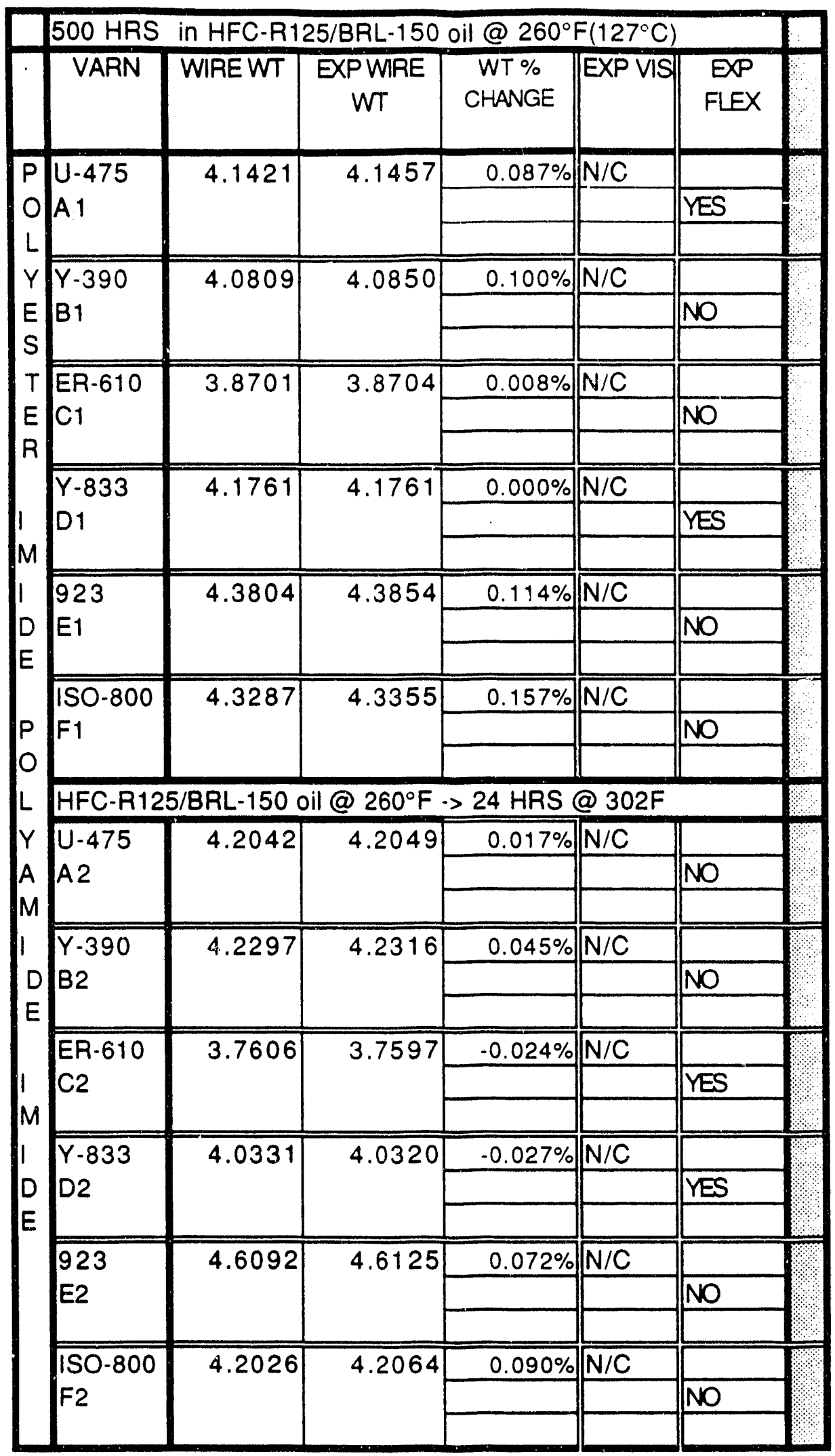




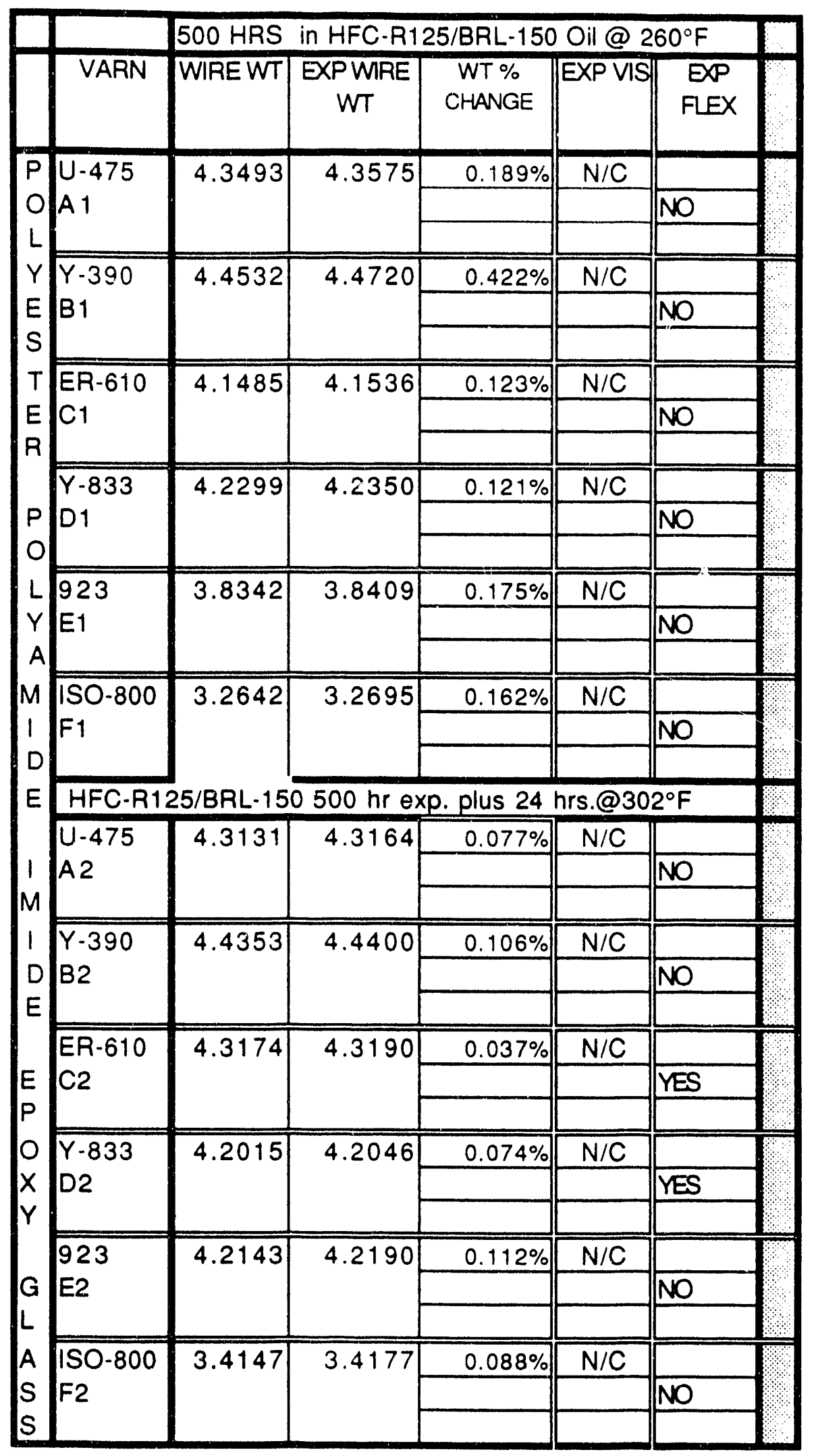




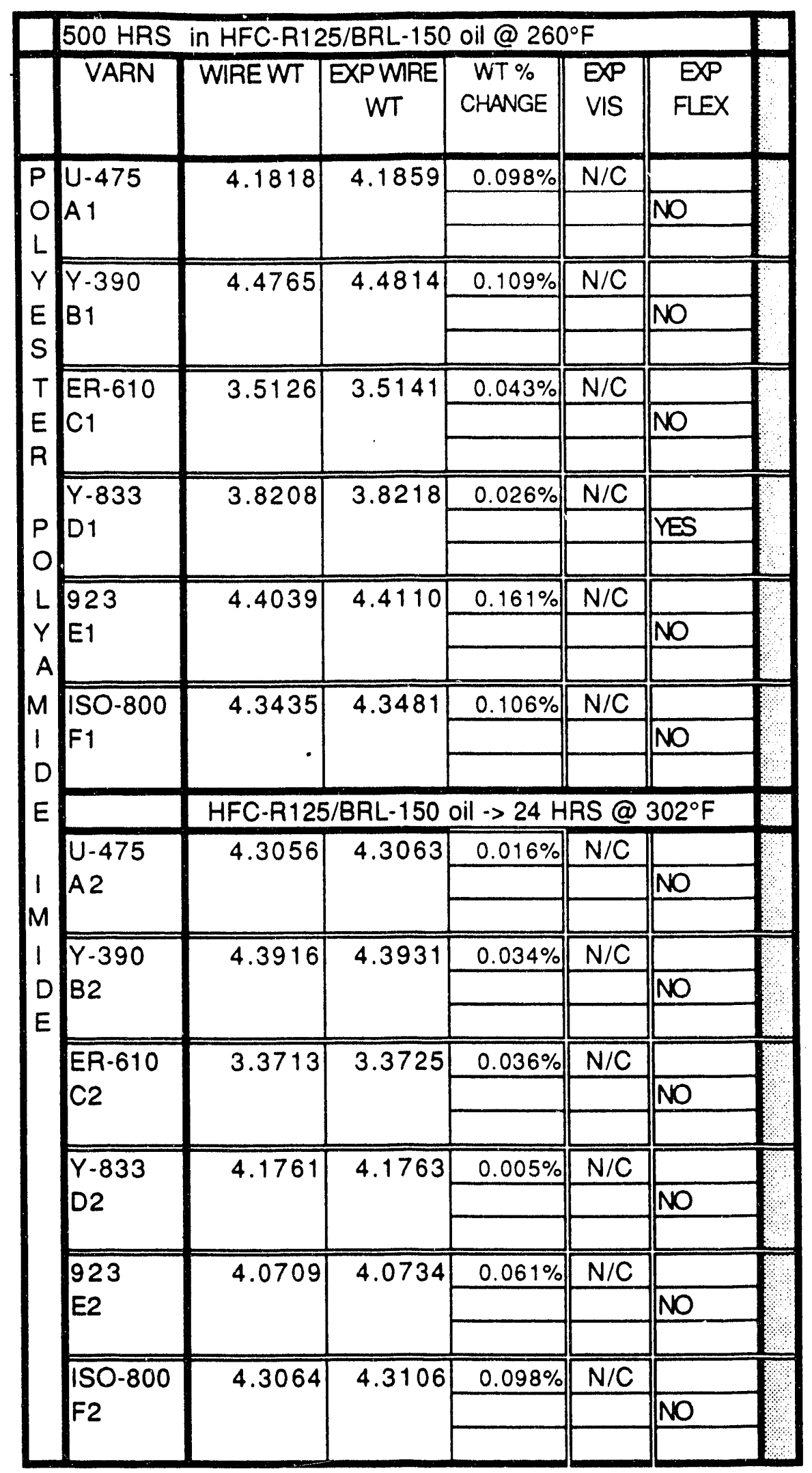




\begin{tabular}{|c|c|c|c|c|c|}
\hline & 500 HRS IN & R125/BRL-1 & $50 @ 260^{\circ}$ & & \\
\hline ID & WT & EXPWT & $\begin{array}{c}\text { WT \% } \\
\text { CHANGE }\end{array}$ & EXP VIS & EXPFLEX \\
\hline U-475 & 3.1403 & 3.1608 & $0.65 \%$ & Surface & $\mathrm{N} / \mathrm{C}$ \\
\hline A 1 & & & & Cloudy & \\
\hline & & & & S. Warpeo & \\
\hline$Y-390$ & 2.9379 & 3.0191 & $2.76 \%$ & $N / C$ & $\mathrm{~N} / \mathrm{C}$ \\
\hline B1 & & & & & \\
\hline ER-610 & 2.3721 & 2.4645 & $3.90 \%$ & $N / C$ & $\mathrm{~N} / \mathrm{C}$ \\
\hline C1 & & & & & \\
\hline$Y \cdot 833$ & 3.2340 & 3.3694 & $4.19 \%$ & $\mathrm{~N} / \mathrm{C}$ & $\mathrm{N} / \mathrm{C}$ \\
\hline D1 & & & & & \\
\hline 923 & 3.1078 & 3.1894 & $2.63 \%$ & & $N / C$ \\
\hline E1 & & & & Warped & \\
\hline $150-800$ & 1.6159 & 1.6402 & $1.50 \%$ & Slightly & $N / C$ \\
\hline & & & & warped & \\
\hline & R125/BRL- & 50 at $\cdots->24$ & hour at 3 & $302^{\circ} \mathrm{F}$. & \\
\hline$U-475$ & 2.5539 & 2.5083 & $-1.79 \%$ & Surface & $\mathrm{N} / \mathrm{C}$ \\
\hline A2 & & & & Cloudy & \\
\hline & & & & Darkened & \\
\hline$Y-390$ & 2.6137 & 2.6490 & $1.35 \%$ & Slightly & $N / C$ \\
\hline B2 & & & & warped & \\
\hline & & & & Darkened & \\
\hline ER-610 & 2.4751 & 2.4911 & $0.65 \%$ & Darkened & $\mathrm{N} / \mathrm{C}$ \\
\hline $\mathrm{C2}$ & & & & & \\
\hline$\overline{Y-833}$ & 29479 & 29441 & $.013 \%$ & & $N / C$ \\
\hline D2 & $2.94 / 9$ & 2.9441 & $-0.13 \%$ & \begin{tabular}{|l} 
Some \\
Somed
\end{tabular} & $N / C$ \\
\hline & & & & Pockets & \\
\hline 923 & 2.2108 & 2.2341 & $1.05 \%$ & Warped & \\
\hline E2 & & & & Darkened & $N / C$ \\
\hline & 1.8138 & 1.7934 & $.112 \%$ & slightly & \\
\hline $\mathrm{F} 2$ & 1.0100 & 1.1004 & $1.12 \%$ & warped & $\sqrt[N]{N / C}$ \\
\hline & & & & Darkened & \\
\hline
\end{tabular}




\begin{tabular}{|c|c|c|c|c|c|c|c|c|c|}
\hline & $500 \mathrm{HRS}$ & 3 in $\mathrm{HFC}$ & R125/BRL- & 150 Oil@ & $260 \mathrm{~F}$ & & & & \\
\hline ID & $\mathrm{WT}$ & EXPWT & $\begin{array}{c}\text { WT \% } \\
\text { CHANGE }\end{array}$ & $\begin{array}{l}\text { SAMPLE } \\
\text { WIDTH }\end{array}$ & $\begin{array}{l}\text { BREAK } \\
\text { LOAD }\end{array}$ & $\begin{array}{c}\text { SAMPLE } \\
\text { THICKNESS } \\
\text { Mils }\end{array}$ & $\begin{array}{c}\text { TENSILE } \\
\text { STR } \\
\text { BASE }\end{array}$ & $\begin{array}{l}\text { TENSIL.E } \\
\text { STREXP }\end{array}$ & $\begin{array}{c}\% \text { CHANGE } \\
\text { TENSILE }\end{array}$ \\
\hline NO/MY/NO & 4.8268 & 5.1964 & $7.66 \%$ & 0.711 & 246.20 & 21 & 17.4 & 16.49 & \\
\hline A1 & & & & 0.613 & 190.70 & 21 & 17.4 & 14.81 & $-11.14 \%$ \\
\hline & & & & 0.651 & 206.20 & 21 & 17.4 & 15.08 & \\
\hline$D A / M Y / D A$ & 4.7849 & 4.8810 & $2.01 \%$ & 0.374 & 99.30 & 21 & 13.7 & 12.64 & \\
\hline B1 & & & & 0.380 & 113.20 & 21 & 13.7 & 14.19 & $-6.91 \%$ \\
\hline & & & & 0.361 & 86.65 & 21 & 13.7 & 11.43 & \\
\hline MYAR MO & 2.2637 & 2.3271 & $2.80 \%$ & 0.432 & 71.20 & 10 & 21.7 & 16.48 & \\
\hline $\mathrm{Cr}$ & & & & 0.453 & 78.95 & 10 & 21.7 & 17.43 & $-22.19 \%$ \\
\hline & & & & 0.442 & 74.00 & 10 & 21.7 & 16.74 & \\
\hline NO 410 & 2.3887 & 2.7257 & $14.11 \%$ & 0.597 & 106.70 & 10 & 18.7 & 17.87 & \\
\hline D1 & & & & 0.399 & 52.12 & 10 & 18.7 & 13.06 & $-11.59 \%$ \\
\hline & & & & 0.422 & 78.75 & 10 & 18.7 & 18.66 & \\
\hline NO MI 418 & 2.1997 & 2.7361 & $24.39 \%$ & 0.530 & 29.25 & 9 & 7.5 & 6.13 & \\
\hline E1 & & & & 0.508 & 29.00 & 9 & 7.5 & 6.34 & $-15.79 \%$ \\
\hline & & & & 0.509 & 29.65 & 9 & 7.5 & 6.47 & \\
\hline MEL 228 & 2.8250 & 2.9133 & $3.13 \%$ & 0.410 & lost & 10 & 21.7 & & \\
\hline F1 & & & & 0.540 & 73.80 & 10 & 21.7 & 13.67 & $-27.76 \%$ \\
\hline & & & & 0.490 & 86.65 & 10 & 21.7 & 17.68 & \\
\hline & $500 \mathrm{HR}$ & $S$ in HFC & R125/BRL & 150 Oil@ & $260 \mathrm{~F}->$ & >24 HRS@ & $302^{\circ} \mathrm{F}$ & & \\
\hline NO/MY/NO & 5.1955 & 5.2968 & $1.95 \%$ & 0.578 & 102.30 & 21 & 17.4 & 8.43 & \\
\hline$A 2$ & & & & 0.537 & 102.30 & 21 & 17.4 & 9.07 & $.52 .36 \%$ \\
\hline & & & & 0.661 & 102.30 & 21 & 17.4 & 7.37 & \\
\hline$D A / M Y / D A$ & 4.7094 & 4.6673 & $-0.89 \%$ & 0.347 & 97.60 & 21 & 13.7 & 13.39 & \\
\hline $\mathrm{B2}$ & & & & 0.407 & 102.30 & 21 & 13.7 & 11.97 & $-6.93 \%$ \\
\hline & & & & 0.378 & 102.30 & 21 & 13.7 & 12.89 & \\
\hline MYLAR MO & 2.1826 & 2.1783 & $-0.20 \%$ & 0.435 & 74.62 & 9 & 21.7 & 19.06 & \\
\hline C2 & & & & 0.467 & 79.80 & 9 & 21.7 & 18.99 & $-11.55 \%$ \\
\hline & & & & 0.388 & 68.22 & 9 & 21.7 & 19.54 & \\
\hline NOMEX 410 & 2.2779 & 2.3669 & $3.91 \%$ & 0.440 & 89.17 & 10 & 18.7 & 20.27 & \\
\hline $\mathrm{D} 2$ & & & & 0.500 & 96.85 & 10 & 18.7 & 19.37 & $3.44 \%$ \\
\hline & & & & 0.527 & 96.95 & 10 & 18.7 & 18.40 & \\
\hline NO/MI 418 & 2.4057 & 2.4422 & $1.52 \%$ & 0.527 & 31.35 & 10 & 7.5 & 5.95 & \\
\hline & & & & 0.475 & 24.85 & 10 & 7.5 & 5.23 & $-25.03 \%$ \\
\hline & & & & 0.458 & 26.05 & 10 & 7.5 & 5.69 & \\
\hline MEL 228 & 2.7661 & 2.7734 & $0.26 \%$ & 0.525 & 88.80 & 10 & 21.7 & 16.91 & \\
\hline $\mathrm{F} 2$ & & & & 0.342 & 59.67 & 10 & 21.7 & 17.45 & $-22.90 \%$ \\
\hline & & & & 0.423 & 66.97 & 10 & 21.7 & 15.83 & \\
\hline
\end{tabular}




\begin{tabular}{|c|c|c|c|c|c|c|c|c|}
\hline & 500 HRS & in $\mathrm{HFC} \cdot \mathrm{F}$ & 125/BRL. & -1500il@ & $D 260 \mathrm{~F}$ & & & \\
\hline ID & $\begin{array}{c}\text { STRETCH } \\
\text { (inch) }\end{array}$ & $\begin{array}{c}\% \\
\text { ELONG }\end{array}$ & $\begin{array}{l}\text { BASE } \\
\text { ELONG } \\
\text { (AVE) }\end{array}$ & $\begin{array}{l}\text { ELONG \% } \\
\text { CHANGE }\end{array}$ & $\begin{array}{c}\text { BASE } \\
\text { DIE } \\
\text { (AVE) }\end{array}$ & EXPDIE & $\begin{array}{c}\text { DIE \% } \\
\text { CHANGE }\end{array}$ & VISUAL EXP \\
\hline NO/MY/NO & 0.43 & $10.8 \%$ & $20.0 \%$ & & $>18.97$ & flash & & $N / C$ \\
\hline A1 & 0.35 & $8.8 \%$ & $20.0 \%$ & $.51 .3 \%$ & $>18.97$ & flash & flash & \\
\hline & 0.55 & $13.8 \%$ & $20.0 \%$ & & $>18.97$ & flash & & \\
\hline$D A / M Y / D A$ & 0.49 & $24.5 \%$ & $46.0 \%$ & & $>15.27$ & flash & & Yellowed \\
\hline B1 & 0.42 & $21.0 \%$ & $46.0 \%$ & $.55 .8 \%$ & $>15.27$ & flash & flash & slightly \\
\hline & 0.31 & $15.5 \%$ & $46.0 \%$ & & $>15.27$ & flash & & warped \\
\hline MYAR MO & 1.81 & $90.5 \%$ & $131.0 \%$ & & $>14.91$ & flash & & $\mathrm{N} / \mathrm{C}$ \\
\hline C1 & 2.39 & $119.5 \%$ & $131.0 \%$ & $.8 .9 \%$ & $>14.91$ & flash & flash & \\
\hline & 2.96 & $148.0 \%$ & $131.0 \%$ & & $>14.91$ & flash & & \\
\hline NO 410 & 0.38 & $19.0 \%$ & $17.0 \%$ & & 10.67 & 13.69 & & $\mathrm{~N} / \mathrm{C}$ \\
\hline D1 & 0.18 & $4.5 \%$ & $17.0 \%$ & $.32 .4 \%$ & 10.67 & 13.70 & $28.7 \%$ & \\
\hline & 0.44 & $11.0 \%$ & $17.0 \%$ & & 10.67 & 13.82 & & \\
\hline NO Ml 418 & 0.05 & $1.3 \%$ & $4.0 \%$ & & 10.23 & 10.36 & & $\mathrm{~N} / \mathrm{C}$ \\
\hline$E 1$ & 0.04 & $1.0 \%$ & $4.0 \%$ & $.68 .8 \%$ & 10.23 & 11.50 & $1.7 \%$ & \\
\hline & 0.06 & $1.5 \%$ & $4.0 \%$ & & 10.23 & 9.36 & & \\
\hline MEL 228 & 3.54 & $177.0 \%$ & $160.0 \%$ & & $>14.22$ & flash & & slightly \\
\hline $\mathrm{F} 1$ & 1.39 & $69.5 \%$ & $160.0 \%$ & $-23.0 \%$ & $\geq 14.22$ & flash & flash & darkened \\
\hline & & & $160.0 \%$ & & $>14.22$ & flash & & \\
\hline & 500 HAS & in HFC. & 125/BRL. & -1500il@ & $260 \mathrm{~F}$ & $>24$ HRS & @ 302 $\mathrm{F}$ & \\
\hline NO/MY/NO & 0.11 & $2.8 \%$ & $20.0 \%$ & & $>18.97$ & flash & & Delamination \\
\hline $\mathrm{A} 2$ & 0.15 & $3.8 \%$ & $20.0 \%$ & $.69 .2 \%$ & $>18.97$ & flash & flash & big pockets \\
\hline & 0.48 & $12.0 \%$ & $20.0 \%$ & & $>18.97$ & flash & & \\
\hline DA/MY/DA & 0.55 & $27.5 \%$ & $46.0 \%$ & & $>15.27$ & flash & & Browned \\
\hline $\mathrm{B} 2$ & 0.55 & $27.5 \%$ & $46.0 \%$ & $.40 .6 \%$ & $>15.27$ & flash & flash & slightly \\
\hline & 0.54 & $27.0 \%$ & $46.0 \%$ & & $>15.27$ & flash & & warped \\
\hline MYAR MO & 2.87 & $143.5 \%$ & $131.0 \%$ & & $>14.91$ & flash & & $\mathrm{N} / \mathrm{C}$ \\
\hline $\mathrm{C} 2$ & 2.42 & $121.0 \%$ & $131.0 \%$ & $3.1 \%$ & $>14.91$ & flash & flash & \\
\hline & 2.81 & $140.5 \%$ & $131.0 \%$ & & $>14.91$ & flash & & \\
\hline NOMEX 410 & 0.51 & $12.8 \%$ & $17.0 \%$ & & 10.67 & 6.93 & & slightly \\
\hline D2 & 0.43 & $10.8 \%$ & $17.0 \%$ & $-36.8 \%$ & 10.67 & 10.86 & $-22.6 \%$ & Darkened \\
\hline & 0.35 & $8.8 \%$ & $17.0 \%$ & & 10.67 & 6.99 & & \\
\hline $\mathrm{NO} / \mathrm{Nil} 418$ & 0.04 & $1.0 \%$ & $4.0 \%$ & & 10.23 & 11.19 & & $\mathrm{~N} / \mathrm{C}$ \\
\hline E2 & 0.03 & $0.8 \%$ & $4.0 \%$ & $.77 .1 \%$ & 10.23 & 9.29 & $1.2 \%$ & \\
\hline & 0.04 & $1.0 \%$ & $4.0 \%$ & & 10.23 & 10.59 & & \\
\hline MEL 228 & 0.13 & $6.5 \%$ & $160.0 \%$ & & $>14.22$ & flash & & browned \\
\hline F2 & 3.01 & $150.5 \%$ & $160.0 \%$ & $-65.9 \%$ & $>14.22$ & flash & flash & darkened \\
\hline & 0.13 & $6.5 \%$ & $160.0 \%$ & & $\mid>14.22$ & flash & & \\
\hline
\end{tabular}




\begin{tabular}{|c|c|c|c|c|}
\hline & \multicolumn{4}{|c|}{500 HRS in HFC-R125/BRL-150@260 0} \\
\hline & WT & EYPWT & $\begin{array}{c}\% \\
\text { CHANGE }\end{array}$ & EXPVS \\
\hline A1 & 0.5445 & 0.6276 & $15.26 \%$ & $\mathrm{~N} / \mathrm{C}$ \\
\hline $\begin{array}{l}\text { B1 } \\
\text { MYLAR }\end{array}$ & 0.4931 & 0.5125 & $3.93 \%$ & \begin{tabular}{|c|} 
small Pockets \\
when rubbed between fingers \\
be bacame cloudy in appearanc
\end{tabular} \\
\hline & 0.3966 & 0.4277 & $7.84 \%$ & some small pockets \\
\hline & HFC-R125/B & L-150 oil & $260^{\circ} \mathrm{F} \mathrm{pl}$ & us24 hrs@302F \\
\hline & 0.5451 & 0.5753 & $5.54 \%$ & Slightly Darkened \\
\hline B2 & 0.4451 & 0.4460 & $0.20 \%$ & Big pockets \\
\hline MYLAR & & & & Darkened \\
\hline $\mathrm{C} 2$ & 0.3922 & 0.3962 & $1.02 \%$ & Nomex delaminated from mylar \\
\hline NO/MY & & & & some pockets where mylar \\
\hline & & & & pulled away. \\
\hline
\end{tabular}




\begin{tabular}{|c|c|c|c|c|c|c|c|c|c|}
\hline \multicolumn{10}{|c|}{$5500 \mathrm{HRS}$ in HFC.134a/BRL-150@ $260 \mathrm{~F}$} \\
\hline ID & WT & EXPWT & $\begin{array}{c}\text { WT \% } \\
\text { CHANGE }\end{array}$ & $\begin{array}{c}\text { BREAK } \\
\text { LOAD } \\
\text { (AVE) }\end{array}$ & $\begin{array}{l}\text { BREAK } \\
\text { LOAD } \\
\text { EPP }\end{array}$ & $\begin{array}{c}\% \\
\text { CHANGE } \\
\text { BRK } \\
\text { LOAD }\end{array}$ & $\begin{array}{c}\text { STRETOH } \\
(\text { INCH) }\end{array}$ & $\%$ ELONG & EXP VIS \\
\hline \multirow{3}{*}{$\begin{array}{l}\text { A } 1 \\
\text { Glass }\end{array}$} & \multirow[t]{3}{*}{1.6620} & \multirow[t]{3}{*}{1.6689} & \multirow[t]{3}{*}{$0.42 \%$} & 39.02 & 66.57 & & 0.08 & $4.00 \%$ & $\mathrm{~N} / \mathrm{C}$ \\
\hline & & & & 39.02 & 51.65 & $38.28 \%$ & 0.06 & $3.00 \%$ & \\
\hline & & & & 39.02 & 43.65 & & 0.06 & $3.00 \%$ & \\
\hline \multirow{3}{*}{$\begin{array}{l}\text { B1 } \\
\text { Polyester }\end{array}$} & \multirow[t]{3}{*}{0.7040} & \multirow[t]{3}{*}{0.7083} & \multirow[t]{3}{*}{$0.61 \%$} & 56.12 & 57.70 & & 0.62 & $31.00 \%$ & $\mathrm{~N} / \mathrm{C}$ \\
\hline & & & & 56.12 & 58.15 & $3.65 \%$ & 0.65 & $32.50 \%$ & \\
\hline & & & & 56.12 & 58.65 & & 0.70 & $35.00 \%$ & \\
\hline \multirow{3}{*}{$\begin{array}{l}\text { C1 } \\
\text { Permacel }\end{array}$} & \multirow[t]{3}{*}{1.5619} & \multirow[t]{3}{*}{1.9732} & \multirow[t]{3}{*}{$26.33 \%$} & 88.50 & 63.30 & & 0.14 & $7.00 \%$ & Slightly \\
\hline & & & & 88.50 & 85.20 & $-21.68 \%$ & 0.14 & $7.00 \%$ & darkened \\
\hline & & & & 88.50 & 59.45 & & 0.12 & $6.00 \%$ & Curled up \\
\hline & \multicolumn{7}{|c|}{ 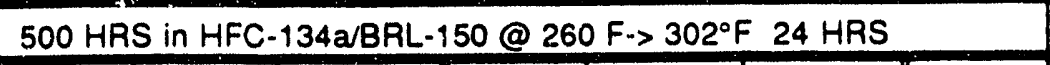 } & & \\
\hline \multirow{3}{*}{$\begin{array}{l}\text { A2 } \\
\text { Glass }\end{array}$} & \multirow[t]{3}{*}{1.4762} & \multirow[t]{3}{*}{1.4758} & \multirow[t]{3}{*}{$.0 .03 \%$} & 39.02 & 66.60 & & 0.07 & $3.50 \%$ & $\mathrm{~N} / \mathrm{C}$ \\
\hline & & & & 39.02 & 58.20 & $65.51 \%$ & 0.06 & $3.00 \%$ & \\
\hline & & & & 39.02 & 68.95 & & 0.07 & $3.50 \%$ & \\
\hline \multirow{3}{*}{$\begin{array}{l}\text { B2 } \\
\text { Polyester }\end{array}$} & \multirow[t]{3}{*}{0.6662} & \multirow[t]{3}{*}{0.6668} & \multirow[t]{3}{*}{$0.09 \%$} & 56.12 & 56.80 & & 0.45 & $22.50 \%$ & $\mathrm{~N} / \mathrm{C}$ \\
\hline & & & & 56.12 & 56.55 & $1.36 \%$ & 0.53 & $26.50 \%$ & \\
\hline & & & & 56.12 & 57.30 & & 0.58 & $29.00 \%$ & \\
\hline \multirow{3}{*}{$\begin{array}{l}\mathrm{C} 2 \\
\text { Permacel }\end{array}$} & \multirow[t]{3}{*}{1.3299} & \multirow[t]{3}{*}{1.2823} & \multirow[t]{3}{*}{$-3.58 \%$} & 88.50 & 66.35 & & 0.07 & $3.50 \%$ & Slightly \\
\hline & & & & 88.50 & 87.75 & $-2.37 \%$ & 0.10 & $5.00 \%$ & Darkened \\
\hline & & & & .88 .50 & 105.10 & & 0.12 & $6.00 \%$ & \\
\hline
\end{tabular}


TIE CORD--HFC-R125/Allied Signal BRL-150 oil @ $260^{\circ} \mathrm{F}\left(127^{\circ} \mathrm{C}\right)$

\begin{tabular}{|c|c|c|c|c|c|c|c|c|c|}
\hline \multicolumn{10}{|c|}{500 HRS IN R125/BRL-150@260 F } \\
\hline 10 & WT & EXPWT & $\begin{array}{c}\text { WT \% } \\
\text { CHANGE }\end{array}$ & $\begin{array}{l}\text { EP } \\
\text { VIS }\end{array}$ & $\begin{array}{l}\text { BREAK } \\
\text { LOAD } \\
\text { (AVE) }\end{array}$ & $\begin{array}{l}\text { BREAK } \\
\text { LOAD } \\
\text { EPP } \\
\text { (ave) }\end{array}$ & $\begin{array}{c}\% \\
\text { CHANGE } \\
\text { BAK } \\
\text { LOAD }\end{array}$ & $\begin{array}{c}\text { STRETCH } \\
\text { (Inch) }\end{array}$ & $\begin{array}{c}\% \\
\text { ELONG }\end{array}$ \\
\hline \multirow[t]{4}{*}{ A1 } & \multirow[t]{3}{*}{0.3063} & \multirow[t]{3}{*}{0.3228} & \multirow[t]{3}{*}{$5.39 \%$} & $\mathrm{~N} / \mathrm{C}$ & 28.36 & 34.20 & & 0.45 & $22.5 \%$ \\
\hline & & & & & 28.36 & 30.65 & $13.72 \%$ & 0.41 & $20.5 \%$ \\
\hline & & & & & 28.36 & 31.90 & & 0.40 & $20.0 \%$ \\
\hline & \multicolumn{9}{|c|}{$500 \mathrm{HRS}$ IN R125/BRL-150 -> 24 HRS @ 302 $\mathrm{F}$} \\
\hline \multirow[t]{3}{*}{ A2 } & \multirow[t]{3}{*}{0.3345} & \multirow[t]{3}{*}{0.3321} & \multirow[t]{3}{*}{$-0.72 \%$} & $\mathrm{~N} / \mathrm{C}$ & 28.36 & 38.15 & & 0.56 & $28.0 \%$ \\
\hline & & & & & 28.36 & 32.05 & $20.20 \%$ & 0.54 & $27.0 \%$ \\
\hline & & & & & 28.36 & 32.07 & & 0.44 & $22.0 \%$ \\
\hline
\end{tabular}


LEAD WIRE INSULATION-HFC-R125/Allied Signal BRL-150@260 $\mathrm{F}\left(127^{\circ} \mathrm{C}\right)$

\begin{tabular}{|c|c|c|c|c|c|c|c|}
\hline & 500 HRS IN & $=C-R 125 / B F$ & LL-150@ & $260^{\circ} \mathrm{F}$ & & & \\
\hline ID & WT & EXPWT & $\begin{array}{c}\text { WT \% } \\
\text { CHANGE }\end{array}$ & EXP VIS & $\begin{array}{c}\text { BASE DIE } \\
\text { (AVE) }\end{array}$ & EXPDIE & $\begin{array}{c}\text { DIE\% } \\
\text { CHANGE }\end{array}$ \\
\hline DMD & 4.4123 & 4.6555 & $5.51 \%$ & $\mathrm{~N} / \mathrm{C}$ & 9.61 & 10.10 & \\
\hline$A 1$ & & & & & 9.61 & 12.24 & $10.65 \%$ \\
\hline & & & & & 9.61 & 9.56 & \\
\hline DTMD & 4.2983 & 4.4866 & $4.38 \%$ & $\mathrm{~N} / \mathrm{C}$ & 9.95 & 14.52 & \\
\hline$B 1$ & & & & & 9.95 & 14.46 & $41.94 \%$ \\
\hline & & & & & 9.95 & 13.39 & \\
\hline & HFC-R125/B & $-150 @ 26$ & $0^{\circ} \mathrm{F} \cdot>24$ & HRS@3 & & & \\
\hline DMD & 4.5536 & 4.6624 & $2.39 \%$ & $N / C$ & 9.61 & 11.22 & \\
\hline$A 2$ & & & & & 9.61 & 11.93 & $12.56 \%$ \\
\hline & & & & & 9.61 & 9.30 & \\
\hline DTMD & 4.3389 & 4.3653 & $0.61 \%$ & $\mathrm{~N} / \mathrm{C}$ & 9.95 & 8.83 & \\
\hline $\mathrm{B} 2$ & & & & & 9.95 & 9.40 & $-1.54 \%$ \\
\hline & & & & & 9.95 & 11.16 & \\
\hline
\end{tabular}




\section{Appendix R}

Experimental Data for HFC-134a/Dow P425 Exposure at $126^{\circ} \mathrm{C}\left(260^{\circ} \mathrm{F}\right)$ 


\begin{tabular}{|c|c|c|c|c|c|c|c|c|c|c|}
\hline & $500 \mathrm{~h}$ & $\Gamma$ & ow $\mathrm{r}$ & 5 oll & 200 & & & & & \\
\hline ID & WT & EXPWT & $\begin{array}{l}\text { WT \% } \\
\text { CHANGE }\end{array}$ & EXP VIS & $\begin{array}{c}\text { BASE BRN } \\
\text { OUT } \\
\text { (AVE) }\end{array}$ & $\begin{array}{c}\text { EXP BRN } \\
\text { OUT }\end{array}$ & $\begin{array}{c}\text { BRN OUT } \\
\% \\
\text { CHANGE }\end{array}$ & $\begin{array}{c}\text { BASEDIE } \\
\text { (AVE) }\end{array}$ & EXPDIE & $\begin{array}{c}\text { DIE \% } \\
\text { CHANGE }\end{array}$ \\
\hline A1 & 30.1313 & 30.1467 & $0.051 \%$ & $\mathrm{~N} / \mathrm{C}$ & 576 & 561 & & 15.80 & 15.95 & \\
\hline & & & & & 576 & 474 & $-12.2 \%$ & 15.80 & 18.30 & $-0.2 \%$ \\
\hline & & & & & 576 & 483 & & 15.80 & 13.05 & \\
\hline$B 1$ & 22.6165 & 22.7138 & $0.430 \%$ & $N / C$ & 736 & 730 & & 11.62 & 15.06 & \\
\hline & & & & & 736 & 599 & $-6.7 \%$ & 11.62 & 15.50 & $29.7 \%$ \\
\hline & & & & & 736 & 731 & & 11.62 & 14.66 & \\
\hline $\mathrm{C} 1$ & 22.5881 & 22.6005 & $0.055 \%$ & $\mathrm{~N} / \mathrm{C}$ & 579 & 468 & & 16.58 & 13.81 & \\
\hline & & & & & 579 & 462 & $-22.6 \%$ & 16.58 & 12.34 & $-12.2 \%$ \\
\hline & & & & & 579 & 415 & & 16.58 & 17.50 & \\
\hline & 500 hours i & HFC-134 & a/Dow P-4 & 125 oil at & $260^{\circ} \mathrm{F}(127$ & $\left.{ }^{\circ} \mathrm{C}\right)$ plus 2 & 24 hours & it $302^{\circ} \mathrm{F}(1$ & $\left.50^{\circ} \mathrm{C}\right)$ & \\
\hline$A 2$ & 23.9251 & 23.9273 & $0.009 \%$ & $\mathrm{~N} / \mathrm{C}$ & 576 & 469 & & 15.80 & 11.43 & \\
\hline & & & & & 576 & 511 & $-15.3 \%$ & 15.80 & 8.78 & $-30.0 \%$ \\
\hline & & & & & 576 & 483 & & 15.80 & 12.95 & \\
\hline$B 2$ & 24.5775 & 24.5663 & $-0.046 \%$ & $N / C$ & 736 & 729 & & 11.62 & 10.23 & \\
\hline & & & & & 736 & 727 & $-1.1 \%$ & 11.62 & 10.27 & $-6.8 \%$ \\
\hline & & & & & 736 & 727 & & 11.62 & 11.98 & \\
\hline $\mathrm{C} 2$ & 23.037 & 23.0743 & $0.162 \%$ & $\mathrm{~N} / \mathrm{C}$ & 579 & 525 & & 16.58 & 12.13 & \\
\hline & & & & & 579 & 456 & $-17.7 \%$ & 16.58 & 13.50 & $-27.9 \%$ \\
\hline & & & & & 579 & 448 & & 16.58 & 10.22 & \\
\hline
\end{tabular}


TWISTED PAIRSMIRE AWITH VARNISH-HFC-134a/Dow P.425 oil @260 F $\left(127^{\circ} \mathrm{C}\right)$

500 hours in HFC-134a/P.425 oil @ 260 $\mathrm{F}\left(127^{\circ} \mathrm{C}\right)$

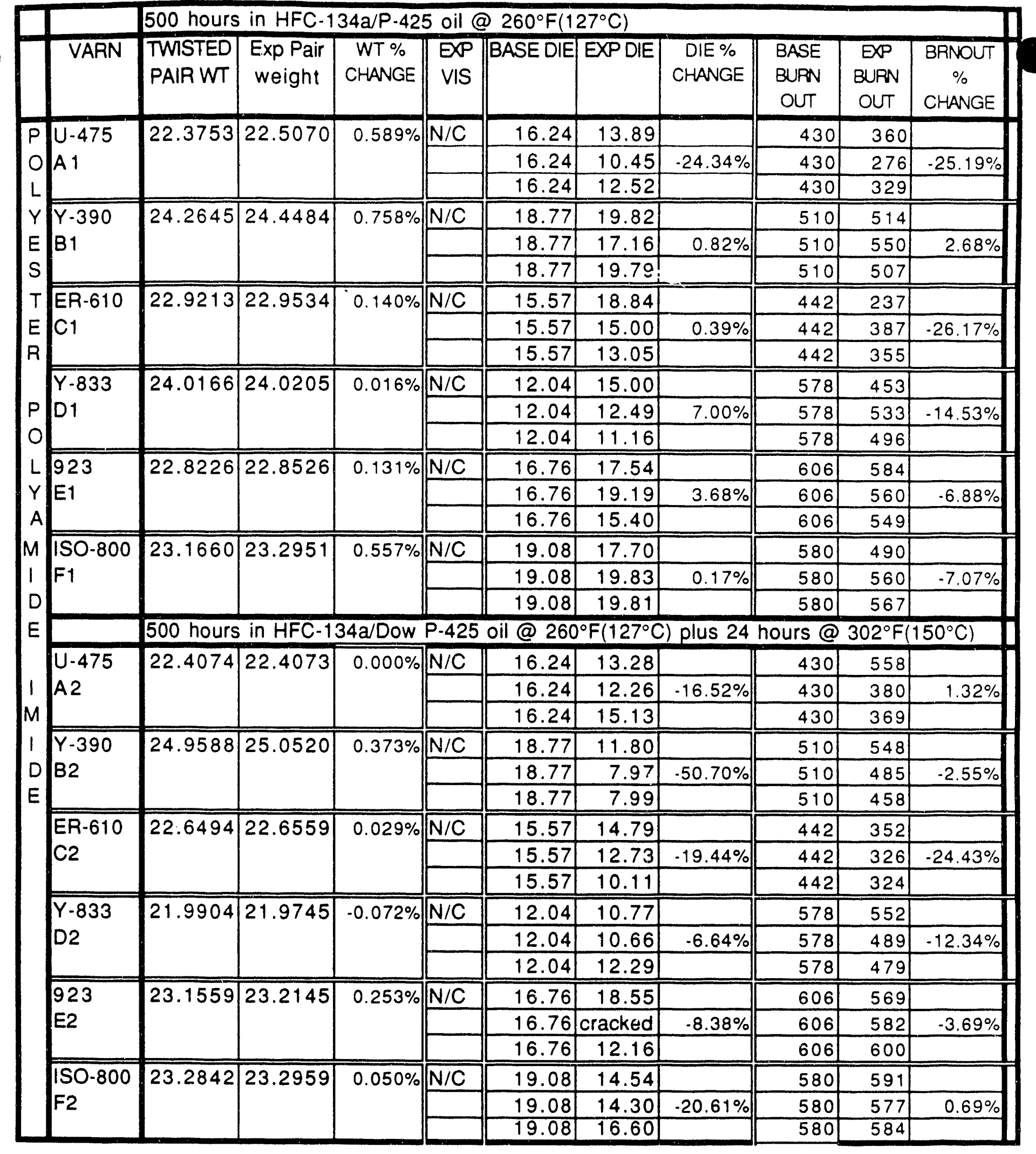




\begin{tabular}{|c|c|c|c|c|c|c|c|c|c|c|c|}
\hline & \multirow[b]{2}{*}{ VARN } & \multicolumn{10}{|c|}{500 HOURS in HFC-134a/Dow P425 oil @ 260 $\mathrm{F}\left(127^{\circ} \mathrm{C}\right)$} \\
\hline & & $\begin{array}{l}\text { TWISTED } \\
\text { PAIR WT }\end{array}$ & $\begin{array}{c}\text { WT\% } \\
\text { Change }\end{array}$ & $\begin{array}{c}\text { WT \% } \\
\text { CHANGE }\end{array}$ & $\begin{array}{l}\text { EXP } \\
\text { VIS }\end{array}$ & $\begin{array}{c}\text { BASE } \\
\text { DIE }\end{array}$ & $\begin{array}{l}\text { EPP } \\
D I E\end{array}$ & $\begin{array}{c}\text { DIE \% } \\
\text { CHANGE }\end{array}$ & \begin{tabular}{|c|} 
BASE \\
BURN \\
OUT
\end{tabular} & \begin{tabular}{|l|} 
EXP \\
BURN \\
OUT \\
\end{tabular} & $\begin{array}{c}\text { BRNOUT } \\
\% \\
\text { CHANGE }\end{array}$ \\
\hline \multirow{3}{*}{$\begin{array}{ll}P & 1 \\
\text { O } & A \\
\text { L }\end{array}$} & \multirow{3}{*}{$\begin{array}{l}\text { U. } 475 \\
\text { A } 1\end{array}$} & \multirow[t]{3}{*}{26.2392} & \multirow[t]{3}{*}{26.4183} & \multirow[t]{3}{*}{$0.68 \%$} & $\mathrm{~N} / \mathrm{C}$ & 13.32 & 19.87 & & 746 & 739 & \\
\hline & & & & & & 13.32 & 19.99 & \multirow{2}{*}{$49.20 \%$} & 746 & 738 & $-1.12 \%$ \\
\hline & & & & & & 13.32 & 19.76 & & 746 & 736 & \\
\hline \multirow{3}{*}{$\begin{array}{l}Y \\
E \\
S\end{array}$} & \multirow{3}{*}{$\begin{array}{l}Y .390 \\
B 1\end{array}$} & \multirow[t]{3}{*}{25.2938} & \multirow[t]{3}{*}{25.4414} & \multirow[t]{3}{*}{$0.58 \%$} & $\mathrm{~N} / \mathrm{C}$ & 12.28 & 19.52 & & 755 & 734 & \\
\hline & & & & & & 12.28 & 19.90 & $60.56 \%$ & 755 & 732 & $-2.87 \%$ \\
\hline & & & & & & 12.28 & 19.73 & & 755 & 734 & \\
\hline$T$ & ER-610 & 26.0024 & 26.0958 & $0.36 \%$ & $\mathrm{~N} / \mathrm{C}$ & 12.73 & 16.02 & & 734 & 729 & \\
\hline$E$ & $C_{1}$ & & & & & 12.73 & 16.37 & $24.46 \%$ & 734 & 731 & $.0 .54 \%$ \\
\hline$R$ & & & & & & 12.73 & 15.14 & & 734 & 730 & \\
\hline & $Y-833$ & 25.7868 & 25.8020 & $0.06 \%$ & $\mathrm{~N} / \mathrm{C}$ & 12.49 & 13.88 & & 734 & 728 & \\
\hline $\mathrm{P}$ & D1 & & & & & 12.49 & 15.20 & $14.20 \%$ & 734 & 731 & $-0.64 \%$ \\
\hline 0 & & & & & & 12.49 & 13.71 & & 734 & 729 & \\
\hline$L$ & 923 & 26.0802 & 26.3279 & $0.95 \%$ & $\mathrm{~N} / \mathrm{C}$ & 14.38 & 15.84 & & 742 & 749 & \\
\hline $\mathrm{Y}$ & E1 & & & & & 14.38 & 16.37 & $8.74 \%$ & 742 & 728 & $-0.63 \%$ \\
\hline A & & & & & & 14.38 & 14.70 & & 742 & 735 & \\
\hline$M$ & $150-800$ & 25.4189 & 25.6384 & $0.86 \%$ & $\mathrm{~N} / \mathrm{C}$ & 12.29 & 15.52 & & 747 & 728 & \\
\hline 1 & $\mathrm{~F} 1$ & & & & & 12.29 & 18.07 & $38.38 \%$ & 747 & 735 & $-1.61 \%$ \\
\hline$D$ & & & & & & 12.29 & 17.43 & & 747 & 742 & \\
\hline$E$ & & HFC-134 & a/Dow P.42 & 25 oil@ & $260^{\circ} \mathrm{F}$ & $\left.127^{\circ} \mathrm{C}\right)$ & olus 241 & hours@ & $302^{\circ} \mathrm{F}$ & $\left(150^{\circ} \mathrm{C}\right.$ & \\
\hline & U-475 & 26.7264 & 26.7493 & $0.09 \%$ & $\mathrm{~N} / \mathrm{C}$ & 13.32 & 12.46 & & 746 & 735 & \\
\hline 1 & $\mathrm{~A} 2$ & & & & & 13.32 & 13.01 & $-9.48 \%$ & 746 & 735 & $-1.03 \%$ \\
\hline$M$ & & & & & & 13.32 & 10.70 & & 746 & 745 & \\
\hline 1 & $Y-390$ & 25.5947 & 25.6985 & $0.41 \%$ & $\mathrm{~N} / \mathrm{C}$ & 12.28 & 11.37 & & 755 & 746 & \\
\hline$D$ & B2 & & & & & 12.28 & 11.62 & $-3.34 \%$ & 755 & 743 & $-1.39 \%$ \\
\hline$E$ & & & & & & 12.28 & 12.62 & & 755 & 740 & \\
\hline & ER-610 & 26.6207 & 26.6263 & $0.02 \%$ & $N / C$ & 12.73 & 12.29 & & 734 & 729 & \\
\hline$E$ & & & & & & 12.73 & 12.85 & $-5.68 \%$ & 734 & 732 & $-0.59 \%$ \\
\hline$P$ & & & & & & 12.73 & 10.88 & & 734 & 728 & \\
\hline 0 & $Y-833$ & 25.7958 & 25.7685 & $-0.11 \%$ & $\mathrm{~N} / \mathrm{C}$ & 12.49 & 12.10 & & 734 & 729 & \\
\hline$x$ & D2 & & & & & 12.49 & 11.90 & $-3.68 \%$ & 734 & 732 & $-0.64 \%$ \\
\hline$Y$ & & & & & & 12.49 & 12.09 & & 734 & 727 & \\
\hline & 923 & 26.9501 & 27.0757 & $0.47 \%$ & $\mathrm{~N} / \mathrm{C}$ & 14.38 & 11.67 & & 742 & 752 & \\
\hline$G$ & E2 & & & & & 14.38 & 11.23 & $-14.19 \%$ & 742 & 745 & $1.35 \%$ \\
\hline$L$ & & & & & & 14.38 & 14.12 & & 742 & 742 & \\
\hline$A$ & ISO-800 & 25.6914 & 25.7309 & $0.15 \%$ & & 12.29 & 10.47 & & 747 & 733 & \\
\hline S & $\mathrm{F} 2$ & & & & $\mathrm{~N} / \mathrm{C}$ & 12.29 & 9.40 & $-16.25 \%$ & 747 & 745 & $-1.07 \%$ \\
\hline$S$ & & & & & & 12.29 & 11.01 & & 747 & 736 & \\
\hline
\end{tabular}




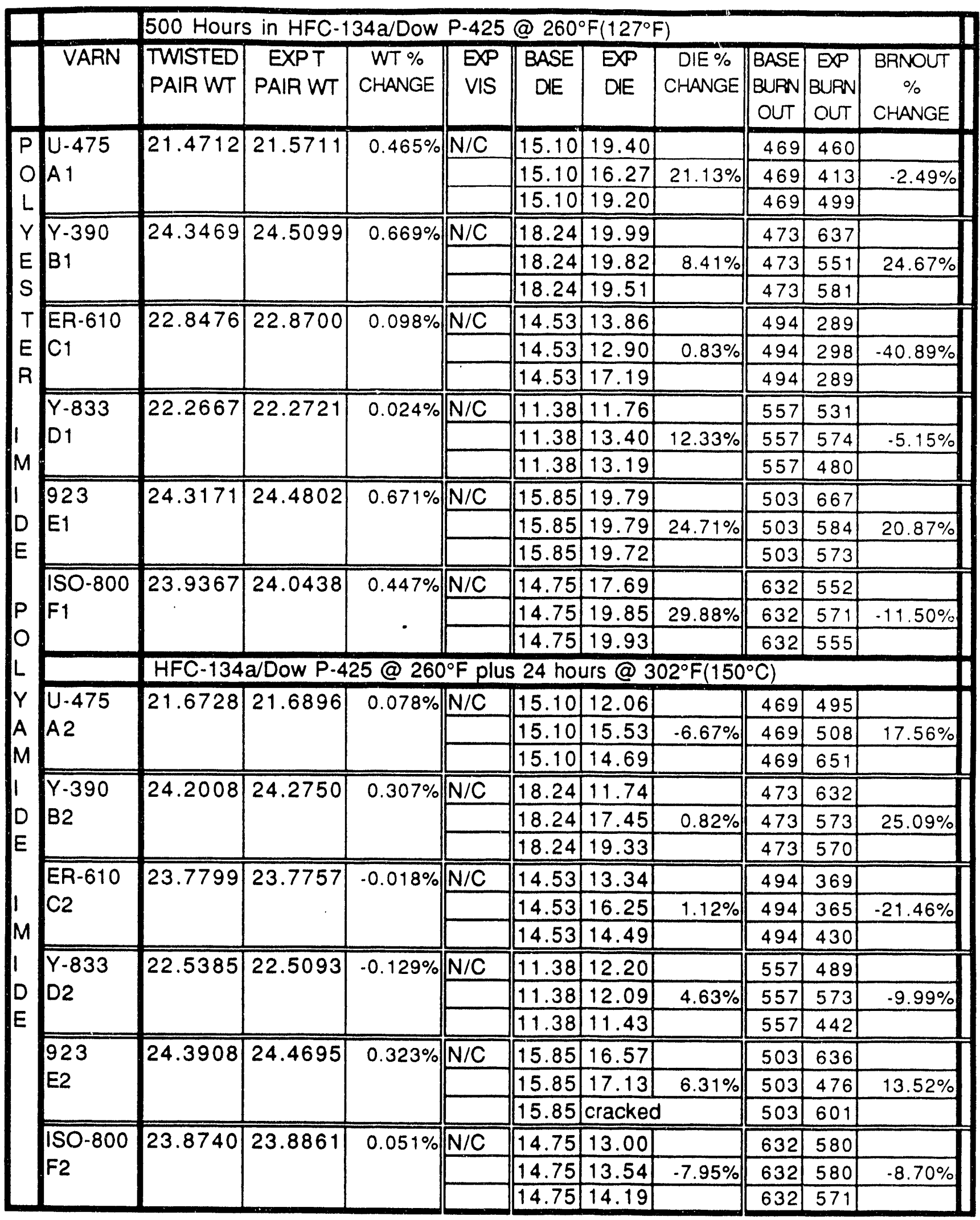




\begin{tabular}{|c|c|c|c|c|c|c|c|c|}
\hline & & 500 HRS & in HFC- 13 & a/P-425@ & $260 \mathrm{~F}$ & & & \\
\hline & VARN & $\begin{array}{l}\text { COIL } \\
W T\end{array}$ & $\begin{array}{c}\text { EXP COIL } \\
W T\end{array}$ & $\begin{array}{c}\text { WT \% } \\
\text { CHANGE }\end{array}$ & EXPVIS| & $\begin{array}{c}\text { BASE } \\
\text { BND STR } \\
(A \vee E)\end{array}$ & $\begin{array}{c}\text { EXPBND } \\
\text { STR }\end{array}$ & $\begin{array}{c}\text { BND STR } \\
\% \\
\text { CHANGE }\end{array}$ \\
\hline$P$ & $U-475$ & 40.6214 & 40.8400 & $0.538 \%$ & $\mathrm{~N} / \mathrm{C}$ & 73.73 & 53.57 & \\
\hline 0 & $A 1$ & & & & & 73.73 & 42.35 & $-35.41 \%$ \\
\hline$L$ & & & & & & 73.73 & 46.95 & \\
\hline$Y$ & $Y-390$ & 39.5701 & 39.8134 & $0.615 \%$ & $\mathrm{~N} / \mathrm{C}$ & 43.78 & 50.70 & \\
\hline$E$ & B1 & & & & & 43.78 & 52.75 & $14.41 \%$ \\
\hline S & & & & & & 43.78 & 46.82 & \\
\hline$T$ & ER-610 & 37.8822 & 38.0086 & $0.334 \%$ & $\mathrm{~N} / \mathrm{C}$ & 51.81 & 60.97 & \\
\hline$E$ & C1 & & & & & 51.81 & 58.20 & $11.38 \%$ \\
\hline $\mathrm{R}$ & & & & & & 51.81 & 53.95 & \\
\hline & $Y-833$ & 40.5884 & 40.5790 & $-0.023 \%$ & $\mathrm{~N} / \mathrm{C}$ & 9.85 & 38.22 & \\
\hline$P$ & D1 & & & & & 9.85 & 42.47 & $316.55 \%$ \\
\hline 0 & & & & & & 9.85 & 42.40 & \\
\hline$L$ & 923 & 39.1250 & 39.2760 & $0.386 \%$ & $N / C$ & 41.28 & 27.10 & \\
\hline Y & E1 & & & & & 41.28 & 36.52 & $-29.97 \%$ \\
\hline A & & & & & & 41.28 & 23.10 & \\
\hline$M$ & ISO-800 & 37.9819 & 38.2106 & $0.602 \%$ & $\mathrm{~N} / \mathrm{C}$ & 45.01 & 30.67 & \\
\hline & $F 1$ & & & & & 45.01 & 38.70 & $-22.94 \%$ \\
\hline D & & & & & & 45.01 & 38.47 & \\
\hline$E$ & & $500 \mathrm{HRS}$ & in $\mathrm{HFC}-13$ & a/Dow P-4 & $25 @ 260$ & $\mathrm{~F}->24 \mathrm{H}$ & RS $302^{\circ} \mathrm{F}$ & \\
\hline & U-475 & 40.2297 & 40.3043 & $0.185 \%$ & $\mathrm{~N} / \mathrm{C}$ & 73.73 & 1.20 & \\
\hline & A2 & & & & & 73.73 & 1.00 & $-62.87 \%$ \\
\hline$M$ & & & & & & 73.73 & 53.75 & \\
\hline I & Y-390 & 41.6526 & 41.7990 & $0.351 \%$ & $\mathrm{~N} / \mathrm{C}$ & 43.78 & 19.52 & \\
\hline D & B2 & & & & & 43.78 & 20.27 & $-24.55 \%$ \\
\hline$E$ & & & & & & 43.78 & 59.30 & \\
\hline & ER-610 & 38.9143 & 38.9520 & $0.097 \%$ & $N / C$ & 51.81 & 67.77 & \\
\hline & $\mathrm{C} 2$ & & & & & 51.81 & 59.77 & $23.14 \%$ \\
\hline & & & & & & 51.81 & 63.85 & \\
\hline & $Y-833$ & 40.9302 & 40.9148 & $-0.038 \%$ & $\mathrm{~N} / \mathrm{C}$ & 9.85 & 26.82 & \\
\hline & $\mathrm{D} 2$ & & & & & 9.85 & 35.30 & $204.70 \%$ \\
\hline & & & & & & 9.85 & 27.92 & \\
\hline & 923 & 39.1009 & 39.1500 & $0.126 \%$ & $\mathrm{~N} / \mathrm{C}$ & 41.28 & 38.70 & \\
\hline & E2 & & & & & 41.28 & 20.67 & $-26.32 \%$ \\
\hline & & & & & & 41.28 & 31.87 & \\
\hline & ISO-800 & 39.2333 & 39.3320 & $0.252 \%$ & $\mathrm{~N} / \mathrm{C}$ & 45.01 & 30.77 & \\
\hline & F2 & & & & & 45.01 & 38.85 & $-18.89 \%$ \\
\hline & & & & & & 45.01 & 39.90 & \\
\hline
\end{tabular}




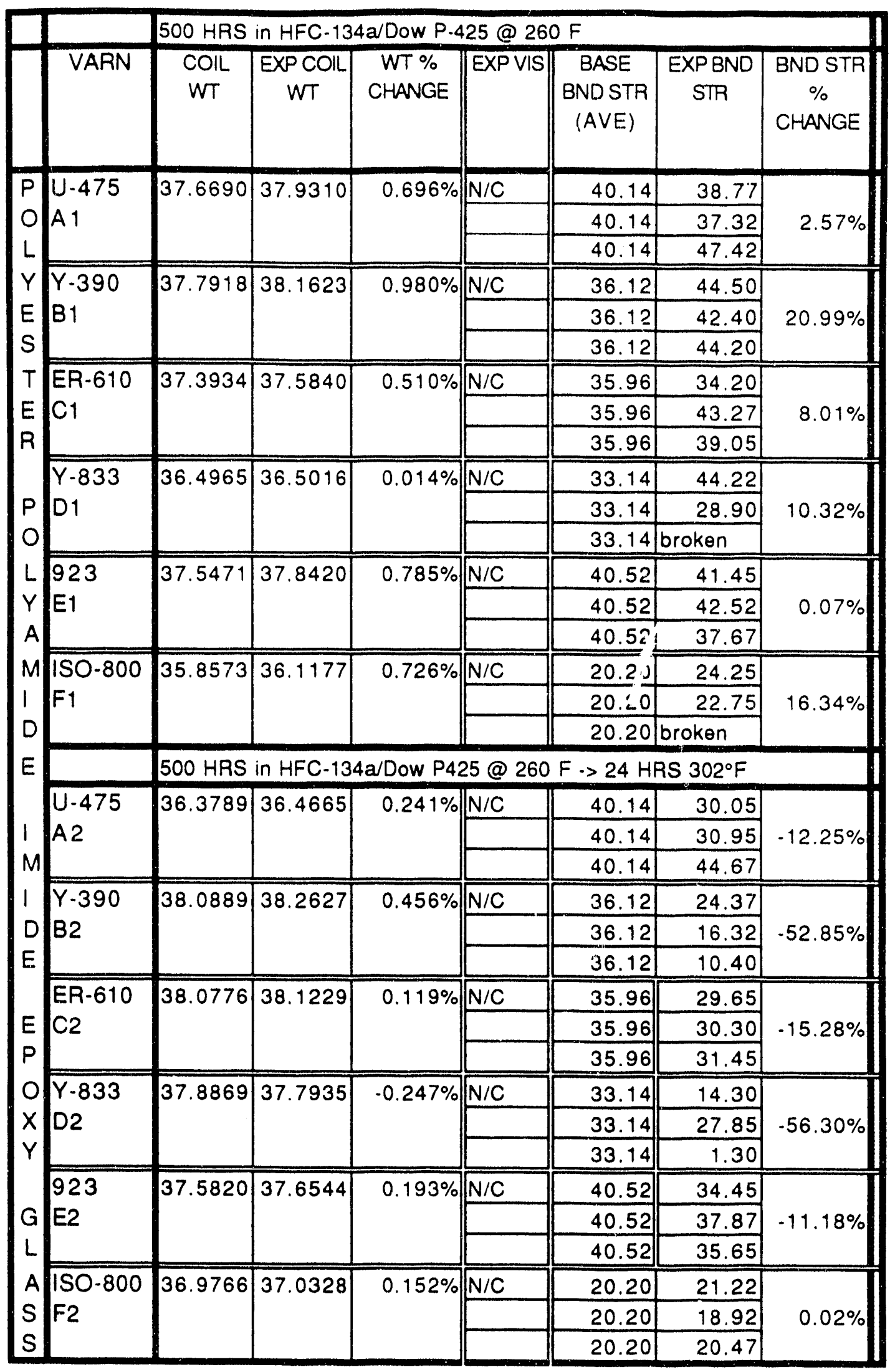




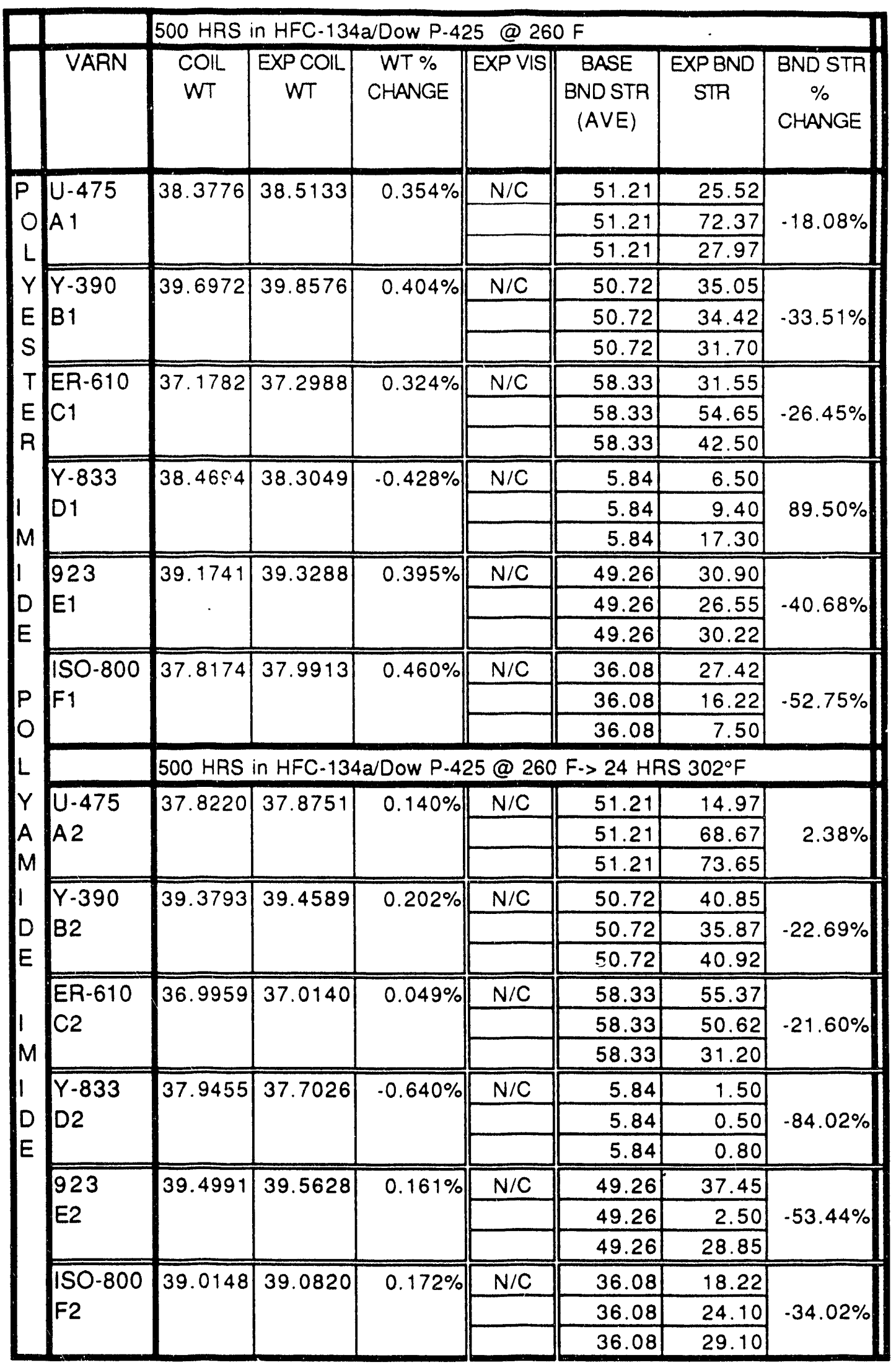




\begin{tabular}{|c|c|c|c|c|c|}
\hline 500 & is in $\mathrm{HF}$ & $-134 a / D o v$ & P425 oil @ & $260^{\circ} \mathrm{F}(1$ & $\left.7^{\circ} \mathrm{C}\right)$ \\
\hline ID & WT & EXPWT & $\begin{array}{l}\text { WT \% } \\
\text { CHANGE }\end{array}$ & EXP VIS & EXP FLEX \\
\hline A 1 & 3.3507 & 3.3542 & $0.104 \%$ & $\mathrm{~N} / \mathrm{C}$ & \\
\hline & & & & & YES \\
\hline$B 1$ & 4.1783 & 4.1896 & $0.270 \%$ & $N / C$ & \\
\hline & & & & & YES \\
\hline C1 & 3.6186 & 3.6209 & $0.064 \%$ & $\mathrm{~N} / \mathrm{C}$ & \\
\hline & & & & & YES \\
\hline 50 & $\mathrm{RS}$ in $\mathrm{H}$ & C-134a/P-. & 25 oil- $>30$ & $F$ for 24 & HRS \\
\hline A2 & 3.3379 & 3.338 & $0.003 \%$ & $\mathrm{~N} / \mathrm{C}$ & \\
\hline & & & & & YES \\
\hline B2 & 3.6683 & 3.6662 & $-0.057 \%$ & $\mathrm{~N} / \mathrm{C}$ & \\
\hline & & & & & YES \\
\hline $\mathrm{C} 2$ & 3.6322 & 3.6328 & $0.017 \%$ & $N / C$ & \\
\hline & & & & & YES \\
\hline & & & & & \\
\hline
\end{tabular}




\begin{tabular}{|c|c|c|c|c|c|c|}
\hline & $\begin{array}{l}500 \text { HRS } \\
\text { VARN }\end{array}$ & $\frac{\text { in HFC-136 }}{\text { WIREWT }}$ & $\begin{array}{c}\text { 4a/P-425 oil } \\
\text { EXPWRE } \\
W T\end{array}$ & $\begin{array}{l}\frac{260^{\circ} \mathrm{F}(1}{\text { WT }} \\
\text { CHANGE } \\
\text { CHA }\end{array}$ & $\frac{\left.27^{\circ} \mathrm{C}\right)}{\text { EXPVS }}$ & $\begin{array}{l}\text { EXP } \\
\text { FLEX }\end{array}$ \\
\hline \multirow{3}{*}{\begin{tabular}{l|l}
$P$ & $L$ \\
$O$ & $A$ \\
$L$
\end{tabular}} & \multirow{3}{*}{$\begin{array}{l}\text { U-475 } \\
\text { A } 1 \\
\end{array}$} & \multirow[t]{3}{*}{4.1503} & \multirow[t]{3}{*}{4.1547} & $0.106 \%$ & $N / C$ & \\
\hline & & & & & & YES \\
\hline & & & & & & \\
\hline \multirow{3}{*}{$\begin{array}{l}Y \\
E \\
S\end{array}$} & \multirow{3}{*}{$\begin{array}{l}Y-390 \\
B 1\end{array}$} & \multirow[t]{3}{*}{3.8787} & \multirow[t]{3}{*}{3.8847} & $0.155 \%$ & $\mathrm{~N} / \mathrm{C}$ & \\
\hline & & & & & & NO \\
\hline & & & & & & \\
\hline \multirow{3}{*}{$\begin{array}{l}T \\
E \\
R\end{array}$} & \multirow{2}{*}{$\begin{array}{l}\text { ER-610 } \\
\text { C1 }\end{array}$} & \multirow[t]{2}{*}{3.9041} & \multirow{2}{*}{3.9102} & $0.156 \%$ & $N / C$ & \\
\hline & & & & & & YES \\
\hline & \multirow{3}{*}{$\begin{array}{l}Y \cdot 833 \\
D 1\end{array}$} & \multirow[t]{3}{*}{3.8760} & \multirow[t]{3}{*}{3.8872} & $0.289 \%$ & $\mathrm{~N} / \mathrm{C}$ & \\
\hline \multirow{2}{*}{$\begin{array}{l}Y \\
D \\
\end{array}$} & & & & & & YES \\
\hline & & & & & & \\
\hline \multirow{3}{*}{$\begin{array}{l}1 \\
D \\
E\end{array}$} & \multirow{2}{*}{$\begin{array}{l}923 \\
E 1\end{array}$} & \multirow[t]{2}{*}{4.5965} & \multirow[t]{2}{*}{4.5999} & $0.074 \%$ & $\mathrm{~N} / \mathrm{C}$ & \\
\hline & & & & & & NO \\
\hline & \multirow{3}{*}{\begin{tabular}{|l}
$150-800$ \\
$\mathrm{~F} 1$ \\
\end{tabular}} & \multirow[t]{3}{*}{4.0680} & \multirow[t]{3}{*}{4.0714} & $0.084 \%$ & $\mathrm{~N} / \mathrm{C}$ & \\
\hline \multirow{3}{*}{$\begin{array}{l}P \\
O\end{array}$} & & & & & & YES \\
\hline & & & & & & \\
\hline & \multicolumn{6}{|c|}{ HFC-134a/P-425 oil @ 260 $\mathrm{F} \rightarrow 24 \mathrm{HRS} @ 302 \mathrm{~F}$} \\
\hline$Y$ & \multirow{3}{*}{$\begin{array}{l}-475 \\
\text { A2 } \\
\end{array}$} & \multirow{3}{*}{4.1642} & \multirow[t]{3}{*}{4.1594} & $-0.115 \%$ & $\mathrm{~N} / \mathrm{C}$ & \\
\hline$A$ & & & & & & YES \\
\hline$M$ & & & & & & \\
\hline \multirow{5}{*}{$\begin{array}{ll}1 \\
D \\
E \\
E\end{array}$} & $\int_{B 2}^{Y-390}$ & 3.7229 & 3.7284 & $0.148 \%$ & $\mathrm{~N} / \mathrm{C}$ & $\overline{N O}$ \\
\hline & & & & & & \\
\hline & ER-610 & 3.9741 & 3.9735 & $-0.015 \%$ & $N / C$ & \\
\hline & & & & & & YES \\
\hline & & & & & & \\
\hline 1 & $Y-833$ & 3.7825 & 3.7810 & $-0.040 \%$ & $\mathrm{~N} / \mathrm{C}$ & \\
\hline$D$ & & & & & & YES \\
\hline$E$ & & & & & & \\
\hline & $\begin{array}{l}923 \\
E 2\end{array}$ & 4.7126 & 4.7311 & $0.393 \%$ & $N / C$ & NO \\
\hline & & & & & & \\
\hline & $150-800$ & 4.0493 & 4.0510 & $0.042 \%$ & $N / C$ & \\
\hline & & & & & & NO \\
\hline & & & & & & \\
\hline
\end{tabular}




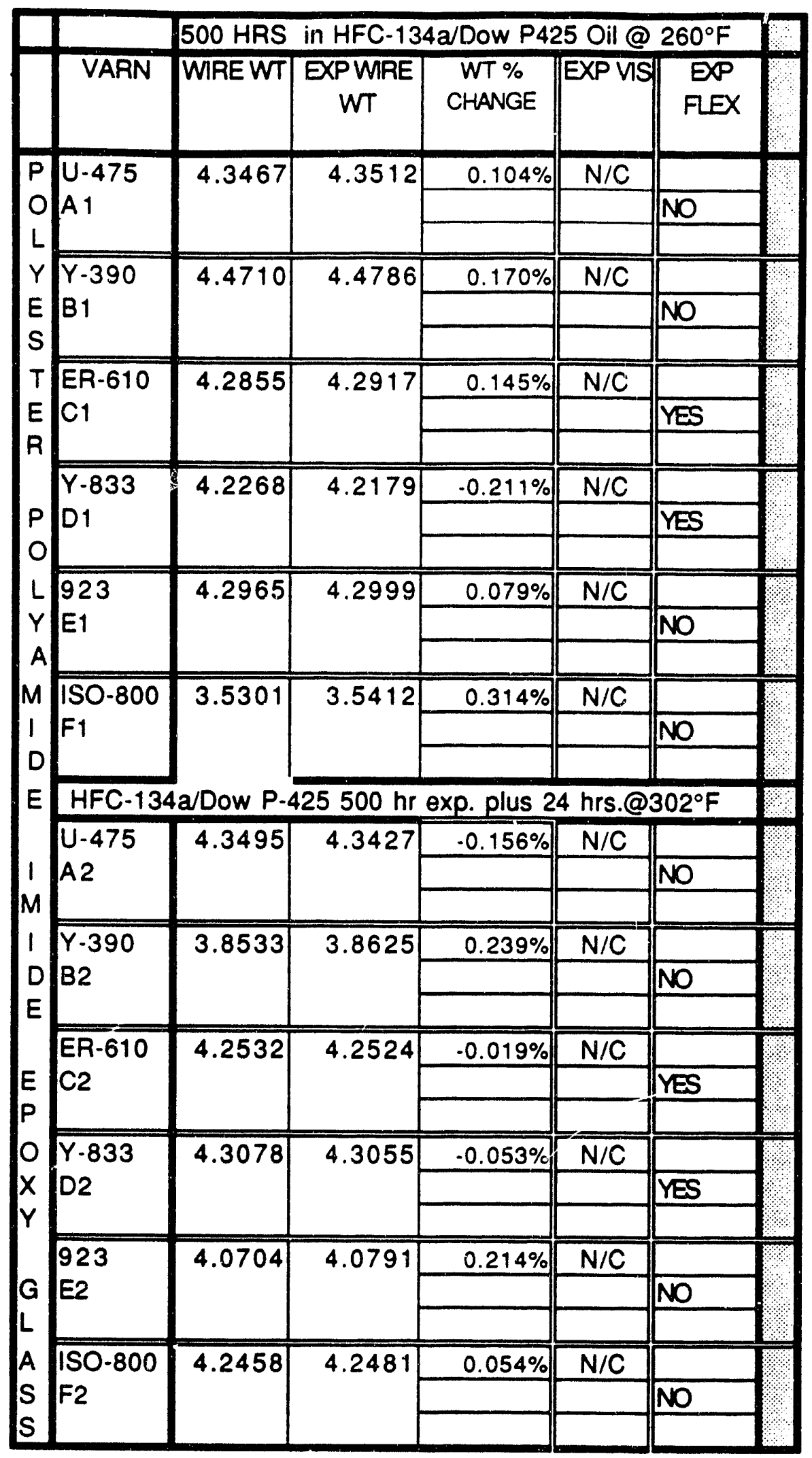




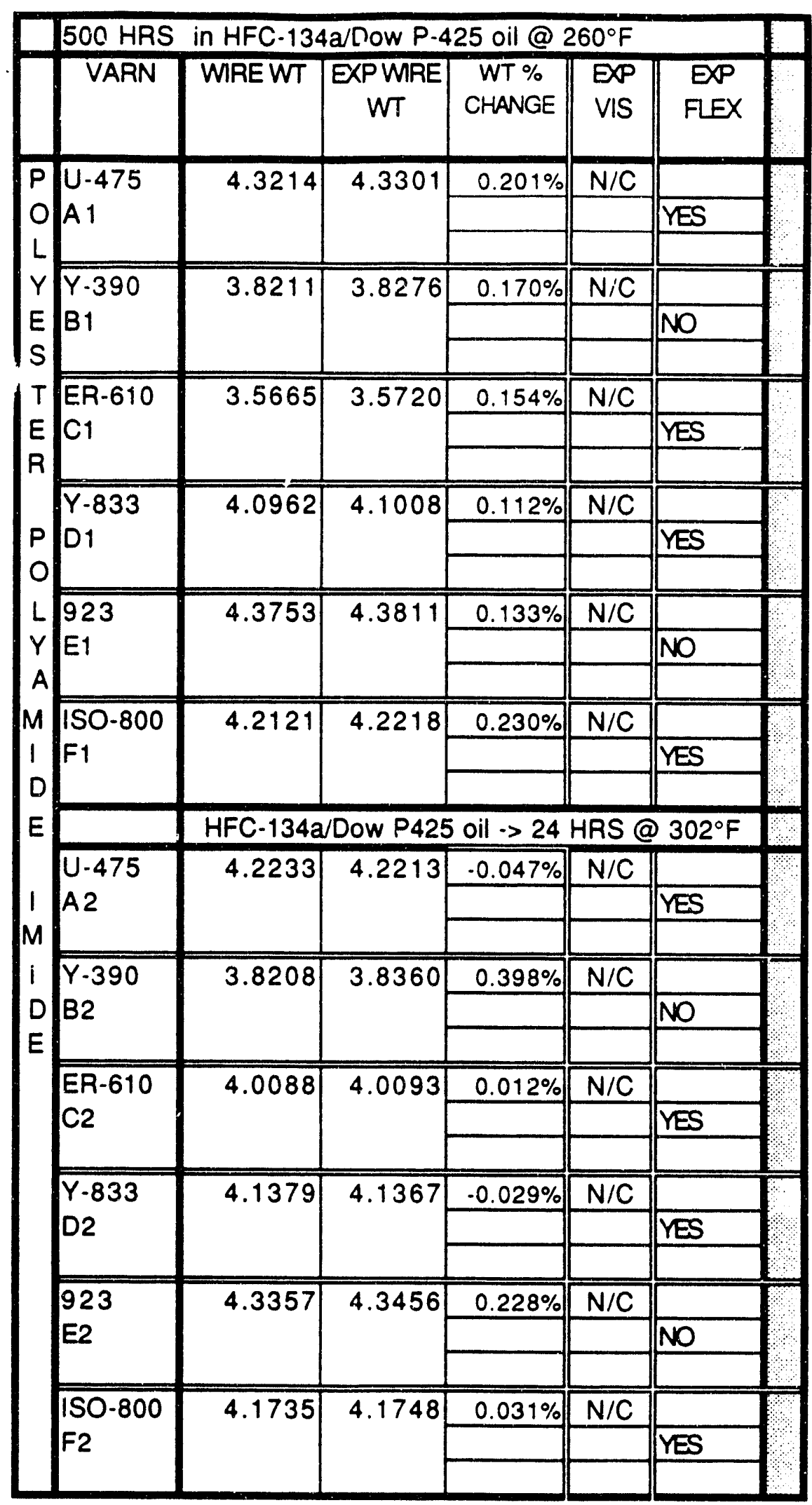




\begin{tabular}{|c|c|c|c|c|c|}
\hline & 500 HRS IN & Q-134a/Dow & P-425@2 & $260^{\circ} \mathrm{F}$ & \\
\hline D & WT & EXPWT & $\begin{array}{l}\text { WT \% } \\
\text { CHANGE }\end{array}$ & EXPVIS & EXPFLEX \\
\hline U-475 & 2.3489 & 2.6007 & $10.72 \% \mid[S$ & Surface & $\mathrm{N} / \mathrm{C}$ \\
\hline A 1 & & & & Cloudy & \\
\hline & & & & S. Warped & \\
\hline$Y \cdot 390$ & 1.4581 & 1.7230 & $18.17 \%[$ & $\mathrm{N} / \mathrm{C}$ & $\mathrm{N} / \mathrm{C}$ \\
\hline & & & & & \\
\hline ER-610 & 2.3853 & 2.5741 & $7.92 \%$, & $\mathrm{N} / \mathrm{C}$ & $\mathrm{N} / \mathrm{C}$ \\
\hline C1 & & & & & \\
\hline$Y \cdot 833$ & 3.0528 & 3.3104 & $8.44 \%$ & Slightly & $\mathrm{N} / \mathrm{C}$ \\
\hline D1 & & & & warped & \\
\hline & & & & ight green & \\
\hline 923 & 2.2642 & 2.5669 & $13.37 \%$ & & $\mathrm{~N} / \mathrm{C}$ \\
\hline E1 & & & & Warped & \\
\hline & 27004 & 33993 & $2588 \%$ & $N / C$ & $N / C$ \\
\hline & & & & & \\
\hline & R-134a/Dov & P.425 at $\cdots$ & $\rightarrow 24$ hour & at $302^{\circ} \mathrm{F}$ & \\
\hline$U-475$ & 2.4376 & 2.5508 & $4.64 \%$ & Darkened | & $\mathrm{N} / \mathrm{C}$ \\
\hline A2 & & & & & \\
\hline$Y-390$ & 1.4398 & 16963 & $1781 \%$ & $N / C$ & $\sqrt{N / C}$ \\
\hline$B 2$ & & & & & \\
\hline ER-610 & 2.6061 & 2.6952 & $3.42 \%$ & Darkened & $\mathrm{N} / \mathrm{C}$ \\
\hline & & & & & \\
\hline & $275+01$ & 20151 & 401010 & natronent & ther \\
\hline $\begin{array}{l}y-833 \\
D 2 \\
D 2\end{array}$ & 3.7518 & 3.9145 & $4.34 \% \|$ & DLarkenea & dive \\
\hline 923 & 2.0059 & 2.1592 & $7.64 \%$ & Warped & \\
\hline E2 & & & & Darkened & $N / C$ \\
\hline & & & & Tarkened & \\
\hline $\begin{array}{l}\text { ISO-800 } \\
\text { F2 }\end{array}$ & 1.7138 & 1.9353 & $12.92 \% \|$ & darkened & $\mathrm{N} / \mathrm{C}$ \\
\hline & & & & & \\
\hline
\end{tabular}




\begin{tabular}{|c|c|c|c|c|c|c|c|c|c|}
\hline \multirow{2}{*}{$\mathrm{ID}$} & \multicolumn{9}{|c|}{500 HRS in HFC-134a/Dow P.425 Oil @260 F } \\
\hline & WT & EXPWT & $\begin{array}{c}\text { WT \% } \\
\text { CHANGE }\end{array}$ & $\begin{array}{l}\text { SAMPLE } \\
\text { WIDTH }\end{array}$ & $\begin{array}{c}\text { BREAK } \\
\text { LOAD }\end{array}$ & $\begin{array}{c}\text { SAMPLE } \\
\text { THICKNESS } \\
\text { Mils }\end{array}$ & $\begin{array}{c}\text { TENSILE } \\
\text { STR } \\
\text { BASE }\end{array}$ & $\begin{array}{l}\text { TENSILE } \\
\text { STREXP }\end{array}$ & $\begin{array}{c}\% \text { CHANGE } \\
\text { TENSILE }\end{array}$ \\
\hline \multirow{3}{*}{$\begin{array}{l}\text { NO/MYINO } \\
\text { A1 }\end{array}$} & \multirow{3}{*}{4.5920} & \multirow[t]{3}{*}{4.9779} & \multirow[t]{3}{*}{$8.40 \%$} & 0.401 & 124.70 & 21 & 17.4 & 14.81 & \\
\hline & & & & 0.453 & 145.20 & 21 & 17.4 & 15.26 & $-13.15 \%$ \\
\hline & & & & 0.437 & 140.10 & 21 & 17.4 & 15.27 & \\
\hline \multirow{3}{*}{$\begin{array}{l}D A / M Y / D A \\
B 1\end{array}$} & \multirow{3}{*}{4.5551} & \multirow[t]{3}{*}{4.4015} & \multirow[t]{3}{*}{$-3.37 \%$} & 0.425 & 102.30 & 21 & 13.7 & 11.46 & \\
\hline & & & & 0.450 & 106.80 & 21 & 13.7 & 11.30 & $-16.91 \%$ \\
\hline & & & & 0.378 & 90.40 & 21 & 13.7 & 11.39 & \\
\hline \multirow{3}{*}{$\begin{array}{l}\text { MYLAR MO } \\
\text { C1 }\end{array}$} & \multirow{3}{*}{2.1785} & \multirow[t]{3}{*}{2.2287} & \multirow[t]{3}{*}{$2.30 \%$} & 0.424 & 72.70 & 10 & 21.7 & 17.15 & \\
\hline & & & & 0.373 & 61.10 & 10 & 21.7 & 16.38 & $-23.23 \%$ \\
\hline & & & & 0.462 & 76.00 & 10 & 21.7 & 16.45 & \\
\hline \multirow{3}{*}{$\begin{array}{l}\text { NO } 410 \\
\text { D1 }\end{array}$} & \multirow[t]{3}{*}{2.1551} & \multirow[t]{3}{*}{2.4530} & \multirow[t]{3}{*}{$13.82 \%$} & 0.503 & 93.80 & 10 & 18.7 & 18.65 & \\
\hline & & & & 0.486 & 88.24 & 10 & 18.7 & 18.16 & $-2.50 \%$ \\
\hline & & & & 0.517 & 92.50 & 10 & 18.7 & 17.89 & \\
\hline \multirow{3}{*}{$\begin{array}{l}\text { NO MI } 418 \\
\text { E1 }\end{array}$} & \multirow[t]{3}{*}{2.0663} & \multirow[t]{3}{*}{2.5399} & \multirow[t]{3}{*}{$22.92 \%$} & 0.501 & 30.75 & 10 & 7.5 & 6.14 & \\
\hline & & & & 0.506 & 31.00 & 10 & 7.5 & 6.13 & $-17.87 \%$ \\
\hline & & & & 0.494 & 30.70 & 10 & 7.5 & 6.21 & \\
\hline \multirow{3}{*}{$\begin{array}{l}\text { MEL } 228 \\
\text { F1 }\end{array}$} & \multirow[t]{3}{*}{2.3513} & \multirow[t]{3}{*}{2.4106} & $2.52 \%$ & 0.444 & 68.50 & 10 & 21.7 & 15.43 & \\
\hline & & & & 0.364 & 56.70 & 10 & 21.7 & 15.58 & $-28.67 \%$ \\
\hline & & & & 0.420 & 64.80 & 10 & 21.7 & 15.43 & \\
\hline & $500 \mathrm{HAS}$ & Mitiा & $4 a / D o w f$ & P.425 Oil & @260 F & $->24$ HRS & $2302^{\circ} \mathrm{F}$ & & \\
\hline NO/MY/NO & 4.4997 & 4.5757 & $1.69 \%$ & 0.418 & 137.20 & 21 & 17.4 & 15.63 & \\
\hline A2 & & & & 0.368 & 118.90 & 21 & 17.4 & 15.39 & $-12.39 \%$ \\
\hline & & & & 0.419 & 129.50 & 21 & 17.4 & 14.72 & \\
\hline$D A / M Y / D A$ & 4.5247 & 4.5247 & $0.00 \%$ & 0.405 & 98.20 & 21 & 13.7 & 11.55 & \\
\hline $\mathrm{B} 2$ & & & & 0.405 & 96.75 & 21 & 13.7 & 11.38 & $-16.54 \%$ \\
\hline & & & & 0.495 & 118.30 & 21 & 13.7 & 11.38 & \\
\hline MYLAR MO & 2.3218 & 2.3255 & $0.16 \%$ & 0.401 & 30.30 & 10 & 21.7 & 7.56 & \\
\hline $\mathrm{C2}$ & & & & 0.390 & 39.30 & 10 & 21.7 & 10.08 & $-48.61 \%$ \\
\hline & & & & 0.482 & 76.25 & 10 & 21.7 & 15.82 & \\
\hline NOMEX 410 & 2.3539 & 2.4169 & $2.68 \%$ & 0.455 & 87.90 & 10 & 18.7 & 19.32 & \\
\hline $\mathrm{D} 2$ & & & & 0.528 & 101.60 & 10 & 18.7 & 19.24 & $3.20 \%$ \\
\hline & & & & 0.495 & 95.70 & 10 & 18.7 & 19.33 & \\
\hline NO/MI 418 & 2.1829 & 2.2012 & $0.84 \%$ & 0.486 & 23.2 .0 & 10 & 7.5 & 4.77 & \\
\hline E2 & & & & 0.479 & 27.05 & 10 & 7.5 & 5.65 & $-29.07 \%$ \\
\hline & & & & 0.492 & 27.25 & 10 & 7.5 & 5.54 & \\
\hline MEL 228 & 2.2705 & 2.4207 & $6.62 \%$ & 0.415 & 59.05 & 10 & 21.7 & 14.23 & \\
\hline$F 2$ & & & & 0.455 & 76.55 & 10 & 21.7 & 16.82 & $-27.00 \%$ \\
\hline & & & & 0.402 & 66.20 & 10 & 21.7 & 16.47 & \\
\hline
\end{tabular}




\begin{tabular}{|c|c|c|c|c|c|c|c|c|}
\hline & $500 \mathrm{HRS}$ & in HFC. & 34a/Dow & P.425 Oil & $1 @ 260$ & & & \\
\hline $\mathbb{D}$ & $\begin{array}{c}\text { STRETCH } \\
\text { (inch) }\end{array}$ & $\begin{array}{c}\% \\
\text { ELONG }\end{array}$ & $\begin{array}{l}\text { BASE } \\
\text { ELONG } \\
\text { (AVE) }\end{array}$ & $\begin{array}{c}\text { ELONG \% } \\
\text { CHANGE }\end{array}$ & $\begin{array}{c}\text { BASE } \\
\text { DIE } \\
(A \vee E)\end{array}$ & EXPDIE & $\begin{array}{c}\text { DIE \% } \\
\text { CHANGE }\end{array}$ & VISUAL EXP \\
\hline NO/MY/NO & 0.38 & $9.5 \%$ & $20.0 \%$ & & $>18.97$ & flash & & Nomex totally \\
\hline A1 & 0.41 & $10.3 \%$ & $20.0 \%$ & $.50 .6 \%$ & $>18.97$ & flash & flash & delaminated from \\
\hline & 0.34 & $8.5 \%$ & $20.0 \%$ & & $>18.97$ & flash & & the mylar, glue still \\
\hline$D A / M Y / D A$ & 0.39 & $19.5 \%$ & $46.0 \%$ & & $>15.27$ & flash & & Dacron totally \\
\hline $\mathrm{Bi}$ & 0.39 & $19.5 \%$ & $46.0 \%$ & $-57.6 \%$ & $>15.27$ & flash & flash & delaminated from \\
\hline & 0.39 & $19.5 \%$ & $46.0 \%$ & & $>15.27$ & flash & & the mylar, glue gone \\
\hline MYLAR MO & 2.27 & $113.5 \%$ & $131.0 \%$ & & $>14.91$ & flash & & $N / C$ \\
\hline C1 & 2.24 & $112.0 \%$ & $131.0 \%$ & $-16.3 \%$ & $>14.91$ & flash & flash & \\
\hline & 2.07 & $103.5 \%$ & $131.0 \%$ & & $>14.91$ & flash & & \\
\hline NO 410 & 0.36 & $18.0 \%$ & $17.0 \%$ & & 10.67 & 12.41 & & $\mathrm{~N} / \mathrm{C}$ \\
\hline D1 & 0.34 & $8.5 \%$ & $17.0 \%$ & $-33.3 \%$ & 10.67 & 13.12 & $16.8 \%$ & \\
\hline & 0.30 & $7.5 \%$ & $17.0 \%$ & & 10.67 & 11.86 & & \\
\hline NO Ml 418 & 0.05 & $1.3 \%$ & $4.0 \%$ & & 10.23 & 10.69 & & $\mathrm{~N} / \mathrm{C}$ \\
\hline E1 & 0.05 & $1.3 \%$ & $4.0 \%$ & $.68 .8 \%$ & 10.23 & 11.33 & $9.0 \%$ & \\
\hline & 0.05 & $1.3 \%$ & $4.0 \%$ & & 10.23 & 11.42 & & \\
\hline MEL 228 & 2.69 & $134.5 \%$ & $160.0 \%$ & & $>14.22$ & flash & & slightly \\
\hline$F_{1}$ & 2.70 & $135.0 \%$ & $160.0 \%$ & $.32 .2 \%$ & $>14.22$ & flash & flash & yellowed \\
\hline & 1.12 & $56.0 \%$ & $160.0 \%$ & & $>14.22$ & flash & & \\
\hline & 500 HRS & in HFC. & 34a/Dow & P.425 Oil & |@ 260 & $F->24 H$ & IRS @ 30 & $2^{\circ} \mathrm{F}$ \\
\hline NO/MY/NO & 0.36 & $9.0 \%$ & $20.0 \%$ & & $>18.97$ & flash & & Delamiriation \\
\hline$A 2$ & 0.33 & $8.3 \%$ & $20.0 \%$ & $.61 .3 \%$ & $>18.97$ & flash & flash & as above \\
\hline & 0.24 & $6.0 \%$ & $20.0 \%$ & & $>18.97$ & flash & & also darkened \\
\hline$D A / M Y / D A$ & 0.34 & $17.0 \%$ & $46.0 \%$ & & $>15.27$ & flash & & Delamination \\
\hline B2 & 0.34 & $17.0 \%$ & $46.0 \%$ & $.67 .4 \%$ & $>15.27$ & flash & flash & as above \\
\hline & 0.22 & $11.0 \%$ & $46.0 \%$ & & $>15.27$ & flash & & darkened \\
\hline MYLAR MO & 0.03 & $1.5 \%$ & $131.0 \%$ & & $>14.91$ & flash & & slightly more \\
\hline $\mathrm{C} 2$ & 0.04 & $2.0 \%$ & $131.0 \%$ & $.72 .3 \%$ & $>14.91$ & flash & flash & yellowed \\
\hline & 2.11 & $105.5 \%$ & $131.0 \%$ & & $>14.91$ & flash & & \\
\hline NOMEX 410 & 0.39 & $9.8 \%$ & $17.0 \%$ & & 10.67 & 8.70 & & slightly \\
\hline $\mathrm{D} 2$ & 0.39 & $9.8 \%$ & $17.0 \%$ & $-44.6 \%$ & 10.67 & 10.54 & $-21.7 \%$ & Darkened \\
\hline & 0.35 & $8.8 \%$ & $17.0 \%$ & & 10.67 & 5.82 & & few pockets \\
\hline NO/MI 418 & 0.03 & $0.8 \%$ & $4.0 \%$ & & 10.23 & 10.14 & & $\mathrm{~N} / \mathrm{C}$ \\
\hline E2 & 0.04 & $1.0 \%$ & $4.0 \%$ & $-79.2 \%$ & 10.23 & 8.54 & $-6.5 \%$ & \\
\hline & 0.03 & $0.8 \%$ & $4.0 \%$ & & 10.23 & 10.00 & & \\
\hline MEL 228 & 0.07 & $3.5 \%$ & $160.0 \%$ & & $>14.22$ & flash & & \\
\hline F2 & 0.13 & $6.5 \%$ & $160.0 \%$ & $-96.5 \%$ & $>14.22$ & flash & flash & yellowed \\
\hline & 0.14 & $7.0 \%$ & $160.0 \%$ & & $\|>14.22$ & flash & & \\
\hline
\end{tabular}




\begin{tabular}{|c|c|c|c|c|}
\hline & \multicolumn{4}{|c|}{500 HRS in HFC-134a/Dow P-425 @ 260 F } \\
\hline & WT & EXPWT & $\begin{array}{c}\% \\
\text { CHANGE }\end{array}$ & EXP VIS \\
\hline $\begin{array}{l}\text { A } 1 \\
\text { NOMEX }\end{array}$ & 0.5645 & 0.6522 & $15.54 \%$ & $\mathrm{~N} / \mathrm{C}$ \\
\hline $\begin{array}{l}\text { B1 } \\
\text { MYLAR }\end{array}$ & 0.4999 & 0.5149 & $3.00 \%$ & $\begin{array}{l}\text { small Pockets } \\
\text { when rubbed between fingers } \\
\text { e bacame cloudy in appearan }\end{array}$ \\
\hline $\begin{array}{l}\mathrm{C} 1 \\
\mathrm{NO} / \mathrm{MY}\end{array}$ & 0.3863 & 0.4063 & $5.18 \%$ & some small pockets \\
\hline & $\mathrm{HFC}-134 \mathrm{a} / \mathrm{DC}$ & V P-425 oil & @ 260 $\mathrm{F}$ & olus24 hrs@ @302 $\mathrm{F}$ \\
\hline NOMEX & 0.5351 & 0.5499 & $2.77 \%$ & Slightly Darkened \\
\hline \begin{tabular}{|l|} 
B2 \\
MYLAR
\end{tabular} & 0.4911 & 0.4867 & $-0.90 \%$ & $\begin{array}{c}\text { no pockets } \\
\text { warped } \\
\text { Darkened }\end{array}$ \\
\hline $\begin{array}{l}\mathrm{C} 2 \\
\mathrm{NO} / \mathrm{MY}\end{array}$ & 0.3912 & 0.3816 & $-2.45 \%$ & $\begin{array}{c}\text { slightly darkened } \\
\text { some pockets where mylar } \\
\text { pulled away. }\end{array}$ \\
\hline
\end{tabular}




\begin{tabular}{|c|c|c|c|c|c|c|c|c|c|}
\hline & \multicolumn{9}{|c|}{500 HRS in HFC-134a/Dow P.425 @ 260 F } \\
\hline ID & WT & EXPWT & $\begin{array}{c}\text { WT \% } \\
\text { CHANGE }\end{array}$ & $\begin{array}{l}\text { BREAK } \\
\text { LOAD } \\
\text { (AVE) }\end{array}$ & $\begin{array}{l}\text { BREAK } \\
\text { LOAD } \\
\text { EP }\end{array}$ & $\begin{array}{c}\% \\
\text { CHANGE } \\
\text { BRK } \\
\text { LOAD }\end{array}$ & $\begin{array}{c}\text { STRETCH } \\
(\text { INCH) }\end{array}$ & \%ELONG & EXPVIS \\
\hline \multirow{3}{*}{$\begin{array}{l}\text { A } 1 \\
\text { Glass }\end{array}$} & \multirow[t]{3}{*}{1.7011} & \multirow[t]{3}{*}{1.7082} & \multirow[t]{3}{*}{$0.42 \%$} & 39.02 & 67.37 & & 0.07 & $3.50 \%$ & $\mathrm{~N} / \mathrm{C}$ \\
\hline & & & & 39.02 & 57.20 & $52.20 \%$ & 0.06 & $3.00 \%$ & \\
\hline & & & & 39.02 & 53.60 & & 0.07 & $3.50 \%$ & \\
\hline \multirow{3}{*}{$\begin{array}{l}\text { B1 } \\
\text { Polyester }\end{array}$} & \multirow[t]{3}{*}{0.7173} & \multirow[t]{3}{*}{0.7295} & \multirow[t]{3}{*}{$1.70 \%$} & 56.12 & 60.77 & & 0.78 & $39.00 \%$ & $\mathrm{~N} / \mathrm{C}$ \\
\hline & & & & 56.12 & 62.05 & $8.20 \%$ & 0.79 & $39.50 \%$ & \\
\hline & & & & 56.12 & 59.35 & & 0.72 & $36.00 \%$ & \\
\hline \multirow{3}{*}{$\begin{array}{l}\text { C1 } \\
\text { Permacel }\end{array}$} & \multirow[t]{3}{*}{1.4783} & \multirow[t]{3}{*}{1.9850} & \multirow[t]{3}{*}{$34.28 \%$} & 88.50 & 44.20 & & 0.15 & $7.50 \%$ & Slightly \\
\hline & & & & 88.50 & 48.92 & $-47.05 \%$ & 0.18 & $9.00 \%$ & darkened \\
\hline & & & & 88.50 & 47.47 & & 0.14 & $7.00 \%$ & Curled up \\
\hline & \multicolumn{7}{|c|}{500 HRS in HFC-134a/Dow P-425 @ 260 F.>302 ${ }^{\circ} F 24$ HRS } & & \\
\hline \multirow{3}{*}{$\begin{array}{l}\text { A2 } \\
\text { Glass }\end{array}$} & \multirow[t]{3}{*}{1.4832} & \multirow{3}{*}{1.4863} & \multirow[t]{3}{*}{$0.21 \%$} & 39.02 & 58.40 & & 0.06 & $3.00 \%$ & $\mathrm{~N} / \mathrm{C}$ \\
\hline & & & & 39.02 & 45.95 & $42.11 \%$ & 0.05 & $2.50 \%$ & \\
\hline & & & & 39.02 & 62.00 & & 0.07 & $3.50 \%$ & \\
\hline \multirow{3}{*}{$\begin{array}{l}\text { B2 } \\
\text { Polyester }\end{array}$} & \multirow[t]{3}{*}{0.6729} & \multirow[t]{3}{*}{0.6651} & \multirow{3}{*}{$-1.16 \%$} & 56.12 & 48.60 & & 0.42 & $21.00 \%$ & $\mathrm{~N} / \mathrm{C}$ \\
\hline & & & & 56.12 & 49.45 & $-12.54 \%$ & 0.47 & $23.50 \%$ & \\
\hline & & & & 56.12 & 49.20 & & 0.44 & $22.00 \%$ & \\
\hline \multirow{3}{*}{$\begin{array}{l}\mathrm{C2} \\
\text { Permacel }\end{array}$} & \multirow[t]{3}{*}{1.2966} & \multirow[t]{3}{*}{1.1624} & \multirow{3}{*}{$-10.35 \%$} & 88.50 & 76.85 & & 0.10 & $5.00 \%$ & Slightly \\
\hline & & & & 88.50 & 79.45 & $-0.90 \%$ & 0.08 & $4.00 \%$ & Darkened \\
\hline & & & & 88.50 & 106.80 & & 0.11 & $5.50 \%$ & \\
\hline
\end{tabular}




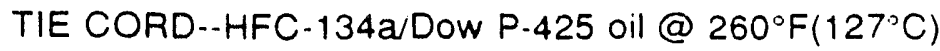

\begin{tabular}{|c|c|c|c|c|c|c|c|c|c|}
\hline \multicolumn{10}{|c|}{500 HRS IN R-134a/Dow P-425@260. F } \\
\hline ID & WT & EXPWT & $\begin{array}{c}\text { WT \% } \\
\text { CHANGE }\end{array}$ & $\begin{array}{l}E X P \\
\text { VIS }\end{array}$ & $\begin{array}{l}\text { BREAK } \\
\text { LOAD } \\
\text { (AVE) }\end{array}$ & $\begin{array}{c}\text { BREAK } \\
\text { LOAD } \\
\text { EXP } \\
(\text { ave })\end{array}$ & $\begin{array}{c}\% \\
\text { CHANGE } \\
\text { BRK } \\
\text { LOAD }\end{array}$ & $\begin{array}{c}\text { STRETCH } \\
\text { (Inch) }\end{array}$ & $\begin{array}{c}\% \\
\text { ELONG }\end{array}$ \\
\hline \multirow[t]{4}{*}{ A 1} & \multirow[t]{3}{*}{0.3325} & \multirow[t]{3}{*}{0.3385} & \multirow[t]{3}{*}{$1.80 \%$} & $\mathrm{~N} / \mathrm{C}$ & 28.36 & 32.10 & & 0.36 & $18.0 \%$ \\
\hline & & & & & 28.36 & 34.32 & $16.17 \%$ & 0.42 & $21.0 \%$ \\
\hline & & & & & 28.36 & 32.42 & & 0.40 & $20.0 \%$ \\
\hline & \multicolumn{9}{|c|}{500 HRS IN R-134a/Dow P-425 ->24 HRS @ 302 F } \\
\hline \multirow[t]{3}{*}{ A2 } & \multirow[t]{3}{*}{0.3176} & \multirow[t]{3}{*}{0.3156} & \multirow[t]{3}{*}{$-0.630 \%$} & $\mathrm{~N} / \mathrm{C}$ & 28.36 & 35.30 & & 0.45 & $22.5 \%$ \\
\hline & & & & & 28.36 & 36.45 & $23.53 \%$ & 0.53 & $26.5 \%$ \\
\hline & & & & & 28.36 & 33.35 & & 0.47 & $23.5 \%$ \\
\hline
\end{tabular}




\begin{tabular}{|c|c|c|c|c|c|c|c|}
\hline & $500 \mathrm{HRS}$ IN & C. $-134 a / D O$ & wP $P-425$ & @ $260^{\circ} \mathrm{F}$ & & & \\
\hline ID & WT & EXP WT & $\begin{array}{c}\text { WT \% } \\
\text { CHANGE }\end{array}$ & EXP VIS & $\begin{array}{c}\text { BASE DIE } \\
\text { (AVE) }\end{array}$ & EXP DIE & $\begin{array}{c}\text { DIE\% } \\
\text { CHANGE }\end{array}$ \\
\hline DMD & 4.0906 & 4.2629 & $4.21 \%$ & $\mathrm{~N} / \mathrm{C}$ & 9.61 & 9.68 & \\
\hline A1 & & & & & 9.61 & 9.10 & $-4.34 \%$ \\
\hline & & & & & 9.61 & 8.80 & \\
\hline DTMD & 4.2861 & 4.4238 & $3.21 \%$ & $\mathrm{~N} / \mathrm{C}$ & 9.95 & 12.13 & \\
\hline B1 & & & & & 9.95 & 11.93 & $17.96 \%$ \\
\hline & & & & & 9.95 & 11.15 & \\
\hline & HFC-134a/Dc & P.425@ & $260^{\circ} \mathrm{F} \rightarrow 2$ & 4 HRS@ & $302^{\circ} \mathrm{F}$ & & \\
\hline DMD & 4.1027 & 4.1824 & $1.94 \%$ & $N / C$ & 9.61 & 9.41 & \\
\hline$A 2$ & & & & & 9.61 & 9.00 & $-5.72 \%$ \\
\hline & & & & & 9.61 & 8.77 & \\
\hline DTMD & 4.3339 & 4.3812 & $1.09 \%$ & $\mathrm{~N} / \mathrm{C}$ & 9.95 & 11.17 & \\
\hline B2 & & & & & 9.95 & 10.47 & $2.31 \%$ \\
\hline & & & & & 9.95 & 8.90 & \\
\hline
\end{tabular}



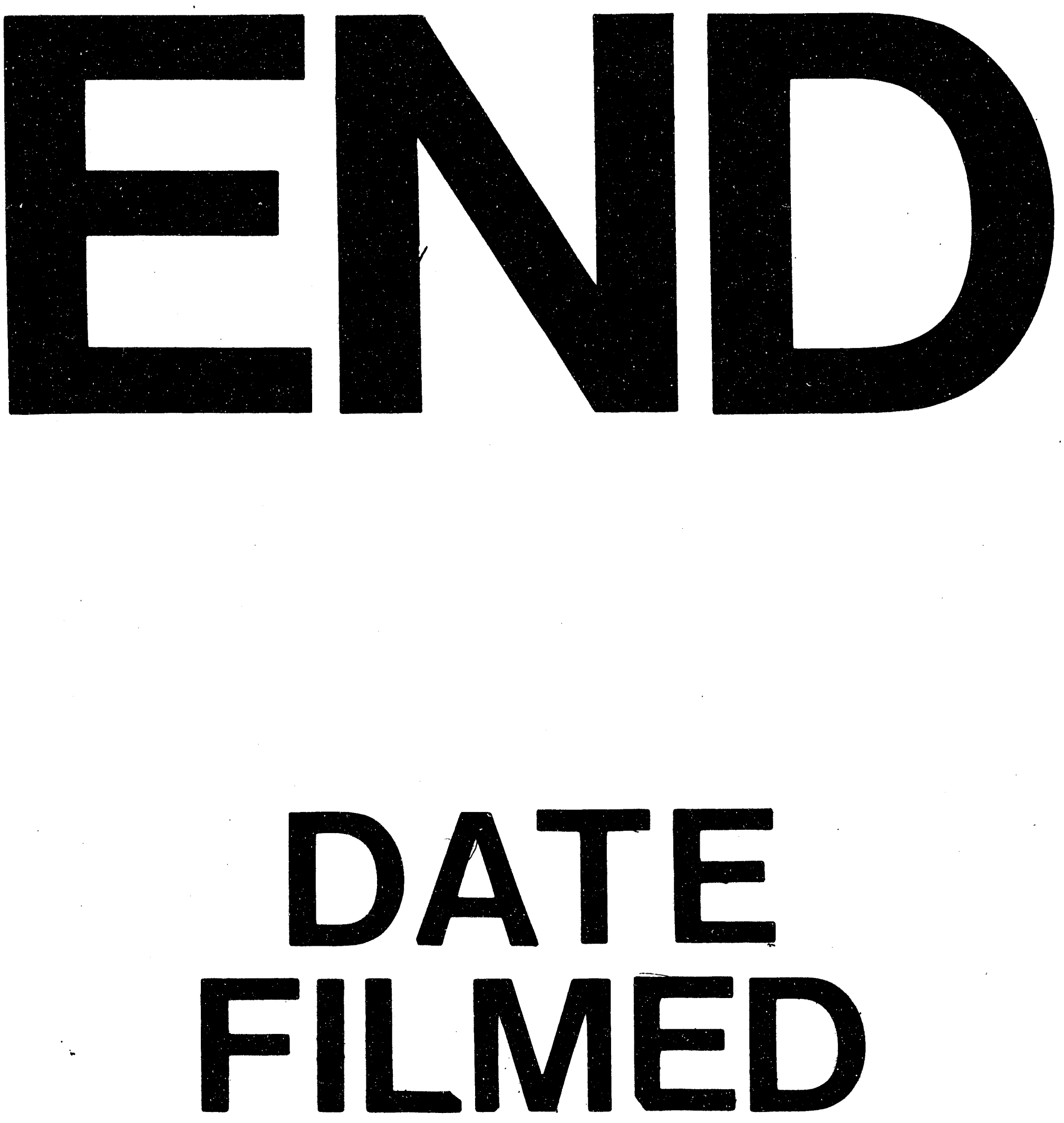

1

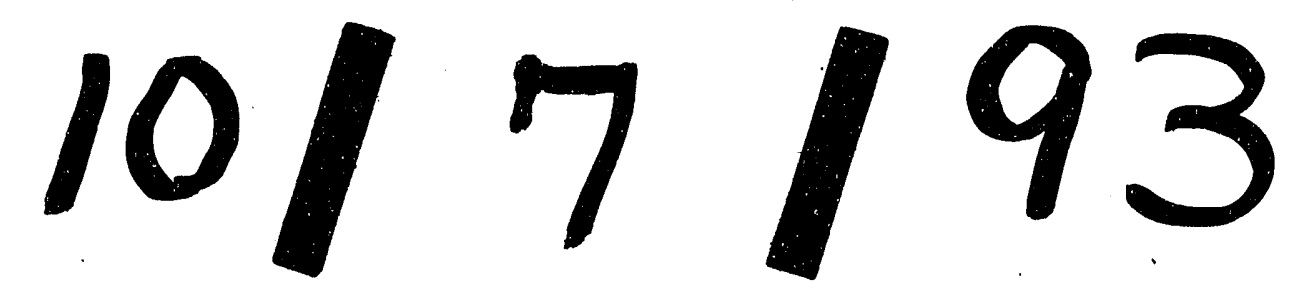


